\title{
A Fossil History of Southern African Land Mammals
}

\author{
D. Margaret Avery
}

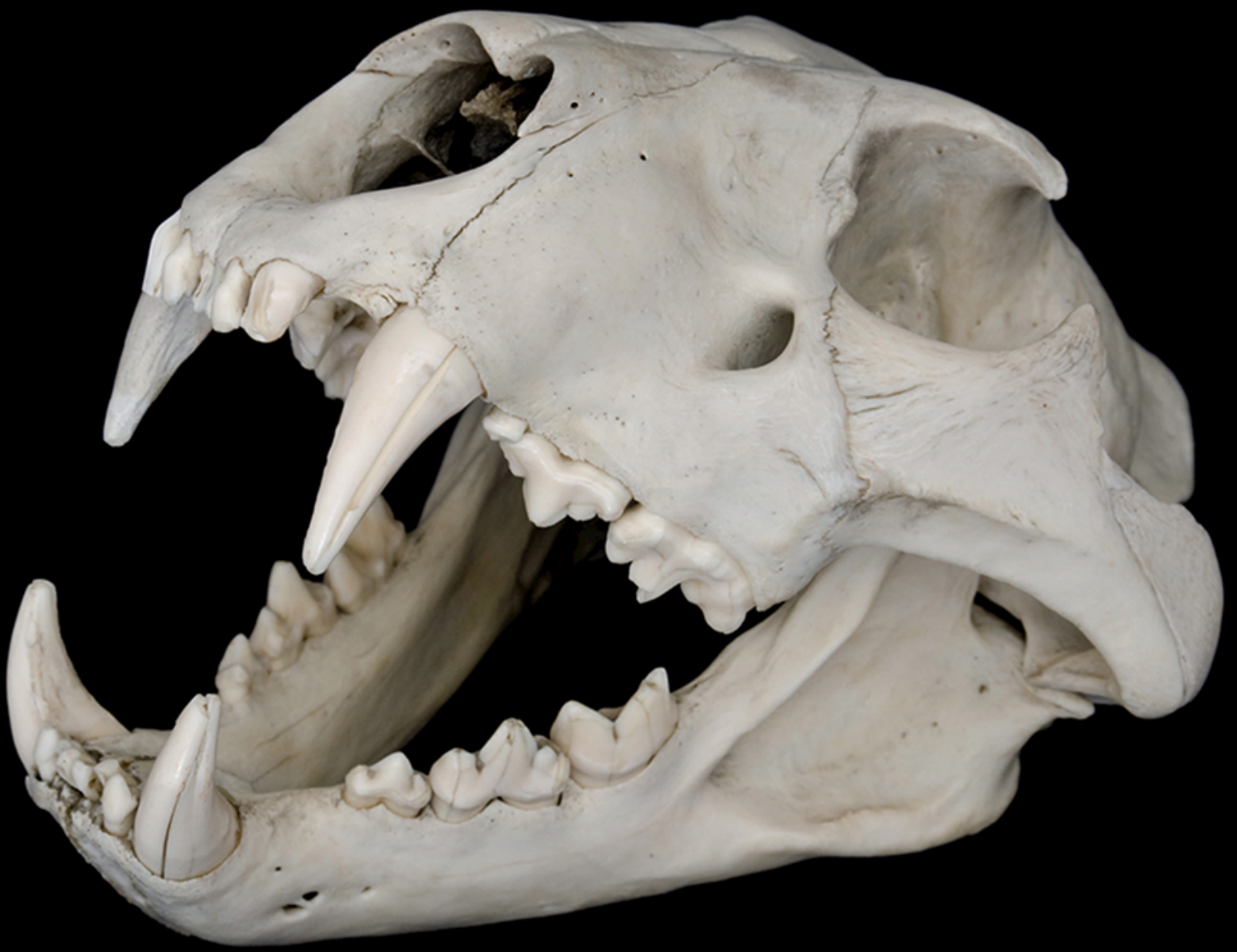




\section{A Fossil History of Southern African Land Mammals}

There is an ever-growing wealth of mammalian fossil material being collected from palaeontological and archaeological sites in southern Africa. This reference provides comprehensive information on the taxonomy and distribution in time and space of all currently recognised southern African fossil mammals. After an introductory background chapter on southern Africa, mammals, sites and dating, the following chapters are presented by epoch, covering the Eocene, Miocene, Pliocene, Pleistocene and Holocene. Individual maps provide information on where in the landscape specific taxa have been found, and a comprehensive index lists all the fauna and site locations. It ends with a chapter on how the book can be used, and lines of future research. Collecting a vast amount of information together in an accessible format, this is an essential reference for non-specialist taxonomists and palaeontologists, as well as for those using fossil data for other applications, such as archaeology, neontology and nature conservation. This title is also available as Open Access on Cambridge Core.

D. Margaret Avery is Emeritus Associate of Cenozoic Studies at Iziko Museums of South Africa, and Honorary Researcher at the Evolutionary Studies Institute at the University of Witwatersrand. Her research interests include understanding the background of human evolution, as well as modern micromammals. She was the President of the International Union for Quaternary Research (INQUA) between 2011 and 2015, and is a Fellow of the Royal Society of South Africa. 


\section{A Fossil History of Southern African Land Mammals}

D. MARGARET AVERY

Iziko Museums of South Africa 


\section{CAMBRIDGE UNIVERSITY PRESS}

University Printing House, Cambridge CB2 8BS, United Kingdom

One Liberty Plaza, 20th Floor, New York, NY 10006, USA

477 Williamstown Road, Port Melbourne, VIC 3207, Australia

314-321, 3rd Floor, Plot 3, Splendor Forum, Jasola District Centre, New Delhi - 110025, India

79 Anson Road, \#06-04/06, Singapore 079906

Cambridge University Press is part of the University of Cambridge.

It furthers the University's mission by disseminating knowledge in the pursuit of education, learning, and research at the highest international levels of excellence.

www.cambridge.org

Information on this title: www.cambridge.org/9781108480888

DOI: $10.1017 / 9781108647243$

(C) D. Margaret Avery 2019

This work is in copyright. It is subject to statutory exceptions and to the provisions of relevant licensing agreements; with the exception of the Creative Commons version the link for which is provided below, no reproduction of any part of this work may take place without the written permission of Cambridge University Press.

An online version of this work is published at doi.org/10.1017/9781108647243 under a Creative Commons Open Access licence CC-BY-NC-ND 4.0 which permits re-use, distribution and reproduction in any medium for non-commercial purposes providing appropriate credit to the original work is given. You may not distribute derivative works without permission. To view a copy of this licence, visit https://creativecommons.org/licenses/by-nc-nd/4.0

All versions of this work may contain content reproduced under license from third parties.

Permission to reproduce this third-party content must be obtained from these third-parties directly.

When citing this work, please include a reference to the DOI 10.1017/9781108647243

First published 2019

A catalogue record for this publication is available from the British Library.

Library of Congress Cataloging-in-Publication Data

Names: Avery, D. Margaret, author.

Title: A fossil history of southern African land mammals / D. Margaret Avery, Iziko Museums of South Africa.

Description: Cambridge, United Kingdom ; New York, NY : Cambridge University Press, [2019] | Includes bibliographical references and index.

Identifiers: LCCN 2018045348 | ISBN 9781108480888 (hardback)

Subjects: LCSH: Mammals, Fossil-Africa, Southern.

Classification: LCC QE881 .A94 2019 | DDC 569.0968-dc23

LC record available at https://lccn.loc.gov/2018045348

ISBN 978-1-108-48088-8 Hardback

Cambridge University Press has no responsibility for the persistence or accuracy of URLs for external or third-party internet websites referred to in this publication and does not guarantee that any content on such websites is, or will remain, accurate or appropriate. 
To all those whose hard work made this book necessary, and in memory of my friend,

H. Basil S. Cooke 


\section{Contents}

Acknowledgements

page viii

1. Background

1.1 History and Rationale

1.2 Southern Africa

1.3 Mammals

1.4 Sites

1.5 Mapping

1.6 Dating

2. The Eocene

2.1 Eocene Mammals

2.2 Eocene Sites

3. The Miocene

3.1 Miocene Mammals

3.2 Miocene Sites

4. The Pliocene

4.1 Pliocene Mammals

4.2 Pliocene Sites

\section{The Pleistocene}

5.1 Pleistocene Mammals

5.2 Pleistocene Sites

6. The Holocene

6.1 Holocene Mammals

6.2 Holocene Sites

7. Present and Future

7.1 The Current Situation

7.2 The Way Forward 


\section{Acknowledgements}

Thanks go to the authors and librarians who responded to requests for reprints and information, especially Martin Pickford, Sorbonne Universités, who answered numerous queries. Tribute must also be paid to various indispensable online archives, particularly the Biodiversity Heritage Library (www.biodiversitylibrary.org) and Gallica (http://gallica.bnf.fr). The University of Edinburgh provided access to JSTOR through its Alumni Access Program. Richard G. Klein, Stanford University, kindly reviewed an early draft and drew my attention to several important references. Andries van Aarde, AOSIS, made excellent suggestions for improving the text. Open Access publication was made possible through grants from the University of the Witwatersrand Centre of Excellence for Palaeosciences and the Palaeontological Scientific Trust (PAST), both in Johannesburg. 


\section{CHAPTER 1}

\section{Background}

\subsection{HISTORY AND RATIONALE}

Mammalian palaeontological and archaeological work in southern African has a history reaching back nearly two centuries, though this initially took the form of what Underhill (2011) calls a 'Victorian penchant [for] the recognition and collection of artefacts'. Probably the first published report of a fossil from South Africa was made in 1839 (Bain 1839; Seeley 1891), while T. H. Bowker excavated artefacts from near the mouth of the Fish River in 1857, and a Palaeolithic stone implement from Cape Town was sent to England in 1866 (Malan 1970). It was not until early in the twentieth century that palaeontological work began in earnest (e.g. Broom 1909a, 1909b and Péringuey 1911) published seminal works on the Stone Ages of South Africa. What might be described as the modern era began some 20 years later. South African mammalian palaeontology received a major boost with the report of Australopithecus africanus from Taung (Dart 1925), and by the second half of the 1930s Broom (e.g. 1936a, 1936b, 1937a, 1937b) had begun to describe material from the extremely rich limestone caves of the then Transvaal (now Gauteng), which continue to yield new forms (e.g. Berger et al. 2015; Fourvel 2018). The palaeontological importance of Namibia (then South West Africa) only began to be appreciated during the 1920s (e.g. Stromer 1921), but the importance of this country for Eocene and Miocene mammalian evolution has since become abundantly clear through the ongoing work of Martin Pickford and colleagues, especially Brigitte Senut, Jorge Morales and Pierre Mein. The systematic study of southern African archaeology has a similar history, beginning with the work of Goodwin and van Riet Lowe (e.g. 1929). However, archaeologists were not at first concerned with the faunal element, apart from human remains, which received attention from very early on (e.g. Shrubsall 1911). One of the earliest general faunal reports was that of Brain (1969) on material from Bushman Rock Shelter. From these beginnings there has developed exponentially a body of information on around 650 taxa collected from over 600 sites in more than 150 degree-squares dating from the Eocene onwards.

With such an ever-growing wealth of data it has become increasingly difficult, especially for non-specialists, to find information on specific taxa. Unannotated maps of large mammal distributions during the last 30000 years have been published (Plug and Badenhorst 2001), but these required updating and their coverage expanding. In particular, the taxonomy of all included genera and species needed attention. The taxonomic data are often hard to locate, with incomplete citations to original descriptions and older publications difficult to find. Moreover, some groups have been updated and it is not always easy for non-specialists to track taxa through to their current nomenclature. To help solve these problems it seemed that the time was right to bring 
together available taxonomic and distributional information on terrestrial mammalian species or genera recorded from palaeontological and archaeological sites in mainland southern Africa.

This compilation is also intended to complement others covering modern distributions (e.g. Skinner and Chimimba 2005) and historical records (e.g. Boshoff et al. 2016; Rookmaker 1989; Skead 2011) and, in so doing, extend knowledge of the distribution of many extant taxa into the past. Although there is some temporal overlap between the historical records and the late Holocene (Iron Age) archaeological records, a distinction can be made according to the source of the records. Thus, historical records are written reports of sightings of live or recently dead animals, mainly by early European travellers. Palaeontological and archaeological records, on the other hand, relate solely to excavated fossil and sub-fossil material. It is information from the latter sources that has been included here. Apart from any other consideration, it is hoped that making the historical evidence available will encourage neontologists to incorporate these data into their studies more regularly. Conservationists will also find it a useful source when they decide whether it is appropriate to re-introduce extirpated species onto a reserve.

\subsection{SOUTHERN AFRICA}

Mainland southern Africa is usually thought of in terms of countries, which, in this case, would include Botswana, Lesotho, Mozambique (southern half), Namibia, South Africa, Swaziland and Zimbabwe. However, political boundaries have been disregarded here because they have no biological significance. Instead, for the purposes of this study, southern Africa is taken to comprise mainland Africa south of the Kunene (Cunene) and Zambezi Rivers, that is, south of approximately $15^{\circ} \mathrm{S}$ (Figure 1.1). Among the various delimitations of southern Africa, this one was chosen because the significant barrier formed by these rivers is likely to have greatly influenced animal movements at various times during the period covered. For those wishing to know more about the major factors affecting mammalian distribution patterns, the geomorphological setting is provided by Partridge (2010), while Feakins and DeMenocal (2010) and Jacobs et al. (2010) describe the other major, and interlinked, influencing factors of climate and vegetation respectively.

\subsection{MAMMALS}

The full taxonomy of animals has a great many levels, as can be seen in The Taxonomicon (http://taxonomicon.taxonomy.nl). However, it is usual (and conventional) for general purposes to disregard ranks higher than Class Mammalia when considering the mammals. Below this level one may include as many or as few ranks as required. Here, only the supra-generic ranks of Order and Family (with suborder and subfamily where these are recognised) are given, both because many workers do not include a complete taxonomy and because further levels are not normally necessary in non-taxonomic works. Only taxa identified to species or genus in the palaeontological and archaeological samples are included. Details for the lowest level of identification are given in the text so that genera are only listed separately when material was not identified to species level. Identifications above the generic level have been disregarded pending more precise identification. Thus, although an author may have listed the family Canidae (dogs), for 


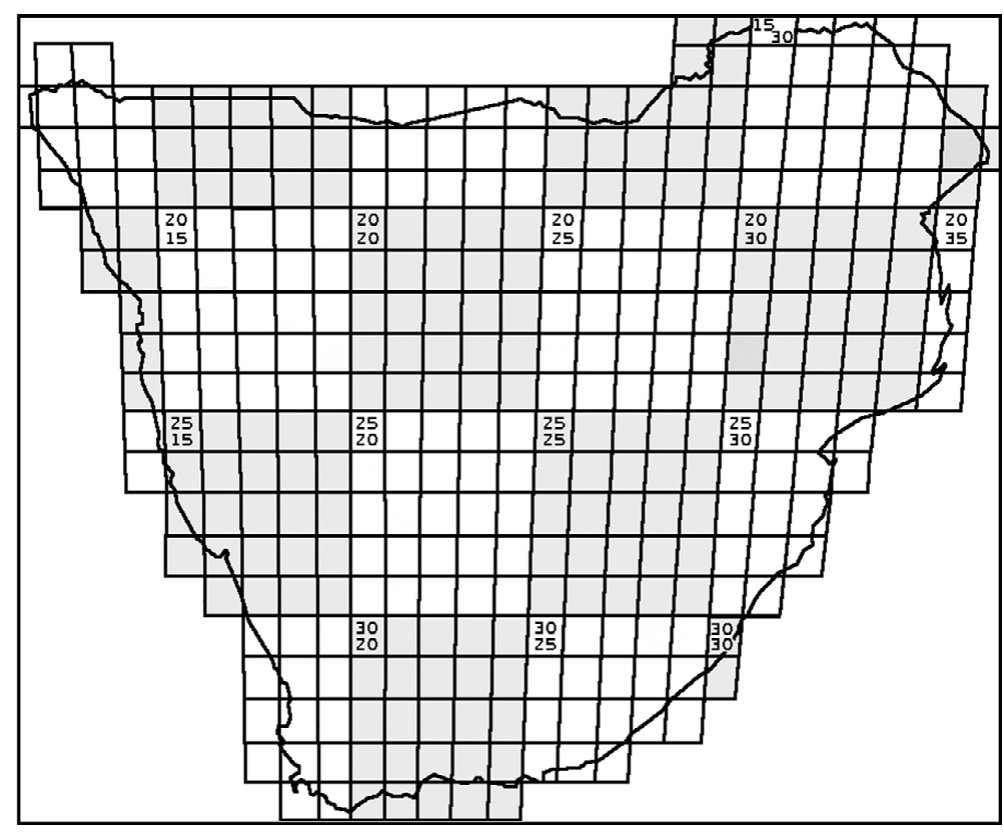

Figure 1.1 Southern Africa base map marked in degree squares and shaded in five-degree squares. Numbers in the top left-hand corner of each five-degree block indicate degree latitude above and longitude below of the degree square in that corner. As an example, 2015 indicates the square is $20^{\circ} \mathrm{S} 15^{\circ} \mathrm{E}$.

example, this will not appear as a separate record. Extinct taxa are identified by the conventional symbol $\uparrow$. Author and citation details are given for genera and species but not higher taxa. Common names, which are included for extant species, follow Wilson and Reeder (2005). Listed synonyms comprise those given in the literature from which data were collected for this study and are intended as an aid to updating and correlating faunal lists, not as full taxonomic synonymies. The database relies on published records of taxa so far represented in the fossil record, that is, material excavated or otherwise recovered from palaeontological and archaeological sites. Unless there is reason for doubt, in which case a comment is included under the taxon description, published identifications have been accepted as accurate. Unpublished data, apart from personal records, have been omitted, partly because not all are in the public domain.

The order of presentation is based on Wilson and Reeder (2005), augmented as necessary for extinct and newly erected taxa. General works consulted include Allen (1939), Bronner et al. (2003), Ellerman et al. (1953), McKenna and Bell (1997), Roberts (1951) and Werdelin and Sanders (2010). The latter generally provide the most recent treatments of modern and fossil mammalian taxa in Africa, and these have been followed except in cases where subsequent changes have been published. Volumes of certain journals, such as the Proceedings of the Zoological Society of London, were originally numbered by year, although the year of publication was the following year. On occasion, this has caused some confusion in the citation, but it would appear that the volume year, rather than the publication year, is now generally accepted for this purpose. 


\subsection{SITES}

There is an exponential increase in the number of samples from the earliest to the latest epoch, despite the fact that the epochs are of decreasing duration through time (Table 1.1). Some sites have produced material dated to more than one epoch so that the total number of sites is slightly lower than that of the samples. Location of the sites is based on published information in most cases. Although precision of coordinates varies, it is generally adequate for the construction of the distribution maps. In all cases latitude is given before longitude. Thus, as an example, $2925: 2228$ is $29^{\circ} 25^{\prime} \mathrm{S} ; 22^{\circ} 28^{\prime} \mathrm{E}$. When more precise coordinates are unavailable, $3218 \mathrm{BB}$ or 3218 , for example, indicate the quarter degree or degree square in which a site is located. In some cases, several different latitudes and longitudes have been found for one site, but these are generally within a few seconds of each other. Primary sources should be consulted.

Table 1.1 Boundaries of Cenozoic epochs from the Eocene upwards, according to Cohen et al. (2013, updated), and number of sites providing data in each epoch. The Oligocene is not yet definitively represented in southern Africa.

\begin{tabular}{|l|l|l|}
\hline Epoch & Date (Ma) & \multicolumn{2}{l|}{ No. sites } \\
\hline Holocene & $<0.0117$ & 417 \\
\hline Pleistocene & $0.0117-2.58$ & 204 \\
\hline Pliocene & $2.58-5.333$ & 23 \\
\hline Miocene & $5.333-23.03$ & 25 \\
\hline Oligocene & $23.03-33.9$ & $1 ?$ \\
\hline Eocene & $33.9-56.0$ & 5 \\
\hline
\end{tabular}

\subsection{MAPPING}

For the purposes of mapping taxa, distribution is recorded at the level of one-degree squares, that is, one degree of latitude by one degree of longitude. This has been done even where more precise localities are recorded because of the size of the database and the area covered. There are 350 degree-squares in the region (Figure 1.1), and excavations in 153 of these have so far yielded remains of mammals. Only type localities for fossil genera and species occurring within southern Africa are listed, and these are shown in black on the distribution maps. The faunal lists for each site are those given in the references cited, except where they have been updated as necessary (see text for more information).

\subsection{DATING}

Sites have been dated to various levels of precision from a few decades in later sites to epochs in earlier sites. Epochs are intermediate-level groupings in the International Chronostratigraphic Chart (Cohen et al. 2013, updated). This level is convenient for present mapping purposes even where greater dating precision for individual sites is available. The period spanned by epochs is progressively shorter towards the present 
(Table 1.1). So far, the Oligocene, which lies between the Eocene and the Miocene at 33.9-23.03 Ma, is not yet conclusively represented. If, however, Sallam and Seiffert (2016) are correct in proposing that Protophiomys algeriensis is of Oligocene rather than Eocene age, it would be the first material of this age recovered from southern Africa.

In most cases there is no problem with assigning a site to a particular epoch. However, confusion can arise over assignment of some sites, notably in the Cradle of Humankind, that have been labelled informally as Plio-Pleistocene. This practice, which arose at a time when the boundary between the two epochs was placed at $1.80 \mathrm{Ma}$, has been continued by some palaeoanthropologists even though it is no longer useful in view of the revised boundary of 2.58 Ma (Cohen et al. 2013, updated), which allows almost all the Cradle sites to be placed within the Pleistocene. 


\section{CHAPTER 2}

\section{The Eocene}

\subsection{EOCENE MAMMALS}

The Eocene mammalian fauna is notable for its high proportion of extinct forms.

Of the eight Orders represented, Cimolesta, Embrithopoda and Creodonta are extinct, as are all of the genera and species and most of the families. Many new species were recently described from the region (Pickford 2015a-2015f, 2018a), thereby adding significantly to the known fauna of the epoch. Despite this, the diversity of taxa known from each Order is much lower than is the case with younger material. Whether this results from the paucity of sites (see below) remains to be seen. The Order Rodentia (rodents) is by far the most diverse, with eight families. Only Afrosoricida (tenrecs and golden moles) and Hyracoidea (hyraxes) have more than one, and, at three each, this is the most diverse at the family level they have been in the region. A fruit bat possibly belonging to the subfamily Propottininae has been described from Black Crow (Pickford 2018b) but not assigned to genus or species. It is significant in that it is the only member of the suborder Megachiroptera so far recovered from the region, although it cannot be included in the lists until a full identification has been published.

\section{ORDER: †CIMOLESTA}

FAMILY: †TODRALESTIDAE

†Namalestes gheerbranti Pickford, Senut, Morales, Mein and Sanchez, 2008. Geol. Surv. Namibia Mem. 20: 468.

Type locality: Black Crow.

\section{ORDER: AFROSORICIDA Suborder: Tenrecomorpha FAMILY: POTAMOGALIDAE}

†Namagale grandis Pickford, 2015. Comm. Geol. Surv. Namibia 16: 119. Type locality: Eocliff.

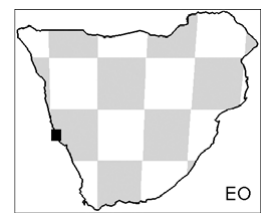


FAMILY: TENRECIDAE

†Arenagale calcareus Pickford, 2015. Comm. Geol. Surv. Namibia 16: 140. Type locality: Eocliff.

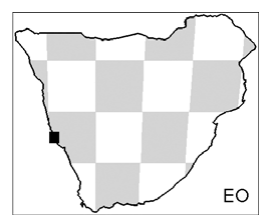

†Sperrgale minutus Pickford, 2015. Comm. Geol. Surv. Namibia 16: 130. Type locality: Eocliff.

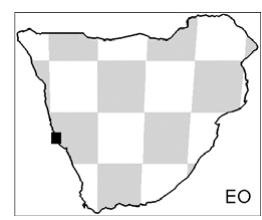

Suborder: Chrysochloridea

FAMILY: CHRYSOCHLORIDAE

†Diamantochloris inconcessus Pickford, 2015. Comm. Geol. Surv. Namibia 16: 109.

Type locality: Black Crow.

†Namachloris arenatans Pickford, 2015. Comm. Geol. Surv. Namibia 16: 148.

Type locality: Eocliff.
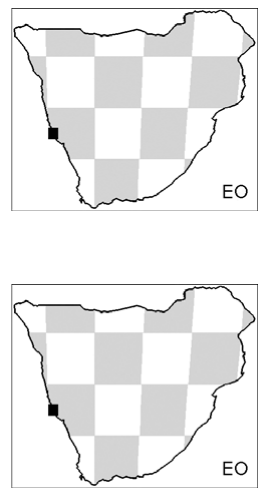

ORDER: MACROSCELIDEA

FAMILY: MACROSCELIDIDAE

Subfamily: †Myohyracinae

†Myohyrax Andrews, 1914. Quart. J. Geol. Soc. Lond. 70: 171.

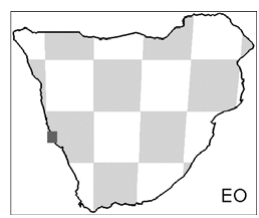

ORDER: †EMBRITHOPODA

FAMILY: †ARSINOITHERIIDAE

†Namatherium blackcrowense Pickford, Senut, Morales, Mein and

Sanchez, 2008. Geol. Surv. Namibia Mem. 20: 479.

Type locality: Black Crow.

Additional references: Gheerbrandt et al. (2018); Sanders et al. (2010b).

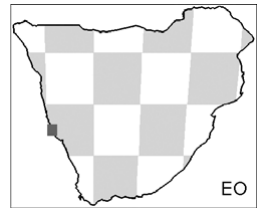




\section{ORDER: HYRACOIDEA}

FAMILY: †GENIOHYIDAE

†Namahyrax corvus Pickford, Senut, Morales, Mein and Sanchez, 2008. Geol. Surv. Namibia Mem. 20: 474.

Type locality: Black Crow.

Comments: this taxon was originally assigned to Namahyracidae but is now placed in Geniohyidae by Pickford (2018c).

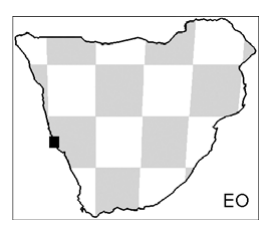

Additional references: Pickford (2015e).

\section{FAMILY: PROCAVIIDAE}

†Rupestrohyrax palustris Pickford, 2015. Comm. Geol. Surv. Namibia 16: 206.

Type locality: Eoridge.

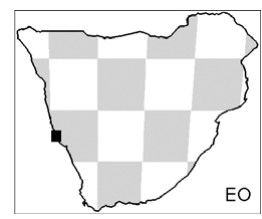

\section{ORDER: PRIMATES}

†Notnamaia bogenfelsi Pickford, Senut, Morales, Mein and Sanchez, 2008. Geol. Surv. Namibia Mem. 20: 487.

Type locality: Black Crow.

Synonyms: Namaia.

Additional references: Pickford and Uhen (2014).

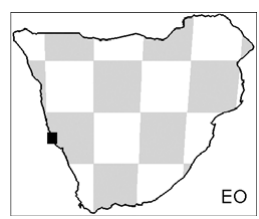

\section{Suborder: Strepsirrhini}

FAMILY: LORISIDAE

†Namaloris rupestris Pickford, 2015. Comm. Geol. Surv. Namibia 16: 196. Type locality: Eocliff.

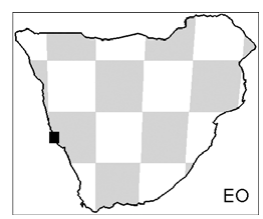

ORDER: RODENTIA

FAMILY: †PARAMYIDAE

Subfamily: †Reithroparamyinae

†Namaparamys inexpectatus Mein and Pickford, 2018. Comm. Geol.

Surv. Namibia 18: 40, 41.

Type locality: Black Crow.

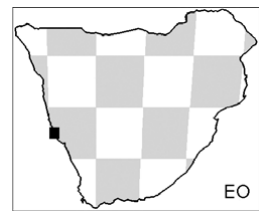

\section{Suborder: Sciuravida}

FAMILY: †CHAPATTIMYIDAE

Subfamily: †Protophiomyinae

†Protophiomys algeriensis Jaeger, Denys and Coiffait, 1985. In: Luckett and Hartenberger, Evolutionary Relationships Among Rodents: 569.

Comments: these Protophiomys specimens may be Oligocene (Sallam and Seiffert 2016) or even Miocene in age (Marivaux et al. 2014).

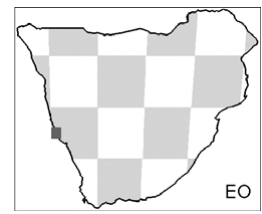




\section{Suborder: Anomaluromorpha \\ FAMILY: †ZEGDOUMYIDAE}

†Clibia namibiensis Pickford, Senut, Morales, Mein and Sanchez, 2008.

Geol. Surv. Namibia Mem. 20: 488.

Type locality: Black Crow.

Comments: this species is considered to belong to the genus Zegdoumys

by Marivaux et al. $(2011,2015)$.

†Tsaukhaebmys calcareus Pickford, 2018. Comm. Geol. Surv. Namibia

18: 50, 51.

Type locality: Black Crow.

\section{Suborder: Hystricomorpha}

FAMILY: †DIAMANTOMYIDAE

Subfamily: $†$ Metaphiomyinae

†Metaphiomys schaubi Wood, 1968. Bull. Peabody Mus. Nat. Hist. 28:

58.

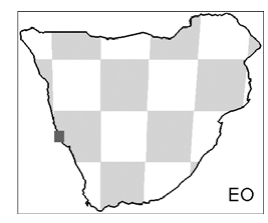

†Prepomonomys bogenfelsi Pickford, Senut, Morales, Mein and Sanchez,

2008. Geol. Surv. Namibia Mem. 20: 490.

Type locality: Silica North.

FAMILY: †BATHYERGOIDIDAE

†Bathyergoides Stromer, 1923. Sitz. Math.-Physik. Klasse Bayer. Akad.

Wiss. München 1923(II): 263.

Type locality: Sperrgebiet.
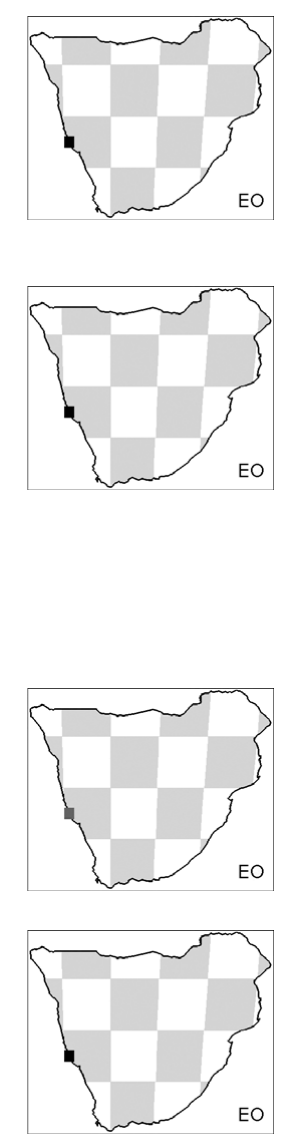

EO

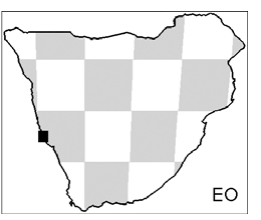

FAMILY: †MYOPHIOMYIDAE

Subfamily: †Phiocricetomyinae

†Silicamys cingulatus Pickford, Senut, Morales, Mein and Sanchez, 2008.

Geol. Surv. Namibia Mem. 20: 489.

Type locality: Silica North.

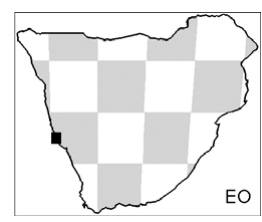

†Talahphiomys lavocati Wood, 1968. Bull. Peabody Mus. Nat.

Hist. 20: 45.

Synonyms: Phiomys.

Additional references: Jaeger et al. (2010).

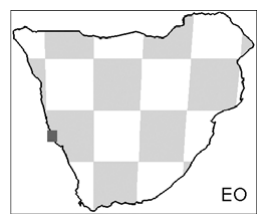




\section{FAMILY: THRYONOMYIDAE}

†Apodecter stromeri Hopwood, 1929. Amer. Mus. Novit. 344: 3.

Type locality: Lüderitz Bay (south of) (?Langental: Mein and Pickford [2008c]).

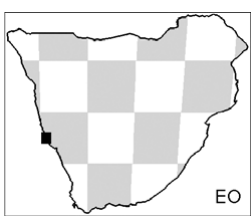

†Gaudeamus Wood, 1968. Bull. Peabody Mus. Nat. Hist. 20: 68.

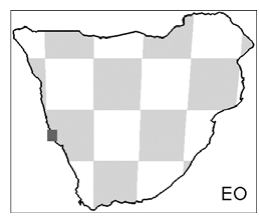

†Namaphiomys Mein and Pickford, unpublished.

Comments: Namaphiomys is a nomen nudum, awaiting publication, according to M. Pickford (pers. comm. 2016).

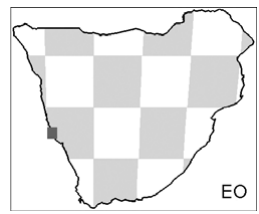

†Phiomys phiomyoides Wood, 1968. Bull. Peabody Mus. Nat. Hist. 20: 41.

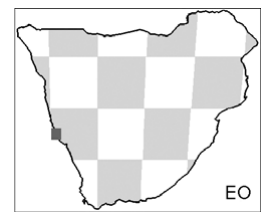

FAMILY: †TUFAMYIDAE

Comments: Pickford (2018f) places this family within the Infraorder Hystricognathi (Suborder Ctenohystrica according to Huchon et al. [2000, 2002]) but Hystricognathi is included in the Suborder Hystricomorpha by Wilson and Reeder (2005), whose arrangement is followed here.

†Efeldomys Mein and Pickford 2008. Geol. Surv. Namibia Mem. 20: 257. Type locality: Elisabethfeld.

Additional references: Pickford (2018f).

Comments: this genus was originally placed in Bathyergidae but is now transferred to Tufamyidae (Pickford, 2018f).

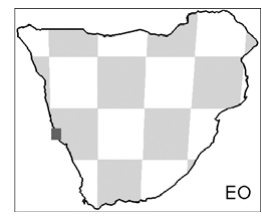

†Tufamys woodi Pickford, 2018. Comm. Geol. Surv. Namibia 19: 75. Type locality: Eocliff.

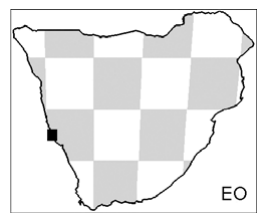

\section{ORDER: †CREODONTA}

FAMILY: †HYAENODONTIDAE

Subfamily: †Hyainailourinae

†Pterodon De Blainville, 1839. Ann. Franç. Etran. Anat. Physiol. 3: 23. Additional references: Holroyd (1999); Lewis and Morlo (2010).

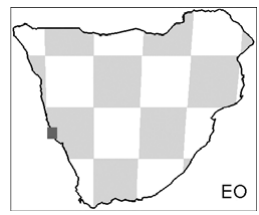




\section{ORDER: ARTIODACTYLA}

\section{FAMILY: †ANTHRACOTHERIIDAE}

†Bothriogenys gorringei Andrews and Beadnell, 1902. A preliminary note on some new mammals from the Upper Eocene of Egypt: 7.

Additional references: Holroyd et al. (2010); Lihoreau and Ducroca (2007); Pickford (2015f).

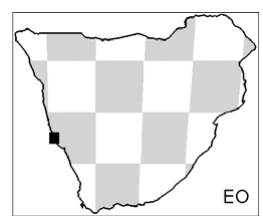

\subsection{EOCENE SITES}

Eocene sites (Figure 2.1) so far discovered in southern Africa have a very restricted distribution, all four of them being within the 2715-degree square. These earliest sites are also the most recent to have been discovered in the region.

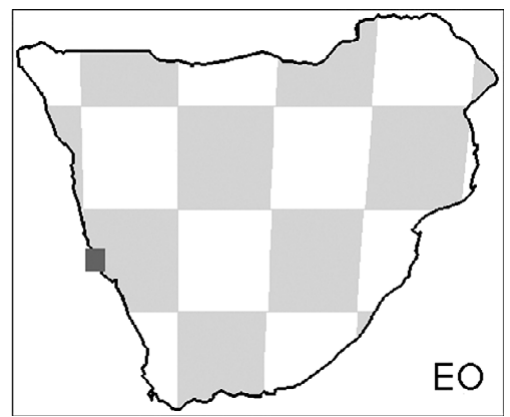

Figure 2.1 Location of Eocene sites.

Black Crow (2723:1528). Taxa: Diamantochloris inconcessus; Glibia namibiensis; Namahyrax corvus; Namalestes gheerbranti; Namaparamys inexpectatus; Namatherium blackcrowense; Notnamaia bogenfelsi; Pterodon; Tsaukhaebmys calcareus. References: Pickford (2015a, 2018a-2018d); Pickford et al. (2008, 2014).

Eocliff (2721:1536). Taxa: Arenagale calcareus; Efeldomys; Gaudeamus; Metaphiomys schaubi cf.; Myohyrax aff.; Namachloris arenatans; Namagale grandis; Namaloris rupestris; Namaphiomys; Phiomys lavocati aff.; Phiomys phiomyoides aff.; Prepomonomys bogenfelsi; Protophiomys algeriensis cf.; Silicamys cingulatus; Sperrgale minutus; Talahphiomys; Tufamys woodi. References: Pickford (2015b, 2015c); Pickford et al. (2008, 2014).

Eoridge (2721:1537). Taxa: Bothriogenys gorringei; Rupestrohyrax palustris; Silicamys cingulatus; Sperrgale minutus. References: Pickford (2015e, 2015f); Pickford et al. (2014).

Silica North and South $(2715: 1525 ; 2716: 1525)$. Taxa: Apodecter stromeri cf.; Bathyergoides cf.; Prepomonomys bogenfelsi; Protophiomys algeriensis cf.; Silicamys cingulatus;

Talahphiomys. References: Pickford et al. (2008, 2014). 


\section{CHAPTER 3}

\section{The Miocene}

\subsection{MIOCENE MAMMALS}

During the Miocene only one extinct Order, Creodonta, is represented in southern Africa, but the total number of Orders rose to 14, with only the Soricomorpha (shrews) and Pholidota (pangolins) not present. This epoch is notable for having the largest number of families, of which slightly more than a third are extinct (Table 1.1), and for the diversity of elephants (Proboscidea), of which there are nine genera and eight species in four families. The Rodentia continued to be the most diverse Order, with 36 genera and 41 species in 12 families. Next most diverse at the family level, with six families each, are the Chiroptera (bats), Carnivora (carnivores) and Artiodactyla (even-toed ungulates), the first two of which appeared for the first time.

\section{ORDER: AFROSORICIDA \\ Suborder: Tenrecomorpha \\ FAMILY: TENRECIDAE}

†Promicrogale namibiensis Pickford, 2018. Comm. Geol. Surv. Namibia

18: $88,89$.

Type locality: Elisabethfeld.

Comments: Pickford (2018d) places the Tenrecidae in Order Soricomorpha, but points out (personal communication, 2018) that there is considerable disagreement on the allocation of this family. For now, Wilson and

Reeder (2005) are followed in placing Tenrecidae in Afrosoricida.

\section{Subfamily: †Protenrecinae}

†Protenrec butleri Mein and Pickford, 2003. Mem. Geol. Soc. Namibia 19: 145.

Type locality: Arrisdrift.

Additional references: Butler and Hopwood, (1957); Mein and Pickford (2008a).
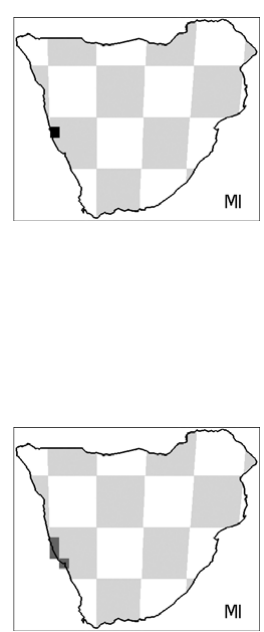

Suborder: Chrysochloridea

FAMILY: CHRYSOCHLORIDAE

Subfamily: †Prochrysochlorinae

†Prochrysochloris miocaenicus Butler and Hopwood, 1957. Foss. Mamm. Afr. 13: 11.

Additional references: Butler (1984); Mein and Pickford (2008a).

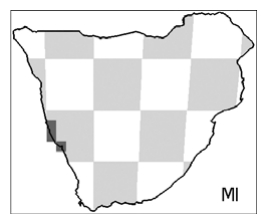




\section{ORDER: MACROSCELIDEA}

FAMILY: MACROSCELIDIDAE

Subfamily: Macroscelidinae

Elephantulus Thomas and Schwann, 1906. Abstr. Proc. Zool. Soc. Lond. 33: 10.

Additional references: Corbet and Hanks (1968); Evans (1942); Holroyd (2010a); Patterson (1965); Van der Horst (1944).

†Miorhynchocyon gariepensis Senut, 2003. Geol. Soc. Namibia Mem. 19: 126.

Type locality: Arrisdrift.

†Palaeothentoides Stromer, 1931. Sitzungsber. Bayer. Akad. Wiss.

München. Math.-Nat. Abt. 1931: 185.

Type locality: Kleinzee.

Additional references: Holroyd (2010a); Patterson (1965).

Subfamily: †Myohyracinae

†Myohyrax oswaldi Andrews, 1914. Quart. J. Geol. Soc. Lond. 70: 171. Synonyms: doederleini; osborni.

Additional references: Holroyd (2010a); Leakey (1943a); Patterson

(1965); Stromer (1923).

†Myohyrax pickfordi Senut, 2008. Geol. Surv. Namibia Mem. 20: 189. Type locality: Langental.
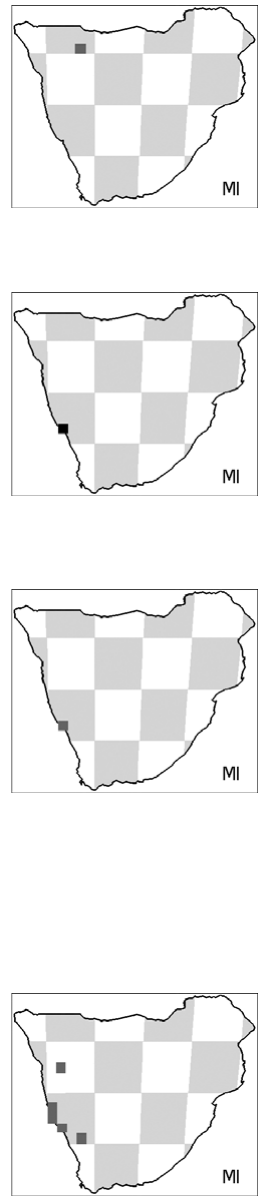

MI

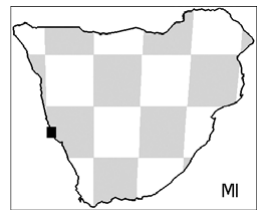

†Protypotheroides beetzi Stromer, 1921. Sitz. Math.-Physik. Klasse Bayer. Akad. Wiss. München 1921: 333.

Synonyms: osborni.

Type locality: Langental.

Additional references: Holroyd (2010a); Patterson (1965); Stromer

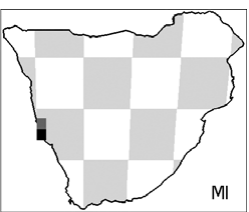
(1923).

Comments: Stromer (1923: 258) refers to this taxon as Protypotheroides beetzi Stromer (1922, S. 333), but see note in Section 1.3 regarding journal volume numbers. 
Subfamily: Rhinchocyoninae

†Brevirhynchocyon gariepensis Senut, 2003. Geol. Surv. Namibia Mem.

19: 126.

Synonyms: Brachyrhynchocyon, Miorhynchocyon.

Type locality: Arrisdrift.

Additional references: Butler (1984); Senut and Georgalis (2014).

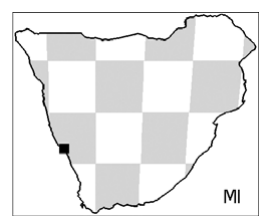

†Brevirhynchocyon jacobi Senut, 2008. Geol. Surv. Namibia Mem.

20: 192.

Synonyms: Brachyrhynchocyon.

Type locality: Elisabethfeld.

Additional references: Senut and Georgalis (2014).

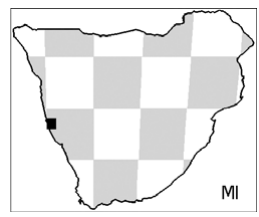

†Hypsorhynchocyon burrelli Senut, 2008. Geol. Surv. Namibia Mem. 20: 194.

Type locality: Grillental 6.

\section{ORDER: TUBULIDENTATA}

\section{FAMILY: ORYCTOPODIDAE}

†Amphiorycteropus Lehmann, 2009. Zool. J. Linn. Soc. 145: 665.

Synonyms: Myorycteropus minutus; Orycteropus.

Additional references: Holroyd (2010b); Pickford (1975, 1996b, 2003a).

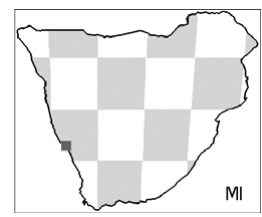

Orycteropus Cuvier, 1798. Tabl. Elem. Hist. Nat. Anim. 1798: 144. Additional references: Holroyd (2010b); Kitching (1963); Lehmann

(2007); Pickford (2008a).

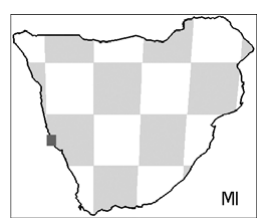

\section{ORDER: HYRACOIDEA}

FAMILY: †PLIOHYRACIDAE

†Parapliohyrax Lavocat, 1961. Notes Mém. Serv. Géol. Maroc 155: 87. Additional references: Pickford (1996a).

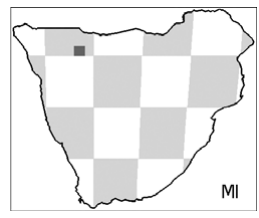

†Parapliohyrax ngororaensis Pickford and Fischer, 1987. N. Jhb. Geol. Palaeontol. Abh. 175: 212.

Additional references: Pickford (2003b); Rasmussen and Gutiérrez (2010).

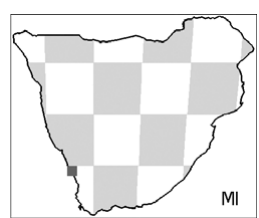


†Prohyrax hendeyi Pickford, 1994. Geol. Surv. Namibia Comm. 9: 45. Type locality: Arrisdrift.

Additional references: Pickford (2003b); Rasmussen and Gutiérrez

(2010).

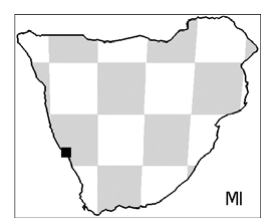

†Prohyrax tertiarius Stromer, 1923. Sitz. Math.-Physik. Klasse Bayer. Akad.

Wiss. München 1923(II): 256.

Type locality: Langental.

Additional references: Rasmussen and Gutiérrez (2010).

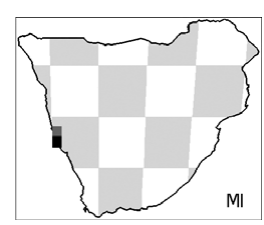

\section{FAMILY: PROCAVIIDAE}

†Heterohyrax auricampensis Rasmussen, Pickford, Mein, Senut and Conroy, 1996. J. Mammal. 77(3): 746.

Type locality: Berg Aukas 1.

Additional references: Rasmussen and Gutiérrez (2010).

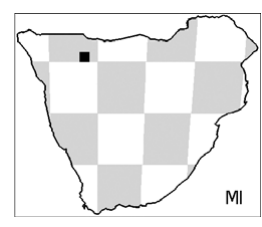

FAMILY: †TITANOHYRACIDAE

†Afrohyrax namibiensis Pickford, 2008. Mem. Geol. Surv. Namibia 20: 314.

Type locality: Grillental 6.

Additional references: Pickford and Senut (2018).

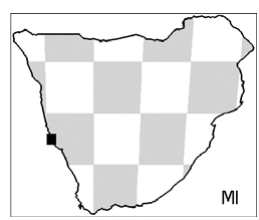

ORDER: PROBOSCIDEA

FAMILY: †DEINOTHERIIDAE

Subfamily: †Deinotheriinae

†Prodeinotherium hobleyi Andrews, 1911. Proc. Zool. Soc. Lond. 1911:

944.

Synonyms: Deinotherium; Dinotherium.

Additional references: Éhik (1930); Harris (1977); Sanders et al. (2010a).

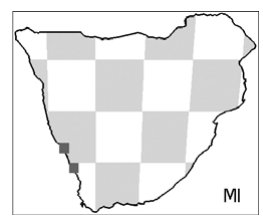

FAMILY: ELEPHANTIDAE

Subfamily: Elephantinae

Loxodonta Anonymous, 1827. Zool. J. 3: 140.

Synonyms: Tetralophodon?.

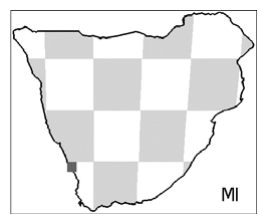

†Loxodonta cookei Sanders, 2007. Trans. R. Soc. S. Afr. 62(1): 7.

Synonyms: Lukeino Stage.

Type locality: Langebaanweg.

Additional references: Pickford and Senut (1997); Sanders et al. (2010a);

Todd (2010).

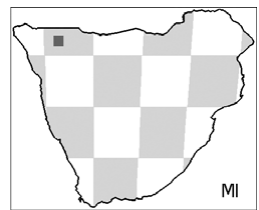




\section{FAMILY: †GOMPHOTHERIIDAE}

Subfamily: †Amebelodontinae

†Afromastodon coppensi Pickford, 2003. Geol. Surv. Namibia Mem. 19:

219.

Type locality: Arrisdrift.

Additional references: Sanders et al. (2010a).

†Progomphotherium maraisi Pickford, 2003c. Geol. Surv. Namibia Mem.

19: 211.

Type locality: Auchas.

Additional references: Osborn (1934); Sanders et al. (2010b).

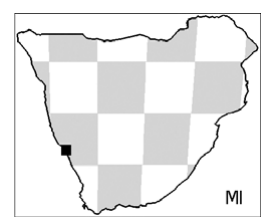

Subfamily: †Anancinae

†Anancus kenyensis Maclnnes, 1942 . Trans. Zool. Soc. Lond. 25(2):

33-106.

Additional references: Cooke (1993b); Hautier et al. (2009); Pickford and Senut (1997); Sanders et al. (2010b); Tassy (1986).

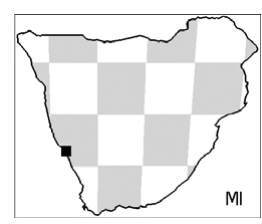

Subfamily: $†$ Choerolophodontinae

†Afrochoerodon kisumuensis Maclnnes, 1942. Trans. Zool. Soc. Lond. 25(2): 51.

Additional references: Pickford (2001a, 2005a); Tassy (1986).

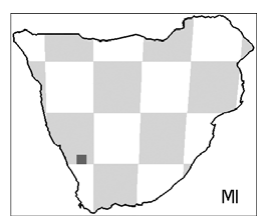

†Gomphotherium pygmaeus Depéret, 1897. Bull. Soc. Géol. France, Series

3, 25: 520 .

Synonyms: Choerolophodon, Mastodon; angustidens.

Additional references: Beck (1906); Fraas (1907); Hamilton (1973);

Pickford (2005a); Sanders et al. (2010a).
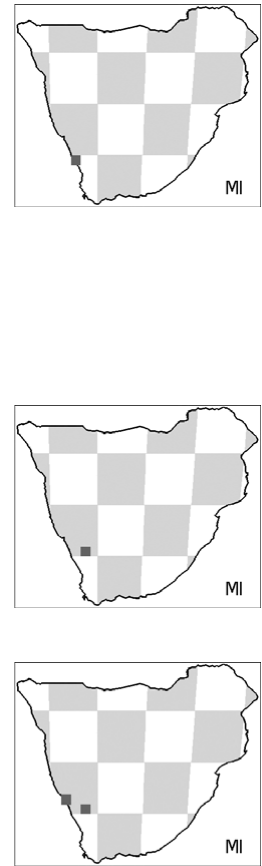

\section{FAMILY: †MAMMUTHIDAE}

†Eozygodon morotoensis Pickford and Tassy, 1980. Neues Jahrb. Geol.

Palaontol., Monatsch. 4: 242.

Synonyms: Zygolophodon aegyptensis cf.

Additional references: Pickford (2007, 2008c).

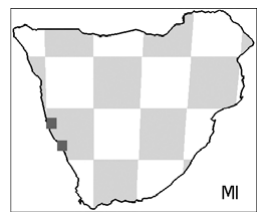




\section{ORDER: PRIMATES}

Suborder: Haplorrhini

FAMILY: CERCOPITHECIDAE

Subfamily: Colobinae

†Microcolobus Benefit and Pickford, 1986. Amer. Phys. Anthrop. 69: 446.

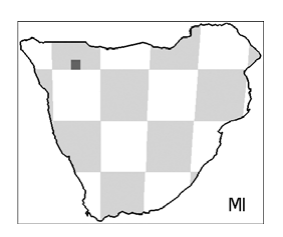

FAMILY: HOMINIDAE

Subfamily: Homininae

†Otavipithecus namibiensis Conroy, Pickford, Senut, Van Couvering and Mein 1992. Nature 356: 144.

Type locality: Berg Aukas I.

Additional references: Gommery (2000); Harrison (2010); Pickford et al. (1992 1997); Singleton (2000).

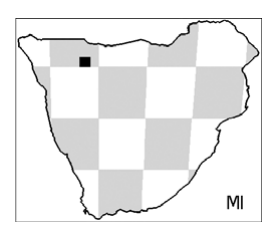

Subfamily: †Kenyapithecinae

†Kenyapithecus Leakey, 1961. Ann. Mag. Nat. Hist., Series 13, 4: 690.

Additional references: McCrossin and Benefit (1997); Pickford and Senut (1997).

Comments: specimen listed as Hominoidea by Senut et al. (1997) and as

a proconsulid (Nyanzapithecinae indet.) by Harrison (2010).

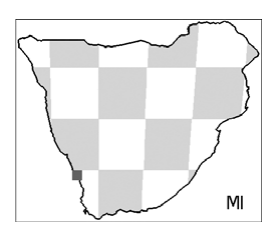

\section{ORDER: RODENTIA \\ Suborder: Sciuromorpha \\ FAMILY: SCIURIDAE \\ Subfamily: Xerinae}

†Heteroxerus karsticus Mein, Pickford and Senut, 2000. Comm. Geol.

Surv. Namibia 12: 381.

Synonyms: Vulcanisciurus.

Type locality: Harasib 3a.

Additional references: Viriot et al. (2011).

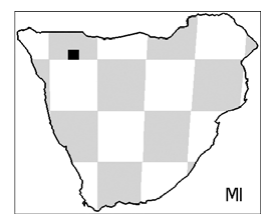

Comments: the version of this publication available on the Geological

Survey of Namibia website at www.mme.gov.na/files/publications/

5dd_Mein\%20et\%20al_Late\%20Miocene\%20micromammals_

Harasib.pdf appears to be wrongly paginated.

†Vulcanisciurus Lavocat, 1973. Mem. Trav. Inst. Montpellier Ecole Prat. Hautes Etudes 1: 1-284.

Comments: Lavocat (1973) not seen.

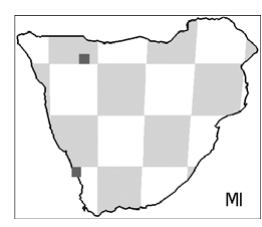


†Vulcanisciurus africanus Lavocat, 1973. Mem. Trav. Inst. Montpellier

Ecole Prat. Hautes Etudes 1: 1-284.

Additional references: Viriot et al. (2011).

Comments: Lavocat (1973) not seen.

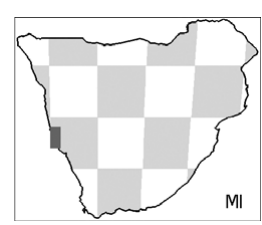

\section{Suborder: Myomorpha}

FAMILY: GLIRIDAE

Subfamily: Graphiurinae

†Otaviglis daamsi Mein, Pickford and Senut, 2000. Comm. Geol. Surv. Namibia 12: 383.

Type locality: Harasib $3 a$.

Comments: the version of this publication available on the Geological Survey of Namibia website at www.mme.gov.na/files/publications/ 5dd_Mein\%20et\%20al_Late\%20Miocene\%20micromammals_ Harasib.pdf appears to be wrongly paginated.

FAMILY: SPALACIDAE

Subfamily: Rhizomyinae

†Harasibomys Mein, Pickford and Senut, 2000. Comm. Geol. Surv. Namibia, 12: 387.

Synonyms: Brachyuromys cf.

Type locality: Harasib 3a.

Comments: the version of this publication available on the Geological

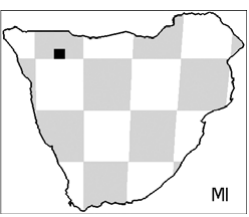

Survey of Namibia website at www.mme.gov.na/files/publications/

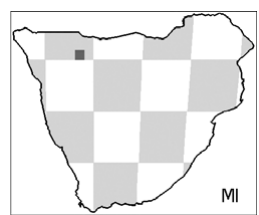

5dd_Mein\%20et\%20al_Late\%20Miocene\%20micromammals_

Harasib.pdf appears to be wrongly paginated.

†Harasibomys petteri Mein, Pickford and Senut, 2000. Comm. Geol. Surv. Namibia, 12: 387.

Synonyms: Brachyuromys cf.

Type locality: Harasib 3a.

Comments: the version of this publication available on the Geological

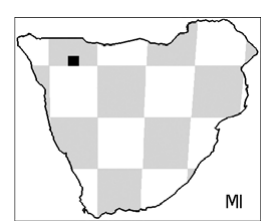

Survey of Namibia website at www.mme.gov.na/files/publications/

5dd_Mein\%20et\%20al_Late\%20Miocene\%20micromammals_

Harasib.pdf appears to be wrongly paginated.

†Nakalimys lavocati Flynn and Sabatier, 1984. J. Paleontol. 3: 161.

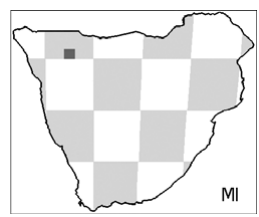


FAMILY: NESOMYIDAE

Subfamily: Afrocricetodontinae

†Notocricetodon Lavocat, 1973. Mem. Trav. Inst. Montpellier Ecole Prat. Hautes Etudes 1: 1-284.

Comments: Lavocat (1973) not seen.

†Protarsomys Lavocat, 1973. Mem. Trav. Inst. Montpellier Ecole Prat.

Hautes Etudes 1: 1-284.

Comments: Lavocat (1973) not seen.

†Protarsomys lavocati Mein and Pickford, 2003. Geol. Soc. Namibia Mem. 19: 148.

Type locality: Arrisdrift.

†Protarsomys macinnesi Lavocat, 1973. Mem. Trav. Inst. Montpellier Ecole Prat. Hautes Etudes 1: 1-284.

Comments: Lavocat (1973) not seen.

Subfamily: Cricetomyinae

Saccostomus Peters, 1846. Bericht Verhandl. K. Preuss. Akad. Wiss. Berlin 11: 258.

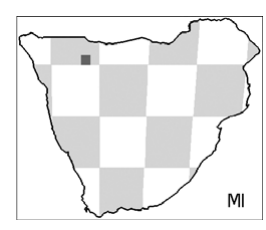

†Saccostomus geraadsi Mein, Pickford and Senut, 2004. Comm. Geol.

Soc. Namibia 13: 43.

Synonyms: major; “K."majus Geraads, 2001.

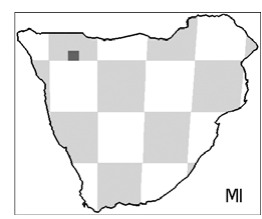

Subfamily: Dendromurinae

†Dendromus denysae Mein, Pickford and Senut, 2004. Comm. Geol. Surv.

Namibia 13: 49.

Type locality: Harasib 3a.

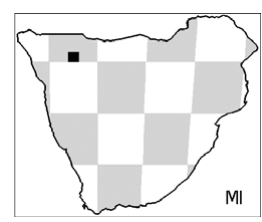


Steatomys Peters, 1846. Bericht Verhandl. K. Preuss. Akad. Wiss. Berlin 11: 258.

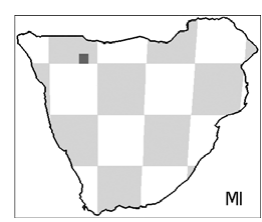

†Steatomys harasibensis Mein, Pickford and Senut, 2004. Comm. Geol. Surv. Namibia 13: 45.

Type locality: Harasib 3a.

†Steatomys jaegeri Mein, Pickford and Senut, 2004. Comm. Geol. Surv. Namibia 13: 48.

Type locality: Harasib 3a.

Subfamily: †Otavimyinae

†Otavimys senegasi Mein, Pickford and Senut, 2004. Comm. Geol. Surv. Namibia 13: 51.

Type locality: Harasib 3a.
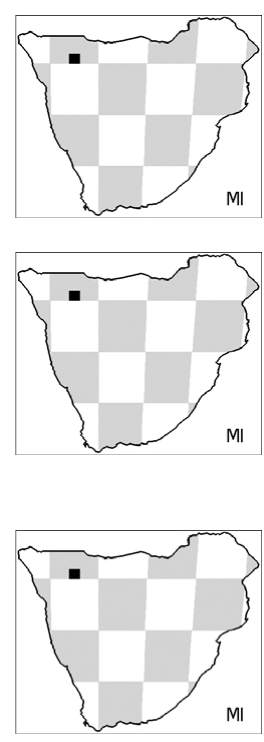

Subfamily: Petromyscinae

†Harimyscus hoali Mein, Pickford and Senut, 2000. Comm. Geol. Surv. Namibia, 12: 395.

Type locality: Harasib $3 a$.

Petromyscus Thomas, 1926. Ann. Mag. Nat. Hist., Series 9, 17: 179. Additional references: Petter (1967).
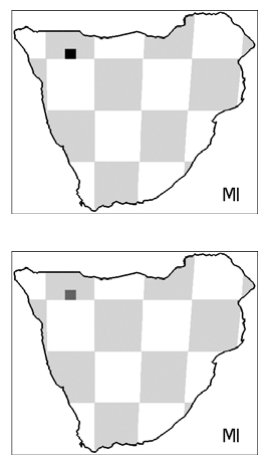

FAMILY: MURIDAE

Subfamily: Cricetodontinae

†Afaromys guillemoti Geraads, 1998. Palaeovertebrata 27(3-4): 205. Additional references: Mein et al. (2004).

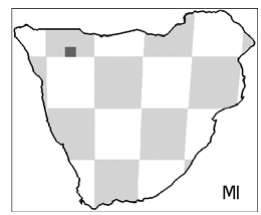

Subfamily: Deomyinae

†Preacomys griffini Mein, Pickford and Senut, 2004. Comm. Geol. Surv. Namibia 13: 57.

Synonyms: Karnimata.

Type locality: Harasib 3a.

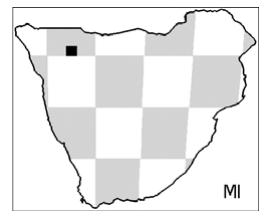


†Preacomys karsticus Mein, Pickford and Senut, 2004. Comm. Geol. Surv. Namibia 13: 57.

Type locality: Harasib 3a.

†Preacomys kikiae Geraads, 2001. Palaeovertebrata 30: 91. Additional references: Mein et al. (2004).
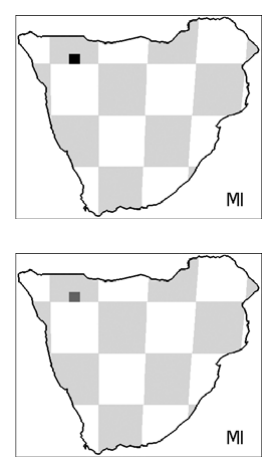

Subfamily: Gerbillinae

†Dakkamyoides Lindsay, 1988. Palaeovertebrata 18(2): 123.

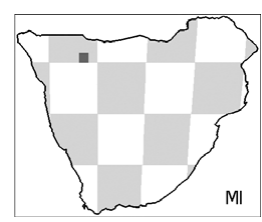

Subfamily: $†$ Myocricetodontinae †Myocricetodon Lavocat, 1952. C. R. Acad. Sci. Paris 235: 190.

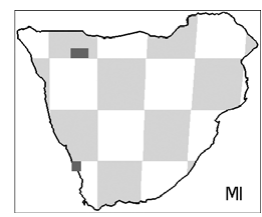

†Mioharimys milleri Mein, Pickford and Senut, 2000. Comm. Geol. Surv. Namibia 12: 391.

Type locality: Harasib 3a.

†Mioharimys schneideri Mein, Pickford and Senut, 2000. Comm. Geol.

Surv. Namibia 12: 394.

Type locality: Harasib 3a.
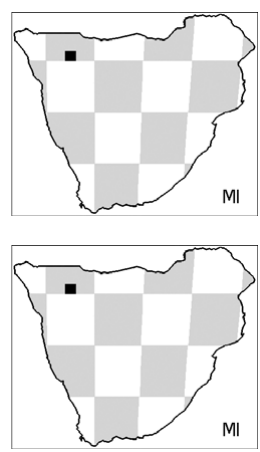

Subfamily: $†$ Namibimyinae

†Namibimys angustidens Mein, Pickford and Senut, 2000. Comm. Geol.

Soc. Namibia 12: 398.

Type locality: Berg Aukas I.

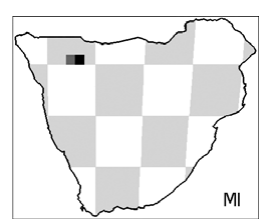

\section{Suborder: Anomaluromorpha}

FAMILY: †PARAPEDETIDAE

†Parapedetes Stromer, 1923. Sitz. Math.-Physik. Abt. Bayer. Akad. Wiss.

München 1923 (II): 261.

Type locality: Elisabethfeld.

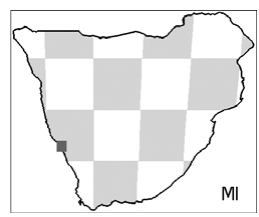


†Parapedetes namaquensis Stromer, 1923. Sitz. Math.-Physik. Klasse

Bayer. Akad. Wiss. München 1923(II): 261.

Type locality: Elisabethfeld.

Additional references: Pickford and Mein (2011).

\section{FAMILY: PEDETIDAE}

†Megapedetes gariepensis Mein and Senut, 2003. Geol. Soc. Namibia Mem. 19: 162.

Type locality: Arrisdrift.

Additional references: Pickford and Mein (2011).

†Oldrichpedetes brigittae Pickford and Mein, 2011. Estud. Geol. 67(2): 465.

Type locality: Zebra Hill.

†Oldrichpedetes pickfordi Mein and Senut, 2003. Geol. Soc. Namibia Mem. 19: 166.

Synonyms: Megapedetes.

Type locality: Arrisdrift.

Additional references: Pickford and Mein (2011).

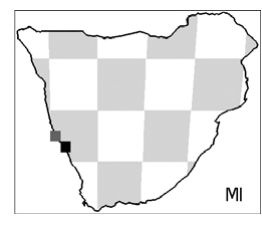

†Propedetes Mein and Pickford, 2008. Geol. Surv. Namibia Mem. 20: 242. Type locality: Elisabethfeld.
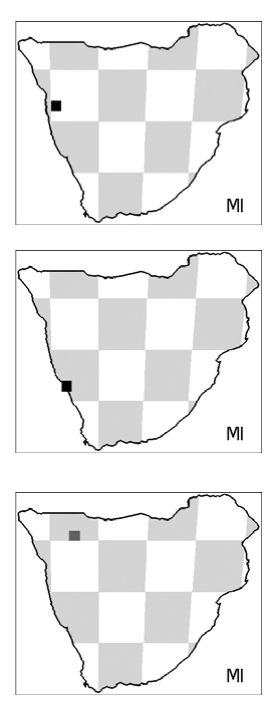

†Propedetes efeldensis Mein and Pickford, 2008. Geol. Surv. Namibia Mem. 20: 242.

Type locality: Elisabethfeld.

†Propedetes Iaetoliensis Davies, 1987. In: Leakey and Harris. The Pliocene

Site of Laetoli, Northern Tanzania: 172.

Synonyms: Pedetes.

Additional references: Pickford and Mein (2011).
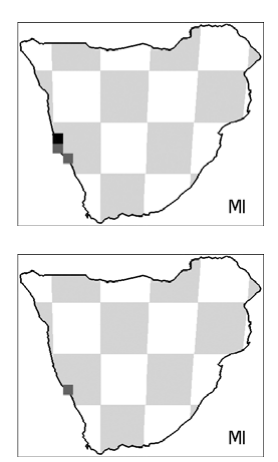

\section{Suborder: Hystricomorpha}

FAMILY: †DIAMANTOMYIDAE

Subfamily: †Diamantomyinae

†Diamantomys luederitzi Stromer, 1921. Sitz. Math.-Physik. Klasse Bayer. Akad. Wiss. München 1921: 334.

Type locality: Sperrgebiet.

Comments: Stromer (1923: 265) refers to this taxon as Diamantomys luederitzi Stromer (1922, S. 334), but see note in Section 1.3 regarding

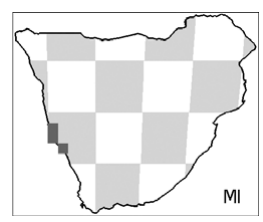
journal volume numbers. 
†Pomonomys dubius Stromer, 1921. Sitz. Math.-Physik. Klasse Bayer. Akad. Wiss. München 1921: 334.

Type locality: Grillental.

Comments: Stromer (1923: 265) refers to this taxon as Pomonomys dubius Stromer (1922, S. 334/5), but see note in Section 1.3 regarding

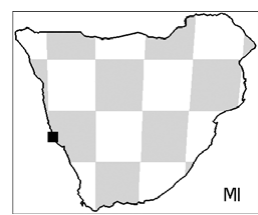
journal volume numbers.

\section{FAMILY: †BATHYERGOIDIDAE}

†Bathyergoides Stromer, 1923. Sitz. Math.-Physik. Klasse Bayer. Akad. Wiss. München 1923(II): 263.

Type locality: Sperrgebiet.

† Bathyergoides neotertiarius Stromer, 1923. Sitz. Math.-Physik. Klasse

Bayer. Akad. Wiss. München 1923(II): 263.

Type locality: Sperrgebiet.

Mem. 19: 144

Type locality: Arrisdrift.
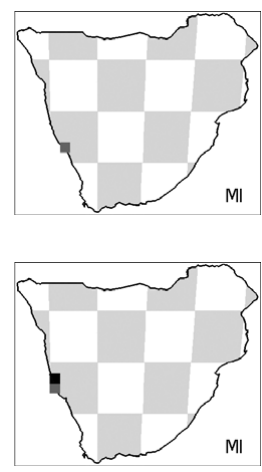

†Geofossor corvinusae Mein and Pickford, 2003. Geol. Soc. Namibia

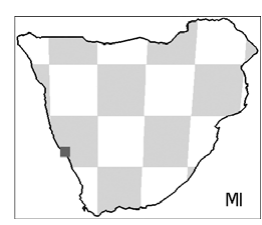

†Ceofossor moralesi Mein and Pickford, 2008. Geol. Surv. Namibia Mem. 20: 262.

Type locality: Elisabethfeld.

Microfossor biradiculatus Mein and Pickford, 2008. Geol. Surv. Namibia Mem. 20: 262.

Type locality: Elisabethfeld.
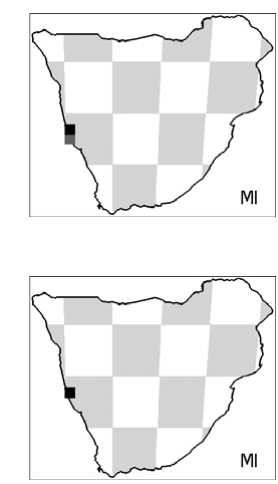

\section{FAMILY: BATHYERGIDAE}

Subfamily: Bathyerginae

†Proheliophobius Lavocat, 1973. Mem. Trav. Inst. Montpellier Ecole Prat. Hautes Etudes 1: 1-284.

Synonyms: Paracryptomys mackennae (see comment below).

Comments: Lavocat (1973) not seen. Mein et al. (2000a: 386) state:

'Denys and Jaeger (1992) provide measurements of a bathyergid (AD

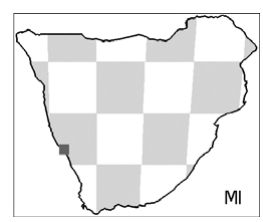

1638) from Arrisdrift, Namibia which they identify as Paracryptomys mackennae, citing Hamilton and Van Couvering (1977) as the source of these measurements. There are two points that should be made, 
firstly, the Arrisdrift species is far too small to belong to Paracryptomys mackennae, which is a giant bathyergid, possibly synonymous with Bathyergoides neotertiarius Stromer, and secondly, the site of Arrisdrift was unknown to Hamilton and Van Couvering as it was discovered in 1976 after they had submitted their manuscript for publication. In our opinion the Arrisdrift species is close to Proheliophobius but is slightly larger than the type species P. leakeyi Lavocat, 1973. The version of Mein et al. (2000a) available on the Geological Survey of Namibia website at www.mme.gov.na/files/publications/5dd_Mein\%20et\% 20al_Late\%20Miocene\%20micromammals_Harasib.pdf appears to be wrongly paginated.

FAMILY: †MYOPHIOMYIDAE

Subfamily: †Myophiomyinae

†Phiomyoides humilis Stromer, 1923. Sitz. Math.-Physik. Klasse Bayer. Akad. Wiss. München 1923(II): 264.

Type locality: Sperrgebiet.

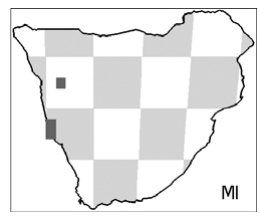

\section{FAMILY: THRYONOMYIDAE}

†Apodecter Hopwood, 1929. Amer. Mus. Novit. 344: 3.

Type locality: Lüderitz Bay (south of ).

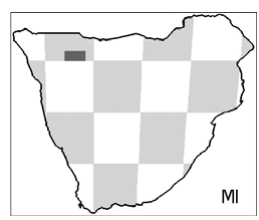

†Apodecter stromeri Hopwood, 1929. Amer. Mus. Novit. 344: 3.

Type locality: Lüderitz Bay (south of) (?Langental: Mein and Pickford, 2008c).

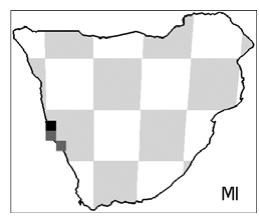

†Neosciuromys africanus Stromer, 1921. Sitz. Math.-Physik. Klass. Bayer. Akad. Wiss. München 1921 (II): 333.

Synonyms: fractus.

Type locality: Sperrgebiet.

Additional references: Stromer (1923).

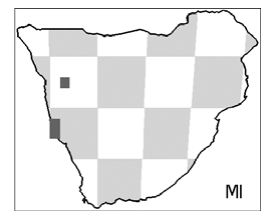

†Paraphiomys australis Mein, Pickford and Senut, 2000. Comm. Geol. Surv. Namibia 12: 378.

Type locality: Harasib $3 a$.

Comments: the version of this publication available on the Geological

Survey of Namibia website at www.mme.gov.na/files/publications/ 5dd_Mein\%20et\%20al_Late\%20Miocene\%20micromammals_ Harasib.pdf appears to be wrongly paginated.

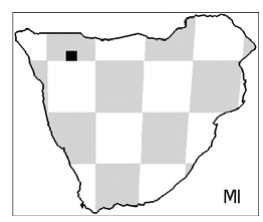


†Paraphiomys orangeus Mein and Pickford, 2003. Geol. Soc. Namibia Mem. 19: 143.

Type locality: Arrisdrift.

†Paraphiomys roessneri Mein, Pickford and Senut, 2000. Comm. Geol. Surv. Namibia 12: 375.

Synonyms: Apodecter cf.

Type locality: Harasib 3a.

Comments: the version of this publication available on the Geological

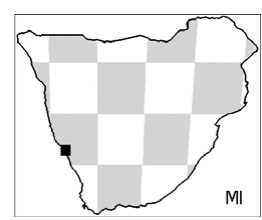

Survey of Namibia website at www.mme.gov.na/files/publications/

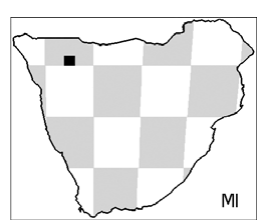

5dd_Mein\%20et\%20al_Late\%20Miocene\%20micromammals_

Harasib.pdf appears to be wrongly paginated.

†Paraulacodus johanesi Jaeger, Michaux and Sabatier, 1980.

Palaeovertebrata 9 ext. Mem. Jubil. R. Lavocat: 367.

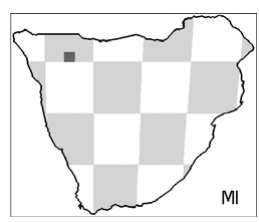

†Phthinylla fracta Hopwood, 1929. Amer. Mus. Novit. 344: 4.

Synonyms: Neosciuromys.

Type locality: Lüderitz Bay (south of ) (?Langental: Mein and Pickford, 2008c).

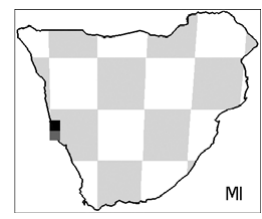

FAMILY: TUFAMYIDAE

†Efeldomys Ioliae Mein and Pickford, 2008. Geol. Surv. Namibia Mem. 20: 259.

Type locality: Elisabethfeld.

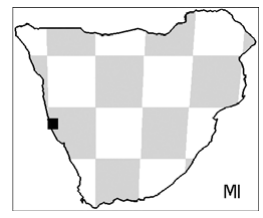

ORDER: LAGOMORPHA

FAMILY: OCHOTONIDAE

†Austrolagomys hendeyi Mein and Pickford, 2003. Geol. Soc. Namibia Mem. 19: 172.

Synonyms: Kenyalagomys.

Type locality: Arrisdrift.

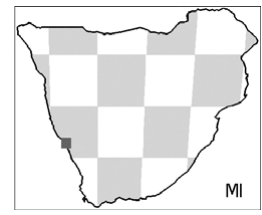

†Austrolagomys inexpectatus Stromer, 1923. Sitz. Math.-Physik. Klasse Bayer. Akad. Wiss. München 1923(II): 261.

Type locality: Sperrgebiet.

Synonyms: Simpsoni.

Additional references: Hopwood (1929); Mein and Pickford (2003d,

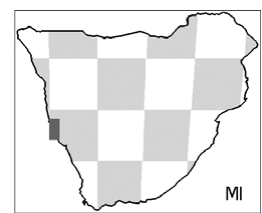

2008b). 


\section{ORDER: ERINACEOMORPHA}

FAMILY: ERINACEIDAE

Subfamily: Erinaceinae

†Amphechinus rusingensis Butler, 1956. Foss. Mamm. Africa 11: 54.

Additional references: Butler (1984, 2010); Mein and Pickford (2008a).

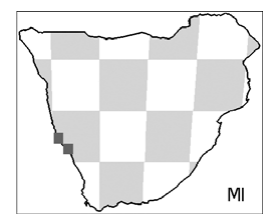

†Gymnurechinus leakeyi Butler, 1956. Foss. Mamm. Afr. 11: 3. Additional references: Mein and Pickford (2008a).

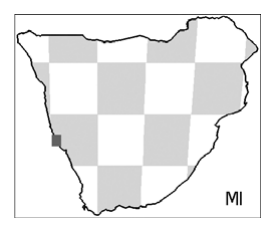

Subfamily: †Galericinae

†Galerix Pomel, 1848. Arch. Sci. Phys. Nat. Genève 9: 164.

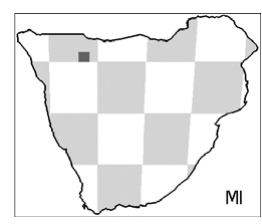

\section{ORDER: CHIROPTERA \\ Suborder: Microchiroptera \\ FAMILY: RHINOLOPHIDAE}

Rhinolophus Lacépède, 1799. Tableau des Divisions, Sous-divisions, Ordres, et Genres des Mammifères: 15.

Additional references: Andersen 1905); Taylor et al. (2012).

Comments: Lacépède, 1799 not seen. Citation according to Wilson and Reeder (2005).

†Rhinolophus contrarius Mein and Pickford, 2003. Geol. Surv. Namibia Mem. 19: 115.

Type locality: Arrisdrift.
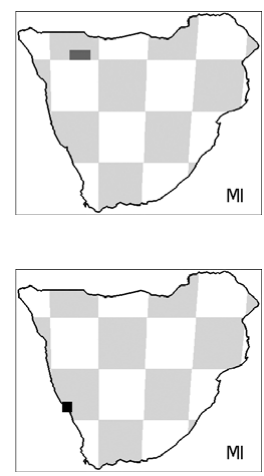

FAMILY: HIPPOSIDERIDAE

Asellia Gray, 1838. Mag. Zool. Bot. 2: 493.

Additional references: Thomas (1904a).

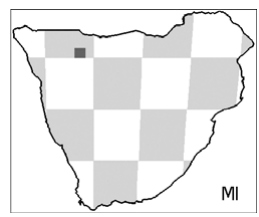

Hipposideros gigas Wagner, 1845. Arch. Naturgesch. 11(1): 148. Giant leaf-nosed bat.

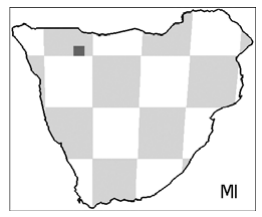


FAMILY: MEGADERMATIDAE

Megaderma Geoffroy Saint-Hilaire, 1810. Ann. Mus. Hist. Nat. Paris 14: 197.

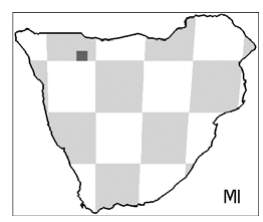

FAMILY: EMBALLONURIDAE

†Taphozous incognita Butler and Hopwood, 1957. Foss. Mamm. Africa 13: 29.

Synonyms: Saccolaimus.

Additional references: Butler (1984).

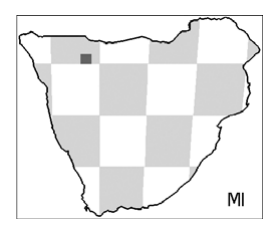

FAMILY: MOLOSSIDAE

Subfamily: Molossinae

Tadarida Rafinesque, 1814. Précis des découvertes et travaux somiologiques: 55 .

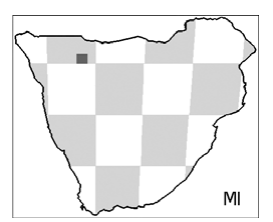

\section{FAMILY: VESPERTILIONIDAE}

Subfamily: Myotinae

Myotis Kaup, 1829. Skizz. Entwickel.-Gesch. Nat. Syst. Europ. Thierwelt 1: 106.

Additional references: Gray (1842).

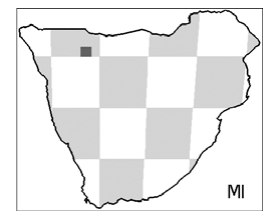

\section{ORDER: †CREODONTA}

FAMILY: †HYAENODONTIDAE

Subfamily: †Hyaenodontinae

†Metapterodon kaiseri Stromer, 1923. Sitz. Math.-Physik. Klasse Bayer. Akad. Wiss. München 1923(II): 254.

Synonyms: Isohyaenodon.

Type locality: Elisabethfeld.

Additional references: Lewis and Morlo (2010); Morales et al. (2008a);

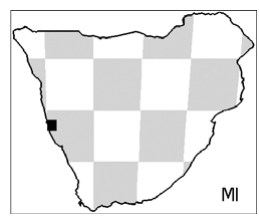

Savage (1965).

† Metapterodon stromeri Morales, Pickford and Soria, 1998. C.R. Acad.

Sci. Paris, Sci. Terre Plan. 327: 634.

Synonyms: Pterodon.

Type locality: Langental.

Additional references: Lewis and Morlo (2010); Morales et al. (2008a).

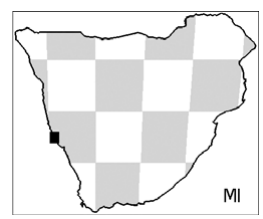


Subfamily: †Hyainailourinae

†Hyainailouros sulzeri Biedermann, 1863. Petrefakten aus der Umgegend von Winterthur. Zweites Heft. 20.

Additional references: Helbing (1924); Morales et al. (1998b, 2003d);

Morlo et al. (2007); Sach and Heizmann (2001).

Comments: Biedermann (1863) not seen.

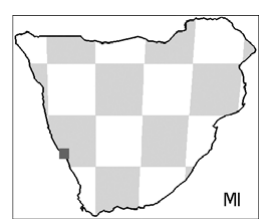

FAMILY: †PRIONOGALIDAE

†Namasector soriae Morales, Pickford and Salesa, 2008. Geol. Surv. Namibia Mem. 20: 295.

Type locality: Elisabethfeld (Tortoise Site).

Comments: Werdelin and Cote (2010) are doubtful that this family should be placed in Creodonta and prefer to retain Prionogalidae as Mammalia incertae sedis.

\section{ORDER: CARNIVORA \\ Suborder: Feliformia \\ FAMILY: †BARBOUROFELIDAE}

†Afrosmilus africanus Andrews, 1914. Quart. J. Geol. Soc. Lond. 70: 179. Synonyms: Pseudaelurus; Metailurus.

Additional references: Savage (1965); Werdelin and Peigné (2010).

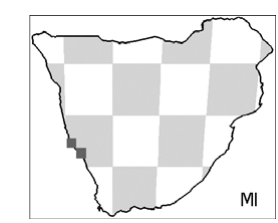

Subfamily: †Barbourofelinae

†Ginsburgsmilus napakensis Morales, Salesa, Pickford and Soria, 2001.

Trans. R. Soc. Edinburgh 92: 98.

Additional references: Morales and Pickford (2018).

\section{FAMILY: FELIDAE}

†Diamantofelis ferox Morales, Pickford, Soria and Fraile, 1998. Eclogae

Geol. Helv. 91(1): 36.

Type locality: Arrisdrift.

Additional references: Morales et al. (2003d).

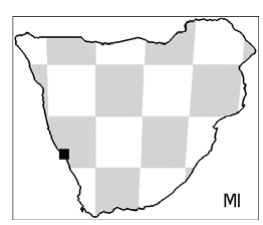

†Namafelis minor Morales, Pickford, Fraile, Salesa and Soria, 2003. Mem.

Geol. Surv. Namibia 19: 184.

Type locality: Arrisdrift.

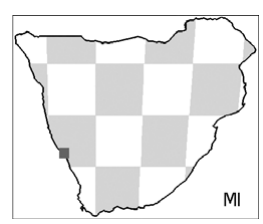


Subfamily: Felinae

†Metailurus Zdansky, 1924. Palaeontol. Sinica, Series C, 2(1): 123.

Comments: publication not seen but citation confirmed by L. Werdelin (personal communication, 12 December 2016).

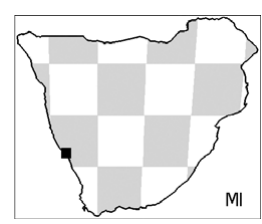

Subfamily: Pantherinae

Panthera leo Linnaeus, 1758. Systema Naturae Regnum Animale, 10th edition, 1, Lion.

Additional references: Haas et al. (2005); Lacruz (2009).

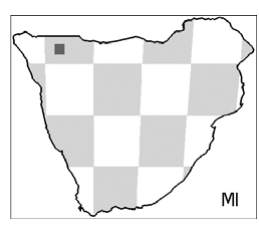

FAMILY: †STENOPLESICTIDAE

†Orangictis gariepensis Morales, Pickford, Soria and Fraile, 2001.

Palaeontol. Afr. 37: 99.

Type locality: Arrisdrift.

Additional references: Werdelin and Peigné (2010).

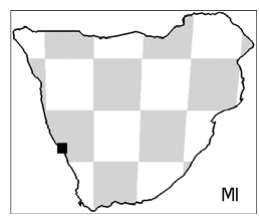

Subfamily: $†$ Stenoplesictinae

†Africanictis hyaenoides Morales, Pickford, Fraile, Salesa and Soria, 2003.

Geol. Soc. Namibia Mem. 19: 183.

Type locality: Arrisdrift.

†Africanictis meini Morales, Pickford, Soria and Fraile, 1998. Eclogae

Geol. Helv. 91: 34.

Type locality: Arrisdrift.

†Africanictis schmidtkittleri Morales, Pickford, Soria and Fraile, 1998.

Eclogae Geol. Helv. 91: 35.

Type locality: Arrisdrift.

Additional references: Werdelin and Peigné (2010).
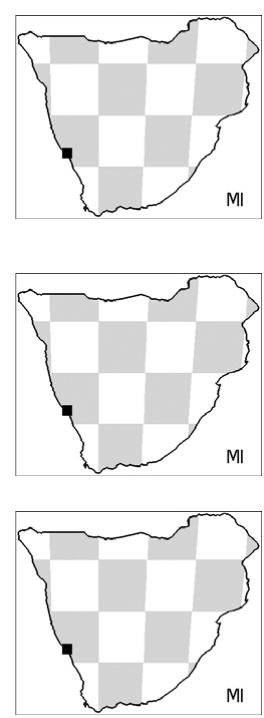

\section{FAMILY: VIVERRIDAE}

†Leptoplesictis namibiensis Morales, Pickford and Salesa, 2008. Geol. Surv. Namibia Mem. 20: 304.

Type locality: Langental.

Comments: according to Werdelin and Peigné (2010), Leptoplesictis is generally considered to be a herpestid rather than a viverrid, although

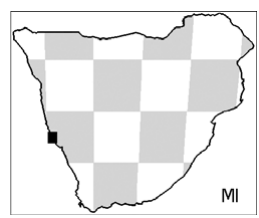
it was placed in the latter Family by Morales et al. (2008a). 
†Leptoplesictis senutae Morales, Pickford and Salesa, 2008. Geol. Surv. Namibia Mem. 20: 303.

Type locality: Grillental.

Comments: see under Leptoplesictis namibiensis.

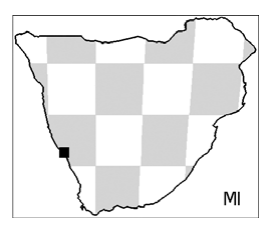

\section{Suborder: Caniformia}

FAMILY: †AMPHICYONIDAE

†Agnotherium Kaup, 1833. Déscription d'Ossements Fossiles de Mammifères 2: 28.

Additional references: Kurtén (1976); Werdelin and Peigné (2010).

Subfamily †Amphicyoninae

†Amphicyon giganteus Schinz, 1825. In: Cuvier, Thierreich IV: 342. Synonyms: Pseudocyon; steinheimensis.

Additional references: Werdelin and Peigné (2010).

†Hecubides euryodon Savage, 1965. Foss. Mamm. Africa 19: 189. Additional references: Morales et al. (2016).

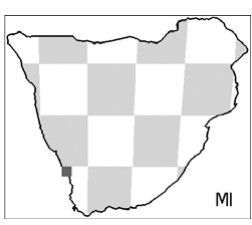

†Ysengrinia Ginsburg, 1965. Bull. Mus. Natl d'Hist. Nat., Series 2, 37(4): 727.

Additional references: Werdelin and Peigné (2010).

†Ysengrinia ginsburgi Morales, Pickford, Soria, and Fraile, 1998. Eclogae

Geol. Helv. 91(1): 30.

Type locality: Arrisdrift.
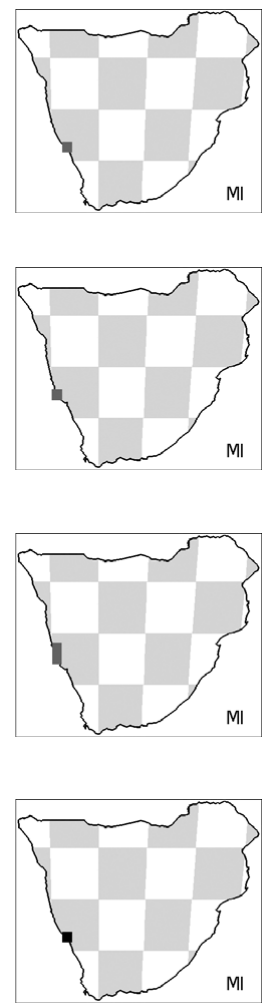

FAMILY: MUSTELIDAE

†Namibictis senuti Morales, Pickford, Soria, and Fraile, 1998. Eclogae Geol. Helv. 91(1): 32.

Type locality: Arrisdrift.

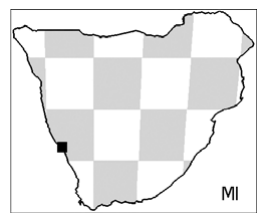




\section{ORDER: PERISSODACTYLA}

FAMILY: EQUIDAE

†Eurygnathohippus cornelianus Van Hoepen, 1930. Paleontol. Nav. Nas. Mus. Bloemfontein 2(2): 23.

Synonyms: Hipparion; Stylohipparion; hipkini; libycum; sitifense in part?; steytleri.

Type locality: Cornelia.

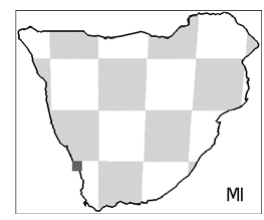

Additional references: Bernor et al. (2010); Churcher (1970, 2000); Churcher and Watson (1993); Franz-Odendaal et al. (2003); Pomel (1897); Van Hoepen (1932a).

Comments: the material from Hondeklip Bay was doubtfully ascribed by Pickford and Senut (1997) to Hipparion sitifense, which Bernor et al. (2010) consider a nomen dubium. See also the comment under Hipparion below.

FAMILY: RHINOCEROTIDAE

Subfamily: Rhinocerotinae

†Brachypotherium Roger, 1902. Ber. Naturwiss. Ver. Schwaben und Neuburg 36: 12.

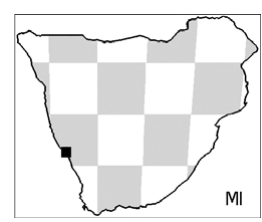

†Brachypotherium heinzelini Hooijer, 1963. Ann. Mus. Roy. Afr. Cent., Tervuren Sci. Géol. 46: 45.

Synonyms: lewisi?.

Additional references: Geraads (2010a); Guérin (2008); Heissig (1971); Hooijer (1973).

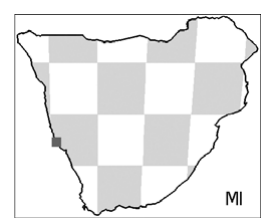

†Chilotheridium pattersoni Hooijer, 1971. Bull. Mus. Comp. Zool. 144: 342.

Additional references: Geraads (2010a); Guérin (2003, 2008); Hooijer (1973).

†Diceros australis Guérin, 2000. Palaeontol. Afr. 36: 122.

Synonyms: Dicerorhinus.

Type locality: Arrisdrift.

Additional references: Geraads (2010a); Guérin (2003).

†Diceros praecox Hooijer and Patterson, 1972. Bull. Mus. Comp. Zool. 144: 19.

Synonyms: Ceratotherium.

Additional references: Geraads (2005, 2010a); Guérin (1987); Hooijer (1972 1973); Pickford and Senut (1997).
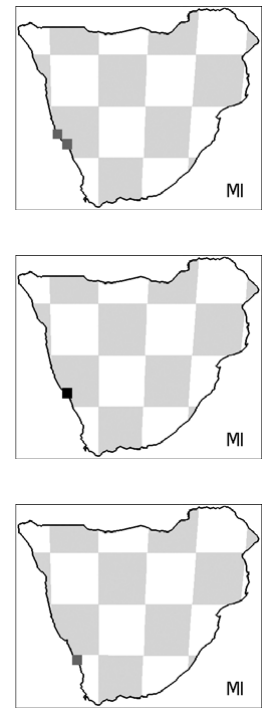
†Turkanatherium acutirostrum Deraniyagala, 1951. Spol. Zeyl. 26(2):

134.

Synonyms: Aceratatherium.

Additional references: Geraads (2010a); Guérin (2008); Hooijer (1973).

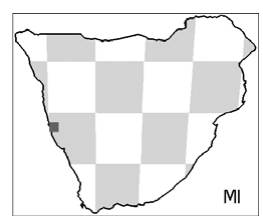

\section{ORDER: ARTIODACTYLA}

†Namibiomeryx senuti Morales, Soria and Pickford, 1995. C. R. Acad. Sci. Paris, Série lla, 321: 1211.

Type locality: Elisabethfeld.

Additional references: Cote (2010).

†Namibiomeryx spaggiarii Morales, Soria and Pickford, 2008. Mem.

Geol. Surv. 20: 442.

Type locality: Langental.
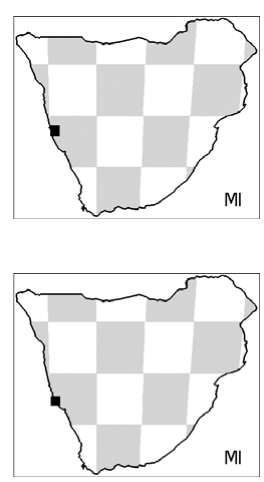

†Orangemeryx hendeyi Morales, Soria and Pickford, 1999. Geodiversitas,

Series 21, 2: 241.

Synonyms: Climacoceras.

Type locality: Arrisdrift.

Additional references: Azanza et al. (2003); Cote (2010); Hamilton

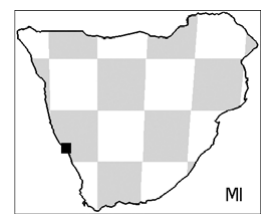

(1978); Maclnnes (1936); Morales et al. (2003d).

Subfamily: $†$ Sperrgebietomerycinae

†Sperrgebietomeryx wardi Morales, Soria and Pickford, 1999.

Geodiversitas, Series 21, 2: 232.

Type locality: Elisabethfeld.

Additional references: Cote (2010).

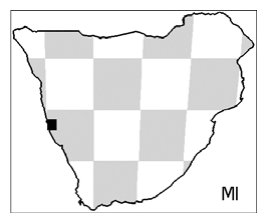

FAMILY: †CLIMACOCERATIDAE

Subfamily: †Propalaeorycinae

†Propalaeoryx austroafricanus Stromer, 1923. Sitz. Math.-Physik. Klasse

Bayer. Akad. Wiss. München 1923(II): 256.

Type locality: Elisabethfeld.

Additional references: Cote (2010).

†Propalaeoryx stromeri Morales, Soria and Pickford, 2008. Geol. Surv.

Namibia Mem. 20: 410.

Type locality: Langental.
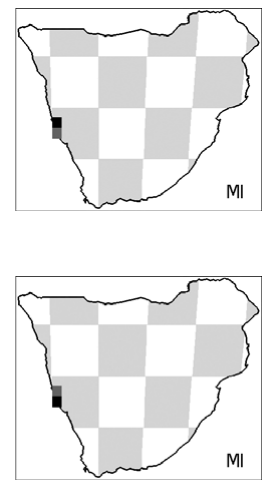
FAMILY: SUIDAE

Subfamily: †Kubanochoerinae

†Kenyasus namaquensis Pickford and Senut, 1997. Palaeontol. Afr.

34: 208.

Type locality: Ryskop.

Additional references: Bishop (2010); Pickford (1986).

†Kenyasus rusingensis Pickford, 1986. Tert. Res. Spec. Pap. 7: 24. Additional references: Bishop (2010); Pickford and Senut (1997).

†Nguruwe namibensis Pickford, 1986. Tert. Res. Spec. Pap. 7: 25. Synonyms: Hyotherium; Kenyasus; kijivium.

Type locality: Langental.

Additional references: Bishop (2010); Pickford (1997, 2003e, 2008e).
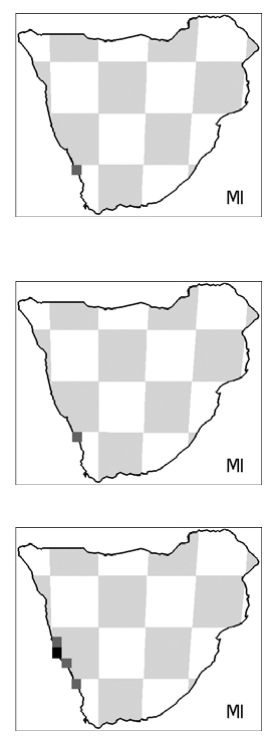

Subfamily: †Namachoerinae

†Namachoerus moruoroti Wilkinson, 1976. Foss. Vert. Afr. 4: 173-282. Synonyms: Lopholistriodon.

Additional references: Pickford (1987, 1995, 2003f).

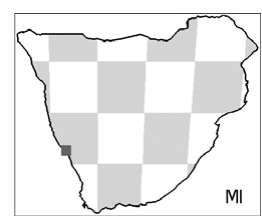

Subfamily: †Tetraconodontinae

†Nyanzachoerus kanamensis Leakey, 1958. Foss. Mamm. Afr. 14: 10. Additional references: Bishop (2010); Harris and White (1979).

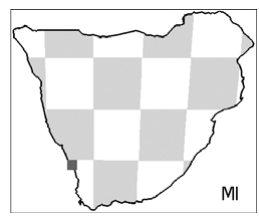

FAMILY: †ANTHRACOTHERIIDAE

†Brachyodus aequatorialis Maclnnes, 1951. Foss. Mamm. Afr. 4: 3. Synonyms: Masritherium.

Additional references: Holroyd et al. (2010); Madden et al. (1983);

Pickford (1991a, 1996b, 2015f).

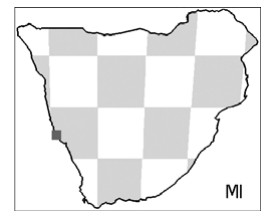

†Brachyodus depereti Fourtau, 1918. Contribution à l'étude des vertébrés miocènes de l'Egypte: 64.

Synonyms: Masritherium.

Additional references: Holroyd et al. (2010); Miller et al. (2014); Pickford (2003d, 2008d).

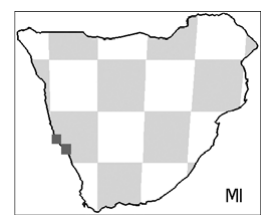

Comments: Fourtau (1918) not seen. M. Pickford (personal communication 1 December 2016) believes that the earliest evidence for the transfer of this species to Brachyodus is that given by Dineur (1982). 
FAMILY: †SANITHERIIDAE

†Diamantohyus africanus Stromer, 1921. Sitz. Math.-Physik. Klass. Bayer. Akad. Wiss. München 1921 (II): 332.

Type locality: Sperrgebiet?

Additional references: Bishop (2010); Pickford (1984, 1997, 2004, 2008f).

Comments: this taxon has been ascribed to Stromer (1926) by Bishop (2010) and others.

\section{FAMILY: TRAGULIDAE}

†Dorcatherium moruorotensis Pickford, 2001. Geobios 34(4): 437. Additional references: Geraads (2010b); Quiralte et al. (2008).

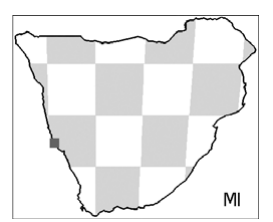

†Dorcatherium parvum Whitworth, 1958. Foss. Mamm. Afr. 15: 11. Additional references: Geraads (2010b); Quiralte et al. (2008).

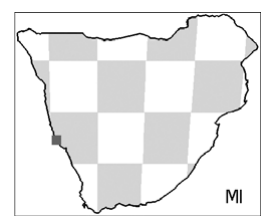

†Dorcatherium pigotti Whitworth, 1958. Foss. Mamm. Afr. 15: 9.

Additional references: Geraads (2010b); Morales et al. (2003d); Pickford et al. (1996); Quiralte et al. (2008).

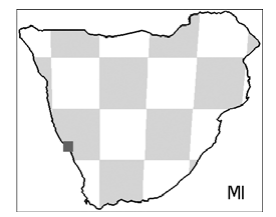

†Dorcatherium songhorensis Whitworth, 1958. Foss. Mamm. Afr. 15: 14. Additional references: Quiralte et al. (2008).

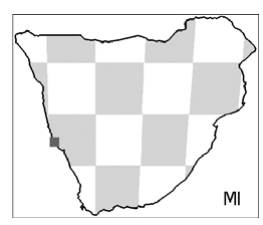

FAMILY: BOVIDAE

†Namacerus gariepensis Morales, Soria, Pickford and Nieto, 2003. Geol. Surv. Namibia Mem. 19: 372.

Type locality: Arrisdrift.

Additional references: Gentry (2010).

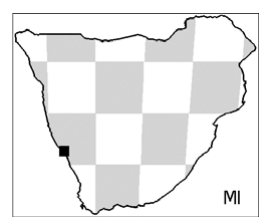

Subfamily: Alcelaphinae

†Damalacra acalla Gentry, 1980. Ann. S. Afr. Mus. 79(8): 272.

Type locality: Langebaanweg.

Additional references: Brink and Stynder (2009).

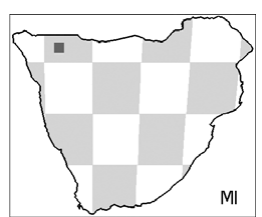


Subfamily: Antilopinae

†Homoiodorcas Thomas, 1981. Proc. Kon. Nederl. Akad. Weten. Ser.

B. Phys. Sci. 84(3): 364.

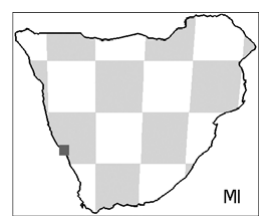

Subfamily: Bovinae

†Ugandax demissum Gentry, 1980. Ann. S. Afr. Mus. 79(8): 233.

Synonyms: Simatherium.

Type locality: Langebaanweg.

Additional references: Gentry (2006, 2010); Geraads (1992); Pickford and

Senut (1997).

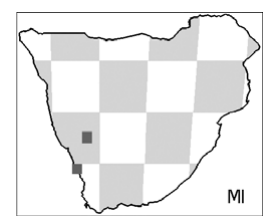

\subsection{MIOCENE SITES}

The 25 Miocene sites are restricted to west of $20^{\circ} \mathrm{E}$ and north of about $30^{\circ} \mathrm{S}$ (Figure 3.1). Particularly rich sites are Harasib $3 a$ in the north, and Arrisdrift, Langental and Grillental in the south. Exploration of the southern African Miocene began fairly early, notably with the work of Stromer (1921), but Corvinus (1978) and Hendey (1978c) started what might be described as the modern era. However, the discovery of Otavipithecus namibiensis at Berg Aukas (Conroy et al. 1996) provided the impetus for the subsequent work undertaken by Martin Pickford and colleagues (see references), which greatly expanded coverage of the Miocene in the region.

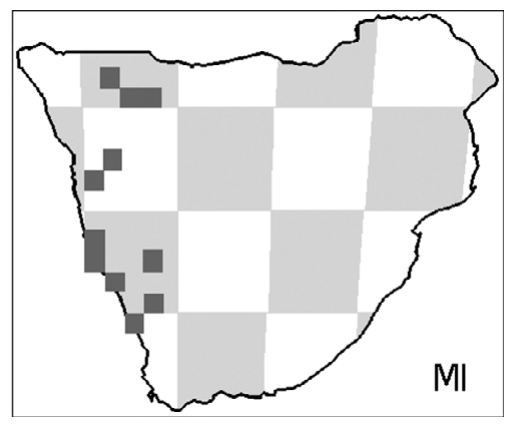

Figure 3.1 Location of Miocene sites.

Arrisdrift (2828:1642). Taxa: Africanictis hyaenoides; Africanictis meini; Africanictis schmidtkittleri; Afromastodon coppensi; Afrosmilus africanus; Amphechinus rusingensis; Amphicyon giganteus; Amphiorycteropus; Apodecter stromeri cf.; Austrolagomys hendeyi; Bathyergoides; Brevirhynchocyon jacobi; Chilotheridium pattersoni cf.; Choerolophodon pygmaeus; Diamantofelis ferox; Diamantomys luederitzi; Diceros australis; Dorcatherium pigotti; Eozygodon morotoensis; Geofossor corvinusae; Homoiodorcas cf.; Hyainailouros sulzeri; Megapedetes gariepensis; Metailurus; Miorhynchocyon gariepensis; Myohyrax oswaldi; Namacerus gariepensis; Namachoerus moruoroti; Namafelis minor; Namibictis senuti; Nguruwe namibensis; Oldrichpedetes pickfordi; Orangemeryx hendeyi; Orangictis 
gariepensis; Palaeothentoides?; Parapedetes; Paraphiomys orangeus; Prochrysochloris miocaenicus cf.; Prodeinotherium hobleyi; Proheliophobius; Prohyrax hendeyi; Protarsomys lavocati; Protenrec butleri; Rhinolophus contrarius; Vulcanisciurus; Ysengrinia ginsburgi. References: Corvinus (1978); Guérin (2003); Harris (1977); Hendey (1978c, 1984); Mein and Pickford (2003b, 2003c); Morales et al. (2001a); Morales et al. (2003b-2003d); Pickford (2003a, 2003c, 2003e, 2005a); Pickford et al. (1996); Pickford and Senut (1999, 2003); Senut (2003); Senut and Georgalis (2014).

Auchas (2816BC?). Taxa: Brachyodus depereti; Brachypotherium?; Diamantofelis; Diamantomys luederitzi; Eozygodon morotoensis; Megapedetes; Prodeinotherium hobleyi; Progomphotherium maraisi; Prohyrax hendeyi. References: Guérin (2003); Morales et al. (2008a); Pickford (2003b, 2003c); Pickford and Senut (2003); Pickford et al. (1995).

Baken (2829:1647). Taxa: Prohyrax hendeyi. References: Pickford (2003b).

Berg Aukas (1931:1815). Taxa: Apodecter; Asellia; Dakkamyoides; Elephantulus; Galerix; Harasibomys; Heterohyrax auricampensis; Hipposideros gigas cf.; Megaderma; Myocricetodon; Myotis; Namibimys angustidens; Notocricetodon; Otavipithecus namibiensis; Parapliohyrax; Protarsomys; Rhinolophus; Saccostomus cf.; Steatomys cf.; Tadarida; Taphozous incognita; Vulcanisciurus. References: Conroy et al. (1996); Grine et al. (1995); Mein et al. (2000b); Pickford and Senut (2010); Senut et al. (1992).

Bogenfels (2728:1523). Taxa: Propalaeoryx austroafricanus. References: Cooke (1955). Bohrloch des Betriebes 4 (2205:1617). Taxa: Myohyrax oswaldi; Neosciuromys africanus; Phiomyoides humilis. References: Mein and Pickford (2008c); Senut (2003).

Bosluis Pan (2953:1858). Taxa: Afrochoerodon kisumuensis aff.; Choerolophodon pygmaeus; Myohyrax oswaldi. References: Pickford (2005a); Senut et al. (1996).

E-Bay [Elisabethbucht?](2615CC). Taxa: Apodecter stromeri; Phiomyoides humilis; Protarsomys macinnesi. References: Mein and Pickford (2008c).

Elisabethfeld (2658:1515). Taxa: Afrohyrax namibiensis; Apodecter stromeri; Austrolagomys inexpectatus; Bathyergoides neotertiarius; Brevirhynchocyon jacobi; Diamantohyus africanus; Diamantomys luederitzi; Efeldomys loliae; Eozygodon morotoensis; Geofossor moralesi; Hecubides euryodon; Mesochoerus lategani; Metapterodon kaiseri; Microfossor biradiculatus; Myohyrax oswaldi; Namasector soriae; Namibiomeryx senuti; Neosciuromys africanus; Nguruwe namibensis; Parapedetes namaquensis; Phiomyoides humilis; Phthinylla fracta; Prochrysochloris miocaenicus cf.; Prohyrax tertiarius; Promicrogale namibiensis; Propalaeoryx austroafricanus; Propedetes efeldensis; Protarsomys macinnesi; Protenrec butleri; Protypotheroides beetzi; Sperrgebietomeryx wardi; Vulcanisciurus africanus; Ysengrinia. References: Mein and Pickford (2008a, 2008c); Morales et al. (2008a, 2008b, 2016); Pickford (2008b, 2008c, 2008e); Pickford and Senut (2008, 2018); Senut (2008); Senut and Georgalis (2014).

Etosha Pan (1816CA). Taxa: Damalacra acalla; Loxodonta cookei cf.; Panthera leo cf. References: Pickford et al. (2014).

Fiskus (2615CA). Taxa: Afrohyrax namibiensis; Afrosmilus africanus; Bathyergoides neotertiarius; Diamantohyus africanus; Myohyrax oswaldi; Neosciuromys africanus; Nguruwe namibensis; Propalaeoryx stromeri; Protypotheroides beetzi; Turkanatherium acutirostrum; Ysengrinia. References: Guérin (2008); Morales et al. (2008a, 2008b); Pickford (2008e); Pickford and Senut (2008, 2018); Senut (2008). 
Glastal (2715). Taxa: Diamantomys luederitzi; Neosciuromys africanus. References: Mein and Pickford (2008c).

GP Pan North (2829:1632). Taxa: Propedetes laetoliensis. References: Pickford and Mein (2011).

Grillental (2715AB). Taxa: Afrohyrax namibiensis; Afrosmilus africanus; Apodecter stromeri; Austrolagomys inexpectatus; Bathyergoides neotertiarius; Brachyodus aequatorialis; Brachyodus depereti; Brachypotherium heinzelini; Chilotheridium pattersoni; Diamantohyus africanus; Diamantomys luederitzi; Dorcatherium moruorotensis cf.; Dorcatherium songhorensis; Geofossor moralesi; Ginsburgsmilus napakensis; Gymnurechinus leakeyi; Hecubides euryodon; Hypsorhynchocyon burrelli; Leptoplesictis senutae; Metapterodon; Myohyrax oswaldi; Neosciuromys africanus; Nguruwe namibensis; Orycteropus; Phiomyoides humilis; Phthinylla fracta; Pomonomys dubius; Prohyrax tertiarius; Propalaeoryx stromeri; Protarsomys macinnesi; Protenrec butleri; Protypotheroides beetzi; Vulcanisciurus africanus; Ysengrinia. References: Guérin (2008); Mein and Pickford (2008a-2008c); Morales and Pickford (2018); Morales et al. (2008a, 2016); Pickford (2008a, 2008b, 2008d-2008f); Pickford and Senut (2008); Senut (2008).

Groenrivier (2718BD). Taxa: Ugandax demissum. References: Pickford and Senut (1997). Harasib 3a (1934:1748). Taxa: Afaromys guillemoti cf.; Apodecter cf.; Dendromus denysae; Harasibomys petteri; Harimyscus hoali; Heteroxerus karsticus; Microcolobus?; Mioharimys milleri; Mioharimys schneideri; Myocricetodon; Nakalimys lavocati; Namibimys angustidens; Otaviglis daamsi; Otavimys senegasi; Paraphiomys australis; Paraphiomys roessneri; Paraulacodus johanesi cf.; Petromyscus; Preacomys griffini; Preacomys karsticus; Preacomys kikiae; Propedetes; Rhinolophus; Saccostomus geraadsi; Steatomys harasibensis; Steatomys jaegeri. References: Conroy et al. (1993); Pickford and Mein (2011); Pickford and Senut (2010); Pickford et al. (1994); Senut et al. (1992).

Hondeklip Bay (3017AD). Taxa: Agnotherium; Anancus kenyensis; Diceros praecox; Eurygnathohippus cornelianus cf.; Loxodonta; Nyanzachoerus kanamensis; Ugandax demissum?. References: Pether (1994); Pickford and Senut (1997).

Karingarab (2812:1621). Taxa: Propedetes laetoliensis. References: Pickford and Mein (2011).

Langental (2724:1524). Taxa: Afrosmilus africanus; Amphechinus rusingensis; Apodecter stromeri; Austrolagomys inexpectatus; Bathyergoides neotertiarius; Brachyodus aequatorialis; Brachypotherium heinzelini; Diamantohyus africanus; Diamantomys luederitzi; Dorcatherium parvum cf.; Dorcatherium songhorensis; Geofossor moralesi; Gymnurechinus leakeyi; Hecubides euryodon; Leptoplesictis namibiensis; Megapedetes gariepensis; Metapterodon stromeri; Myohyrax oswaldi; Myohyrax pickfordi; Namibiomeryx spaggiarii; Neosciuromys africanus; Nguruwe namibensis; Phiomyoides humilis; Phthinylla fracta; Pomonomys dubius; Prochrysochloris miocaenicus cf.; Prohyrax tertiarius; Propalaeoryx stromeri; Propedetes efeldensis; Protarsomys macinnesi; Protenrec butleri; Protypotheroides beetzi; Vulcanisciurus africanus; Ysengrinia. References: Guérin (2008); Mein and Pickford (2008a, 2008b); Morales et al. (2008a, 2016); Pickford (2003b, 2003d-2003f); Pickford and Senut (2008); Senut (2008).

Lüderitz Bay (south of) (2615). Taxa: Apodecter stromeri; Austrolagomys inexpectatus Phthinylla fracta. References: Hopwood (1929); Mein and Pickford (2008c). Nova (3017AD). Taxa: Parapliohyrax ngororaensis. References: Pickford (2003f). 
Rooilepel (2818:1635). Taxa: Amphiorycteropus; Propedetes efeldensis; Propedetes laetoliensis. References: Lehmann (2009); Pickford (1996b); Pickford and Mein (2011).

Ryskop (3018:1718). Taxa: Kenyapithecus cf.; Kenyasus namaquensis; Kenyasus rusingensis; Nguruwe namibensis; Prodeinotherium hobleyi; Ugandax demissum. References: Pickford (2003e); Pickford and Senut (1997); Senut et al. (1997).

Swartlintjies 2 (3018:1718). Taxa: Anancus kenyensis; Diceros praecox. References: Pickford and Senut (1997).

Zebra Hill (2337:1538). Taxa: Oldrichpedetes brigittae. References: Pickford and Mein (2011). 


\section{CHAPTER 4}

\section{The Pliocene}

\subsection{PLIOCENE MAMMALS}

By the Pliocene, all Orders were extant and only four extinct families remained, two in the Proboscidea and one each in the Carnivora and the Perissodactyla (odd-toed ungulates). However, at lower taxonomic ranks, there remained a high proportion of extinct taxa, e.g. 50 per cent of genera and 69 per cent of species in the Carnivora, and 47 per cent and 73 per cent respectively in the Artiodactyla (see Table 7.1). The Tubulidentata (aardvarks) and Soricomorpha were not represented and the number of Rodentia families was at its lowest level. Conversely, the number of Carnivora families increased to seven, its highest at any time, making it the most diverse Order during the epoch. The number of genera rose to 28 and species more than trebled compared to the Miocene, while the number of Artiodactyla genera was double that in the Miocene. Although there were fewer Rodentia families, those present contained far fewer extinct genera, and more species overall.

\section{ORDER: AFROSORICIDA \\ Suborder: Chrysochloridea \\ FAMILY: CHRYSOCHLORIDAE \\ Subfamily: Chrysochlorinae}

†Chrysochloris arenosa Asher and Avery, 2010. Palaeontol. Electr. 13(1)

3A: 5.

Type locality: Langebaanweg.

†Chrysochloris bronneri Asher and Avery, 2010. Palaeontol. Electr. 13(1) 3A: 8.

Type locality: Langebaanweg.
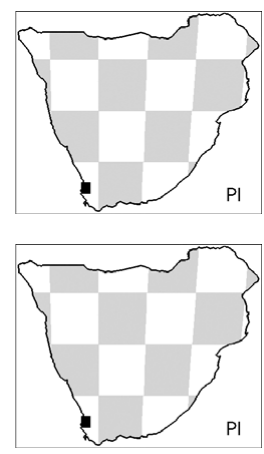

Subfamily: Amblysomyinae

†Amblysomus hamiltoni De Graaff, 1958. Palaeontol. Afr. 5: 22. Synonyms: Chrysosticha.

Type locality: Limeworks Makapansgat dumps.

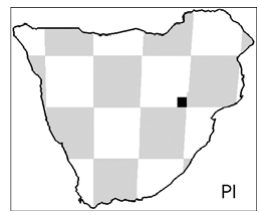




\section{ORDER: MACROSCELIDEA}

FAMILY: MACROSCELIDIDAE

Subfamily: Macroscelidinae

†Elephantulus antiquus Broom, 1948. Ann. Transvaal Mus. 21(1): 5.

Type locality: Bolt's Farm.

Additional references: Patterson (1965).

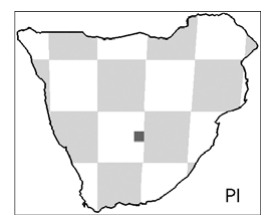

Elephantulus brachyrhynchus Smith, 1836. Report of the Expedition for Exploring Central Africa: 42. Short-snouted elephant shrew.

Synonyms: Macroscelides.

Type locality: Kuruman to southern Botswana.

Additional references: Smith (1849).

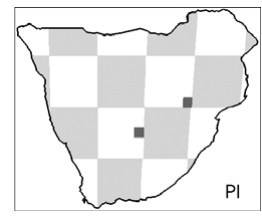

†Elephantulus broomi Corbet and Hanks, 1968. Bull. Brit. Mus. Nat. Hist. Zool. 16: 54.

Synonyms: Elephantomys langi.

Type locality: Schurveberg.

Elephantulus fuscus Peters, 1851. Reise nach Mossambique, Säugethiere:

87. Dusky elephant shrew.

Type locality: Boror.
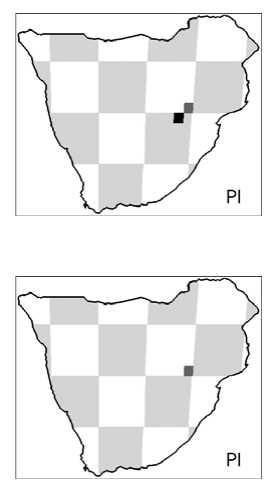

Macroscelides proboscideus Shaw, 1800. Gen. Zool. Syst. Nat. Hist. 1(2)

Mammalia: 536. Short-eared elephant shrew.

Synonyms: typicus.

Additional references: Roberts $(1933,1938)$.

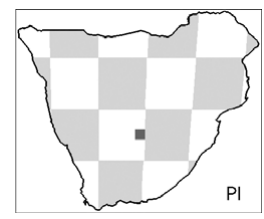

†Palaeothentoides africanus Stromer, 1931. Sitzungsber. Bayer. Akad.

Wiss. München. Math.-Nat. Abt. 1931: 185.

Type locality: Kleinzee.

Additional references: Holroyd (2010a); Patterson (1965).

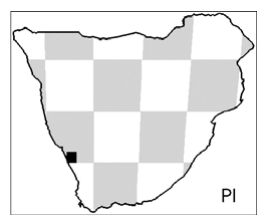

Subfamily: Mylomygalinae

†Mylomygale spiersi Broom, 1948. Ann. Transvaal Mus. 21(1): 8.

Type locality: Taung.

Additional references: Holroyd (2010a); Patterson (1965).

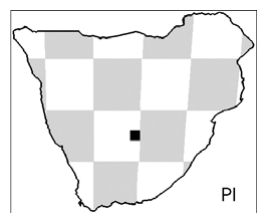


ORDER: HYRACOIDEA

FAMILY: PROCAVIIDAE

†Gigantohyrax maguirei Kitching, 1965. Palaeontol. Afr. 9: 91.

Type locality: Limeworks Makapansgat.

Additional references: Rasmussen and Gutiérrez (2010).

†Procavia antiqua Broom, 1934. S. Afr. J. Sci. 31: 472.

Synonyms: Prohyrax; robertsi.

Type locality: Taung.

Additional references: Broom (1948a); Churcher (1956); McMahon and

Thackeray (1994); Rasmussen and Gutiérrez (2010); Schwartz (1997).

†Procavia pliocenica Pickford, 2005. Palaeontol. Afr. 41: 142.

Type locality: Langebaanweg.
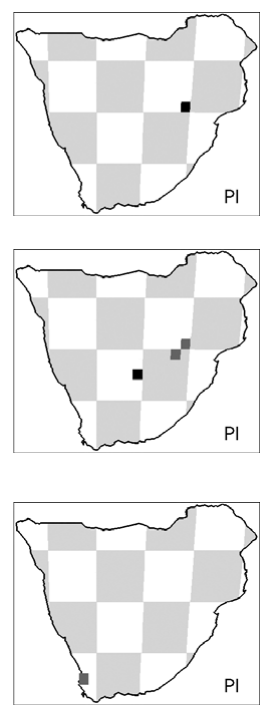

†Procavia transvaalensis Shaw, 1937. J. Dental Res. 16: 40.

Synonyms: obermeyerae.

Type locality: Taung.

Additional references: Churcher (1956); McMahon and Thackeray

(1994).

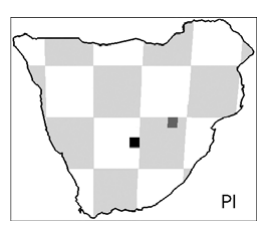

ORDER: PROBOSCIDEA

FAMILY: †DEINOTHERIIDAE

Subfamily: †Deinotheriinae

†Prodeinotherium hobleyi Andrews, 1911. Proc. Zool. Soc. Lond. 1911:

944.

Synonyms: Deinotherium; Dinotherium.

Additional references: Éhik (1930); Harris (1977); Sanders et al. (2010a).

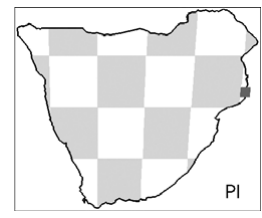

FAMILY: ELEPHANTIDAE

Subfamily: Elephantinae

Elephas Linnaeus, 1758. Systema Naturae Regnum Animale, 10th edition, 1: 33.

Synonyms: Archidiskodon; Palaeoloxodon.

Additional references: Cooke (1961); Todd (2010).

†Loxodonta cookei Sanders, 2007. Trans. R. Soc. S. Afr. 62(1): 7.

Synonyms: Lukeino Stage.

Type locality: Langebaanweg.

Additional references: Pickford and Senut (1997); Sanders et al. (2010a);

Todd (2010).

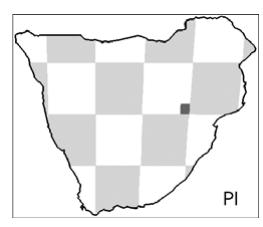


†Mammuthus subplanifrons Osborn, 1928. Nature 121: 672-673. Synonyms: Archidiskodon; Elephas; Mastodon; Stegodon; andrewsi; milletti; proplanifrons; scotti; vanalpheni.

Type locality: Sydney-on-Vaal.

Additional references: Dart (1929b); Maglio and Hendey (1970); Meiring (1955); Osborn (1934, 1942); Sanders (2007); Sanders et al. (2010a); Todd (2010).

\section{FAMILY: †GOMPHOTHERIIDAE Subfamily: †Anancinae}

†Anancus capensis Sanders, 2007. Trans. R. Soc. S. Afr. 62(1): 1. Type locality: Langebaanweg.

Additional references: Sanders et al. (2010a).

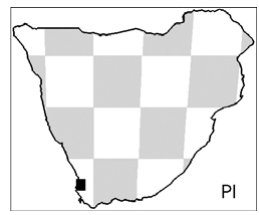

\section{ORDER: PRIMATES \\ Suborder: Haplorrhini \\ FAMILY: CERCOPITHECIDAE \\ Subfamily: Cercopithecinae}

Cercocebus Geoffroy Saint-Hilaire, 1812. Ann Mus. Hist. Nat. Paris 19: 97. Additional references: Jablonsky and Frost (2010).

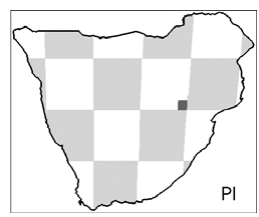

†Dinopithecus ingens Broom, 1937. S. Afr. J. Sci. 33: 753.

Synonyms: Papio.

Type locality: Schurveberg.

Additional references: Freedman and Brain (1977); Gilbert (2013);

Gommery and Bento da Costa (2016); Jablonsky and Frost (2010).

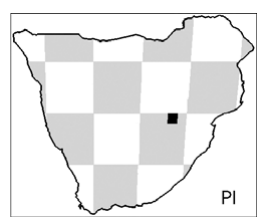

†Papio izodi Gear, 1926. S. Afr. J. Sci. 23: 746.

Synonyms: Gorgopithecus; Parapapio; angusticeps; wellsi.

Type locality: Taung.

Additional references: Adams et al. (2013); Cooke (1990); Freedman

(1965); Gilbert (2013); Gilbert et al. (2015); Gilbert et al. (2016b);

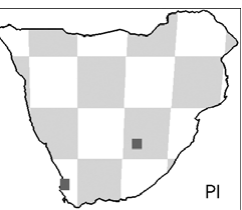

Heaton (2006); Jablonsky and Frost (2010); McKee (1993b); McKee and Keyser (1994).

Comments: known material from Kromdraai is referred to Papio (hamadryas) angusticeps and a new specimen to Papio hamadryas ssp. indet. by Singleton et al. (2016). 
†Parapapio Jones, 1937. S. Afr. J. Sci. 33: 726.

Type locality: Sterkfontein.

Additional references: Grine and Hendey (1981); Jablonsky and Frost

(2010).

†Parapapio broomi Jones, 1937. S. Afr. J. Sci. 33: 727.

Synonyms: Brachygnathopithecus peppercorni; Gorgopithecus; makapani. Type locality: Sterkfontein.

Additional references: Freedman $(1965,1976)$; Freedman and Stenhouse (1972); Gilbert (2013); Gommery and Bento da Costa (2016); Heaton (2006); Jablonsky and Frost (2010); Maier (1970); Thackeray and Myer (2004).

†Parapapio jonesi Broom, 1940. Ann. Transvaal Mus. 20: 93.

Type locality: Sterkfontein.

Additional references: Freedman and Brain (1977); Freedman and

Stenhouse (1972); Gilbert (2013); Heaton (2006); Jablonsky and Frost (2010); Maier (1970); Thackeray and Myer (2004).

†Parapapio whitei Broom, 1940. Ann. Transvaal Mus. 20: 90.

Type locality: Sterkfontein.

Additional references: Freedman (1965); Freedman and Stenhouse

(1972); Gilbert (2013); Heaton (2006); Jablonsky and Frost (2010);

Maier (1970).

†Procercocebus antiquus Haughton, 1924. Trans. R. Soc. S. Afr. 12: Ixviii. Synonyms: Gorgopithecus; Papio; Parapapio.

Type locality: Taung.

Additional references: Cooke (1990); Freedman (1965, 1976); Gilbert

(2007a, 2013); Gilbert et al. (2016b); Gommery and Bento da Costa (2016); Heaton (2006); Maier (1971).

†Theropithecus darti Broom and Jensen, 1946. Ann. Transvaal Mus. 20: 340.

Synonyms: Papio; Simopithecus.

Type locality: Limeworks Makapansgat.

Additional references: Gilbert (2013); Jablonsky and Frost (2010); Maier (1970, 1972).

Comments: Gilbert (2013) accepts the case for making this species a subspecies of T. oswaldi though Gommery and Bento da Costa (2016) continue to consider it a full species.

†Theropithecus oswaldi Andrews, 1916. Ann. Mag. Nat. Hist., Series 8, 18: 417.

Synonyms: Parapapio coronatus?; Simopithecus; danieli; leakeyi.

Additional references: Broom and Robinson (1948); Dechow and Singer (1984); Freedman (1957, 1976); Freedman and Brain (1977); Frost
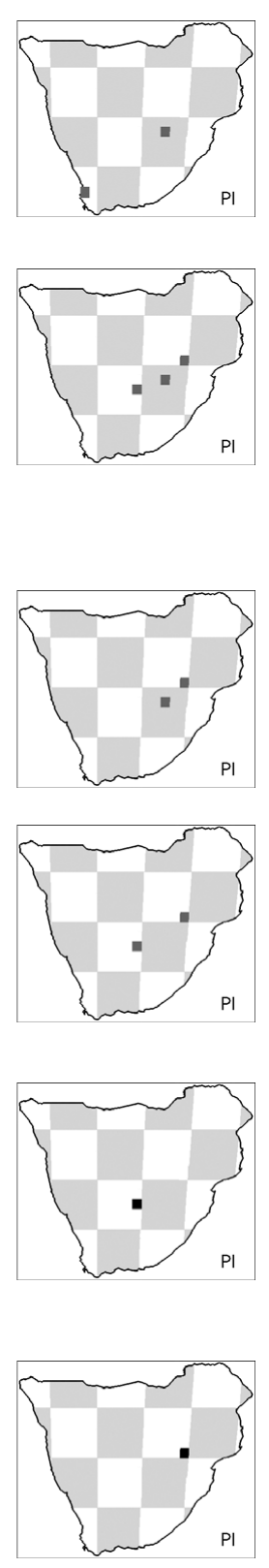

et al. (2017); Gilbert (2007b, 2013); Heaton (2006); Hopwood (1934); Jablonsky and Frost (2010); Leakey (1943a). 


\section{Subfamily: Colobinae}

†Cercopithecoides williamsi Mollett, 1947. S. Afr. J. Sci. 43: 298.

Synonyms: molletti.

Type locality: Limeworks Makapansgat.

Additional references: Freedman (1961, 1965); Freedman and Brain

(1977); Gommery and Bento da Costa (2016); Jablonsky and

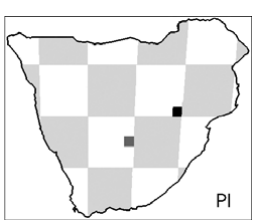

Frost (2010); Kuykendall and Rae (2008); Maier (1970); Von Mayer (1998).

\section{FAMILY: HOMINIDAE}

Subfamily: Homininae

†Australopithecus Dart, 1925. Nature 114: 195-199.

Type locality: Taung.

Additional references: Clarke (1999, 2002, 2008).

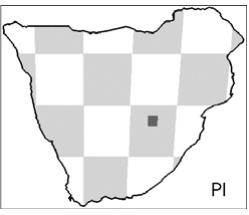

†Australopithecus africanus Dart, 1925. Nature 114: 195-199.

Synonyms: Plesianthropus; prometheus; transvaalensis.

Type locality: Taung.

Additional references: Broom (1929, 1939a); Broom and Robinson

(1949a, 1949c); Clarke and Tobias (1995); Dart (1929a, 1948a, 1954,

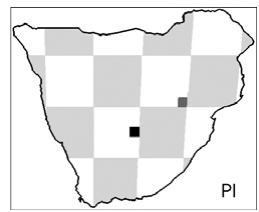

1959); Häusler and Berger (2001); Lockwood and Tobias (1999,

2002); MacLatchy et al. (2010); Toussaint et al. (2003).

\section{ORDER: RODENTIA \\ Suborder: Myomorpha \\ FAMILY: NESOMYIDAE}

Subfamily: Delanymyinae

†Stenodontomys darti Lavocat, 1956. Palaeontol. Afr. 4: 71.

Synonyms: Mystromys.

Type locality: Limeworks Makapansgat.

†Stenodontomys saldanhae Pocock, 1987. Palaeontol. Afr. 26(7): 89.

Type locality: Langebaanweg.

Additional references: Denys (1994b).
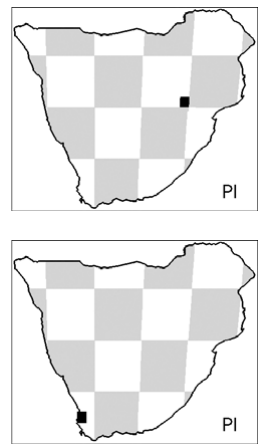

Subfamily: Dendromurinae

†Dendromus antiquus Broom, 1946. Mem. Transvaal Mus. 2: 29.

Type locality: Taung.

Comments: originally named by Broom (1946) but not formally described according to De Graaff (1961a).

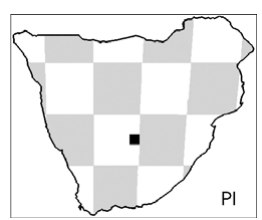


†Dendromus averyi Denys, 1994. Palaeovertebrata 23(1-4): 163. Type locality: Langebaanweg.

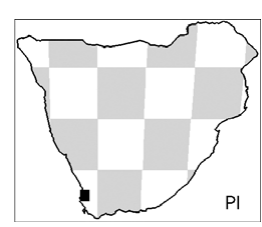

†Dendromus darti Denys, 1994. Palaeovertebrata 23(1-4): 163. Type locality: Langebaanweg.

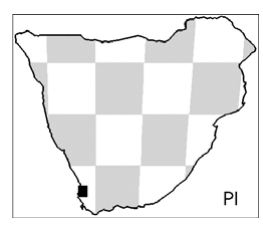

Dendromus mesomelas Brants, 1827. Het Geslacht der Muizen door Linnaeus Opgesteld: 122. Brants' African climbing mouse.

Synonyms: ayresi; typica.

Type locality: Sunday's River, east of Port Elizabeth.

Additional references: Smith (1849).

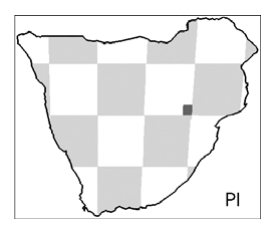

†Malacothrix makapani De Graaff, 1961. Palaeontol. Afr. 7: 86.

Type locality: Limeworks Makapansgat.

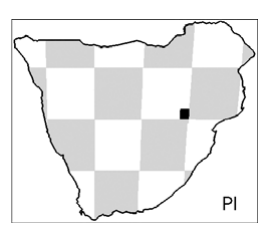

Malacothrix typica Smith, 1834. S. Afr. Quart. J., Series 2, 2: 148. Largeeared African desert mouse.

Type locality: Graaff Reinet District.

Additional references: Roberts (1932).

Steatomys pratensis Peters, 1846. Bericht Verhandl. K. Preuss. Akad. Wiss. Berlin 11: 258. Common African fat mouse.

Synonyms: natalensis; opimus.

Type locality: Tete.

Comments: material from Botswana was identified as Steatomys opimus

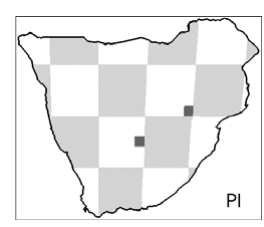
but, as currently understood, this species is almost entirely confined to West Africa (Musser and Carleton 2005).

\section{Subfamily: Mystromyinae}

Mystromys albicaudatus Smith, 1834. S. Afr. Quart. J., Series 2, 2: 148. African white-tailed rat. Synonyms: Otomys; antiquus.

Type locality: Albany District.

Additional references: De Winton (1898); Grubb (2004); Smith (1849).

†Mystromys hausleitneri Broom, 1937. S. Afr. J. Sci. 33: 766.

Synonyms: hauslichtneri.

Type locality: Schurveberg.

Additional references: Broom (1948a).

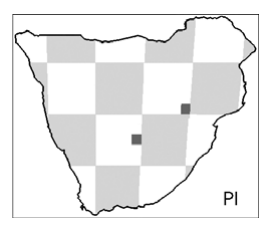


†Mystromys pocockei Denys, 1991. C. R. Acad. Sci. Paris, Série lla, 313: 1337.

Type locality: Langebaanweg.

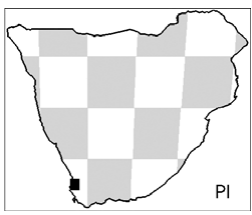

†Proodontomys cookei Pocock, 1987. Palaeontol. Afr. 26(7): 82.

Type locality: Limeworks Makapansgat.

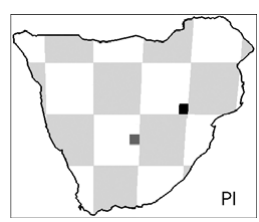

FAMILY: MURIDAE

Subfamily: Deomyinae

Acomys Geoffroy Saint-Hilaire, 1838. Ann. Sci. Nat. Zool. Paris, Series 2, 10: 126.

Additional references: Chevret et al. (1993a); Dippenaar and Rautenbach (1986).

†Acomys mabele Denys, 1990. Palaeontographica Abt. A 210(1-3): 82. Type locality: Langebaanweg.

Acomys spinosissimus Peters, 1852. Naturwissenschaftliche Reise nach Mossambique: 160 . Southern African spiny mouse.

Synonyms: Cahirinus selousi; transvaalensis.

Type locality: Tete and Buio.

Additional references: Dippenaar and Rautenbach (1986).
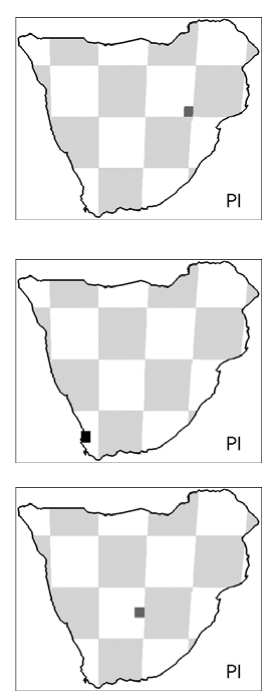

Subfamily: Gerbillinae

Desmodillus Thomas and Schwann, 1904. Abstr. Proc. Zool. Soc. Lond. 1904(2): 6.

Synonyms: Gerbillus; Pachyuromys.

Additional references: Pavlinov (2001).

Desmodillus auricularis Smith, 1834. S. Afr. Quart. J., Series 2, 2: 160.

Short-eared gerbil.

Synonyms: Gerbillus; brevicaudatus.

Type locality: Kamiesberg.

Additional references: Griffin (1990); Qumsiyeh (1986); Smith (1849).

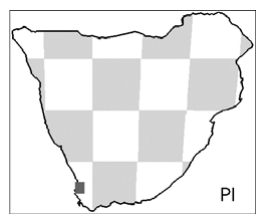

†Desmodillus magnus Denys and Matthews, 2017. Palaeovertebrata

41(1): e1-5.

Type locality: Langebaanweg.
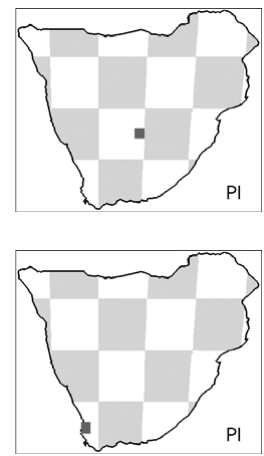
Gerbilliscus Thomas, 1897. Proc. Zool. Soc. Lond. 1897: 433. Synonyms: Gerbillus; Tatera.

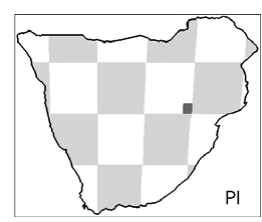

Gerbilliscus brantsii Smith, 1836. Report of the Expedition for Exploring Central Africa: 43. Highveld gerbil.

Synonyms: Gerbillus; Tatera; montanus.

Type locality: Ladybrand.

Additional references: Davis (1949, 1965); Griffin (1990); Qumsiyeh

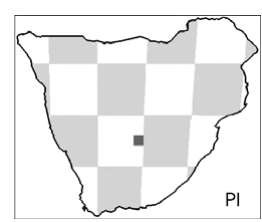

(1986); Roberts (1926); Smith (1849).

Subfamily: Murinae

†Aethomys adamanticola Denys, 1990. Ann. Paleontol. 76(1): 45.

Type locality: Langebaanweg.

Additional references: Matthews and Stynder (2011a).

†Aethomys modernis Denys, 1990. Ann. Paleontol. 76(1): 52.

Type locality: Langebaanweg.

Additional references: Matthews and Stynder (2011a).

Dasymys incomtus Sundevall, 1846. Vetenskaps-Akademiens Förhandlingar 3: 120. Common dasymys.

Type locality: Durban.

Additional references: Gordon (1991); Mullin et al. (2004).

Comments: much of the material identified as D. incomtus was identified
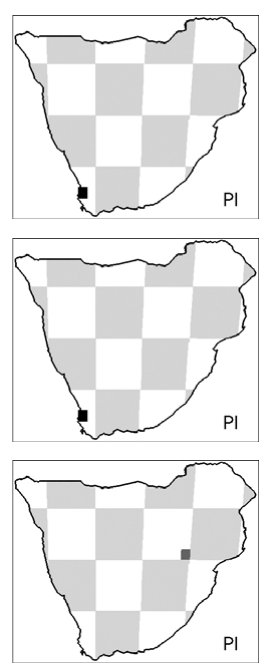
at a time when this species was the only one recognised in southern

Africa. For this reason, this material should be considered as

D. incomtus sensu lato until such time as it is re-examined.

†Dasymys 'lavocati' Broom, unpublished.

Type locality: Bolt's Farm.

Comments: Nomen nudum.

Grammomys dolichurus Smuts, 1832. Dissertation Zoologica,

Ennumerationem Mammalium Capensium: 38. Common grammomys. Synonyms: Thamnomys.

Type locality: near Cape Town.

Additional references: Roberts (1931).

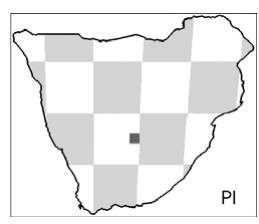

Lemniscomys Troussaert, 1881. Bull. Soc. Etudes Sci. Angers 10: 124.
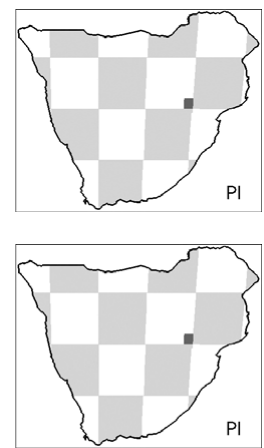
Mastomys natalensis Smith, 1834. S. Afr. Quart. J., Series 2, 2: 146. Natal mastomys.

Synonyms: Praomys.

Type locality: Port Natal = Durban.

Additional references: Bronner et al. (2007); Dippenaar et al. (1993); Green et al. (1980); Smit and Van der Bank (2001); Smith (1849).

Comments: this material was identified as $M$. natalensis at a time when $M$. coucha was not recognised as a separate species.

Micaelamys namaquensis Smith, 1834. S. Afr. Quart. J., Series 2, 2: 160. Namaqua micaelamys.

Synonyms: Aethomys; lehocla.

Type locality: Cape of Good Hope: restricted to Witwater.

Additional references: Chimimba (2001); Chimimba and Dippenaar (1994); Roberts (1926, 1946); Russo (2009); Smith (1849); Visser and Robinson (1986).

Mus minutoides Smith, 1834. S. Afr. Quart. J., Series 2, 2: 147. Southern African pygmy mouse.

Synonyms: Leggada.

Type locality: Cape Town.

Additional references: Britton-Davidian et al. (2012).

Comments: some of the specimens from the more arid areas may be $M$. indutus, but see comment under that species.

Myomyscus Shortridge, 1942. Ann. S. Afr. Mus. 36: 93.

Synonyms: Praomys; Myomys.

Type locality: Kliphuis.

Pelomys fallax Peters, 1852. Bericht Verhandl. K. Preuss. Akad. Wiss. Berlin

17: 275. East African pelomys.

Type locality: Caia District.

Rhabdomys Thomas, 1916. Ann. Mag. Nat. Hist., Series 8, 18: 69.
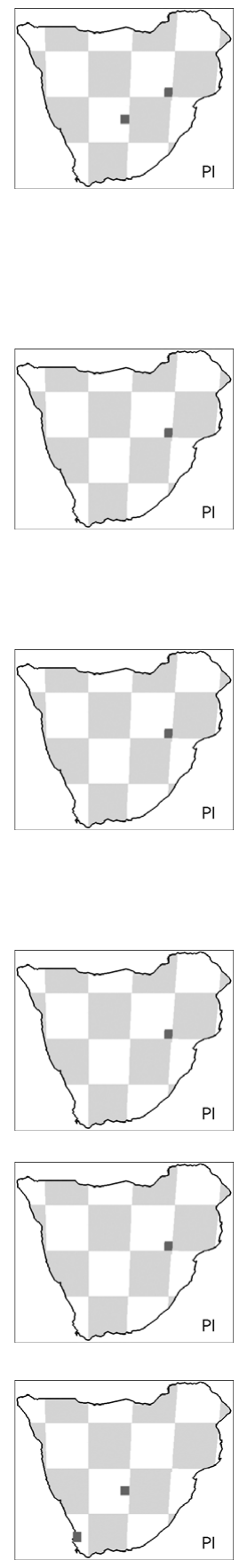

Rhabdomys pumilio Sparrman, 1784. K. Svenska Vet.-Akad. Handl.:

236. Xeric four-striped grass rat.

Type locality: Slangrivier, east of Knysna.

Additional references: Castiglia et al. (2012); Le Grange et al. (2015);

Roberts (1946); Smith (1849); Wroughton (1905).

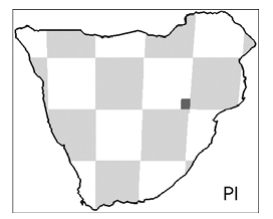

Comments: samples recorded here should be regarded as belonging to Rhabdomys pumilio sensu lato and are very likely to include some material that would currently be regarded as $R$. dilectus. 
Thallomys Thomas, 1920. Ann. Mag. Nat. Hist., Series 9, 5: 141.

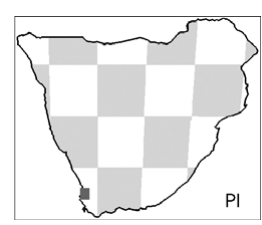

†Thallomys debruyni Broom, 1948. Ann. Transvaal Mus. 21: 35. Type locality: Taung Hrdlicka's Cave.

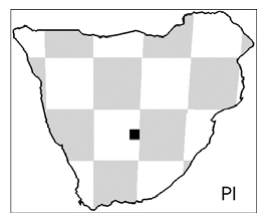

Zelotomys Osgood, 1910. Field Mus. Nat. Hist. Publ. Zool. Ser. 10: 7.

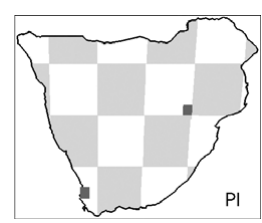

Subfamily: $†$ Myocricetodontinae

†Boltimys broomi Sénégas and Michaux, 2000. C. R. Acad. Sci. Paris, Sci.

Terre Plan. 330: 522.

Type locality: Waypoint 160.

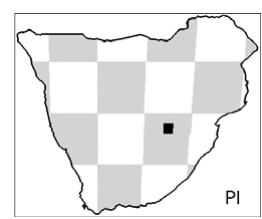

Subfamily: Otomyinae

†Euryotomys bolti Sénégas and Avery, 1998. S. Afr. J. Sci. 94: 503.

Type locality: Waypoint 160.

Additional references: Sénégas (2001).

†Euryotomys pelomyoides Pocock, 1976. S. Afr. J. Sci. 72: 58.

Type locality: Langebaanweg.

Additional references: Denys et al. (1987).
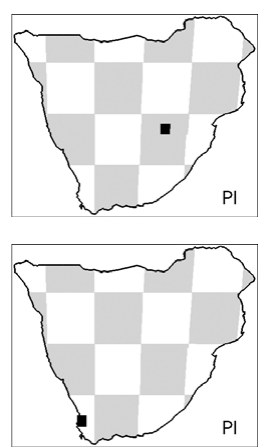

†Myotomys campbelli Broom and Schepers, 1946. Transvl Mus. Mem. 2: 29.

Type locality: Taung.

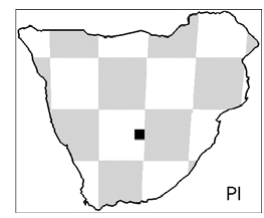

Myotomys sloggetti Thomas, 1902. Ann. Mag. Nat. Hist., Series 7, 10:

311. Rock karoo rat.

Synonyms: Otomys.

Type locality: Deelfontein.

Comments: it seems unlikely that the Pliocene record is correct.

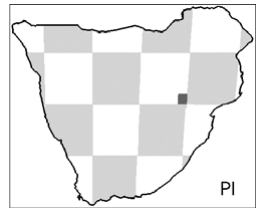

Additional references: Taylor et al. (2004). 
†Otomys gracilis Broom, 1937. S. Afr. J. Sci. 33: 761.

Synonyms: Palaeotomys.

Type locality: Schurveberg.

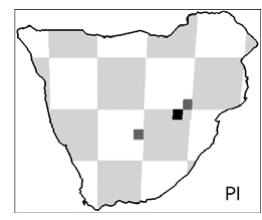

\section{Suborder: Anomaluromorpha FAMILY: PEDETIDAE}

Pedetes capensis Forster, 1778. K. Svenska Vet.-Akad. Handl. 39: 109.

South African spring hare.

Type locality: Cape of Good Hope.

Additional references: Roberts (1946).

†Pedetes gracilis Broom, 1934. S. Afr. J. Sci. 31: 476.

Type locality: Taung.

Additional references: Pickford and Mein (2011).

†Propedetes efeldensis Mein and Pickford, 2008. Geol. Surv. Namibia Mem. 20: 242.

Type locality: Elisabethfeld.

†Propedetes Iaetoliensis Davies, 1987. In: Leakey and Harris. The Pliocene

Site of Laetoli, Northern Tanzania: 172.

Synonyms: Pedetes.

Additional references: Pickford and Mein (2011).
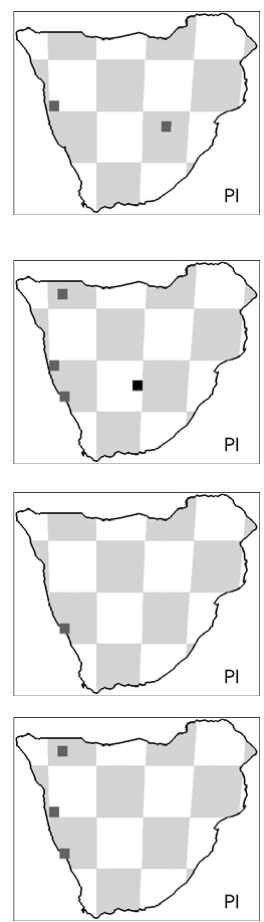

\section{Suborder: Hystricomorpha}

FAMILY: BATHYERGIDAE

Subfamily: Bathyerginae

†Bathyergus hendeyi Denys, 1998. Ann. S. Afr. Mus. 105(5): 268.

Type locality: Langebaanweg.

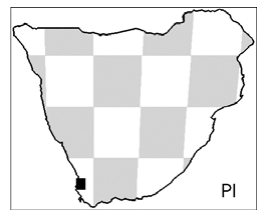

†Cryptomys broomi Denys, 1998. Ann. S. Afr. Mus. 105(5): 280.

Type locality: Langebaanweg.

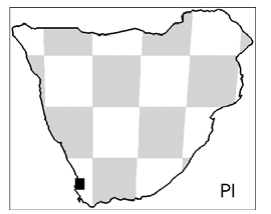

Cryptomys hottentotus Lesson, 1826. Zool. 1: 166. Southern African mole-rat.

Type locality: near Paarl.

Additional references: Avery (2004); Denys (1988a); Roberts (1913, 1946).

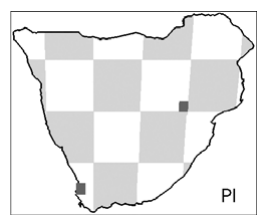


Comments: it is possible that some of the material may be referable to Fukomys damarensis, which was not recognised as a separate species when many of the samples were identified.

†Cryptomys robertsi Broom, 1937. S. Afr. J. Sci. 33: 760.

Type locality: Schurveberg.

Comments: it has been suggested that this taxon is inseparable from Georychus capensis (Avery, 1998).

†Gypsorhychus darti Broom, 1934. S. Afr. J. Sci. 31: 474.

Type locality: Taung.
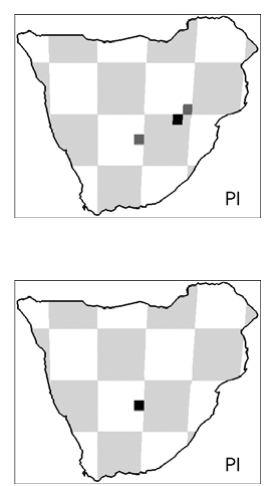

†Gyporhychus makapani Broom, 1948. Ann. Transvaal Mus. 21: 49. Type locality: Limeworks Makapansgat.

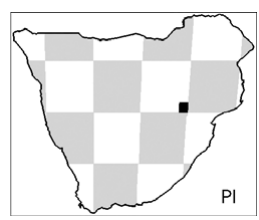

†Gypsorhychus minor Broom, 1948. Ann. Transvaal Mus. 21: 48. Type locality: Taung.

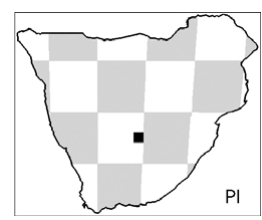

Heterocephalus Rüppell, 1842. Mus. Senckenbergianum 3(2): 99.

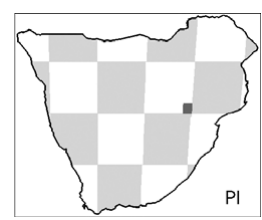

FAMILY: HYSTRICIDAE

Subfamily: Hystricinae

Hystrix africaeaustralis Peters, 1852. Naturwissenschaftliche Reise nach Mossambique: 170. Cape porcupine.

Additional references: Maguire (1976).

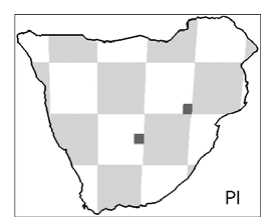

†Hystrix makapanensis Greenwood, 1958. Ann. Mag. Nat. Hist. 13: 365. Synonyms: major.

Type locality: Limeworks Makapansgat.

Additional references: Adams (2012a); Greenwood (1955).

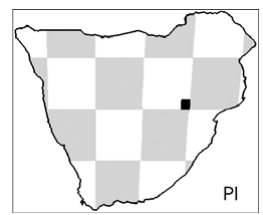


†Xenohystrix crassidens Greenwood, 1955. Palaeontol. Afr. 3: 81. Type locality: Limeworks Makapansgat.

Additional references: Collings et al. (1976).

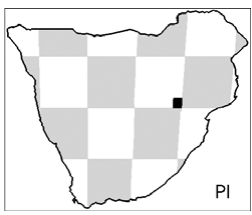

FAMILY: PETROMURIDAE

†Petromus antiquus Sénégas, 2004. J. Vert. Paleontol. 24(3): 757.

Type locality: Waypoint 160.

†Petromus minor Broom, 1939. Ann. Transvaal Mus. 19: 316.

Synonyms: Palaepetromys.

Type locality: Taung.
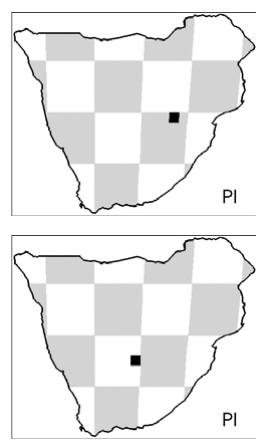

\section{ORDER: LAGOMORPHA}

FAMILY: LEPORIDAE

Lepus Linnaeus, 1758. Systema Naturae Regnum Animale, 10th edition, 1: 57.

Additional references: Robinson and Dippenaar (1987); Robinson and Matthee (2005).

Lepus capensis Linnaeus, 1758. Systema Naturae Regnum Animale, 10th edition, 1: 58. Cape hare.

Type locality: Cape of Good Hope.

Additional references: Roberts (1932).

Pronolagus Lyon, 1904. Smithson. Misc. Coll. 45: 416.

Additional references: Robinson and Matthee (2005).
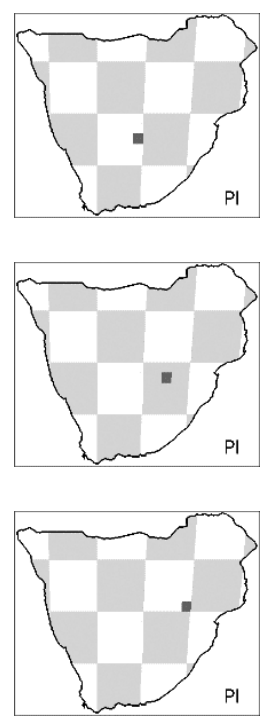

\section{ORDER: SORICOMORPHA}

\section{FAMILY: SORICIDAE}

Subfamily: Crocidurinae

Crocidura fuscomurina Heuglin, 1865. Nouv. Acta Acad. Caes. Leop.-

Carol. 32: 36. Bicolored musk shrew.

Synonyms: bicolor.

Additional references: Hutterer (1983).

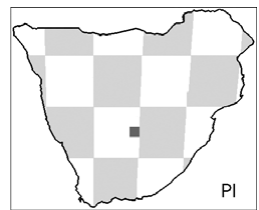


†Crocidura taungensis Broom, 1948. Ann. Transvaal Mus. 21: 10. Synonyms: Suncus.

Type locality: Taung.

Additional references: Meester (1954).

†Diplomesodon fossorius Repenning, 1965. J. Mammal. 46(2): 190. Type locality: Limeworks Makapansgat.

Suncus infinitesimus Heller, 1912. Smithson. Misc. Coll. 60(12): 5. Least dwarf shrew.
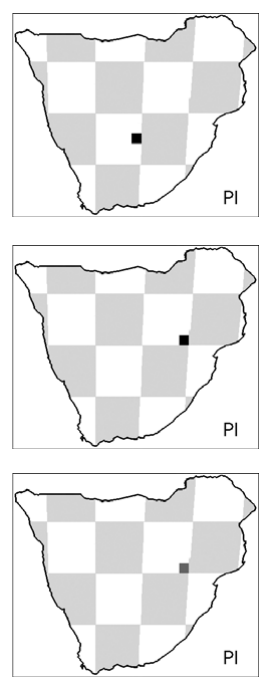

Suncus varilla Thomas, 1895. Ann. Mag. Nat. Hist., Series 6, 16: 54.

Lesser dwarf shrew.

Type locality: East London.

Additional references: Roberts (1946).

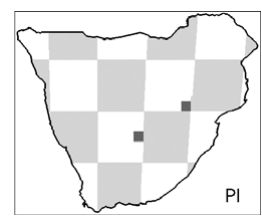

Subfamily: Myosoricinae

†Myosorex robinsoni Meester, 1954. Ann. Transvaal Mus. 22: 272. Type locality: Swartkrans.

Additional references: Butler (2010); Butler and Greenwood (1979).

Myosorex varius Smuts, 1832. Dissertation Zoologica, Ennumerationem Mammalium Capensium: 108. Forest shrew.

Synonyms: Sorex.

Type locality: Algoa Bay: Port Elizabeth.

Additional references: Roberts (1924); Smith (1849).
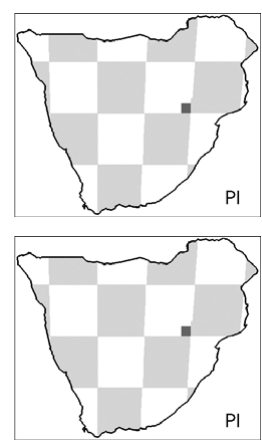

\section{ORDER: CHIROPTERA Suborder: Microchiroptera \\ FAMILY: RHINOLOPHIDAE}

Rhinolophus capensis Lichtenstein, 1823. Verzeichniss der Doubletten des zoologischen Museums der Königl. Universität zu Berlin: 4. Cape horseshoe bat.

Type locality: Cape of Good Hope.

Rhinolophus clivosus Cretzschmar, 1826. In: Rüppell, Atlas zu der Reise im nördlichen Afrika Zoologie: 47. Geoffroy Saint-Hilaire's horseshoe bat. Synonyms: geoffroyi.
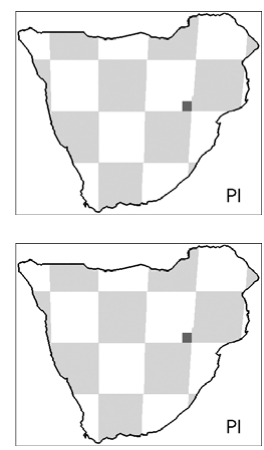
Rhinolophus darlingi Andersen, 1905. Ann. Mag. Nat. Hist., Series 7, 15:

70. Darling's horseshoe bat.

Type locality: Mazoe.

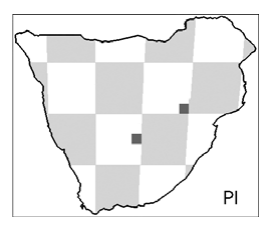

FAMILY: VESPERTILIONIDAE

Subfamily: Vespertilioninae

Eptesicus hottentotus Smith, 1833. S. Afr. Quart. J., Series 2, 1: 59.

Long-tailed serotine.

Type locality: Uitenhage.

Additional references: Hill and Harrison (1987); Kearney et al. (2002).

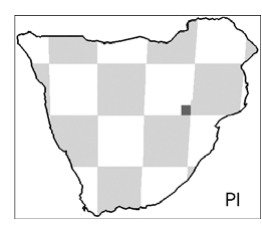

Subfamily: Miniopterinae

Miniopterus Bonaparte, 1837. Iconografia della fauna italica: 20.

Additional references: Miller-Butterworth et al. (2007).

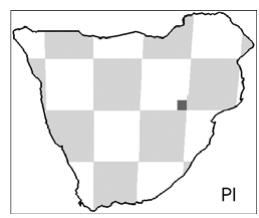

\section{ORDER: PHOLIDOTA}

FAMILY: MANIDAE

Subfamily: Smutsiinae

†Smutsia gigantea Illiger, 1815. Abh. Phys. Klasse K. Pruess Konigl. Akad.

Wiss. 1804-1811: 84.

Synonyms: Manis.

Additional references: Botha and Gaudin (2007); Gaudin (2010).

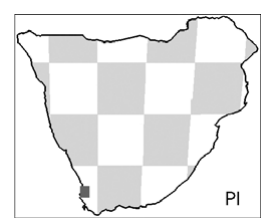

\section{ORDER: CARNIVORA \\ Suborder: Feliformia}

FAMILY: FELIDAE

†Amphimachairodus Kretzoi, 1929. 10th International Congress of Zoology: 1316.

Synonyms: Homotherium; Machairodus.

Additional references: Sardella and Werdelin (2007); Werdelin and

Peigné (2010); Werdelin and Sardella (2006).

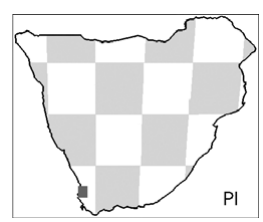

Subfamily: Felinae

Acinonyx Brookes, 1828. A Catalogue of the Anatomical \& Zoological Museums of Joshua Brookes, Esq., F.R.S., F.L.S. etc. Part 1: 16, 33.

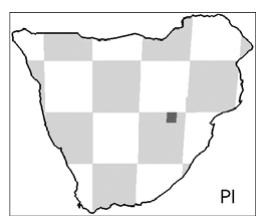


Caracal caracal Schreber, 1776. Die Säugethiere 3 (16): pl. 110 [1776], see also text 3 (24): 413, 587 [1777]. Caracal.

Synonyms: Felis.

Type locality: Table Mountain, near Cape Town.

Additional references: Roberts (1926); Werdelin and Peigné (2010).

Comments: citation according to Wilson and Reeder (2005). Volume 3 was published in 1778 according to the version reproduced in the Biodiversity Heritage Library (www.biodiversitylibrary.org/item/ 135003\#page/141/mode/1 up) and Felis caracal is listed on p. 587. Plate 110 appears in an undated volume containing plates 81-165 (www.biodiversitylibrary.org/item/97341\#page/131/mode/1up).

†Dinofelis barlowi Broom, 1937. S. Afr. J. Sci. 33: 757.

Synonyms: Machaerodus; Megantereon; Therailurus; darti; transvaalensis. Type locality: Sterkfontein.

Additional references: Collings (1973); Collings et al. (1976);

Cooke (1991); Ewer (1955c, 1957a); Hemmer (1965); Lacruz et al. (2006).

†Dinofelis diastemata Astre, 1929. Bull. Soc. Géol. France 29: 203. Additional references: Lacruz et al. (2006).

†Felis issiodorensis Croizet and Jobert, 1828. Recherches sur les ossemens fossiles du Département du Puy-de-Dome: 200.

†Homotherium crenatidens Weithofer, 1889. Jahrbuch der Kaiserlich Königlichen Geologischen Reichsanstalt 39(1): 65.

Synonyms: Machairodus; altidens.

Comments: there appears to be disagreement as to which species of Homotherium is represented at Sterkfontein: Turner (1987a) considers the material to be $H$. crenatidens, whereas Reynolds (2010b) assigns it to H. altidens.

†Homotherium problematicum Collings, 1972. Palaeontol. Afr. 14: 87. Synonyms: Megantereon; eyrnodon, nestianus.

Type locality: Limeworks Makapansgat.

Additional references: Berta and Galiano (1983); Collings (1973);

Collings et al. (1976); Ewer (1955c).
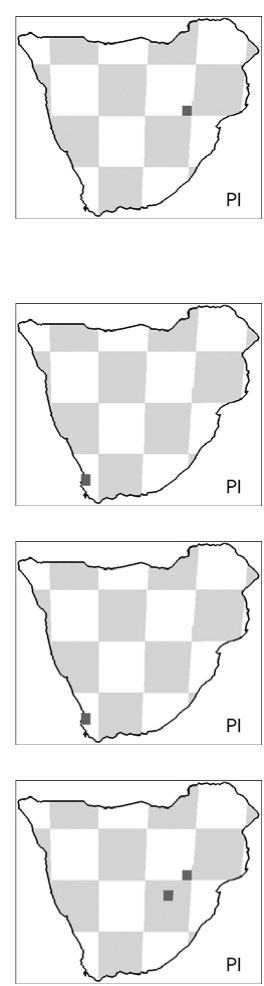

Leptailurus serval Schreber, 1776. Die Säugethiere 3 (16): pl. 108 [1776], see also text 3 (23): 407 [1777]. Serval.

Synonyms: Felis; spelaeus.

Type locality: restricted to the 'Cape region of South Africa'.

Additional references: Werdelin and Peigné (2010).
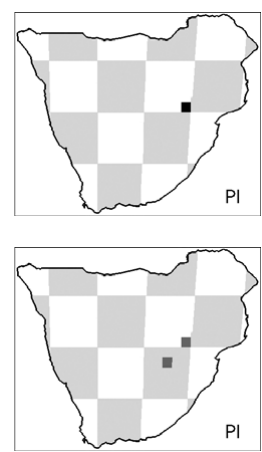
Comments: citation according to Wilson and Reeder (2005). Volume 3 was published in 1778 according to the version reproduced in the Biodiversity Heritage Library (www.biodiversitylibrary.org/item/ 135003\#page/135/mode/1 up) and Felis serval is listed on p. 587. Plate 107 appears in an undated volume containing plates 81-165 (www.biodiversitylibrary.org/item/97341\#page/125/mode/1 up).

†Metailurus obscurus Hendey, 1974. Ann. S. Afr. Mus. 63: 164.

Synonyms: Felis; Adelphailurus; Megantereon obscura.

Type locality: Langebaanweg.

Additional references: Werdelin and Peigné (2010).

Subfamily: †Machairodontinae

†Megantereon whitei Broom, 1937. S. Afr. J. Sci. 33: 756.

Synonyms: Felis; cultridens.

Type locality: Schurveberg.

Additional references: Broom (1939c, 1946); Hartstone-Rose et al.

(2007); Turner (1987b); Werdelin and Peigné (2010).
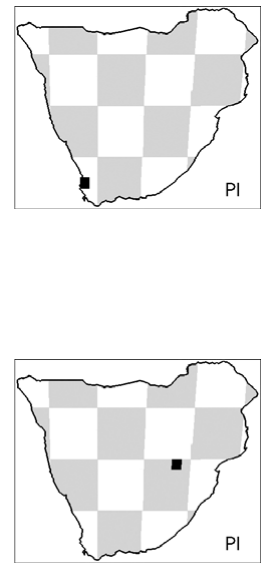

Subfamily: Pantherinae

Panthera leo Linnaeus, 1758. Systema Naturae Regnum Animale, 10th edition, 1: 41. Lion.

Additional references: Haas et al. (2005); Lacruz (2009).

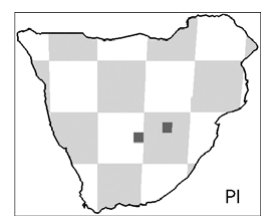

Panthera pardus Linnaeus, 1758. Systema Naturae Regnum Animale, 10th edition, 1: 41. Leopard.

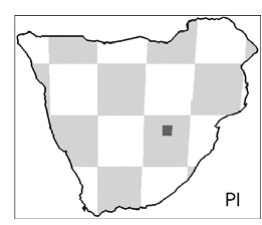

FAMILY: HERPESTIDAE

†Atilax mesotes Ewer, 1956. Proc. Zool. Soc. Lond. 126: 259-274.

Synonyms: Herpestes.

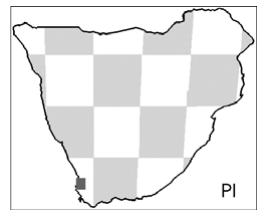

Cynictis penicillata Cuvier, 1829. Le Règne Animal distribué d'après son Organisation, Nouvelle édition, 1: 158. Yellow mongoose.

Synonyms: lepturus; ogilbyii.

Type locality: restricted to 'Uitenhage, CP'.

Additional references: Ewer (1956a, 1957a); Lundholm (1954); Roberts

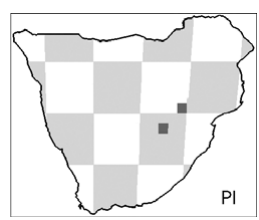

(1932); Smith (1849); Taylor and Meester (1993). 


\section{FAMILY: HYAENIDAE}

†Lycyaenops silberbergi Broom, 1946. Mem. Transvaal Mus. 2: 83.

Synonyms: Chasmaporthetes; Lycyaena.

Type locality: Sterkfontein.

Additional references: Broom (1948a); Werdelin and Peigné (2010).

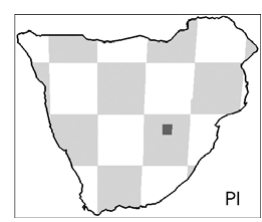

Subfamily: Hyaeninae

†Chasmaporthetes australis Hendey, 1974. Ann. S. Afr. Mus. 63: 91. Synonyms: Euryboas; Percrocuta.

Type locality: Langebaanweg.

Additional references: Hendey (1978a); Werdelin and Peigné (2010).

†Chasmaporthetes nitidula Ewer, 1955. Proc. Zool. Soc. Lond. 124: 842. Synonyms: Euryboas; Lycyaeon.

Type locality: Sterkfontein.

Additional references: Hendey (1978a).

Crocuta crocuta Erxleben, 1777. Systema Regni Animales per Classes: 578. Spotted hyaena.

Synonyms: spelaea.

Additional references: Broom (1939c); Turner (1984).

†Crocuta dietrichi Petter and Howell, 1989. C.R. Acad. Sci. Paris 308: 1038.

Additional references: Morales et al. (2011).

Hyaena hyaena Linnaeus, 1758. Systema Naturae Regnum Animale, 10th edition, 1: 40. Striped hyaena.

Synonyms: striata.

Additional references: Turner (1988).

†Hyaena makapani Toerien, 1952. S. Afr. J. Sci. 48: 293.

Type locality: Limeworks Makapansgat.

Additional references: Toerien (1955).

†Hyaenictis hendeyi Werdelin, Turner and Solounias, 1994. Zool. J. Linn. Soc. 111: 214.

Type locality: Langebaanweg.
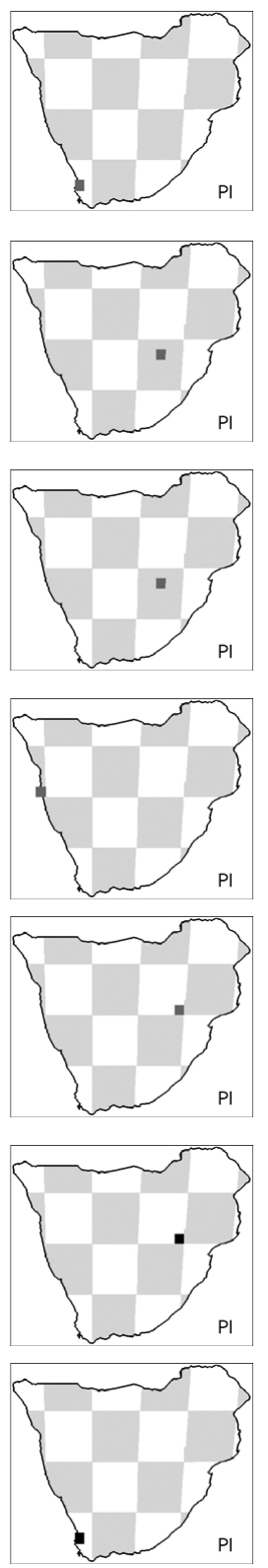

†Hyaenictis preforfex Hendey, 1974. Ann. S. Afr. Mus. 63: 125.

Synonyms: Ictitherium; Leecyaena forfex.

Type locality: Langebaanweg.

Additional references: Ewer (1955a, 1955b); Hendey (1978a).

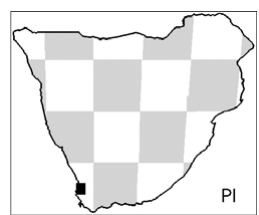


Comments: Werdelin et al. (1994) maintain that this taxon is a synonym of Ikelohyaena abronia.

†Hyaenictitherium namaquensis Stromer, 1931. Bayer. Akad. Wiss.

München Sitz. Math.-Naturwiss. Klasse 1931: 26.

Synonyms: Hyaenictis; Hyaena; namaquense.

Type locality: Kleinzee.

Additional references: Hendey (1978a); Werdelin and Solounias (1991).

†lkelohyaena abronia Hendey, 1974. Ann. S. Afr. Mus. 63: 103.

Synonyms: Hyaena.

Type locality: Langebaanweg.

†Pachycrocuta brevirostris Gervais, 1850. Zool. Paléontol. Françaises 1: 122.

Synonyms: Canis; Hyaena bellax.

Additional references: Alba et al. (2015); Ewer (1954, 1956b); Mutter et al. (2001); Turner and Antón (1996); Werdelin and Peigné (2010).

Comments: according to Alba et al. (2015), this taxon has long been incorrectly attributed to Aymard since his 1846 paper neither describes nor names the taxon.

Parahyaena brunnea Thunberg, 1820. K. Svenska Vet.-Akad. Handl. 8: 59. Brown hyaena.

Synonyms: Hyaena.

Type locality: Cape of Good Hope.

Additional references: Grubb (2004); Hendey (1973a); Mills (1982);
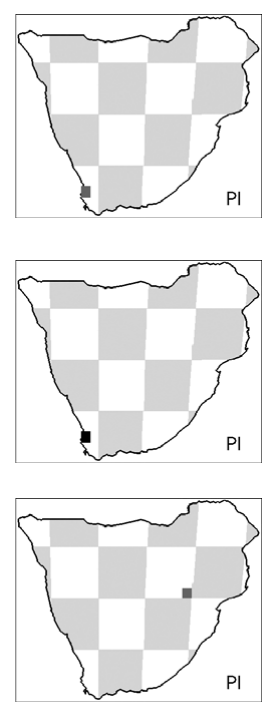

Werdelin and Peigné (2010).

FAMILY: VIVERRIDAE

Subfamily: Viverrinae

Genetta Cuvier, 1817. Le Règne Animal distribué d'après son Organisation: 156.

Additional references: De Meneses Cabral (1966); Gaubert et al. (2005).

Genetta genetta Linnaeus, 1758. Systema Naturae Regnum Animale, 10th edition, 1: 45. Common genet.

Additional references: Gaubert et al. (2005); Larivière and Calzada (2001).

†Viverra leakeyi Petter, 1963. Bull. Soc. Géol. France, Series 7, 3: 270. Additional references: Werdelin and Peigné (2010).
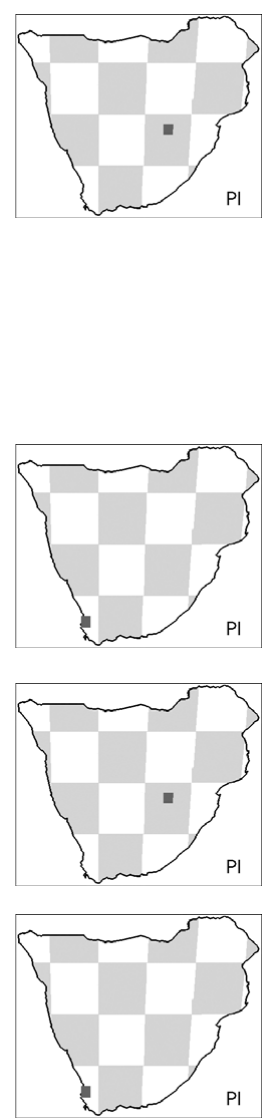


\section{Suborder: Caniformia \\ FAMILY: CANIDAE \\ Subfamily: Caninae}

Canis mesomelas Schreber, 1775. Die Säugethiere 2 (14): pl. 95 [1775], text 3 (21): 370 [1776], 586 [1777]. Black-backed jackal.

Type locality: Cape of Good Hope.

Comments: citation according to Wilson and Reeder (2005). Volume 3 was published in 1778 according to the version reproduced in the Biodiversity Heritage Library (www.biodiversitylibrary.org/item/ 135003\#page/98/mode/1 up) and Canis mesomelas is listed on p. 586. Plate 95 appears in an undated volume containing plates 81-165 (www.biodiversitylibrary.org/item/97341\#page/57/mode/1 up).

Vulpes Frisch, 1775. Das Natur-System der Vierfüssigen Thiere: 14. Additional references: Werdelin and Peigné (2010).

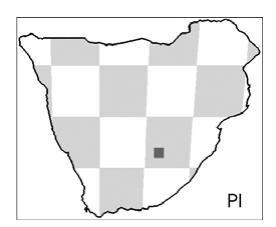

Vulpes chama Smith, 1833. S. Afr. Quart. J., Series 2, 2: 89. Cape fox. Type locality: fixed as 'Port Nolloth'.

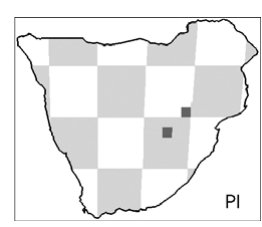

†Vulpes pattisoni Broom, 1948. Ann. Transvaal Mus. 21(1): 23. Type locality: Taung.

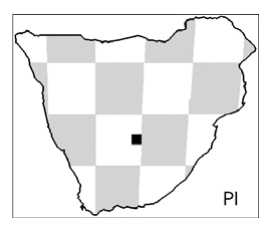

FAMILY: URSIDAE

†Agriotherium africanum Hendey, 1972. Ann. S. Afr. Mus. 59: 126. Type locality: Langebaanweg.

Additional references: Hendey (1980); Werdelin and Peigné (2010).

\section{FAMILY: MUSTELIDAE}

†Plesiogulo monspessulanus Viret, 1939. Trav. Lab. Géol. Fac. Sci. Lyon: 37. Additional references: Hendey (1978b); Montoya et al. (2011); Werdelin and Peigné (2010).

Comments: Viret (1939) not seen, pagination therefore not verified.

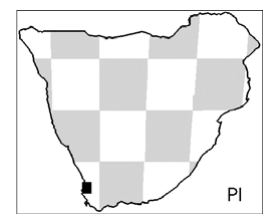


Subfamily: Lutrinae

†Sivaonyx africanus Stromer, 1931. Sitz. Math.-Naturwiss. Klasse Bayer. Akad. Wiss. München 1931: 19.

Synonyms: Enhydriodon.

†Sivaonyx hendeyi Morales, Pickford and Soria, 2005. Rev. Soc. Geol.

España 18(1-2): 56.

Synonyms: Enhydriodon.

Type locality: Langebaanweg.

†Mellivora benfieldi Hendey, 1978. Ann. S. Afr. Mus. 76(10): 343.

Synonyms: punjabiensis.

Type locality: Langebaanweg.

Additional references: Werdelin and Peigné (2010).
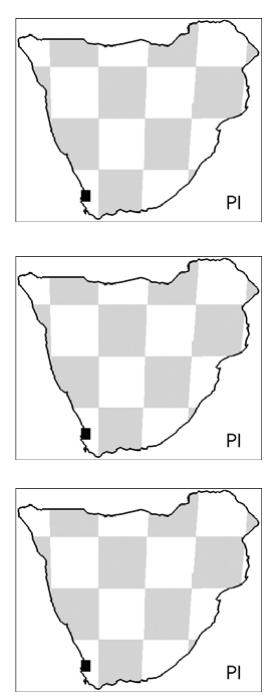

ORDER: PERISSODACTYLA

FAMILY: †CHALICOTHERIIDAE

†Metaschizotherium transvaalensis George, 1950. S. Afr. J. Sci. 46(8):

241.

Type locality: Limeworks Makapansgat.

Additional references: Cooke (1950); Coombs and Cote (2010); Webb

(1965).

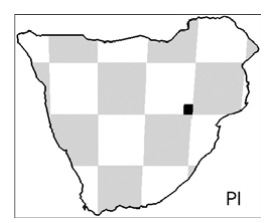

Subfamily: Schizotheriinae

†Ancylotherium Gaudry, 1863. Animaux Fossiles et Géologie de l'Attique: 129.

Additional references: Butler (1965); Coombs and Cote (2010).

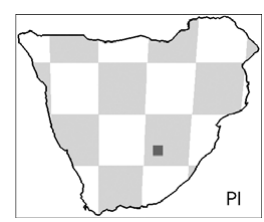

\section{FAMILY: EQUIDAE}

†Eurygnathohippus Van Hoepen, 1930. Paleontol. Nav. Nas. Mus. Bloemfontein 2(2): 23.

Synonyms: Hipparion; Stylohipparion.

Additional references: Boné and Singer (1965); Hooijer (1975, 1976).

†Eurygnathohippus cornelianus Van Hoepen, 1930. Paleontol. Nav. Nas. Mus. Bloemfontein 2(2): 23.

Synonyms: Hipparion; Stylohipparion; hipkini; libycum; sitifense in part?; steytleri.

Type locality: Cornelia.

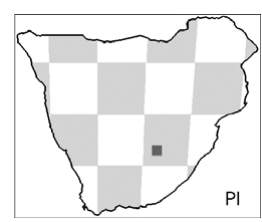

Additional references: Bernor et al. (2010); Churcher (1970, 2000);

Churcher and Watson (1993); Franz-Odendaal et al. (2003); Pomel (1897); Van Hoepen (1932a). 
†Eurygnathohippus hooijeri Bernor and Kaiser, 2006. Mitt. Hamb. Zool. Mus. Inst. 103: 148.

Synonyms: Hipparion baardi cf.

Type locality: Langebaanweg.

Additional references: Bernor et al. (2010); Franz-Odendaal et al. (2003); Hooijer (1976); Pickford and Senut (1997).

†Eurygnathohippus namaquense Haughton, 1932. Ann. S. Afr. Mus. 28: 421.

Synonyms: Hipparion; Notohipparion.

Type locality: Areb.

†Hipparion De Christol, 1832. Ann. Sci. Indust. Midi France 1: 181. Additional references: Boné and Singer (1965); MacFadden (1984).

Comments: there is no certain record of Hipparion, as currently understood, from Africa (Bernor et al. 2010) and it is therefore most likely that this record should be †Eurygnathohippus cornelianus.
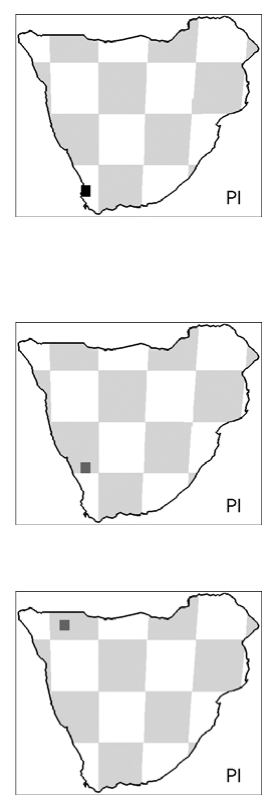

FAMILY: RHINOCEROTIDAE

Subfamily: Rhinocerotinae

Ceratotherium simum Burchell, 1817. Bull. Sci. Soc. Philom. Paris 1817-1819: 97. White rhinoceros.

Synonyms: Diceros; Rhinoceros; simus.

Type locality: since identified as Chue Spring = Heuningvlei. Additional references: Geraads (2010a); Gray (1867); Groves

(1972); Hooijer (1959, 1973); Hooijer and Singer (1960); Smith (1849).

†Diceros praecox Hooijer and Patterson, 1972. Bull. Mus. Comp. Zool. 144: 19.

Synonyms: Ceratotherium.

Additional references: Geraads (2005, 2010a); Guérin (1987); Hooijer (1972, 1973); Pickford and Senut (1997).
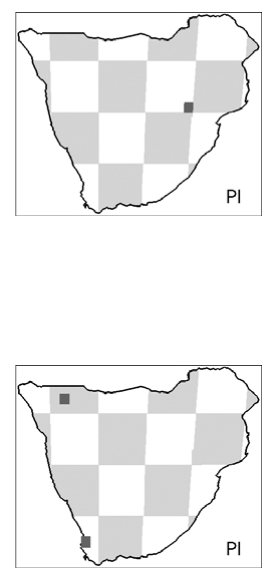

\section{ORDER: ARTIODACTYLA}

FAMILY: SUIDAE

Subfamily: †Namachoerinae

†Cainochoerus africanus Hendey, 1976. Science 192(4241): 789.

Synonyms: Pecarichoerus?.

Type locality: Langebaanweg.

Additional references: Bishop (2010); Pickford (1988).

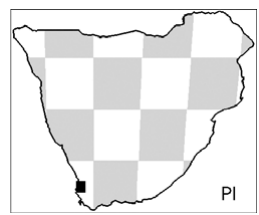


Subfamily: Suinae

†Kolpochoerus paiceae Broom, 1931. Rec. Albany Mus. 4: 168.

Synonyms: Notochoerus.

Type locality: Windsorton.

Additional references: Hendey and Cooke (1985); Pickford (2012).

†Metridiochoerus andrewsi Hopwood, 1926. Ann. Mag. Nat. Hist. 9: 267.

Synonyms: Notochoerus; Tapinochoerus; capensis; meadowsi.

Additional references: Bender (1990); Bishop (2010); Broom (1948a);

Cooke (1993a); Ewer (1956b); Harris and White (1979); Herries et al.

(2006); Hopwood (1926); Keen and Singer (1956); Pickford (2012, 2013a, 2003b); Shaw (1938).

†Potamochoeroides hypsodon Dale, 1948. S. Afr. Sci. 2(5): 116.

Synonyms: Metridiochoerus; Pronotochoerus; shawi.

Additional references: Bender (1990, 1992); Cooke (1993a, 2005);

Ewer (1956b, 1958d); Pickford (2013a); Pickford and Gommery

(2016).
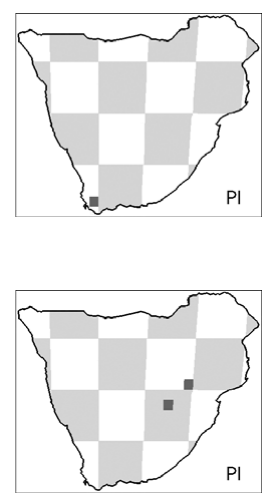

Potamochoerus Iarvatus Cuvier, 1822. Mem. Mus. Hist. Nat. Paris 8: 447.

Bush-pig.

Synonyms: Koiropotamus; porcus.

Additional references: Grubb (2004); Hopwood (1934); Leakey (1942).
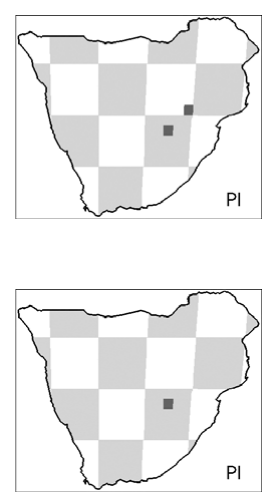

Subfamily: Tetraconodontinae

†Notochoerus capensis Broom, 1925. Rec. Albany Mus. 3(4): 308.

Synonyms: scotti.

Type locality: Longlands.

Additional references: Harris and White (1979); Pickford and Gommery

(2016); Shaw (1938).

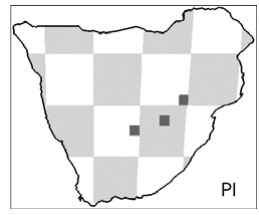

†Notochoerus jaegeri Coppens, 1971. C. R. Hebdom. Scé. Acad. Sci. Paris 272: 3266.

Synonyms: Nyanzachoerus.

Additional references: Bishop (2010); Cooke and Hendey (1992); Harris and White (1979).

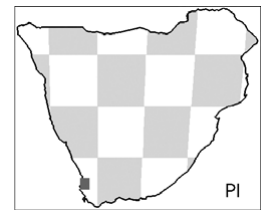

†Nyanzachoerus kanamensis Leakey, 1958. Foss. Mamm. Afr. 14: 10. Additional references: Bishop (2010); Harris and White (1979).

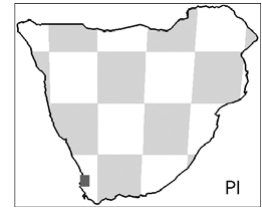




\section{FAMILY: HIPPOPOTAMIDAE}

Hippopotamus Linnaeus, 1758. Systema Naturae Regnum Animale, 10th edition, 1: 74.

Additional references: Boisserie (2005).

Hippopotamus amphibius Linnaeus, 1758. Systema Naturae Regnum Animale, 10th edition, 1: 74. Common hippopotamus.

Synonyms: capensis; poderosus.

Additional references: Fraas (1907); Hooijer and Singer (1961); Scott (1907); Smith (1849).
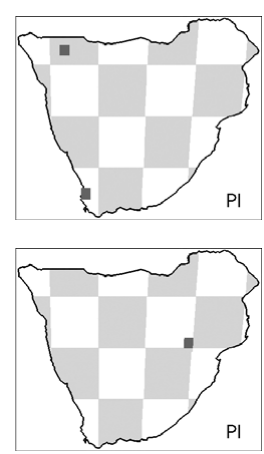

FAMILY: GIRAFFIDAE

Subfamily: Giraffinae

Giraffa Brisson, 1762. Regnum animale in classes IX. Distributum, sive Synopsis Methodica 2nd edition: 12, 37.

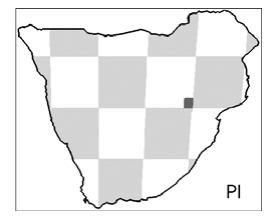

†Giraffa stillei Dietrich, 1942. Palaeontographica A 94: 43-133. Synonyms: gracilis.

Additional references: Arambourg (1947); Harris et al. (2010).

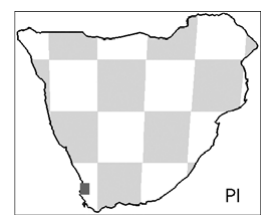

Subfamily: $†$ Sivatheriinae

†Sivatherium Falconer and Cautley, 1836. J. Asiatic Soc. Bengal 1836: 38.

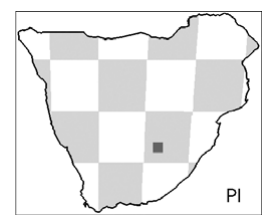

†Sivatherium hendeyi Harris, 1976. Ann. S. Afr. Mus. 69(12): 328. Synonyms: Helladotherium; Libytherium olduvaiensis. Type locality: Langebaanweg.

Additional references: Harris et al. (2010); Hopwood (1934).

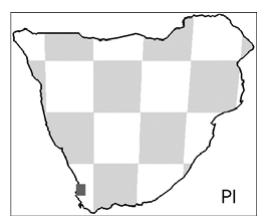

FAMILY: BOVIDAE

Subfamily: Alcelaphinae

Alcelaphus caama Geoffroy Saint-Hilaire, 1803. Catalogue des Mammifères du Muséum National d'Histoire Naturelle: 269. Red hartebeest.

Synonyms: Antilope; Bubalus; bubalis; buselaphus caama. Type locality: since restricted to syntype locality Steynsburg.

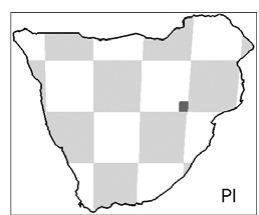
Additional references: Gray (1850a, 1850b); Grubb (2004); Hoffman (1953); Smith (1849). 
†Alcelaphus robustus Cooke, 1949. Mem. Geol. Surv. S. Afr. 35: 20.

Type locality: Larsen's Main Workings.

Comments: see Parmularius braini below.

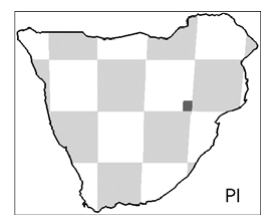

Connochaetes taurinus Burchell, 1824. Travels in the Interior of Southern Africa 2: 278 (footnote) [1824]. Blue wildebeest.

Synonyms: Catoblepas.

Type locality: apparently 'Kosi Fountain', but lectotype came from 'Chue

Spring, Maadji Mtn [Klein Heuningvlei]'.

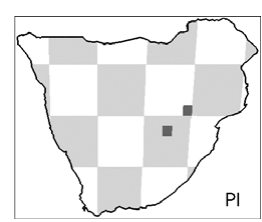

Additional references: Smith (1849).

†Damalacra Gentry, 1980. Ann. S. Afr. Mus. 79(8): 264.

Type locality: Langebaanweg.

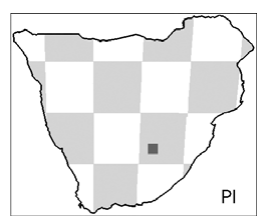

†Damalacra acalla Gentry, 1980. Ann. S. Afr. Mus. 79(8): 272.

Type locality: Langebaanweg.

Additional references: Brink and Stynder (2009).

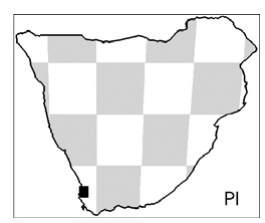

†Damalacra neanica Gentry, 1980. Ann. S. Afr. Mus. 79(8): 265.

Type locality: Langebaanweg.

Additional references: Brink and Stynder (2009).

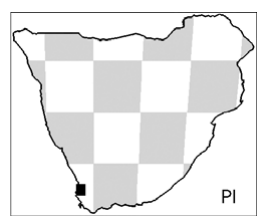

†Damaliscus gentryi Vrba, 1977. Palaeontol. Afr. 20: 143.

Type locality: Limeworks Makapansgat.

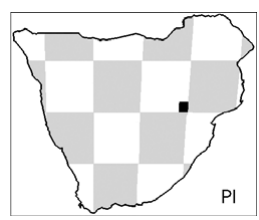

†Megalotragus Van Hoepen, 1932. Paleontol. Nav. Nas. Mus.

Bloemfontein 2(5): 63.

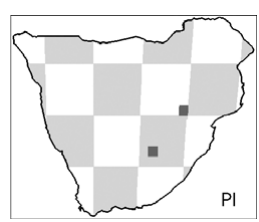

†Parmularius braini Vrba, 1977. Palaeontol. Afr. 20: 140.

Type locality: Limeworks Makapansgat.

Comments: according to Gentry (2010), Alcelaphus robustus from this site is synonymous with this taxon in the Pliocene.

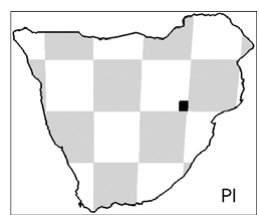


Subfamily: Antilopinae

†Antidorcas bondi Cooke and Wells, 1951. S. Afr. J. Sci. 47: 207.

Synonyms: Gazella.

Type locality: Chelmer.

Additional references: Gentry (2010); Plug and Peters (1991); Vrba (1973).

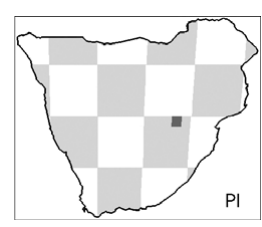

†Antidorcas recki Schwarz, 1932. Zentr. Mineral., Geol. Paläontol. B 1932:

1.

Synonyms: Gazella; wellsi.

Additional references: Gentry (2010).

Gazella De Blainville, 1816. Bull. Sci. Soc. Philom. Paris 1816: 75.

Additional references: Gentry (2010).

†Gazella vanhoepeni Wells and Cooke, 1957. Palaeontol. Afr. 4: 43.

Synonyms: gracilior?.

Type locality: Limeworks Makapansgat.
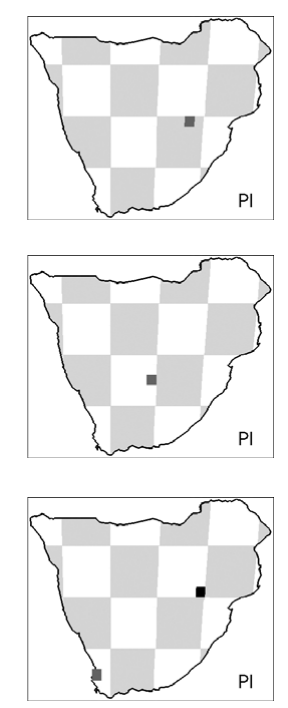

Oreotragus oreotragus Zimmermann, 1783. Geographische Geschichte des Menschen und der Allgemein Verbreiteten Vierfüssigen Thiere 3: 269. Klipspringer.

Synonyms: Palaeotragiscus longiceps; major.

Type locality: now known to be False Bay.

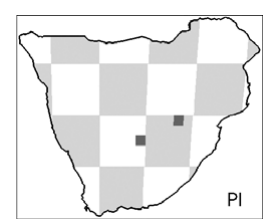

Additional references: Broom (1934); Gentry (2010); Gray (1850a,

1850b); Watson and Plug (1995); Wells (1951).

Raphicerus Smith, 1827. In: Griffith et al., The Animal Kingdom Arranged in Conformity with its Organization by the Baron Cuvier 5: 342.

Additional references: Klein (1976c).

†Raphicerus paralus Gentry, 1980. Ann. S. Afr. Mus. 79(8): 300.

Type locality: Langebaanweg.
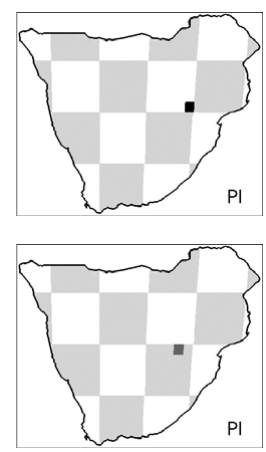

Subfamily: Bovinae

†Miotragoceros acrae Gentry, 1974. Ann. S. Afr. Mus. 65(5): 146.

Synonyms: Mesembriportax; Tragoportax.

Type locality: Langebaanweg.

Additional references: Gentry (1980, 2010).

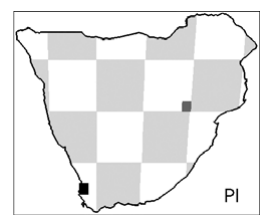


Syncerus Hodgson, 1847. J. Asiatic Soc. Bengal, Series 2, 16: 709. Synonyms: Bos; Bubalis; Pelorovis.

Additional references: Martínez-Navarro et al. (2007).

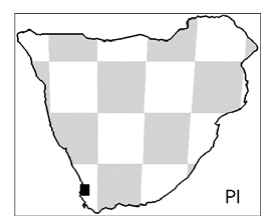

†Syncerus aceolotus Gentry and Gentry, 1978. Bull. Brit. Mus. Nat. Hist. Geol. Ser. 29: 313.

Additional references: Gentry (2010).

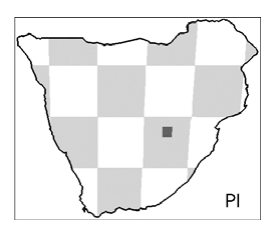

Tragelaphus De Blainville, 1816. Bull. Sci. Soc. Philom. Paris 1816: 75.

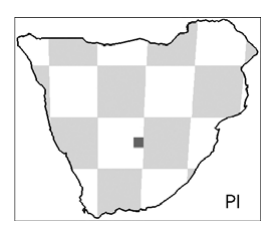

Tragelaphus angasii Angas, 1848. Proc. Zool. Soc. Lond. 1848: 89. Nyala.

Type locality: 'Hills that border: upon the northern shores of St Lucia Bay'.

Additional references: Gray (1850a, 1850b); Grubb (2004); Willows-

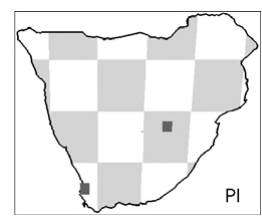

Munro et al. (2005).

†Tragelaphus pricei Wells and Cooke, 1957. Palaeontol. Afr. 4: 12. Synonyms: Cephalophus.

Type locality: Limeworks Makapansgat.

Additional references: Gentry (2010).

Tragelaphus strepsiceros Pallas, 1766. P.S. Pallas Medecinae Doctoris Miscellanea Zoologica: 9. Greater kudu.

Synonyms: Damalis; Strepsiceros; capensis.

Type locality: restricted to eastern part of western Cape Province. Additional references: Smith (1849); Willows-Munro et al.

(2005).

†Ugandax demissum Gentry, 1980. Ann. S. Afr. Mus. 79(8): 233.

Synonyms: Simatherium.

Type locality: Langebaanweg.

Additional references: Gentry (2006, 2010); Geraads (1992); Pickford and

Senut (1997).
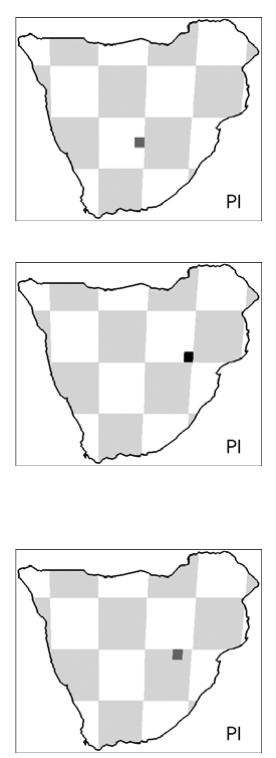

Subfamily: Caprinae

†'Makapania' broomi Wells and Cooke, 1957. Palaeontol. Afr. 4: 26.

Synonyms: Bos; Hippotragus; Hippotragoides; makapaani.

Type locality: Limeworks Makapansgat.

Additional references: Gentry (1970, 2010).

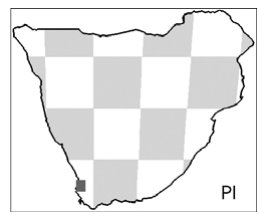


Subfamily: Cephalophinae

†Cephalophus parvus Broom, 1934. S. Afr. J. Sci. 31: 477.

Additional references: Gentry (2010).

Sylvicapra Ogilby, 1836. Proc. Zool. Soc. Lond. 1836: 138.
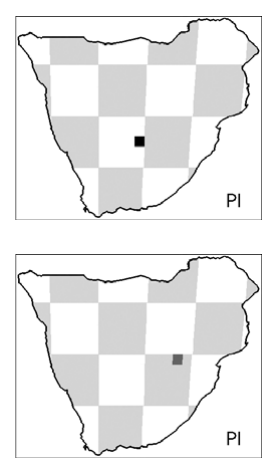

Subfamily: Hippotraginae

†Hippotragus cookei Vrba, 1987. Palaeontol. Afr. 26(5): 49.

Type locality: Limeworks Makapansgat.

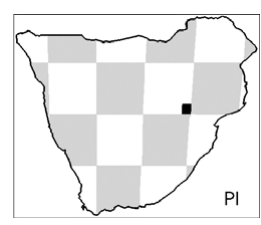

Hippotragus niger Harris, 1838. Proc. Zool. Soc. Lond. 1838: 2. Sable antelope.

Synonyms: harrisi.

Type locality: since specified as Magaliesberg near Krugersdorp and

Rustenburg.

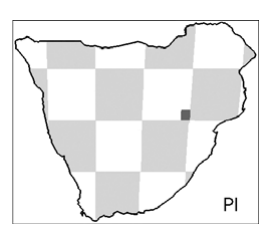

Additional references: Gray (1850a, 1850b); Harris (1838a).

†Wellsiana torticornuta Vrba, 1987. Palaeontol. Afr. 26(5): 53.

Type locality: Limeworks Makapansgat.

Additional references: Gentry (2010).

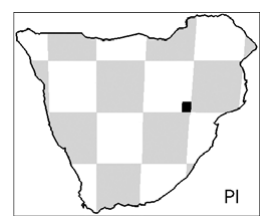

Subfamily: Reduncinae

Kobus Smith, 1840. Illustrations of the Zoology of South Africa,

Part 12: pl. 28, plus text.

Synonyms: Aigoceros; Onotragus.

Additional references: Birungi and Arctander (2001).

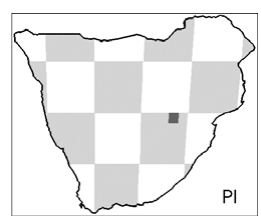

†Kobus subdolus Gentry, 1980. Ann. S. Afr. Mus. 79(8): 248.

Type locality: Langebaanweg.

Additional references: Gentry (2010).

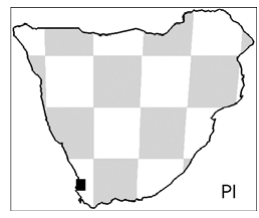

Redunca Smith, 1827. In: Griffith et al., The Animal Kingdom Arranged in Conformity with its Organization by the Baron Cuvier 5: 337.

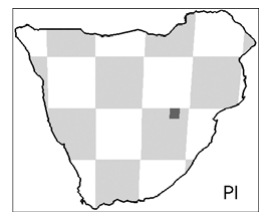


†Redunca darti Wells and Cooke, 1957. Palaeontol. Afr. 4: 17. Type locality: Limeworks Makapansgat.

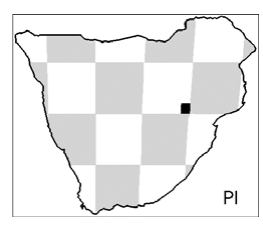

\subsection{PLIOCENE SITES}

The distribution of Pliocene sites is noticeably broader than was the case in previous epochs (Figure 4.1). There are three particularly important and rich sites that have provided much of the available information. These are Langebaanweg (3218) in the southwest, and Taung (2724) and Limeworks Makapansgat (2429) to the northeast. Praia de Morrugusu (2335) may only have produced a specimen of Deinotherium bozasi (Harris 1977), but it is notable for its location in the extreme east of the region.

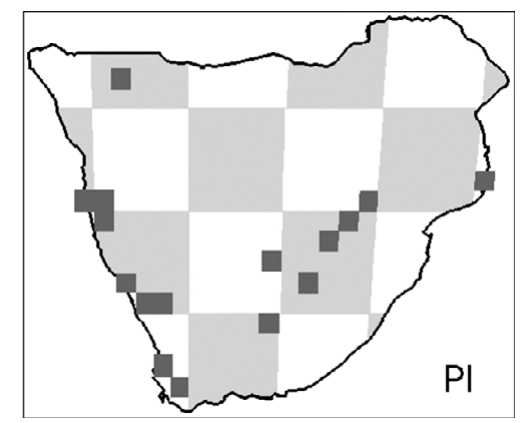

Figure 4.1. Location of Pliocene sites in the region.

Areb (2931:1815). Taxa: Eurygnathohippus namaquense. References: Cooke (1955); Pickford et al. (1999).

Aves Cave 1 (2627). Taxa: Canis mesomelas cf.; Dinofelis cf.; Felis silvestris; Notochoerus capensis; Panthera leo cf.; Panthera pardus cf.; Parahyaena cf.; Potamochoeroides hypsodon. References: Gommery et al. (2016); Pickford and Gommery (2016).

Awasib (2518:1539). Taxa: Pedetes gracilis cf. References: Pickford and Mein (2011).

Bushman Hill (W) (2509:1544). Taxa: Pedetes gracilis. References: Pickford and Mein (2011).

Daberas Dune (2808:1639). Taxa: Pedetes gracilis. References: Pickford and Mein (2011). Ekuma (1837:1600). Taxa: Pedetes gracilis cf.; Propedetes laetoliensis. References: Pickford and Mein (2011).

Etosha Pan (1816CA). Taxa: Diceros praecox; Hipparion; Hippopotamus; Loxodonta cookei cf.; Propedetes; Redunca darti aff. References: Pickford et al. (2014).

Hoogland (2549:2800). Taxa: Acinonyx?; Antidorcas bondi; Antidorcas recki; Felis?; Kobus; Oreotragus oreotragus; Procavia antiqua; Procavia transvaalensis; Raphicerus; Redunca; Sylvicapra cf.; Theropithecus oswaldi; Tragelaphus strepsiceros. References: Adams et al. (2010).

Jacovec Cavern (2601:2744). Taxa: Australopithecus; Canis mesomelas; Caracal caracal; Chasmaporthetes nitidula; Connochaetes taurinus; Crocuta crocuta; Cynictis penicillata; 
Genetta genetta; Homotherium crenatidens; Lepus capensis; Lycyaenops silberbergi; Panthera leo; Panthera pardus; Parahyaena brunnea; Parapapio broomi; Parapapio jonesi; Pedetes capensis; Potamochoerus larvatus; Syncerus cf.; Vulpes chama. References: Kibii (2006); Reynolds (2010b).

Kleinzee (2941:1704). Taxa: Palaeothentoides africanus. References: Pickford and Senut (1997).

Langebaanweg (3258:1809). Taxa: Acomys mabele; Aethomys adamanticola; Aethomys modernis; Agriotherium africanum; Amphimachairodus; Anancus capensis; Atilax mesotes; Bathyergus hendeyi; Cainochoerus africanus; Chasmaporthetes australis; Chrysochloris arenosa; Chrysochloris bronneri; Cryptomys broomi; Cryptomys hottentotus; Damalacra acalla; Damalacra neanica; Dendromus averyi; Dendromus darti; Desmodillus magnus; Diceros praecox; Dinofelis diastemata; Eurygnathohippus hooijeri; Euryotomys pelomyoides; Felis issiodorensis; Gazella vanhoepeni aff.; Genetta; Giraffa stillei cf.; Hyaenictis hendeyi; Hyaenictis preforfex; Hyaenictitherium namaquensis; Ikelohyaena abronia; Kobus subdolus; Loxodonta cookei; Mammuthus subplanifrons; Mellivora benfieldi; Metailurus obscurus; Miotragoceros acrae; Mystromys hausleitneri; Mystromys pocockei; Notochoerus jaegeri; Nyanzachoerus kanamensis; Parapapio; Plesiogulo monspessulanus; Procavia antiqua aff.; Procavia pliocenica; Raphicerus paralus; Rhabdomys; Sivaonyx africanus; Sivaonyx hendeyi; Sivatherium hendeyi; Stenodontomys saldanhae; Thallomys; Tragelaphus; Ugandax demissum; Viverra leakeyi; Zelotomys. References: Cooke and Hendey (1992); FranzOdendaal et al. (2003); Franz-Odendaal and Salounias (2004); Grine and Hendey (1981); Hendey (1969, 1970, 1973b, 1976b, 1978b, 1981, 1984); Hooijer (1972, 1976a); Klein (1974b); Matthews et al. (2007); Pickford (1988); Sardella and Werdelin (2007); Werdelin and Sardella (2006); Werdelin et al. (1994).

Limeworks Makapansgat (2408:2911). Taxa: Acinonyx jubatus; Acomys; Alcelaphus caama cf.; Alcelaphus robustus cf.; Amblysomus hamiltoni; Australopithecus africanus; Caracal caracal; Cephalophus; Ceratotherium simum cf.; Cercocebus cf.; Cercopithecoides williamsi; Connochaetes taurinus cf.; Cryptomys hottentotus; Cryptomys robertsi; Cynictis penicillata; Damaliscus gentryi; Dasymys incomtus cf.; Dendromus mesomelas cf.; Dinofelis barlowi; Diplomesodon fossorius; Elephantulus brachyrhynchus cf.; Elephantulus broomi; Elephas cf.; Eptesicus hottentotus; Eurygnathohippus cornelianus; Gazella vanhoepeni; Gerbilliscus; Gigantohyrax maguirei; Giraffa; Grammomys dolichurus cf.; Gypsorhychus makapani; Herpestes; Heterocephalus; Hippopotamus amphibius cf.; Hippotragus cookei; Hippotragus niger; Homotherium crenatidens; Homotherium problematicum; Hyaena hyaena; Hyaena makapani; Hystrix africaeaustralis; Hystrix makapanensis; Lemniscomys; Leptailurus serval; 'Makapania' broomi; Malacothrix makapani; Malacothrix typica; Mastomys natalensis; Megalotragus; Metaschizotherium transvaalensis; Metridiochoerus andrewsi; Micaelamys namaquensis cf.; Miniopterus; Mus minutoides cf.; Myomyscus; Myosorex robinsoni; Myosorex varius; Myotomys sloggetti; Mystromys albicaudatus; Mystromys hausleitneri; Notochoerus capensis; Otomys gracilis; Pachycrocuta brevirostris; Parapapio broomi; Parapapio jonesi cf.; Parapapio whitei; Parmularius braini; Pelomys fallax cf.; Potamochoeroides hypsodon; Procavia antiqua; Pronolagus; Proodontomys cookei; Raphicerus paralus; Redunca darti; Rhabdomys pumilio cf.; Rhinolophus capensis cf.; Rhinolophus clivosus cf.; Rhinolophus darlingi cf.; Steatomys; Steatomys pratensis cf.; Stenodontomys darti; Suncus infinitesimus; Suncus varilla; Theropithecus darti; Tragelaphus pricei; Vulpes chama; Wellsiana torticornuta; Xenohystrix crassidens; Zelotomys?. References: Bender (1992); Boné and Dart (1955); Broom and Robinson (1950a); Brophy et al. (2014); De 
Graaff (1961c); Denys (1999); Ewer (1957a, 1958b, 1958d); Freedman (1970); Hopley et al. (2006); Lavocat (1957); Maguire (1985); McKee et al. (1995); Reed et al. (1993); Turner et al. (1999); Vrba (1987a).

Matjihabeng (2807:2655). Taxa: Ancylotherium; Damalacra cf.; Eurygnathohippus; Mammuthus subplanifrons; Megalotragus; Sivatherium; Vulpes cf. References: De Ruiter et al. (2010).

Meob (2439:1444). Taxa: Crocuta dietrichi. References: Morales et al. (2011).

Milo's Pit A (2602:2743). Taxa: Hippopotamus cf.; Metridiochoerus andrewsi; Tragelaphus cf. References: Gommery et al. (2012a).

Praia de Morrugusu (2320:3522). Taxa: Deinotherium bozasi. References: Harris (1977).

Rooilepel (2818:1635). Taxa: Propedetes efeldensis; Propedetes laetoliensis. References: Lehmann (2009); Pickford (1996b); Pickford and Mein (2011).

Schurveberg (2528). Taxa: Cryptomys robertsi; Dinopithecus ingens; Elephantulus broomi; Mystromys hausleitneri; Megantereon whitei; Otomys gracilis. References: Broom (1937a, 1948a); Freedman (1970).

Skuurwerug (3319). Taxa: Kolpochoerus paiceae. References: Broom (1937a, 1948a).

Taung (2737:2437). Taxa: Acomys spinosissimus; Australopithecus africanus; Canis mesomelas cf.; Cephalophus parvus; Cercopithecoides williamsi; Crocidura fuscomurina cf.; Crocidura taungensis; Cryptomys robertsi; Dasymys 'lavocati'; Dendromus antiquus; Desmodillus auricularis; Elephantulus antiquus; Elephantulus brachyrhynchus cf.; Gazella; Gerbilliscus brantsii cf.; Gypsorhychus darti; Gypsorhychus minor; Hystrix africaeaustralis; Lepus cf.; Macroscelides proboscideus; Malacothrix typica cf.; Mastomys natalensis cf.; Mylomygale spiersi; Myotomys campbelli; Mystromys albicaudatus; Notochoerus capensis cf.; Oreotragus oreotragus; Otomys gracilis; Panthera leo cf.; Papio izodi; Parapapio broomi; Parapapio whitei; Pedetes gracilis; Petromus minor; Procavia antiqua; Procavia transvaalensis; Procercocebus antiquus; Proodontomys cookei; Rhabdomys cf.; Rhinolophus capensis cf.; Rhinolophus darlingi cf.; Suncus varilla; Syncerus aceolotus cf.; Thallomys debruyni; Tragelaphus angasii cf.; Vulpes pattisoni. References: Broom (1934, 1939b, 1948a); Cooke (1990); Davis (1961); De Graaff (1961a); Freedman (1965, 1970, 1976); McKee (1993a); McKee et al. (1995).

Tree Pan (2555:1556). Taxa: Pedetes gracilis cf. References: Pickford and Mein (2011).

Tsauchab (2430:1543). Taxa: Pedetes capensis cf.; Pedetes laetoliensis. References: Pickford and Mein (2011).

Waypoint 160 (2602:2743). Taxa: Boltimys broomi; Euryotomys bolti; Parapapio; Petromus antiquus. References: Gommery et al. (2014); Gommery et al. (2008c, 2009). 


\section{CHAPTER 5}

\section{The Pleistocene}

\subsection{PLEISTOCENE MAMMALS}

More families, genera and species have been recorded from the Pleistocene than any other epoch in southern Africa (see Table 7.1). Proportions of extinct taxa vary at different levels. Thus, all 15 extant Orders are represented, and all of the 38 families are extant, but the situation is different at the generic and specific levels. Here there is a clear diversification in certain Orders, combined with high proportions of extinct taxa. For instance, in the Artiodactyla, 32 per cent of the genera and 49 per cent of the species are now extinct, while the numbers are 23 per cent and 43 per cent respectively in the Carnivora. On the other hand, the Rodentia contain only 8 per cent extinct genera and 17 per cent extinct species, while all the Chiroptera are extant.

\section{ORDER: AFROSORICIDA \\ Suborder: Chrysochloridea \\ FAMILY: CHRYSOCHLORIDAE \\ Subfamily: Chrysochlorinae}

Chlorotalpa duthiae Broom, 1907. Trans. S. Afr. Philos. Soc. 18: 292.

Duthie's golden mole.

Type locality: Knysna.

Additional references: Bronner (1995a, 1995b).

Chlorotalpa sclateri Broom, 1907. Ann. Mag. Nat. Hist., Series 7, 19: 263.

Sclater's golden mole.

Type locality: Beaufort West.

Additional references: Bronner (1995a, 1995b); Broom (1907b, 1946, 1950).

†Chlorotalpa spelea Broom, 1941. Ann. Transvaal Mus. 20: 214.

Type locality: Sterkfontein.
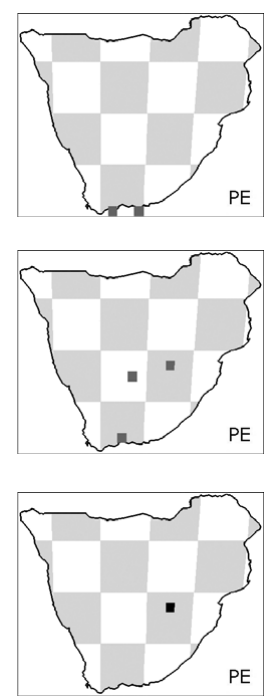

Chrysochloris asiatica Linnaeus, 1758. Systema Naturae Regnum Animale, 10th edition, 1: 53. Cape golden mole.

Type locality: usually taken as Cape of Good Hope.

Additional references: Broom (1907b, 1909c, 1910, 1946, 1950); Petter (1981); Roberts (1919).

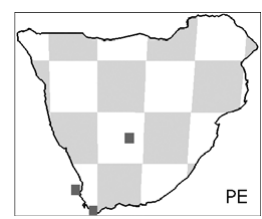


Chrysospalax Gill, 1884. Stand. Nat. Hist. 5 (Mamm): 137.

Type locality: None given.

Additional references: Petter (1981).

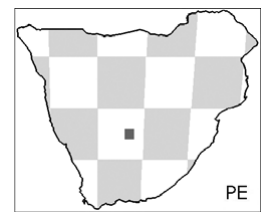

Chrysospalax villosus Smith, 1833. S. Afr. Quart. J., Series 2, 2: 81.

Rough-haired golden mole.

Synonyms: Chrysochloris villosa.

Type locality: near Durban.

Additional references: Broom (1909c, 1913a); Gray (1865a); Meester

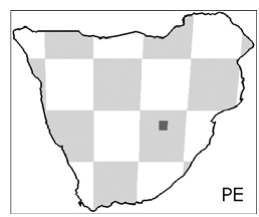

(1953b); Smith (1849).

Subfamily: Amblysomyinae

Amblysomus hottentotus Smith, 1829. Zool. J. 4: 436. Hottentot golden mole. Synonyms: iris.

Type locality: Grahamstown.

Additional references: Bronner (1996); Broom (1908, 1909c); Capanna et al. (1989); Mynhardt et al. (2015); Petter (1981); Roberts (1946).

Neamblysomus gunningi Broom, 1908. Ann. Transvaal Mus. 1: 14.

Gunning's golden mole.

Synonyms: Amblysomus.

Type locality: Woodbush.

Additional references: Bronner (1995b, 2013); Bronner and Jenkins (2005).

†Proamblysomus antiquus Broom, 1941 Ann. Transvaal Mus. 20: 214.

Type locality: Sterkfontein
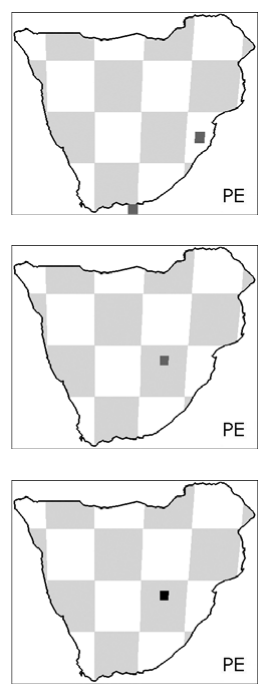

ORDER: MACROSCELIDEA

FAMILY: MACROSCELIDIDAE

Subfamily: Macroscelidinae

Elephantulus Thomas and Schwann, 1906. Abstr. Proc. Zool. Soc. Lond. 33: 10.

Additional references: Corbet and Hanks (1968); Evans (1942); Holroyd

(2010a); Patterson (1965); Van der Horst (1944).

†Elephantulus antiquus Broom, 1948. Ann. Transvaal Mus. 21(1): 5.

Type locality: Bolt's Farm.

Additional references: Patterson (1965).

Elephantulus brachyrhynchus Smith, 1836. Report of the Expedition for Exploring Central Africa: 42. Short-snouted elephant shrew.

Synonyms: Macroscelides.

Type locality: Kuruman to southern Botswana.

Additional references: Smith (1849).
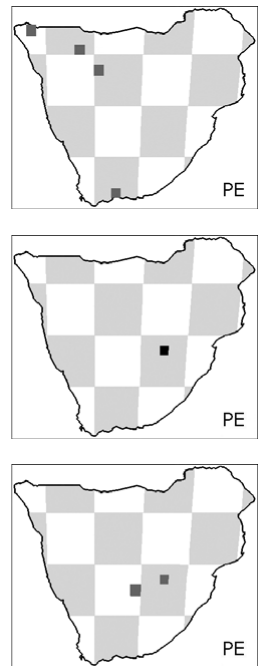
†Elephantulus broomi Corbet and Hanks, 1968. Bull. Brit. Mus. Nat. Hist. Zool. 16: 54.

Synonyms: Elephantomys langi.

Type locality: Schurveberg.

Elephantulus edwardii Smith, 1839. Illustrations of the Zoology of South Africa: pl. 14. Cape elephant shrew.

Synonyms: Macroscelides.

Type locality: Oliphants River.

Additional references: Roberts (1924); Smith (1849).

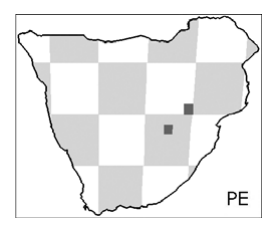

Elephantulus fuscus Peters, 1851. Reise nach Mossambique, Säugethiere: 87. Dusky elephant shrew.

Type locality: Boror.

Elephantulus intufi Smith, 1836. Report of the Expedition for Exploring Central Africa: 42. Bushveld elephant shrew.

Synonyms: Macroscelides.

Type locality: flats beyond Kurrichaine.

Additional references: Smith (1849).
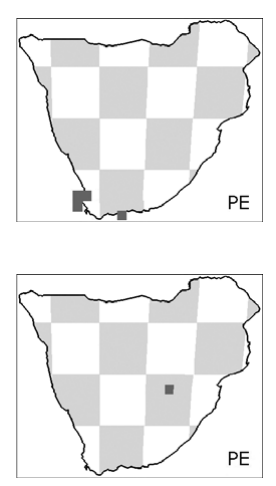

Elephantulus myurus Thomas and Schwann, 1906. Proc. Zool. Soc. Lond. 1906: 586. Eastern rock elephant shrew.

Type locality: Woodbush.

Elephantulus rupestris Smith, 1830. Proc. Commmittee. Sci. Corr. Zool. Soc. Lond. 1: 11. Western rock elephant shrew.

Synonyms: Macroscelides.

Type locality: mountains near mouth of Orange River.

Additional references: Broom (1938b); Roberts (1938, 1946); Smith
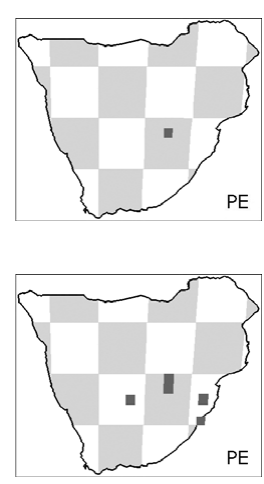
(1849).

Macroscelides Smith, 1829. Zool. J. Lond. 4: 435.

Type locality: none given.

Additional references: Corbet and Hanks (1968); Evans (1942); Patterson (1965); Smith (1849).

Comments: this genus is considered to be monospecific, so

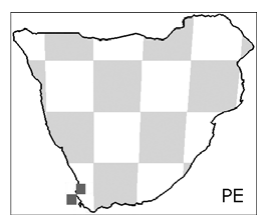

this material should almost certainly be assigned to

M. proboscideus.

Macroscelides proboscideus Shaw, 1800. Gen. Zool. Syst. Nat. Hist. 1(2)

Mammalia: 536. Short-eared elephant shrew.

Synonyms: typicus.

Type locality: Roodeval.

Additional references: Roberts $(1933,1938)$.
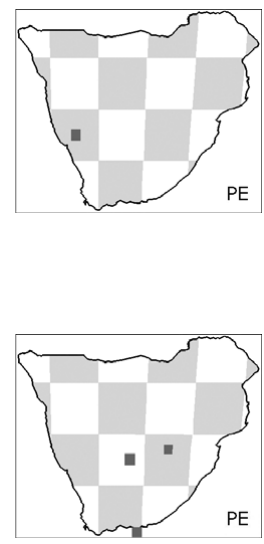
Subfamily: †Mylomygalinae

†Mylomygale spiersi Broom, 1948. Ann. Transvaal Mus. 21(1): 8.

Type locality: Taung.

Additional references: Holroyd (2010a); Patterson (1965).

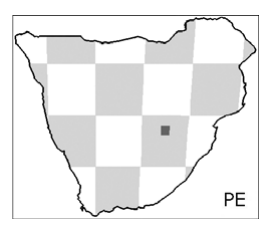

\section{ORDER: TUBULIDENTATA}

FAMILY: ORYCTOPODIDAE

Orycteropus Cuvier, 1798. Tableau Elémentaire de l'Histoire Naturelle des Animaux 1798: 144.

Additional references: Holroyd (2010b); Kitching (1963); Lehmann (2007); Pickford (2008a).

Orycteropus afer Pallas, 1766. P.S. Pallas Medecinae Doctoris Miscellanea Zoologica: 64. Aardvark.

Type locality: Cape of Good Hope.

Additional references: Kitching (1963); Lehmann (2004, 2007); Pickford

(2005b); Shoshani et al. (1988).
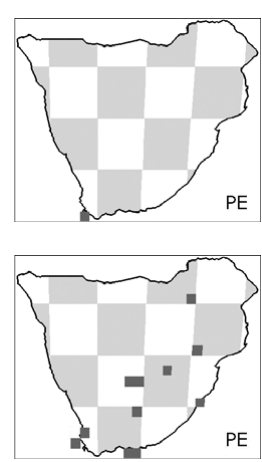

ORDER: HYRACOIDEA

FAMILY: PROCAVIIDAE

Dendrohyrax arboreus Smith, 1827. Trans. Linn. Soc. Lond. 14: 468.

Southern tree hyrax.

Type locality: Cape of Good Hope.

Additional references: Gray (1873); Thomas (1892).

Procavia Storr, 1780. Prodromus Methodi Mammalium: 40.
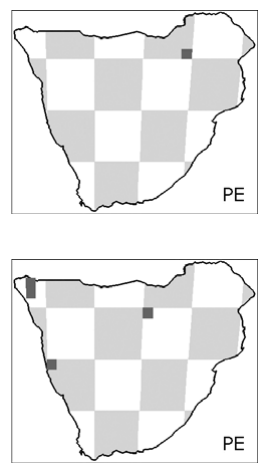

†Procavia antiqua Broom, 1934. S. Afr. J. Sci. 31: 472.

Synonyms: Prohyrax; robertsi.

Type locality: Taung.

Additional references: Broom (1948a); Churcher (1956); McMahon and Thackeray (1994); Rasmussen and Gutiérrez (2010); Schwartz

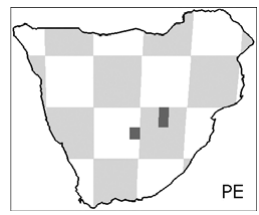
(1997).

Procavia capensis Pallas, 1766. P.S. Pallas Medecinae Doctoris Miscellanea Zoologica:30. Rock hyrax.

Synonyms: Heterohyrax; coombsi; syriacus; welwitschii.

Type locality: Cape of Good Hope.

Additional references: Maswanganye et al. (2017); McMahon and

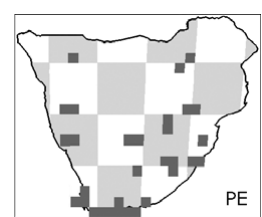

Thackeray (1994); Thomas (1892). 
†Procavia transvaalensis Shaw, 1937. J. Dental Res. 16: 40.

Synonyms: obermeyerae.

Type locality: Taung.

Additional references: Churcher (1956); McMahon and Thackeray (1994).

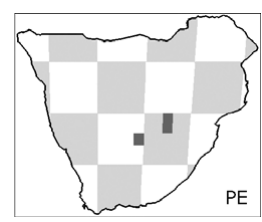

\section{ORDER: PROBOSCIDEA}

FAMILY: ELEPHANTIDAE

Subfamily: Elephantinae

Elephas Linnaeus, 1758. Systema Naturae Regnum Animale, 10th edition, 1: 33.

Synonyms: Archidiskodon; Palaeoloxodon.

Additional references: Cooke (1961); Todd (2010).

†Elephas iolensis Pomel, 1895. Les éléphants Quaternaires: Carte Géologique de l'Algérie: 32.

Synonyms: Archidiskodon; Mammuthus; Palaeoloxodon; pilgrimia;

archidiskodontoides; broomi; hanekomi; kuhni; sheppardi;

transvaalensis; wilmani; yorki.

Additional references: Cooke (1993b); Dart (1927, 1929b); Haughton (1932b); Hendey (1967); Osborn (1942); Sanders et al. (2010a); Todd (2010).

Comments: Pomel (1895) named this species E. jolensis.

†Elephas recki Dietrich, 1915. Arch. Biontol. 4(1): 22.

Synonyms: Archidiskodon; Mammuthus; Palaeoloxodon; griqua; antiquus recki.

Additional references: Arambourg (1942); Cooke (1993b); Dart (1929b); Haughton (1921); Osborn (1942); Sanders et al. (2010a); Todd (2005, 2010).

Loxodonta, Anonymous, 1827. Zool. J. 3: 140.

Synonyms: Tetralophodon?
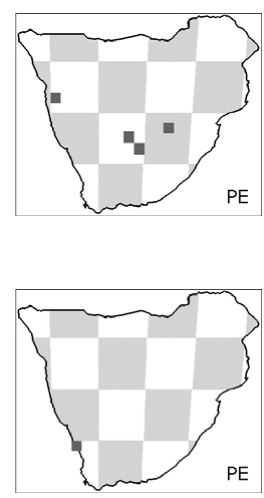

Loxodonta africana Blumenbach, 1797. D. Joh. Fr. Blumenbach's Handbuch der Naturgeschichte., 5th edition: 125. African bush elephant.

Synonyms: Archidiskodon; Palaeoloxodon; Elephas; Mammuthus; loxodontoides; zulu.
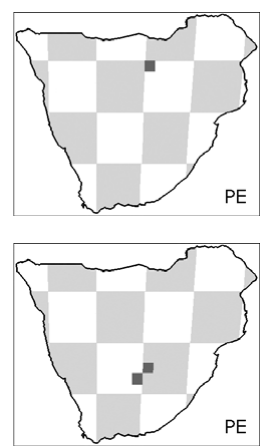

PE

Type locality: Orange River, South Africa.

Additional references: Brook et al. (2014); Burmeister (1837); Dart

(1929b); Sanders et al. (2010b); Todd (2010).

†Loxodonta atlantica Pomel, 1879. Bull. Soc. Géol. France, Series 3, 7: 51. Synonyms: Archidiskodon; Palaeoloxodon; zulu.

Additional references: Sanders et al. (2010b); Scott (1907).

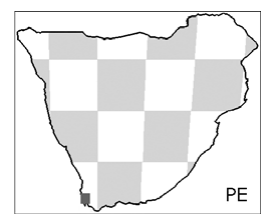


†Loxodonta cookei Sanders, 2007. Trans. R. Soc. S. Afr. 62(1): 7. Synonyms: Lukeino Stage.

Type locality: Langebaanweg.

Additional references: Pickford and Senut (1997); Sanders et al. (2010a);

Todd (2010).

†Mammuthus subplanifrons Osborn, 1928. Nature 121:

672-673.

Synonyms: Archidiskodon; Elephas; Mastodon; Stegodon; andrewsi;

milletti; proplanifrons; scotti; vanalpheni.

Type locality: Sydney-on-Vaal.

Additional references: Dart (1929b); Maglio and Hendey (1970); Meiring (1955); Osborn (1934, 1942); Sanders (2007); Sanders et al. (2010a); Todd (2010).

\section{ORDER: PRIMATES \\ Suborder: Haplorrhini \\ FAMILY: CERCOPITHECIDAE \\ Subfamily: Cercopithecinae}

Cercopithecus Linnaeus, 1758. Systema Naturae Regnum Animale, 10th edition, 1: 26.

Additional references: Jablonsky and Frost (2010).

Comments: material originally identified as Cercopithecus sp. is not mapped because it may be assigned to this genus or to Chlorocebus as currently understood (Wilson and Reeder 2005).

Cercopithecus albogularis Sykes, 1831. Proc. Committee Sci. Corr. Zool. Soc. Lond. 1L. Sykes' monkey.

Synonyms: mitis.

Additional references: Dalton et al. (2015); Lawes (1990).

Chlorocebus aethiops Linnaeus, 1758, Systema Naturae Regnum Animale, 10th edition, 1: 28. Grivet.

Synonyms: Cercopithecus.

Chlorocebus pygerythrus Cuvier, 1821. Hist. Nat. Mamm. 24: 2. Vervet monkey.

Synonyms: aethiops.
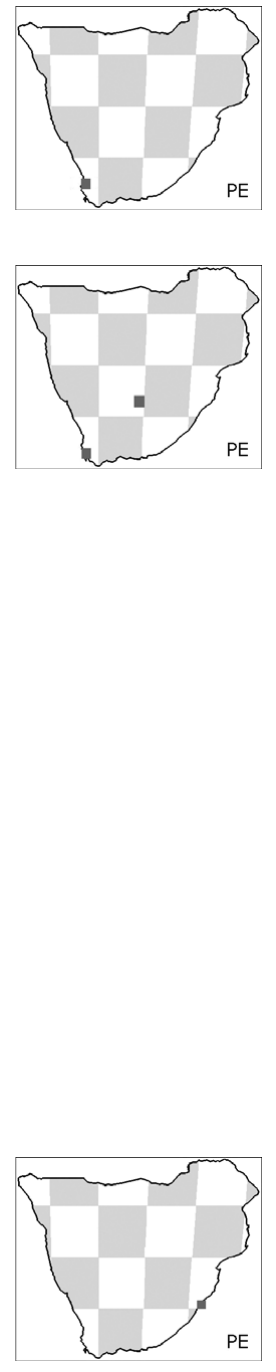

PE
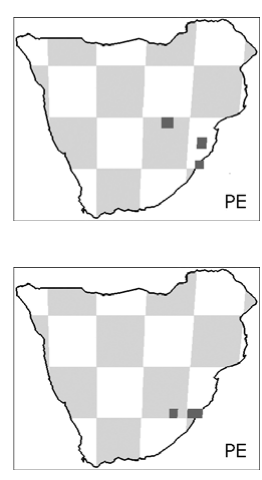

†Dinopithecus ingens Broom, 1937. S. Afr. J. Sci. 33: 753.

Synonyms: Papio.

Type locality: Schurveberg.

Additional references: Freedman and Brain (1977); Gilbert (2013);

Jablonsky and Frost (2010).

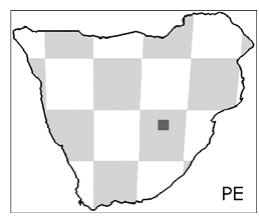


† Gorgopithecus major Broom and Robinson, 1949. Proc. Zool. Soc. Lond. 119: 386.

Synonyms: Parapapio

Type locality: Kromdraai.

Additional references: Gilbert (2013); Gilbert et al. (2016a); Gommery and Bento da Costa (2016); Jablonsky and Frost (2010).

Papio Erxleben, 1777. Systema Regni Animales per Classes : Mammalia: $\mathrm{xxx}, 15$.

Additional references: Williams et al. (2012).

†Papio izodi Gear, 1926. S. Afr. J. Sci. 23: 746.

Synonyms: Gorgopithecus; Parapapio; angusticeps; wellsi.

Type locality: Taung.

Additional references: Adams et al. (2013); Cooke (1990); Freedman (1965); Gilbert (2013); Gilbert et al. (2015); Gilbert et al. (2016b);
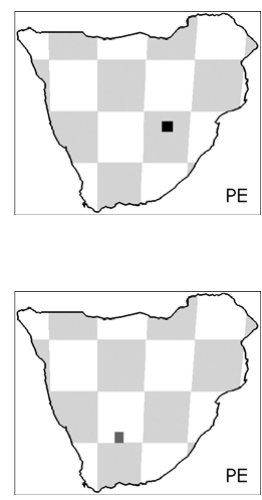

Gommery and Bento da Costa (2016); Heaton (2006); Jablonsky and Frost (2010); McKee (1993b); McKee and Keyser (1994).

Comments: known material from Kromdraai is referred to Papio (hamadryas) angusticeps and a new specimen to Papio hamadryas spp. indet. by Singleton et al. (2016).

†Papio robinsoni Freedman, 1957. Ann. Transvaal Mus. 23(2): 183. Synonyms: Dinopithecus; Parapapio; hamadryas robinsoni.

Type locality: Swartkrans.

Additional references: Gommery and Bento da Costa (2016); Gommery et al. (2008b); Freedman (1965); Freedman and Brain (1977); Jablonsky and Frost (2010).

Comments: Gommery and Bento da Costa (2016) consider this taxon to be a subspecies of Papio hamadryas.

†Papio spelaeus Broom, 1936. Ann. Transvaal Mus. 18: 396.

Type locality: Pretoria.

Additional references: Freedman (1957).

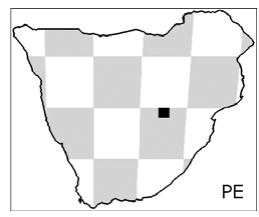

Papio ursinus Kerr, 1792. The Animal Kingdom, or Zoological System of the Celebrated Sir Charles Linnaeus: 63. Chacma baboon.

Synonyms: cynocephalus; comatus; hamadryas; porcarius; rhodesiae.

Type locality: Cape of Good Hope.

Additional references: Freedman $(1954,1965)$; Jablonsky and Frost
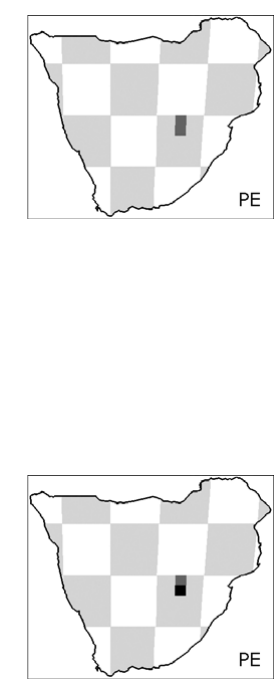

(2010); Roberts (1932); Williams et al. (2012).

Comments: material from the Koanaka Hills in Botswana is assigned to P. hamadryas by Williams et al. (2012), probably because some authors (e.g. Singleton et al. 2016) consider ursinus to be a subspecies of P. hamadryas. Here, Wilson and Reeder (2005) are followed in considering ursinus to be a full species. 
†Parapapio Jones, 1937. S. Afr. J. Sci. 33: 726.

Type locality: Sterkfontein.

Additional references: Grine and Hendey (1981); Jablonsky and Frost

(2010).

†Parapapio broomi Jones, 1937. S. Afr. J. Sci. 33: 727.

Synonyms: Brachygnathopithecus peppercorni; Gorgopithecus; makapani. Type locality: Sterkfontein.

Additional references: Freedman (1965); Freedman and Stenhouse (1972);

Gilbert (2013); Gommery and Bento da Costa (2016); Heaton (2006);

Jablonsky and Frost (2010); Maier (1970); Thackeray and Myer (2004).

†Parapapio jonesi Broom, 1940. Ann. Transvaal Mus. 20: 93.

Type locality: Sterkfontein.

Additional references: Freedman and Brain (1977); Freedman and

Stenhouse (1972); Gilbert (2013); Gommery and Bento da Costa

(2016); Heaton (2006); Jablonsky and Frost (2010); Maier (1970);

Thackeray and Myer (2004).

†Parapapio whitei Broom, 1940. Ann. Transvaal Mus. 20: 90.

Type locality: Sterkfontein.

Additional references: Freedman (1965); Freedman and Stenhouse

(1972); Gilbert (2013); Gommery and Bento da Costa (2016); Heaton (2006); Jablonsky and Frost (2010); Maier (1970).

†Theropithecus oswaldi Andrews, 1916. Ann. Mag. Nat. Hist., Series 8,

18: 417.

Synonyms: Parapapio coronatus?; Simopithecus; danieli; leakeyi.

Additional references: Broom and Robinson (1948); Dechow and Singer

(1984); Freedman (1957, 1976); Freedman and Brain (1977); Frost

et al. (2017); Gilbert (2007b, 2013); Heaton (2006); Jablonsky and

Frost (2010); Leakey (1943a).

Subfamily: Colobinae

†Cercopithecoides haasgati McKee, Von Mayer and Kuykendall, 2011.

J. Human Evol. 60: 90.

Type locality: Haasgat.

Additional references: Adams et al. (2013); Kegley et al. (2011); Von

Mayer (1998).

†Cercopithecoides williamsi Mollett, 1947. S. Afr. J. Sci. 43: 298.

Synonyms: molletti.

Type locality: Limeworks Makapansgat.

Additional references: Freedman (1961, 1965); Freedman and Brain

(1977); Jablonsky and Frost (2010); Kuykendall and Rae (2008); Maier
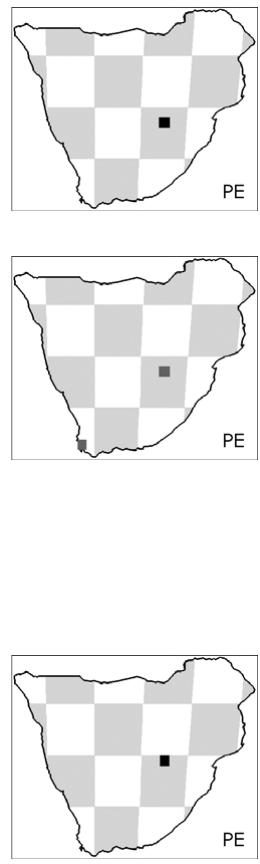

(1970); Von Mayer (1998).

†Microcolobus Benefit and Pickford 1986. Amer. Phys. Anthrop. 69: 446.

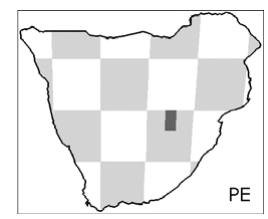


FAMILY: HOMINIDAE

Subfamily: Homininae

†Australopithecus africanus Dart, 1925. Nature 114: 198.

Synonyms: Plesianthropus; prometheus; transvaalensis.

Type locality: Taung.

Additional references: Broom (1929, 1939a); Broom and Robinson

(1949a, 1949c); Clarke and Tobias (1995); Dart (1929a, 1948a, 1954, 1959); Häusler and Berger (2001); Lockwood and Tobias (1999, 2002); MacLatchy et al. (2010); Toussaint et al. (2003).

†Australopithecus sediba Berger, De Ruiter, Churchill, Schmid, Carlson, Dirks and Kibii, 2010. Science 328: 195.

Type locality: Malapa.

Additional references: Carlson et al. (2016); De Ruiter et al. (2013);

Pickering et al. (2011).

Homo Linnaeus, 1758. Systema Naturae Regnum Animale, 10th edition, 1: 20.

Additional references: Clarke et al. (1970); Curnoe (2001); Curnoe and Tobias (2006); Grine (1993, 2005); Smith and Grine (2008).

†Homo erectus Dubois, 1894. Pithecanthropus erectus: ein meschenähnliche Übergangsform aus Java.

Synonyms: ergaster.

Additional references: MacLatchy et al. (2010); Susman (1993).

Comments: Dubois (1894) not seen.

†Homo gautengensis Curnoe, 2010. Homo- J. Comp. Human Biol. 61: 172.

Synonyms: habilis.

Type locality: Sterkfontein.

Additional references: Curnoe and Tobias (2006).
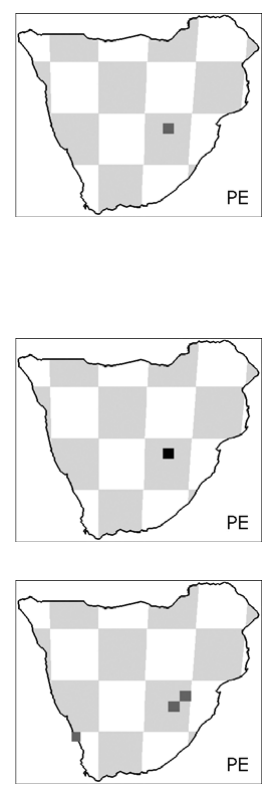

†Homo naledi Berger, Hawks, de Ruiter, Churchill et al. 2015. eLIFE 2015, 4: e09560: 1.

Type locality: Dinaledi Chamber.

Additional references: Dembo et al. (2016); Holloway et al. (2018).

†Homo rhodesiensis Woodward, 1921. Nature 108 (2716): 371-372. Synonyms: heidelbergensis.

Additional references: Dart (1948a, 1948b); MacLatchy et al. (2010); Singer (1956).

Comments: there is disagreement among specialists as to whether the
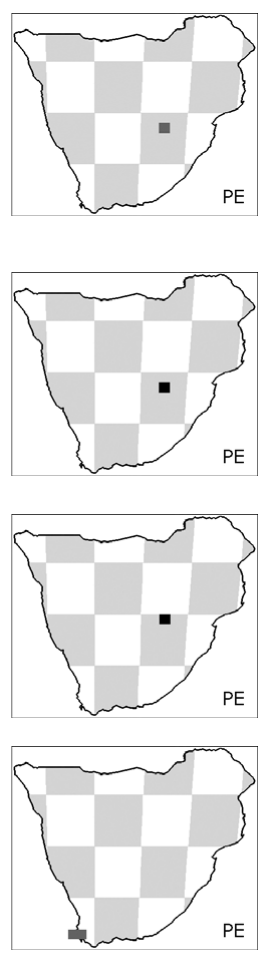

African material should be assigned to $H$. heidelbergensis or to

H. rhodesiensis (MacLatchy et al. 2010).

Homo sapiens Linnaeus, 1758. Systema Naturae Regnum Animale, 10th edition, 1: 20. Modern human.

Synonyms: capensis; helmei.

Additional references: Ackermann et al. (2016); Beaumont et al. (1978);

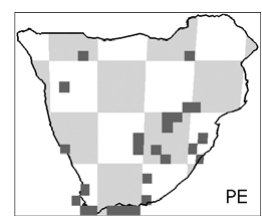


Bräuer et al. (1992); Bräuer and Singer (1996); Broom (1918);

Churchill et al. (1996); Dart (1940); De Villers (1973, 1974, 1976a, 1976b); Drennan (1937, 1953, 1955); Dreyer (1935); Galloway (1937a, 1937b); Grine (1998, 2000, 2012); Grine et al. (1995, 2007, 2010); Haughton et al. (1917); Hughes (1990); L'Abbé et al. (2008); Pycraft (1925); Keith (1933); MacLatchy et al. (2010); Pearson and Grine (1996); Pickford and Senut (1998); Rightmire (1978, 1979b); Rightmire and Deacon (1991); Rightmire et al. (2006); Singer (1954); Smith et al. (2012); Stynder et al. (2001); Trinkaus et al. (1999).

†Paranthropus robustus Broom, 1938. Nature 142: 379.

Synonyms: Australopithecus; crassidens.

Type locality: Kromdraai.

Additional references: Broom (1939a, 1939d, 1939e, 1949a); Broom and Robinson (1950a, 1950b, 1952); Clarke (1988); Clarke et al. (1970);

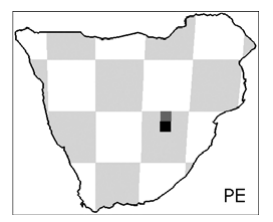

De Ruiter et al. (2006); Gommery (2000); Grine (1981, 1982, 1993);

Grine and Daegling (1993); Grine and Klein (1985); Grine and Susman (1991); Grine et al. (2012); Keyser (2000); Kuykendall and Conroy (1999); MacLatchy et al. (2010); Prat and Gommery (2012); Prat et al. (2014); Robinson (1970); Steininger et al. (2008).

\section{ORDER: RODENTIA Suborder: Sciuromorpha \\ FAMILY: SCIURIDAE \\ Subfamily: Xerinae}

Paraxerus palliatus Peters, 1852. Bericht Verhandl. K. Preuss. Akad. Wiss. Berlin 17: 273. Red bush squirrel.

Type locality: Quintangonha.

Xerus inauris Zimmermann, 1780. Geographische Geschichte des

Menschen und der Allgemein Verbreiteten Vierfüssigen Thiere 2: 344.

South African ground squirrel.

Synonyms: Geosciurus capensis.

Type locality: Kaffirland, 100 miles $(160 \mathrm{~km})$ north of Cape of Good Hope.

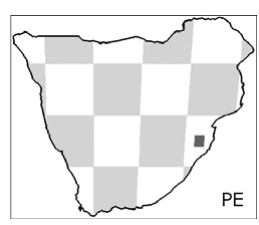

Additional references: Skurski and Waterman (2005).

\section{Suborder: Myomorpha}

FAMILY: GLIRIDAE

Subfamily: Graphiurinae

Graphiurus Smuts, 1832. Dissertation Zoologica, Ennumerationem Mammalium Capensium: 32-33.

Synonyms: Claviglis; Gliriscus; Myoxus.

Additional references: Daams and De Bruijn (1995); Holden (1996);

Montgelard et al. (2003); Webb and Skinner (1995).

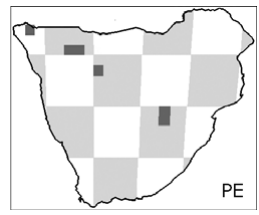


Graphiurus microtis Noack, 1887. Zool. Jahrb. 2: 248. Large savanna African dormouse.

Synonyms: griselda; littoralis; schneideri; streeteri.

Additional references: Roberts (1938).

Graphiurus murinus Desmarest, 1822. Mammalogie ou descriptions des espèces de mammifères, Part 2 (suppl.): 542. Forest African dormouse. Synonyms: alticola; tasmani; woosnami; vandami; zuluensis.

Type locality: Cape of Good Hope.

Additional references: Holden (1996); Kryštufek et al. (2004); Roberts (1931, 1938).

Graphiurus ocularis Smith, 1829. Zool. J. 4: 439. Spectacled African dormouse.

Synonyms: Myoxus; capensis.

Type locality: near Plettenberg Bay.

Additional references: De Winton (1898); Holden (1996); Smith (1849).
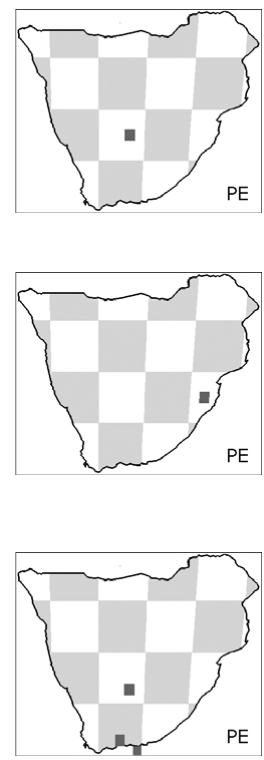

FAMILY: NESOMYIDAE

Subfamily: Cricetomyinae

Cricetomys ansorgei Thomas, 1904. Ann. Mag. Nat. Hist., Series 7, 13:

412. Southern giant pouched rat.

Synonyms: gambianus.

Saccostomus Peters, 1846. Bericht Verhandl. K. Preuss. Akad. Wiss. Berlin 11: 258.

Comments: the Holocene material should almost certainly be assigned to S. campestris since this is the only species currently understood to occur in southern Africa (Wilson and Reeder 2005).
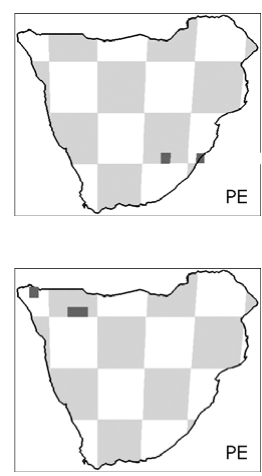

Saccostomus campestris Peters, 1846. Bericht Verhandl. K. Preuss. Akad. Wiss. Berlin 11: 258. Southern African pouched mouse.

Synonyms: anderssoni; hildae; limpopoensis; mashonae; pagei; streeteri. Type locality: Tete.

Additional references: Denys (1988b); Roberts (1914).

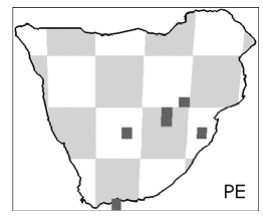

Subfamily: Delanymyinae

†Stenodontomys Pocock, 1987. Palaeontol. Afr. 26(7): 86.

Type locality: Limeworks Makapansgat.

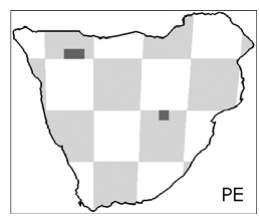




\section{Subfamily: Dendromurinae}

Dendromus Smith, 1829. Zool. J. 4: 438.

Synonyms: Poemys.

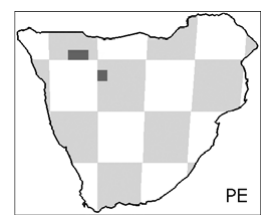

Dendromus melanotis Smith, 1834. S. Afr. Quart. J., Series 2, 2: 148. Grey African climbing mouse.

Synonyms: arenarius; concinnus; nigrifrons.

Type locality: near Port Natal: Durban.

Additional references: Roberts (1931); Smith (1849).

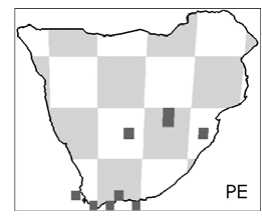

Dendromus mesomelas Brants, 1827. Het Geslacht der Muizen door

Linnaeus Opgesteld: 122. Brants' African climbing mouse.

Synonyms: ayresi; typica.

Type locality: Sunday's River, east of Port Elizabeth.

Additional references: Smith (1849).

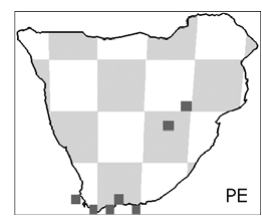

Dendromus mystacalis Heuglin, 1863. Nov. Act. Acad. Caes. Leop.-Carol. 30: 2 suppl.: 5. Chestnut African climbing mouse

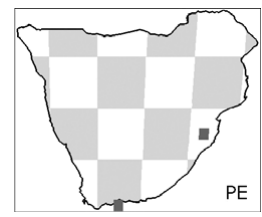

Dendromus nyikae Wroughton, 1909. Ann. Mag. Nat. Hist., Series 8, 3: 248. Nyika African climbing mouse.

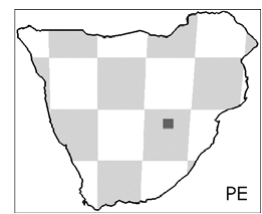

Malacothrix Wagner, 1843. In: Schreber, Die Säugethiere in Abbildungen nach der Natur, mit Beschreibungen, Suppl. 3: 496.

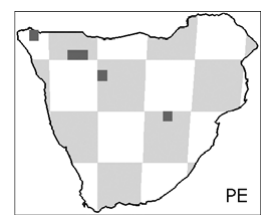

Malacothrix typica Smith, 1834. S. Afr. Quart. J., Series 2, 2: 148. Largeeared African desert mouse.

Type locality: Graaff Reinet District.

Additional references: Roberts (1932).

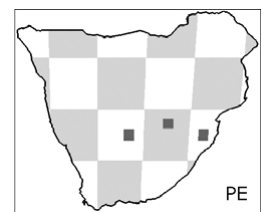

Steatomys Peters, 1846. Bericht Verhandl. K. Preuss. Akad. Wiss. Berlin 11: 258.

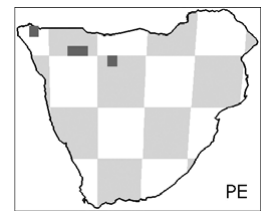


Steatomys krebsii Peters, 1852. Reise nach Mossambique, Säugethiere:

165. Krebs's African fat mouse.

Synonyms: chiversi; pentonyx; pratensis.

Type locality: Kaffraria.

Additional references: Roberts (1931).

Steatomys pratensis Peters, 1846. Bericht Verhandl. K. Preuss. Akad. Wiss. Berlin 11: 258. Common African fat mouse.

Synonyms: natalensis; opimus.

Type locality: Tete.

Comments: material from Botswana was identified as Steatomys opimus but, as currently understood, this species is almost entirely confined to West Africa (Musser and Carleton 2005).

\section{Subfamily: Mystromyinae}

Mystromys Wagner, 1841. Gelehrte Anz. I. K. Bayer. Akad. Wiss. München 12(54): col. 434.

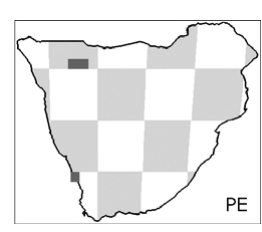

Mystromys albicaudatus Smith, 1834. S. Afr. Quart. J., Series 2, 2: 148.

African white-tailed rat.

Synonyms: Otomys; antiquus.

Type locality: Albany District.

Additional references: De Winton (1898); Grubb (2004); Smith (1849).

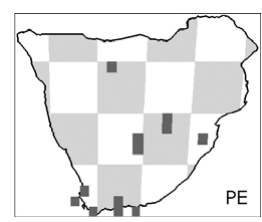

†Mystromys hausleitneri Broom, 1937. S. Afr. J. Sci. 33: 766.

Synonyms: hauslichtneri.

Type locality: Schurveberg.

Additional references: Broom (1948a).

Comments: there is disagreement about the identity of some Pleistocene

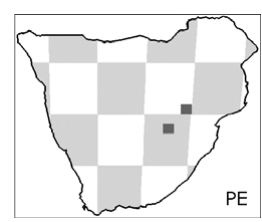
samples, which have been variously assigned to this species or to M. albicaudatus. See Avery (1998) for further discussion.

†Proodontomys cookei Pocock, 1987. Palaeontol. Afr. 26(7): 82.

Type locality: Limeworks Makapansgat

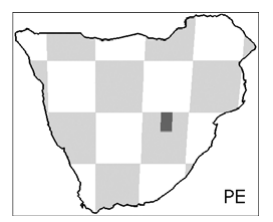

Subfamily: Petromyscinae

Petromyscus Thomas, 1926. Ann. Mag. Nat. Hist., Series 9, 17: 179. Additional references: Petter (1967).

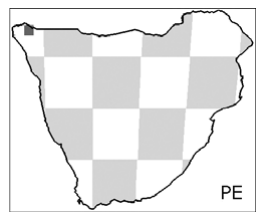


FAMILY: MURIDAE

Subfamily: Deomyinae

Acomys Geoffroy Saint-Hilaire, 1838. Ann. Sci. Nat. Zool. Paris, Series 2:

10: 126.

Additional references: Chevret et al. (1993a); Dippenaar and Rautenbach (1986).

Acomys spinosissimus Peters, 1852. Reise nach Mossambique,

Saugethiere: 160. Southern African spiny mouse.

Synonyms: cahirinus selousi; transvaalensis.

Type locality: Tete and Buio.

Additional references: Dippenaar and Rautenbach (1986).

Acomys subspinosus Waterhouse, 1837. Proc. Zool. Soc. Lond. 1837: 104.

Cape spiny mouse.

Type locality: Cape of Good Hope.

Additional references: Dippenaar and Rautenbach (1986).
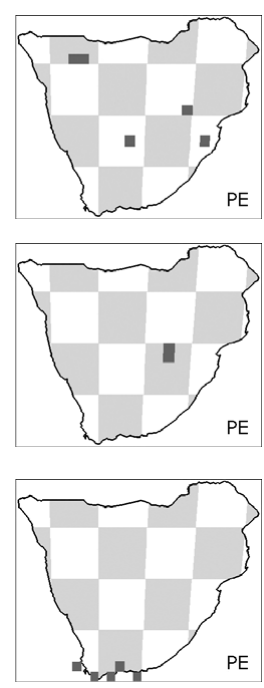

Subfamily: Gerbillinae

Desmodillus Thomas and Schwann, 1904. Abstr. Proc. Zool. Soc.

Lond. 2: 6.

Synonyms: Gerbillus; Pachyuromys.

Additional references: Pavlinov (2001).

Desmodillus auricularis Smith, 1834. S. Afr. Quart. J., Series 2, 2: 160.

Short-eared gerbil.

Synonyms: Gerbillus; brevicaudatus.

Type locality: Kamiesberg.

Additional references: Griffin (1990); Qumsiyeh (1986); Smith (1849).

Gerbilliscus Thomas, 1897. Proc. Zool. Soc. Lond. 1897: 433.

Synonyms: Gerbillus; Tatera.
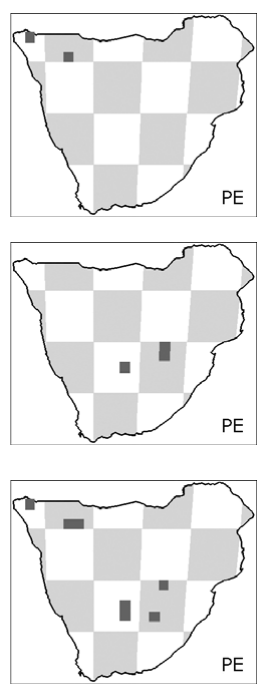

Gerbilliscus afra Gray, 1830. Spicil. Zool. 2: 10. Cape gerbil.

Synonyms: Gerbillus; Meriones; Tatera; africanus.

Type locality: vicinity of Cape Town.

Additional references: Chacornac (1999); Cuvier (1841); Davis (1949,

1965); Qumsiyeh (1986); Smith (1849); Smuts (1832).

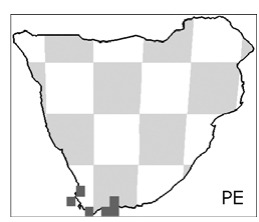

Comments: Gray, 1830 not seen. Citation according to Wilson and

Reeder (2005).

Gerbilliscus brantsii Smith, 1836. Report of the Expedition for Exploring

Central Africa: 43. Highveld gerbil.

Synonyms: Gerbillus; Tatera; montanus.

Type locality: Ladybrand.

Additional references: Davis (1949, 1965); Griffin (1990); Qumsiyeh

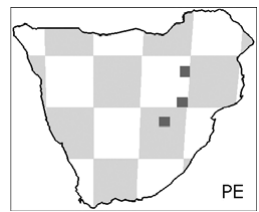

(1986); Roberts (1926); Smith (1849). 
Gerbilliscus leucogaster Peters, 1852. Bericht Verhandl. K. Preuss., Akad. Wiss. Berlin 17: 274. Bushveld gerbil.

Synonyms: Gerbillus; Tatera.

Type locality: Boror.

Additional references: Davis (1949, 1965); Griffin (1990); Qumsiyeh (1986).

Gerbillurus Shortridge, 1942. Ann. S. Afr. Mus. 36(1): 52.

Additional references: Qumsiyeh et al. (1991).
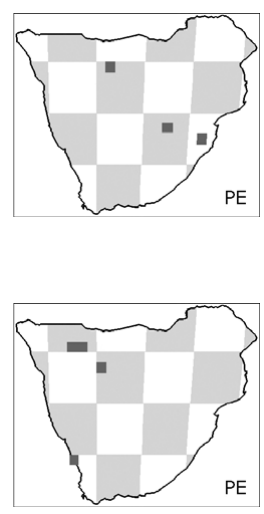

Gerbillurus paeba Smith, 1836. Report of the Expedition for Exploring Central Africa: 43. Paeba hairy-footed gerbil.

Synonyms: Gerbillus; tenuis.

Type locality: Vryberg.

Additional references: Chacornac (1999); De Winton (1898); Griffin

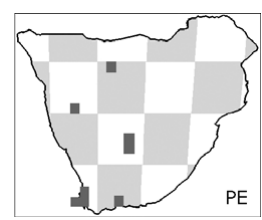

(1990); Perrin et al. (1999); Qumsiyeh (1986); Taylor (2000).

FAMILY: MURIDAE

Subfamily: Murinae

Aethomys Thomas, 1915. Ann. Mag. Nat. Hist., Series 8, 16: 477.

Additional references: Chimimba (1997, 1998, 2005); Chimimba and

Dippenaar (1994); Chimimba et al. (1999); Visser and Robinson (1986).

Comments: many specimens were identified as Aethomys sp. at a time when Micaelamys was not recognised as a separate genus. For this reason, distributions that rely on material not identified to species have not been mapped, pending re-identification.

Aethomys chrysophilus De Winton, 1896. Proc. Zool. Soc. Lond. 1896: 801. Red veld aethomys.

Additional references: Chimimba (2000); Chimimba and Dippenaar (1994); Roberts $(1926,1946)$.

Arvicanthis Lesson, 1842. Nouveau Tableau du Règne Animal: 147. Additional references: Grubb (2004).
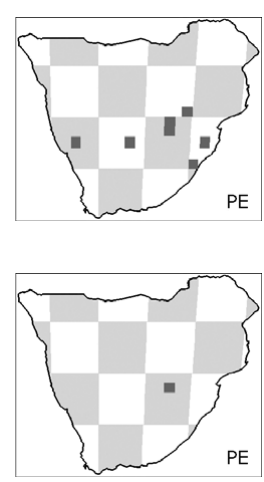

Dasymys Peters, 1875. Monatsb. K. Preuss. Akad. Wiss. Berlin 1875: 12. Additional references: Verheyen et al. (2003).

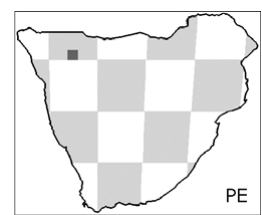


†Dasymys 'bolti' Broom, unpublished.

Type locality: Bolt's Farm.

Additional references: Denys (1990a).

Comments: Nomen nudum.

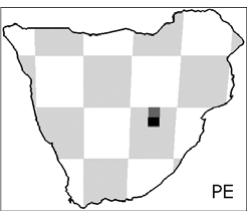

†Dasymys 'broomi' Broom, unpublished.

Synonyms: brevirostris.

Type locality: Sterkfontein.

Comments: Nomen nudum.

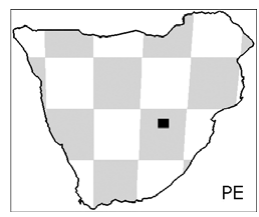

Dasymys incomtus Sundevall, 1846. Ofv. K. Svenska Vet.-Akad. Forhandl, 3: 120. Common dasymys.

Type locality: Durban.

Additional references: Gordon (1991); Mullin et al. (2004).

Comments: much of the material identified as D. incomtus was identified

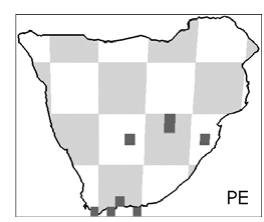
at a time when this species was the only one recognised in southern Africa. For this reason, this material should be considered as $D$. incomtus sensu lato until such time as it is re-examined.

†Dasymys 'lavocati' Broom, unpublished.

Type locality: Bolt's Farm.

Comments: Nomen nudum.

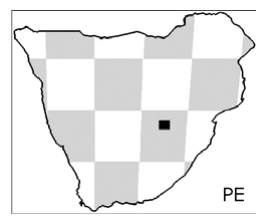

Grammomys Thomas, 1915. Ann. Mag. Nat. Hist. (8)16: 150. Synonyms: Thamnomys.

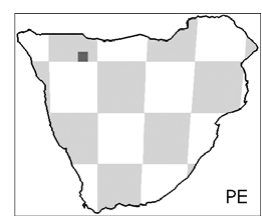

Grammomys dolichurus Smuts, 1832. Dissertation Zoologica, Ennumerationem Mammalium Capensium: 38. Common grammomys. Type locality: near Cape Town.

Additional references: Roberts (1931).

Lemniscomys rosalia Thomas, 1904. Ann. Mag. Nat. Hist., Series 7, 13:

414. Single-striped lemniscomys.

Synonyms: dorsalis; griselda.

Additional references: Smith (1849).

Mastomys Thomas, 1915. Ann. Mag. Nat. Hist., Series 8, 16: 477. Synonyms: Praomys.

Additional references: Britton-Davidian et al. (1995); Granjon et al. (1997); Grubb (2004); Taylor (2000).
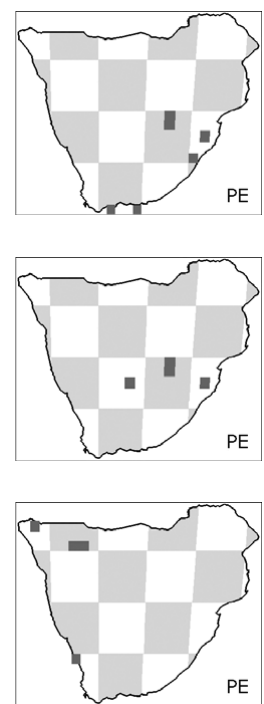
Mastomys coucha Smith, 1834. Report of the Expedition for Exploring Central Africa: 43. Southern African mastomys.

Synonyms: Praomys; natalensis.

Type locality: between Orange River and Tropic of Capricorn.

Additional references: Bronner et al. (2007); Dippenaar et al. (1993); Smit and Van der Bank (2001).

Comments: this material was originally identified as M. natalensis at a time when $M$. coucha was not recognised as a separate species.

Mastomys natalensis Smith, 1834. S. Afr. Quart. J., Series 2, 2: 146. Natal mastomys.

Synonyms: Praomys.

Type locality: Port Natal = Durban.

Additional references: Bronner et al. (2007); Dippenaar et al. (1993);

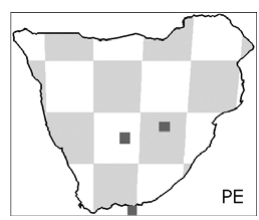

Green et al. (1980); Smit and Van der Bank (2001); Smith (1849).

Comments: see comment under M. coucha above.

Micaelamys Ellerman, 1941. Families and Genera of Living Rodents 2: 170. Synonyms: Aethomys.

Type locality: not given by Ellerman (1941) but presumably Deelfontein since this is the type locality of the type species M. granti.

Micaelamys namaquensis Smith, 1834. S. Afr. Quart. J., Series 2, 2: 160. Namaqua micaelamys.

Synonyms: Aethomys; lehocla.

Type locality: Cape of Good Hope: restricted to Witwater.

Additional references: Chimimba (2001); Chimimba and Dippenaar

(1994); Roberts (1926, 1946); Russo (2009); Smith (1849); Visser and Robinson (1986).

Mus Linnaeus, 1758. Systema Naturae Regnum Animale, 10th edition, 1: 59.

Additional references: Britton-Davidian et al. (2012); Veyrunes et al. (2005).

†Mus major Broom, ?unpublished.

Type locality: Bolt's Farm.

Comments: Nomen nudum?
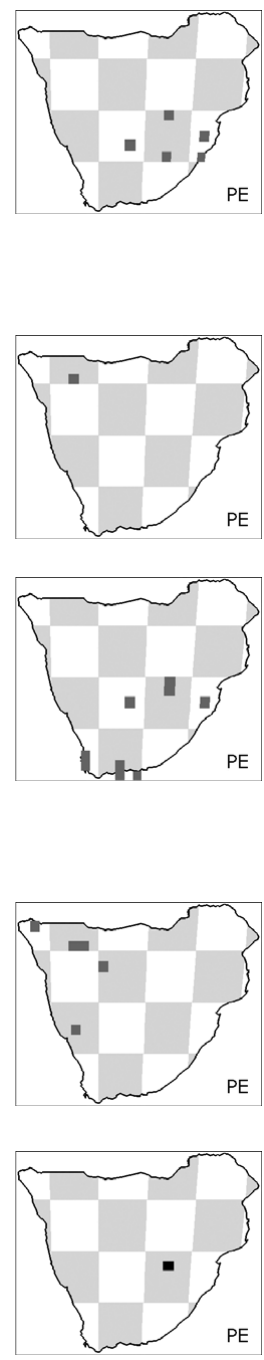

Mus minutoides Smith, 1834. S. Afr. Quart. J., Series 2, 2: 147. Southern African pygmy mouse.

Synonyms: Leggada.

Type locality: Cape Town.

Additional references: Britton-Davidian et al. (2012).

Comments: some of the specimens from the more arid areas may be $M$. indutus, but see comment under that species.

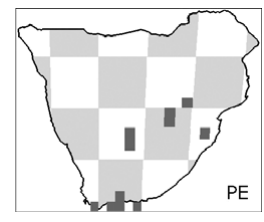


Mus triton Thomas, 1909. Ann. Mag. Nat. Hist., Series 8, 4: 548. Greybellied mouse.

Additional references: Britton-Davidian et al. (2012).

Myomyscus verreauxii Smith, 1834. S. Afr. Quart. J., Series 2, 2: 146.

Verreaux's white-footed rat.

Synonyms: Praomys; Myomys; colonus.

Type locality: near Cape Town.

Additional references: Grubb (2004); Smith (1849).

Pelomys fallax Peters, 1852. Bericht Verhandl. K. Preuss. Akad. Wiss. Berlin

17: 275. East African pelomys.

Type locality: Caia District.

Rhabdomys Thomas, 1916. Ann. Mag. Nat. Hist., Series 8, 18: 69.
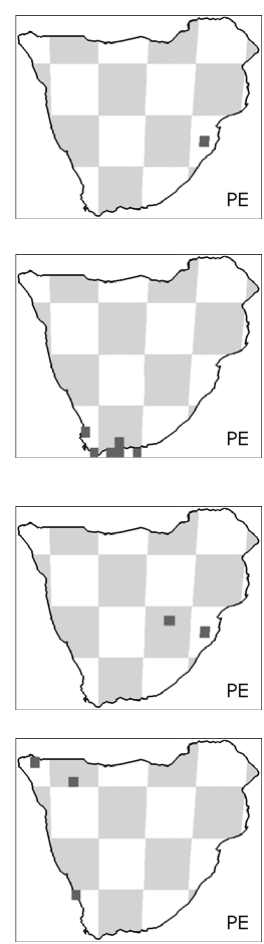

Rhabdomys dilectus De Winton, 1896. Proc. Zool. Soc. Lond. 1896: 803. Mesic four-striped grass rat.

Type locality: Mazoe.

Comments: Rhabdomys dilectus is again regarded (Wilson and Reeder 2005) as a separate species from $R$. pumilio, but at the time that most of the fossil material was identified only $R$. pumilio was recognised as a full species. No fossil material has yet been identified as $R$. dilectus, but see comments under R. pumilio below.

Rhabdomys pumilio Sparrman, 1784. K. Svenska Vet.-Akad. Handl.: 236. Xeric four-striped grass rat.

Type locality: Slangrivier: east of Knysna.

Additional references: Castiglia et al. (2012); Le Grange et al. (2015);

Roberts (1946); Smith (1849); Wroughton (1905).

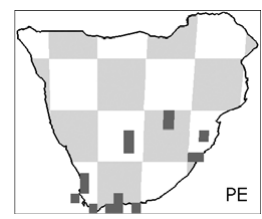

Comments: samples recorded here should be regarded as belonging to Rhabdomys pumilio sensu lato and are very likely to include some material that would currently be regarded as $R$. dilectus.

Thallomys Thomas, 1920. Ann. Mag. Nat. Hist., Series 9, 5: 141.

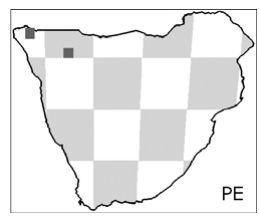

†Thallomys debruyni Broom, 1948. Ann. Transvaal Mus. 21: 35. Type locality: Taung Hrdlicka's Cave.

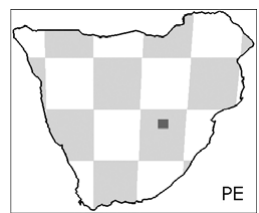


Thallomys paedulcus Sundevall, 1846. Ofv. K. Svenska Vet.-Akad.

Forhandl. 3: 120.

Synonyms: Acacia thallomys.

Type locality: provisionally fixed as Crocodile Drift, Brits.

Additional references: Taylor (2000); Taylor et al. (1995).

Comments: this material was identified as T. paedulcus at a time when only the one species was recognised, but some would now almost certainly be identified as T. nigricauda.

Zelotomys Osgood, 1910. Field Mus. Nat. Hist. Publ., Zool. Series 10: 7.
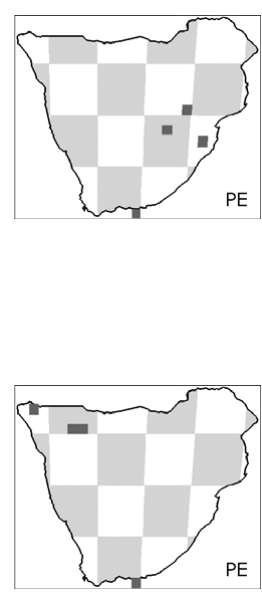

Zelotomys woosnami Schwann, 1906. Proc. Zool. Soc. Lond. 1906: 108.

Woosnam's zelotomys.

Type locality: Molopo River.

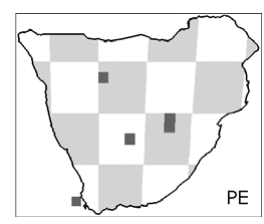

Subfamily: †Myocricetodontinae

†Myocricetodon Lavocat, 1952. C. R. Acad. Sci. Paris, 235: 190.

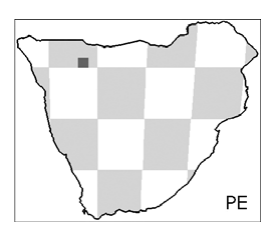

Subfamily: Otomyinae

†Myotomys campbelli Broom and Schepers, 1946. Transvaal Mus. Mem. 2: 29.

Type locality: Taung.

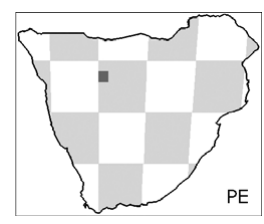

Myotomys sloggetti Thomas, 1902. Ann. Mag. Nat. Hist., Series 7, 10:

311. Rock karoo rat.

Synonyms: Otomys.

Type locality: Deelfontein.

Additional references: Taylor et al. (2004).

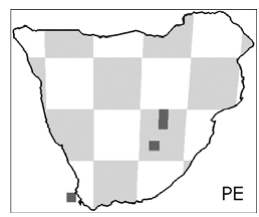

Myotomys unisulcatus Cuvier, 1829. In: Geoffroy Saint-Hilaire and

Cuvier, Histoire Naturelle des Mammifères: 6 LX Otomys cafre. Bush karoo rat.

Synonyms: Euryotis; Otomys.

Type locality: Matjiesfontein.

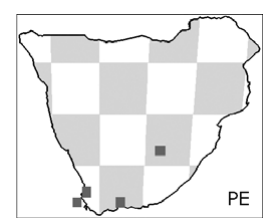

Additional references: Edwards (2009); Edwards et al. (2011); Smith

(1849); Taylor et al. (1989, 2004).

Otomys Cuvier, 1825. Des Dents des Mammifères Considérées comme Caractères Zoologiques: 168.

Additional references: Chevret et al. (1993b); Maree (2002); Meester et al. (1992). 
Comments: many specimens identified as Otomys sp. were identified at a time when Myotomys was not recognised as a separate genus. For this reason, distributions that rely on material not identified to species have not been mapped, pending further identification.

Otomys angoniensis Wroughton, 1906. Ann. Mag. Nat. Hist., Series 7, 18: 274. Angoni vlei rat.

Additional references: Bronner and Meester (1988); Taylor et al. (2004).

†Otomys gracilis Broom, 1937. S. Afr. J. Sci. 33: 761.

Synonyms: Palaeotomys.

Type locality: Schurveberg.

Otomys irroratus Brants, 1827. Het Geslacht der Muizen door Linnaeus

Opgesteld: 94. Southern African vlei rat.

Synonyms: Euryotis; Mus.

Type locality: Cape Town District.

Additional references: Bronner et al. (1988); Engelbrecht et al. (2011);

Smith (1849); Taylor (2000); Taylor et al. (1989, 2009).

Otomys Iaminatus Thomas and Schwann, 1905. Abst. Proc. Zool. Soc.

Lond. 1905(i)(18): 23. KwaZulu vlei rat.

Type locality: Sibudeni.

Additional references: Roberts $(1919,1932)$.

Otomys saundersiae Roberts, 1929. Ann. Transvaal Mus. 13: 114.

Saunders' vlei rat.

Synonyms: karoensis.

Type locality: Grahamstown.

Additional references: Roberts (1931); Taylor et al. (1993, 2009).
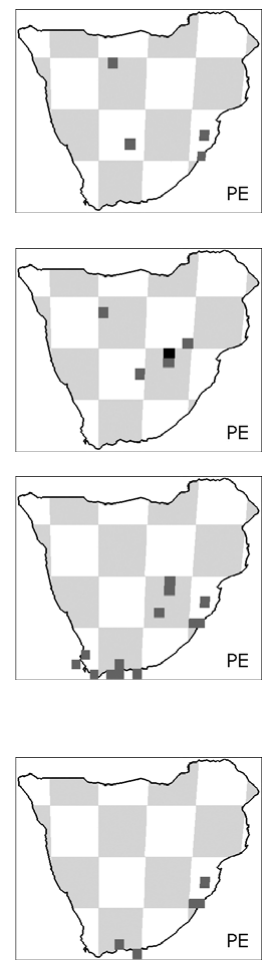

Parotomys Thomas, 1918. Ann. Mag. Nat. Hist., Series 9, 2: 205.

Additional references: Maree (2002); Meester et al. (1992).
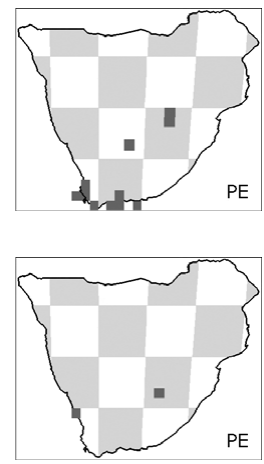

Parotomys brantsii Smith, 1834. S. Afr. Quart. J., Series 2, 2: 150. Brants' whistling rat.

Synonyms: Euryotis.

Type locality: 'towards the mouth of the Orange River'.

Additional references: Rookmaker and Meester (1988); Smith (1849);

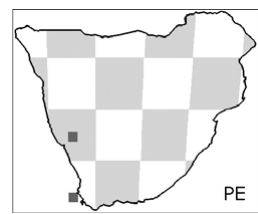

Taylor et al. (1989, 2004).

Parotomys littledalei Thomas, 1918. Ann. Mag. Nat. Hist., Series 9, 2:

205. Littledale's whistling rat.

Type locality: Tuin Kenhardt.

Additional references: Roberts (1933); Taylor et al. (1989, 2004).

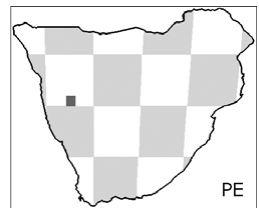




\section{Suborder: Anomaluromorpha \\ FAMILY: PEDETIDAE}

Pedetes Illiger, 1811. Prodromus Systematis Mammaliam et Avium: 81.

Pedetes capensis Forster, 1778. K. Svenska Vet.-Akad. Handl. 39: 109.

South African spring hare.

Type locality: Cape of Good Hope.

Additional references: Roberts (1946).

†Pedetes hagenstadti Dreyer and Lyle, 1931. New Fossil Mammals and Man from South Africa: 40.

Type locality: Florisbad.
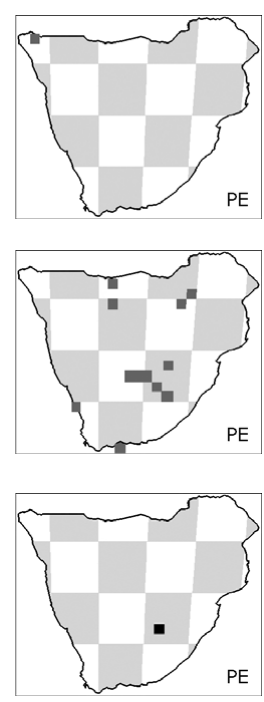

\section{Suborder: Hystricomorpha}

FAMILY: BATHYERGIDAE

Subfamily: Bathyerginae

Bathyergus Illiger, 1811. Prodromus Systematis Mammaliam et Avium: 86. Additional references: De Graaff (1965); Faulkes et al. (2004).

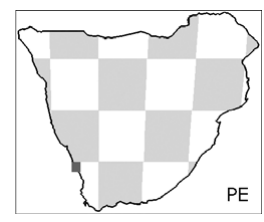

Bathyergus janetta Thomas and Schwann, 1904, Abstr. Proc. Zool. Soc.

Lond. 2: 6. Namaqua dune mole-rat.

Type locality: Port Nolloth.

Additional references: Thomas and Schwann (1904b).

Bathyergus suillus Schreber, 1782. Die Säugethiere in Abbildungen nach der Natur, mit Beschreibungen 4: 714. Cape dune mole-rat.

Type locality: Cape of Good Hope.

Comments: volume 4 was published in 1792 according to the version reproduced in the Biodiversity Heritage Library (www.biodiversity library.org/item/135004\#page/137/mode/1 up; accessed 19 May

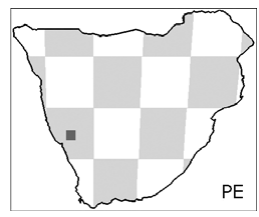

2017) and 'Der Sandmoll' is discussed on pp. 715-716, not 714, and Mus suillus is listed on p. 932. Plate 204B appears in an undated volume containing plates 166-280 (www.biodiversitylibrary.org/ item/97330\#page/125/mode/1 up). Bennett et al. (2009) give the reference for this species as Schreber, J.C.D. 1782. Die Säugthiere in Abbildungen nach der Natur, mit Beschreibungen. Supplementband III [Dritte Abtheilung: Die Beutelthiere und Rage]. Wolfgang Walther, Erlangen, Germany. This publication was not seen. 
Cryptomys Gray, 1864. Proc. Zool. Soc. Lond. 1864: 124.

Additional references: De Graaff (1965); Faulkes et al. (2004); Ingram et al. (2004); Kock et al. (2006); Thomas (1917).

Comments: some specimens previously identified as Cryptomys sp. may be referable to Fukomys sp. as currently understood.

Cryptomys hottentotus Lesson, 1826. Zool. 1: 166. Southern African mole-rat.

Type locality: near Paarl.

Additional references: Avery (2004); Denys (1988a); Roberts (1913, 1946). Comments: it is possible that some of the material may be referable to
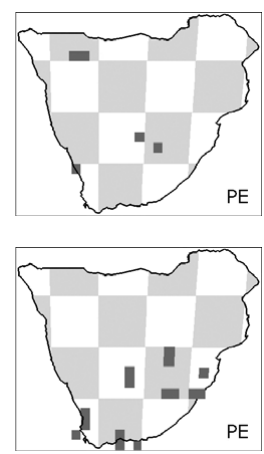
Fukomys damarensis, which was not recognised as a separate species when many of the samples were identified.

†Cryptomys robertsi Broom, 1937. S. Afr. J. Sci. 33: 760.

Type locality: Schurveberg.

Comments: it has been suggested that this taxon is inseparable from Georychus capensis (Avery, 1998).

Georychus Illiger, 1811. Prodromus Systematis Mammaliam et Avium: 87. Additional references: De Graaff (1965); De Winton (1898); Faulkes et al. (2004); Gray (1864a); Thomas (1895b, 1917).

Comments: these should almost certainly be assigned to G. capensis.

Georychus capensis Pallas, 1778. Njova. Spec. Quad. Glir. Ord. 76: 172. Cape mole-rat.

Type locality: Cape of Good Hope.

Additional references: Bennett et al. (2006, 2016).

Comments: the Holocene occurrence in 2032 may be considered unlikely: this taxon is currently endemic to South Africa (Maree et al. 2017), whereas Cryptomys hottentotus occurs in southern Zimbabwe (Maree and Faulkes 2016).

FAMILY: HYSTRICIDAE

Subfamily: Hystricinae

Hystrix africaeaustralis Peters, 1852. Reise nach Mossambique, Saugethiere: 170. Cape porcupine.

Additional references: Maguire (1976).

Hystrix cristata Linnaeus, 1758. Systema Naturae Regnum Animale, 10th edition, 1: 56. Crested porcupine.

Additional references: Maguire (1976).

†Hystrix makapanensis Greenwood, 1958. Ann. Mag. Nat. Hist., Series 13, 1: 365.

Synonyms: major.

Type locality: Limeworks Makapansgat.

Additional references: Adams (2012a); Greenwood (1955).

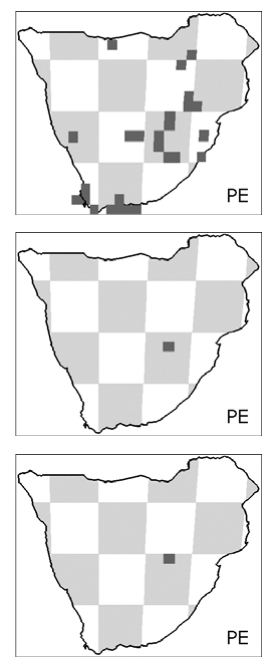


FAMILY: PETROMURIDAE

Petromus Smith, 1831. S. Afr. Quart. J. 1(5): 10

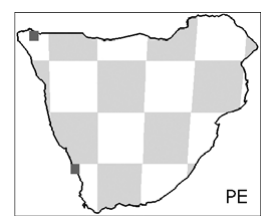

Petromus typicus Smith, 1831. S. Afr. Quart. J. 1(5): 11. Dassie rat. Type locality: 'Mountains towards mouth of Orange River'. Additional references: Roberts (1938, 1946); Smith (1849).

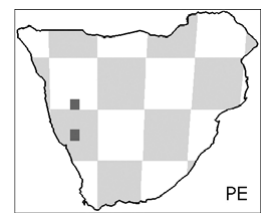

\section{FAMILY THRYONOMYIDAE}

Thryonomys Fitzinger, 1867. Sitzb. Akad. Wiss. Wein 56(1): 141.

Thryonomys swinderianus Temminck, 1827. Monographies de Mammalogie 1: 248. Greater cane rat. Additional references: Van der Merwe (2007).

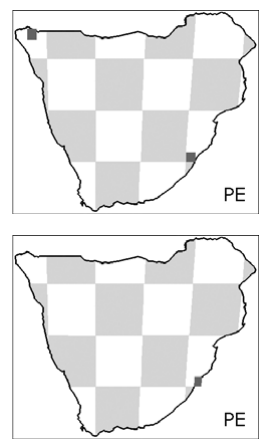

\section{ORDER: LAGOMORPHA}

FAMILY: LEPORIDAE

Bunolagus monticularis Thomas, 1903. Ann. Mag. Nat. Hist., Series 7, 11:

78. Riverine rabbit.

Synonyms: Lepus.

Type locality: Deelfontein.

Additional references: Robinson and Dippenaar (1987); Robinson and

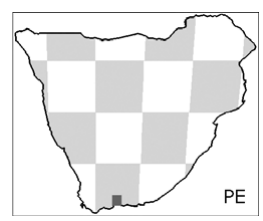

Matthee (2005); Robinson and Skinner (1983).

Lepus Linnaeus, 1758. Systema Naturae Regnum Animale, 10th edition, 1: 57.

Additional references: Robinson and Dippenaar (1987); Robinson and Matthee (2005).

Lepus capensis Linnaeus, 1758. Systema Naturae Regnum Animale, 10th edition, 1: 58. Cape hare.

Type locality: Cape of Good Hope.

Additional references: Roberts (1932).

Lepus saxatilis Cuvier, 1823. Dict. Sci. Nat. 26: 309. Scrub hare. Type locality: 'à trois journées au nord du cap de Bonne-Espérance' (north of Cape of Good Hope).

Additional references: Kolbe (1948); Roberts (1932); Robinson and

Dippenaar (1983).
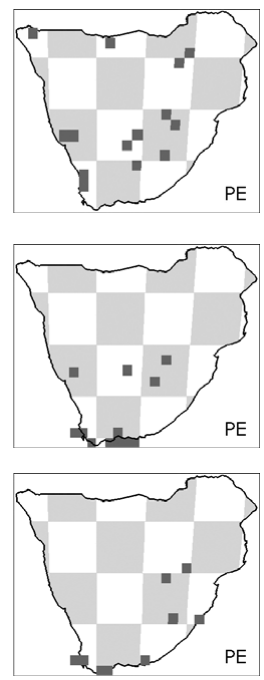
Pronolagus Lyon, 1904. Smithson. Misc. Coll. 45: 416. Additional references: Robinson and Matthee (2005).

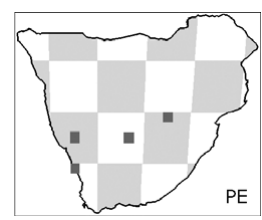

Pronolagus crassicaudatus Geoffroy Saint-Hilaire, 1832. Mag. Zool. Paris

2: cl. 1, pl. 9 and text. Natal red rock hare.

Synonyms: Oryctolagus.

Type locality: 'Port Natal' (Durban).

Pronolagus randensis Jameson, 1907. Ann. Mag. Nat. Hist., Series 7, 20: 404. Jameson's red rock hare.

Type locality: 'Observatory Kopje Johannesburg'.

Pronolagus rupestris Smith, 1834. S. Afr. Quart. J., Series 2, 2: 174.

Smith's red rock hare.

Synonyms: crassicaudatus.

Type locality: Probably Van Rhynsdorp District.

Additional references: Roberts (1938).
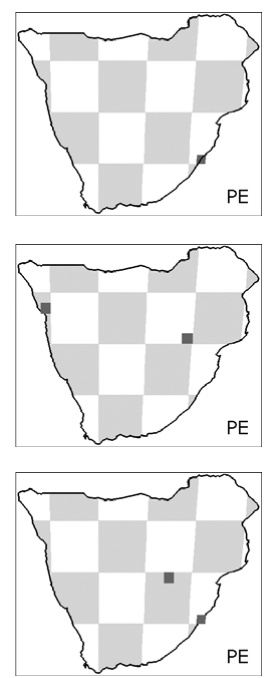

\section{ORDER: ERINACEOMORPHA}

FAMILY: ERINACEIDAE

Subfamily: Erinaceinae

Atelerix frontalis Smith, 1831. S. Afr. Quart. J. 1(5): 10, 29. Southern

African hedgehog.

Synonyms: Erinaceus capensis.

Type locality: northern parts of the Graaff Reinet district.

Additional references: Smith (1830, 1838, 1849).

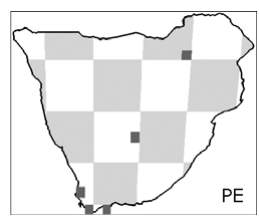

†Erinaceus broomi Butler and Greenwood, 1973. Foss. Vert. Afr. 3: 7-42.

Synonyms: Atelerix major.

Type locality: Bolt's Farm (A. major).

Additional references: Broom (1937b, 1948a).

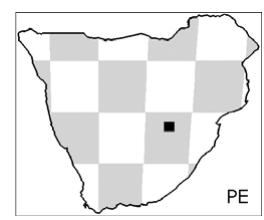

\section{ORDER: SORICOMORPHA \\ FAMILY: SORICIDAE \\ Subfamily: Crocidurinae}

Crocidura Wagler, 1832. Isis von Oken 25: 275.

Additional references: Butler et al. (1989); Jenkins et al. (1998); Meester (1953a, 1961b, 1963); Meester et al. (1985).

Crocidura cyanea Duvernoy, 1838. Mem. Soc. Hist. Nat. Strasbourg 2: 2. Reddish-grey musk shrew.

Type locality: Citrusdal Citrusdal fide Shortridge (1942: 27).
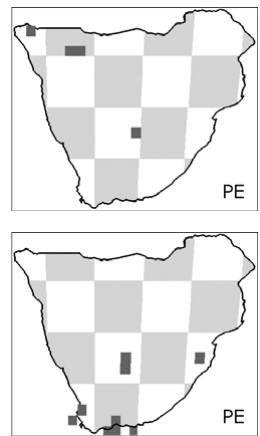
Crocidura flavescens Geoffroy Saint-Hilaire, 1827. Dict. Class. Hist. Nat. 11: 324. Greater red musk shrew.

Synonyms: Sorex; capensis.

Type locality: King William's Town.

Additional references: Smith (1849).

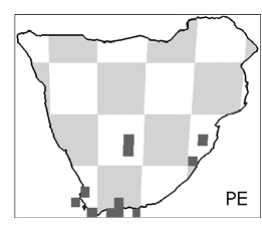

Crocidura fuscomurina Heuglin, 1865. Nov. Acta Acad. Caes. Leop.-Carol.

32: 36. Bicolored musk shrew.

Synonyms: bicolor.

Additional references: Hutterer (1983).

Crocidura hirta Peters, 1852. Reise nach Mossambique, Saugethiere: 78. Lesser red musk shrew.

Type locality: Tete.

Crocidura mariquensis Smith, 1844. Illustrations of the Zoology of South Africa: pl. 44, fig. 1. Swamp musk shrew.

Synonyms: Sorex.

Type locality: near Marico River.

Additional references: Dippenaar (1977, 1979); Meester (1964b); Smith (1849).

Comments: citation as given in Wilson and Reeder (2005). There is clearly considerable uncertainty surrounding the publication dates of Smith's Illustrations of South African Zoology (Low and Evenhuis 2014).

Crocidura silacea Thomas, 1895. Ann. Mag. Nat. Hist., Series 6, 16: 53.

Lesser grey-brown musk shrew.

Type locality: Figtree Creek.

†Crocidura taungensis Broom, 1948. Ann. Transvaal Mus. 21: 10.

Synonyms: Suncus.

Type locality: Taung.

Additional references: Meester (1954).

Suncus Ehrenberg, 1832. In Hemprich and Ehrenberg, Symbolae Physicae, seu, Icones et Descriptiones 2: k.

Additional references: Jenkins et al. (1998); Meester (1953a); Meester and Lambrechts (1971); Meester and Meyer (1972); Quérouil et al. (2001).

Comments: there is doubt about authorship of this series of publications. See discussion on www.zoonomen.net/mammtax/cit/jours.html.

Suncus infinitesimus Heller, 1912. Smithson. Misc. Coll. 60(12): 5. Least dwarf shrew.
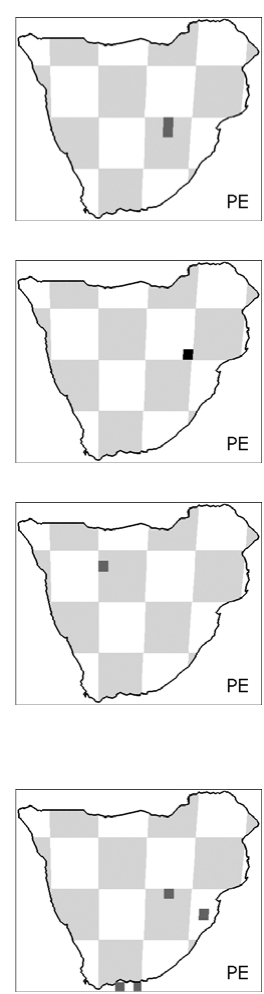
Suncus lixus Thomas, 1897. Proc. Zool. Soc. Lond. 1897: 930. Greater dwarf shrew.

Synonyms: Crocidura.

Suncus varilla Thomas, 1895. Ann. Mag. Nat. Hist., Series 6, 16: 54.

Lesser dwarf shrew.

Type locality: East London.

Additional references: Roberts (1946).

Subfamily: Myosoricinae

Myosorex Gray, 1837. Proc. Zool. Soc. Lond. 1837: 124.

Additional references: Matthews and Stynder (2011b); Meester (1953a,

1958); Quérouil et al. (2001); Willows-Munro and Matthee (2009).

Myosorex cafer Sundevall, 1846. Ofv. K. Svenska Vet.-Akad. Forhandl.

3: 119. Dark-footed mouse shrew.

Synonyms: tenuis.

Type locality: 'E Caffraria interiore et Port-Natal'.

Additional references: Avery (1998).

†Myosorex robinsoni Meester, 1954. Ann. Transvaal Mus. 22: 272.

Type locality: Swartkrans.

Additional references: Butler (2010); Butler and Greenwood (1979).

Myosorex varius Smuts, 1832. Dissertation Zoologica, Ennumerationem

Mammalium Capensium: 108. Forest shrew.

Synonyms: Sorex.

Type locality: Algoa Bay, Port Elizabeth.

Additional references: Roberts (1924); Smith (1849).
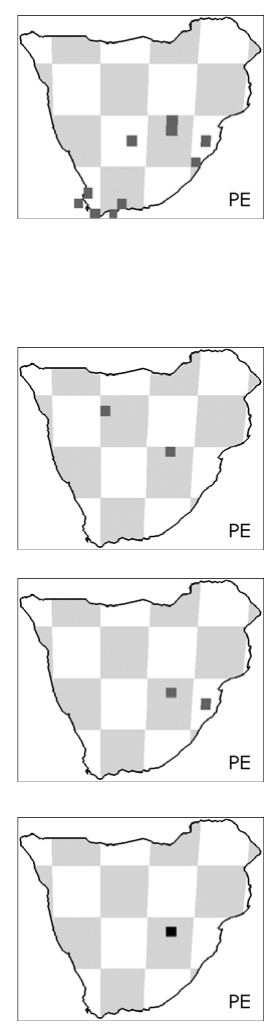

\section{ORDER: CHIROPTERA Suborder: Microchiroptera \\ FAMILY: RHINOLOPHIDAE}

Rhinolophus Lacépède, 1799. Tableau des Divisions, Sous-divisions, Ordres, et Genres des Mammifères: 15.

Additional references: Andersen (1905); Taylor et al. (2012).

Comments: Lacépède, 1799 not seen. Citation according to Wilson and Reeder (2005).

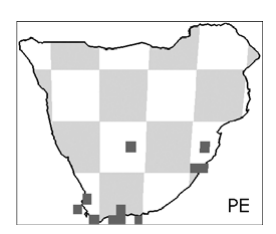

Rhinolophus blasii Peters, 1866. Monatsb. K. Preuss. Akad. Wiss. Berlin 1866: 17. Blasius' horseshoe bat.
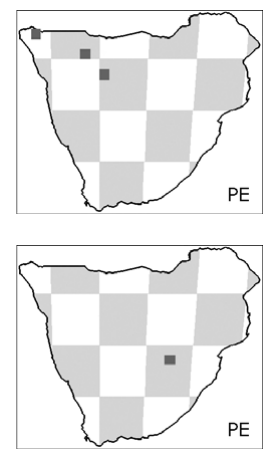
Rhinolophus capensis Lichtenstein, 1823. Verzeichniss der Doubletten des zoologischen Museums der Königl. Universität zu Berlin: 4. Cape horseshoe bat.

Type locality: Cape of Good Hope.

Rhinolophus clivosus Cretzschmar, 1826. In: Rüppell, Atlas zu der Reise im nördlichen Afrika Zoologie: 47. Geoffroy Saint-Hilaire's horseshoe bat. Synonyms: geoffroyi.

Rhinolophus darlingi Andersen, 1905. Ann. Mag. Nat. Hist., Series 7, 15: 70. Darling's horseshoe.

Type locality: Mazoe.

Rhinolophus hildebrandtii Peters, 1878. Monatsb. K. Preuss. Akad. Wiss. Berlin 1878: 195. Hildebrandt's horseshoe bat. Additional references: Taylor et al. (2012).

FAMILY: HIPPOSIDERIDAE

Hipposideros Gray, 1831. Zoological Miscellany 1: 37.
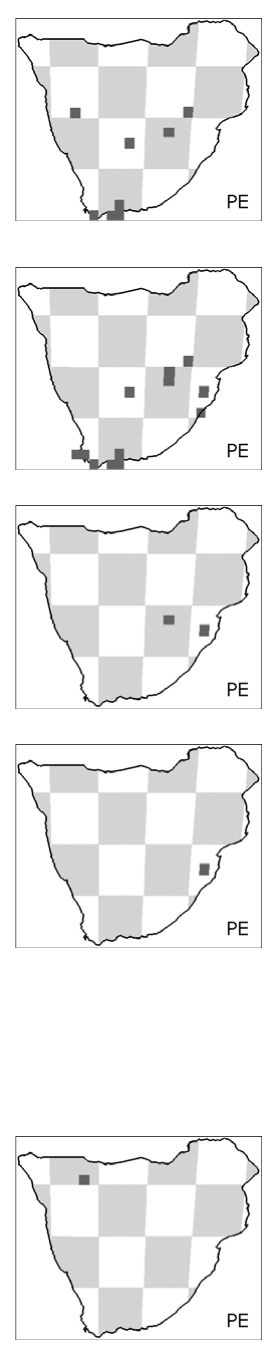

Hipposideros caffer Sundevall, 1846. Ofv. K. Svenska Vet.-Akad. Forhandl. 3: 118. Sundevall's leaf-nosed bat.

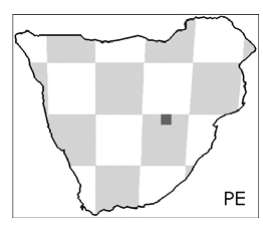

Hipposideros gigas Wagner, 1845. Arch. Naturgesch. 11(1): 148. Giant leaf-nosed bat.

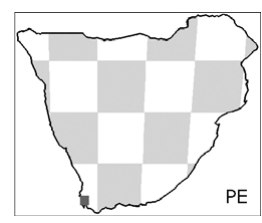

Hipposideros vittatus Peters, 1852. Naturwissenschaftliche Reise nach Mossambique: 32. Striped leaf-nosed bat.

Synonyms: Rhinolophus; commersoni.

Additional references: Geoffroy Saint-Hilaire (1813); Thomas (1904a).

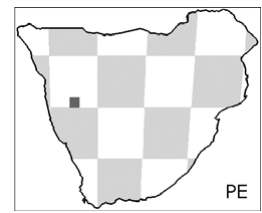


Nycteris Geoffroy Saint-Hilaire and Cuvier, 1795. Mag. Encyclop. 2: 186.

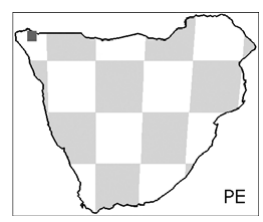

Nycteris thebaica Geoffroy Saint-Hilaire, 1818. Description des

Mammifères qui se trouvent en Egypte 2: 119. Egyptian slit-faced bat. Additional references: Gray et al. (1999).

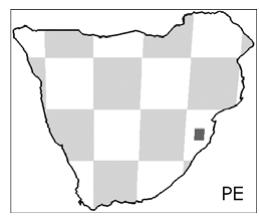

FAMILY: EMBALLONURIDAE

Taphozous Geoffroy Saint-Hilaire, 1818. Description des Mammifères qui se trouvent en Egypte 2: 113.

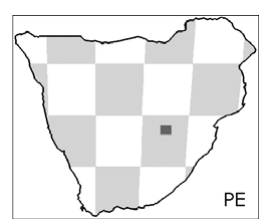

FAMILY: MOLOSSIDAE

Subfamily: Molossinae

Tadarida Rafinesque, 1814. Précis des découvertes et travaux somiologiques: 55 .

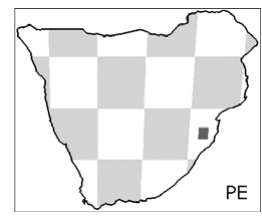

Tadarida aegyptiaca Geoffroy Saint-Hilaire, 1818. Description des Mammifères qui se trouvent en Egypte 2: 128. Egyptian free-tailed bat.

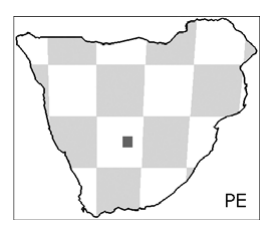

FAMILY: VESPERTILIONIDAE

Subfamily: Vespertilioninae

Eptesicus Rafinesque, 1820. Ann. Nature 1: 2.

Comments: distribution of material assigned only to genus has been disregarded because it may well belong to Neoromicia as presently understood.

Eptesicus hottentotus Smith, 1833. S. Afr. Quart. J., Series 2, 1: 59. Longtailed serotine.

Type locality: Uitenhage.

Additional references: Hill and Harrison (1987); Kearney et al. (2002).

Glauconycteris variegata Tomes, 1861. Proc. Zool. Soc. Lond. 1861: 36. Variegated butterfly bat.
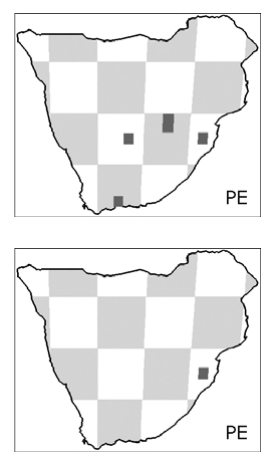
Neoromicia capensis Smith, 1829. Zool. J. 4: 435. Cape serotine. Additional references: Riccucci and Lanza (2008).

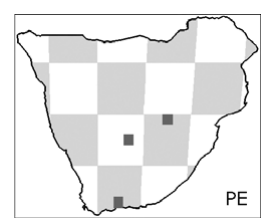

Scotophilus nigrita Schreber, 1775. Die Säugethiere in Abbildungen nach der Natur, mit Beschreibungen 1: 171. Giant house bat.

Synonyms: dinganii.

Additional references: Robbins (1978); Robbins et al. (1985).

Comments: volume 1 was published in 1775 (not 1774) according to

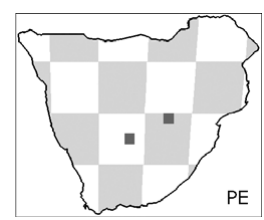
the version obtained from the University of Heidelberg (http:// digi.ub.uni-heidelberg.de/schreber1875textbd) and Vespertilio nigrita is listed on p. 190. Plate 58 appears in an undated volume containing plates 1-80 (www.biodiversitylibrary.org/item/97331\#page/279/ mode/1up).

Subfamily: Myotinae

Cistugo lesueuri Roberts, 1919. Ann. Transvaal Mus. 6: 112. Lesueur's wing-gland bat.

Synonyms: Myotis.

Type locality: Lormarins.

Additional references: Kearney and Van Schalkwyk (2009).

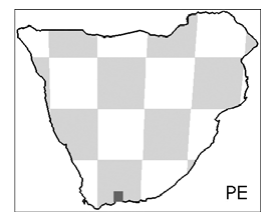

Myotis Kaup, 1829. Skizz. Entwickel.-Gesch. Nat. Syst. Europ. Thierwelt 1: 106.

Additional references: Gray (1842).

Myotis tricolor Temminck, 1832. In: Smuts, Dissertation Zoologica, Ennumerationem Mammalium Capensium: 106. Temminck's myotis. Type locality: Cape Town.

Additional references: Kearney et al. (2002).

Myotis welwitschii Gray, 1866. Proc. Zool. Soc. Lond. 1866: 211.

Welwitsch's myotis.

Synonyms: Scotophilus.

Additional references: Gray (1866); Ratcliffe (2002).
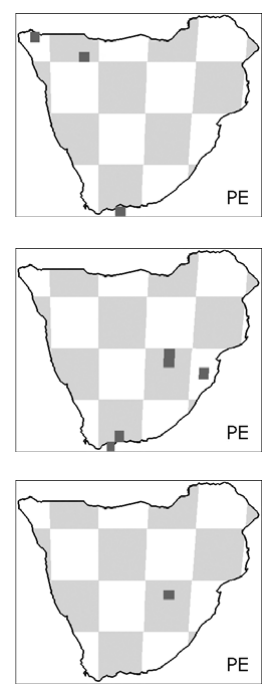

Subfamily: Miniopterinae

Miniopterus Bonaparte, 1837. Iconografia della Fauna Italica 1: fasc. 20. Additional references: Miller-Butterworth et al. (2007).

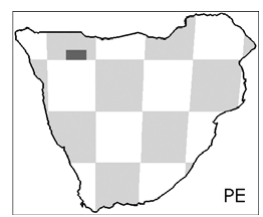


Miniopterus schreibersii Kuhl, 1817. Die Deutschen Fledermäuse: 14. Schreibers's long-fingered bat.

Additional references: Miller-Butterworth et al. (2005); Smith (1849). Comments: some of the material would perhaps now be assigned to Miniopterus natalensis, which was previously considered to be a subspecies of M. schreibersii.

\section{ORDER: PHOLIDOTA}

FAMILY: MANIDAE

Subfamily: Smutsiinae

Smutsia Gray, 1865. Proc. Zool. Soc. Lond. 1865: 360.

Synonyms: Manis.

Additional references: Gaudin et al. (2009).

Smutsia temminckii Smuts, 1832. Dissertation Zoologica,

Ennumerationem Mammalium Capensium: 54. Ground pangolin.

Synonyms: Manis.

Type locality: Latakou = Litakun.

Additional references: Gaudin (2010); Gaudin et al. (2009); Gray

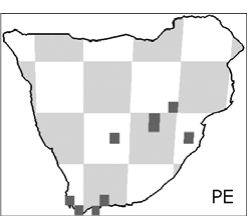

(1865b); Smith (1849); Sundevall (1842).

\section{ORDER: CARNIVORA \\ Suborder: Feliformia}

FAMILY: FELIDAE

Subfamily: Felinae

Acinonyx jubatus Schreber, 1775. Die Säugethiere in Abbildungen nach der Natur, mit Beschreibungen 2(14): pl. 105 [1775], also see text 3 (22): 392 [1777]. Cheetah.

Type locality: Cape of Good Hope.

Additional references: Krausman and Morales (2005).

Comments: citation according to Wilson and Reeder (2005). Volume 3 was published in 1778 according to the version reproduced in the Biodiversity Heritage Library (www.biodiversitylibrary.org/item/ 135003\#page/120/mode/1 up) and Felis jubata is listed on p. 586. Plate 105 appears in an undated volume containing plates 81-165 (www.biodiversitylibrary.org/item/97341\#page/107/mode/1up).

Caracal caracal Schreber, 1776. Die Säugethiere in Abbildungen nach der Natur, mit Beschreibungen 3(16): pl. 110 [1776], see also text 3(24): 413, 587 [1777]. Caracal.

Synonyms: Felis.

Type locality: Table Mountain, near Cape Town.
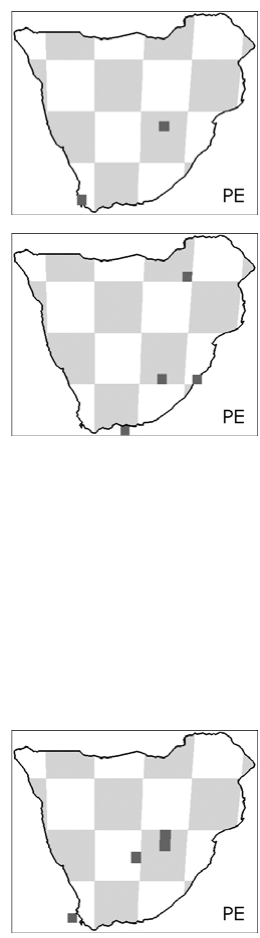

Additional references: Roberts (1926); Werdelin and Peigné (2010).

Comments: citation according to Wilson and Reeder (2005). Volume 3 was published in 1778 according to the version reproduced in the Biodiversity Heritage Library (www.biodiversitylibrary.org/item/ 135003\#page/141/mode/1 up) and Felis caracal is listed on p. 587. 
Plate 110 appears in an undated volume containing plates 81-165

(www.biodiversitylibrary.org/item/97341\#page/131/mode/1up).

†Dinofelis aronoki Werdelin and Lewis, 2001. Zool. J. Linn. Soc. 132: 239, 250.

Synonyms: Therailurus.

Additional references: Hemmer (1965); Lacruz et al. (2006); O'Regan and Menter (2009); O'Regan and Steininger (2017).

Comments: the material from Cooper's was originally assigned to

D. piveteaui (Ewer 1955c) by O'Regan and Menter (2009).

Dinofelis barlowi Broom, 1937. S. Afr. J. Sci. 33: 757.

Synonyms: Machaerodus; Megatereon; Therailurus; darti; transvaalensis. Type locality: Sterkfontein.

Additional references: Collings (1973); Collings et al. (1976); Cooke (1991);

Ewer (1955c, 1957a); Hemmer (1965); Lacruz et al. (2006).

†Dinofelis piveteaui Ewer, 1955. Proc. Zool. Soc. Lond. 125: 588.

Synonyms: Therailurus.

Additional references: Piveteau (1948).

Felis Linnaeus, 1758. Systema Naturae Regnum Animale, 10th edition, 1: 41 .

Comments: material identified as Felis sp. has not been mapped because, in many cases, this identification included what are currently ascribed to other genera such as Leptailurus and Caracal.

†Felis crassidens Broom, 1948. Ann. Transvaal Mus. 21: 14.

Type locality: Kromdraai.

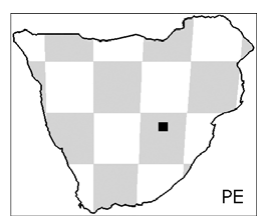

Felis nigripes Burchell, 1824. Travels in the Interior of Southern Africa 2: 592. Black-footed cat.

Type locality: implied country of the 'Bachapins', presumably in the capital Litákun: Letárkoon.

Additional references: Renard et al. (2015); Roberts (1926).

†Felis shawi Broom, 1948. Ann. Transvaal Mus. 21(1): 14.

Synonyms: Panthera.

Type locality: Sterkfontein.

Additional references: Ewer (1956a).

Felis silvestris Schreber, 1777. Die Säugethiere in Abbildungen nach der Natur, mit Beschreibungen 3(23): 397. Wild cat.

Synonyms: libyca.

Additional references: Grubb (2004).

Comments: volume 3 was published in 1778 according to the version
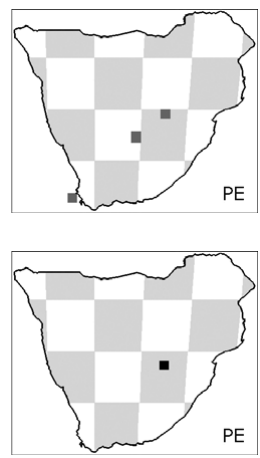
reproduced in the Biodiversity Heritage Library (www.biodiversitylibrary.org/item/135003\#page/125/mode/1 up)

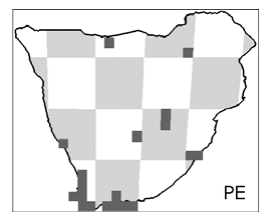


and Felis catus is listed on p. 587. Plate 107 appears in an undated volume containing plates 81-165 (www.biodiversitylibrary.org/item/ 97341\#page/115/mode/1up).

†Homotherium crenatidens Weithofer, 1889. Jahrbuch der Kaiserlich Königlichen Geologischen Reichsanstalt 39(1): 65.

Synonyms: Machairodus; latidens.

Comments: there appears to be disagreement as to which species of Homotherium is represented at Sterkfontein: Turner (1987a) considers the material to be $H$. crenatidens, whereas Reynolds (2010b) assigns it to H. latidens.

Leptailurus serval Schreber, 1776. Die Säugethiere in Abbildungen nach der Natur, mit Beschreibungen 3(16): pl. 108 [1776], see also text 3 (23): 407[1777]. Serval.

Synonyms: Felis; spelaeus.

Type locality: restricted to the 'Cape region of South Africa'.

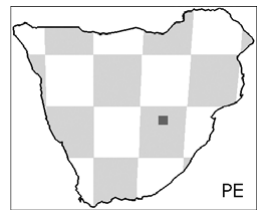

Additional references: Werdelin and Peigné (2010).

Comments: citation according to Wilson and Reeder (2005). Volume 3 was published in 1778 according to the version reproduced in the Biodiversity Heritage Library (www.biodiversitylibrary.org/item/ 135003\#page/135/mode/1 up) and Felis serval is listed on p. 587. Plate 107 appears in an undated volume containing plates 81-165 (www.biodiversitylibrary.org/item/97341\#page/125/mode/1up).

\section{Subfamily: †Machairodontinae}

†Megantereon gracile Broom, 1948. Ann. Transvaal Mus. 21: 11. Type locality: Sterkfontein.

†Megantereon whitei Broom, 1937. S. Afr. J. Sci. 33: 756. Synonyms: Felis; cultridens.

Type locality: Schurveberg. Additional references: Broom (1939c, 1946); Hartstone-Rose et al. (2007); Turner (1987b); Werdelin and Peigné (2010).
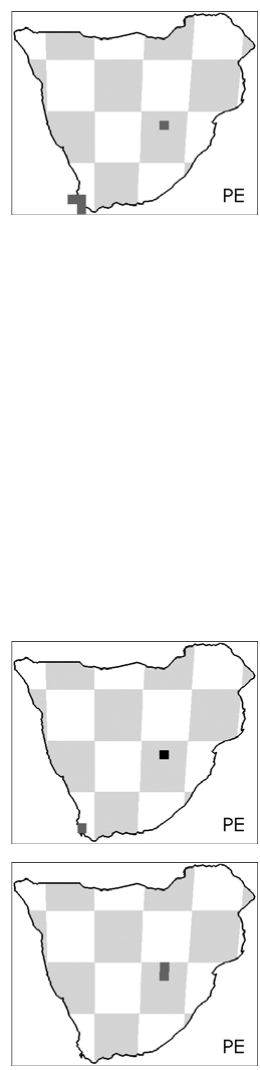

Subfamily: Pantherinae

Panthera leo Linnaeus, 1758. Systema Naturae Regnum Animale, 10th edition, 1: 41. Lion.

Additional references: Haas et al. (2005); Lacruz (2009).

Panthera pardus Linnaeus, 1758. Systema Naturae Regnum Animale, 10th edition, 1: 41. Leopard.

Additional references: Ewer (1956a).
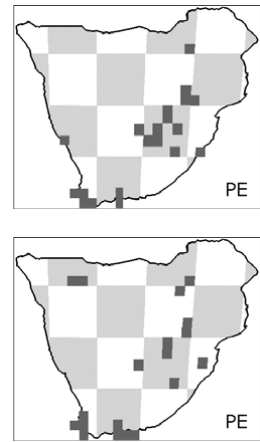
FAMILY: HERPESTIDAE

†Atilax mesotes Ewer, 1956. Proc. Zool. Soc. Lond. 126: 259-274. Synonyms: Herpestes.

Atilax paludinosus Cuvier, 1829. Le Règne Animal distribué d'après son Organisation, Nouvelle édition, 1: 158. Marsh mongoose.

Synonyms: Herpestes.

Type locality: Cape of Good Hope.

Additional references: Baker (1992); Cuvier (1824).

†Crossarchus transvaalensis Broom, 1937. Ann. Mag. Nat. Hist., Series 10, 20: 512.

Type locality: Bolt's Farm.

Additional references: Broom (1939c); Werdelin and Peigné (2010).

Cynictis penicillata Cuvier, 1829. Le Règne Animal distribué d'après son

Organisation, Nouvelle édition 1: 158. Yellow mongoose.

Synonyms: lepturus; ogilbyii.

Type locality: restricted to 'Uitenhage, $C P$ '.

Additional references: Ewer (1956a, 1957a); Lundholm (1954); Roberts

(1932); Smith (1849); Taylor and Meester (1993).

Galerella pulverulenta Wagner, 1839. Gelehrte. Anz. I. K. Bayer. Akad.

Wiss. München 9: 426. Cape grey mongoose.

Type locality: Cape of Good Hope.

Additional references: Cavallini (1992); Lynch (1981).

Galerella sanguinea Rüppell, 1835. Neue Wirbelthiere zu der Fauna von Abyssinien gehörig. 1: 27. Slender mongoose.

Synonyms: Herpestes sanguineus; punctulatus.

Additional references: Gray (1849); Roberts (1932); Taylor (1975).

Herpestes Illiger, 1811. Prodromus Systematis Mammaliam et Avium: 135. Additional references: Hendey (1973a); Werdelin and Peigné (2010).

Comments: this genus has not been mapped because material was identified at a time when Galerella, as currently understood, was not recognised as a separate genus.

Herpestes ichneumon Linnaeus, 1758. Systema Naturae Regnum Animale, 10th edition, 1: 43. Egyptian mongoose.

Synonyms: Ichneumon ratlamuchi; Herpestes badius.

Additional references: Smith $(1838,1849)$.

Ichneumia albicauda Cuvier, 1829. Le Règne Animal distribué d'après son

Organisation, Nouvelle édition, 1: 158. White-tailed mongoose.

Additional references: Geoffroy Saint-Hilaire (1837); Taylor (1972). 
Mungos mungo Gmelin, 1788. In: Linnaeus, Systema Naturae, 13th edition, 1: 84. Banded mongoose.

Type locality: believed to be eastern part of South Africa, (former) Cape Province.

Rhynchogale Thomas, 1894. Proc. Zool. Soc. Lond. 1894: 139

†Suricata major Hendey, 1974. Ann. S. Afr. Mus. 63: 267.

Type locality: Elandsfontein.

Suricata suricatta Schreber, 1776. Die Säugethiere in Abbildungen nach der Natur, mit Beschreibungen: pl. 117 [1776]. Meerkat.

Type locality: restricted to 'Deelfontein'.

Additional references: Van Staaden (1994).

Comments: citation according to Wilson and Reeder (2005). Version of
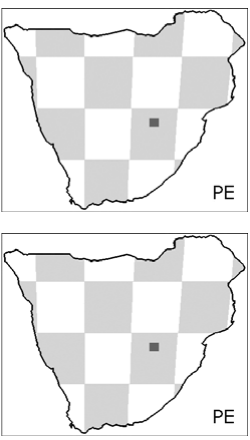
text volume 3 at (www.biodiversitylibrary.org/item/135003\#page/5/

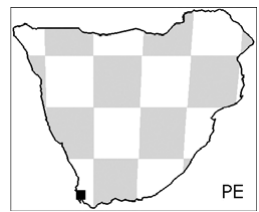
mode/1up) is dated 1778, while the volume in which the plate appears is undated (www.biodiversitylibrary.org/item/97341\#page/ $1 /$ mode/1 up).

\section{FAMILY: HYAENIDAE}

†Lycyaenops silberbergi Broom and Schepers, 1946. Transvaal Mem. Mus. 2: 83.

Synonyms: Chasmaporthetes; Lycyaena.

Type locality: Sterkfontein.

Additional references: Broom (1948a); Werdelin and Peigné (2010).

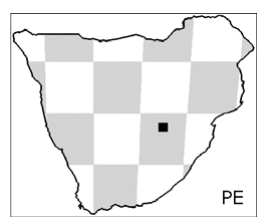

Subfamily: Hyaeninae

†Chasmaporthetes nitidula Ewer, 1955. Proc. Zool. Soc. Lond. 124: 842. Synonyms: Euryboas; Lycyaeon.

Type locality: Sterkfontein.

Additional references: Hendey (1978a).

Crocuta Kaup, 1828. Oken's Isis. Encyclop. Zeit. 21(11): col. 1145

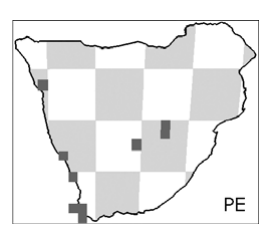


†Crocuta ultra Ewer, 1954. Proc. Zool. Soc. Lond. 124: 570.

Type locality: Kromdraai.

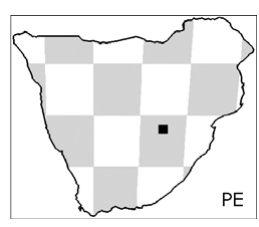

Hyaena hyaena Linnaeus, 1758. Systema Naturae Regnum Animale, 10th edition, 1: 40. Striped hyaena.

Synonyms: striata.

Additional references: Turner (1988).

†Pachycrocuta brevirostris Gervais, 1850. Zool. Paléontol. Françaises 1: 122.

Synonyms: Hyaena bellax.

Additional references: Alba et al. (2015); Ewer (1954, 1956b); Mutter et al. (2001); Turner and Antón (1996); Werdelin and Peigné (2010).

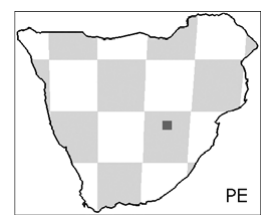

Comments: according to Alba et al. (2015), this taxon has long been incorrectly attributed to Aymard since his 1846 paper neither describes nor names the taxon.

Parahyaena brunnea Thunberg, 1820. K. Svenska Vet.-Akad. Handl., Series 3, 8: 59. Brown hyaena.

Synonyms: Hyaena.

Type locality: Cape of Good Hope.

Additional references: Grubb (2004); Hendey (1973a); Mills (1982);

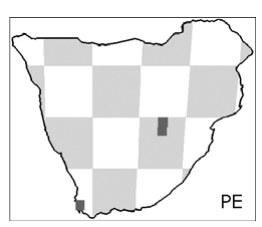

Werdelin and Peigné (2010).

Subfamily: Protelinae

Proteles cristata Sparrman, 1783. Resa Goda-Hopps-Udden, Soedra Polkretsen Och Omkring Jordklotet: 581. Aardwolf.

Synonyms: cristatus; lalandii.

Type locality: listed as 'Near Little Fish River, Somerset East'.

†Proteles transvaalensis Hendey, 1974. Ann. Transvaal Mus. 29(3): 35. Type locality: Swartkrans.
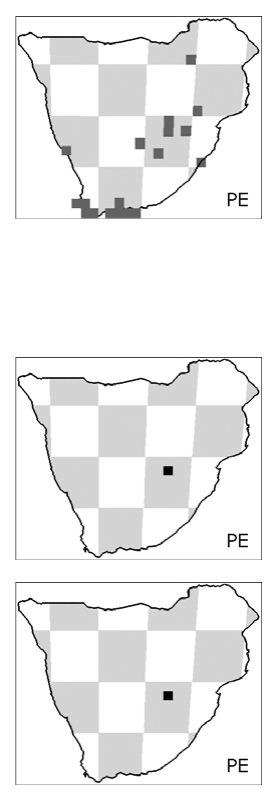

FAMILY: VIVERRIDAE

Subfamily: Viverrinae

†Civettictis braini Fourvel, 2018. C. R. Palevol. 17: 369.

Type locality: Kromdraai.

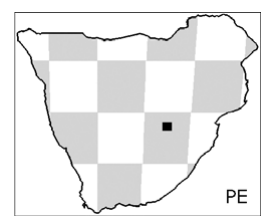

Genetta Cuvier, 1817. Le Règne Animal distribué d'après son Organisation 1: 156.

Additional references: De Meneses Cabral (1966); Gaubert et al. (2005).

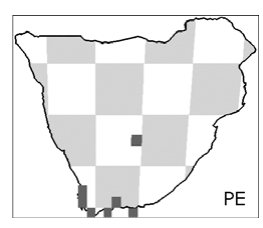


Genetta genetta Linnaeus, 1758. Systema Naturae Regnum Animale, 10th edition, 1: 45. Common genet.

Additional references: Gaubert et al. (2005); Larivière and Calzada (2001).

Genetta maculata Gray, 1830. Spicil. Zool. 2: 9. Rusty-spotted genet. Additional references: Crawford-Cabral and Fernandes (2001); Gaubert et al. (2003a, 2003b, 2005); Grubb (2004).

Genetta tigrina Schreber, 1776. Die Säugethiere in Abbildungen nach der Natur, mit Beschreibungen (3)17: pl. 114 [1776]; see also text, 3(25): 425 [1777]. Cape genet.

Type locality: Cape of Good Hope.

Additional references: Gaubert et al. (2005); Grubb (2004).
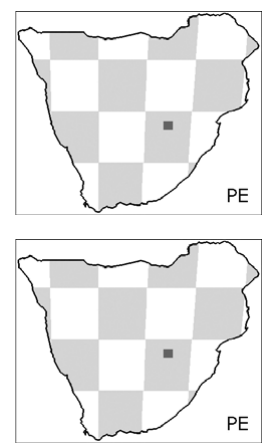

Comments: citation according to Wilson and Reeder (2005). Volume 3 was published in 1778 according to the version reproduced in the Biodiversity Heritage Library (www.biodiversitylibrary.org/item/ 135003\#page/153/mode/1 up) and Viverra tigrina is listed on p. 587. Plate 114 appears in an undated volume containing plates 81-165 (www.biodiversitylibrary.org/item/97341\#page/147/mode/1up).

\section{Suborder: Caniformia \\ FAMILY: CANIDAE \\ Subfamily: Caninae}

Canis Linnaeus, 1758. Systema Naturae Regnum Animale, 10th edition, 1: 38.

Additional references: Werdelin and Peigné (2010).

Canis adustus Sundevall, 1846. Ofv. K. Vet.-Akad. Forhandl. 3: 121. Sidestriped jackal.

Type locality: 'Magaliesberg'.

†Canis antiquus Broom, 1937. Ann. Mag. Nat. Hist., Series 10, 20: 511. Synonyms: Thos.

Type locality: Sterkfontein.

Additional references: Broom (1939c); Ewer (1956b).

†Canis atrox Broom, 1948. Ann. Transvaal Mus. 21: 19.

Type locality: Kromdraai.
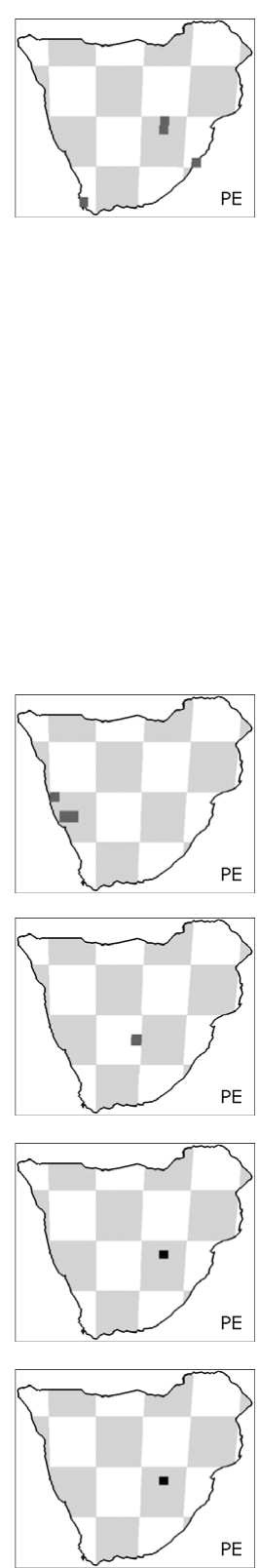
Canis mesomelas Schreber, 1775. Die Säugethiere in Abbildungen nach der Natur, mit Beschreibungen 2(14): pl. 95 [1775]; text 3(21): 370 [1776], 586 [1777]. Black-backed jackal.

Type locality: Cape of Good Hope.

Comments: citation according to Wilson and Reeder (2005). Volume 3 was

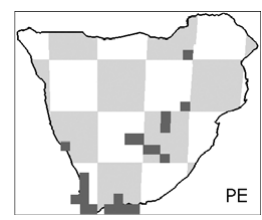
published in 1778 according to the version reproduced in the Biodiversity Heritage Library (www. biodiversitylibrary.org/item/ 135003\#page/98/mode/1 up) and Canis mesomelas is listed on p. 586. Plate 95 appears in an undated volume containing plates 81-165 (www.biodiversitylibrary.org/item/97341\#page/57/mode/1up).

Lycaon Brookes, 1827. In Griffiths et al., The Animal Kingdom Arranged in Conformity with its Organization by the Baron Cuvier 5: 151.

Additional references: Brookes (1828).

Comments: Griffiths et al. attribute this identification to Brookes, whose name they spell Brooks. There appears to be no formal description by Brookes himself.

Lycaon pictus Temminck, 1820. Ann. Gen. Sci. Phys. 3: p. 46, pl. 35. African wild dog.

Type locality: 'á la côte de Mosambique'.

†Lycaon sekowei Hartstone-Rose, Werdelin, De Ruiter, Berger and Churchill, 2010. J. Paleontol. 84(2): 301.

Type locality: Cooper's.

†Nyctereutes terblanchei Broom, 1948. Ann. Transvaal Mus. 21(1): 21. Synonyms: Canis; Thos.

Type locality: Kromdraai.

Additional references: Ficcarelli et al. (1984); Reynolds (2012).

Otocyon megalotis Desmarest, 1822. Mammalogie ou descriptions des espèces de mammifères, Part 2 (suppl.): 538. Bat-eared fox.

Type locality: Cape of Good Hope.

Additional references: Clark (2005).

†Otocyon recki Pohle, 1928. Wiss. Ergebnisse Oldoway-Exped, 1913 (N.F.): 45-54
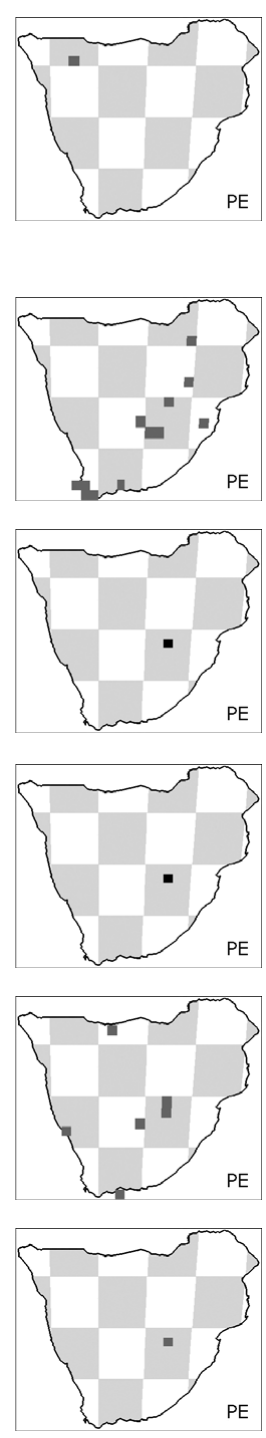

Vulpes chama Smith, 1833. S. Afr. Quart. J., Series 2, 1: 89. Cape fox. Type locality: fixed as 'Port Nolloth'.

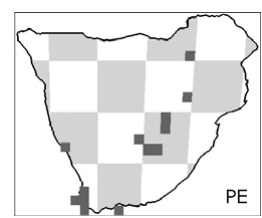


†Vulpes pulcher Broom, 1939. Ann. Transvaal Mus. 19: 336.

Type locality: Kromdraai.

Additional references: Ewer (1956b).

† Vulpes skinneri Hartstone-Rose, Kuhn, Nalla, Werdelin and Berger (2013). Trans. R. Soc. S. Afr. 68(1): 3.

Type locality: Malapa.

\section{FAMILY: MUSTELIDAE \\ Subfamily: Lutrinae}

Aonyx Lesson, 1827. Manuel de Mammalogie ou l'Histoire Naturelle des Mammifères: 147.

Additional references: Werdelin and Peigné (2010).

Aonyx capensis Schinz, 1821. In: Cuvier, Das Thierreich eingetheilt nach dem Bau der Thiere 1: 211. African clawless otter.

Synonyms: robustus.

Type locality: (former) Cape Province.

Additional references: Brink (1987); Dreyer and Lyle (1931); Ewer
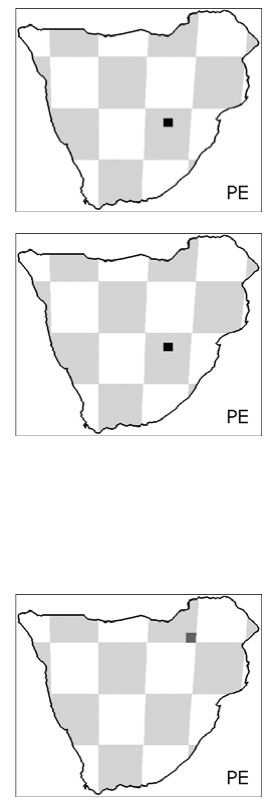

(1958c); Larivière (2001a).

Subfamily: Mellivorinae

Mellivora capensis Schreber, 1776. Die Säugethiere in Abbildungen nach der Natur, mit Beschreibungen 3(18): pl. 125 [1776], see also text 3 (26): 450 [1777]. Honey badger.

Synonyms: sivalensis.

Type locality: Cape of Good Hope.

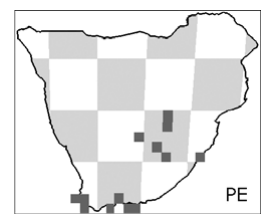

Additional references: O'Regan et al. (2013).

Comments: citation according to Wilson and Reeder (2005). Volume 3 was published in 1778 according to the version reproduced in the Biodiversity Heritage Library (www.biodiversitylibrary.org/item/ 135003\#page/178/mode/1 up) and Viverra capensis is listed on p. 588. Plate 125 appears in an undated volume containing plates 81-165 (www.biodiversitylibrary.org/item/97341\#page/191/mode/1up).

Subfamily: Mustelinae

Ictonyx striatus Perry, 1811. Arcana, or, the Museum of Natural History: Containing the Most Recent Discovered Objects, Signature Y: Fig. 41 [1810]. Striped polecat.

Type locality: fixed as 'Cape of Good Hope'.

Additional references: Larivière (2002); Roberts (1932).

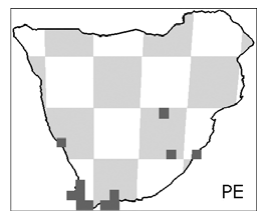


Poecilogale albinucha Gray, 1864. Proc. Zool. Soc. Lond. 1864: 69, pl.

$X$. African striped weasel.

Synonyms: Ictonyx.

Type locality: fixed as 'Cape Colony'.

Additional references: Larivière (2001b); Roberts (1931); Thomas

(1883).

†Prepoecilogale bolti Cooke, 1985. S. Afr. J. Sci. 81: 618-619.

Synonyms: Ictonyx; Propoecilogale.

Type locality: Bolt's Farm.

Additional references: O’Regan et al. (2013); Petter (1987).
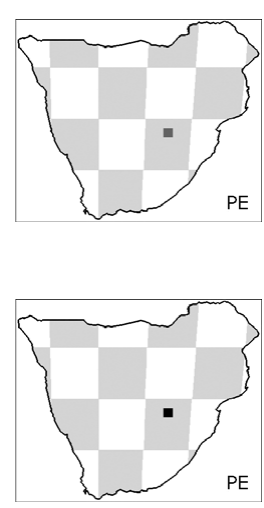

\section{ORDER: PERISSODACTYLA}

\section{FAMILY: EQUIDAE}

Equus Linnaeus, 1758. Systema Naturae Regnum Animale, 10th edition, 1: 73.

Additional references: Eisenmann and Baylac (2000); Thackeray (2010); Wells (1959a).

Comments: Brink (1994) assigned some equid material from Florisbad and Vlakkraal Thermal Springs to the subgenus Equus (Asinus).

Equus burchellii Gray, 1824. Zool. J. 1: 247. Burchell's zebra. Synonyms: Iylei; platyconus; simplicissimus.

Type locality: now identified as Little Klibbolikhonni Fontein. Additional references: Churcher (1970); Churcher and Watson (1993); Grubb (1981); Mendrez (1966); Reynolds and Bishop (2006); Van

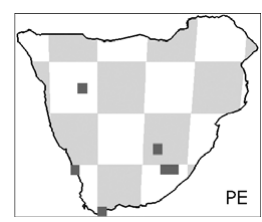
Hoepen (1930a).

†Equus capensis Broom, 1909. Ann. S. Afr. Mus. 7: 281.

Synonyms: Kolpohippus; cawoodi; fowleri?; gigas?; harrisi; helmei; kuhni; plicatus; poweri; zietsmani.

Type locality: Ysterplaat.

Additional references: Bernor et al. (2010); Broom (1913b); Churcher (1970, 2000, 2006); Churcher and Watson (1993); Eisenmann (2000); Haughton (1932a); Orlando et al. (2009); Van Hoepen (1930a); Wells (1940).

†Equus quagga Boddaert, 1785. Elenchus Animalium 1: 160. Quagga.

Type locality: locality of paralectotype now identified as Seekoei River. Additional references: Bernor et al. (2010); Churcher (1970); Eisenmann and Brink (2000); Haughton (1932a); Klein and Cruz-Uribe (1999); Lundholm (1951).

†Equus sandwithi Haughton, 1932. Ann. S. Afr. Mus. 28: 419. Type locality: Usakos.
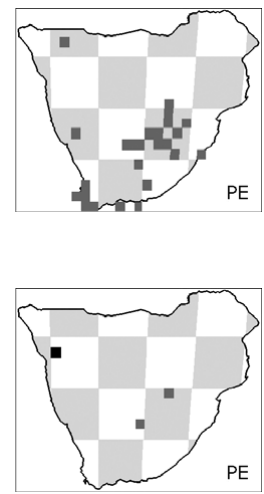
Equus zebra Linnaeus, 1758. Systema Naturae Regnum Animale, 10th edition, 1: 74. Mountain zebra.

Synonyms: Hippotigris.

Type locality: since restricted to Perdekop.

Additional references: Bernor et al. (2010); Fraas (1907); Hooijer (1945); Lundholm (1952); Penzhorn (1988).

†Eurygnathohippus Van Hoepen, 1930. Paleontol. Nav. Nas. Mus. Bloemfontein 2(2): 23.

Synonyms: Hipparion; Stylohipparion.

Additional references: Boné and Singer (1965); Hooijer (1975, 1976).

†Eurygnathohippus cornelianus Van Hoepen, 1930. Paleontol. Nav. Nas. Mus. Bloemfontein 2(2): 23.

Synonyms: Hipparion; Stylohipparion; hipkini; libycum; sitifense in part?; steytleri.

Type locality: Cornelia.

Additional references: Bernor et al. (2010); Churcher (1970, 2000); Churcher and Watson (1993); Franz-Odendaal et al. (2003); Pomel (1897); Van Hoepen (1932a).

Comments: the material from Hondeklip Bay was doubtfully ascribed by Pickford and Senut (1997) to Hipparion sitifense, which Bernor et al. (2010) consider a nomen dubium. See also the comment under Hipparion below.

†Eurygnathohippus hooijeri Bernor and Kaiser, 2006. Mitt. Hamb. Zool. Mus. Inst. 103: 148.

Synonyms: Hipparion baardi cf.

Type locality: Langebaanweg.

Additional references: Bernor et al. (2010); Franz-Odendaal et al. (2003);
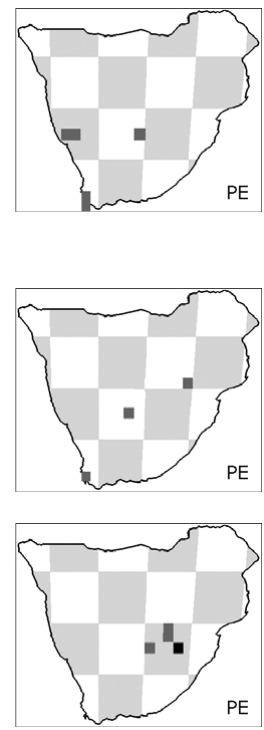
Hooijer (1976); Pickford and Senut (1997).

\section{FAMILY: RHINOCEROTIDAE}

Subfamily: Rhinocerotinae

Ceratotherium simum Burchell, 1817. Bull. Sci. Soc. Philom. Paris 1817-1819: 97. White rhinoceros.

Synonyms: Diceros; Rhinoceros; simus.

Type locality: since identified as Chue Spring = Heuningvlei.

Additional references: Geraads (2010a); Gray (1867); Groves (1972);

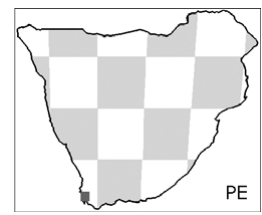

Hooijer (1959, 1973); Hooijer and Singer (1960); Smith (1849).

Diceros bicornis Linnaeus, 1758. Systema Naturae Regnum Animale, 10th edition, 1: 56. Black rhinoceros.

Synonyms: Opsiceros simplicidens.

Type locality: now identified as Cape of Good Hope.

Additional references: Gray (1867); Hillman-Smith and Groves (1994);

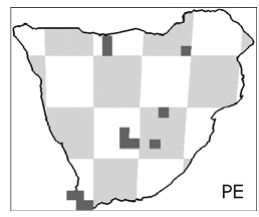

Hooijer (1959, 1973); Hooijer and Singer (1960); Scott (1907). 


\section{ORDER: ARTIODACTYLA}

FAMILY: SUIDAE

Subfamily: Suinae

†Kolpochoerus heseloni Leakey, 1943. J. E. Afr. Nat. Hist. Soc. 17: 55. Synonyms: Mesochoerus.

Additional references: Bender (1992); Bishop (2010); Cooke (1997); Keen and Singer (1956); Pickford (2012).

†Kolpochoerus paiceae Broom, 1931. Rec. Albany Mus. 4: 168.

Synonyms: Notochoerus.

Type locality: Windsorton.

Additional references: Hendey and Cooke (1985); Pickford (2012).

†Mesochoerus lategani Singer and Keen, 1955. Ann. S. Afr. Mus. 42: 170. Type locality: Elandsfontein.

†Metridiochoerus Hopwood, 1926. Ann. Mag. Nat. Hist., Series 9, 18: 266-272.

Additional references: Cooke (2005).

†Metridiochoerus andrewsi Hopwood, 1926. Ann. Mag. Nat. Hist., Series 9, 18: 266-272.

Synonyms: Notochoerus; Tapinochoerus; capensis; meadowsi.

Additional references: Bender (1990); Bishop (2010); Broom (1948a);

Cooke (1993a); Ewer (1956b); Harris and White (1979); Herries et al.

(2006); Hopwood (1926); Keen and Singer (1956); Pickford (2012, 2013a, 2013b); Shaw (1938).

†Metridiochoerus compactus Van Hoepen and Van Hoepen, 1932.

Paleontol. Nav. Nas. Mus. Bloemfontein 2(4): 53.

Synonyms: Phacochoerus; Stylochoerus.

Type locality: Cornelia.

Additional references: Bishop (2010); Harris and White (1979); Hopwood
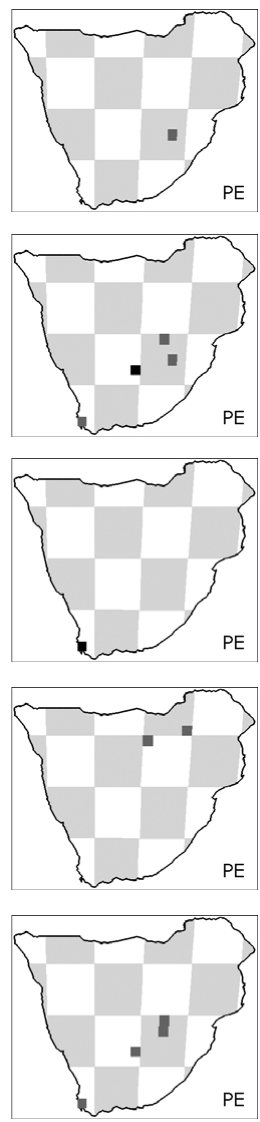

(1926).

†Metridiochoerus modestus Van Hoepen and Van Hoepen, 1932.

Paleontol. Nav. Nas. Mus. Bloemfontein 2(4): 58.

Synonyms: Tapinochoerus.

Type locality: Cornelia.

Additional references: Bishop (2010); Cooke (1993a, 1994); Harris and

White (1979); Hopwood (1926).

Phacochoerus Cuvier, 1826. Dict. Sci. Nat. 39: 383.

Comments: the Holocene material is likely to be $P$. africanus since this is the only species currently recognised in the sub-region.
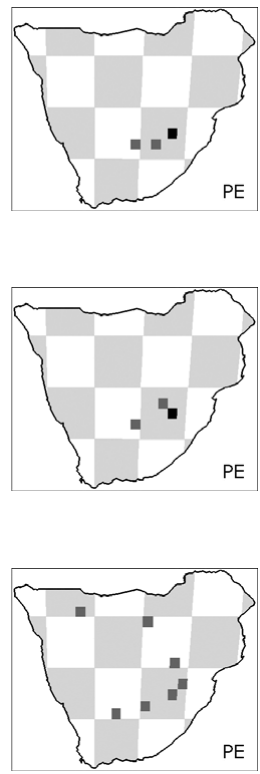
Phacochoerus africanus Gmelin, 1788. In Linnaeus, C. Systema Naturae, 13th edition, 1: 220. Common warthog.

Synonyms: aethiopicus; dreyeri?; helmei; laticolumnatus; venteri.

Additional references: Cooke (1949b); Ewer (1956b, 1957b); Grubb and d'Huart (2010); Pia (1930).

†Phacochoerus altidens Shaw and Cooke, 1940. Trans. R. Soc. S. Afr. 28(4): 296.

Type locality: Pneil.

†Phacochoerus antiquus Broom, 1948. Ann. Transvaal Mus. 21: 31. Type locality: Sterkfontein.

Additional references: Harris and White (1979).

†Potamochoeroides hypsodon Dale, 1948. S. Afr. Sci. 2(5): 116. Synonyms: Metridiochoerus; Pronotochoerus; shawi.

Additional references: Cooke (1993a, 2005); Ewer (1956b, 1958d);

Pickford (2013a); Pickford and Gommery (2016).

Potamochoerus larvatus Cuvier, 1822. Mem. Mus. Hist. Nat. Paris 8: 447. Bush-pig.

Synonyms: Koiropotamus; porcus.

Additional references: Grubb (2004); Hopwood (1934); Leakey (1942).
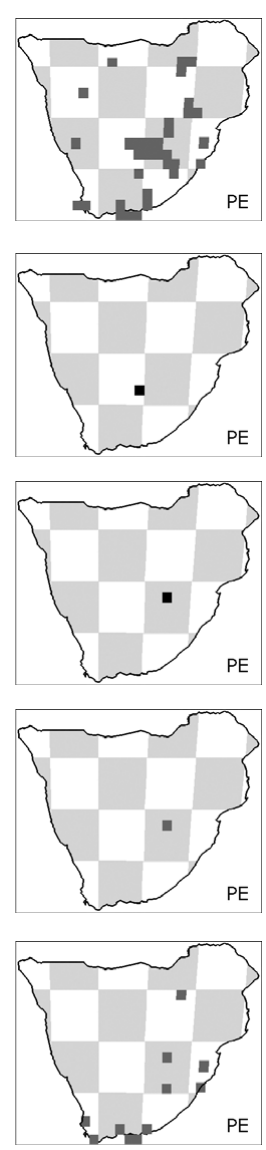

\section{Subfamily: †Tetraconodontinae}

†Notochoerus capensis Broom, 1925. Rec. Albany Mus. 3(4): 308.

Synonyms: scotti.

Type locality: Longlands.

Additional references: Harris and White (1979); Shaw (1938).

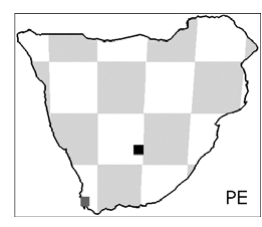

\section{FAMILY: HIPPOPOTAMIDAE}

Hippopotamus Linnaeus, 1758. Systema Naturae Regnum Animale, 10th edition, 1: 74.

Additional references: Boisserie (2005).

Hippopotamus amphibius Linnaeus, 1758. Systema Naturae Regnum Animale, 10th edition, 1: 74. Common hippopotamus.

Synonyms: capensis; poderosus.

Additional references: Fraas (1907); Hooijer and Singer (1961); Scott (1907); Smith (1849).
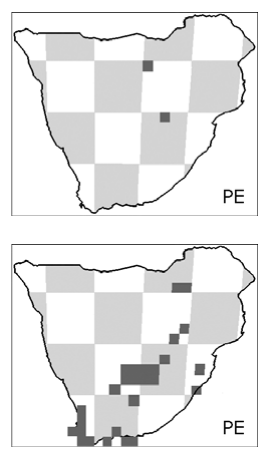
†Hippopotamus gorgops Dietrich, 1926. Forsch. und Fortsch. 2: 121. Additional references: Dietrich (1928); Hooijer (1958); Weston and Boisserie (2010).

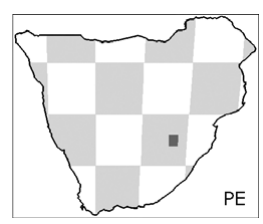

\section{FAMILY: GIRAFFIDAE}

Subfamily: Giraffinae

Giraffa Brisson, 1762. Regnum animale in classes IX. Distributum, sive Synopsis Methodica, 2nd edition: 12, 37

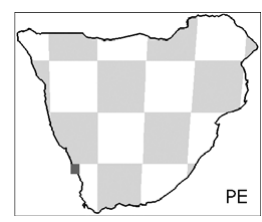

Giraffa camelopardalis Linnaeus, 1758. Systema Naturae Regnum Animale, 10th edition, 1: 66. Giraffe.

Additional references: Dagg (1971); Harris et al. (2010); Singer and Boné (1960).

Comments: Cooke (1955) lists this taxon from Bloembosch (3318), but

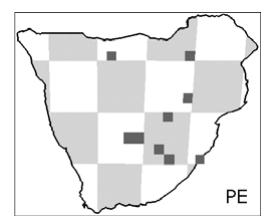

R.G. Klein (personal communication, 2016) is of the opinion that the specimen is probably referable to Giraffa stillei, which occurs at Langebaanweg. Recently, Helm et al. (2018) have reported giraffe tracks from near Still Bay (3421), but this occurrence has not been plotted, pending the collection of physical remains of this taxon.

Subfamily: Sivatheriinae

†Sivatherium maurisium Pomel, 1892. C.R. Hebd. Acad. Sci. Paris 114: 100. Synonyms: Griquatherium, Libytherium; cingulatum.

Additional references: Churcher (1974); Haughton (1921); Harris et al. (2010); Singer and Boné (1960).

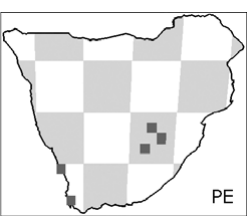

FAMILY: BOVIDAE

Subfamily: Aepycerotinae

†Aepyceros helmoedi Van Hoepen, 1932. Palentol. Nav. Nas. Mus. Bloemfontein 2(5): 65.

Synonyms: Gazella.

Type locality: Cornelia.

Aepyceros melampus Lichtenstein, 1812. Reisen im südlichen Africa in en Jahren 1803, 1804, 1805 und 1806 2: pl. 4 opp. p. 544. Impala.

Type locality: now identified as Khosis.

Additional references: Reynolds (2010a).
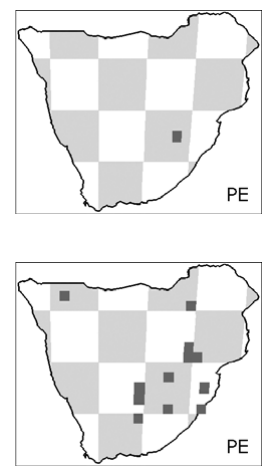
Subfamily: Alcelaphinae

Alcelaphus De Blainville, 1816. Bull. Sci. Soc. Philom. Paris 1816: 75.

Alcelaphus buselaphus Pallas, 1766. P.S. Pallas Medecinae Doctoris Miscellanea Zoologica: 7. Hartebeest.

Alcelaphus caama Geoffroy Saint-Hilaire, 1803. Catalogue des Mammifères du Muséum National d'Histoire Naturelle: 269. Red hartebeest.

Synonyms: Antilope; Bubalus; bubalis; buselaphus caama.

Type locality: since restricted to syntype locality Steynsburg.

Additional references: Gray (1850a, 1850b); Grubb (2004); Hoffman

(1953); Smith (1849).

†Alcelaphus robustus Cooke, 1949. Mem. Geol. Surv. S. Afr. 35: 20. Type locality: Larsen's Main Workings.

Comments: see Parmularius braini below.

Beatragus Heller, 1912. Smithson. Misc. Coll. 60(8): 8.

Additional references: Gentry (2010); Vrba (1974b, 1997).

Connochaetes Lichtenstein, 1812. Mag. Ges. Naturf. Fr. Berlin 6: 152. Synonyms: Gorgon.
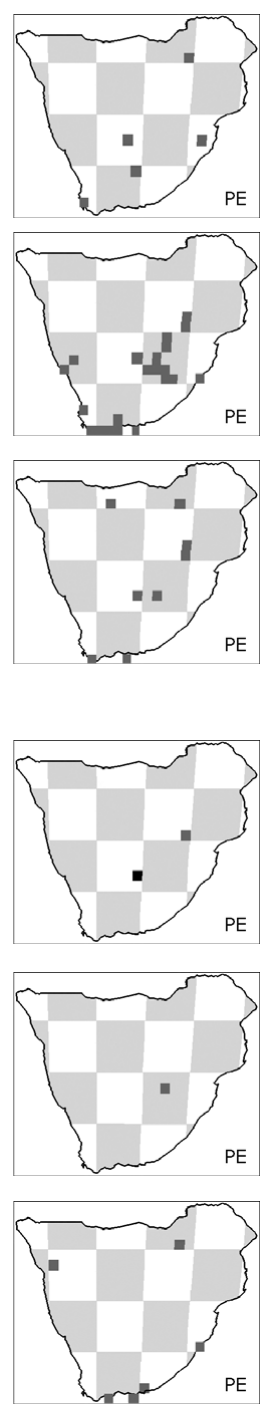

†Connochaetes antiquus Broom, 1913. Ann. S. Afr. Mus. 12: 14. Type locality: Florisbad (Hagenstad).

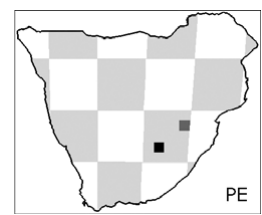

Connochaetes gnou Zimmermann, 1780. Geographische Geschichte des Menschen und der Allgemein Verbreiteten Vierfüssigen Thiere 2: 102. Black wildebeest.

Synonyms: laticornutus.

Type locality: since selected as Agterbruintjieshoogte.

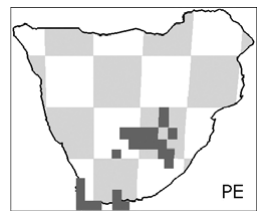

Additional references: Brink (1993, 2005); Gentry (2010); Gray (1850a, 1850b); Von Richter (1974).

Comments: Gentry (2010) proposes that Connochaetes laticornutus should be retained as a distinct species. 
Connochaetes taurinus Burchell, 1824. Travels in the Interior of Southern Africa 2: 278 (footnote). Blue wildebeest.

Synonyms: Catoblepas.

Type locality: Apparently 'Kosi Fountain', but lectotype came from ‘Chue

Spring, Maadji Mtn [Klein Heuningvlei]'.

Additional references: Smith (1849).

Damaliscus Sclater and Thomas, 1894. The Book of Antelopes 1: 51.

Additional references: Fraas (1907).
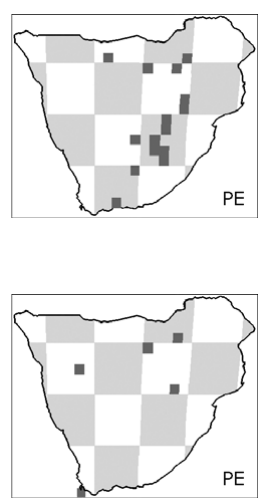

Damaliscus lunatus Burchell, 1824. Travels in the Interior of Southern Africa 2: 334. Common tsessebe.

Synonyms: Acronotus; Bubalus.

Type locality: 'Makkwarin' (Matlhwareng) River.

Additional references: Cotterill (2003); Gray (1850a, 1850b); Groves and

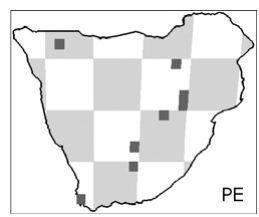
Grubb (2011); Smith (1849).

†Damaliscus niro Hopwood, 1936. Ann. Mag. Nat. Hist., Series 10, 17: 640.

Synonyms: Hippotragus.

Additional references: Gentry (1965); Thackeray and Brink (2004).

Damaliscus pygargus Pallas, 1767. Spicil. Zool. 1: 10. Bontebok. Synonyms: albifrons; dorcas phillipsi; dorcas; 'hipkini'.

Type locality: since restricted to Swart River.

Additional references: Faith et al. (2012); Gentry (2010); Gray

(1850a, 1850b); Groves and Grubb (2011); Grubb (2004);

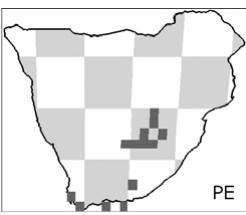

Vrba (1997).

Comments: according to Vrba (1997), Wells intended to name

Damaliscus 'hipkini', but never did. Groves and Grubb (2011) propose that Damaliscus phillipsi (blesbok) be a full species separate from D. pygargus (bontebok).

†Megalotragus Van Hoepen, 1932. Paleontol. Nav. Nas. Mus. Bloemfontein 2(5): 63.

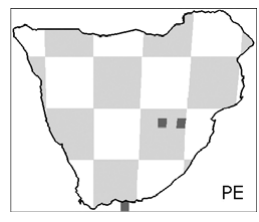

†Megalotragus priscus Broom, 1909. Ann. S. Afr. Mus. 7: 279.

Synonyms: Bubalis; Connochaetes; Lunatoceras; Peleoroceras; broomi;

elegans; eucornutus; grandis; helmei; kattwinkeli; mirum.

Type locality: Modder River.

Additional references: Brink et al. (2015a); Gentry et al. (1995); Hoffman

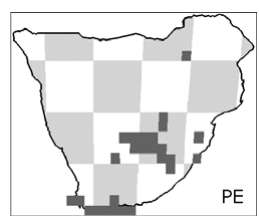

(1953); Seeley (1891); Van Hoepen (1932b, 1947); Wells (1959b, 1964). 
†Parmularius Hopwood, 1934. Ann. Mag. Nat. Hist., Series 10, 14: 550.

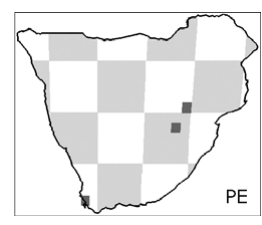

†Parmularius parvus Vrba, 1978. Ann. Transvaal Mus. 31(3): 23.

Type locality: Kromdraai.

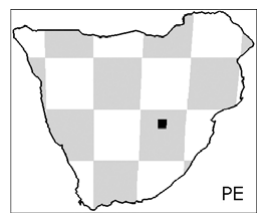

Subfamily: Antilopinae

Antidorcas Sundevall, 1845. K. Svenska Vet.-Akad. Handl. 1845: 271.

†Antidorcas australis Hendey and Hendey, 1968. Ann. S. Afr. Mus. 52(2): 56.

Synonyms: marsupialis australis.

Type locality: Swartklp.

Additional references: Gentry (2010); Peters and Brink (1992); Vrba

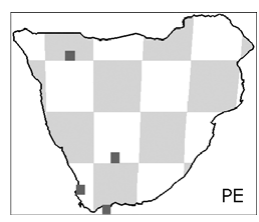
(1973).

†Antidorcas bondi Cooke and Wells, 1951. S. Afr. J. Sci. 47: 207. Synonyms: Gazella.

Type locality: Chelmer.

Additional references: Gentry (2010); Plug and Peters (1991); Vrba (1973).

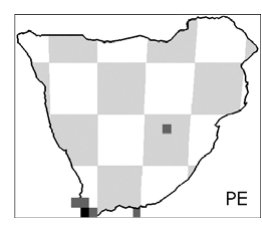

Antidorcas marsupialis Zimmermann, 1780. Geographische Geschichte des Menschen und der Allgemein Verbreiteten Vierfüssigen Thiere 2: 427. Springbok

Type locality: since restricted to 'Cape Colony [Cape Province]'. Additional references: Cain et al. (2004); Gentry (2010); Gray (1850a,

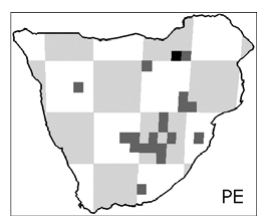
1850b); Plug and Peters (1991); Vrba, (1970).

†Antidorcas recki Schwarz, 1932. Zentr. Mineral., Geol. Paläontol. B 1932: 1. Synonyms: Gazella; wellsi.

Additional references: Gentry (2010).

Gazella De Blainville, 1816. Bull. Sci. Soc. Philom. Paris 1816: 75. Additional references: Gentry (2010).
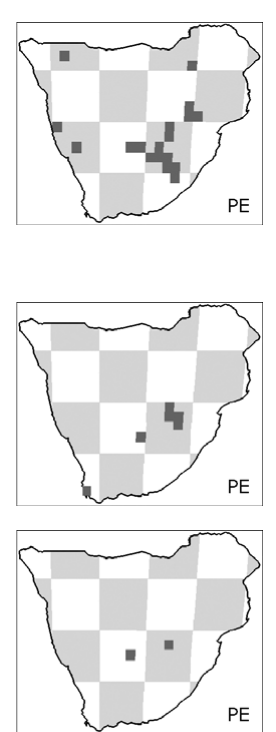
†Gazella praethomsoni Arambourg, 1947. Mission Scient. Omo 1. Géol. Anthrop. 3: 237.

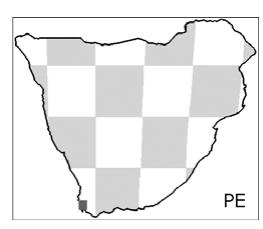

†Gazella vanhoepeni Wells and Cooke, 1957. Palaeontol. Afr. 4: 43. Synonyms: gracilior?.

Type locality: Limeworks Makapansgat.

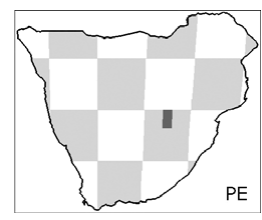

Oreotragus oreotragus Zimmermann, 1783. Geographische Geschichte des Menschen und der Allgemein Verbreiteten Vierfüssigen Thiere 3: 269. Klipspringer.

Synonyms: Palaeotragiscus longiceps; major.

Type locality: now known to be False Bay.

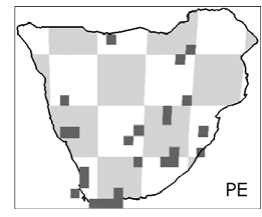

Additional references: Broom (1934); Gentry (2010); Gray (1850a, 1850b); Watson and Plug (1995); Wells (1951).

Ourebia Laurillard, 1842. In: d'Orbigny, Dictionnaire Universel d'Histoire Naturelle 1: 622.

Type locality: none given.

Ourebia ourebi Zimmermann, 1783. Geographische Geschichte des Menschen und der Allgemein Verbreiteten Vierfüssigen Thiere 3: 268. Oribi.

Type locality: since restricted to Bruintjieshoogte. Additional references: Gray (1850a 1850b).
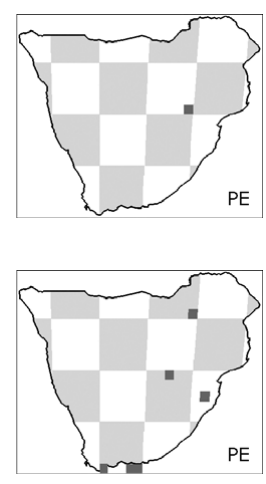

Raphicerus Smith, 1827. In: Griffith et al., The Animal Kingdom Arranged in Conformity with its Organization by the Baron Cuvier 5: 342. Additional references: Klein (1976c).

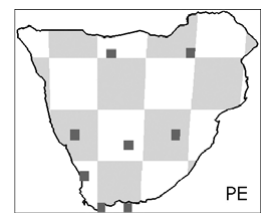

Raphicerus campestris Thunberg, 1811. Mem. Acad. Imp. Sci. St. Petersbourg 3: 313. Steenbok.

Type locality: since selected as Malmesbury District.

Additional references: Gray (1850a 1850b).

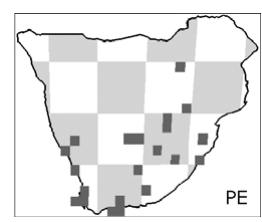

Raphicerus melanotis Thunberg, 1811. Mem. Acad. Imp. Sci. St. Petersbourg 3: 312. Cape grysbok.

Type locality: since selected as Cape Peninsula. Additional references: Gray (1850a, 1850b).

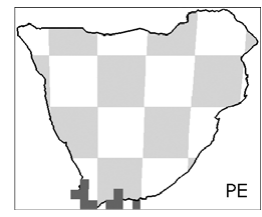


Raphicerus sharpei Thomas, 1896. Proc. Zool. Soc. Lond. 1896: 795, pl. 34. Sharpe's grysbok.

Synonyms: melanotis sharpei

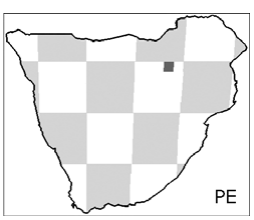

Subfamily: Bovinae

†'Bos' makapani Broom, 1937. S. Afr. J. Sci. 33: 510.

Type locality: Buffalo Cave.

Additional references: Gentry (2010).

†Miotragoceros acrae Gentry, 1974. Ann. S. Afr. Mus. 65(5): 146. Synonyms: Mesembriportax; Tragoportax.

Type locality: Langebaanweg.

Additional references: Gentry (1980, 2010).

†Simatherium kohllarseni Dietrich, 1942. Palaeontographica A 94: 43-133.

Additional references: Gentry (2010).

Syncerus Hodgson, 1847. J. Asiatic Soc. Bengal, Series 2, 16: 709. Synonyms: Bos; Bubalis; Pelorovis.

Additional references: Martínez-Navarro et al. (2007).

†Syncerus antiquus Duvernoy, 1851. C. R. Hebdom. Scé. Acad. Sci. Paris 33: 597.

Synonyms: Bubalis; Homoioceras; Pelorovis; Syncerus; baini.

Additional references: Bate (1949); Gentry (2010); Geraads (1992);

Klein (1994a); Martínez-Navarro et al. (2007); Pomel (1893); Reck
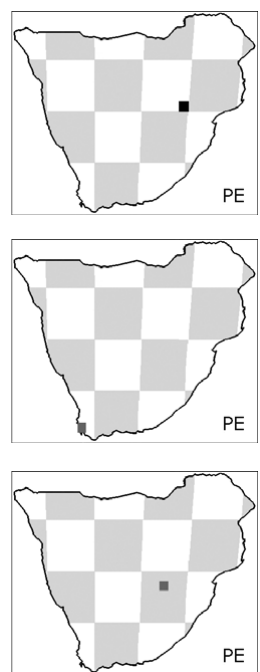
(1928).

Syncerus caffer Sparrman, 1779. K. Svenska Vet.-Akad. Handl. 40: 79. African buffalo.

Synonyms: Bubalis; andersoni.

Type locality: now restricted to Sundays River.

Additional references: Geraads (1992); Scott (1907).
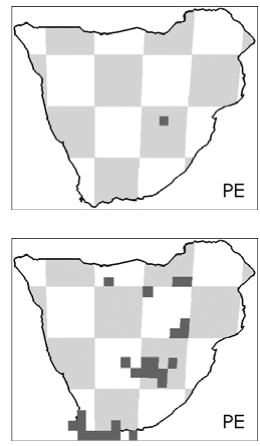

Taurotragus oryx Pallas, 1766. P.S. Pallas Medecinae Doctoris Miscellanea Zoologica: 9. Common eland.

Synonyms: Antilope; Boselaphus; Tragelaphus; oreas.

Type locality: restricted to near Cape Town.

Additional references: Gray (1850a, 1850b); Pappas (2002); Smith

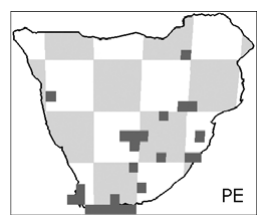

(1849); Willows-Munro et al. (2005). 
Tragelaphus De Blainville, 1816. Bull. Sci. Soc. Philom. Paris 1816: 75.

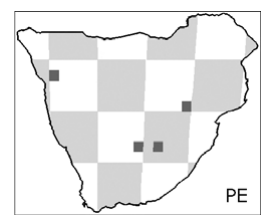

Tragelaphus angasii Angas, 1848. Proc. Zool. Soc. Lond. 1848: 89. Nyala.

Type locality: 'Hills that border: upon the northern shores of St Lucia Bay'.

Additional references: Gray (1850a, 1850b); Grubb (2004); Willows-

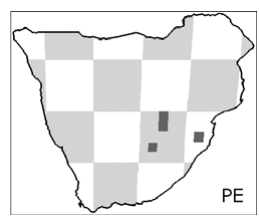
Munro et al. (2005).

Tragelaphus scriptus Pallas, 1766. P.S. Pallas Medecinae Doctoris Miscellanea Zoologica: 8. Bushbuck.

Additional references: Gray (1850a, 1850b); Willows-Munro et al. (2005).

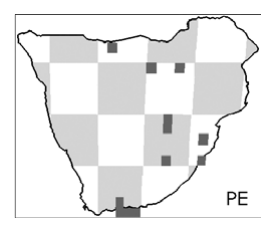

Tragelaphus spekeii Speke, 1863. Journal of the Discovery of the Source of the Nile: 223 (footnote). Sitatunga.

Additional references: Grubb (2004); Willows-Munro et al. (2005).

Tragelaphus strepsiceros Pallas, 1766. P.S. Pallas Medecinae Doctoris Miscellanea Zoologica: 9. Greater kudu.

Synonyms: Damalis; Strepsiceros; capensis.

Type locality: restricted to eastern part of western Cape Province. Additional references: Smith (1849); Willows-Munro et al. (2005).
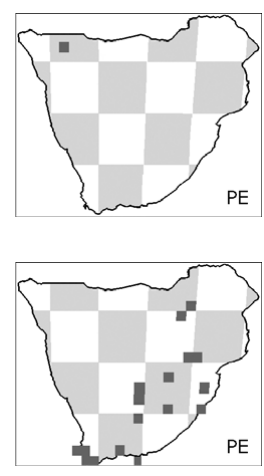

\section{Subfamily: Caprinae}

†'Makapania' Wells and Cooke, 1957. Palaeontol. Afr. 4: 26.

Type locality: Limeworks Makapansgat.

Additional references: Gentry (1970).

†'Makapania' broomi Wells and Cooke, 1957. Palaeontol. Afr. 4: 26. Synonyms: Bos; Hippotragus; Hippotragoides; makapaani.

Type locality: Limeworks Makapansgat.

Additional references: Gentry (1970, 2010).

†Numidocapra arambourgi Ennouchi, 1953. C.R. Somm. Séanc. Soc.

Geol. Fr., Paris, 8: 126.

Synonyms: Rabaticeras.

Additional references: Arambourg (1949).

Comments: Ennouchi (1953) not seen.
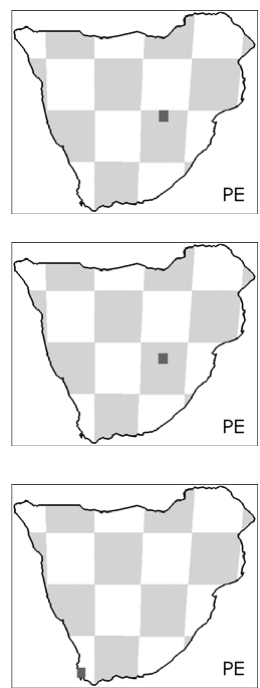
†Numidocapra porrocornutus Vrba, 1971. Ann. Transvaal Mus. 27(5): 59.

Synonyms: Damaliscus; Rabaticeras.

Type locality: Swartkrans.

Additional references: Laubscher et al. (1972); Vrba (1974b).

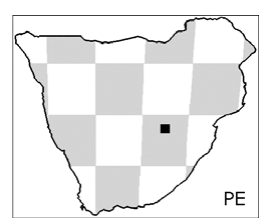

Subfamily: Cephalophinae

Cephalophus Smith, 1827. In: Griffith et al., The Animal Kingdom

Arranged in Conformity with its Organization by the Baron Cuvier 5: 344.

Comments: material only identified to genus level has not been mapped because this could include specimens assignable to Philantomba as currently understood.

Cephalophus natalensis Smith, 1834. S. Afr. Quart. J., Series 2, 2: 217. Red duiker.

Type locality: 'Port Natal' = Durban.

Additional references: Gray (1850a, 1850b); Smith (1849).

Philantomba monticola Thunberg, 1789. Resa uti Europa Africa, Asia, forrattad aren 1770-1779 2: 66. Blue duiker.

Synonyms: Cephalophus; bicolor; caeruleus.

Type locality: since identified as Langkloof.

Additional references: Gray (1850a, 1850b, 1862a, 1862b); Jansen van Vuuren (1999).

Sylvicapra Ogilby, 1836. Proc. Zool. Soc. Lond. 1836: 138.
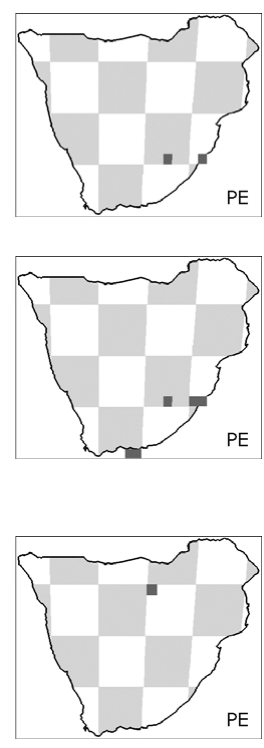

Sylvicapra grimmia Linnaeus, 1758. Systema Naturae Regnum Animale,

10th edition, 1: 70. Bush duiker.

Type locality: now known to be Cape Town.

Additional references: Gentry (2010); Gray (1850a, 1850b).

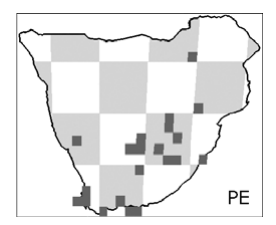

Subfamily: Hippotraginae

Hippotragus Sundevall, 1845. Ofv. K. Svenska Vet.-Akad. Förhand. 1845: 31.

Additional references: Commission on Zoological Nomenclature (2003);

Grubb (2004).

†Hippotragus cookei Vrba, 1987. Palaeontol. Afr. 26(5): 49.

Type locality: Limeworks Makapansgat.
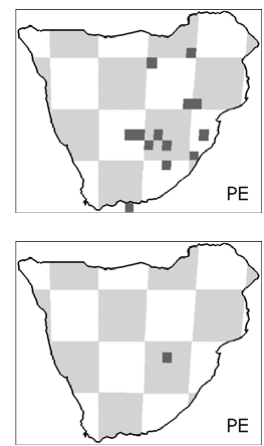
Hippotragus equinus Geoffroy Saint-Hilaire, 1803. Catalogue des Mammifères du Muséum National d'Histoire Naturelle: 259. Roan antelope.

Synonyms: Aigoceros; Antilope; osanne.

Type locality: now thought to be Plettenberg Bay.

Additional references: Gray (1850a, 1850b); Grubb (2004); Smith (1849).

†Hippotragus gigas Leakey, 1965. Olduvai Gorge 1: 49.

Additional references: Gentry (2010).

†Hippotragus leucophaeus Pallas, 1766. P.S. Pallas Medecinae Doctoris Miscellanea Zoologica: 4. Blaaubok, blue antelope.

Synonyms: problematicus.

Type locality: since restricted to Swellendam Dist.

Additional references: Broom (1949b); Cooke (1947); Gentry (2010); Klein (1974a).

Hippotragus niger Harris, 1838. Proc. Zool. Soc. Lond. 1838: 2. Sable antelope.

Synonyms: harrisi.

Type locality: since specified as Magaliesberg near Krugersdorp and Rustenburg.
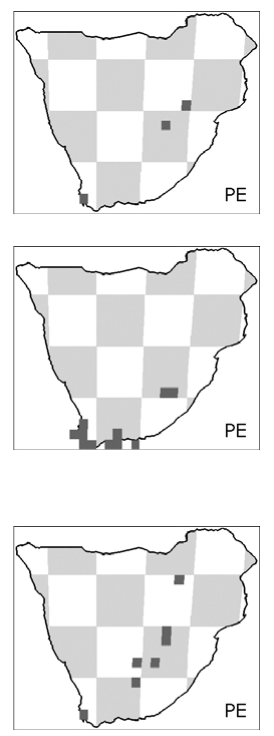

Additional references: Gray (1850a 1850b); Harris (1838a).

Oryx De Blainville, 1816. Bull. Sci. Soc. Philom. Paris 1816: 75.

Type locality: understood to be South Africa.

Comments: this material is likely to be O.gazella since only one species has so far been recognised in the sub-region.

Oryx gazella Linnaeus, 1758. Systema Naturae Regnum Animale, 10th edition, 1: 69. Gemsbok.

Type locality: understood to be South Africa.

Additional references: Gray (1850a, 1850b).
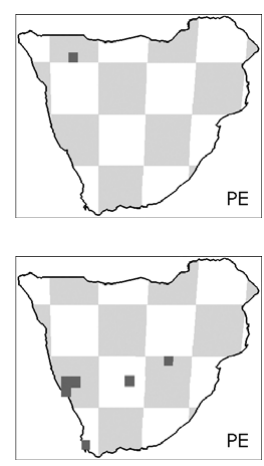

\section{Subfamily: Reduncinae}

Kobus Smith, 1840. Illustrations of the Zoology of South Africa, Part 12: pl. 28 plus text.

Synonyms: Aigoceros; Onotragus.

Additional references: Birungi and Arctander (2001).

†'Kobus' altidens Cooke, 1949. Mem. Geol. Surv. 35(3): 29.

Type locality: Keeble's Paddock.

Comments: according to Wells (1965), this taxon is a 'myth', being a bovine not a reduncine, although he did not reassign the specimen.

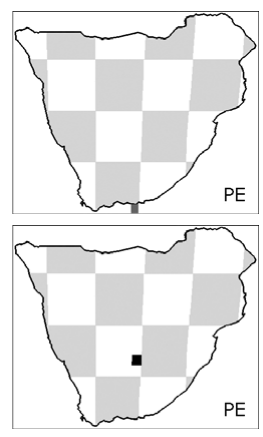


Kobus ellipsiprymnus Ogilby, 1833. Proc. Zool. Soc. Lond. 1833: 47. Waterbuck.

Synonyms: Aigoceros.

Type locality: since restricted to Gaborone.

Additional references: Birungi and Arctander (2001); Gray (1850a, 1850b); Smith (1849).

Kobus leche Gray, 1850. Gleanings from the Menagerie and Aviary at Knowsley Hall 2: 23. Lechwe.

Synonyms: Onotragus; venterae.

Type locality: since identified as Botletle River, near Lake Ngami. Additional references: Birungi and Arctander (2001).

Pelea capreolus Forster, 1790. Le Vaillant's Reise in das Innere von Afrika

1: 71. Vaal rhebok.

Type locality: now specified as Houhoek Pass.

Additional references: Birungi and Arctander (2001); Gray (1850a, 1850b).

Redunca Smith, 1827. In: Griffith et al., The Animal Kingdom Arranged in Conformity with its Organization by the Baron Cuvier 5: 337.

Redunca arundinum Boddaert, 1785. Elenchus Animalium 1: 141. Southern reedbuck.

Type locality: since selected as Bethulie.

Additional references: Gentry (2010); Gray (1850a, 1850b).

†Redunca darti Wells and Cooke, 1957. Palaeontol. Afr. 4: 17. Type locality: Limeworks Makapansgat.
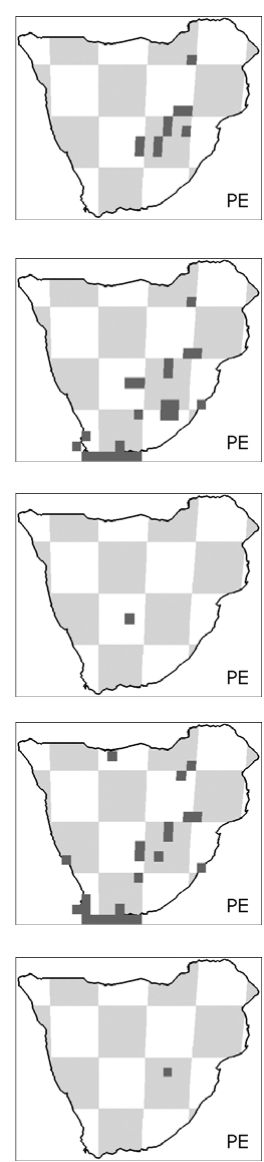

Redunca fulvorufula Afzelius, 1815. Nova Acta Reg. Soc. Sci. Upsala 7: 250. Mountain reedbuck.

Synonyms: Cervicapra.

Type locality: restricted to eastern North Cape Province.

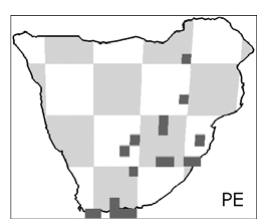

\subsection{PLEISTOCENE SITES}

So far, 201 sites have yielded Pleistocene material, ranging from individual specimens to the major collections. The latter include the Early Pleistocene sites in the Cradle of Humankind and Later Pleistocene ones such as Border Cave, Elandsfontein, Florisbad, Klasies River and Wonderwerk. During this epoch, sites, especially the later ones, increasingly constitute former human habitations. Although the distribution of sites is much broader than it was previously, there remains a large area from which evidence is not yet forthcoming (Figure 5.1). The gap may be real, arising, for instance, from a lack of suitable sites for bone preservation and/or human habitation, or may simply result from a lack of attention to the region by workers. 


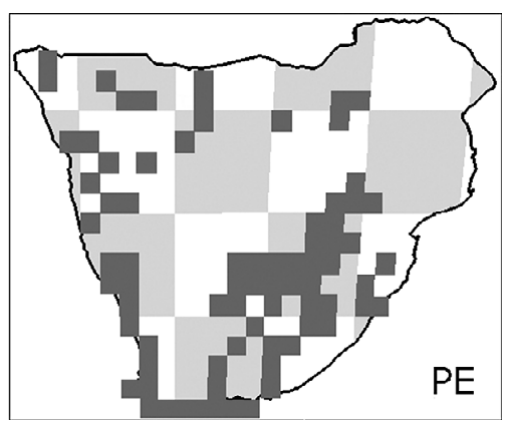

Figure 5.1 Location of Pleistocene sites.

!Ncumtsa Hills (2021). Other names: Koanaka. Taxa: Papio ursinus; Parapapio jonesi cf.; Pedetes capensis. References: Pickford (1990); Pickford and Mein (2011); Pickford et al. (1994); Senut (1996); Williams et al. (2012).

$\neq \mathbf{G i}$ (1938:2100). Taxa: Alcelaphus caama cf.; Ceratotherium simum; Connochaetes taurinus; Equus capensis; Giraffa camelopardalis?; Megalotragus priscus; Phacochoerus africanus; Raphicerus; Syncerus antiquus. References: Brooks et al. (1980); Brooks and Yellen (1977); Wadley (2015).

Abenab (1917:1838). Taxa: Phacochoerus. References: Pia (1930); Pickford and Senut (2010).

Abrahamskraal (2826). Taxa: Megalotragus priscus. References: Hoffman (1953).

Aigamas I and II (1929:1713; 1927:1717). Taxa: Acomys; Crocidura; Desmodillus; Gerbilliscus; Gerbillurus; Graphiurus; Malacothrix; Miniopterus; Mus; Rhabdomys; Saccostomus; Steatomys; Stenodontomys; Thallomys; Zelotomys. References: Pickford and Senut (2010).

Aloes Bone Deposit (3349:2538). Taxa: Antidorcas marsupialis; Connochaetes; Crocuta crocuta; Equus capensis cf.; Phacochoerus africanus; Potamochoerus larvatus. References: Wells (1970a).

Amis (2110:1430). Taxa: Pronolagus randensis; Suricata suricatta. References: Van Neer and Breunig (1999).

Andriesgrond (3218BB). Taxa: Crocidura flavescens; Cryptomys hottentotus; Elephantulus edwardii; Gerbilliscus afra; Gerbillurus paeba; Micaelamys namaquensis; Myosorex varius; Myotomys unisulcatus; Mystromys albicaudatus; Otomys saundersiae; Rhabdomys pumilio; Steatomys krebsii. References: Avery (unpublished); Pfeiffer (2013).

Anyskop Blowout (3358:1809). Taxa: Bathyergus suillus; Equus capensis; Diceros bicornis; Taurotragus oryx. References: Kandel and Conard (2012).

Apollo 11 (2745:1706). Taxa: Aethomys chrysophilus; Antidorcas marsupialis; Canis; Caracal caracal cf.; Crocuta; Equus capensis; Equus quagga cf.; Equus zebra; Hystrix africaeaustralis; Lepus; Macroscelides; Oreotragus oreotragus; Oryx gazella; Petromus typicus cf.; Phacochoerus africanus; Procavia capensis; Pronolagus; Raphicerus; Saccostomus campestris. References: Cruz-Uribe and Klein (1981-1983); Dewar and Stewart (2016); Thackeray (1979); Vogelsang et al. (2010); Wadley (2015).

Asis Ost (1942:1743). Taxa: Antidorcas; Procavia capensis. References: Pickford and Senut (2002).

Austin's Rush (2824). Taxa: Equus capensis; Equus sandwithi; Hippopotamus amphibius; Loxodonta africana. References: Cooke (1949a). 
Baard's Quarry (3318). Taxa: Antidorcas; Canis?; Ceratotherium; Diceros bicornis; Equus capensis cf.; Eurygnathohippus hooijeri; Gazella praethomsoni cf.; Hippopotamus; Hippotragus leucophaeus; Mammuthus subplanifrons; Mellivora capensis; Miotragoceros acrae; Orycteropus; Pachycrocuta brevirostris cf.; Panthera; Parahyaena brunnea aff.; Raphicerus; Sivatherium maurisium?. References: Hendey (1969, 1978d); Klein (1974b).

Barkly West (2824DA). Taxa: Damaliscus pygargus; Equus capensis; Equus quagga; Hippopotamus amphibius cf. References: Fraas (1907); Wells (1959c).

Berg Aukas (1931:1815). Taxa: Crocidura; Cryptomys; Dendromus; Elephantulus; Gerbilliscus; Grammomys; Graphiurus; Hipposideros; Homo sapiens; Malacothrix; Mastomys; Mus; Myocricetodon; Mystromys; Rhinolophus; Saccostomus; Steatomys; Stenodontomys; Zelotomys. References: Conroy et al. (1996); Grine et al. (1995); Mein et al. (2000b); Pickford and Senut (2010); Senut et al. (1992).

Bestpan (2824). Taxa: Connochaetes; Megalotragus priscus. References: Cooke (1949a). Black Earth Cave (2737:2437). Taxa: Aepyceros melampus; Antidorcas bondi; Antidorcas marsupialis; Canis adustus; Canis mesomelas; Connochaetes gnou; Crocidura; Cryptomys; Damaliscus pygargus; Equus burchellii; Equus capensis; Homo sapiens; Hystrix; Kobus leche; Lycaon; Panthera leo cf.; Parahyaena brunnea; Pelea capreolus; Phacochoerus africanus; Procavia capensis; Raphicerus; Redunca arundinum; Redunca fulvorufula; Syncerus caffer; Vulpes. References: Kuhn et al. (2016); McKee (1994).

Bloembos[ch] (3317:1811). Taxa: Connochaetes; Crocuta crocuta; Diceros bicornis; Equus capensis; Giraffa camelopardalis; Hippopotamus amphibius cf.; Hippotragus leucophaeus; Hippotragus niger cf.; Syncerus antiquus; Syncerus caffer. References: Cooke (1947, 1955); Broom (1913c).

Bloemhof (2725DA). Taxa: Connochaetes gnou; Elephas iolensis; Equus capensis; Hippopotamus amphibius; Syncerus antiquus. References: Cooke (1949a); Osborn (1942). Bloemhof Site. See Sheppard Island.

Blombos Cave (3425:2113). Taxa: Acomys subspinosus; Alcelaphus buselaphus; Antidorcas; Aonyx capensis; Atelerix frontalis; Bathyergus suillus; Canis mesomelas; Chlorotalpa duthiae; Connochaetes; Crocidura cyanea; Crocidura flavescens; Damaliscus pygargus; Dasymys incomtus; Dendromus melanotis; Dendromus mesomelas; Diceros bicornis; Equus capensis; Felis silvestris; Galerella pulverulenta; Genetta; Georychus capensis; Gerbilliscus afra; Hippopotamus amphibius; Hippotragus leucophaeus; Homo sapiens; Hystrix africaeaustralis; Ictonyx striatus; Lepus capensis; Lepus saxatilis; Mellivora capensis; Miniopterus natalensis; Mus minutoides; Myomyscus verreauxii; Myosorex varius; Oreotragus oreotragus; Otomys irroratus; Otomys saundersiae; Papio ursinus; Pelea capreolus; Procavia capensis; Raphicerus campestris; Raphicerus melanotis; Redunca arundinum; Rhabdomys pumilio; Rhinolophus capensis; Rhinolophus clivosus; Steatomys krebsii; Suncus varilla; Syncerus antiquus; Syncerus caffer; Taurotragus oryx. References: Badenhorst et al. (2016); Discamps and Henshilwood (2015); Henshilwood (1995, 1996); Henshilwood et al. (2001); Hillestad Nel and Henshilwood (2016); Wadley (2015).

Boegoeberg 1 (2846:1634). Taxa: Antidorcas marsupialis; Bathyergus janetta; Canis mesomelas; Connochaetes taurinus; Diceros bicornis; Felis silvestris; Galerella pulverulenta; Ictonyx striatus; Mellivora capensis; Oryx gazella; Otocyon megalotis; Panthera leo?; Parahyaena brunnea; Raphicerus campestris; Redunca arundinum; Suricata suricatta; Vulpes chama. References: Klein et al. (1999b).

Bolt's Farm (2602:2753). Taxa: Acomys spinosissimus; Caracal caracal; Cercopithecoides williamsi; Crocidura fuscomurina; Crossarchus transvaalensis; Cryptomys robertsi; Dasymys 
'bolti'; Dasymys 'lavocati'; Dendromus melanotis; Desmodillus auricularis; Dinofelis barlowi; Elephantulus antiquus; Elephantulus brachyrhynchus cf.; Elephantulus broomi; Elephas recki cf.; Equus burchellii?; Equus capensis; Erinaceus broomi; Eurygnathohippus cornelianus; Felis shawi; Gerbilliscus; Lemniscomys rosalia cf.; Leptailurus serval; Malacothrix typica cf.; Metridiochoerus andrewsi; Metridiochoerus modestus; Mus major; Mus minutoides; Myosorex robinsoni; Myotis cf.; Mystromys hausleitneri; Otomys gracilis; Otomys irroratus aff.; Papio izodi; Parapapio broomi; Parapapio whitei; Potamochoeroides hypsodon; Proamblysomus antiquus; Proodontomys cookei; Proteles cristata; Rhabdomys pumilio cf.; Rhinolophus capensis cf.; Steatomys pratensis; Thallomys debruyni; Theropithecus oswaldi. References: Broom (1937b, 1939c, 1948a); Churcher (1970); Cooke (1985, 1991, 1993a, 1993b); Davis (1961); Freedman (1965, 1970); Gilbert (2007b); Gingerich (1974); Gommery et al. (2012a); Lacruz et al. (2006); Meester (1961a); Reynolds (2010b); Thackeray et al. (2008).

Boomplaas Cave (3323:2211). Taxa: Acomys subspinosus; Alcelaphus buselaphus; Antidorcas marsupialis cf.; Bunolagus monticularis; Canis mesomelas; Caracal caracal cf.; Chlorotalpa sclateri; Connochaetes gnou cf.; Connochaetes taurinus cf.; Crocidura cyanea; Crocidura flavescens; Cryptomys hottentotus; Damaliscus pygargus; Dasymys incomtus; Dendromus melanotis; Dendromus mesomelas; Elephantulus; Equus capensis; Felis silvestris; Galerella pulverulenta; Genetta; Gerbilliscus afra; Gerbillurus paeba; Graphiurus ocularis; Herpestes ichneumon; Hippotragus equinus; Hippotragus leucophaeus; Hystrix africaeaustralis; Lepus capensis; Lycaon pictus; Megalotragus priscus; Mellivora capensis; Micaelamys namaquensis; Mus minutoides; Myomyscus verreauxii; Myosorex varius; Myotomys unisulcatus; Mystromys albicaudatus; Oreotragus oreotragus; Otomys irroratus; Otomys laminatus; Otomys saundersiae; Panthera pardus; Papio ursinus; Parahyaena brunnea cf.; Pelea capreolus; Phacochoerus africanus; Potamochoerus larvatus; Procavia capensis; Raphicerus campestris; Raphicerus melanotis; Redunca arundinum; Redunca fulvorufula; Rhabdomys pumilio; Suncus varilla; Syncerus caffer; Taurotragus oryx; Tragelaphus strepsiceros. References: Avery (1977, 1982b); Brophy et al. (2014); Faith (2013); Klein (1978a, 1994b); Von den Driesch and Deacon (1985); Wadley (2015).

Border Cave (2701:3159). Taxa: Acomys; Aepyceros melampus; Aethomys chrysophilus; Alcelaphus; Amblysomus hottentotus; Antidorcas bondi; Chlorocebus aethiops; Connochaetes taurinus; Crocidura cyanea; Crocidura flavescens; Crocidura fuscomurina; Crocidura hirta; Crocidura mariquensis; Cryptomys hottentotus; Damaliscus pygargus cf.; Dasymys incomtus; Dendromus melanotis; Dendromus mystacalis; Elephantulus myurus; Eptesicus hottentotus; Equus burchellii; Equus capensis; Gerbilliscus; Gerbilliscus leucogaster cf.; Glauconycteris variegata; Grammomys dolichurus; Graphiurus murinus; Hippopotamus amphibius; Hippotragus; Homo sapiens; Hystrix africaeaustralis; Kobus ellipsiprymnus; Lemniscomys rosalia; Lepus; Loxodonta africana?; Lycaon pictus; Malacothrix typica; Mastomys natalensis; Mellivora capensis; Micaelamys namaquensis; Miniopterus natalensis; Mus minutoides; Mus triton; Myosorex cafer; Myosorex varius; Mystromys albicaudatus; Neoromicia capensis; Nycteris thebaica; Oreotragus oreotragus; Otomys angoniensis; Otomys irroratus; Otomys laminatus; Ourebia ourebi; Panthera pardus; Papio ursinus; Paraxerus palliatus; Pelomys fallax; Phacochoerus africanus; Potamochoerus larvatus; Procavia capensis; Raphicerus campestris cf.; Redunca fulvorufula; Rhabdomys pumilio; Rhinolophus clivosus cf.; Rhinolophus darlingi?; Rhinolophus hildebrandtii cf.; Saccostomus campestris; Scotophilus nigrita; Steatomys pratensis; Suncus infinitesimus; Suncus varilla; Syncerus caffer; Tadarida; Taurotragus oryx; Thallomys paedulcus; Tragelaphus angasii; Tragelaphus 
scriptus; Tragelaphus strepsiceros. References: Avery (1982a, 1991b, 1992a); Cooke et al. (1945); De Villiers (1974, 1976a); Klein (1977); Rightmire (1979b); Wadley (2015).

Boskop (2634:2707). Taxa: Homo sapiens. References: Broom (1918); Dusseldorp et al. (2013).

Brakfontein (3149:2301). Taxa: Diceros bicornis. References: Cooke (1955).

Buffalo Cave (2408:2911). Taxa: 'Bos' makapani; Connochaetes; Damaliscus; Equus; Eurygnathohippus; Hippotragus; Panthera leo cf.; Parmularius cf.; Phacochoerus; Redunca; Syncerus; Tragelaphus cf. References: Kuykendall et al. (1995).

Bulawayo (2010:2840). Taxa: Pedetes capensis. References: Pickford and Mein (2011).

Bundu Farm (2945:2212). Taxa: Antidorcas; Connochaetes gnou; Damaliscus pygargus; Equus capensis; Hippopotamus amphibius; Megalotragus priscus; Papio; Phacochoerus. References: Hutson (2016); Kiberd (2006).

Bushman Rockshelter (3038:2435). Taxa: Aepyceros melampus; Alcelaphus; Connochaetes taurinus; Damaliscus lunatus; Equus burchellii; Equus capensis; Equus quagga; Hippotragus equinus; Hippotragus niger; Hippopotamus amphibius; Lepus; Megalotragus priscus cf.; Oreotragus oreotragus; Orycteropus afer; Papio ursinus; Pelea capreolus cf.; Phacochoerus africanus; Procavia capensis; Raphicerus campestris; Redunca arundinum; Redunca fulvorufula; Sylvicapra grimmia; Syncerus caffer cf.; Taurotragus oryx; Tragelaphus strepsiceros. References: Badenhorst and Plug (2012); Brain (1969, 1981); Dusseldorp et al. (2013); Plug (1981); Wadley (2015).

Calabria 630 (2808:2656). Taxa: Antidorcas bondi; Connochaetes gnou; Equus burchellii; Hippopotamus amphibius; Phacochoerus africanus. References: De Ruiter et al. (2011).

Cango Caves (3324:2212). Taxa: Connochaetes; Procavia capensis; Tragelaphus scriptus cf. References: Cooke (1955).

Canteen Koppie (2824DA). Taxa: Homo sapiens. References: Smith et al. (2012).

Cave of Hearths (2409:2904). Taxa: Acomys; Aepyceros melampus; Aethomys chrysophilus cf.; Alcelaphus buselaphus; Alcelaphus caama; Alcelaphus robustus; Antidorcas bondi; Antidorcas marsupialis; Canis mesomelas; Caracal caracal; Connochaetes taurinus; Crocidura taungensis; Crocuta crocuta; Cryptomys robertsi; Damaliscus lunatus; Dendromus mesomelas; Elephantulus broomi; Equus burchellii; Equus capensis; Gerbilliscus brantsii cf.; Herpestes ichneumon; Homo sapiens; Hystrix africaeaustralis; Kobus leche; Lepus saxatilis; Megalotragus priscus; Miniopterus natalensis cf.; Mus minutoides cf.; Mystromys hausleitneri; Oreotragus oreotragus; Otomys gracilis; Ourebia cf.; Panthera leo; Panthera pardus; Papio ursinus; Pelea capreolus; Phacochoerus africanus; Procavia capensis; Pronolagus randensis; Raphicerus campestris; Redunca arundinum; Rhinolophus capensis cf.; Rhinolophus clivosus cf.; Saccostomus campestris; Steatomys pratensis; Syncerus antiquus; Syncerus caffer; Taurotragus oryx; Thallomys paedulcus cf.; Tragelaphus strepsiceros. References: Brophy et al. (2014); Cooke (1962, 1988); De Graaff (1961c, 1988); McKee et al. (1995); Tobias (1971); Wadley (2015); Wells (1988).

Chelmer (1959:2830). Taxa: Alcelaphus caama; Antidorcas bondi; Connochaetes; Damaliscus; Equus capensis; Hippopotamus amphibius; Megalotragus priscus; Phacochoerus africanus cf.; Syncerus antiquus; Taurotragus oryx cf. References: Cooke (1962); Cooke and Wells (1951); Wadley (2015); Wells and Cooke (1955).

Christiana (2725CC). Taxa: Connochaetes gnou; Elephas iolensis; Equus quagga cf.; Eurygnathohippus cornelianus; Hippopotamus amphibius; Loxodonta africana. References: Cooke (1949a). 
Cooper's (2600:2745). Taxa: Acinonyx jubatus; Antidorcas marsupialis; Antidorcas recki; Australopithecus africanus; Canis mesomelas; Caracal caracal; Cercopithecoides williamsi; Chasmaporthetes nitidula; Connochaetes taurinus cf.; Crocuta crocuta; Crocuta ultra; Cynictis penicillata; Damaliscus pygargus cf.; Dinofelis aronoki cf.; Equus burchellii; Equus capensis; Felis silvestris; Herpestes ichneumon; Hippotragus; Homo; Hystrix africaeaustralis; Lepus; Lycaon sekowei; Megalotragus; Megantereon whitei; Metridiochoerus andrewsi; Metridiochoerus modestus; Panthera leo; Panthera pardus; Papio izodi; Papio robinsoni; Paranthropus robustus; Parahyaena brunnea; Pedetes; Pelea; Poecilogale albinucha; Procavia antiqua; Procavia transvaalensis; Proteles cristata; Raphicerus; Redunca fulvorufula; Simatherium kohllarseni; Sivatherium maurisium; Suricata; Syncerus; Taurotragus oryx; Theropithecus oswaldi; Tragelaphus scriptus cf.; Tragelaphus strepsiceros cf. References: Berger et al. (1995, 2003); Churcher (1970); De Ruiter et al. (2009); Freedman (1970); Kuhn et al. (2017); O'Regan and Menter (2009); O’Regan and Steininger (2017); Reynolds (2010b).

Cornelia-Uitzoek (2710:2852). Taxa: Aepyceros helmoedi; Antidorcas bondi; Antidorcas recki; Connochaetes gnou; Damaliscus niro; Equus burchellii cf.; Equus capensis; Equus quagga cf.; Eurygnathohippus cornelianus; Hippopotamus gorgops; Homo; Kolpochoerus heseloni; Kolpochoerus paiceae; Megalotragus priscus; Metridiochoerus compactus; Metridiochoerus modestus; Panthera leo; Phacochoerus; Raphicerus; Sivatherium maurisium; Sylvicapra grimmia; Syncerus antiquus. References: Bender and Brink (1992); Brink (2004, 2005); Brink et al. (2012); Brink and Rossouw (2000); Brophy et al. (2014); Cooke (1974); McKee et al. (1995).

David's Drift (2824). Taxa: Megalotragus priscus. References: Cooke (1949a).

De Hoop 120 (2828:2644). Taxa: Antidorcas bondi. References: De Ruiter et al. (2011).

Delport's Hope (2824). Taxa: Aepyceros melampus; Damaliscus; Elephas iolensis; Equus capensis cf.; Hippopotamus; Loxodonta africana; Megalotragus priscus; Taurotragus. References: Cooke (1949a).

Die Kelders (3432:1922). Taxa: Acomys subspinosus; Alcelaphus buselaphus; Antidorcas australis; Atelerix frontalis; Atilax paludinosus; Bathyergus suillus; Canis mesomelas; Caracal caracal; Ceratotherium simum; Chrysochloris asiatica; Connochaetes gnou; Crocidura flavescens; Damaliscus niro; Damaliscus pygargus; Dasymys incomtus; Dendromus melanotis; Dendromus mesomelas; Diceros bicornis; Equus capensis; Equus quagga; Felis silvestris; Galerella pulverulenta; Genetta; Georychus capensis; Gerbilliscus afra; Herpestes ichneumon; Hippopotamus amphibius; Hippotragus leucophaeus; Homo sapiens; Hystrix africaeaustralis; Ictonyx striatus; Lepus capensis; Lycaon pictus; Mellivora capensis; Mus minutoides; Myomyscus verreauxii; Myosorex varius; Mystromys albicaudatus; Oreotragus oreotragus; Otomys irroratus; Otomys saundersiae; Panthera leo; Papio ursinus; Parahyaena brunnea; Pelea capreolus; Potamochoerus larvatus; Procavia capensis; Raphicerus melanotis; Redunca arundinum; Redunca fulvorufula; Rhabdomys pumilio; Suncus varilla; Syncerus antiquus; Syncerus caffer; Taurotragus oryx; Tragelaphus strepsiceros. References: Armstrong (2016); Avery (1977, 1979); Avery et al. (1997); Brophy et al. (2014); Grine et al. (1991); Klein (1975b); Klein and Cruz-Uribe (2000); Marean et al. (2000); Rightmire (1979a); Schweitzer (1974, 1979); Schweitzer and Scott (1973); Wadley (2015).

Diepkloof (3223:1827). Taxa: Antidorcas; Atelerix frontalis; Bathyergus suillus; Canis mesomelas; Connochaetes gnou; Equus capensis; Felis silvestris; Galerella pulverulenta; Genetta; Hippopotamus amphibius; Hippotragus leucophaeus; Homo sapiens; Hystrix 
africaeaustralis; Lepus; Mellivora capensis; Oreotragus oreotragus; Orycteropus afer; Panthera pardus; Papio ursinus; Pelea capreolus; Procavia capensis; Raphicerus campestris; Raphicerus melanotis; Redunca arundinum; Syncerus antiquus; Taurotragus oryx; Vulpes chama. References: Klein and Steele (2008); Steele and Klein (2013); Wadley (2015).

Dikbosch 1 (2839:2354). Taxa: Aethomys chrysophilus; Alcelaphus buselaphus; Ceratotherium simum?; Crocidura cyanea; Crocidura flavescens; Crocidura hirta; Cryptomys hottentotus; Equus quagga cf.; Gerbilliscus; Gerbillurus paeba; Graphiurus ocularis; Hippopotamus amphibius; Lepus; Mystromys natalensis; Mus minutoides; Mystromys albicaudatus; Oreotragus oreotragus; Otomys saundersiae; Procavia capensis; Raphicerus; Redunca fulvorufula; Rhabdomys pumilio; Sylvicapra grimmia?; Steatomys; Suncus lixus; Taurotragus oryx. References: Avery and Avery (2011); Humphreys (1974); Humphreys and Thackeray (1983); Klein (1979a).

Dinaledi Chamber (2601:2743). Taxa: Homo naledi. References: Berger et al. (2015). Doring River (2810:2648). Taxa: Alcelaphus buselaphus; Antidorcas bondi; Connochaetes taurinus; Damaliscus niro; Damaliscus pygargus; Megalotragus priscus; Syncerus antiquus. References: Brink et al. (1999).

Draaihoek (2824). Taxa: Diceros bicornis cf.; Megalotragus priscus. References: Cooke (1949a).

Driefontein (3225). Taxa: Antidorcas bondi; Damaliscus niro cf.; Equus capensis cf.; Equus quagga cf.; Megalotragus priscus; Oryx gazella cf.; Phacochoerus africanus cf.; Raphicerus campestris; Redunca arundinum cf.; Syncerus antiquus; Taurotragus oryx. References: Wells (1970b).

Drimolen (2527). Taxa: Acomys spinosissimus aff.; Antidorcas recki; Canis; Caracal caracal; Cercopithecoides; Chasmaporthetes nitidula?; Connochaetes; Cryptomys hottentotus cf.; Damaliscus; Dasymys 'bolti'; Dendromus melanotis cf.; Desmodillus auricularis; Dinofelis piveteaui aff.; Elephantulus; Eurygnathohippus cornelianus cf.; Gazella vanhoepeni aff.; Gerbilliscus; Grammomys dolichurus; Graphiurus; Hippotragus; Homo; Malacothrix; Mastomys natalensis; Megalotragus; Metridiochoerus; Mus; Myosorex; Mystromys hausleitneri; Oreotragus; Otomys gracilis; Papio robinsoni; Paranthropus robustus; Pelea; Praomys; Proodontomys cookei; Redunca cf.; Rhabdomys pumilio aff.; Steatomys pratensis cf.; Suncus; Tragelaphus; Vulpes chama. References: Dusseldorp et al. (2013); Keyser et al. (2000); Moggi-Cecchi et al. (2010); O'Regan and Menter (2009); Reynolds (2010b); Rovinsky et al. (2015); Sénégas et al. (2005).

Drotsky's Cave (2002:2125). Taxa: Equus burchellii; Gerbilliscus brantsii cf.; Gerbillurus paeba; Mystromys albicaudatus; Otomys angoniensis cf.; Pedetes capensis. References: Robbins et al. (1996); Yellen et al. (1987).

Duinefontein (3343:1827). Taxa: Antidorcas australis; Bathyergus suillus; Canis mesomelas; Caracal caracal; Ceratotherium simum; Crocuta crocuta; Damaliscus pygargus; Diceros bicornis; Equus quagga; Felis silvestris; Galerella pulverulenta; Genetta; Herpestes ichneumon; Hippopotamus amphibius; Hippotragus leucophaeus; Hystrix africaeaustralis; Ictonyx striatus; Leptailurus serval; Lepus capensis; Loxodonta africana; Megalotragus priscus; Oryx gazella; Panthera leo; Parahyaena brunnea; Raphicerus melanotis; Redunca arundinum; Syncerus antiquus; Taurotragus oryx; Tragelaphus strepsiceros. References: Brophy et al. (2014); Cruz-Uribe et al. (2003); Klein (1976b); Klein and Cruz-Uribe (1991); Klein et al. (1999a).

Elands Bay Cave (3218:1820). Taxa: Alcelaphus buselaphus; Antidorcas; Atelerix frontalis; Bathyergus suillus; Canis mesomelas; Caracal caracal; Crocidura cyanea; Crocidura 
flavescens; Cryptomys hottentotus; Diceros bicornis; Elephantulus edwardii; Elephantulus rupestris; Equus capensis; Equus quagga; Felis silvestris; Gerbilliscus afra; Gerbillurus paeba; Herpestes ichneumon; Hippopotamus amphibius; Hippotragus leucophaeus; Hystrix africaeaustralis; Ictonyx striatus; Lepus; Loxodonta africana; Loxodonta cookei; Mellivora capensis; Micaelamys namaquensis; Myosorex varius; Mystromys albicaudatus; Myotomys unisulcatus; Oreotragus oreotragus; Orycteropus afer; Otomys irroratus; Otomys saundersiae; Panthera pardus; Papio ursinus; Potamochoerus larvatus; Procavia capensis; Raphicerus campestris; Raphicerus melanotis; Redunca arundinum; Rhabdomys pumilio; Steatomys krebsii; Suncus varilla; Sylvicapra grimmia; Syncerus caffer; Taurotragus oryx; Vulpes chama. References: Avery (unpublished); Dusseldorp et al. (2013); Jerardino et al. (2013); Klein and Cruz-Uribe (1987); Klein et al. (2007); Pfeiffer (2013).

Elandsfontein (3305:1815). Taxa: Alcelaphus; Antidorcas australis; Antidorcas recki; Aonyx capensis; Atilax paludinosus; Bathyergus suillus; Canis mesomelas; Caracal caracal; Ceratotherium simum; Connochaetes gnou; Crocuta crocuta; Damaliscus lunatus cf.; Damaliscus niro; Diceros bicornis; Equus capensis; Equus quagga; Felis silvestris; Gazella; Herpestes ichneumon; Hippopotamus amphibius; Hippotragus gigas; Hippotragus leucophaeus; Homo rhodesiensis; Hystrix africaeaustralis; Ictonyx striatus; Kolpochoerus paiceae; Lepus capensis; Loxodonta africana; Loxodonta atlantica; Lycaon pictus; Megalotragus priscus; Megantereon gracile; Mellivora capensis; Metridiochoerus andrewsi; Numidocapra arambourgi; Oryx gazella; Panthera leo; Panthera pardus; Parahyaena brunnea; Parmularius?; Phacochoerus africanus; Raphicerus melanotis; Redunca arundinum; Sivatherium maurisium; Smutsia; Suricata major; Suricata suricatta; Syncerus antiquus; Taurotragus oryx; Theropithecus oswaldi; Tragelaphus strepsiceros; Vulpes chama. References: Braun et al. (2013); Brophy et al. (2014); Drennan (1953); Ewer and Singer (1956); Hendey (1969); Klein (1974b, 1978c); Klein and Cruz-Uribe (1991); Klein et al. (2007); McKee et al. (1995); Singer (1956).

Equus Cave (2737:2438). Taxa: Acinonyx jubatus; Alcelaphus buselaphus; Antidorcas bondi; Antidorcas marsupialis; Aonyx capensis; Atelerix frontalis; Atilax paludinosus; Canis mesomelas; Caracal caracal; Connochaetes taurinus; Crocuta crocuta; Cynictis penicillata; Damaliscus pygargus; Diceros bicornis; Equus burchellii; Equus capensis; Felis nigripes; Felis silvestris; Genetta; Giraffa camelopardalis; Herpestes ichneumon; Hippopotamus amphibius; Hippotragus; Homo sapiens; Hystrix africaeaustralis; Kobus leche; Lepus; Lycaon pictus; Megalotragus priscus; Mellivora capensis; Orycteropus afer; Otocyon megalotis; Panthera leo; Panthera pardus; Papio ursinus; Parahyaena brunnea; Pedetes capensis; Pelea capreolus; Phacochoerus africanus; Procavia capensis; Raphicerus campestris; Redunca fulvorufula; Suricata suricatta; Sylvicapra grimmia; Syncerus caffer; Taurotragus oryx; Tragelaphus strepsiceros; Vulpes chama. References: Brophy et al. (2014); Cruz-Uribe (1991); Grine and Klein (1985); Klein et al. (1991); McKee (1994); McKee et al. (1995).

Erfkroon (2852:2536). Taxa: Alcelaphus buselaphus; Antidorcas bondi; Antidorcas marsupialis; Atilax paludinosus; Canis mesomelas; Connochaetes gnou; Crocuta crocuta; Cynictis penicillata; Damaliscus niro; Damaliscus pygargus; Equus capensis; Galerella sanguinea; Hippopotamus amphibius; Hippotragus; Kobus ellipsiprymnus; Lycaon pictus cf.; Megalotragus priscus; Panthera leo; Phacochoerus; Syncerus antiquus; Vulpes chama. References: Bousman and Brink (2014); Brophy et al. (2014); Churchill et al. (2000b).

Etosha Pan (1816CA). Taxa: Aepyceros melampus; Antidorcas marsupialis; Damaliscus lunatus; Equus quagga; Taurotragus oryx; Tragelaphus spekeii. References: Pickford et al. (2014). 
Faraoskop Rock Shelter (3207:1836). Taxa: Procavia capensis. References: Manhire (1993); Pfeiffer (2013).

Femur Dump (2602:2742). Taxa: Acomys spinosissimus; Aethomys chrysophilus cf.; Cercopithecoides williamsi; Cryptomys hottentotus cf.; Dasymys 'bolti'; Dendromus melanotis; Dinofelis barlowi; Mastomys cf.; Micaelamys namaquensis cf.; Mus; Mystromys hausleitneri; Otomys gracilis cf.; Pachycrocuta brevirostris; Papio robinsoni; Proodontomys cookei; Rhabdomys pumilio cf.; Steatomys pratensis. References: Gommery et al. (2008a); Reynolds (2010b).

Florisbad (2846:2604). Taxa: Alcelaphus buselaphus; Alcelaphus caama cf.; Antidorcas bondi; Antidorcas marsupialis; Aonyx capensis; Atilax paludinosus; Canis mesomelas; Cephalophus cf.; Ceratotherium simum; Connochaetes antiquus; Connochaetes gnou; Crocuta crocuta; Cryptomys?; Damaliscus niro; Damaliscus pygargus; Diceros bicornis; Equus (Asinus); Equus burchellii; Equus capensis; Equus quagga; Galerella sanguinea; Gerbilliscus; Giraffa camelopardalis; Hippopotamus amphibius; Hippotragus; Homo sapiens; Kobus ellipsiprymnus; Kobus leche; Lepus capensis; Lycaon pictus; Megalotragus priscus; Metridiochoerus cf.; Myotomys sloggetti cf.; Myotomys unisulcatus cf.; Otomys irroratus cf.; Panthera leo; Parahyaena brunnea; Parotomys; Pedetes capensis; Pedetes hagenstadti; Phacochoerus africanus; Raphicerus campestris; Redunca arundinum; Sivatherium maurisium; Sylvicapra grimmia cf.; Syncerus antiquus; Taurotragus oryx; Tragelaphus angasii cf.; Xerus inauris. References: Brink (1988, 1994, 2005); Brophy et al. (2014); Churchill et al. (2000b); Cooke (1955); Dreyer (1935); Ewer (1957b); Hoffman (1953); Kuman and Clarke (1986); Lewis et al. (2011); McKee et al. (1995); Scott and Brink (1992); Singer (1956); Wadley (2015).

Forlorn Hope (2824). Taxa: Equus burchellii cf.; Equus capensis; Hippopotamus. References: Cooke (1949a).

Friesenberg Hilltop (1917:1740). Taxa: Lycaon cf. References: Pickford and Senut (2010); Sénégas (1996).

Garage Ravine Cave (2602:2752). Taxa: Canis mesomelas; Equus capensis; Taurotragus oryx. References: Badenhorst et al. (2011b).

Gcwihaba C Hill (2121:2001). Taxa: Dendromus; Elephantulus; Georychus; Gerbilliscus brantsii cf.; Gerbillurus; Graphiurus; Malacothrix; Mus; Myosorex; Myotomys campbelli; Otomys gracilis; Rhinolophus; Steatomys pratensis; Suncus; Zelotomys woosnami aff. References: Pickford (1990); Pickford and Mein (1988); Pickford and Senut (2003); Pickford et al. (1994).

Geelbek Dunes (3311:1810). Taxa: Bathyergus suillus; Connochaetes gnou; Crocuta crocuta; Damaliscus; Equus capensis; Hippopotamus amphibius; Hippotragus leucophaeus; Lepus capensis; Loxodonta africana; Megalotragus priscus; Mellivora capensis; Raphicerus campestris; Sylvicapra grimmia; Syncerus antiquus; Taurotragus oryx. References: Conard and Kandel (2006); Kandel and Conard (2012).

Geelwal Karoo (3131:1804). Taxa: Canis mesomelas. References: Cooke (1955).

Gladysvale (2553:2746). Other names: Uitkomst. Taxa: Acomys spinosissimus; Aepyceros melampus cf.; Aethomys chrysophilus; Antidorcas bondi; Antidorcas marsupialis; Antidorcas recki; Alcelaphus buselaphus; Australopithecus africanus cf.; Canis mesomelas; Cercopithecoides williamsi; Connochaetes gnou cf.; Connochaetes taurinus; Crocidura fuscomurina cf.; Crocuta ultra cf.; Cryptomys hottentotus; Cryptomys robertsi; Damaliscus lunatus; Damaliscus pygargus; Dasymys incomtus; Dendromus melanotis; Desmodillus auricularis; Diceros bicornis; Dinofelis barlowi; Dinofelis piveteaui cf.; Elephantulus myurus; 
Elephas; Eptesicus hottentotus; Equus burchellii; Equus capensis; Eurygnathohippus; Gerbilliscus; Grammomys; Hipposideros caffer; Hippotragus equinus; Hippotragus niger; Homo; Hystrix africaeaustralis; Kobus leche; Kolpochoerus paiceae?; Lemniscomys rosalia; 'Makapania' broomi; Mastomys; Megalotragus; Miniopterus natalensis; Mus minutoides; Myosorex robinsoni; Myotis tricolor; Myotomys sloggetti; Mystromys albicaudatus; Mystromys hausleitneri; Neoromicia capensis; Nyctereutes terblanchei cf.; Oreotragus oreotragus; Oryx gazella cf.; Otomys saundersiae; Pachycrocuta brevirostris; Otomys gracilis; Panthera leo; Panthera cf. pardus; Papio izodi cf.; Papio robinsoni cf.; Pelea capreolus; Phacochoerus antiquus cf.; Potamochoeroides hypsodon cf.; Potamochoerus larvatus; Procavia antiqua; Procavia capensis; Procavia transvaalensis; Proodontomys cookei; Raphicerus campestris; Redunca arundinum; Redunca darti; Redunca fulvorufula; Rhabdomys pumilio; Rhinolophus clivosus; Saccostomus campestris; Scotophilus nigrita; Steatomys; Stenodontomys; Suncus infinitesimus; Suncus varilla; Syncerus; Taurotragus oryx; Thallomys debruyni; Theropithecus oswaldi; Tragelaphus angasii; Tragelaphus strepsiceros. References: Avery (1995a); Berger (1992, 1993); Berger et al. (1993); Broom (1937a, 1948a); Churcher (1956); Cooke (1962); Freedman (1970); Lacruz et al. (2002, 2003); Meester (1961a); Reynolds (2010b).

Gobabis townlands (2227:1858). Taxa: Antidorcas bondi; Crocuta crocuta; Damaliscus; Equus; Megalotragus priscus cf.; Phacochoerus africanus. References: Jacobson (1978). Gondolin (2549:2750). Taxa: Antidorcas recki; Canis mesomelas; Ceratotherium simum; Connochaetes; Crocuta crocuta; Damaliscus niro?; Damaliscus pygargus; Equus capensis cf.; Eurygnathohippus cornelianus cf.; Hippopotamus; Hippotragus equinus; Hystrix makapanensis; Metridiochoerus andrewsi; Oreotragus oreotragus; Procavia antiqua; Procavia transvaalensis; Redunca arundinum; Taurotragus oryx; Tragelaphus angasii; Tragelaphus strepsiceros. References: Adams and Conroy (2005); Adams et al. (2007b); Kuykendall and Conroy (1999); Menter et al. (1999); Watson (1993b).

Gong-Gong (2824). Taxa: Equus capensis; Mammuthus subplanifrons; Megalotragus priscus. References: Cooke (1949a).

Groot Kloof (2411:2821). Taxa: Damaliscus; Equus; Kobus leche; Syncerus antiquus. References: Curnoe et al. (2006).

Haasgat (2551:2750). Taxa: Alcelaphus buselaphus; Antidorcas bondi; Antidorcas marsupialis; Australopithecus?; Canis; Cercopithecoides haasgati; Cercopithecoides williamsi; Chasmaporthetes nitidula?; Connochaetes gnou; Connochaetes taurinus; Damaliscus pygargus; Dinofelis cf.; Equus burchellii; Equus capensis; Equus quagga cf.; Giraffa camelopardalis; Hippotragus equinus; Homo?; Hystrix africaeaustralis; Kobus ellipsiprymnus; Kobus leche; Megalotragus; Metridiochoerus cf.; Oreotragus oreotragus; Ourebia ourebi; Papio izodi; Parapapio broomi; Pelea capreolus; Procavia antiqua; Procavia capensis; Procavia transvaalensis; Pronolagus rupestris; Raphicerus; Redunca arundinum; Redunca fulvorufula; Sylvicapra grimmia; Taurotragus oryx; Tragelaphus strepsiceros. References: Adams (2012b); Brophy et al. (2014); Keyser (1991); Keyser and Martini (1991); Leece et al. (2016); McKee and Keyser (1994); Plug and Keyser (1993); Reynolds (2010b).

Hadeco. See Minaar's Cave.

Halliwell's Workings (2824). Taxa: Damaliscus lunatus cf.; Connochaetes; Equus burchellii cf.; Equus capensis; Equus quagga cf.; Hippopotamus amphibius; Loxodonta; Megalotragus priscus; Metridiochoerus compactus; Syncerus antiquus. References: Cooke (1949a).

Hawston (3419AD). Taxa: Diceros bicornis; Loxodonta africana. References: Cooke (1955). Hennops River (2551:2756). Taxa: Mystromys hausleitneri. References: Broom (1948a). 
Herolds Bay (3315:2226). Taxa: Alcelaphus buselaphus; Antidorcas marsupialis; Aonyx capensis; Canis mesomelas; Caracal caracal; Connochaetes gnou; Damaliscus; Equus; Hippopotamus amphibius; Hippotragus leucophaeus; Hystrix africaeaustralis; Ictonyx striatus; Lepus capensis; Lycaon pictus; Mellivora capensis; Oreotragus oreotragus; Panthera leo; Papio ursinus; Parahyaena brunnea; Pelea capreolus; Potamochoerus larvatus; Procavia capensis; Raphicerus melanotis; Redunca arundinum; Redunca fulvorufula; Sylvicapra grimmia; Syncerus antiquus; Syncerus caffer; Taurotragus oryx. References: Brink and Deacon (1982).

Hettie 582 (2808:2658). Taxa: Antidorcas marsupialis; Connochaetes gnou; Equus burchellii; Megalotragus priscus; Phacochoerus africanus. References: De Ruiter et al. (2011).

Heuningneskrans (2436:3039). Taxa: Aepyceros melampus; Antidorcas bondi; Antidorcas marsupialis; Equus burchellii; Hippotragus; Homo sapiens; Hystrix africaeaustralis; Orycteropus afer; Panthera leo; Papio ursinus; Parahyaena brunnea; Pelea capreolus; Phacochoerus africanus; Procavia capensis; Redunca arundinum; Sylvicapra grimmia; Syncerus caffer; Tragelaphus strepsiceros. References: Klein (1984a).

Hoedjiesbaai (3317BD). Taxa: Canis mesomelas; Procavia capensis; Raphicerus campestris; Suricata?. References: Cooke (1955).

Hoedjiespunt 1 (3303:1758). Taxa: Acomys subspinosus; Antidorcas australis; Aonyx capensis; Atilax paludinosus; Bathyergus suillus; Canis mesomelas; Ceratotherium simum; Crocidura cyanea; Crocuta crocuta; Cryptomys hottentotus; Damaliscus pygargus; Elephantulus rupestris; Equus capensis; Equus quagga; Felis nigripes; Felis silvestris; Genetta tigrina; Gerbilliscus afra; Herpestes ichneumon; Homo rhodesiensis; Hystrix africaeaustralis; Ictonyx striatus; Lepus capensis; Lepus saxatilis; Lycaon pictus; Megalotragus priscus; Mellivora capensis; Micaelamys namaquensis; Myomyscus verreauxii; Myosorex varius; Myotomys sloggetti; Myotomys unisulcatus; Mystromys albicaudatus; Otomys irroratus; Otomys saundersiae; Panthera leo; Panthera pardus; Parahyaena brunnea; Parotomys brantsii; Pelea capreolus; Procavia capensis; Raphicerus; Redunca arundinum; Rhabdomys pumilio; Rhinolophus clivosus; Suricata suricatta; Syncerus antiquus; Syncerus caffer; Taurotragus oryx; Tragelaphus strepsiceros; Vulpes chama; Zelotomys woosnami. References: Berger and Parkington (1995); Brophy et al. (2014); Churchill et al. (2000a); Matthews et al. (2005, 2006); Stynder (1997).

Hofmeyr Cave (3125DB). Taxa: Homo sapiens. References: Dusseldorp et al. (2013); Grine et al. (2007).

Homestead Area (2824). Taxa: Aepyceros melampus; Equus capensis; Phacochoerus africanus. References: Cooke (1949a).

Hoogstede (3151:2607). Taxa: Equus burchellii; Megalotragus priscus cf. References: Cooke (1955).

Jack's Camp (2033:2512) Other names: SAAN-0036. Taxa: Hippopotamus; Hippotragus; Kobus ellipsiprymnus cf.; Syncerus antiquus; Tragelaphus. References: Berger and Brink (2001).

Jägersquelle (1925:1804). Taxa: Acomys; Crocidura; Cryptomys; Gerbillurus; Malacothrix; Micaelamys; Miniopterus; Mus; Mystromys; Panthera pardus; Parapapio; Rhinolophus; Steatomys; Zelotomys. References: Pickford and Senut (2010).

Jakkalsfontein (3123AC). Taxa: Equus burchellii; Equus capensis. References: Cooke (1955). Kalk Bay (3418AB). Taxa: Hippopotamus amphibius; Loxodonta africana. References: Cooke (1955).

Kalk Plateau (2417DB). Taxa: Equus burchellii. References: Cooke (1955). 
Kalkbank (2332:2921). Taxa: Aepyceros melampus; Alcelaphus buselaphus; Alcelaphus caama cf.; Antidorcas bondi; Antidorcas marsupialis; Connochaetes taurinus; Crocuta crocuta; Damaliscus lunatus cf.; Diceros bicornis; Equus burchellii; Equus capensis; Georychus capensis; Giraffa camelopardalis; Hippopotamus amphibius; Hystrix africaeaustralis; Ichneumia albicauda; Kobus ellipsiprymnus; Loxodonta africana; Lycaon pictus; Megalotragus priscus; Panthera leo; Panthera pardus; Phacochoerus africanus; Redunca fulvorufula; Syncerus antiquus; Taurotragus oryx; Vulpes chama cf. References: Brown (1988); Cooke (1962); Ewer (1958a, 1962); Hutson (2006); Hutson and Cain (2008); Wadley (2015).

Kalkoenkrans 225 (2810:2645). Taxa: Antidorcas marsupialis; Connochaetes gnou. References: De Ruiter et al. (2011).

Kathu Pan (2740:2301). Taxa: Alcelaphus; Antidorcas bondi; Antidorcas marsupialis; Ceratotherium simum; Elephas recki; Equus burchellii; Equus capensis; Giraffa camelopardalis; Hippopotamus amphibius; Hippotragus cf.; Megalotragus priscus; Orycteropus afer; Pedetes capensis; Pelea capreolus; Phacochoerus africanus; Redunca; Syncerus antiquus; Syncerus caffer; Tragelaphus. References: Klein (1988); Wadley (2015).

Keeble's Paddock (2824). Taxa: Connochaetes gnou cf.; Equus burchellii; Equus capensis; Hippopotamus amphibius; Hippotragus niger; 'Kobus' altidens; Phacochoerus. References: Cooke (1949a).

Klasies River (3406:2424). Taxa: Acomys subspinosus; Alcelaphus buselaphus cf.; Amblysomus hottentotus; Antidorcas australis; Aonyx capensis; Atilax paludinosus; Bathyergus suillus; Canis mesomelas; Caracal caracal cf.; Chlorotalpa duthiae; Connochaetes; Crocidura cyanea; Crocidura flavescens; Cryptomys hottentotus; Damaliscus niro; Damaliscus pygargus; Dasymys incomtus; Dendromus melanotis; Dendromus mesomelas; Diceros bicornis; Equus quagga; Felis silvestris; Galerella pulverulenta; Genetta; Georychus capensis; Grammomys dolichurus; Graphiurus ocularis; Herpestes ichneumon; Hippopotamus amphibius; Hippotragus leucophaeus; Homo sapiens; Hystrix africaeaustralis; Kobus; Lepus capensis; Loxodonta africana; Macroscelides proboscideus; Mastomys coucha; Megalotragus priscus; Mellivora capensis; Micaelamys namaquensis; Mus minutoides; Myomyscus verreauxii; Myosorex varius; Mystromys albicaudatus; Orycteropus afer; Otomys irroratus; Otomys laminatus; Otomys saundersiae; Ourebia ourebi; Panthera pardus; Papio ursinus; Parahyaena brunnea; Pelea capreolus; Phacochoerus africanus; Philantomba monticola; Potamochoerus larvatus; Procavia capensis; Raphicerus melanotis; Redunca arundinum; Redunca fulvorufula; Rhabdomys pumilio; Suncus infinitesimus; Sylvicapra grimmia; Syncerus antiquus; Syncerus caffer; Taurotragus oryx; Thallomys paedulcus; Tragelaphus scriptus; Tragelaphus strepsiceros. References: Avery (1987b, 1995b); Brophy et al. (2014); Grine et al. (1998); Henderson (1992); Klein (1975b, 1976a); McKee et al. (1995); Pearce (2008); Rightmire and Deacon (1991); Van Pletzen (2000); Wadley (2015).

Klipdrift Complex (3427:2044). Taxa: Alcelaphus buselaphus; Antidorcas marsupialis cf.; Bathyergus suillus; Connochaetes gnou; Damaliscus pygargus; Diceros bicornis; Equus; Lepus saxatilis; Oreotragus oreotragus; Ourebia ourebi; Parahyaena brunnea; Pelea capreolus; Procavia capensis; Raphicerus; Redunca arundinum; Redunca fulvorufula; Sylvicapra grimmia; Syncerus antiquus; Syncerus caffer; Taurotragus oryx. References: Harvati et al. (2015); Henshilwood et al. (2014); Reynard et al. (2016a, 2016b); Wadley (2015).

Koanaka Hills. See !Ncumtsa.

Koffiefontein (2925AC). Taxa: Equus capensis. References: Wells (1940). 
Kombat E900 (1942:1743). Taxa: Antidorcas; Procavia. References: Pickford and Senut (2010).

Kraanvogelvallei Breakwater (2824). Taxa: Hippopotamus amphibius. References: Cooke (1949a).

Kramleeg (3017:1724). Taxa: Pedetes capensis cf. References: Pickford and Mein (2011).

Kranskraal (2928AB). Taxa: Megalotragus priscus. References: Hoffman (1953).

Kromdraai (2600:2745). Taxa: Acomys; Arvicanthis; Canis antiquus; Canis atrox; Chrysospalax; Connochaetes taurinus cf.; Crocuta crocuta; Crocuta ultra; Crossarchus transvaalensis cf.; Cryptomys robertsi; Dasymys; Dendromus; Desmodillus; Elephantulus antiquus; Elephantulus brachyrhynchus; Elephantulus broomi; Eptesicus hottentotus cf.; Equus burchellii; Equus capensis; Equus quagga; Eurygnathohippus cornelianus; Felis crassidens; Gerbilliscus leucogaster cf.; Gorgopithecus major; Grammomys dolichurus cf.; Graphiurus; Hyaena hyaena; Lemniscomys; Macroscelides proboscideus; Malacothrix; Mastomys natalensis cf.; Mellivora; Metridiochoerus andrewsi; Metridiochoerus modestus; Micaelamys namaquensis cf.; Mus; Myosorex robinsoni; Myotis tricolor cf.; Myotis welwitschii cf.; Mystromys hausleitneri; Nyctereutes terblanchei; Otomys gracilis; Panthera leo; Papio izodi; Parahyaena brunnea; Paranthropus robustus; Parmularius parvus; Potamochoeroides hypsodon; Proamblysomus antiquus; Procavia transvaalensis; Proodontomys cookei; Rhabdomys pumilio cf.; Rhinolophus capensis cf.; Steatomys; Suncus varilla cf.; Taphozous; Thallomys paedulcus cf.; Theropithecus oswaldi; Vulpes pulcher; Zelotomys. References: Broom (1939c, 1940, 1948a); Broom and Robinson (1948); Brophy et al. (2014); Churcher (1970); De Graaff (1961b); De Ruiter (2004); De Ruiter et al. (2008b); Ewer (1958b); Freedman (1970); Freedman and Brain (1972); Gommery et al. (2008b); McCrae and Potze (2006); Pickford (2013b); Pocock (1985, 1987); Reynolds (2010b); Singleton et al. (2016); Thackeray et al. (2001, 2005); Turner (1986); Vrba (1981).

Larsen's Pits, Main Workings and Shaft 47 (2820:2444). Taxa: Alcelaphus robustus; Connochaetes; Damaliscus pygargus cf.; Equus burchellii; Equus capensis; Equus sandwithi; Hippopotamus amphibius; Metridiochoerus compactus; Phacochoerus africanus; Phacochoerus altidens; Syncerus antiquus; Syncerus caffer. References: Cooke (1949a, 1949b); Shaw and Cooke (1940).

LeRoux 717 (2811:2659). Taxa: Antidorcas marsupialis. References: De Ruiter et al. (2011).

Lesedi Chamber (2601:2743). Taxa: Homo naledi. References: Hawks et al. (2017).

Lincoln Cave (2627BA). Taxa: Antidorcas; Canis mesomelas; Damaliscus niro; Equus burchellii; Hippopotamus amphibius; Hippotragus; Homo erectus cf.; Hystrix cf.; Otocyon megalotis; Papio ursinus cf.; Pedetes capensis; Pelea capreolus; Phacochoerus africanus; Procavia capensis; Raphicerus; Suricata suricatta; Taurotragus oryx cf.; Tragelaphus scriptus cf.; Vulpes chama. References: Reynolds et al. (2007); Wadley (2015).

Linkerhandsgat (3419). Taxa: Alcelaphus caama cf.; Canis mesomelas; Crocuta. References: Cooke (1955).

Little England (3222BD). Taxa: Equus burchellii cf. References: Cooke (1955).

Longlands (2824). Taxa: Crocuta crocuta; Equus burchellii; Equus capensis; Hippopotamus amphibius; Metridiochoerus andrewsi; Notochoerus capensis. References: Broom (1925, 1928); Cooke (1949a).

Lower Kemp's Cave (2605:2742). Taxa: Aepyceros melampus; Alcelaphus buselaphus; Antidorcas marsupialis; Canis mesomelas; Connochaetes gnou; Connochaetes taurinus; Crocuta crocuta; Damaliscus pygargus; Equus burchellii; Equus capensis; Genetta genetta; Hippotragus niger cf.; Hystrix africaeaustralis; Leptailurus serval; Lepus saxatilis; Panthera 
pardus; Papio ursinus; Parahyaena brunnea; Pedetes capensis; Pelea capreolus; Phacochoerus africanus; Potamochoerus larvatus; Procavia capensis; Pronolagus; Raphicerus campestris; Redunca arundinum; Taurotragus oryx; Tragelaphus strepsiceros; Vulpes chama. References: Swanepoel (2003).

Luleche (2550:2751). Taxa: Oreotragus oreotragus; Redunca; Tragelaphus angasii. References: Adams et al. (2007a).

Mahemspan (2746:2609). Taxa: Alcelaphus buselaphus; Antidorcas bondi; Antidorcas marsupialis; Connochaetes gnou; Connochaetes taurinus; Crocuta crocuta; Damaliscus niro; Damaliscus pygargus; Equus capensis; Equus quagga cf.; Hippopotamus amphibius; Hippotragus; Hystrix africaeaustralis; Kobus leche; Megalotragus priscus; Panthera leo; Phacochoerus africanus; Syncerus antiquus; Taurotragus oryx. References: Brink (1993, 2005); Brophy et al. (2014); Hoffman (1953); Van Hoepen (1947).

Malapa (2553:2748). Taxa: Atilax mesotes; Australopithecus sediba; Cynictis cf.; Dinofelis barlowi; Elephantulus; Equus; Felis nigripes; Felis silvestris; Genetta; Lepus; Lycaon; Megantereon whitei; Mungos; Oreotragus; Panthera pardus; Papio; Parahyaena brunnea; Rhynchogale cf.; Tragelaphus scriptus cf.; Tragelaphus strepsiceros cf.; Vulpes chama; Vulpes skinneri. References: Dirks et al. (2010); Kuhn et al. (2011, 2016); Val et al. (2011, 2014).

Meerholtzkop (2727BB). Taxa: Megalotragus priscus. References: Hoffman (1953).

Melkbos (3342:1824). Taxa: Antidorcas australis; Bathyergus suillus; Canis mesomelas; Ceratotherium simum; Connochaetes; Diceros bicornis; Equus capensis; Hippopotamus amphibius; Loxodonta africana; Panthera leo; Parahyaena brunnea; Raphicerus; Redunca arundinum; Syncerus; Taurotragus oryx; Tragelaphus strepsiceros. References: Hendey (1968, 1969); Klein (1974b).

Mimosa 559 (2827:2638). Taxa: Antidorcas bondi; Antidorcas marsupialis; Connochaetes gnou; Damaliscus pygargus; Equus burchellii; Equus capensis; Giraffa camelopardalis; Hippotragus niger; Hystrix africaeaustralis; Kobus ellipsiprymnus; Kobus leche; Megalotragus priscus; Phacochoerus africanus. References: De Ruiter et al. (2011).

Minaar's Cave (2559:2746). Other names: SAAN-0004; Hadeco. Taxa: Canis mesomelas; Crocuta crocuta; Equus capensis; Hystrix africaeaustralis; Megalotragus; Oreotragus oreotragus; Panthera pardus; Papio izodi cf.; Procavia antiqua; Tragelaphus strepsiceros. References: Brain (1981); Berger and Brink, (2001); Churcher (1970); Freedman (1970); Gommery et al. (2012b).

Mitasrust Farm (2826). Taxa: Connochaetes antiquus cf.; Damaliscus pygargus; Equus burchellii; Equus capensis cf.; Megalotragus priscus; Phacochoerus africanus; Syncerus antiquus. References: Rossouw (2006).

Mockesdam (2928AB). Taxa: Megalotragus priscus. References: Hoffman (1953).

Modder River (2825). Megalotragus priscus; Syncerus antiquus. References: Cooke (1955).

Morris Draai (2824). Taxa: Equus capensis cf.; Hippopotamus amphibius; Hippotragus niger cf.; Tragelaphus strepsiceros. References: Cooke (1949a).

Motsetse (2554:2749). Taxa: Antidorcas; Canis mesomelas; Connochaetes taurinus; Crocuta crocuta; Damaliscus; Dinofelis piveteaui; Equus; Genetta; Oreotragus oreotragus cf.; Pelea capreolus cf.; Procavia capensis; Redunca; Tragelaphus strepsiceros. References: Berger and Lacruz (2003); Reynolds (2010b).

Namib IV (2347:1520). Taxa: Elephas recki; Equus burchellii cf.; Syncerus caffer cf. References: Klein (1988); Shackley $(1980,1985)$.

Nelson Bay Cave (3406:2322). Taxa: Alcelaphus caama cf.; Antidorcas marsupialis cf.; Aonyx capensis; Atilax paludinosus; Canis mesomelas; Caracal caracal; Connochaetes gnou cf.; 
Damaliscus pygargus; Equus capensis; Felis silvestris; Galerella pulverulenta; Herpestes ichneumon; Hippopotamus amphibius; Hippotragus; Homo sapiens; Hystrix africaeaustralis; Lepus capensis; Megalotragus; Mellivora capensis; Orycteropus afer; Ourebia ourebi; Panthera pardus; Papio ursinus; Parahyaena brunnea; Pelea capreolus; Phacochoerus africanus; Philantomba monticola; Potamochoerus larvatus; Procavia capensis; Raphicerus; Redunca arundinum; Redunca fulvorufula; Smutsia temminckii cf.; Sylvicapra grimmia; Syncerus caffer; Taurotragus oryx; Tragelaphus scriptus. References: Brophy et al. (2014); Klein (1972, 1974b); Pearce (2008).

Niekerk's Rush (2824). Taxa: Equus burchellii. References: Cooke (1949a).

Nooitgedacht (2830). Taxa: Homo sapiens. References: Morris et al. (1995).

Nooitgedacht (3419). Taxa: Aepyceros melampus; Redunca arundinum. References: Cooke (1955).

Nos (2530:1530). Taxa: Antidorcas marsupialis; Canis; Procavia. References: Thackeray (1979).

Nosib (1925:1748). Taxa: Crocidura; Cryptomys; Dasymys; Dendromus; Gerbilliscus; Gerbillurus; Graphiurus; Malacothrix; Mastomys; Mus; Mystromys; Rhabdomys; Rhinolophus; Steatomys; Stenodontomys; Zelotomys. References: Pickford and Senut (2010).

Nxazini Pans (2005:2522). Other names: SAAN-0034, 0035. Taxa: Antidorcas bondi; Connochaetes taurinus cf.; Damaliscus; Elephas; Equus burchellii; Equus capensis cf.; Hippopotamus; Metridiochoerus; Phacochoerus; Procavia; Sylvicapra cf.; Syncerus; Tragelaphus scriptus. References: Berger and Brink (2001).

Old Pont Site (2824). Taxa: Elephas iolensis; Equus burchellii; Hippopotamus amphibius; Tragelaphus strepsiceros. References: Cooke (1949a).

Ondera (1839:1339). Taxa: Procavia. References: Pickford and Senut (2010); Pickford et al. (1993).

Ongers River (2957:2311). Taxa: Megalotragus priscus. References: Brink et al. (1995). Oranjemund (2833:1624). Taxa: Homo sapiens. References: Senut et al. (2000). Otjiseva (2216BD). Taxa: Homo sapiens. References: De Villiers (1972a); Sydow (1969). Peers Cave (3407:1825). Taxa: Equus capensis; Equus zebra; Homo sapiens. References: Cooke (1955); Pfeiffer (2013).

Pinnacle Point (3422AA). Taxa: Alcelaphus buselaphus; Antidorcas marsupialis; Bathyergus suillus; Canis mesomelas; Caracal caracal; Connochaetes gnou; Crocidura cyanea; Crocidura flavescens; Cryptomys hottentotus; Damaliscus niro; Damaliscus pygargus; Dendromus mystacalis; Diceros bicornis; Elephantulus; Elephantulus edwardii; Equus capensis cf.; Equus quagga cf.; Felis silvestris; Galerella pulverulenta; Genetta; Georychus capensis; Gerbilliscus afra; Grammomys dolichurus; Hippotragus leucophaeus; Homo sapiens; Hystrix africaeaustralis; Ictonyx striatus; Lepus capensis; Megalotragus priscus; Mellivora capensis; Mus minutoides; Myomyscus verreauxii; Myosorex varius; Myotis; Mystromys albicaudatus; Oreotragus oreotragus; Otocyon megalotis; Otomys irroratus; Otomys saundersiae; Panthera leo; Panthera pardus; Papio ursinus; Parahyaena brunnea; Pedetes capensis; Pelea capreolus; Phacochoerus africanus; Procavia capensis; Raphicerus campestris; Raphicerus melanotis; Redunca arundinum; Redunca fulvorufula; Rhabdomys pumilio; Rhinolophus clivosus; Saccostomus campestris; Steatomys krebsii; Suncus infinitesimus; Suncus varilla; Syncerus antiquus; Syncerus caffer; Taurotragus oryx; Tragelaphus scriptus cf.; Vulpes chama; Zelotomys. References: Armstrong (2016); Brophy et al. (2014); Marean et al. (2004); Matthews et al. (2009, 2011); McGrath et al. (2015); Rector and Reed (2010); Wadley (2015). 
Plovers Lake (2559:2747). Taxa: Aethomys chrysophilus; Alcelaphus buselaphus; Antidorcas bondi; Antidorcas marsupialis; Aonyx capensis; Atilax paludinosus; Canis mesomelas; Chlorocebus aethiops; Connochaetes gnou; Connochaetes taurinus; Crocidura silacea; Crocuta crocuta cf.; Cryptomys hottentotus; Cynictis penicillata cf.; Damaliscus niro; Damaliscus pygargus; Dendromus melanotis; Elephantulus; Equus burchellii; Equus capensis; Felis silvestris; Galerella sanguinea cf.; Genetta tigrina; Gerbilliscus; Hippopotamus; Hippotragus; Homo sapiens; Hystrix africaeaustralis; Ictonyx striatus; Kobus ellipsiprymnus; Lepus; Lycaon pictus; Mastomys natalensis; Megalotragus priscus; Mellivora capensis; Metridiochoerus andrewsi; Micaelamys namaquensis cf.; Mungos mungo cf.; Mus minutoides; Mystromys albicaudatus; Otocyon megalotis; Otomys irroratus; Panthera leo cf.; Panthera pardus cf.; Papio robinsoni; Papio ursinus; Parahyaena brunnea; Pelea capreolus; Phacochoerus africanus; Procavia capensis; Procavia transvaalensis; Raphicerus campestris; Redunca arundinum; Redunca fulvorufula; Steatomys pratensis; Suricata suricatta; Syncerus caffer; Taurotragus oryx; Tragelaphus strepsiceros; Vulpes chama; Zelotomys woosnami. References: Brophy et al. (2006, 2014); De Ruiter et al. (2008a); McKee et al. (1995); Reynolds (2010b); Thackeray and Watson (1994); Wadley (2015).

Pneil (2824BD). Taxa: Connochaetes gnou; Damaliscus niro; Damaliscus pygargus; Elephas iolensis; Equus burchellii; Equus capensis; Hippopotamus amphibius; Kobus leche cf.; Kolpochoerus paiceae; Loxodonta africana; Megalotragus priscus; Phacochoerus africanus; Phacochoerus altidens; Syncerus caffer; Taurotragus. References: Broom (1928); Cooke (1939); Cooke and Wells (1946); Hoffman (1953); Hutson (2016); Shaw and Cooke (1940).

Pockenbank (2713:1631). Taxa: Canis; Equus zebra cf.; Lepus; Oreotragus oreotragus; Oryx gazella; Procavia capensis. References: Thackeray (1979); Wadley (2015).

Pomongwe (2032:2830). Taxa: Connochaetes taurinus cf.; Damaliscus lunatus cf.; Equus burchellii; Equus capensis; Hippotragus equinus; Hippotragus niger; Hystrix africaeaustralis; Kobus ellipsiprymnus; Lepus; Mellivora capensis; Oreotragus oreotragus; Panthera pardus; Pedetes capensis; Phacochoerus africanus; Potamochoerus larvatus; Procavia capensis; Raphicerus campestris; Raphicerus sharpei cf.; Redunca arundinum; Taurotragus oryx; Tragelaphus scriptus; Tragelaphus strepsiceros. References: Brain (1981); Wadley (2015).

Power's Site (2832:2428DA). Taxa: Alcelaphus caama cf.; Antidorcas bondi; Antidorcas recki; Ceratotherium simum; Connochaetes; Damaliscus pygargus cf.; Elephas iolensis; Elephas recki; Equus burchellii; Equus capensis; Equus quagga cf.; Equus sandwithi cf.; Hippopotamus amphibius; Loxodonta; Megalotragus priscus; Metridiochoerus andrewsi; Metridiochoerus compactus; Phacochoerus africanus; Phacochoerus altidens; Redunca arundinum; Sylvicapra grimmia; Syncerus antiquus; Syncerus caffer cf.; Taurotragus oryx. References: Cooke (1949a); Klein (1988); Power (1955).

Pretoria (2528). Taxa: Papio spelaeus. References: Broom (1936b); Freedman (1970).

Prieska (2922DA). Taxa: Megalotragus priscus. References: Hoffman (1953).

Putslaagte 8 (3218). Taxa: Bathyergus suillus; Caracal caracal; Equus; Hippotragus leucophaeus; Hystrix africaeaustralis; Oreotragus oreotragus; Procavia capensis; Raphicerus; Redunca; Sylvicapra grimmia; Syncerus caffer. References: Mackay et al. (2015).

Ravenscraig (3000:2747). Taxa: Equus; Oreotragus oreotragus; Pelea capreolus. References: Plug (1997b).

Redcliff (1901:2946). Taxa: Aepyceros melampus; Alcelaphus cf.; Antidorcas bondi; Antidorcas marsupialis; Aonyx; Atelerix frontalis; Canis mesomelas; Ceratotherium simum; Connochaetes taurinus; Crocuta crocuta; Damaliscus pygargus; Dendrohyrax arboreus; Equus 
burchellii; Equus capensis; Felis silvestris; Galerella sanguinea; Giraffa camelopardalis; Hippopotamus amphibius; Hippotragus; Homo sapiens; Hystrix africaeaustralis; Ichneumia albicauda; Kobus ellipsiprymnus; Kobus leche; Lepus; Loxodonta africana; Lycaon pictus; Megalotragus priscus; Mellivora capensis; Metridiochoerus; Oreotragus oreotragus; Orycteropus afer; Ourebia ourebi; Panthera leo; Panthera pardus; Papio ursinus; Parahyaena brunnea; Pedetes capensis; Pelea capreolus; Phacochoerus africanus; Procavia capensis; Raphicerus; Redunca arundinum; Redunca fulvorufula; Smutsia temminckii; Sylvicapra grimmia; Syncerus antiquus; Syncerus caffer; Taurotragus oryx; Tragelaphus strepsiceros; Vulpes chama. References: Cruz-Uribe (1983); Klein (1978b); Wadley (2015).

Reunion Rocks (2930DD). Taxa: Loxodonta africana. References: Ramsay et al. (1993). Riet River (2614:2948). Taxa: Connochaetes antiquus; Equus quagga; Homo; Kobus leche; Megalotragus cf.; Parahyaena brunnea; Phacochoerus. References: Berger and Brink (1996).

Rietfontein (1942:1753). Taxa: Antidorcas; Oryx; Panthera pardus; Procavia. References: Pickford and Senut (2010).

River-bed above the Island (2824). Taxa: Equus capensis; Hippopotamus amphibius; Loxodonta africana cf.; Syncerus caffer. References: Cooke (1949a).

River-bed below the Island (2824). Taxa: Elephas iolensis; Hippopotamus amphibius. References: Cooke (1949a).

Riverton (2831:2442). Taxa: Equus burchellii; Equus capensis; Hippopotamus amphibius; Phacochoerus africanus. References: Cooke (1949a).

Rocky II and III (1748:1342). Taxa: Crocidura; Desmodillus; Elephantulus; Gerbilliscus; Graphiurus; Lepus; Malacothrix; Mastomys; Mus; Myotis; Nycteris; Pedetes; Petromus; Petromyscus; Procavia; Rhabdomys; Rhinolophus; Saccostomus; Steatomys; Thallomys; Thryonomys; Zelotomys. References: Pickford and Senut (2010); Pickford et al. (1993).

Rose Cottage Cave (2913:2728). Taxa: Aepyceros melampus cf.; Alcelaphus buselaphus; Antidorcas bondi; Antidorcas marsupialis; Aonyx capensis cf.; Cephalophus natalensis; Connochaetes gnou; Connochaetes taurinus; Cricetomys ansorgei cf.; Cryptomys hottentotus; Damaliscus pygargus; Equus burchellii; Equus capensis; Giraffa camelopardalis cf.; Herpestes; Hippotragus leucophaeus cf.; Homo sapiens; Hystrix africaeaustralis; Lepus; Mastomys natalensis; Megalotragus priscus; Papio ursinus; Pedetes capensis; Pelea capreolus cf.; Phacochoerus africanus; Philantomba monticola; Potamochoerus larvatus; Procavia capensis; Redunca fulvorufula; Smutsia temminckii; Sylvicapra grimmia cf.; Syncerus antiquus cf.; Syncerus caffer; Taurotragus oryx; Tragelaphus scriptus; Tragelaphus strepsiceros. References: Avery (1997b); Brophy et al. (2014); Plug (1997b); Plug and Engela (1992); Welbourne (1988); Wells (2006).

SAAN-0005 (2559:2746). Taxa: Equus capensis; Megalotragus; Oreotragus oreotragus; Procavia capensis. References: Berger and Brink (2001).

SAAN-0034, 0035. See Nxazini Pans.

SAAN-0036. See Jack's Camp.

SAAN-004. See Minaar's Cave.

SAAN-0042, 0043. See Thomeng.

Saldanha Bay (3300:1757). Taxa: Equus capensis. References: Cooke (1955); Freedman (1970).

Saldanha Bay Yacht Club (3317BB). Taxa: Bathyergus suillus; Chrysochloris asiatica; Crocidura cyanea; Crocidura flavescens; Cryptomys hottentotus; Dendromus melanotis; Dendromus mesomelas; Elephantulus edwardii; Gerbilliscus afra; Gerbillurus paeba; Myosorex varius; Myotomys unisulcatus; Mystromys albicaudatus; Otomys irroratus; Otomys 
saundersiae; Rhabdomys pumilio; Rhinolophus clivosus; Steatomys krebsii; Suncus varilla. References: Manthi (2002); Matthews et al. (2007).

Sand River (2826AA). Taxa: Megalotragus priscus. References: Hoffman (1953); Van Hoepen (1947).

Schmidt's Drift (2824DA). Taxa: Equus capensis. References: Cooke (1949a).

Schoolplaats No. 1 (2824BB). Taxa: Equus capensis. References: Cooke (1949a).

Sea Harvest (3301:1757). Taxa: Acinonyx jubatus; Antidorcas australis cf.; Aonyx capensis; Bathyergus suillus; Canis mesomelas; Ceratotherium simum; Crocuta crocuta; Damaliscus pygargus; Equus capensis; Equus quagga; Felis silvestris; Galerella pulverulenta; Genetta tigrina cf.; Herpestes ichneumon; Hippopotamus amphibius; Hippotragus leucophaeus; Homo sapiens; Hystrix africaeaustralis; Ictonyx striatus; Leptailurus serval cf.; Leptis capensis; Lepus saxatilis; Loxodonta africana; Lycaon pictus; Mellivora capensis; Oreotragus oreotragus; Orycteropus afer; Panthera leo; Panthera pardus; Papio ursinus; Parahyaena brunnea; Pelea capreolus; Phacochoerus africanus; Procavia capensis; Raphicerus campestris; Raphicerus melanotis; Redunca arundinum; Sylvicapra grimmia; Taurotragus oryx; Tragelaphus strepsiceros. References: Brophy et al. (2014); Grine and Klein (1993).

Sehonghong (2946:2847). Taxa: Alcelaphus buselaphus; Antidorcas marsupialis; Canis mesomelas; Caracal caracal; Chlorocebus pygerythrus; Connochaetes gnou; Cryptomys hottentotus; Damaliscus pygargus; Equus capensis; Equus quagga; Hippotragus equinus; Hippotragus leucophaeus; Hystrix africaeaustralis; Ictonyx striatus; Lepus saxatilis; Megalotragus priscus; Oreotragus oreotragus; Panthera leo; Panthera pardus; Papio ursinus; Pelea capreolus; Phacochoerus africanus; Procavia capensis; Raphicerus campestris; Redunca fulvorufula; Sylvicapra grimmia; Taurotragus oryx. References: Horsburgh et al. (2016); Plug and Mitchell (2008); Wadley (2015).

Sheppard Island (2725DA). Other names: Bloemhof site. Taxa: Elephas iolensis; Equus burchellii; Equus capensis; Equus sandwithi; Hippopotamus amphibius; Megalotragus priscus; Phacochoerus africanus cf.; Syncerus antiquus; Syncerus caffer; Tragelaphus?. References: Cooke (1949a, 1955).

Sibudu (2931:3105). Taxa: Aepyceros melampus; Alcelaphus buselaphus; Aonyx capensis cf.; Atilax paludinosus; Cephalophus natalensis; Cercopithecus albogularis; Chlorocebus aethiops; Chlorocebus pygerythrus; Connochaetes taurinus; Cricetomys ansorgei; Cryptomys hottentotus; Damaliscus pygargus; Elephantulus myurus; Equus burchellii cf.; Equus capensis cf.; Equus quagga; Felis silvestris; Galerella sanguinea; Genetta tigrina cf.; Georychus capensis; Giraffa camelopardalis; Hippopotamus amphibius; Hippotragus equinus; Homo sapiens; Hystrix africaeaustralis cf.; Ictonyx striatus cf.; Kobus ellipsiprymnus; Lepus saxatilis; Loxodonta africana; Mastomys natalensis; Megalotragus priscus; Myosorex varius; Oreotragus oreotragus; Orycteropus afer; Otomys angoniensis; Otomys irroratus; Otomys laminatus; Panthera leo; Papio ursinus; Parahyaena brunnea; Pelea capreolus; Phacochoerus africanus; Philantomba monticola; Potamochoerus larvatus; Procavia capensis; Pronolagus crassicaudatus; Pronolagus rupestris; Raphicerus campestris; Redunca arundinum; Redunca fulvorufula; Rhabdomys pumilio; Rhinolophus clivosus; Smutsia temminckii; Sylvicapra grimmia; Syncerus antiquus; Syncerus caffer; Taurotragus oryx; Thryonomys swinderianus; Tragelaphus scriptus; Tragelaphus strepsiceros. References: Cain (2006); Clark and Plug (2008); Glenny (2006); Le Roux and Badenhorst (2016); Plug (1997d, 2004); Wadley et al. (2008); Wells (2006).

Silberberg Grotto (2627). Taxa: Acinonyx jubatus; Australopithecus; Homotherium crenatidens; Lycyaenops silberbergi; Megantereon whitei; Panthera pardus. References: Reynolds (2010b). 
SK400 (2941:1704). Other names: Springbok Midden. Taxa: Antidorcas marsupialis; Canis. References: Dewar (2007); Dewar et al. (2006).

Spitzkloof A Rockshelter (2852:1705). Taxa: Alcelaphus buselaphus; Antidorcas marsupialis; Bathyergus janetta; Canis mesomelas; Lepus capensis; Mus; Oreotragus oreotragus; Oryx gazella; Parotomys brantsii; Procavia capensis; Raphicerus campestris; Sylvicapra grimmia. References: Dewar and Stewart (2012, 2016, 2017); Wadley (2015).

Spitskop B (2827:2748). Taxa: Antidorcas bondi; Antidorcas marsupialis; Connochaetes gnou; Connochaetes taurinus; Damaliscus niro; Damaliscus pygargus; Equus capensis; Equus quagga cf.; Hippotragus; Megalotragus priscus; Phacochoerus africanus; Syncerus antiquus; Taurotragus oryx. References: Brink (2005).

Spreeuwal (3318AA). Taxa: Bathyergus suillus; Connochaetes gnou; Damaliscus pygargus; Diceros bicornis; Equus capensis; Equus quagga; Gerbillurus paeba; Hippotragus leucophaeus; Lepus saxatilis; Megalotragus priscus; Oryx gazella; Otomys saundersiae; Parahyaena brunnea; Raphicerus campestris; Redunca arundinum; Syncerus antiquus; Taurotragus oryx. References: Avery (1997); Brophy et al. (2014); Klein et al. (2007).

Springbok Flats. See Tuinplaas.

Springbok Midden. See SK400.

Sterkfontein (2601:2744). Taxa: Acomys spinosissimus cf.; Aepyceros; Aethomys chrysophilus; Amblysomus; Antidorcas bondi; Antidorcas marsupialis; Antidorcas recki; Arvicanthis?; Australopithecus africanus; Canis antiquus; Canis mesomelas; Cercopithecoides williamsi cf.; Chasmaporthetes nitidula; Chlorotalpa sclateri; Chlorotalpa spelea; Chrysospalax villosus; Connochaetes gnou cf.; Connochaetes taurinus; Crocidura silacea cf.; Crocuta crocuta; Cryptomys hottentotus; Cryptomys robertsi; Damaliscus pygargus cf.; Dasymys 'broomi'; Dasymys incomtus cf.; Dendromus melanotis; Dendromus mesomelas cf.; Dinofelis barlowi; Elephantulus antiquus; Elephantulus brachyrhynchus cf.; Elephantulus broomi; Elephantulus fuscus; Elephantulus intufi; Elephas recki; Equus burchellii; Equus capensis;

Eurygnathohippus cornelianus; Gazella?; Georychus capensis; Gerbilliscus brantsii cf.; Gerbilliscus leucogaster cf.; Graphiurus; Herpestes ichneumon; Hippopotamus amphibius; Hippotragus cookei; Hippotragus equinus; Hippotragus niger; Homo erectus; Homo gautengensis; Homotherium crenatidens; Hystrix africaeaustralis; Hystrix cristata?; Lepus capensis; Lycyaenops silberbergi; Macroscelides proboscideus; 'Makapania' broomi cf.; Malacothrix typica; Mastomys natalensis sl.; Megalotragus cf.; Megantereon gracile; Megantereon whitei; Metridiochoerus modestus; Micaelamys namaquensis cf.; Miniopterus natalensis; Mungos mungo cf.; Mus minutoides cf.; Mylomygale spiersi?; Myosorex cafer; Myosorex robinsoni; Myotis tricolor; Myotomys sloggetti; Mystromys albicaudatus; Mystromys hausleitneri; Oreotragus oreotragus; Otocyon megalotis; Otomys gracilis; Otomys irroratus; Otomys saundersiae; Pachycrocuta brevirostris; Panthera leo; Panthera pardus; Papio izodi; Papio robinsoni; Papio ursinus; Parahyaena brunnea; Paranthropus robustus; Parapapio broomi; Parapapio jonesi; Parapapio whitei; Pedetes capensis; Pelea capreolus; Pelomys fallax cf.?; Phacochoerus africanus; Phacochoerus antiquus; Proamblysomus antiquus; Procavia antiqua; Procavia capensis; Procavia transvaalensis; Pronolagus; Proodontomys cookei; Proteles; Raphicerus campestris; Redunca darti; Rhabdomys pumilio; Rhinolophus capensis cf.; Rhinolophus clivosus cf.; Rhinolophus darlingi cf.; Saccostomus campestris; Steatomys pratensis; Suncus varilla; Suricata suricatta; Sylvicapra grimmia; Syncerus; Thallomys paedulcus cf.; Theropithecus oswaldi; Tragelaphus angasii aff.; Tragelaphus scriptus cf.; Tragelaphus strepsiceros; Vulpes chama; Zelotomys woosnami cf. References: Avery (2000, 2001); Berger et al. (2002); Berger and Tobias (1994); Broom (1937a, 1937b, 1939c, 
1940, 1948a); Broom and Robinson (1949a); Brophy et al. (2014); Churcher (1970); Cooke (1947); De Graaff (1961c); De Ruiter (2004); De Ruiter et al. (2008b); Ewer (1958b); Freedman (1970); Kibii (2006); Lavocat (1957); McKee (1991); McKee et al. (1995); Moggi-Cecchi et al. (1998, 2006); Ogola (2009); Pocock (1969, 1987); O’Regan (2007); Reynolds (2010b); Reynolds and Kibii (2011); Reynolds et al. (2003, 2007); Turner (1986, 1987a, 1997); Val and Stratford (2015); Vrba (1974a).

Strathalan B (3059:2823). Taxa: Antidorcas marsupialis; Connochaetes gnou; Damaliscus pygargus; Equus; Hippotragus; Oreotragus oreotragus; Pelea capreolus; Phacochoerus africanus; Procavia capensis; Taurotragus oryx. References: Opperman (1992, 1996); Wadley (2015).

Sunnyside Pan (2839:2609). Taxa: Connochaetes gnou; Connochaetes taurinus; Crocuta crocuta; Damaliscus niro. References: Brink (2005).

Swart Duinen (3000:1702). Taxa: Bathyergus; Equus; Gerbillurus; Giraffa; Mastomys; Mystromys; Parotomys; Petromus; Pronolagus; Raphicerus campestris; Rhabdomys; Suricata suricatta. References: Pickford and Senut (1997).

Swartklip (3405:1841). Other names: Zwartklip. Taxa: Antidorcas australis; Aonyx capensis; Atilax paludinosus; Bathyergus suillus; Canis mesomelas; Ceratotherium simum; Connochaetes gnou; Damaliscus; Equus capensis; Equus quagga; Felis silvestris; Herpestes ichneumon; Hippopotamus amphibius; Hippotragus leucophaeus; Ictonyx striatus; Leptailurus serval; Lycaon pictus; Mellivora capensis; Panthera leo; Panthera pardus; Parahyaena brunnea; Pelea capreolus; Raphicerus melanotis; Redunca arundinum; Suricata suricatta; Syncerus antiquus; Taurotragus oryx; Tragelaphus strepsiceros; Vulpes chama. References: Brophy et al. (2014); Cruz-Uribe (1991); Ewer (1958c); Hendey (1968, 1969); Klein (1974b, 1975a); Klein and Cruz-Uribe (1991); Singer and Fuller (1962).

Swartkrans (2602:2743). Taxa: Acinonyx jubatus; Acomys spinosissimus; Aepyceros melampus; Aethomys chrysophilus cf.; Antidorcas australis; Antidorcas bondi; Aonyx capensis; Atilax mesotes; Canis mesomelas; Caracal caracal; Cercopithecoides williamsi; Chasmaporthetes nitidula; Chrysospalax villosus cf.; Connochaetes taurinus cf.; Crocuta crocuta; Cryptomys hottentotus; Cryptomys robertsi; Cynictis penicillata; Damaliscus niro?; Damaliscus pygargus cf.; Dasymys; Dendromus nyikae?; Dinofelis; Dinopithecus ingens; Elephantulus broomi; Elephantulus fuscus; Elephas; Eptesicus hottentotus cf.; Equus burchellii; Equus capensis; Equus quagga; Eurygnathohippus cornelianus; Felis silvestris; Galerella sanguinea; Gazella; Genetta tigrina; Gerbilliscus; Graphiurus; Herpestes ichneumon; Hippopotamus; Beatragus cf.; Hippotragus gigas cf.; Hippotragus niger cf.; Homo gautengensis; Homotherium?; Hyaena hyaena; Hystrix africaeaustralis; Kobus leche cf.; Leptailurus serval; Macroscelides proboscideus cf.; 'Makapania'; Malacothrix typica; Mastomys coucha; Megalotragus; Megantereon whitei; Mellivora capensis; Metridiochoerus andrewsi; Micaelamys namaquensis cf.; Mus; Myosorex cafer cf.; Myosorex robinsoni; Myotis tricolor; Mystromys albicaudatus; Neamblysomus gunningi cf.; Numidocapra porrocornutus; Oreotragus oreotragus; Orycteropus afer; Otocyon recki; Otomys gracilis; Otomys saundersiae cf.; Panthera leo; Panthera pardus; Papio robinsoni; Parahyaena brunnea; Paranthropus robustus; Parapapio broomi; Parapapio jonesi; Parapapio whitei; Pedetes; Pelea capreolus; Phacochoerus antiquus; Procavia transvaalensis; Proodontomys cookei; Proteles transvaalensis; Raphicerus campestris; Redunca arundinum cf.; Redunca darti; Rhabdomys pumilio; Rhinolophus blasii cf.; Rhinolophus capensis cf.; Smutsia; Steatomys pratensis; Suncus varilla; Suricata suricatta; Syncerus; Taurotragus oryx; Thallomys; Theropithecus 
oswaldi; Tragelaphus angasii aff.; Tragelaphus scriptus; Tragelaphus strepsiceros; Vulpes pulcher. References: Avery (1995b, 1998, 2001); Brain (1970, 1976); Brain et al. (1974, 1988); Brain and Watson (1992); Brophy et al. (2014); Churcher (1970); De Ruiter (2003); De Ruiter et al. (2006); De Ruiter et al. (2008b); Ewer (1958b); Freedman (1970); Gommery (2008); Grine (1989, 1993, 2005); Grine and Daegling (1993); Grine and Strait (1994); Grine and Susman (1991); Hendey (1974b); McKee et al. (1995); Pickering et al. (2012); Reynolds (2010b); Susman and De Ruiter (2004); Stynder (1997); Susman et al. (2001); Turner (1993); Watson (1993a).

Swartlintjies 1 (3017). Taxa: Cryptomys; Equus; Homo; Loxodonta; Pronolagus; Sivatherium maurisium. References: Pickford and Senut (1997).

Sydney-on-Vaal (2827:2419). Taxa: Damaliscus; Elephas iolensis; Elephas recki; Equus burchellii cf.; Hippopotamus amphibius; Loxodonta africana; Mammuthus subplanifrons. References: Cooke (1949a); Dart (1929b); Osborn (1942); Wells (1964).

The Bend (2824DA). Taxa: Equus capensis. References: Broom (1928); Cooke (1949a). Thomeng (2742:2436). Other names: SAAN-0042, 0043. Taxa: Hystrix; Lepus; Oreotragus; Procavia. References: Berger and Brink (2001).

Tierfontein (2826AA). Taxa: Sivatherium maurisium. References: Singer and Boné (1960). Tim's Cave (1747:1359). Taxa: Lepus; Procavia. References: Pickford and Senut (2010). Tloutle (2928:2746). Taxa: Damaliscus pygargus; Equus burchellii; Hystrix africaeaustralis; Procavia capensis; Redunca fulvorufula. References: Mitchell (1993); Plug (1993d, 1997b).

Tobias Cave (2737:2437). Taxa: Alcelaphus buselaphus; Antidorcas marsupialis; Canis mesomelas; Caracal caracal; Connochaetes gnou; Crocuta crocuta; Damaliscus pygargus; Diceros bicornis; Equus burchellii; Equus capensis; Homo sapiens; Hystrix africaeaustralis; Oreotragus oreotragus; Otocyon megalotis; Panthera leo; Panthera pardus; Papio ursinus; Parahyaena brunnea; Pelea capreolus; Phacochoerus africanus; Procavia capensis; Raphicerus campestris; Redunca fulvorufula; Sylvicapra grimmia; Syncerus caffer; Taurotragus oryx; Tragelaphus strepsiceros; Vulpes chama. References: McKee (1994).

Tuinplaas (2555:2845). Other names: Springbok Flats. Taxa: Equus capensis; Homo sapiens. References: Dusseldorp et al. (2013); Haughton (1932a); Schepers (1941).

Uitkomst. See Gladysvale.

Umhlatuzana (2948:3045). Taxa: Aethomys chrysophilus; Chlorocebus pygerythrus; Crocidura flavescens; Cryptomys hottentotus; Felis silvestris; Grammomys dolichurus; Hippotragus; Myosorex varius; Otomys irroratus; Otomys laminatus; Philantomba monticola; Procavia capensis; Redunca fulvorufula; Rhabdomys pumilio; Suncus varilla; Syncerus caffer; Thryonomys. References: Avery (1991b); Kaplan (1990).

Usakos (2159:1535). Taxa: Connochaetes; Equus sandwithi; Tragelaphus. References: Cooke (1955).

Vanwyksfontein (2724CD). Taxa: Equus zebra. References: Lundholm (1952).

Varsche Rivier 003 (3118DA). Taxa: Cryptomys hottentotus; Felis silvestris; Hippopotamus amphibius; Lepus; Oreotragus oreotragus; Raphicerus; Rhabdomys pumilio. References: Steele et al. (2012); Wadley (2015).

Virginia 751 (2806:2654). Taxa: Antidorcas marsupialis; Megalotragus priscus. References: Butzer (1973); De Ruiter et al. (2011).

Vlakkraal Thermal Springs (2826CC). Taxa: Alcelaphus caama; Antidorcas marsupialis; Connochaetes taurinus cf.; Crocuta crocuta cf.; Damaliscus pygargus cf.; Equus (Asinus); Equus burchellii; Equus capensis; Hippopotamus amphibius cf.; Hystrix; Kobus; Lycaon pictus cf.; Metridiochoerus compactus; Phacochoerus africanus; Redunca arundinum; Sylvicapra 
grimmia cf.; Syncerus antiquus; Taurotragus oryx cf.; Vulpes chama cf. References: Brink (1994); Cooke (1962); Hoffman (1953); Wells et al. (1942).

Vlakplaats (2628). Taxa: Antidorcas recki cf.; Lepus cf.; Parmularius cf.; Tragelaphus scriptus cf. References: Vrba and Panagos (1978).

Waldeck's Plant (2824). Taxa: Equus burchellii cf.; Equus capensis; Equus quagga cf.; Hippopotamus amphibius; Loxodonta africana; Metridiochoerus modestus; Phacochoerus africanus. References: Beck (1906); Cooke (1949a).

Warrenton (2824BB). Taxa: Crocuta crocuta; Equus burchellii; Syncerus antiquus. References: Cooke (1949a).

White Paintings Rock Shelter (1825:2130). Taxa: Ceratotherium simum; Crocidura hirta cf.; Equus capensis; Felis silvestris; Hystrix africaeaustralis; Lepus; Megalotragus priscus; Oreotragus oreotragus; Otocyon megalotis; Pedetes capensis; Redunca arundinum; Tragelaphus scriptus. References: Robbins (1990); Robbins et al. (2000).

Willowbank (2824). Taxa: Aepyceros melampus; Connochaetes; Megalotragus priscus; Syncerus caffer. References: Cooke (1949a).

Windsorton (2824BB). Taxa: Equus capensis; Kolpochoerus paiceae. References: Broom (1931); Cooke (1949a).

Winter's Rush (2824). Taxa: Equus capensis. References: Broom (1928); Cooke (1949a). Witkrans Cave (2736:2436). Taxa: Elephantulus; Homo sapiens; Otomys gracilis. References: Davis (1961); Dusseldorp et al. (2013); Humphreys (1978); McCrossin (1992); Meester (1961a).

Wonderkrater (2426:2845). Taxa: Damaliscus; Equus quagga; Hippopotamus amphibius; Megalotragus priscus; Phacochoerus; Syncerus antiquus. References: Backwell et al. (2014).

Wonderwerk (2751:2333). Taxa: Acomys; Alcelaphus; Antidorcas; Canis mesomelas; Chlorotalpa sclateri; Chrysochloris asiatica; Chrysospalax; Connochaetes gnou?; Crocidura cyanea; Crocidura flavescens; Crocidura fuscomurina; Crocidura hirta cf.; Cryptomys hottentotus; Damaliscus pygargus; Dasymys incomtus; Dendromus melanotis; Desmodillus auricularis; Elephantulus myurus; Eptesicus hottentotus; Equus burchellii; Equus capensis; Eurygnathohippus; Gazella; Raphicerus campestris; Gerbilliscus; Gerbillurus paeba; Graphiurus microtis cf.; Hystrix africaeaustralis; Lemniscomys rosalia; Lepus capensis; Macroscelides proboscideus; Malacothrix typica; Mastomys coucha; Megalotragus priscus; Micaelamys namaquensis; Miniopterus natalensis; Mus minutoides; Myosorex varius; Mystromys albicaudatus; Neoromicia capensis; Oryx gazella; Otomys angoniensis; Papio ursinus; Pedetes capensis; Pelea capreolus cf.; Phacochoerus africanus; Procavia antiqua; Procavia capensis; Procavia transvaalensis; Pronolagus; Raphicerus; Rhabdomys pumilio; Rhinolophus capensis; Saccostomus campestris; Steatomys krebsii; Suncus varilla; Syncerus antiquus; Syncerus caffer; Tadarida aegyptiaca; Taurotragus oryx; Zelotomys woosnami. References: Avery (1981, 1995b, 2007); Brink et al. (2015, 2016); Fernandez-Jalvo and Avery (2015); Klein (1988); Malan and Cooke (1941); Malan and Wells (1943); Thackeray (1984, 2015); Wells et al. (1942); Vrba (1973); Wadley (2015).

X Cave (2602:2743). Taxa: Aepyceros melampus; Antidorcas bondi; Connochaetes; Hippotragus; Oreotragus oreotragus; Pelea capreolus; Raphicerus campestris; Redunca; Syncerus; Taurotragus oryx. References: Van Zyl et al. (2016).

Yatala 73 (2807:2646). Taxa: Antidorcas marsupialis; Connochaetes gnou. References: De Ruiter et al. (2011).

Ysterfontein 1 (3320:1809). Taxa: Bathyergus suillus; Canis mesomelas; Caracal caracal; Connochaetes gnou; Diceros bicornis; Equus; Felis silvestris; Hippotragus leucophaeus; Hystrix 
africaeaustralis; Lepus; Mellivora capensis; Procavia capensis; Raphicerus campestris; Redunca arundinum; Syncerus antiquus; Taurotragus oryx; Tragelaphus strepsiceros. References: Avery et al. (2008); Halkett et al. (2003); Klein et al. (2004); Wadley (2015).

Ysterplaat (3318CD). Taxa: Equus capensis. References: Cooke (1955).

Zais (2401:1608). Taxa: Procavia capensis. References: Cruz-Uribe and Klein (1981-1983).

Zebrarivier (2431:1716). Taxa: Gerbillurus paeba; Hipposideros vittatus; Oreotragus oreotragus; Parotomys littledalei cf.; Petromus typicus; Procavia capensis; Rhinolophus capensis. References: Avery (1984b); Broom (1928); Cruz-Uribe and Klein (1981-1983).

Zwartklip. See Swartklip. 


\section{CHAPTER 6}

\section{The Holocene}

\subsection{HOLOCENE MAMMALS}

By the beginning of the Holocene, all taxa, apart from five species (see Chapter 7), were extant. A notable feature of the Holocene fauna is the appearance of various introduced taxa, both domestic and commensal. The domestic animals were, of course, introduced intentionally and their distribution expanded greatly as their human owners spread across the landscape. The distribution of two of the unintentionally introduced commensals indicates their arrival from the north; the roof rat Rattus rattus follows the route taken by Iron Age immigrants, while the house mouse Mus musculus almost certainly followed the same route, though there is only one record (Wadley and Turner 1987). The third taxon is the black rat Rattus norvegicus, which occurs only in the vicinity of Cape Town. It must have arrived from Europe on ships and seems not to have extended its range very far beyond its port of entry (Avery 1985).

\section{ORDER: AFROSORICIDA \\ Suborder: Chrysochloridea \\ FAMILY: CHRYSOCHLORIDAE \\ Subfamily: Chrysochlorinae}

Chlorotalpa sclateri Broom, 1907. Ann. Mag. Nat. Hist., Series 7, 19: 263.

Sclater's golden mole.

Type locality: Beaufort West.

Additional references: Bronner (1995a, 1995b); Broom (1907b, 1946, 1950).

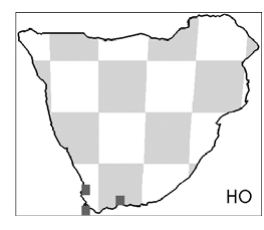

Chrysochloris asiatica Linnaeus 1758. Systema Naturae Regnum Animale, 10th edition, 1: 53. Cape golden mole.

Type locality: usually taken as Cape of Good Hope.

Additional references: Broom (1907b, 1909c, 1910, 1946, 1950); Petter (1981); Roberts (1919).

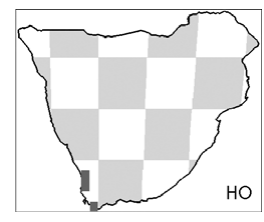

Chrysospalax villosus Smith, 1833. S. Afr. Quart. J., Series 2, 1: 81. Rough-haired golden mole.

Synonyms: Chrysochloris villosa.

Type locality: near Durban.

Additional references: Broom (1909c, 1913a); Gray (1865a); Meester

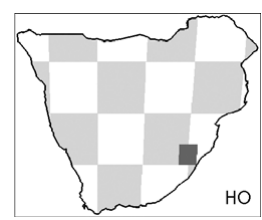

(1953b); Smith (1849). 
Cryptochloris zyli Shortridge and Carter, 1938. Ann. S. Afr. Mus. 32: 284. Van Zyl's golden mole.

Type locality: Compagnie's Drift.

Additional references: Broom (1946).

Eremitalpa granti Broom, 1907. Ann. Mag. Nat. Hist., Series 7, 19: 265. Grant's golden mole.

Type locality: Garies.

Additional references: Broom (1946, 1950); Meester (1964a); Perrin and Fielden (1999); Petter (1981).
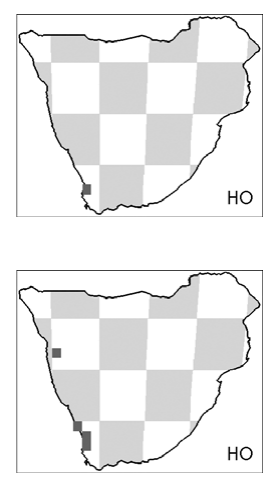

Subfamily: Amblysomyinae

Amblysomus hottentotus Smith, 1829. Zool. J. 4: 436. Hottentot golden mole.

Synonyms: iris.

Type locality: Grahamstown.

Additional references: Bronner (1996); Broom (1908, 1909c);

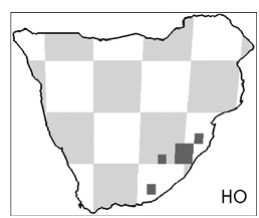

Capanna et al. (1989); Mynhardt et al. (2015); Petter (1981);

Roberts (1946).

\section{ORDER: MACROSCELIDEA \\ FAMILY: MACROSCELIDIDAE \\ Subfamily: Macroscelidinae}

Elephantulus Thomas and Schwann, 1906. Abstr. Proc. Zool. Soc. Lond. 33: 10.

Additional references: Corbet and Hanks (1968); Evans (1942);

Holroyd, (2010a); Patterson (1965); Van der Horst (1944).

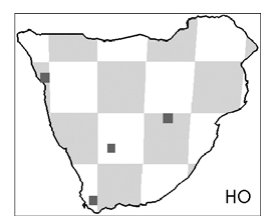

Elephantulus edwardii Smith, 1839. Illustrations of the Zoology of South Africa: pl. 14. Cape elephant shrew.

Synonyms: Macroscelides.

Type locality: Oliphants River.

Additional references: Roberts (1924); Smith (1849).

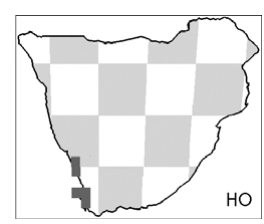

Elephantulus intufi Smith, 1836. Report of the Expedition for Exploring Central Africa: 42. Bushveld elephant shrew.

Synonyms: Macroscelides.

Type locality: flats beyond Kurrichaine.

Additional references: Smith (1849).

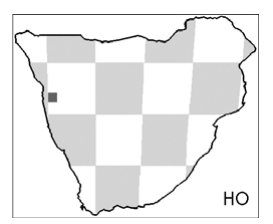

Elephantulus myurus Thomas and Schwann, 1906. Proc. Zool. Soc. Lond. 1906: 586. Eastern rock elephant shrew.

Type locality: Woodbush.

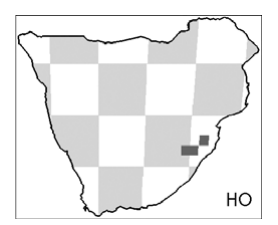


Elephantulus rupestris Smith, 1830. Proc. Commmittee Sci. Corr. Zool. Soc. Lond. 1: 11. Western rock elephant shrew.

Synonyms: Macroscelides.

Type locality: mountains near mouth of Orange River.

Additional references: Broom (1938b); Roberts (1938, 1946); Smith (1849).

Macroscelides proboscideus Shaw, 1800. Gen. Zool. Syst. Nat. Hist. 1(2) Mammalia: 536. Short-eared elephant shrew.

Synonyms: typicus.

Type locality: Roodeval.

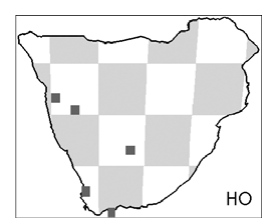

Additional references: Roberts $(1933,1938)$.

\section{ORDER: TUBULIDENTATA}

FAMILY: ORYCTOPODIDAE

Orycteropus afer Pallas, 1766. P.S. Pallas Medecinae Doctoris Miscellanea Zoologica: 64. Aardvark.

Type locality: Cape of Good Hope.

Additional references: Kitching (1963); Lehmann (2004, 2007); Pickford

(2005b); Shoshani et al. (1988).

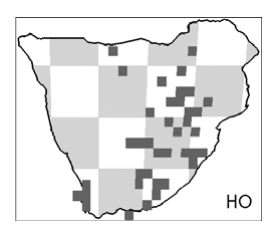

\section{ORDER: HYRACOIDEA}

FAMILY: PROCAVIIDAE

Heterohyrax brucei Gray, 1868. Ann. Mag. Nat. Hist., Series 4, 1: 44.

Yellow-spotted rock hyrax.

Additional references: Barry and Shoshani (2000); Thomas (1892).

Procavia Storr, 1780. Prodromus Methodi Mammalium: 40.
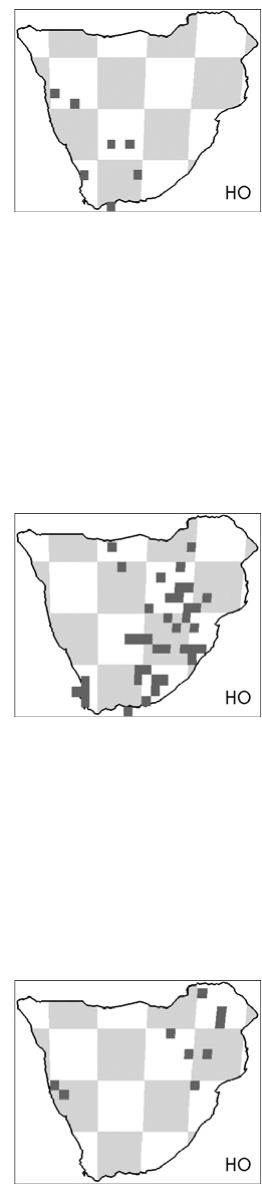

$\mathrm{O}$ 


\section{ORDER: PROBOSCIDEA}

FAMILY: ELEPHANTIDAE

Subfamily: Elephantinae

Loxodonta africana Blumenbach, 1797. D. Joh. Fr. Blumenbach's Handbuch der Naturgeschichte, 5th edition: 125. African bush elephant.

Synonyms: Archidiskodon; Palaeoloxodon; Elephas; Mammuthus; loxodontoides; zulu.

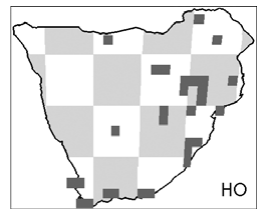

Type locality: Orange River, South Africa.

Additional references: Brook et al. (2014); Burmeister (1837); Dart (1929b); Sanders et al. (2010a); Todd (2010).

\section{ORDER: PRIMATES \\ Suborder: Strepsirrhini \\ FAMILY: GALAGIDAE}

Galago Geoffroy Saint-Hilaire, 1796. Mag. Encyclop. 1: 49.

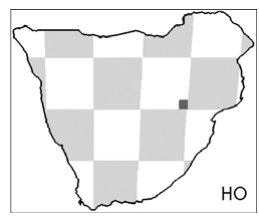

Galago senegalensis Geoffroy Saint-Hilaire, 1796. Mag. Encyclop. 1: 38. Senegal bushbaby.

Comments: as currently understood (Wilson and Reeder (2005), the only species of Galago possibly occurring in the sub-region are G. nyasae, inland to southern Malawi and northern Mozambique, and

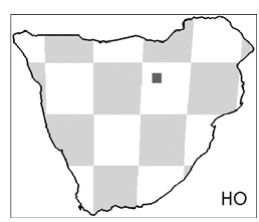

G. zanzibaricus, coastally to southern Mozambique. It therefore seems unlikely that this identification is correct.

Otolemur crassicaudatus Geoffroy Saint-Hilaire, 1812. Ann. Mus. Hist. Nat. Paris 19: 166. Brown greater galago.

Synonyms: Galago.

Type locality: Quelimane.

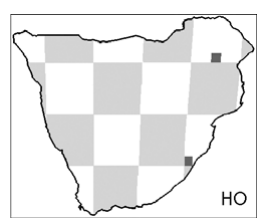

\section{Suborder: Haplorrhini}

FAMILY: CERCOPITHECIDAE

Subfamily: Cercopithecinae

Cercopithecus Linnaeus, 1758. Systema Naturae Regnum Animale, 10th edition, 1: 26.

Additional references: Jablonsky and Frost (2010).

Comments: material originally identified as Cercopithecus sp. is not mapped because it may be assigned to this genus or to Chlorocebus as currently understood (Wilson and Reeder 2005). 
Chlorocebus Linnaeus, 1758. Systema Naturae Regnum Animale, 10th edition, 1: 28.

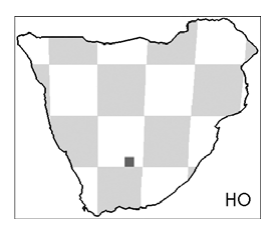

Chlorocebus aethiops Linnaeus, 1758. Systema Naturae Regnum Animale, 10th edition, 1: 28. Grivet.

Synonyms: Cercopithecus.

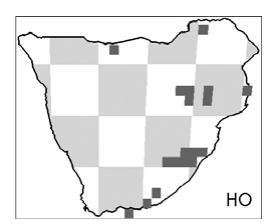

Chlorocebus pygerythrus Cuvier, 1821. Hist. Nat. Mamm. 24: 2. Vervet monkey.

Synonyms: aethiops.

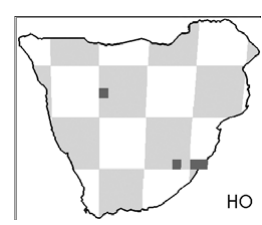

Papio Erxleben, 1777. Systema Regni Animales per Classes 1: Mammalia: $\mathrm{xxx}, 15$.

Additional references: Williams et al. (2012).

Comments: the Holocene material should almost certainly be assigned to $P$. ursinus since this is the only species currently recognised in the sub-region (Wilson and Reeder 2005).

Papio ursinus Kerr, 1792. The Animal Kingdom, or Zoological System of the Celebrated Sir Charles Linnaeus: 63. Chacma baboon.

Synonyms: cynocephalus; comatus; hamadryas; porcarius; rhodesiae. Type locality: Cape of Good Hope.

Additional references: Freedman (1954, 1965); Jablonsky and Frost

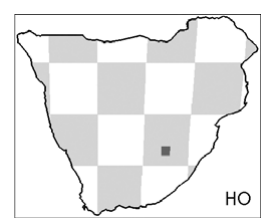
(2010); Roberts (1932); Williams et al. (2012).

Comments: material from the Koanaka Hills in Botswana is assigned to $P$. hamadryas by Williams et al. (2012), probably because some authors (e.g. Singleton et al. 2016) consider ursinus to be a subspecies of P. hamadryas. Here, Wilson and Reeder (2005) are followed in considering ursinus to be a full species.

\section{FAMILY: HOMINIDAE}

Subfamily: Homininae

Homo sapiens Linnaeus, 1758. Systema Naturae Regnum Animale, 10th edition, 1: 20. Modern human.

Synonyms: capensis; helmei.

Additional references: Ackermann et al. (2016); Beaumont et al. (1978);

Bräuer et al. (1992); Bräuer and Singer (1996); Broom (1918);

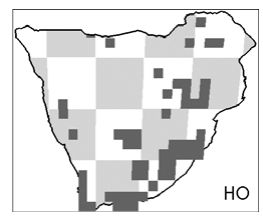

Churchill et al. (1996); Dart (1940); De Villiers (1973, 1974, 1976a, 1976b); Drennan (1937, 1953, 1955); Dreyer (1935); Galloway

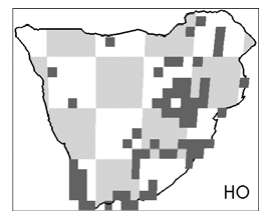


(1937a, 1937b); Grine (1998, 2000, 2012); Grine et al. (1995, 2007, 2010); Haughton et al. (1917); Hughes (1990); L'Abbé et al. (2008); Pycraft (1925); Keith (1933); MacLatchy et al. (2010); Pearson and Grine (1996); Pickford and Senut (1998); Rightmire (1978, 1979b); Rightmire and Deacon (1991); Rightmire et al. (2006); Singer (1954); Smith et al. (2012); Stynder et al. (2001); Trinkaus et al. (1999).

\section{ORDER: RODENTIA \\ Suborder: Sciuromorpha}

FAMILY: SCIURIDAE

Subfamily: Xerinae

Paraxerus cepapi Smith, 1836. Report of the Expedition for Exploring Central Africa: 43. Smith's bush squirrel.

Synonyms: Sciurus.

Type locality: Marico River.

Additional references: Roberts $(1932,1946)$; Smith $(1838,1849)$.

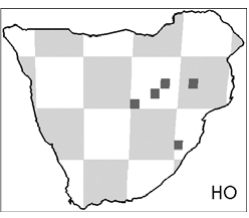

Xerus Ehrenberg, 1833. In: Hemprich and Ehrenberg, Symbolae Physicae, seu, Icones et Descriptiones 1: sig. Ee, pl. 9.

Comments: there is disagreement about the authorship and dating of this series of publications. See discussion at www.zoonomen.net/ mammtax/cit/jours.html.

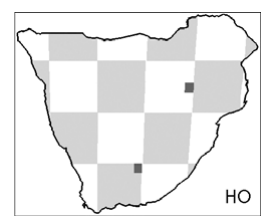

Xerus inauris Zimmermann, 1780. Geographische Geschichte des

Menschen und der Allgemein Verbreiteten Vierfüssigen Thiere 2: 344.

South African ground squirrel.

Synonyms: Geosciurus capensis.

Type locality: Kaffirland, 100 miles $(160 \mathrm{~km})$ north of Cape of Good Hope. Additional references: Skurski and Waterman (2005).

Xerus princeps Thomas, 1929. Proc. Zool. Soc. Lond. 1929: 106. Damara ground squirrel.
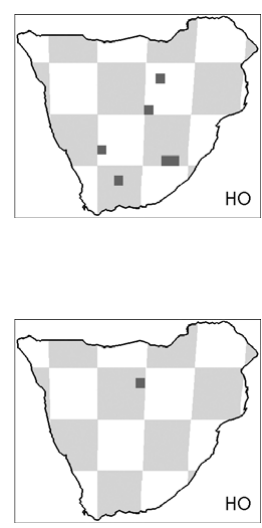

\section{Suborder: Myomorpha}

FAMILY: GLIRIDAE

Subfamily: Graphiurinae

Graphiurus murinus Desmarest, 1822. Mammalogie ou descriptions des espèces de mammifères, Part 2 (suppl.): 542. Forest African dormouse. Synonyms: alticola; tasmani; woosnami; vandami; zuluensis.

Type locality: Cape of Good Hope.

Additional references: Holden (1996); Kryštufek et al. (2004); Roberts

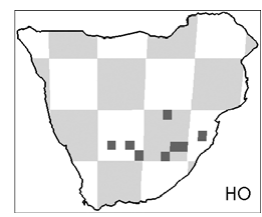
(1931, 1938). 
Graphiurus ocularis Smith, 1829. Zool. J. 4: 439. Spectacled African dormouse.

Synonyms: Myoxus; capensis.

Type locality: near Plettenberg Bay.

Additional references: De Winton (1898); Holden (1996); Smith (1849).

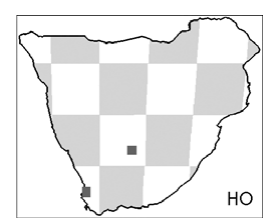

Graphiurus platyops Thomas, 1897. Ann. Mag. Nat. Hist., Series 6, 19: 388. Flat-headed African dormouse.

Synonyms: eastwoodae.

Type locality: Enkeldorn.

Additional references: Holden (1996).

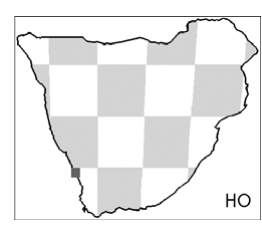

\section{FAMILY: NESOMYIDAE}

Subfamily: Cricetomyinae

Saccostomus Peters, 1846. Bericht Verhandl. K. Preuss. Akad. Wiss.

Berlin 11: 258.

Comments: the Holocene material should almost certainly be assigned to

S. campestris since this is the only species currently understood to occur in southern Africa (Wilson and Reeder 2005).

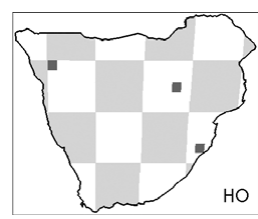

Saccostomus campestris Peters, 1846. Bericht Verhandl. K. Preuss.

Akad. Wiss. Berlin 11: 258. Southern African pouched mouse.

Synonyms: anderssoni; hildae; limpopoensis; mashonae; pagei; streeteri.

Type locality: Tete.

Additional references: Denys (1988b); Roberts (1914).

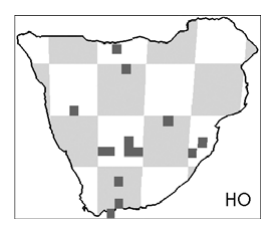

Subfamily: Dendromurinae

Dendromus Smith, 1829. Zool. J. 4: 438.

Synonyms: Poemys.

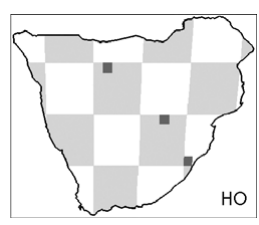

Dendromus melanotis Smith, 1834. S. Afr. Quart. J., Series 2, 2: 148. Grey

African climbing mouse.

Synonyms: arenarius; concinnus; nigrifrons.

Type locality: near Port Natal = Durban.

Additional references: Roberts (1931); Smith (1849).

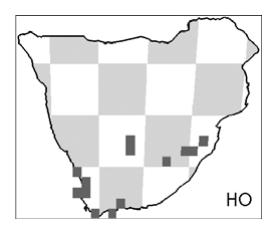

Dendromus mesomelas Brants, 1827. Het Geslacht der Muizen door

Linnaeus Opgesteld: 122. Brants' African climbing mouse.

Synonyms: ayresi; typica.

Type locality: Sunday's River, east of Port Elizabeth.

Additional references: Smith (1849).

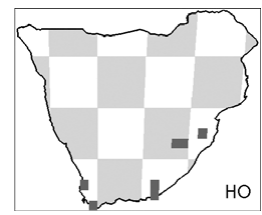


Dendromus mystacalis Heuglin, 1863. Nov. Act. Acad. Caes. Leop.-Carol. 30(2 suppl.): 5. Chestnut African climbing mouse.

Malacothrix typica Smith, 1834. S. Afr. Quart. J., Series 2, 2: 148. Large-eared African desert mouse.

Type locality: Graaff Reinet District.

Additional references: Roberts (1932).

Steatomys Peters, 1846. Bericht Verhandl. K. Preuss. Akad. Wiss. Berlin 11: 258.
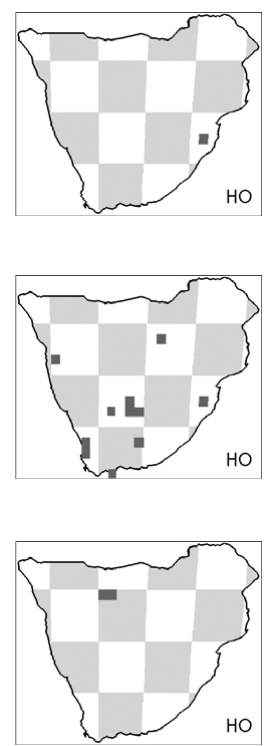

Steatomys krebsii Peters, 1852. Naturwissenschaftliche Reise nach Mossambique: 165. Krebs's African fat mouse.

Synonyms: chiversi; pentonyx; pratensis.

Type locality: Kaffraria.

Additional references: Roberts (1931).

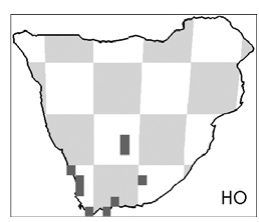

Steatomys parvus Rhoads, 1896. Proc. Acad. Nat. Sci. Philadelphia 48:

529. Tiny African fat mouse.

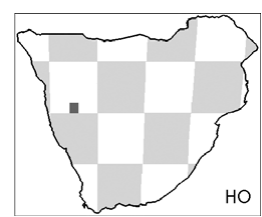

Steatomys pratensis Peters, 1846. Bericht Verhandl. K. Preuss. Akad. Wiss. Berlin 11: 258. Common African fat mouse.

Synonyms: natalensis; opimus.

Type locality: Tete.

Comments: material from Botswana was identified as Steatomys opimus

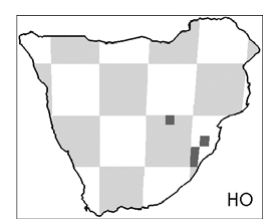
but, as currently understood, this species is almost entirely confined to West Africa (Musser and Carleton 2005).

Subfamily: Mystromyinae

Mystromys albicaudatus Smith, 1834. S. Afr. Quart. J., Series 2, 2: 148. African white-tailed rat. Synonyms: Otomys; antiquus. Type locality: Albany District. Additional references: De Winton (1898); Grubb (2004); Smith (1849).

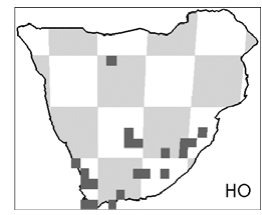


Subfamily: Petromyscinae

Petromyscus Thomas, 1926. Ann. Mag. Nat. Hist., Series 9, 17: 179. Additional references: Petter (1967).

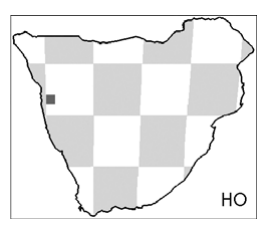

Petromyscus collinus Thomas and Hinton, 1925. Proc. Zool. Soc. Lond. 1925: 237. Pygmy rock mouse.

Synonyms: Praomys.

Type locality: Karibib.

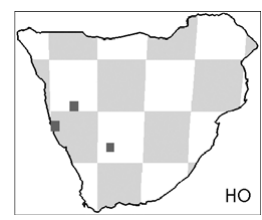

FAMILY: MURIDAE

Subfamily: Deomyinae

Acomys subspinosus Waterhouse, 1837. Proc. Zool. Soc. Lond. 1837: 104.

Cape spiny mouse.

Type locality: Cape of Good Hope.

Additional references: Dippenaar and Rautenbach (1986).

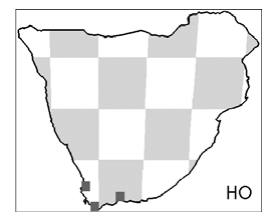

Subfamily: Gerbillinae

Desmodillus auricularis Smith, 1834. S. Afr. Quart. J., Series 2, 2: 160. Short-eared gerbil.

Synonyms: Gerbillus; brevicaudatus.

Type locality: Kamiesberg.

Additional references: Griffin (1990); Qumsiyeh (1986); Smith (1849).

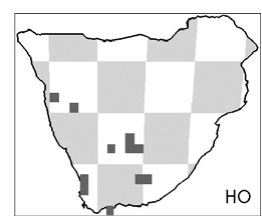

Gerbilliscus Thomas, 1897. Proc. Zool. Soc. Lond. 1897: 433.

Synonyms: Gerbillus; Tatera.

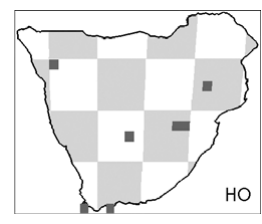

Gerbilliscus afra Gray, 1830. Spicil. Zool. 2: 10. Cape gerbil.

Synonyms: Gerbillus; Meriones; Tatera; africanus.

Type locality: vicinity of Cape Town.

Additional references: Chacornac (1999); Cuvier (1841); Davis (1949, 1965); Qumsiyeh (1986); Smith (1849); Smuts (1832).

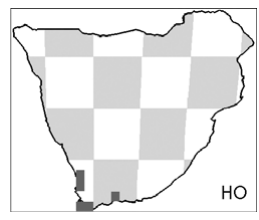

Comments: Gray (1830) not seen. Citation according to Wilson and Reeder (2005).

Gerbilliscus brantsii Smith, 1836. Report of the Expedition for Exploring

Central Africa: 43. Highveld gerbil.

Synonyms: Gerbillus; Tatera; montanus.

Type locality: Ladybrand.

Additional references: Davis (1949, 1965); Griffin (1990); Qumsiyeh

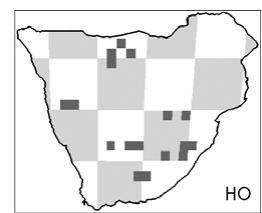

(1986); Roberts (1926); Smith (1849). 
Gerbilliscus leucogaster Peters, 1852. Bericht Verhandl. K. Preuss., Akad. Wiss. Berlin 17: 274. Bushveld gerbil.

Synonyms: Gerbillus; Tatera.

Type locality: Boror.

Additional references: Davis (1949, 1965); Griffin (1990); Qumsiyeh

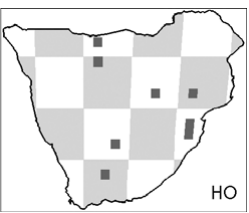
(1986).

Gerbillurus paeba Smith, 1836. Report of the Expedition for Exploring Central Africa: 43. Paeba hairy-footed gerbil.

Synonyms: Gerbillus; tenuis.

Type locality: Vryberg.

Additional references: Chacornac (1999); De Winton (1898);

Griffin (1990); Perrin et al. (1999); Qumsiyeh (1986); Taylor (2000).

Gerbillurus vallinus Thomas, 1918. Ann. Mag. Nat. Hist., Series 9, 2: 148. Brush-tailed hairy-footed gerbil.

Type locality: Tuin Kenhardt.

Additional references: Dempster et al. (1999); Griffin (1990); Qumsiyeh (1986).

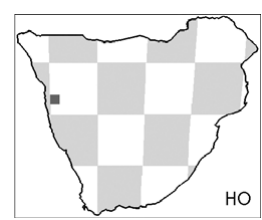

Subfamily: Murinae

Aethomys Thomas, 1915. Ann. Mag. Nat. Hist, Series, 8, 16: 477.

Additional references: Chimimba (1997, 1998, 2005); Chimimba and Dippenaar (1994); Chimimba et al. (1999); Visser and Robinson (1986).

Comments: many specimens were identified as Aethomys sp. at a time when Micaelamys was not recognised as a separate genus. For this reason, distributions that rely on material not identified to species have not been mapped, pending re-identification.

Aethomys chrysophilus De Winton, 1896. Proc. Zool. Soc. Lond. 1896: 801. Red veld aethomys.

Additional references: Chimimba (2000); Chimimba and Dippenaar (1994); Roberts (1926, 1946).

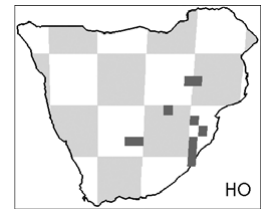

Dasymys incomtus Sundevall, 1846. Ofv. K. Svenska Vet.-Akad. Forhandl. 3: 120. Common dasymys.

Type locality: Durban.

Additional references: Gordon (1991); Mullin et al. (2004).

Comments: much of the material identified as D. incomtus was

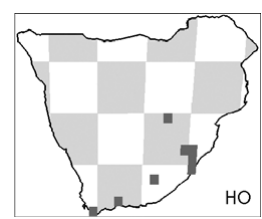
identified at a time when this species was the only one recognised in southern Africa. For this reason, this material should be considered as $D$. incomtus sensu lato until such time as it is re-examined. 
Grammomys cometes Thomas and Wroughton, 1908. Proc. Zool. Soc. Lond. 1908: 549. Mozambique grammomys.

Synonyms: Thamnomys; silindensis.

Type locality: Inhambane.

Additional references: Kryštufek et al. (2008).

Grammomys dolichurus Smuts, 1832. Dissertation Zoologica,

Ennumerationem Mammalium Capensium: 38. Common grammomys.

Synonyms: Thamnomys.

Type locality: near Cape Town.

Additional references: Roberts (1931).

Lemniscomys rosalia Thomas, 1904. Ann. Mag. Nat. Hist., Series 7, 13:

414. Single-striped lemniscomys.

Synonyms: dorsalis; griselda.

Additional references: Smith (1849).

Mastomys Thomas, 1915. Ann. Mag. Nat. Hist., Series 8, 16: 477.

Synonyms: Praomys.

Additional references: Britton-Davidian et al. (1995); Granjon et al.

(1997); Grubb (2004); Taylor (2000).

Mastomys coucha Smith, 1834. Report of the Expedition for Exploring Central Africa: 43. Southern African mastomys.

Synonyms: Praomys; natalensis.

Type locality: between Orange River and Tropic of Capricorn.

Additional references: Bronner et al. (2007); Dippenaar et al. (1993); Smit
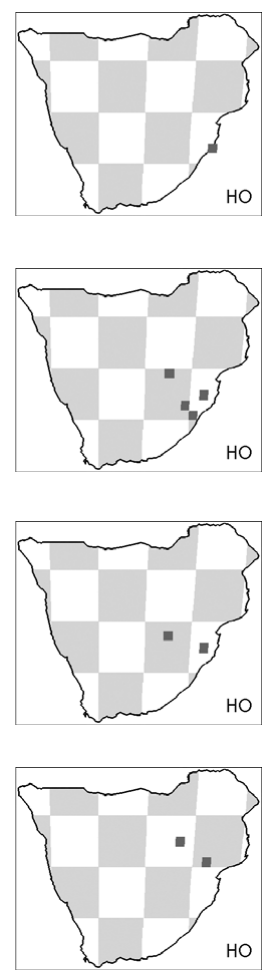
and Van der Bank (2001).

Comments: this material was originally identified as M. natalensis at a time when M. coucha was not recognised as a separate species.

Mastomys natalensis Smith, 1834. S. Afr. Quart. J., Series 2, 2: 146. Natal mastomys.

Synonyms: Praomys.

Type locality: Port Natal = Durban.

Additional references: Bronner et al. (2007); Dippenaar et al. (1993);

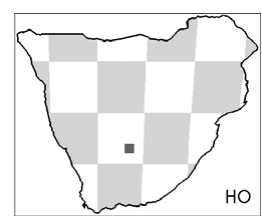

Green et al. (1980); Smit and Van der Bank (2001); Smith (1849).

Comments: see comment under M. coucha above.

Micaelamys namaquensis Smith, 1834. S. Afr. Quart. J., Series 2, 2: 160. Namaqua micaelamys.

Synonyms: Aethomys; lehocla.

Type locality: Cape of Good Hope, restricted to Witwater.

Additional references: Chimimba (2001); Chimimba and Dippenaar

(1994); Roberts (1926, 1946); Russo (2009); Smith (1849); Visser and Robinson (1986). 
Mus Linnaeus, 1758. Systema Naturae Regnum Animale, 10th edition, 1: 59. Additional references: Britton-Davidian et al. (2012); Veyrunes et al. (2005).

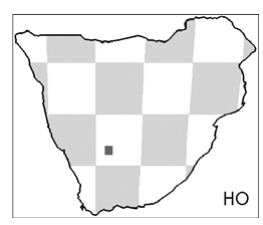

Mus indutus Thomas, 1910. Ann. Mag. Nat. Hist, Series 8, 5: 89. Desert pygmy mouse.

Synonyms: Leggada.

Type locality: Molopo River, west of Morokwen.

Additional references: Britton-Davidian et al. (2012).

Comments: there is no agreement on differences between $M$. indutus and M. minutoides, but it is possible that both species are present at Wonderwork (Avery, unpublished).

Mus minutoides Smith, 1834. S. Afr. Quart. J., Series 2, 2: 147. Southern African pygmy mouse.

Synonyms: Leggada.

Type locality: Cape Town.

Additional references: Britton-Davidian et al. (2012).

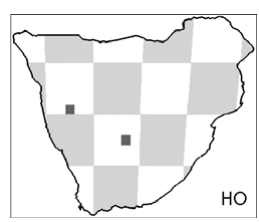

Comments: some of the specimens from the more arid areas may be $M$. indutus, but see comment under that species.

Mus musculus Linnaeus, 1758. Systema Naturae Regnum Animale, 10th edition, 1: 62. House mouse.

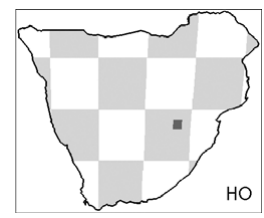

Myomyscus verreauxii Smith, 1834. S. Afr. Quart. J., Series 2, 2: 146.

Verreaux's white-footed rat.

Synonyms: Praomys; Myomys; colonus.

Type locality: near Cape Town.

Additional references: Grubb (2004); Smith (1849).

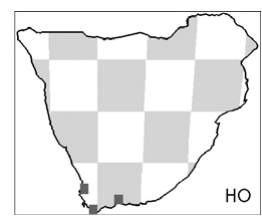

Rattus norvegicus Berkenhout, 1769. Outlines of the Natural History of Great Britain and Ireland 1: 5. Brown rat.

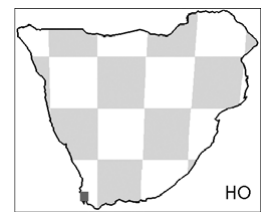

Rattus rattus Linnaeus, 1758. Systema Naturae Regnum Animale, 10th edition, 1: 61. Roof rat.

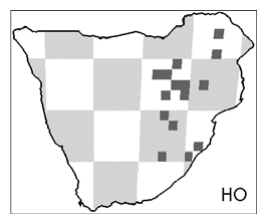


Rhabdomys Thomas, 1916. Ann. Mag. Nat. Hist., Series 8, 18: 69.

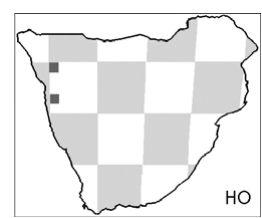

Rhabdomys dilectus De Winton, 1896. Proc. Zool. Soc. Lond. 1896: 803. Mesic four-striped grass rat.

Type locality: Mazoe.

Comments: Rhabdomys dilectus is again regarded (Wilson and Reeder 2005) as a separate species from $R$. pumilio, but at the time that most of the fossil material was identified only R. pumilio was recognised as a full species. No fossil material has yet been identified as $R$. dilectus, but see comments under R. pumilio below.

Rhabdomys pumilio Sparrman, 1784. K. Svenska Vet.-Akad. Handl. 5: 236. Xeric four-striped grass rat.

Type locality: Slangrivier, east of Knysna.

Additional references: Castiglia et al. (2012); Le Grange et al. (2015); Roberts (1946); Smith (1849); Wroughton (1905).

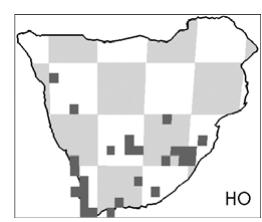

Comments: samples recorded here should be regarded as belonging to Rhabdomys pumilio sensu lato and are very likely to include some material that would currently be regarded as R. dilectus.

Thallomys Thomas, 1920. Ann. Mag. Nat. Hist., Series 9, 5: 141.

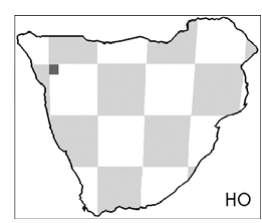

Thallomys paedulcus Sundevall, 1846. Ofv. K. Svenska Vet.-Akad. Forhandl. 3: 120. Acacia thallomys.

Type locality: provisionally fixed as Crocodile Drift, Brits.

Additional references: Taylor (2000); Taylor et al. (1995).

Comments: this material was identified as T. paedulcus at a time when

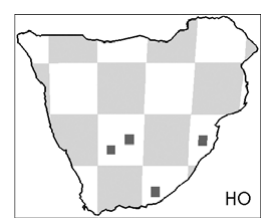
only the one species was recognised, but some would now almost certainly be identified as T. nigricauda.

Zelotomys woosnami Schwann, 1906. Proc. Zool. Soc. Lond. 1906: 108. Woosnam's zelotomys.

Type locality: Molopo River.

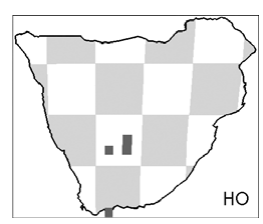




\section{Subfamily: Otomyinae}

Myotomys sloggetti Thomas, 1902. Ann. Mag. Nat. Hist., Series 7, 10:

311. Rock karoo rat.

Synonyms: Otomys.

Type locality: Deelfontein.

Additional references: Taylor et al. (2004).

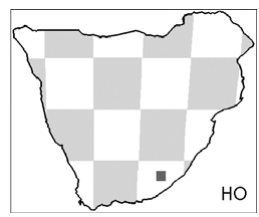

Myotomys unisulcatus Cuvier, 1829. In: Geoffroy Saint-Hilaire and Cuvier Histoire Naturelle des Mammifères, 6 LX: Otomys caffre. Bush karoo rat. Synonyms: Euryotis; Otomys.

Type locality: Matjiesfontein.

Additional references: Edwards (2009); Edwards et al. (2011); Smith

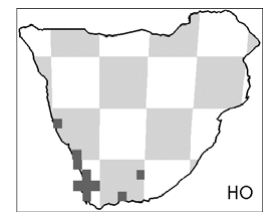

(1849); Taylor et al. (1989, 2004).

Otomys angoniensis Wroughton, 1906. Ann. Mag. Nat. Hist., Series 7, 18: 274. Angoni vlei rat.

Additional references: Bronner and Meester (1988); Taylor et al. (2004).

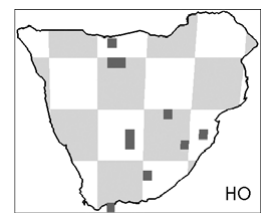

Otomys irroratus Brants, 1827. Het Geslacht der Muizen door Linnaeus Opgesteld: 94. Southern African vlei rat.

Synonyms: Euryotis; Mus.

Type locality: Cape Town District.

Additional references: Bronner et al. (1988); Engelbrecht et al. (2011);

Smith (1849); Taylor (2000); Taylor et al. (1989, 2009).

Otomys laminatus Thomas and Schwann, 1905. Abstr. Proc. Zool. Soc. Lond. 18: 23. KwaZulu vlei rat.

Type locality: Sibudeni.

Additional references: Roberts $(1919,1932)$.

Otomys saundersiae Roberts, 1929. Ann. Transvaal Mus. 13: 114.

Saunders' vlei rat.

Synonyms: karoensis.

Type locality: Grahamstown.

Additional references: Roberts (1931); Taylor et al. (1993, 2009).
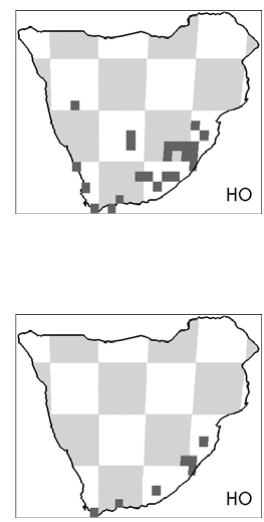

Parotomys brantsii Smith, 1834. S. Afr. Quart. J., Series 2, 2: 150. Brants' whistling rat.

Synonyms: Euryotis.

Type locality: 'toward the mouth of the Orange River'.

Additional references: Rookmaker and Meester (1988); Smith (1849);

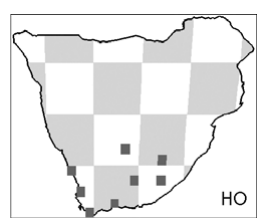

Taylor et al. (1989, 2004).

Parotomys littledalei Thomas, 1918. Ann. Mag. Nat. Hist., Series 9, 2:

205. Littledale's whistling rat.

Type locality: Tuin Kenhardt.

Additional references: Roberts (1933); Taylor et al. (1989, 2004).
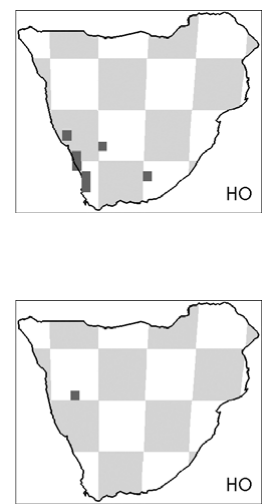


\section{Suborder: Anomaluromorpha \\ FAMILY: PEDETIDAE}

Pedetes capensis Forster, 1778. K. Svenska Vet.-Akad., Handl. 39: 109.

South African spring hare.

Type locality: Cape of Good Hope.

Additional references: Roberts (1946).

\section{Suborder: Hystricomorpha}

FAMILY: BATHYERGIDAE

Subfamily: Bathyerginae

Bathyergus janetta Thomas and Schwann, 1904. Abstr. Proc. Zool. Soc. Lond. 2: 6. Namaqua dune mole-rat.

Type locality: Port Nolloth.

Additional references: Thomas and Schwann (1904b).

Bathyergus suillus Schreber, 1782. Die Säugethiere in Abbildungen nach der Natur, mit Beschreibungen 4: 714. Cape dune mole-rat.

Type locality: Cape of Good Hope.

Comments: volume 4 was published in 1792 according to the version reproduced in the Biodiversity Heritage Library (www.biodiversity

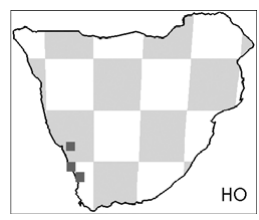
library.org/item/135004\#page/137/mode/1 up; accessed 19 May 2017) and 'Der Sandmoll' is discussed on pp. 715-716, not 714, and Mus suillus is listed on p. 932. Plate 204B appears in an undated volume containing plates 166-280 (www.biodiversitylibrary.org/ item/97330\#page/125/mode/1up). Bennett et al. (2009) give the reference for this species as Schreber, J.C.D. 1782. Die Säugthiere in Abbildungen nach der Natur, mit Beschreibungen. Supplementband III [Dritte Abtheilung: Die Beutelthiere und Rage]. Wolfgang Walther, Erlangen, Germany. This publication was not seen.

Cryptomys Gray, 1864. Proc. Zool. Soc. Lond. 1864: 124.

Additional references: De Graaff (1965); Faulkes et al. (2004); Ingram et al. (2004); Kock et al. (2006); Thomas (1917).

Comments: some specimens previously identified as Cryptomys sp. may be referable to Fukomys sp. as currently understood.

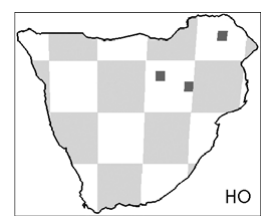

Cryptomys hottentotus Lesson, 1826. Zool. 1: 166. Southern African mole-rat.

Type locality: near Paarl.

Additional references: Avery (2004); Denys (1988a); Roberts (1913, 1946). Comments: it is possible that some of the material may be referable to

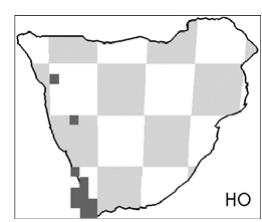

Fukomys damarensis, which was not recognised as a separate species when many of the samples were identified. 
Fukomys damarensis Ogilby, 1838. Proc. Zool. Soc. Lond. 1838: 5.

Damara mole-rat.

Synonyms: Cryptomys.

Type locality: Damaraland.

Additional references: Aguilar (1993); Faulkes et al. (1997, 2004); Ingram

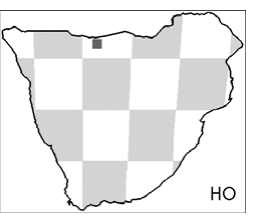

et al. (2004); Kock et al. (2006).

Georychus capensis Pallas, 1778. Njova. Spec. Quad. Glir. Ord. 76: 172. Cape mole-rat.

Type locality: Cape of Good Hope.

Additional references: Bennett et al. (2006, 2016).

Comments: the Holocene occurrence in 2032 may be considered unlikely: this taxon is currently endemic to South Africa (Maree et al. 2017), whereas Cryptomys hottentotus occurs in southern Zimbabwe (Maree and Faulkes 2016).

FAMILY: HYSTRICIDAE

Subfamily: Hystricinae

Hystrix Linnaeus, 1758. Systema Naturae Regnum Animale, 10th edition, 1: 56.

Additional references: Maguire (1976).

Comments: it is probable that this material should be assigned to

H. africaeaustralis according to current taxonomy.
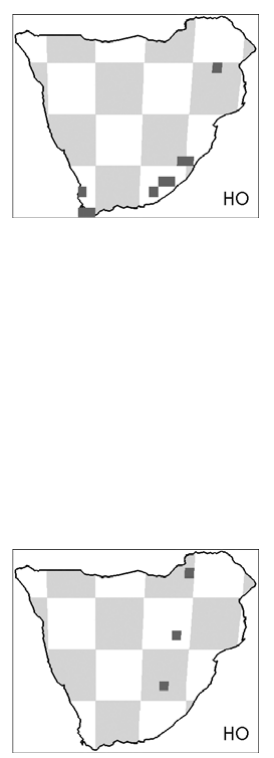

Hystrix africaeaustralis Peters, 1852. Naturwissenschaftliche Reise nach Mossambique: 170. Cape porcupine.

Additional references: Maguire (1976).

\section{FAMILY: PETROMURIDAE}

Petromus Smith, 1831. S. Afr. Quart. J. 1(5): 10.

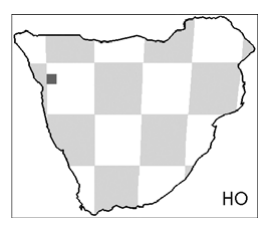

Petromus typicus Smith, 1831. S. Afr. Quart. J. 1(5): 11. Dassie rat. Type locality: 'Mountains towards mouth of Orange River'. Additional references: Roberts $(1938,1946)$; Smith (1849).

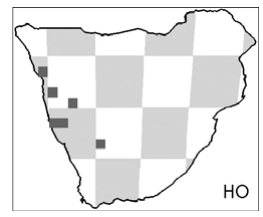




\section{FAMILY THRYONOMYIDAE}

Thryonomys Fitzinger, 1867. Sitzb. Akad. Wiss. Wein 56(1): 141.

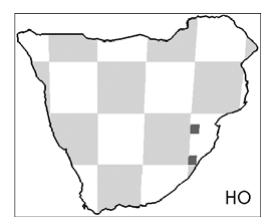

Thryonomys gregorianus Thomas, 1894. Ann. Mag. Nat. Hist., Series 6, 13: 202. Lesser cane rat.

Additional references: Van der Merwe (2007).

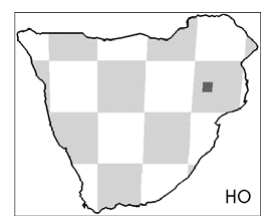

Thryonomys swinderianus Temminck, 1827. Monographies de

Mammalogie 1: 248. Greater cane rat.

Additional references: Van der Merwe (2007).

\section{ORDER: LAGOMORPHA}

FAMILY: LEPORIDAE

Bunolagus monticularis Thomas, 1903. Ann. Mag. Nat. Hist., Series 7, 11:

78. Riverine rabbit.

Synonyms: Lepus.

Type locality: Deelfontein.

Additional references: Robinson and Dippenaar (1987); Robinson and

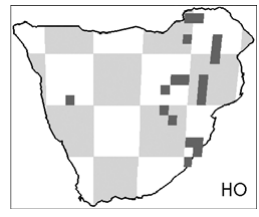

Matthee (2005); Robinson and Skinner (1983).

Lepus Linnaeus, 1758. Systema Naturae Regnum Animale, 10th edition, 1: 57.

Additional references: Robinson and Dippenaar (1987); Robinson and

Matthee (2005).
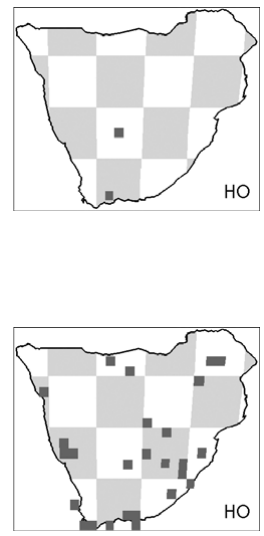

Lepus capensis Linnaeus, 1758. Systema Naturae Regnum Animale, 10th edition, 1: 58. Cape hare.

Type locality: Cape of Good Hope.

Additional references: Roberts (1932).

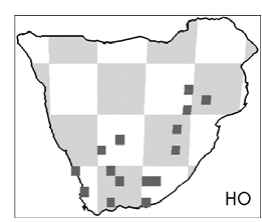

Lepus saxatilis Cuvier, 1823. Dict. Sci. Nat. 26: 309. Scrub hare.

Type locality: 'à trois journées au nord du cap de Bonne-Espérance'

(north of Cape of Good Hope).

Additional references: Kolbe (1948); Roberts (1932); Robinson and

Dippenaar (1983).

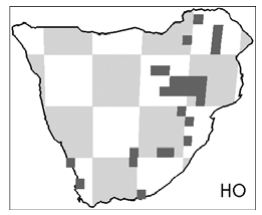


Oryctolagus cuniculus Linnaeus, 1758. Systema Naturae Regnum Animale, 10th edition, 1: 58. European rabbit.

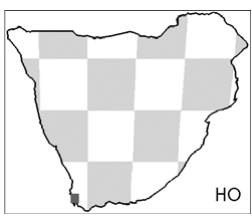

Pronolagus Lyon, 1904. Smithson. Misc. Coll. 45: 416.

Additional references: Robinson and Matthee (2005).

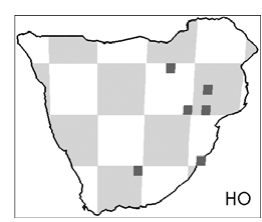

†Pronolagus intermedius Jameson, 1909. Ann. Transvaal Mus. 1: 195.

Type locality: Godwan River.

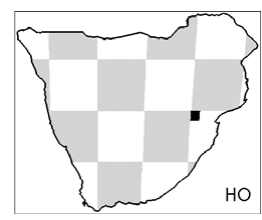

Pronolagus randensis Jameson, 1907. Ann. Mag. Nat. Hist., Series 7, 20:

404. Jameson's red rock hare.

Type locality: 'Observatory Kopje Johannesburg'.

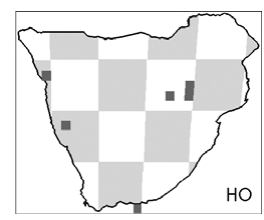

Pronolagus rupestris Smith, 1834. S. Afr. Quart. J., Series 2, 2: 174.

Smith's red rock hare.

Synonyms: crassicaudatus.

Type locality: probably Van Rhynsdorp District.

Additional references: Roberts (1938).

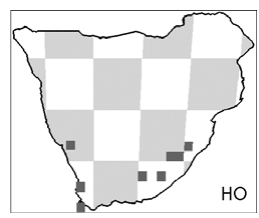

\section{ORDER: ERINACEOMORPHA}

\section{FAMILY: ERINACEIDAE}

Subfamily: Erinaceinae

Atelerix frontalis Smith, 1831. S. Afr. Quart. J. 1(5): 10, 29. Southern

African hedgehog.

Synonyms: Erinaceus capensis.

Type locality: northern parts of the Graaff Reinet district.

Additional references: Smith (1830, 1838, 1849).

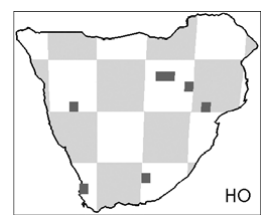

\section{ORDER: SORICOMORPHA}

FAMILY: SORICIDAE

Subfamily: Crocidurinae

Crocidura Wagler, 1832. Isis von Oken: 275.

Additional references: Butler et al. (1989); Jenkins et al. (1998); Meester (1953a, 1961b, 1963); Meester et al. (1985).

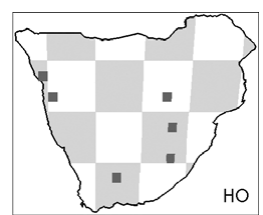


Crocidura cyanea Duvernoy, 1838. Mem. Soc. Hist. Nat. Strasbourg 2: 2. Reddish-grey musk shrew.

Type locality: Citrusdal fide Shortridge (1942: 27).

Crocidura flavescens Geoffroy Saint-Hilaire, 1827. Dict. Class. Hist. Nat.

11: 324. Greater red musk shrew.

Synonyms: Sorex; capensis.

Type locality: King William's Town.

Additional references: Smith (1849).
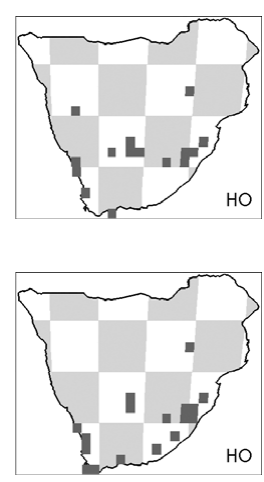

Crocidura fuscomurina Heuglin, 1865. Nov. Act. Acad. Caes. Leop.-Carol.

32: 36. Bicolored musk shrew.

Synonyms: bicolor.

Additional references: Hutterer (1983).

Crocidura hirta Peters, 1852. Naturwissenschaftliche Reise nach

Mossambique: 78. Lesser red musk shrew.

Type locality: Tete.
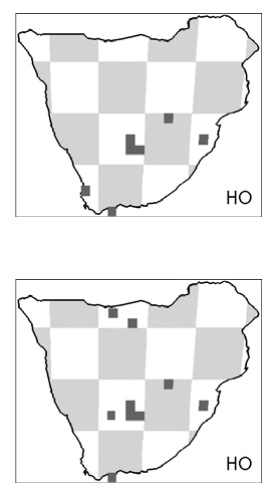

Crocidura mariquensis Smith, 1844. Illustrations of the Zoology of South Africa: pl. 44, fig. 1. Swamp musk shrew.

Synonyms: Sorex.

Type locality: near Marico River.

Additional references: Dippenaar (1977, 1979); Meester (1964b); Smith

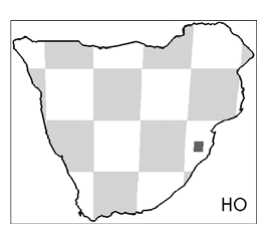
(1849).

Comments: citation as given in Wilson and Reeder (2005). There is clearly considerable uncertainty surrounding the publication dates of Smith's Illustrations of South African Zoology (Low and Evenhuis 2014).

Suncus Ehrenberg, 1832. In Hemprich and Ehrenberg, Symbolae Physicae, seu, Icones et Descriptiones 2: k.

Additional references: Jenkins et al. (1998); Meester (1953a); Meester and Lambrechts (1971); Meester and Meyer (1972); Quérouil et al. (2001).

Comments: there is disagreement about the authorship and dating of this

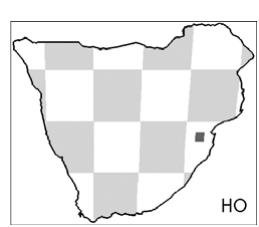
series of publications. See discussion at www.zoonomen.net/ mammtax/cit/jours.html.

Suncus infinitesimus Heller, 1912. Smithson. Misc. Coll. 60(12): 5. Least dwarf shrew.

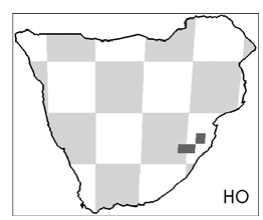


Suncus lixus Thomas, 1897. Proc. Zool. Soc. Lond. 1897: 930. Greater dwarf shrew.

Synonyms: Crocidura.

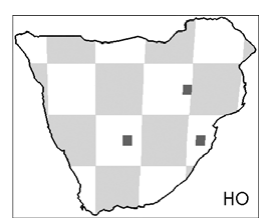

Suncus varilla Thomas, 1895. Ann. Mag. Nat. Hist., Series 6, 16: 54.

Lesser dwarf shrew.

Type locality: East London.

Additional references: Roberts (1946).

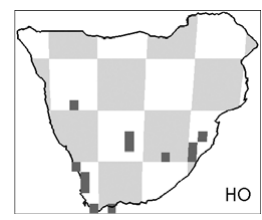

Subfamily: Myosoricinae

Myosorex Gray, 1837. Proc. Zool. Soc. Lond. 1837: 124.

Additional references: Matthews and Stynder (2011b); Meester (1953a,

1958); Willows-Munro and Matthee (2009); Quérouil et al. (2001).

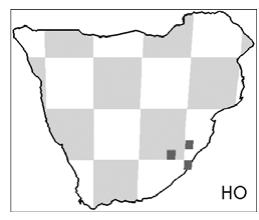

Myosorex varius Smuts, 1832. Dissertation Zoologica, Ennumerationem

Mammalium Capensium: 108. Forest shrew.

Synonyms: Sorex.

Type locality: Algoa Bay: Port Elizabeth.

Additional references: Roberts (1924); Smith (1849).

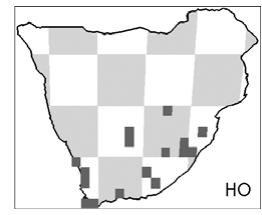

\section{ORDER: CHIROPTERA \\ Suborder: Microchiroptera \\ FAMILY: RHINOLOPHIDAE}

Rhinolophus capensis Lichtenstein, 1823. Verzeichniss der Doubletten des zoologischen Museums der Königl. Universität zu Berlin: 4. Cape horseshoe bat.

Type locality: Cape of Good Hope.

Rhinolophus clivosus Cretzschmar, 1826. In: Rüppell, Atlas zu der Reise im nördlichen Afrika Zoologie: 47. Geoffroy Saint-Hilaire's horseshoe bat. Synonyms: geoffroyi.
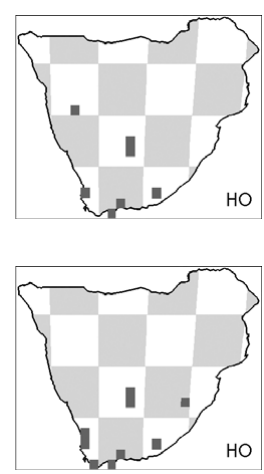

Rhinolophus darlingi Andersen, 1905. Ann. Mag. Nat. Hist., Series 7, 15: 70. Darling's horseshoe bat.

Type locality: Mazoe.

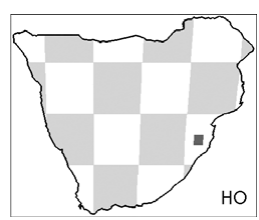


Rhinolophus fumigatus Rüppell, 1842. Mus. Senckenbergianum 3(2):

132. Rüppell's horseshoe bat.

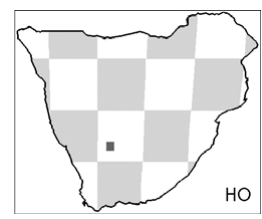

Rhinolophus simulator Andersen, 1904. Ann. Mag. Nat. Hist., Series 7, 14: 384. Bushveld horseshoe bat.

Type locality: Mazoe.

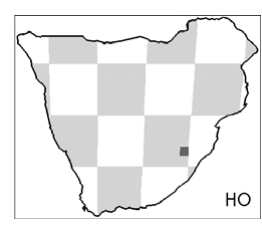

FAMILY: NYCTERIDAE

Nycteris thebaica Geoffroy Saint-Hilaire, 1818. Description des

Mammifères qui se trouvent en Egypte 2: 119. Egyptian slit-faced bat. Additional references: Gray et al. (1999).

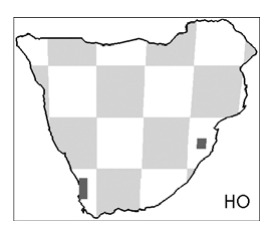

\section{FAMILY: MOLOSSIDAE}

Subfamily: Molossinae

Tadarida aegyptiaca Geoffroy Saint-Hilaire, 1818. Description des

Mammifères qui se trouvent en Egypte 2: 128. Egyptian free-tailed bat.

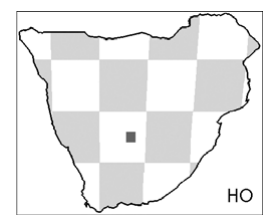

FAMILY: VESPERTILIONIDAE

Subfamily: Vespertilioninae

Eptesicus Rafinesque, 1820. Ann. Nature 1: 2.

Comments: distribution of material assigned only to genus has been disregarded because it may well belong to Neoromicia as presently understood.

Eptesicus hottentotus Smith, 1833. S. Afr. Quart. J., Series 2, 1: 59. Long-tailed serotine.

Type locality: Uitenhage.

Additional references: Hill and Harrison (1987); Kearney et al. (2002).

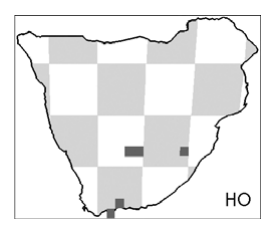

Neoromicia capensis Smith, 1829. Zool. J. 4: 435. Cape serotine.

Additional references: Riccucci and Lanza (2008).

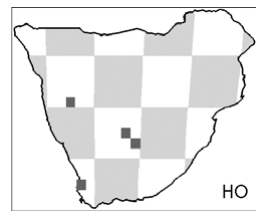


Scotophilus nigrita Schreber, 1775. Die Säugethiere in Abbildungen nach der Natur, mit Beschreibungen 1: 171. Giant house bat.

Synonyms: dinganii.

Additional references: Robbins (1978); Robbins et al. (1985).

Comments: volume 1 was published in 1775 (not 1774) according to the

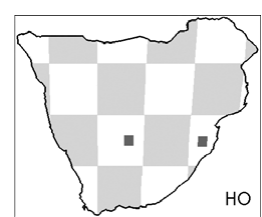
version obtained from the University of Heidelberg (http://digi.ub .uni-heidelberg.de/schreber1875textbd) and Vespertilio nigrita is listed on p. 190. Plate 58 appears in an undated volume containing plates 1-80 (www.biodiversitylibrary.org/item/97331\#page/279/mode/1up).

\section{Subfamily: Myotinae}

Myotis tricolor Temminck, 1832. In: Smuts, Dissertation Zoologica, Ennumerationem Mammalium Capensium: 106. Temminck's myotis. Type locality: Cape Town.

Additional references: Kearney et al. (2002).

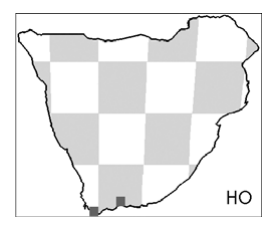

\section{Subfamily: Miniopterinae}

Miniopterus Bonaparte, 1837. Iconografia della Fauna Italica 1: fasc. 20. Additional references: Miller-Butterworth et al. (2007).

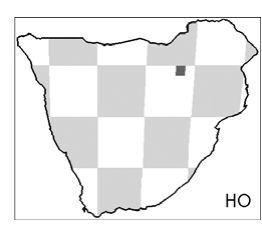

Miniopterus schreibersii Kuhl, 1817. Die Deutschen Fledermäuse: 14. Schreibers' long-fingered bat.

Additional references: Miller-Butterworth et al. (2005); Smith (1849). Comments: some of the material would perhaps now be assigned to Miniopterus natalensis, which was previously considered to be a subspecies of M. schreibersii.

Kervoula argentata Tomes, 1861. Proc. Zool. Soc. Lond. 1861: 32.

Damara woolly bat.

Type locality: Otjoro.
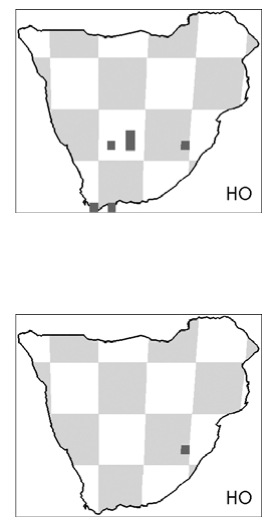

\section{ORDER: PHOLIDOTA}

FAMILY: MANIDAE

Subfamily: Smutsiinae

Smutsia Gray, 1865. Proc. Zool. Soc. Lond. 1865: 360.

Synonyms: Manis.

Additional references: Gaudin et al. (2009).

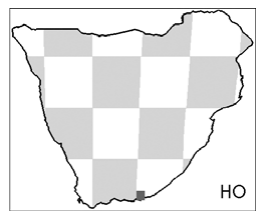


Smutsia temminckii Smuts, 1832. Dissertation Zoologica,

Ennumerationem Mammalium Capensium: 54. Ground pangolin. Synonyms: Manis.

Type locality: Latakou = Litakun.

Additional references: Gaudin (2010); Gaudin et al. (2009); Gray

(1865b); Smith (1849); Sundevall (1842).

\section{ORDER: CARNIVORA \\ Suborder: Feliformia}

FAMILY: FELIDAE

Subfamily: Felinae

Acinonyx jubatus Schreber, 1775. Die Säugethiere in Abbildungen nach der Natur, mit Beschreibungen 2(14): pl. 105 [1775], also see text 3 (22): 392 [1777]. Cheetah.

Type locality: Cape of Good Hope.

Additional references: Krausman and Morales (2005).

Comments: citation according to Wilson and Reeder (2005). Volume

3 was published in 1778 according to the version reproduced in the Biodiversity Heritage Library (www.biodiversitylibrary.org/item/ 135003\#page/120/mode/1 up) and Felis jubata is listed on p. 586. Plate 105 appears in an undated volume containing plates 81-165 (www.biodiversitylibrary.org/item/97341\#page/107/mode/1up).

Caracal caracal Schreber, 1776. Die Säugethiere in Abbildungen nach der Natur, mit Beschreibungen 3(16): pl. 110 [1776], see also text 3(24): 413, 587 [1777]. Caracal.

Synonyms: Felis.

Type locality: Table Mountain, near Cape Town.

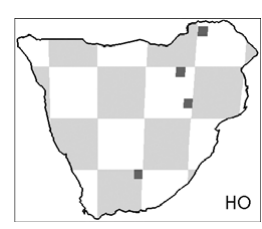

Additional references: Roberts (1926); Werdelin and Peigné (2010).

Comments: citation according to Wilson and Reeder (2005). Volume 3 was published in 1778 according to the version reproduced in the Biodiversity Heritage Library (www.biodiversitylibrary.org/item/ 135003\#page/141/mode/1 up) and Felis caracal is listed on p. 587. Plate 110 appears in an undated volume containing plates 81-165 (www.biodiversitylibrary.org/item/97341\#page/131/mode/1up).

Felis Linnaeus, 1758. Systema Naturae Regnum Animale, 10th edition, 1: 41 .

Comments: material identified as Felis sp. has not been mapped because, in many cases, this identification included what are currently ascribed to other genera such as Leptailurus and Caracal.

Felis nigripes Burchell, 1824. Travels in the Interior of Southern Africa 2: 592. Black-footed cat.

Type locality: implied country of the 'Bachapins', presumably in the capital Litákun = Letárkoon

Additional references: Roberts (1926); Renard et al. (2015).
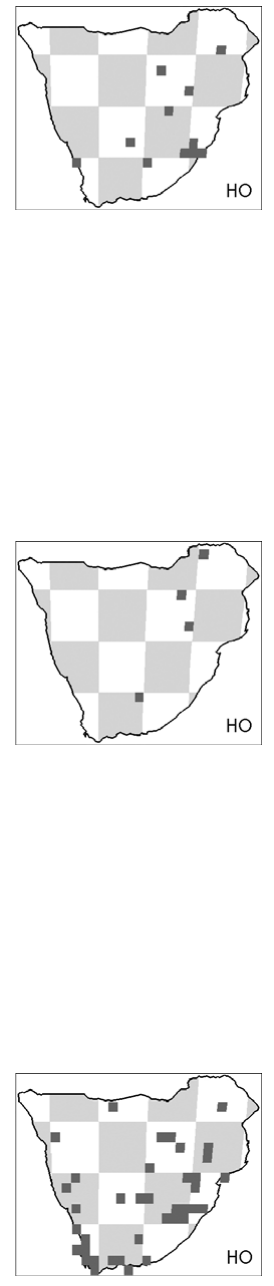
Felis silvestris Schreber, 1777. Die Säugethiere in Abbildungen nach der Natur, mit Beschreibungen 3(23): 397. Wild cat.

Synonyms: libyca.

Additional references: Grubb (2004).

Comments: volume 3 was published in 1778 according to the version reproduced in the Biodiversity Heritage Library (www.biodiversitylibrary.org/item/135003\#page/125/mode/1 up) and Felis catus is listed on p. 587. Plate 107 appears in an undated volume containing plates 81-165 (www.biodiversitylibrary.org/item/ 97341\#page/115/mode/1 up).

Leptailurus serval Schreber, 1776. Die Säugethiere in Abbildungen nach der Natur, mit Beschreibungen 3(16): pl. 108 [1776], see also text 3 (23): 407 [1777]. Serval.

Synonyms: Felis; spelaeus.

Type locality: restricted to the 'Cape region of South Africa'

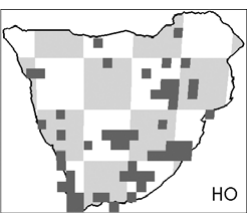
Additional references: Werdelin and Peigné (2010).

Comments: citation according to Wilson and Reeder (2005). Volume 3 was published in 1778 according to the version reproduced in the Biodiversity Heritage Library (www.biodiversitylibrary.org/item/ 135003\#page/135/mode/1 up) and Felis serval is listed on p. 587. Plate 107 appears in an undated volume containing plates 81-165 (www.biodiversitylibrary.org/item/97341\#page/125/mode/1up).

Subfamily: Pantherinae

Panthera leo Linnaeus, 1758. Systema Naturae Regnum Animale, 10th edition, 1: 41. Lion.

Additional references: Haas et al. (2005); Lacruz (2009).

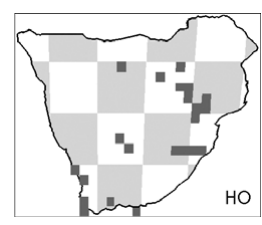

Panthera pardus Linnaeus, 1758. Systema Naturae Regnum Animale, 10th edition, 1: 41. Leopard

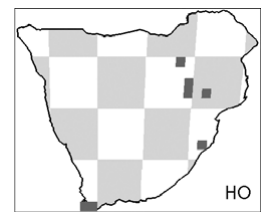

$\mathrm{HO}$

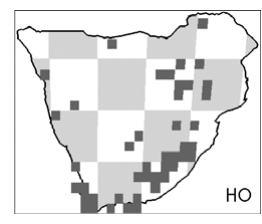

\section{FAMILY: HERPESTIDAE}

Atilax paludinosus Cuvier, 1829. Regnum animale in classes IX.

Distributum, sive Synopsis Methodica, Nouvelle Édition. 1: 158. Marsh mongoose.

Synonyms: Herpestes.

Type locality: Cape of Good Hope.

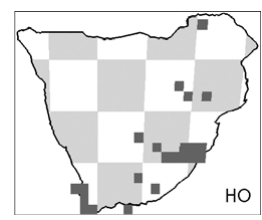

Additional references: Baker (1992); Cuvier (1824). 
Cynictis Ogilby, 1833. Proc. Zool. Soc. Lond. 1833: 48.

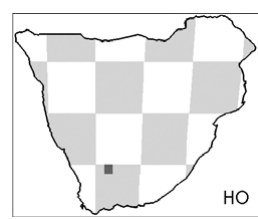

Cynictis penicillata Cuvier, 1829. Le Règne Animal distribué d'après son Organisation, Nouvelle Édition 1: 158. Yellow mongoose.

Synonyms: lepturus; ogilbyii.

Type locality: restricted to 'Uitenhage, CP'.

Additional references: Ewer (1956a, 1957a); Lundholm (1954); Roberts

(1932); Smith (1849); Taylor and Meester (1993).

Galerella Gray, 1864. Proc. Zool. Soc. Lond. 1864: 564.

Additional references: Werdelin and Peigné (2010).
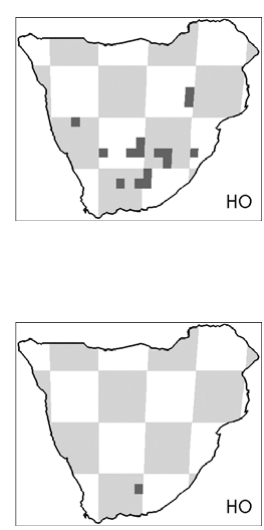

Galerella pulverulenta Wagner, 1839. Gelehrte. Anz. I. K. Bayer. Akad. Wiss. München 9: 426. Cape grey mongoose.

Type locality: Cape of Good Hope.

Additional references: Cavallini (1992); Lynch (1981).

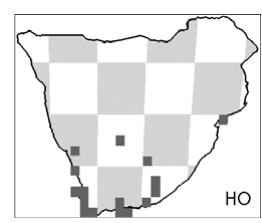

Galerella sanguinea Rüppell, 1835. Neue Wirbelthiere zu der Fauna von Abyssinien gehörig. 1: 27. Slender mongoose.

Synonyms: Herpestes sanguineus; punctulatus.

Additional references: Gray (1849); Roberts (1932); Taylor (1975).

Herpestes Illiger, 1811. Prodromus Systematis Mammaliam et

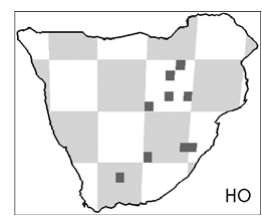
Avium: 135.

Additional references: Hendey (1973a); Werdelin and Peigné (2010).

Comments: this genus has not been mapped because material was identified at a time when Galerella, as currently understood, was not recognised as a separate genus.

Herpestes ichneumon Linnaeus, 1758. Systema Naturae Regnum Animale, 10th edition, 1: 43. Egyptian mongoose.

Synonyms: Ichneumon ratlamuchi; Herpestes badius.

Additional references: Smith $(1838,1849)$.

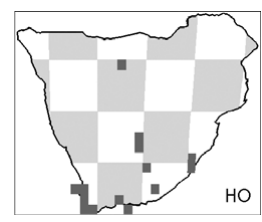

Ichneumia albicauda Cuvier, 1829. Le Règne Animal distribué d'après son

Organisation, Nouvelle Édition, 1: 158. White-tailed mongoose.

Additional references: Geoffroy Saint-Hilaire (1837); Taylor (1972).

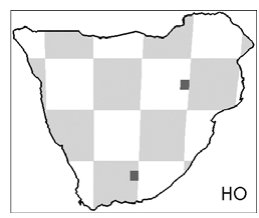


Mungos mungo Gmelin, 1788. In: Linnaeus, C. Systema Naturae 1, 13th edition, 1: 84. Banded mongoose.

Type locality: believed to be eastern part of South Africa, (former) Cape Province.

Rhynchogale melleri Gray, 1864. Proc. Zool. Soc. Lond. 1864: 575. Meller's mongoose.
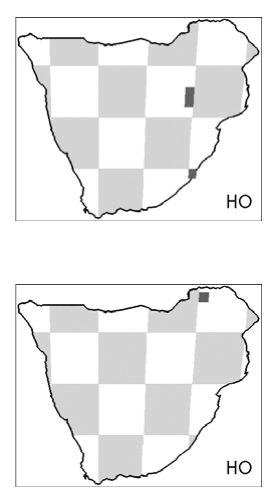

Suricata suricatta Schreber, 1776. Die Säugethiere in Abbildungen nach der Natur, mit Beschreibungen: pl. 117 [1776]. Meerkat.

Type locality: restricted to 'Deelfontein'.

Additional references: Van Staaden (1994).

Comments: citation according to Wilson and Reeder (2005). Version of text

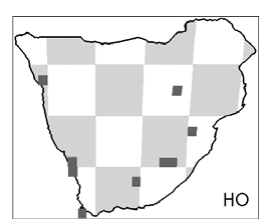
volume 3 at www.biodiversitylibrary.org/item/135003\#page/5/mode/ 1 up is dated 1778, while volume in which the plate appears is undated (www.biodiversitylibrary.org/item/97341\#page/1/mode/1 up).

\section{FAMILY: HYAENIDAE \\ Subfamily: Hyaeninae}

Crocuta crocuta Erxleben, 1777. Systema Regni Animales per Classes 1: 578. Spotted hyaena.

Synonyms: spelaea.

Additional references: Broom (1939c); Turner (1984).

Hyaena Brisson, 1762. Regnum animale in classes IX. Distributum, sive Synopsis Methodica, 2nd edition: 168.

Comments: this material may well be Parahyaena as presently understood.

Parahyaena brunnea Thunberg, 1820. K. Svenska Vet.-Akad. Handl. 8: 59. Brown hyaena.

Synonyms: Hyaena.

Type locality: Cape of Good Hope.

Additional references: Grubb (2004); Hendey (1973a); Mills (1982);
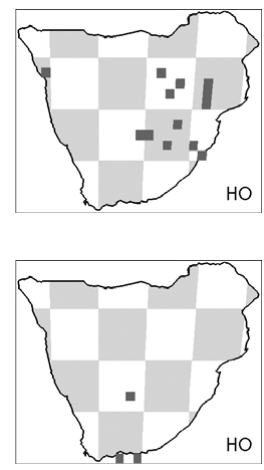

Werdelin and Peigné (2010).

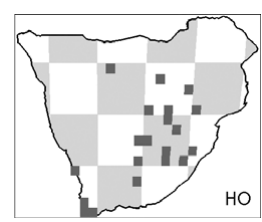

Subfamily: Protelinae

Proteles cristata Sparrman, 1783. Resa Goda-Hopps-Udden, Soedra Polkretsen Och Omkring Jordklotet 1: 581. Aardwolf.

Synonyms: cristatus; lalandii.

Type locality: listed as 'Near Little Fish River, Somerset East'.

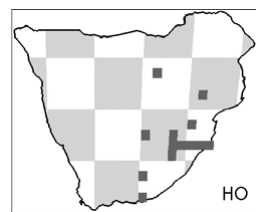


FAMILY: VIVERRIDAE

Subfamily: Viverrinae

Civettictis civetta Schreber, 1776. Die Säugethiere in Abbildungen nach der Natur, mit Beschreibungen 3(16): pl. 111 [1776]; see also text 3(24): 418, 3: index, p. 587 [1777]. African civet.

Synonyms: Viverra.

Additional references: Ray (1995).

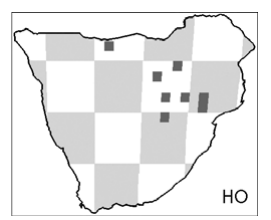

Comments: citation according to Wilson and Reeder (2005). Volume 3 was published in 1778 according to the version reproduced in the Biodiversity Heritage Library (www.biodiversitylibrary.org/item/ 135003\#page/146/mode/1 up) and Viverra civetta is listed on p. 587. Plate 111 appears in an undated volume containing plates 81-165 (www.biodiversitylibrary.org/item/97341\#page/137/ mode/1up).

Genetta Cuvier, 1817. Le Règne Animal distribué d'après son Organisation 1: 156.

Additional references: De Meneses Cabral (1966); Gaubert et al. (2005).

Genetta genetta Linnaeus, 1758. Systema Naturae Regnum Animale, 10th edition, 1: 45. Common genet.

Additional references: Gaubert et al. (2005); Larivière and Calzada (2001).
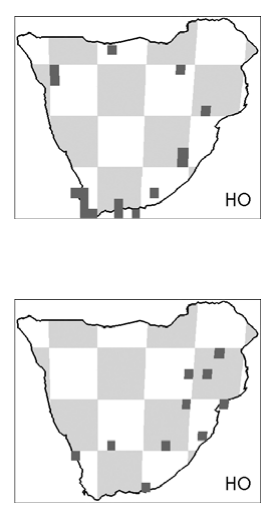

Genetta maculata Gray, 1830. Spicil. Zool. 2: 9. Rusty-spotted genet. Additional references: Crawford-Cabral and Fernandes (2001); Gaubert et al. (2003a, 2003b, 2005); Grubb (2004); Koehler and Richardson (1990).

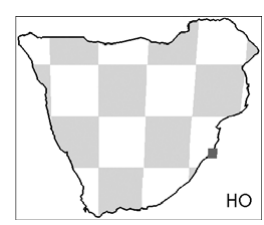

Genetta tigrina Schreber, 1776. Die Säugethiere in Abbildungen nach der Natur, mit Beschreibungen 3(17): pl. 114 [1776]; see also text 3(25): 425 [1777]. Cape genet.

Type locality: Cape of Good Hope.

Additional references: Gaubert et al. (2005); Grubb (2004).

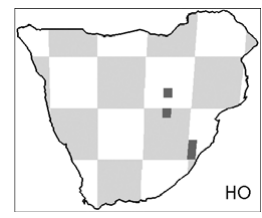

Comments: citation according to Wilson and Reeder (2005). Volume 3 was published in 1778 according to the version reproduced in the Biodiversity Heritage Library (www.biodiversitylibrary.org/item/ 135003\#page/153/mode/1 up) and Viverra tigrina is listed on p. 587. Plate 114 appears in an undated volume containing plates 81-165 (www.biodiversitylibrary.org/item/97341\#page/147/ mode/1up). 


\section{Suborder: Caniformia \\ FAMILY: CANIDAE \\ Subfamily: Caninae}

Canis Linnaeus, 1758. Systema Naturae Regnum Animale, 10th edition, 1: 38.

Additional references: Werdelin and Peigné (2010).

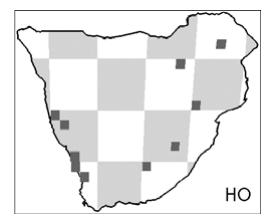

Canis adustus Sundevall, 1846. Ofv. K. Svenska Vet.-Akad. Forhandl. 3:

121. Side-striped jackal.

Type locality: 'Magaliesberg'.

Canis lupus Linnaeus, 1758. Systema Naturae Regnum Animale, 10th edition, 1: 39. Wolf (including domestic dog).

Synonyms: familiaris.
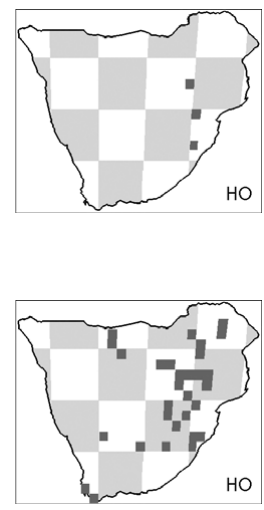

Canis mesomelas Schreber, 1775. Die Säugethiere in Abbildungen nach der Natur, mit Beschreibungen 2(14): pl. 95 [1775], text 3 (21): 370 [1776], 586 [1777]. Black-backed jackal.

Type locality: Cape of Good Hope.

Comments: citation according to Wilson and Reeder (2005). Volume

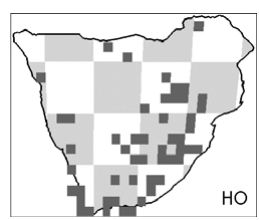

3 was published in 1778 according to the version reproduced in the Biodiversity Heritage Library (www.biodiversitylibrary.org/item/ 135003\#page/98/mode/1 up) and Canis mesomelas is listed on p. 586. Plate 95 appears in an undated volume containing plates 81-165 (www.biodiversitylibrary.org/item/97341\#page/57/ mode/1up).

Lycaon pictus Temminck, 1820. Ann. Gen. Sci. Phys. 3: 46, pl. 35. African wild dog.

Type locality: 'á la côte de Mosambique'.

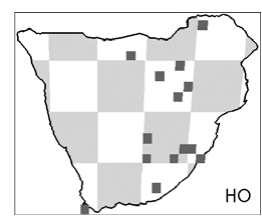

Otocyon megalotis Desmarest, 1822. Mammalogie ou descriptions des espèces de mammifères, Part 2 (suppl.): 538. Bat-eared fox.

Type locality: Cape of Good Hope.

Additional references: Clark (2005).

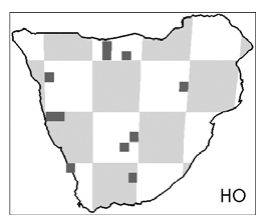


Vulpes chama Smith, 1833. S. Afr. Quart. J., Series 2, 2: 89. Cape fox. Type locality: fixed as 'Port Nolloth'.

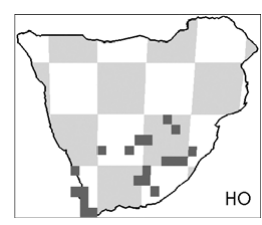

\section{FAMILY: MUSTELIDAE}

Subfamily: Lutrinae

Aonyx capensis Schinz, 1821. In: Cuvier, Das Thierreich eingetheilt nach dem Bau der Thiere 1: 211. African clawless otter.

Synonyms: robustus.

Type locality: (former) Cape Province.

Additional references: Brink (1987); Dreyer and Lyle (1931); Ewer

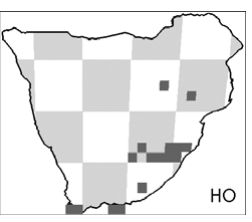

(1958c); Larivière (2001a).

Hydrictis maculicollis Lichtenstein, 1835. Arch. Naturgesch. 1: 89.

Spotted-necked otter.

Synonyms: Lutra.

Type locality: 'Kafferlandes am östlichen Abhange der Bambusberge'.

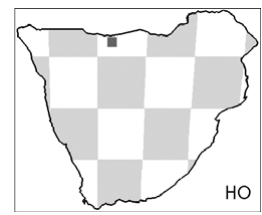

Subfamily: Mustelinae

Ictonyx Kaup, 1835. Das Thierreich in seinen Hauptformen 1: 352.

Type locality: fixed as 'Cape of Good Hope'.

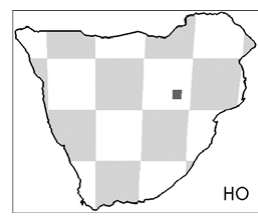

Ictonyx striatus Perry, 1811. Arcana, or, the Museum of Natural History: Containing the Most Recent Discovered Objects, Signature Y: Fig. 41 [1810]. Striped polecat.

Type locality: fixed as 'Cape of Good Hope'.

Additional references: Larivière (2002); Roberts (1932).

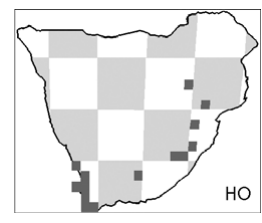

Mellivora capensis Schreber, 1776. Die Säugethiere in Abbildungen nach der Natur, mit Beschreibungen 3(18): pl. 125 [1776], see also text 3 (26): 450 [1777]. Honey badger.

Synonyms: sivalensis.

Type locality: Cape of Good Hope.

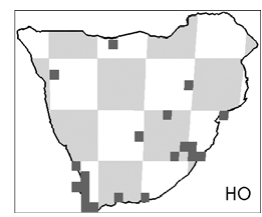

Additional references: O'Regan et al. (2013).

Comments: citation according to Wilson and Reeder (2005). Volume 3 was published in 1778 according to the version reproduced in the Biodiversity Heritage Library (www.biodiversitylibrary.org/item/ 135003\#page/178/mode/1 up) and Viverra capensis is listed on p. 588. Plate 125 appears in an undated volume containing plates 81-165 (www.biodiversitylibrary.org/item/97341\#page/191/ mode/1 up). 


\section{ORDER: PERISSODACTYLA}

FAMILY: EQUIDAE

Equus Linnaeus, 1758. Systema Naturae Regnum Animale, 10th edition, 1: 73. Additional references: Brink (1994); Eisenmann and Baylac (2000); Thackeray (2010); Wells (1959a).

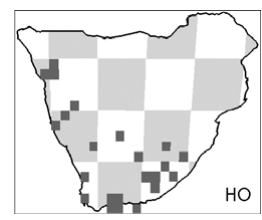

Equus asinus Linnaeus, 1758. Systema Naturae Regnum Animale, 10th edition, 1: 73. Ass (including donkey).

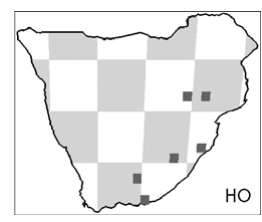

Equus burchellii Gray, 1824. Zool. J. 1: 247. Burchell's zebra. Synonyms: lylei; platyconus; simplicissimus.

Type locality: now identified as Little Klibbolikhonni Fontein. Additional references: Churcher (1970); Churcher and Watson (1993); Grubb (1981); Mendrez (1966); Reynolds and Bishop (2006); Van Hoepen (1930a).

Equus caballus Linnaeus, 1758. Systema Naturae Regnum Animale, 10th edition, 1: 73. Horse (domestic horse).
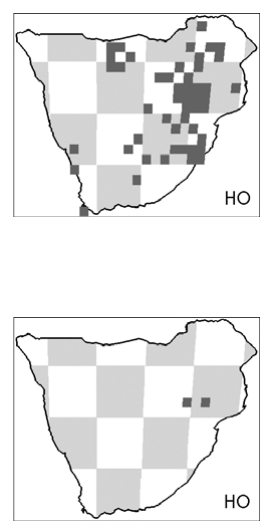

†Equus capensis Broom, 1909. Ann. S. Afr. Mus. 7: 281.

Synonyms: Kolpohippus; cawoodi; fowleri?; gigas?; harrisi; helmei; kuhni; plicatus; poweri; zietsmani.

Type locality: Ysterplaat.

†Equus quagga Boddaert, 1785. Elenchus Animalium 1: 160. Quagga. Type locality: locality of paralectotype now identified as Seekoei River. Additional references: Bernor et al. (2010); Churcher (1970); Eisenmann and Brink (2000); Haughton (1932a); Klein and Cruz-Uribe (1999); Lundholm (1951).
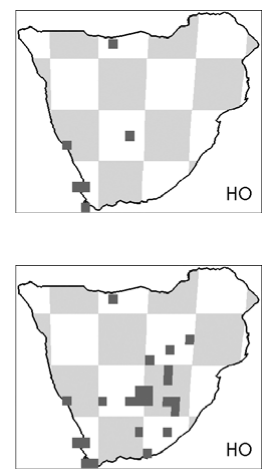

Comments: the version of this book reproduced in the Biodiversity Heritage Library (www.biodiversitylibrary.org/item/89677\#page/7/ mode/1up) is dated 1784 .

Equus zebra Linnaeus, 1758. Systema Naturae Regnum Animale, 10th edition, 1: 74. Mountain zebra.

Synonyms: Hippotigris.

Type locality: since restricted to Perdekop.

Additional references: Bernor et al. (2010); Fraas (1907); Hooijer (1945);

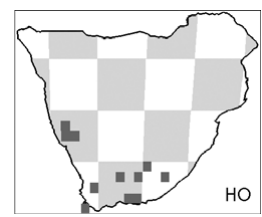
Lundholm (1952); Penzhorn (1988). 


\section{FAMILY: RHINOCEROTIDAE}

Subfamily: Rhinocerotinae

Ceratotherium simum Burchell, 1817. Bull. Sci. Soc. Philom. Paris

1817-1819: 97. White rhinoceros.

Synonyms: Diceros; Rhinoceros; simus.

Type locality: since identified as Chue Spring = Heuningvlei.

Additional references: Geraads (2010a); Gray (1867); Groves (1972);

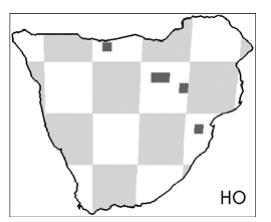

Hooijer (1959, 1973); Hooijer and Singer (1960); Smith (1849).

Diceros bicornis Linnaeus, 1758. Systema Naturae Regnum Animale, 10th edition, 1: 56 . Black rhinoceros.

Synonyms: Opsiceros simplicidens.

Type locality: now identified as Cape of Good Hope.

Additional references: Gray (1867); Hillman-Smith and Groves (1994);

Hooijer (1959, 1973); Hooijer and Singer (1960); Scott (1907).

\section{ORDER: ARTIODACTYLA}

FAMILY: SUIDAE

Subfamily: Suinae

Phacochoerus Cuvier, 1826. Dict. Sci. Nat. 39: 383.

Comments: the Holocene material is likely to be $P$. africanus since this is the only species currently recognised in the sub-region.

Phacochoerus africanus Gmelin, 1788. Linnaeus, C. Systema Naturae, 13th edition, 1: 220. Common warthog.

Synonyms: aethiopicus; dreyeri?; helmei; laticolumnatus; venteri.

Additional references: Cooke (1949b); Ewer (1956b, 1957b); Grubb and d'Huart (2010); Pia (1930).
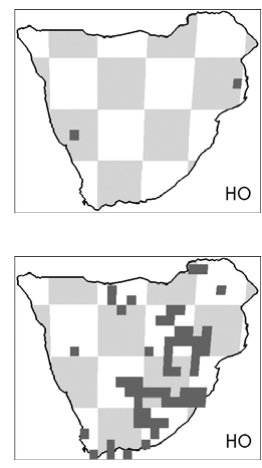

Potamochoerus larvatus Cuvier, 1822. Mem. Mus. Hist. Nat. Paris 8: 447. Bush-pig.

Synonyms: Koiropotamus; porcus.

Additional references: Grubb (2004); Hopwood (1934).

Sus Linnaeus, 1758. Systema Naturae Regnum Animale, 10th edition, 1: 49 .

Comments: it is most likely that this material should be assigned to Sus scrofa, given that this is the only currently accepted species of Sus in southern Africa.
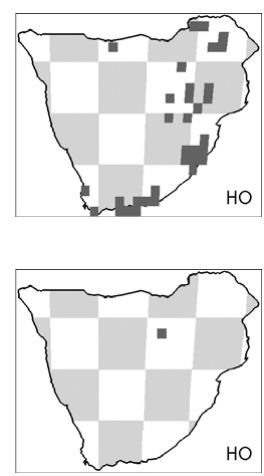

Sus scrofa Linnaeus, 1758. Systema Naturae Regnum Animale, 10th edition, 1: 49. Wild boar (including domestic pig).

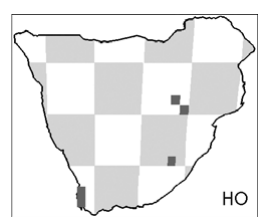




\section{FAMILY: HIPPOPOTAMIDAE}

Hippopotamus amphibius Linnaeus, 1758. Systema Naturae Regnum Animale, 10th edition, 1: 74. Common hippopotamus.

Synonyms: capensis; poderosus.

Additional references: Fraas (1907); Hooijer and Singer (1961); Scott (1907); Smith (1849).

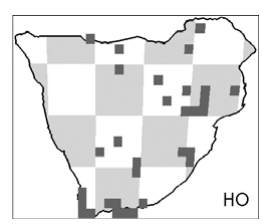

FAMILY: GIRAFFIDAE

Subfamily: Giraffinae

Giraffa camelopardalis Linnaeus, 1758. Systema Naturae Regnum Animale, 10th edition, 1: 66. Giraffe.

Additional references: Dagg (1971); Harris et al. (2010); Singer and Boné (1960).

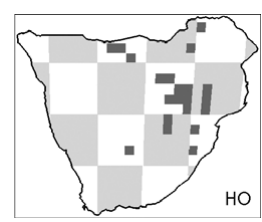

FAMILY: BOVIDAE

Subfamily: Aepycerotinae

Aepyceros melampus Lichtenstein, 1812. Reisen im südlichen Africa in en Jahren 1803, 1804, 1805 und 1806 2: pl. 4 opp. p. 544. Impala.

Type locality: now identified as Khosis.

Additional references: Reynolds (2010a).

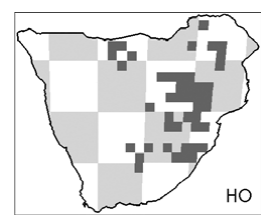

Subfamily: Alcelaphinae

Alcelaphus De Blainville, 1816. Bull. Sci. Soc. Philom. Paris 1816: 75.

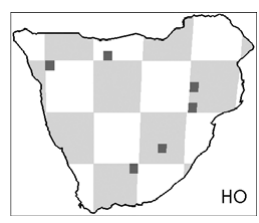

Alcelaphus buselaphus Pallas, 1766. P.S. Pallas Medecinae Doctoris Miscellanea Zoologica: 7. Hartebeest.

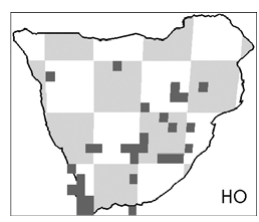

Alcelaphus caama Geoffroy Saint-Hilaire, 1803. Catalogue des Mammifères du Muséum National d'Histoire Naturelle: 269. Red hartebeest.

Synonyms: Antilope; Bubalus; bubalis; buselaphus caama.

Type locality: since restricted to syntype locality Steynsburg.

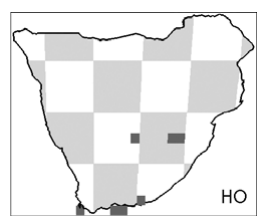

Additional references: Gray (1850a, 1850b); Grubb (2004); Hoffman (1953); Smith (1849). 
Connochaetes Lichtenstein, 1812. Mag. Ges. Naturf. Fr. Berlin 6: 142. Synonyms: Gorgon.

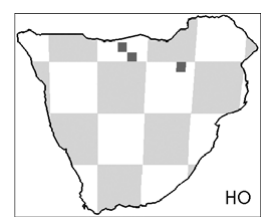

Connochaetes gnou Zimmermann, 1780. Geographische Geschichte des Menschen und der Allgemein Verbreiteten Vierfüssigen Thiere 2: 102. Black wildebeest.

Synonyms: laticornutus.

Type locality: since selected as Agterbruintjieshoogte.

Additional references: Brink (1993, 2005); Gentry (2010); Gray (1850a, 1850b); Von Richter (1974).

Comments: Gentry (2010) proposes that Connochaetes laticornutus should be retained as a distinct species.

Connochaetes taurinus Burchell, 1824. Travels in the Interior of Southern Africa 2: 278 (footnote). Blue wildebeest.

Synonyms: Catoblepas.

Type locality: apparently 'Kosi Fountain', but lectotype came from 'Chue Spring, Maadji Mtn [Klein Heuningvlei]'. Additional references: Smith (1849).

Damaliscus Sclater and Thomas, 1894. The Book of Antelopes 1: 51. Additional references: Fraas (1907).
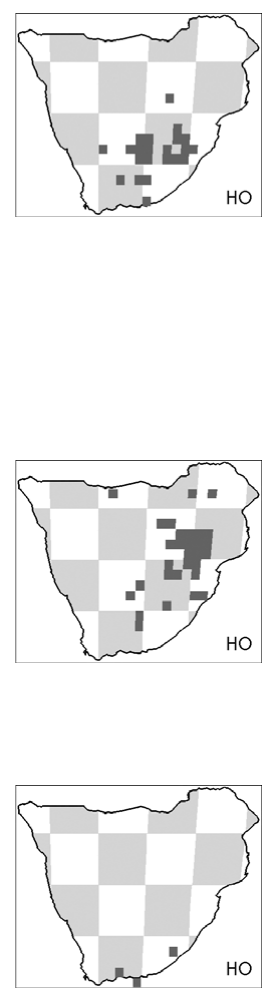

Damaliscus lunatus Burchell, 1824. Travels in the Interior of Southern Africa 2: 334. Common tsessebe.

Synonyms: Acronotus; Bubalus.

Type locality: 'Makkwarin' (Matlhwareng) River.

Additional references: Cotterill (2003); Gray (1850a, 1850b); Groves and

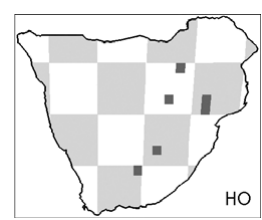

Grubb (2011); Smith (1849).

Damaliscus pygargus Pallas, 1767. Spicil. Zool. 1: 10. Bontebok. Synonyms: albifrons; dorcas phillipsi; dorcas dorcas; 'hipkini'.

Type locality: since restricted to Swart River.

Additional references: Faith et al. (2012); Gentry (2010); Gray (1850a, 1850b); Groves and Grubb (2011); Grubb (2004); Vrba (1997).

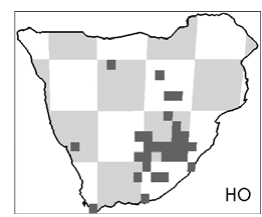

Comments: according to Vrba (1997), Wells intended to name

Damaliscus 'hipkini', but never did. Groves and Grubb (2011) propose that Damaliscus phillipsi (blesbok) be a full species separate from D. pygargus (bontebok). 
Subfamily: Antilopinae

†Antidorcas australis Hendey and Hendey, 1968. Ann. S. Afr. Mus. 52(2): 56.

Synonyms: Marsupialis australis.

Type locality: Swartklp.

Additional references: Gentry (2010); Peters and Brink (1992); Vrba

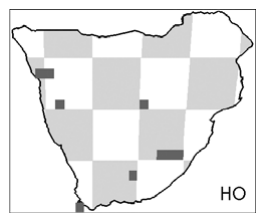

(1973).

†Antidorcas bondi Cooke and Wells, 1951. S. Afr. J. Sci. 47: 207.

Synonyms: Gazella.

Type locality: Chelmer.

Additional references: Gentry (2010); Plug and Peters (1991); Vrba (1973).

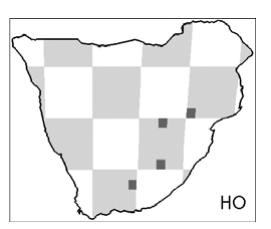

Antidorcas marsupialis Zimmermann, 1780. Geographische Geschichte des Menschen und der Allgemein Verbreiteten Vierfüssigen Thiere 2: 427. Springbok.

Type locality: since restricted to 'Cape Colony [Cape Province]'

References: Cain et al. (2004); Gentry (2010); Gray (1850a, 1850b); Plug

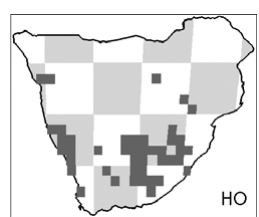
and Peters (1991); Vrba (1970).

Madoqua Ogilby, 1836. Proc. Zool. Soc. Lond. 1836: 137.

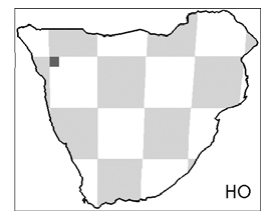

Neotragus moschatus Von Dueben, 1846. In: Sundevall, Ofv. K. Svenska Vet.-Akad. Forhandl. 3(7): 221. Suni.

Synonyms: Nesotragus.

Additional references: Gray (1850a, 1850b).

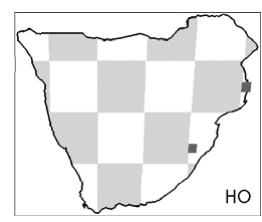

Oreotragus oreotragus Zimmermann, 1783. Geographische Geschichte des Menschen und der Allgemein Verbreiteten Vierfüssigen Thiere 3: 269. Klipspringer.

Synonyms: Palaeotragiscus longiceps; major.

Type locality: now known to be False Bay.

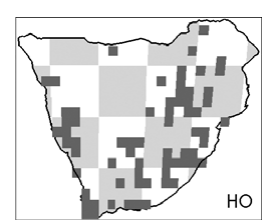

Additional references: Broom (1934); Gentry (2010); Gray (1850a, 1850b); Watson and Plug (1995); Wells (1951).

Ourebia ourebi Zimmermann, 1783. Geographische Geschichte des Menschen und der Allgemein Verbreiteten Vierfüssigen Thiere 3: 268. Oribi.

Type locality: since restricted to Bruintjieshoogte. Additional references: Gray (1850a, 1850b).

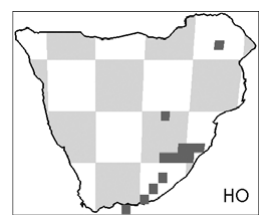


Raphicerus Smith, 1827. In: Griffith et al., The Animal Kingdom Arranged in Conformity with its Organization by the Baron Cuvier with Additional Descriptions 5: 342.

Additional references: Klein (1976c).

Raphicerus campestris Thunberg, 1811. Mem. Acad. Imp. Sci. St. Petersbourg 3: 313. Steenbok.

Type locality: since selected as Malmesbury District.

Additional references: Gray (1850a, 1850b).

Raphicerus melanotis Thunberg, 1811. Mem. Acad. Imp. Sci. St.

Petersbourg 3: 312. Cape grysbok.

Type locality: since selected as Cape Peninsula.

Additional references: Gray (1850a, 1850b).

Raphicerus sharpei Thomas, 1896. Proc. Zool. Soc. Lond. 1896: 795, pl. 34. Sharpe's grysbok.

Synonyms: melanotis sharpei.
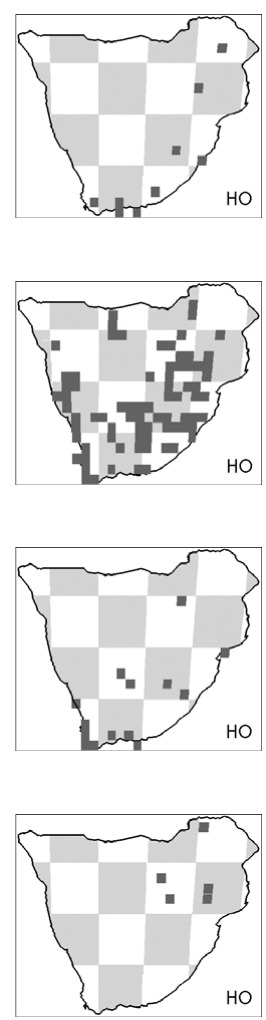

Subfamily: Bovinae

Bos taurus Linnaeus, 1758. Systema Naturae Regnum Animale, 10th edition, 1: 71. Aurochs (including domestic cattle).

Additional references: Horsburgh et al. (2016).

Syncerus Hodgson, 1847. J. Asiatic Soc. Bengal, Series 2, 16: 709.

Synonyms: Bos; Bubalis; Pelorovis.

Additional references: Martínez-Navarro et al. (2007).
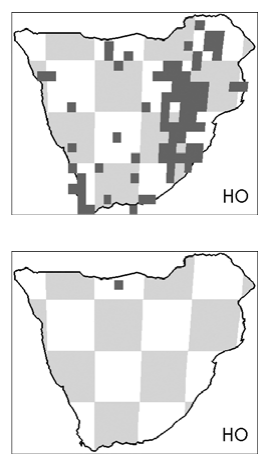

Syncerus caffer Sparrman, 1779. K. Svenska Vet.-Akad. Handl. 40: 79. African buffalo.

Synonyms: Bubalis; andersoni.

Type locality: now restricted to Sundays River.

Additional references: Geraads (1992); Scott (1907).

Taurotragus Wagner, 1855. In: Schreber, Die Säugethiere in Abbildungen nach der Natur, mit Beschreibungen 5: 438.

Comments: given that this genus is monospecific, it is to be assumed that this material should be assigned to T. oryx.
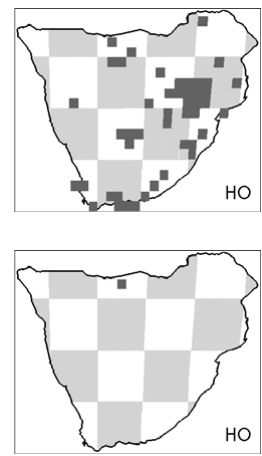
Taurotragus oryx Pallas, 1766. P.S. Pallas Medecinae Doctoris Miscellanea Zoologica: 9. Common eland.

Synonyms: Antilope; Boselaphus; Tragelaphus; oreas.

Type locality: restricted to near Cape Town.

Additional references: Gray (1850a, 1850b); Pappas (2002); Smith

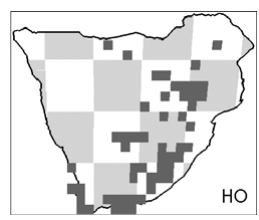

(1849); Willows-Munro et al. (2005).

Tragelaphus angasii Angas, 1848. Proc. Zool. Soc. Lond. 1848: 89. Nyala.

Type locality: 'Hills that border: upon the northern shores of St Lucia Bay'.

Additional references: Gray (1850a, 1850b); Grubb (2004); Willows-

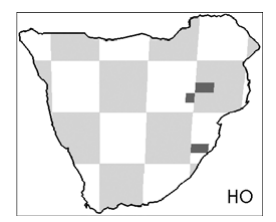
Munro et al. (2005).

Tragelaphus scriptus Pallas, 1766. P.S. Pallas Medecinae Doctoris Miscellanea Zoologica: 8. Bushbuck.

Additional references: Gray (1850a, 1850b); Willows-Munro et al. (2005).

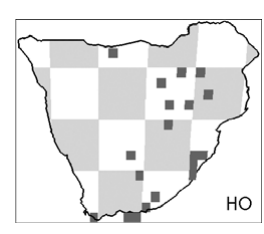

Tragelaphus spekeii Speke, 1863. Journal of the Discovery of the Source of the Nile: 223 (footnote). Sitatunga.

Additional references: Grubb (2004); Willows-Munro et al. (2005).

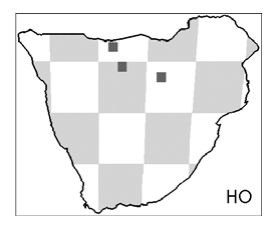

Tragelaphus strepsiceros Pallas, 1766. P.S. Pallas Medecinae Doctoris Miscellanea Zoologica: 9. Greater kudu.

Synonyms: Damalis; Strepsiceros; capensis.

Type locality: restricted to eastern part of Western Cape Province. Additional references: Smith (1849); Willows-Munro et al.

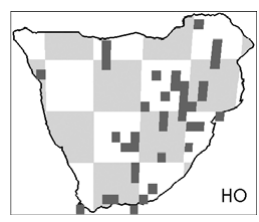
(2005).

\section{Subfamily: Caprinae}

Capra hircus Linnaeus, 1758. Systema Naturae Regnum Animale, 10th edition, 1: 68. Goat.

Additional references: Badenhorst and Plug (2003); Horsburgh et al. (2016).

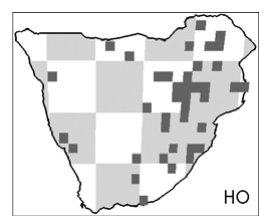

Ovis aries Linnaeus, 1758. Systema Naturae Regnum Animale, 10th edition, 1: 70. Red sheep (including domestic sheep).

Additional references: Horsburgh and Rhines (2010); Horsburgh et al. (2016).

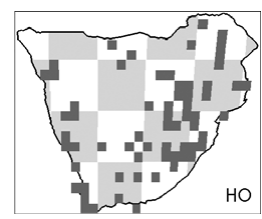


Subfamily: Cephalophinae

Cephalophus Smith, 1827. In: Griffith et al., The Animal Kingdom Arranged in Conformity with its Organization by the Baron Cuvier 5: 344.

Comments: material only identified to genus level has not been mapped because this could include specimens assignable to Philantomba as currently understood

Cephalophus natalensis Smith, 1834. S. Afr. Quart. J., Series 2, 2: 217. Red duiker.

Type locality: 'Port Natal' = Durban.

Additional references: Gray (1850a, 1850b); Smith (1849).

Philantomba monticola Thunberg, 1789. Resa uti Europa Africa, Asia, forrattad aren 1770-1779 2: 66. Blue duiker.

Synonyms: Cephalophus; bicolor; caeruleus.

Type locality: since identified as Langkloof.

Additional references: Gray (1850a, 1850b, 1862a, 1862b); Jansen van Vuuren (1999).

Sylvicapra grimmia Linnaeus, 1758. Systema Naturae Regnum Animale, 10th edition, 1: 70. Bush duiker.

Type locality: now known to be Cape Town.

Additional references: Gentry (2010); Gray (1850a, 1850b).
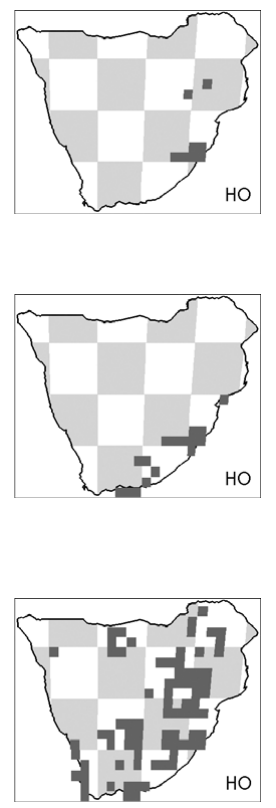

Subfamily: Hippotraginae

Hippotragus Sundevall, 1845. Ofv. K. Svenska Vet.-Akad. Förhand. 1845: 31.

Additional references: Commission on Zoological Nomenclature (2003); Grubb (2004).

Hippotragus equinus Geoffroy Saint-Hilaire, 1803. Catalogue des Mammifères du Muséum National d'Histoire Naturelle: 259. Roan antelope.

Synonyms: Aigoceros; Antilope; osanne.

Type locality: now thought to be Plettenberg Bay.

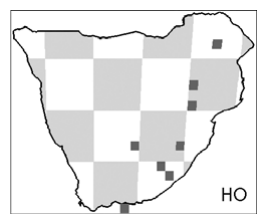

Additional references: Gray (1850a, 1850b); Grubb (2004); Smith (1849).

†Hippotragus leucophaeus Pallas, 1766. P.S. Pallas Medecinae Doctoris Miscellanea Zoologica: 4. Blaaubok, blue antelope.

Synonyms: problematicus.

Type locality: since restricted to Swellendam District.

Additional references: Broom (1949b); Cooke (1947); Gentry (2010);

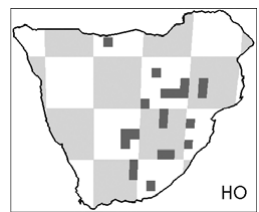

Klein (1974a). 
Hippotragus niger Harris, 1838. Proc. Zool. Soc. Lond. 1838: 2. Sable antelope.

Synonyms: harrisi.

Type locality: since specified as Magaliesberg near Krugersdorp and Rustenburg.

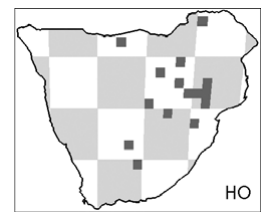

Additional references: Gray (1850a, 1850b); Harris (1838a).

Oryx gazella Linnaeus, 1758. Systema Naturae Regnum Animale, 10th edition, 1: 69. Gemsbok.

Type locality: understood to be South Africa.

Additional references: Gray (1850a, 1850b).

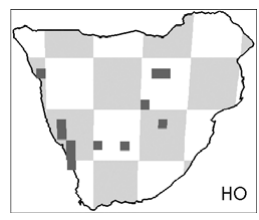

Subfamily: Reduncinae

Kobus ellipsiprymnus Ogilby, 1833. Proc. Zool. Soc. Lond. 1833: 47. Waterbuck.

Synonyms: Aigoceros.

Type locality: since restricted to Gaborone.

Additional references: Birungi and Arctander (2001); Gray (1850a, 1850b); Smith (1849).

Kobus leche Gray, 1850. Gleanings from the Menagerie and Aviary at Knowsley Hall 2: 23. Lechwe.

Synonyms: Onotragus; venterae.

Type locality: since identified as Botletle River, near Lake Ngami. Additional references: Birungi and Arctander (2001).

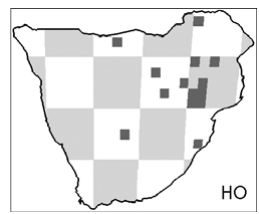

Pelea capreolus Forster, 1790. Le Vaillant's Reise in das Innere von Afrika 1: 71. Vaal rhebok.

Type locality: now specified as Houhoek Pass.

Additional references: Birungi and Arctander (2001); Gray (1850a, 1850b).

Redunca Smith, 1827. In: Griffith et al., The Animal Kingdom Arranged in Conformity with its Organization by the Baron Cuvier 5: 337

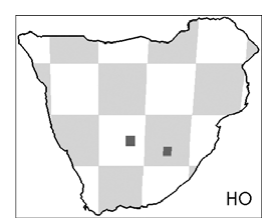

Redunca arundinum Boddaert, 1785. Elenchus Animalium 1: 141. Southern reedbuck.

Type locality: since selected as Bethulie.

Additional references: Gentry (2010); Gray (1850a, 1850b).

Comments: the version of this book reproduced in the Biodiversity
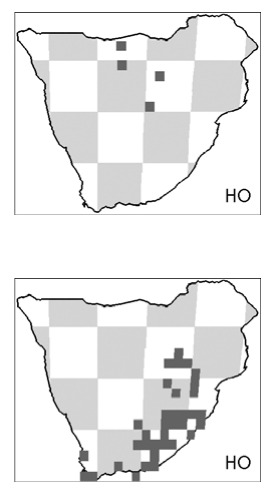

Heritage Library (www.biodiversitylibrary.org/item/89677\#page/7/

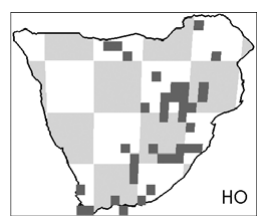

mode/1 up) is dated 1784 . 
Redunca fulvorufula Afzelius, 1815. Nova Acta Reg. Soc. Sci. Upsala 7:

250. Mountain reedbuck.

Synonyms: Cervicapra.

Type locality: restricted to eastern North Cape Province.

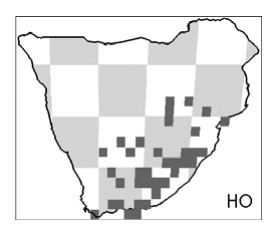

\subsection{HOLOCENE SITES}

So far, records for the Holocene have come from 414 sites (Figure 6.1). These cover much of the region but, as during the Pleistocene, show a gap in what are today arid, sparsely occupied parts of the region. Possible reasons for this gap in the record were mentioned with respect to the Pleistocene and may well apply also to the Holocene. In the Holocene, however, since all material comes from human occupation sites the existence of suitable sites for this purpose must have taken precedence, with bone preservation and attention by archaeologists being secondary explanations. Indeed, variation in the numbers of mammalian taxa represented suggests that this is the case.

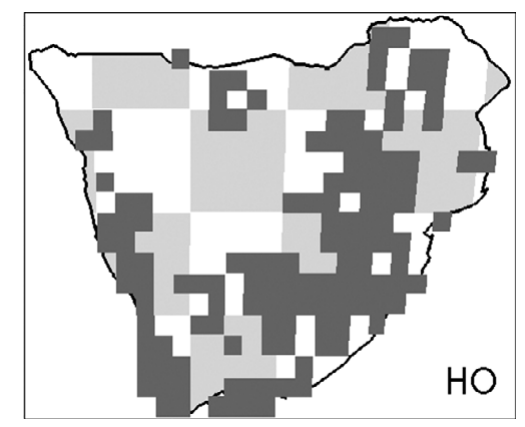

Figure 6.1 Location of Holocene sites.

/hei-/khomas. See Vaalhoek.

2229AD5 (2228:2925). Taxa: Aepyceros melampus; Bos taurus; Canis lupus; Capra hircus; Equus burchellii; Hippotragus equinus; Hystrix africaeaustralis; Kobus ellipsiprymnus; Lepus saxatilis; Ovis aries; Panthera pardus; Phacochoerus africanus; Pronolagus randensis; Sylvicapra grimmia; Syncerus caffer. References: Plug (2000).

2329CD (2329CD). Taxa: Bos taurus; Equus burchellii; Hystrix africaeaustralis; Oreotragus oreotragus; Procavia capensis. References: Loubser (1994).

Aar I and II (2643:1631). Taxa: Equus zebra cf.; Lepus; Oreotragus oreotragus; Oryx gazella; Petromus typicus cf.; Procavia. References: Cruz-Uribe and Klein (1981-1983); Thackeray (1979).

Abbot's Cave (3127:2439). Taxa: Alcelaphus buselaphus; Antidorcas australis; Antidorcas bondi; Atilax paludinosus; Bos taurus; Canis mesomelas; Capra hircus; Caracal caracal; Connochaetes gnou; Connochaetes taurinus cf.; Crocidura cyanea cf.; Crocidura flavescens; Cryptomys hottentotus; Cynictis penicillata; Damaliscus pygargus; Desmodillus auricularis; Equus quagga; Equus zebra cf.; Felis silvestris; Galerella; Gerbilliscus brantsii cf.; Homo sapiens; Ichneumia albicauda; Ictonyx striatus; Lycaon pictus; Macroscelides proboscideus; Malacothrix typica; Micaelamys namaquensis; Myotomys unisulcatus; Mystromys albicaudatus; Oreotragus oreotragus; Otocyon megalotis; Otomys irroratus; Otomys 
saundersiae; Ovis aries; Panthera pardus; Papio ursinus; Parahyaena brunnea; Pelea capreolus; Phacochoerus africanus; Philantomba monticola; Procavia capensis; Raphicerus campestris; Redunca arundinum; Redunca fulvorufula; Rhabdomys pumilio; Suricata suricatta; Sylvicapra grimmia; Taurotragus oryx; Tragelaphus strepsiceros; Vulpes chama. References: Avery (1991a); Plug (1993a, 1999b); Plug and Sampson (1996); Voigt et al. (1995).

Adullam (2828CB). Taxa: Canis; Equus burchellii cf.; Hystrix africaeaustralis; Papio ursinus; Procavia capensis. References: Wadley and Laue (2000).

Amis (2110:1430). Taxa: Antidorcas marsupialis; Crocidura; Crocuta crocuta; Elephantulus; Equus; Oreotragus oreotragus; Oryx gazella; Petromus typicus; Procavia capensis; Pronolagus randensis; Rhabdomys pumilio; Suricata suricatta. References: Van Neer and Breunig (1999).

Andriesgrond (3218BB). Taxa: Acomys subspinosus; Chlorotalpa sclateri; Crocidura cyanea; Crocidura flavescens; Crocidura fuscomurina; Cryptomys hottentotus; Dendromus melanotis; Dendromus mesomelas; Elephantulus edwardii; Gerbilliscus afra; Gerbillurus paeba; Graphiurus ocularis; Homo sapiens; Micaelamys namaquensis; Mus minutoides; Myomyscus verreauxii; Myosorex varius; Myotomys unisulcatus; Mystromys albicaudatus; Nycteris thebaica; Otomys irroratus; Otomys saundersiae; Rhabdomys pumilio; Rhinolophus capensis; Rhinolophus clivosus; Steatomys krebsii. References: Avery (unpublished); Pfeiffer (2013).

Andrieskraal (3325DA). Taxa: Bos taurus; Canis mesomelas; Capra hircus; Diceros bicornis; Equus asinus; Felis silvestris; Galerella pulverulenta; Genetta genetta; Hippopotamus amphibius; Homo sapiens; Hystrix africaeaustralis; Lepus saxatilis; Loxodonta africana; Mellivora capensis; Panthera pardus; Papio ursinus; Philantomba monticola; Potamochoerus larvatus; Procavia capensis; Raphicerus campestris; Sylvicapra grimmia; Tragelaphus scriptus; Tragelaphus strepsiceros. References: De Villiers (1965); Hendey and Singer (1965).

Apollo 11 (2745:1706). Taxa: Antidorcas marsupialis; Equus zebra; Felis silvestris; Lepus; Micaelamys namaquensis; Oreotragus oreotragus; Papio ursinus; Petromus typicus cf.; Phacochoerus; Procavia capensis. References: Cruz-Uribe and Klein (1981-1983); Thackeray (1979); Vogelsang et al. (2010); Wadley (2015).

Aspoort (3230:1931). Taxa: Cryptomys hottentotus; Equus zebra cf.; Gerbillurus paeba; Myotomys unisulcatus; Mystromys albicaudatus; Procavia capensis. References: Smith and Ripp (1978).

Atlantic Beach (3318). Taxa: Alcelaphus; Atilax paludinosus; Bathyergus suillus; Genetta; Hystrix africaeaustralis; Mellivora capensis; Raphicerus melanotis; Sylvicapra grimmia; Taurotragus oryx. References: Sealy et al. (2004).

Austerlitz (2817:1915). Taxa: Alcelaphus buselaphus; Equus; Procavia capensis; Raphicerus campestris. References: Cruz-Uribe and Klein (1981-1983).

Baden-Baden (2821:2530). Taxa: Aepyceros melampus; Alcelaphus buselaphus; Antidorcas marsupialis; Connochaetes gnou; Damaliscus pygargus; Equus quagga; Ovis aries; Phacochoerus africanus. References: Van Aardt et al. (2016).

Badfontein Valley (2520:3020). Taxa: Bos taurus. References: Collett (1982).

Bambata (2030:2830). Taxa: Bos taurus?. References: Walker (1983).

Biesje Poort 2 (2826:2033). Taxa: Antidorcas marsupialis; Connochaetes gnou; Cynictis penicillata; Equus quagga; Procavia capensis; Raphicerus campestris; Saccostomus campestris; Sylvicapra grimmia; Xerus inauris. References: Badenhorst et al. (2015). 
Big Elephant Shelter (2142:1540). Taxa: Antidorcas marsupialis; Bos taurus; Caracal caracal cf.; Felis silvestris cf.; Equus; Genetta; Hystrix africaeaustralis; Mellivora capensis; Oreotragus oreotragus; Otocyon megalotis; Ovis aries; Procavia capensis; Raphicerus campestris cf. References: Wadley (1979).

Blaauheuvel (2925AC). Taxa: Homo sapiens. References: Wells and Gear (1931). Blinkklipkop (2818:2307). Taxa: Canis mesomelas cf.; Crocidura cyanea; Crocidura fuscomurina; Crocidura hirta; Cryptomys hottentotus; Dendromus melanotis; Desmodillus auricularis; Elephantulus rupestris cf.; Eptesicus hottentotus; Equus; Gerbilliscus; Gerbillurus paeba; Hyaena; Hystrix africaeaustralis; Lepus; Macroscelides proboscideus; Malacothrix typica; Mastomys natalensis; Micaelamys namaquensis; Miniopterus natalensis; Mus minutoides; Mystromys albicaudatus; Otomys angoniensis cf.; Otomys irroratus; Panthera pardus; Phacochoerus africanus; Procavia capensis; Rhabdomys pumilio; Rhinolophus capensis; Rhinolophus clivosus; Saccostomus campestris; Steatomys krebsii; Suncus varilla; Zelotomys woosnami. References: Avery (1981); Humphreys and Thackeray (1983); Thackeray et al. (1983).

Blombos Cave (3425:2113). Taxa: Canis mesomelas cf.; Crocidura cyanea; Crocidura fuscomurina; Crocidura hirta; Cryptomys hottentotus; Dendromus melanotis; Desmodillus auricularis; Elephantulus rupestris cf.; Eptesicus hottentotus; Equus; Gerbilliscus; Gerbillurus paeba; Hyaena; Hystrix africaeaustralis; Lepus; Macroscelides proboscideus; Malacothrix typica; Mastomys natalensis; Micaelamys namaquensis; Miniopterus natalensis; Mus minutoides; Mystromys albicaudatus; Otomys angoniensis cf.; Otomys irroratus; Panthera pardus; Phacochoerus africanus; Procavia capensis; Rhabdomys pumilio; Rhinolophus capensis; Rhinolophus clivosus; Saccostomus campestris; Steatomys krebsii; Suncus varilla; Zelotomys woosnami. References: Badenhorst et al. (2016a); Discamps and Henshilwood (2015); Henshilwood (1995, 1996); Henshilwood et al. (2001); Hillestad Nel and Henshilwood (2016); Wadley (2015).

Bloubergstrand (3348:1828). Taxa: Homo sapiens. References: Orton et al. (2015); Pfeiffer (2013).

Bloubos (3105:2450). Taxa: Antidorcas australis; Canis mesomelas; Capra hircus; Damaliscus pygargus; Oreotragus oreotragus; Ovis aries. References: Plug (1999b); Plug and Sampson (1996); Voigt et al. (1995).

Blue Pool Cave (2724). Taxa: Equus burchellii; Syncerus caffer?. References: Humphreys (1978).

Blydefontein (3108:2513). Taxa: Cynictis penicillata; Equus; Felis silvestris; Oreotragus oreotragus; Panthera pardus; Papio ursinus; Pelea capreolus; Procavia capensis; Raphicerus campestris; Redunca fulvorufula; Vulpes chama. References: Avery (unpublished); Horsburgh and Moreno-Mayar (2015); Klein (1979a).

Boitsemangano (2527CA). Taxa: Aepyceros melampus; Aethomys chrysophilus; Alcelaphus buselaphus; Bos taurus; Canis mesomelas cf.; Capra hircus; Connochaetes taurinus; Equus quagga; Homo sapiens; Ovis aries; Pelea capreolus; Potamochoerus larvatus; Raphicerus campestris; Sylvicapra grimmia. References: Plug and Badenhorst (2006).

Bokbaai (3334:1820). Taxa: Homo sapiens. References: Pfeiffer (2013).

Bokvasmaak 3 (2826:2007). Taxa: Alcelaphus buselaphus; Antidorcas marsupialis; Canis lupus; Felis nigripes; Felis silvestris; Hippopotamus amphibius; Lepus capensis; Oryx gazella; Ovis aries; Petromus typicus; Procavia capensis; Raphicerus campestris; Redunca fulvorufula; Sylvicapra grimmia; Vulpes chama; Xerus inauris. References: Badenhorst et al. (2015). 
Bolahla (3004:2824). Taxa: Bos taurus; Capra hircus; Hystrix africaeaustralis; Ovis aries; Panthera pardus; Papio ursinus; Procavia capensis; Redunca fulvorufula; Sylvicapra grimmia; Taurotragus oryx. References: Mitchell et al. (1994); Plug (1997b).

Boleu (2529AB). Taxa: Bos taurus; Equus burchellii; Gerbilliscus brantsii; Homo sapiens; Myotomys sloggetti; Mystromys albicaudatus; Otomys irroratus; Otomys saundersiae; Ovis aries; Potamochoerus larvatus; Raphicerus campestris; Sylvicapra grimmia. References: Badenhorst and Plug (2004/2005).

Bonawe (3120:2746). Taxa: Antidorcas marsupialis; Canis mesomelas cf.; Crocidura flavescens; Cryptomys hottentotus; Equus quagga cf.; Equus zebra cf.; Hippotragus; Hystrix africaeaustralis; Mystromys albicaudatus; Oreotragus oreotragus; Orycteropus afer; Ourebia ourebi; Panthera pardus; Pelea capreolus; Phacochoerus africanus; Procavia capensis; Raphicerus campestris; Redunca fulvorufula; Sylvicapra grimmia; Taurotragus oryx. References: Opperman (1987).

Boomplaas Cave (3323:2211). Taxa: Acomys subspinosus; Canis mesomelas cf.; Caracal caracal cf.; Chlorotalpa sclateri; Cistugo lesueuri; Crocidura flavescens; Cryptomys hottentotus; Damaliscus; Dasymys incomtus; Dendromus melanotis; Diceros bicornis; Elephantulus; Equus; Felis silvestris; Galerella pulverulenta; Genetta; Gerbilliscus afra; Gerbillurus paeba; Herpestes ichneumon; Hippotragus; Homo sapiens; Hystrix africaeaustralis; Loxodonta africana; Mellivora capensis cf.; Micaelamys namaquensis; Mus minutoides; Myomyscus verreauxii; Myosorex varius; Myotomys unisulcatus; Mystromys albicaudatus; Otomys irroratus; Otomys laminatus; Otomys saundersiae; Ovis aries; Panthera pardus; Papio ursinus; Pelea capreolus; Potamochoerus larvatus; Procavia capensis; Raphicerus; Redunca arundinum; Redunca fulvorufula; Rhabdomys pumilio; Saccostomus campestris; Steatomys krebsii; Syncerus antiquus; Syncerus caffer; Taurotragus oryx; Tragelaphus strepsiceros. References: Avery (1977, 1982b); Brophy et al. (2014); Faith (2013); Klein (1978a, 1994b); Von den Driesch and Deacon (1985); Wadley (2015).

Border Cave (2701:3159). Taxa: Aethomys chrysophilus; Amblysomus hottentotus; Crocidura cyanea; Crocidura flavescens; Crocidura fuscomurina; Crocidura hirta; Crocidura mariquensis; Cryptomys hottentotus; Dendromus melanotis; Dendromus mesomelas cf.; Dendromus mystacalis; Elephantulus myurus; Equus burchellii cf.; Gerbilliscus leucogaster cf.; Glauconycteris variegata; Grammomys dolichurus; Graphiurus murinus; Lemniscomys rosalia; Lepus; Malacothrix typica; Mastomys natalensis; Micaelamys namaquensis; Miniopterus natalensis; Mus minutoides; Myosorex varius; Mystromys albicaudatus; Nycteris thebaica; Oreotragus oreotragus; Otomys angoniensis; Otomys irroratus; Otomys laminatus; Ovis aries; Papio ursinus; Potamochoerus larvatus; Rhabdomys pumilio; Rhinolophus clivosus; Rhinolophus darlingi; Saccostomus campestris; Scotophilus nigrita; Steatomys pratensis; Suncus infinitesimus; Suncus lixus; Suncus varilla; Syncerus caffer?; Thallomys paedulcus. References: Avery (1982a, 1991b, 1992a); Cooke et al. (1945); De Villiers (1974, 1976a); Klein (1977); Rightmire (1979b); Wadley (2015).

Borrow Pit Midden (3219:1819). Taxa: Bathyergus suillus; Felis silvestris; Procavia capensis; Raphicerus. References: Jerardino et al. (2009a).

Bosutswe (2157:2637). Taxa: Aepyceros melampus; Alcelaphus buselaphus; Antidorcas marsupialis; Atelerix frontalis; Bos taurus; Canis lupus; Canis mesomelas; Capra hircus; Caracal caracal; Ceratotherium simum cf.; Civettictis civetta; Connochaetes taurinus; Crocuta crocuta; Cryptomys cf.; Damaliscus pygargus; Diceros bicornis cf.; Equus burchellii; Felis silvestris; Galago senegalensis; Giraffa camelopardalis; Hippopotamus amphibius; 
Hippotragus equinus; Hippotragus niger; Homo sapiens; Kobus ellipsiprymnus; Kobus leche cf.; Lepus saxatilis; Loxodonta africana; Lycaon pictus; Malacothrix typica; Oreotragus oreotragus; Orycteropus afer; Oryx gazella; Ovis aries; Panthera leo; Panthera pardus; Papio ursinus; Parahyaena brunnea; Pedetes capensis; Phacochoerus africanus; Proteles cristata; Raphicerus campestris; Raphicerus sharpei; Rattus rattus; Redunca arundinum; Smutsia temminckii; Sus; Sylvicapra grimmia; Syncerus caffer; Taurotragus oryx; Tragelaphus scriptus; Tragelaphus spekeii; Tragelaphus strepsiceros; Xerus inauris. References: Denbow et al. (2008); Plug (1996b).

Boundary Shelter (3130:2435). Taxa: Alcelaphus buselaphus; Antidorcas australis; Canis mesomelas; Capra hircus; Caracal caracal; Connochaetes gnou; Cynictis penicillata; Damaliscus pygargus; Equus quagga; Equus zebra cf.; Hippotragus equinus cf.; Ovis aries; Panthera pardus; Pelea capreolus; Procavia capensis; Raphicerus campestris; Sylvicapra grimmia; Taurotragus oryx. References: Plug (1999b); Plug and Sampson (1996); Voigt et al. (1995).

Bremen (2517CB). Taxa: Bathyergus suillus; Caracal caracal; Equus; Felis silvestris; Hystrix africaeaustralis; Oreotragus oreotragus; Otocyon megalotis; Pedetes capensis; Procavia capensis; Raphicerus campestris. References: Cruz-Uribe and Klein (1981-1983).

Broederstroom 24/73 (2545:2750). Taxa: Bos taurus; Capra hircus; Cryptomys; Damaliscus pygargus; Equus burchellii; Gerbilliscus brantsii cf.; Hippotragus; Loxodonta africana; Mastomys natalensis; Oreotragus oreotragus; Ovis aries; Papio ursinus; Phacochoerus africanus; Procavia capensis; Rattus rattus; Redunca arundinum; Redunca fulvorufula; Thryonomys swinderianus. References: Brown (1981); Welbourne (1973).

Buffelshoek (2747:2222). Taxa: Bos taurus; Bunolagus monticularis cf.; Canis mesomelas cf.; Caracal caracal cf.; Diceros bicornis; Equus; Felis silvestris; Galerella pulverulenta; Hippopotamus amphibius; Hystrix africaeaustralis; Lepus capensis cf.; Loxodonta africana; Oreotragus oreotragus; Panthera leo; Papio ursinus; Phacochoerus africanus; Procavia capensis; Raphicerus campestris; Raphicerus melanotis; Redunca fulvorufula; Syncerus caffer; Taurotragus oryx; Tragelaphus strepsiceros. References: Klein (1978d); Loubser (1985).

Burchell's Shelter (2848:2343). Taxa: Alcelaphus buselaphus; Antidorcas marsupialis; Canis mesomelas cf.; Connochaetes gnou; Equus quagga cf.; Hippotragus; Hystrix africaeaustralis; Procavia capensis; Raphicerus campestris; Redunca fulvorufula; Tragelaphus strepsiceros. References: Humphreys (1975); Klein (1979a).

Bushman Rockshelter (3038:2435). Taxa: Acinonyx jubatus cf.; Aepyceros melampus; Alcelaphus cf.; Connochaetes taurinus; Damaliscus lunatus; Equus burchellii; Hippopotamus amphibius; Hippotragus equinus; Hippotragus niger; Homo sapiens; Hystrix africaeaustralis; Lepus saxatilis; Oreotragus oreotragus; Orycteropus afer; Papio ursinus; Phacochoerus africanus; Procavia capensis; Pronolagus; Raphicerus campestris; Redunca arundinum; Redunca fulvorufula; Sylvicapra grimmia; Taurotragus oryx; Tragelaphus scriptus; Tragelaphus strepsiceros; Xerus. References: Badenhorst and Plug (2012); Brain (1969, 1981); Dusseldorp et al. (2013); Plug (1981); Wadley (2015).

Buzz Shelter (3131:1836). Other names: VR005. Taxa: Alcelaphus buselaphus cf.; Bathyergus suillus; Canis; Caracal caracal; Crocidura flavescens; Cryptomys hottentotus; Desmodillus auricularis; Felis silvestris; Gerbillurus paeba; Hystrix africaeaustralis; Macroscelides proboscideus; Micaelamys namaquensis; Myotomys unisulcatus; Procavia capensis; Raphicerus campestris; Rhabdomys pumilio; Rhinolophus clivosus. References: Avery (unpublished); Orton et al. (2011). 
Byneskranskop (3435:1928). Taxa: Aonyx capensis; Atilax paludinosus; Bathyergus suillus; Canis mesomelas; Caracal caracal; Damaliscus pygargus cf.; Diceros bicornis; Equus quagga cf.; Felis silvestris; Galerella pulverulenta; Herpestes ichneumon; Hippopotamus amphibius; Hippotragus leucophaeus; Homo sapiens; Hystrix africaeaustralis; Lepus; Loxodonta africana; Mellivora capensis; Oreotragus oreotragus; Ovis aries; Panthera pardus; Papio ursinus; Parahyaena brunnea cf.; Pelea capreolus; Phacochoerus africanus; Potamochoerus larvatus; Procavia capensis; Raphicerus campestris; Raphicerus melanotis; Redunca arundinum; Redunca fulvorufula; Syncerus caffer; Taurotragus oryx. References: Avery (1977, 1982b); De Villiers and Wilson (1982); Klein (1981, 1982).

CaeCae (1947:2104). Taxa: Aepyceros melampus; Alcelaphus cf.; Bos taurus; Canis lupus; Equus burchellii; Gerbilliscus brantsii cf.; Otocyon megalotis; Phacochoerus africanus; Raphicerus campestris; Sylvicapra grimmia; Tragelaphus strepsiceros. References: Wilmsen (1989).

Cape Point (3418AD). Taxa: Homo sapiens. References: Pfeiffer (2013).

Cape St Francis (3424DB). Taxa: Homo sapiens. References: De Villiers (1974).

Cape Town (3356:1825). Taxa: Rattus norvegicus. References: Avery (1985).

Chamabvefva (3005:2013). Taxa: Bos taurus. References: Huffman (1979b).

Chibuene (2202:3519). Taxa: Bos taurus; Capra hircus; Chlorocebus aethiops; Homo sapiens; Neotragus moschatus; Ovis aries; Philantomba monticola. References: Badenhorst et al. (2011c).

Chivowa Hill (2010:3030). Taxa: Bos taurus; Capra hircus; Equus burchellii; Homo sapiens; Hystrix africaeaustralis; Kobus ellipsiprymnus; Oreotragus oreotragus; Procavia capensis; Sylvicapra grimmia; Taurotragus oryx; Tragelaphus scriptus cf. References: Sinclair (1991); Welbourne (1985).

Clanwilliam (3211:1853). Taxa: Homo sapiens. References: Pfeiffer (2013).

Clarke's Shelter (2901:2918). Taxa: Canis; Chlorocebus aethiops; Cryptomys hottentotus; Felis silvestris; Genetta; Oreotragus oreotragus; Otomys irroratus cf.; Otomys laminatus; Papio ursinus; Procavia capensis; Raphicerus; Rhabdomys pumilio. References: Avery (1991b); Mazel (1984b).

Collingham (2927:2935). Taxa: Alcelaphus buselaphus; Amblysomus hottentotus cf.; Aonyx capensis; Atilax paludinosus; Canis mesomelas; Caracal caracal; Cephalophus natalensis cf.; Chrysospalax villosus; Connochaetes gnou; Crocidura cyanea cf.; Crocidura flavescens; Cryptomys hottentotus; Damaliscus pygargus; Dendromus melanotis; Dendromus mesomelas; Felis silvestris; Georychus capensis; Gerbilliscus brantsii cf.; Hystrix africaeaustralis; Ictonyx striatus cf.; Myosorex varius; Mystromys albicaudatus; Oreotragus oreotragus; Otomys irroratus; Otomys laminatus; Ourebia ourebi; Panthera pardus; Papio ursinus; Parahyaena brunnea; Pelea capreolus; Potamochoerus larvatus; Procavia capensis; Pronolagus rupestris; Raphicerus campestris; Redunca arundinum; Redunca fulvorufula; Rhabdomys pumilio; Sylvicapra grimmia; Taurotragus oryx; Vulpes chama. References: Avery (1991b, 1992b); Mazel (1992); Plug (1992, 1997b).

Colwinton (3107:2744). Taxa: Antidorcas marsupialis; Canis mesomelas cf.; Damaliscus pygargus; Equus quagga cf.; Equus zebra cf.; Felis silvestris; Hippotragus leucophaeus cf.; Hystrix africaeaustralis; Lepus capensis; Myotomys sloggetti; Oreotragus oreotragus; Otomys irroratus; Otomys saundersiae; Ourebia ourebi; Panthera pardus; Papio ursinus; Pelea capreolus; Phacochoerus africanus; Procavia capensis; Raphicerus campestris; Redunca fulvorufula; Syncerus caffer; Taurotragus oryx. References: Opperman (1982, 1987); Plug (1997b). 
Commando Kop (2208:2910). Taxa: Bos taurus; Canis lupus; Capra hircus; Chlorocebus aethiops; Equus burchellii; Giraffa camelopardalis; Oreotragus oreotragus; Ovis aries; Pedetes capensis; Raphicerus campestris; Syncerus caffer; Thryonomys swinderianus; Tragelaphus strepsiceros. References: Plug (2000); Plug and Voigt (1985); Voigt (1980b); Voigt and Plug (1981).

Connies Limpet Bar (3219:1820). Taxa: Bathyergus suillus; Felis silvestris; Genetta; Lepus; Myotomys unisulcatus; Papio ursinus; Procavia capensis; Raphicerus; Rhabdomys pumilio; Taurotragus oryx. References: Jerardino et al. (2009b); Klein and Cruz-Uribe (1987).

Copper Queen Mine (1731:2919). Taxa: Homo sapiens; Hystrix cf.; Raphicerus campestris cf.; Sylvicapra grimmia cf. References: DeSilva et al. (2013); Swan (2002).

Darling (3323:1823). Taxa: Homo sapiens. References: Pfeiffer (2013).

Deelpan (2911:2545). Taxa: Antidorcas marsupialis; Aonyx capensis; Canis mesomelas; Connochaetes gnou; Damaliscus pygargus; Equus burchellii; Galerella pulverulenta; Galerella sanguinea; Lycaon pictus; Pedetes capensis; Raphicerus campestris. References: Brink (2005); Scott and Brink (1992); Scott and Klein (1981).

Deurspring 16 (3218AB). Taxa: Bathyergus suillus; Canis mesomelas; Lepus capensis; Papio ursinus; Diceros bicornis; Procavia capensis; Raphicerus; Vulpes chama. References: Jerardino et al. (2016).

Diamant (2345:2815). Taxa: Aepyceros melampus; Aethomys chrysophilus; Alcelaphus buselaphus; Bos taurus; Canis lupus; Capra hircus; Connochaetes taurinus; Diceros bicornis; Equus burchellii; Felis silvestris; Giraffa camelopardalis; Homo sapiens; Orycteropus afer; Ovis aries; Raphicerus campestris; Syncerus caffer. References: Plug (2000).

Diamond 1 (2829:2856). Taxa: Caracal caracal; Cryptomys hottentotus; Damaliscus pygargus; Dendromus mesomelas; Equus quagga cf.; Graphiurus murinus cf.; Otomys irroratus cf.; Otomys laminatus; Panthera leo; Papio ursinus; Pelea capreolus; Procavia capensis; Raphicerus; Rhabdomys pumilio. References: Avery (1991b); Mazel (1984b).

Diana's Vow (1835:3210). Taxa: Canis; Heterohyrax brucei; Hippotragus; Homo sapiens; Hystrix africaeaustralis; Ourebia ourebi; Papio ursinus cf.; Phacochoerus africanus; Potamochoerus larvatus; Procavia capensis; Raphicerus; Sylvicapra grimmia. References: Klein (1979b).

Die Kelders (3432:1922). Taxa: Alcelaphus buselaphus; Atilax paludinosus; Bathyergus suillus; Bos taurus?; Canis lupus; Canis mesomelas; Caracal caracal; Chrysochloris asiatica; Crocidura flavescens; Damaliscus pygargus; Dendromus melanotis; Diceros bicornis; Felis silvestris; Galerella pulverulenta; Genetta; Gerbilliscus afra; Herpestes ichneumon; Hippopotamus amphibius; Hippotragus leucophaeus; Homo sapiens; Hystrix africaeaustralis; Ictonyx striatus; Leptailurus serval; Lepus; Loxodonta africana; Mellivora capensis; Myomyscus verreauxii; Myosorex varius; Myotis tricolor; Mystromys albicaudatus; Oreotragus oreotragus; Otomys irroratus; Otomys saundersiae; Ovis aries; Panthera pardus; Papio ursinus; Pelea capreolus; Potamochoerus larvatus; Procavia capensis; Raphicerus campestris; Raphicerus melanotis; Rhabdomys pumilio; Rhinolophus capensis; Rhinolophus clivosus; Steatomys krebsii; Suncus varilla; Syncerus caffer; Tragelaphus scriptus; Vulpes chama. References: Armstrong (2016); Avery (1977, 1979); Avery et al. (1997); Brophy et al. (2014); Grine et al. (1991); Klein (1975b); Klein and Cruz-Uribe (2000); Marean et al. (2000); Rightmire (1979a); Schweitzer (1974, 1979); Schweitzer and Scott (1973); Wadley (2015).

Die Toon (2819:1717). Taxa: Antidorcas marsupialis; Damaliscus pygargus cf.; Equus burchellii cf.; Oreotragus oreotragus; Procavia capensis; Pronolagus rupestris cf. References: Webley et al. (1993). 
Diepkloof (3223:1827). Taxa: Bathyergus suillus; Felis silvestris; Galerella pulverulenta; Oreotragus oreotragus; Ovis aries; Pelea capreolus; Procavia capensis; Raphicerus. References: Klein and Steele (2008); Steele and Klein (2013); Wadley (2015).

Dikbosch 1 (2839:2354). Taxa: Aethomys chrysophilus; Alcelaphus buselaphus; Antidorcas marsupialis; Canis mesomelas?; Crocidura cyanea; Crocidura flavescens; Cryptomys hottentotus; Cynictis penicillata; Desmodillus auricularis?; Equus quagga; Gerbilliscus; Gerbillurus paeba; Graphiurus ocularis; Hystrix africaeaustralis; Lepus; Mastomys coucha; Mus minutoides; Myosorex varius; Mystromys albicaudatus; Otomys angoniensis; Otomys irroratus; Otomys saundersiae cf.; Ovis aries; Papio ursinus; Phacochoerus africanus; Procavia capensis; Raphicerus campestris; Raphicerus melanotis; Rhabdomys pumilio; Redunca fulvorufula?; Steatomys; Suncus varilla; Syncerus caffer; Taurotragus oryx; Vulpes chama. References: Avery and Avery (2011); Humphreys (1974); Humphreys and Thackeray (1983); Klein (1979a).

Divuyu (1845:2144). Taxa: Aepyceros melampus; Bos taurus; Canis mesomelas cf.; Capra hircus; Connochaetes; Felis silvestris; Genetta; Gerbilliscus; Giraffa camelopardalis; Hippopotamus amphibius; Homo sapiens; Hystrix africaeaustralis; Kobus ellipsiprymnus; Kobus leche; Lepus; Otocyon megalotis; Ovis aries; Panthera pardus; Papio ursinus; Pedetes capensis; Redunca arundinum; Sylvicapra grimmia; Syncerus caffer; Taurotragus. References: Denbow (2011); G. Turner (1987a).

Doonside (3004:3052). Taxa: Homo sapiens. References: Galloway (1936).

Doornfontein (2812:2302). Taxa: Aepyceros melampus; Antidorcas marsupialis;

Connochaetes taurinus; Crocidura; Cryptomys hottentotus; Dendromus; Elephantulus; Equus burchellii; Felis silvestris; Gerbilliscus brantsii; Gerbilliscus leucogaster; Gerbillurus paeba; Giraffa camelopardalis; Hippotragus equinus; Hippotragus niger; Homo sapiens; Hystrix africaeaustralis; Lepus; Micaelamys namaquensis?; Mystromys albicaudatus; Oreotragus oreotragus; Oryx gazella; Otocyon megalotis; Otomys irroratus; Panthera leo; Papio ursinus; Phacochoerus africanus; Procavia capensis; Raphicerus campestris; Redunca arundinum; Saccostomus; Sylvicapra grimmia; Syncerus caffer; Tragelaphus scriptus; Tragelaphus strepsiceros; Vulpes chama. References: Beaumont and Boshier (1974); Klein (1979a); Thackeray et al. (1983).

Doringbaai (3149:1814). Taxa: Homo sapiens. References: Pfeiffer (2013).

DP2004-014 (2936:1703). Taxa: Felis silvestris; Oryx gazella; Parotomys brantsii; Pedetes capensis; Raphicerus campestris; Suricata suricatta; Sylvicapra grimmia. References: Dewar (2007).

Drie Susters (3306:1800). Taxa: Bathyergus suillus; Bos taurus; Diceros bicornis; Equus; Lepus capensis; Ovis aries; Procavia capensis; Raphicerus campestris; Sylvicapra grimmia. References: Smith et al. (1991).

Driebos (3306:1903). Taxa: Procavia capensis; Raphicerus. References: Smith et al. (1991).

Driekoppen (3105:2440). Taxa: Antidorcas australis; Canis mesomelas; Capra hircus; Connochaetes gnou; Damaliscus pygargus; Equus zebra cf.; Ictonyx striatus; Ovis aries; Phacochoerus africanus; Procavia capensis; Raphicerus campestris; Redunca fulvorufula; Suricata suricatta. References: Plug (1999b); Plug and Sampson (1996).

Driel Shelter (2844:2917). Taxa: Antidorcas marsupialis; Aonyx capensis; Atilax paludinosus; Canis mesomelas cf.; Damaliscus pygargus; Felis silvestris; Hippopotamus amphibius; Homo sapiens; Hystrix africaeaustralis; Orycteropus afer; Ourebia ourebi; Pelea capreolus; Phacochoerus africanus; Procavia capensis; Proteles cristata; Raphicerus campestris; Redunca arundinum; Taurotragus oryx. References: Klein (1980); Meester (1980). 
Drotsky's Cave (2002:2125). Taxa: Damaliscus pygargus; Dendromus; Diceros bicornis; Equus burchellii; Gerbilliscus brantsii cf.; Gerbilliscus leucogaster cf.; Gerbillurus paeba; Hystrix africaeaustralis; Mystromys albicaudatus; Otomys angoniensis cf.; Parahyaena brunnea; Pedetes capensis; Raphicerus campestris; Steatomys; Sylvicapra grimmia; Syncerus caffer; Tragelaphus strepsiceros. References: Robbins et al. (1996); Yellen et al. (1987).

Duiker Eiland (3405:1832). Taxa: Bathyergus suillus; Chlorotalpa sclateri; Crocidura flavescens; Felis silvestris; Georychus capensis; Gerbilliscus afra; Gerbillurus paeba; Myosorex varius; Myotomys unisulcatus cf.; Raphicerus sp.; Rhabdomys pumilio; Sylvicapra grimmia. References: Robertshaw (1979).

Duinefontein (3343:1827). Taxa: Damaliscus pygargus; Dendromus; Diceros bicornis; Equus burchellii; Gerbilliscus brantsii cf.; Gerbilliscus leucogaster cf.; Gerbillurus paeba; Hystrix africaeaustralis; Mystromys albicaudatus; Otomys angoniensis cf.; Parahyaena brunnea; Pedetes capensis; Raphicerus campestris; Steatomys; Sylvicapra grimmia; Syncerus caffer; Tragelaphus strepsiceros. References: Brophy et al. (2014); Cruz-Uribe et al. (2003); Klein (1976b); Klein and Cruz-Uribe (1991); Klein et al. (1999a); Robbins et al. (1996); Yellen et al. (1987).

Durban Country Club (2950:3102). Taxa: Homo sapiens. References: Galloway (1936).

Dzata (2250:3015). Taxa: Aethomys chrysophilus; Alcelaphus; Bos taurus; Canis lupus; Capra hircus; Connochaetes taurinus; Hippotragus; Lepus saxatilis; Loxodonta africana; Procavia capensis; Sylvicapra grimmia; Syncerus caffer; Taurotragus oryx; Tragelaphus angasii. References: De Wet-Bronner (1995a); Plug (2000).

Dzombo Shelter (2229BA). Taxa: Aepyceros melampus; Hystrix africaeaustralis; Mellivora capensis; Oreotragus oreotragus; Papio ursinus; Procavia capensis; Raphicerus campestris cf.; Sylvicapra grimmia. References: Forssman (2014).

Eiland Salt Works (2330:3025). Taxa: Aepyceros melampus; Bos taurus; Connochaetes taurinus; Equus burchellii; Hippotragus niger; Kobus ellipsiprymnus; Lepus saxatilis; Phacochoerus africanus; Raphicerus campestris; Sylvicapra grimmia; Syncerus caffer; Taurotragus oryx. References: Plug (1999a).

Elands Bay Cave (3218:1820). Taxa: Acomys subspinosus; Alcelaphus buselaphus; Antidorcas marsupialis; Atelerix frontalis; Bathyergus suillus; Canis mesomelas; Caracal caracal; Chrysochloris asiatica; Crocidura cyanea; Crocidura flavescens; Cryptochloris zyli; Cryptomys hottentotus; Dendromus melanotis; Diceros bicornis; Elephantulus edwardii; Elephantulus rupestris; Eremitalpa granti; Equus capensis; Equus quagga; Felis silvestris; Galerella pulverulenta; Genetta; Georychus capensis; Graphiurus ocularis; Herpestes ichneumon; Hippopotamus amphibius; Hippotragus leucophaeus; Homo sapiens; Hystrix africaeaustralis; Ictonyx striatus; Loxodonta africana; Mellivora capensis; Micaelamys namquensis; Mus minutoides; Myomyscus verreauxii; Myosorex varius; Myotomys unisulcatus; Mystromys albicaudatus; Oreotragus oreotragus; Otomys irroratus; Otomys saundersiae; Ovis aries; Panthera pardus; Papio ursinus; Potamochoerus larvatus; Procavia capensis; Raphicerus campestris; Raphicerus melanotis; Redunca arundinum; Rhabdomys pumilio; Steatomys krebsii; Suncus varilla; Sylvicapra grimmia; Syncerus caffer; Taurotragus oryx; Vulpes chama. References: Avery (unpublished); Dusseldorp et al. (2013); Jerardino et al. (2013); Klein and Cruz-Uribe (1987); Klein et al. (2007); Pfeiffer (2013).

Elands Bay Open (3218:1819). Taxa: Alcelaphus buselaphus; Bathyergus suillus; Canis; Caracal caracal; Felis silvestris; Galerella pulverulenta; Herpestes ichneumon; Hystrix africaeaustralis; Mellivora capensis; Ovis aries; Papio ursinus; Procavia capensis; Raphicerus; 
Sylvicapra grimmia; Taurotragus oryx. References: Jerardino et al. (2013); Klein and CruzUribe (1987).

Equus Cave (2737:2438). Taxa: Antidorcas marsupialis; Canis mesomelas; Caracal caracal; Connochaetes taurinus; Crocuta crocuta; Cynictis penicillata; Damaliscus pygargus; Felis nigripes; Felis silvestris; Herpestes ichneumon; Homo sapiens; Hystrix africaeaustralis; Mellivora capensis; Orycteropus afer; Otocyon megalotis; Ovis aries; Panthera pardus; Parahyaena brunnea; Pedetes capensis; Procavia capensis; Raphicerus campestris; Redunca fulvorufula; Sylvicapra grimmia; Syncerus caffer; Taurotragus oryx; Tragelaphus strepsiceros; Vulpes chama. References: Brophy et al. (2014); Cruz-Uribe (1991); Grine and Klein (1985); Klein et al. (1991); Kuhn et al. (2016); McKee (1994); McKee et al. (1995).

Esikhunjini (2529BD). Taxa: Aepyceros melampus cf.; Bos taurus; Connochaetes taurinus cf.; Equus; Homo sapiens; Lepus saxatilis; Orycteropus afer cf.; Ovis aries; Pedetes capensis; Raphicerus campestris; Redunca arundinum cf.; Taurotragus oryx cf.; Tragelaphus strepsiceros. References: Nelson (2008, 2009).

eSinhlonhweni (2851:3010). Taxa: Amblysomus hottentotus; Canis; Felis silvestris; Genetta; Hippotragus; Hystrix africaeaustralis; Lepus; Mastomys natalensis; Micaelamys namaquensis; Mystromys albicaudatus; Oreotragus oreotragus; Papio ursinus; Phacochoerus africanus; Procavia capensis; Raphicerus; Redunca fulvorufula; Sylvicapra grimmia; Tragelaphus scriptus. References: Avery (1991b); Mazel (1986b).

Etemba (2127:1537). Taxa: Bathyergus suillus; Caracal caracal; Equus; Homo sapiens; Pedetes capensis; Procavia capensis; Raphicerus campestris. References: Cruz-Uribe and Klein (1981-1983).

Fackelträger (2134:1532). Taxa: Antidorcas marsupialis; Diceros bicornis; Petromus; Procavia; Raphicerus campestris. References: Brain (1981); Thackeray (1979).

Fairview (3232:2634). Taxa: Amblysomus hottentotus; Antidorcas marsupialis; Aonyx capensis; Atilax paludinosus; Canis mesomelas; Chlorocebus aethiops; Crocidura flavescens; Cryptomys hottentotus; Dasymys incomtus; Dendromus mesomelas; Equus; Felis silvestris; Galerella pulverulenta; Genetta; Georychus capensis; Herpestes ichneumon; Hippotragus equinus cf.; Hippotragus leucophaeus; Hystrix africaeaustralis; Lycaon pictus; Myosorex varius; Orycteropus afer; Otomys irroratus; Otomys laminatus; Ourebia ourebi; Panthera pardus; Papio ursinus; Pelea capreolus; Phacochoerus africanus; Philantomba monticola; Potamochoerus larvatus; Procavia capensis; Raphicerus; Redunca arundinum; Redunca fulvorufula; Rhabdomys pumilio; Rhinolophus capensis; Rhinolophus clivosus; Syncerus caffer; Taurotragus oryx; Thallomys paedulcus; Tragelaphus scriptus; Tragelaphus strepsiceros; Vulpes chama. References: Avery (1984a); Klein (1984b).

Faraoskop Rock Shelter (3207:1836). Taxa: Atelerix frontalis; Homo sapiens; Hystrix africaeaustralis; Phacochoerus africanus?; Procavia capensis. References: Manhire (1993); Pfeiffer (2013).

Ficus Cave (2429). Taxa: Canis lupus; Equus burchellii; Felis silvestris; Homo sapiens; Orycteropus afer; Procavia capensis. References: Partridge (1966).

Forest Hall (3354:2220). Taxa: Hippopotamus amphibius; Hippotragus leucophaeus cf. References: Wilson (1988).

Geduld (2017:1550). Taxa: Alcelaphus; Equus; Genetta; Gerbilliscus; Madoqua; Ovis aries; Rhabdomys; Saccostomus; Steatomys; Sylvicapra grimmia; Thallomys. References: Smith and Jacobson (1995); Smith et al. (1995).

Gehle (2907:2954). Taxa: Canis mesomelas cf.; Caracal caracal; Chlorocebus aethiops; Chrysospalax villosus; Cryptomys hottentotus; Felis silvestris; Georychus capensis; Hystrix 
africaeaustralis; Myosorex; Mystromys albicaudatus; Otomys irroratus cf.; Ourebia ourebi; Procavia capensis; Raphicerus campestris; Redunca fulvorufula; Rhabdomys pumilio; Smutsia temminckii. References: Avery (1991b); Mazel (1984a).

Ghoenkop. See Hill X.

Girls' School (2114). Taxa: Procavia capensis. References: Cruz-Uribe and Klein (1981-1983).

Gladysvale (2553:2746). Other names: Uitkomst. Taxa: Aepyceros melampus; Equus burchellii; Hippotragus equinus; Redunca arundinum. References: Avery (1995a); Berger (1992, 1993); Broom (1937a, 1948a); Churcher (1956); Cooke (1962); Freedman (1970); Lacruz et al. (2002, 2003); Meester (1961a); Reynolds (2010b).

Glen Elliott (3025DC). Taxa: Antidorcas marsupialis; Canis; Cynictis penicillata; Damaliscus pygargus?; Equus; Hystrix africaeaustralis; Orycteropus afer; Papio ursinus; Pedetes capensis; Phacochoerus africanus; Procavia capensis; Raphicerus campestris; Taurotragus oryx. References: Klein (1979a).

Glennel (2229). Taxa: Homo sapiens. References: De Villiers (1980); Steyn and Nienaber (2000).

Glentyre (3401:2240). Taxa: Alcelaphus caama; Equus; Galerella pulverulenta?; Genetta?; Hippopotamus amphibius; Hippotragus leucophaeus?; Panthera pardus?; Papio ursinus; Potamochoerus larvatus; Raphicerus; Syncerus caffer; Taurotragus oryx?. References: Fagan (1960); Wells (1960).

Godwan River (2535:3038). Taxa: Pronolagus intermedius. References: Jameson (1909).

Goedgekloof Middens (3424). Taxa: Alcelaphus buselaphus; Bos taurus cf.; Canis; Damaliscus; Diceros bicornis; Equus; Felis; Hippopotamus amphibius; Lepus; Ovis aries; Panthera leo; Raphicerus; Sylvicapra grimmia; Syncerus; Tragelaphus strepsiceros. References: Binneman (2004/2005).

Gokomere Tunnel (2005:2835). Taxa: Bos taurus; Homo sapiens. References: Shee (1963). Good Hope Shelter (2939:2926). Taxa: Alcelaphus buselaphus; Bos taurus; Damaliscus pygargus; Equus; Oreotragus oreotragus; Ourebia ourebi; Papio ursinus; Pelea capreolus; Phacochoerus africanus; Procavia capensis; Raphicerus melanotis cf.; Redunca fulvorufula; Taurotragus oryx. References: Cable et al. (1980).

Gordons Bay (3409:1852). Taxa: Alcelaphus caama; Atilax paludinosus; Canis; Cryptomys hottentotus; Georychus capensis cf.; Gerbilliscus; Hippotragus leucophaeus cf.; Homo sapiens; Mystromys albicaudatus; Oreotragus oreotragus; Papio; Pelea capreolus?; Procavia capensis; Raphicerus; Vulpes chama?. References: Pfeiffer (2013); Van Noten (1974).

Grassridge (3134:2651). Taxa: Antidorcas marsupialis; Canis mesomelas; Damaliscus dorcas; Equus; Felis silvestris; Galerella pulverulenta; Lepus capensis; Orycteropus afer; Ovis aries; Panthera pardus; Pelea capreolus; Phacochoerus africanus; Procavia capensis; Pronolagus crassicaudatus; Redunca fulvorufula; Sylvicapra grimmia; Taurotragus oryx. References: Collins et al. (2017); Opperman (1987).

Great Zimbabwe (2016:3056). Taxa: Bos taurus; Canis lupus; Felis silvestris cf.; Homo sapiens; Lepus; Panthera pardus; Sylvicapra grimmia; Tragelaphus scriptus. References: Brain (1974).

Green Point Cape Town (3354:1824). Taxa: Homo sapiens. References: Pfeiffer (2013).

Groenriviermond (3030:1721). Taxa: Homo sapiens; Parotomys. References: Jerardino et al. (1992).

Groot Kommandokloof Shelter (3340:2407). Taxa: Equus zebra cf.; Lepus; Oreotragus oreotragus; Procavia capensis; Raphicerus. References: Binneman (1999); Pearce (2008). 
Grootrif G (3210:1819). Taxa: Bathyergus suillus; Canis; Felis silvestris; Genetta; Ovis aries; Procavia capensis; Raphicerus; Sylvicapra grimmia. References: Jerardino (2007).

Gwenzi II Hill (1914:3231). Taxa: Equus burchellii; Lepus saxatilis; Orycteropus afer; Rattus cf.; Smutsia temminckii; Thryonomys swinderianus. References: Katsamudanga (2007a, 2007b); Mupira and Katsamudanga (2007).

Ha Makotoko (2920:2748). Taxa: Antidorcas australis; Canis mesomelas; Connochaetes gnou; Equus burchellii; Hippotragus leucophaeus cf.; Homo sapiens; Hystrix africaeaustralis; Oreotragus oreotragus; Ourebia ourebi; Papio ursinus; Pedetes capensis; Pelea capreolus; Phacochoerus africanus; Philantomba monticola; Procavia capensis; Raphicerus campestris; Redunca fulvorufula; Taurotragus oryx; Xerus. References: Plug (1997b).

Haalenberg (2640:1532). Taxa: Equus; Felis silvestris cf.; Gerbillurus paeba cf.; Myotomys unisulcatus; Oreotragus oreotragus; Petromus typicus; Petromyscus collinus; Procavia capensis; Raphicerus campestris. References: Thackeray (1979).

Haaskraal (3130:2420). Taxa: Antidorcas australis; Atilax paludinosus; Bos taurus; Canis mesomelas; Caracal caracal; Connochaetes gnou; Cynictis penicillata; Damaliscus pygargus; Equus asinus; Equus quagga; Felis silvestris; Galerella; Ichneumia albicauda; Ictonyx striatus; Lycaon pictus; Oreotragus oreotragus; Orycteropus afer; Ovis aries; Panthera pardus; Papio ursinus; Parahyaena brunnea; Pelea capreolus; Phacochoerus africanus; Procavia capensis; Raphicerus campestris; Suricata suricatta; Taurotragus oryx; Vulpes chama. References: Plug (1999b); Plug et al. (1994); Plug and Sampson (1996).

Hailstone Midden (3218:1820). Taxa: Galerella pulverulenta; Papio ursinus; Procavia capensis; Raphicerus. References: Klein and Cruz-Uribe (1987); Noli (1988).

Hamilton (2229AD). Taxa: Homo sapiens. References: Boshoff and Steyn (2000).

Hapi Pan (2225:3120). Taxa: Equus burchellii; Hippopotamus amphibius; Syncerus caffer. References: Plug (2000).

Happy Rest (2303:2947). Taxa: Bos taurus; Capra hircus; Equus burchellii; Lepus; Ovis aries; Panthera leo; Phacochoerus africanus; Sylvicapra grimmia. References: Steyn and Nienaber (2000); Voigt and Plug (1984).

Harleigh Farm (1832CA). Taxa: Bos taurus; Canis lupus; Equus burchellii; Heterohyrax brucei; Lepus; Loxodonta africana; Papio ursinus; Potamochoerus. References: Fagan (1966).

Harmony Salt Factory (2411:3036). Taxa: Aepyceros melampus; Bos taurus; Equus burchellii cf.; Kobus ellipsiprymnus; Phacochoerus africanus; Procavia capensis; Redunca arundinum cf.; Sylvicapra grimmia; Tragelaphus strepsiceros. References: Chatterton et al. (1979); Welbourne $(1974,1979)$.

Heuningneskrans (2436:3039). Taxa: Aepyceros melampus; Alcelaphus; Antidorcas bondi; Antidorcas marsupialis; Canis; Equus burchellii; Hippopotamus amphibius; Hippotragus; Homo sapiens; Hystrix africaeaustralis; Oreotragus oreotragus; Orycteropus afer; Panthera leo; Papio ursinus; Parahyaena brunnea; Pelea capreolus; Phacochoerus africanus; Potamochoerus larvatus; Procavia capensis; Redunca fulvorufula; Sylvicapra grimmia; Syncerus caffer; Tragelaphus strepsiceros. References: Klein (1984a).

Highlands Rock Shelter (3149:2534). Taxa: Antidorcas marsupialis; Atelerix frontalis; Connochaetes gnou cf.; Equus; Hystrix africaeaustralis; Lepus capensis; Oreotragus oreotragus; Phacochoerus africanus; Philantomba monticola; Procavia capensis; Pronolagus rupestris cf.; Proteles cristata?; Raphicerus campestris cf.; Redunca fulvorufula; Vulpes chama. References: Deacon (1976).

Hill X (2355:3105). Other names: Ghoenkop. Taxa: Aepyceros melampus; Bos taurus; Capra hircus; Caracal caracal; Connochaetes taurinus; Damaliscus lunatus; Felis silvestris; Giraffa 
camelopardalis; Homo sapiens; Kobus ellipsiprymnus; Lepus capensis; Oreotragus oreotragus; Panthera leo; Phacochoerus africanus; Raphicerus campestris; Redunca arundinum; Sylvicapra grimmia; Syncerus caffer; Tragelaphus strepsiceros. References: Plug and Pistorius (1999).

Historic Cave (2429AA). Taxa: Bos taurus; Canis lupus; Capra hircus; Equus quagga; Galago; Giraffa camelopardalis; Lepus capensis; Ovis aries; Papio ursinus; Procavia capensis; Pronolagus; Redunca arundinum; Syncerus caffer. References: Le Roux et al. (2013).

Hlamba Mlonga Hill (1829DD). Taxa: Aepyceros melampus; Bos taurus; Canis lupus; Capra hircus cf.; Connochaetes taurinus; Equus burchellii; Giraffa camelopardalis; Hippopotamus amphibius cf.; Lepus saxatilis; Oreotragus oreotragus; Ovis aries cf.; Papio ursinus; Procavia capensis; Raphicerus campestris; Thryonomys swinderianus. References: Thorp (2009).

Hoekfontein (2536:2756). Taxa: Bos taurus; Ovis aries; Procavia capensis; Tragelaphus strepsiceros. References: Van Schalkwyk et al. (1999).

Honingklip I and V (2601:3048). Taxa: Aepyceros melampus; Aethomys chrysophilus; Alcelaphus buselaphus cf.; Bos taurus; Canis mesomelas; Caracal caracal; Connochaetes taurinus; Giraffa camelopardalis; Hippotragus equinus; Hippotragus niger cf.; Homo sapiens; Hystrix africaeaustralis; Ictonyx striatus; Lepus saxatilis; Oreotragus oreotragus; Orycteropus afer; Otomys irroratus cf.; Ovis aries; Panthera pardus; Pedetes capensis; Pelea capreolus; Phacochoerus africanus; Procavia capensis; Proteles cristata; Raphicerus campestris; Redunca arundinum; Redunca fulvorufula; Suricata suricatta; Sylvicapra grimmia; Taurotragus oryx; Thryonomys; Tragelaphus strepsiceros cf. References: Korsman and Plug (1994).

Hope Hill Shelter (2624:2856). Taxa: Aepyceros melampus cf.; Alcelaphus buselaphus; Antidorcas marsupialis; Bos taurus; Canis mesomelas; Connochaetes gnou cf.; Connochaetes taurinus cf.; Crocidura; Crocuta crocuta; Cryptomys hottentotus; Damaliscus lunatus; Damaliscus pygargus; Equus burchellii; Felis silvestris; Gerbilliscus; Gerbillurus paeba; Hystrix africaeaustralis; Lepus capensis; Mastomys natalensis; Mus musculus; Orycteropus afer; Oryx gazella cf.; Ovis aries; Panthera pardus; Parahyaena brunnea; Pedetes capensis; Pelea capreolus; Phacochoerus africanus; Procavia capensis; Raphicerus campestris; Rattus rattus; Sylvicapra grimmia; Thryonomys swinderianus cf.; Vulpes chama. References: Wadley and Turner (1987).

Hout Bay (3403:1821). Taxa: Homo sapiens. References: Pfeiffer (2013).

Icon (2225:2915). Taxa: Aepyceros melampus; Bos taurus; Capra hircus; Giraffa camelopardalis; Homo sapiens; Lepus; Ovis aries cf.; Phacochoerus africanus; Taurotragus oryx. References: Plug (2000); Voigt (1979, 1980b).

Induna Cave (1829DD). Taxa: Aepyceros melampus cf.; Connochaetes taurinus; Equus burchellii; Felis silvestris cf.; Giraffa camelopardalis; Orycteropus afer; Papio ursinus; Potamochoerus larvatus; Procavia capensis cf.; Raphicerus campestris; Sylvicapra grimmia cf. References: Thorp (2010).

iNkolomahashi (2848:3011). Taxa: Aepyceros melampus; Damaliscus pygargus; Genetta tigrina; Hystrix africaeaustralis; Ictonyx striatus; Lepus saxatilis; Loxodonta africana; Oreotragus oreotragus; Ourebia ourebi; Ovis aries; Papio ursinus; Parahyaena brunnea; Pelea capreolus; Phacochoerus africanus; Philantomba monticola; Potamochoerus larvatus; Procavia capensis; Pronolagus rupestris; Raphicerus campestris; Redunca arundinum; Redunca fulvorufula; Smutsia temminckii; Sylvicapra grimmia; Taurotragus oryx; Thryonomys swinderianus; Tragelaphus scriptus; Tragelaphus strepsiceros cf. References: Badenhorst (2003). 
Jagt Pan 7 (3030:2130). Taxa: Antidorcas marsupialis; Canis mesomelas cf.; Cynictis cf.; Hystrix africaeaustralis; Lepus capensis; Procavia capensis cf.; Raphicerus campestris; Redunca fulvorufula. References: Badenhorst et al. (2015).

Jakkalsberg (2810:1653). Taxa: Equus capensis; Ovis aries. References: Brink and Webley (1996).

Jubilee Shelter (2542:2755). Taxa: Aepyceros melampus; Alcelaphus buselaphus; Canis mesomelas cf.; Civettictis civetta; Connochaetes; Crocidura fuscomurina cf.; Crocidura hirta; Crocidura mariquensis cf.; Cryptomys hottentotus; Damaliscus pygargus; Dasymys incomtus; Dendromus; Elephantulus; Equus burchellii; Gerbilliscus; Grammomys dolichurus; Graphiurus murinus cf.; Hippotragus niger cf.; Homo sapiens; Hystrix africaeaustralis; Lemniscomys rosalia; Lepus; Mastomys natalensis; Mellivora capensis; Mus minutoides; Myosorex varius?; Oreotragus oreotragus; Orycteropus afer; Otomys angoniensis cf.; Ourebia ourebi; Papio ursinus; Parahyaena brunnea; Pelea capreolus; Phacochoerus africanus; Procavia capensis; Raphicerus campestris; Rattus rattus; Redunca fulvorufula; Rhabdomys pumilio; Saccostomus campestris; Smutsia temminckii; Steatomys pratensis cf.; Sylvicapra grimmia; Tragelaphus scriptus; Vulpes chama. References: Avery (1987a); G. Turner (1986).

K2 (2225:2915). Taxa: Aepyceros melampus; Aethomys chrysophilus; Bos taurus; Canis lupus; Canis mesomelas; Capra hircus; Connochaetes; Cryptomys; Cynictis penicillata; Equus burchellii; Hippopotamus amphibius; Homo sapiens; Ictonyx striatus; Leptailurus serval; Lepus saxatilis; Loxodonta africana; Mastomys natalensis; Ovis aries; Papio ursinus; Pedetes capensis; Phacochoerus africanus; Potamochoerus larvatus; Pronolagus randensis; Suncus lixus; Sylvicapra grimmia. References: Plug (2000); Steyn and Nienaber (2000); Steyn et al. (1998, 1999); Voigt (1983).

Kabeljous River Cave 1 (3424BB). Taxa: Alcelaphus buselaphus; Equus; Homo sapiens; Hyaena; Pelea capreolus; Philantomba monticola; Procavia capensis; Raphicerus melanotis; Redunca fulvorufula; Sylvicapra grimmia; Syncerus caffer; Taurotragus oryx. References: Binneman (2006/2007).

Kadzi River (1650:3020). Taxa: Acinonyx jubatus; Aepyceros melampus; Atilax paludinosus; Bos taurus; Canis mesomelas cf.; Capra hircus; Chlorocebus aethiops; Equus burchellii; Giraffa camelopardalis; Heterohyrax brucei; Hippopotamus amphibius; Hippotragus niger cf.; Homo sapiens; Kobus ellipsiprymnus; Loxodonta africana; Lycaon pictus; Oreotragus oreotragus; Ovis aries; Panthera pardus; Papio ursinus; Phacochoerus africanus; Potamochoerus larvatus; Raphicerus sharpei; Redunca arundinum; Rhynchogale melleri cf.; Sylvicapra grimmia; Syncerus caffer; Thryonomys swinderianus; Tragelaphus strepsiceros cf. References: Plug (1997a); Pwiti (1996); Shenjere-Nyabezi et al. (2013).

Kamukombe (1630BD). Taxa: Aepyceros melampus; Bos taurus cf.; Kobus ellipsiprymnus cf.; Lepus saxatilis; Phacochoerus africanus; Procavia capensis; Tragelaphus strepsiceros. References: Shenjere-Nyabezi et al. (2013).

Kapako (1755:1940). Taxa: Hippopotamus amphibius. References: Sandelowsky (1979). Kareepan (2730:2536). Taxa: Alcelaphus buselaphus; Antidorcas marsupialis; Canis mesomelas; Caracal caracal; Connochaetes gnou; Crocuta crocuta; Damaliscus pygargus; Equus quagga cf.; Felis silvestris; Hystrix africaeaustralis; Lepus; Lycaon pictus; Orycteropus afer; Parahyaena brunnea; Phacochoerus; Proteles cristata; Raphicerus campestris; Taurotragus oryx; Vulpes chama. References: Brink (2005).

Karridene (3008:3050). Taxa: Homo sapiens. References: Galloway and Wells (1934). Kasteelberg (3249:1757). Taxa: Alcelaphus buselaphus; Atilax paludinosus; Bathyergus suillus; Bos taurus; Canis; Caracal caracal; Diceros bicornis; Equus capensis cf.; Equus 
quagga cf.; Felis silvestris; Galerella pulverulenta; Genetta; Herpestes ichneumon; Hystrix africaeaustralis; Ictonyx striatus; Lepus; Loxodonta africana; Mellivora capensis; Orycteropus afer; Ovis aries; Panthera pardus; Raphicerus campestris; Sylvicapra grimmia; Taurotragus oryx; Vulpes chama. References: Klein (1986); Klein and Cruz-Uribe (1989); Sadr (2007); Smith and Mütti (2013).

Katarakt (2110:1430). Taxa: Canis. References: Van Neer and Breunig (1999).

Khami Hill (2009:2823). Taxa: Acinonyx jubatus; Bos taurus; Canis; Civettictis civetta; Equus burchellii; Felis silvestris; Genetta; Galerella sanguinea; Leptailurus serval; Lycaon pictus; Oreotragus oreotragus; Orycteropus afer; Panthera leo; Panthera pardus; Pedetes capensis; Potamochoerus larvatus; Raphicerus campestris; Rattus rattus cf.; Sylvicapra grimmia. References: Thorp (1984).

Khartoum 1 (2916:2444). Taxa: Antidorcas marsupialis. References: Klein (1979a). Klasies River (3406:2424). Taxa: Alcelaphus buselaphus; Aonyx capensis; Canis mesomelas; Genetta?; Hippopotamus amphibius; Homo sapiens; Hystrix africaeaustralis; Panthera pardus; Papio ursinus; Pelea capreolus; Philantomba monticola; Potamochoerus larvatus; Procavia capensis; Raphicerus melanotis; Redunca fulvorufula; Syncerus caffer; Taurotragus oryx. References: Avery (1987b, 1995b); Brophy et al. (2014); Grine et al. (1998); Henderson (1992); Klein (1975b, 1976a); McKee et al. (1995); Pearce (2008); Rightmire and Deacon (1991); Van Pletzen (2000); Wadley (2015).

Klein Kliphuis (3207:1851). Taxa: Antidorcas marsupialis; Bathyergus suillus; Galerella pulverulenta; Genetta; Lepus saxatilis; Myotomys unisulcatus cf.; Ovis aries; Procavia capensis; Raphicerus melanotis cf. References: Avery (1992).

Klein Spitzkoppe (2152:1503). Taxa: Antidorcas australis; Felis silvestris; Raphicerus campestris. References: Cruz-Uribe and Klein (1981-1983).

Klein Witkrans. See Little Witkrans.

Klingbeil (2506:3030). Taxa: Bos taurus. References: Evers (1980).

Klipfonteinrand (3218BB). Taxa: Acomys subspinosus; Chlorotalpa sclateri; Crocidura cyanea; Crocidura flavescens; Cryptomys hottentotus; Dendromus melanotis; Elephantulus edwardii; Georychus capensis; Gerbilliscus afra; Gerbillurus paeba; Graphiurus ocularis; Homo sapiens; Micaelamys namaquensis; Myomyscus verreauxii; Myosorex varius; Mystromys albicaudatus; Myotomys unisulcatus; Neoromicia capensis; Otomys irroratus; Otomys saundersiae; Rhabdomys pumilio; Steatomys krebsii; Suncus varilla. References: Avery (unpublished); Pfeiffer (2013).

Klipspruit (2530:3020). Taxa: Bos taurus; Canis adustus cf.; Canis lupus cf.; Connochaetes taurinus; Homo sapiens; Loxodonta africana; Pelea capreolus; Phacochoerus africanus. References: Plug (1999a).

KN2005-0041 (3014:1715). Taxa: Bos taurus. References: Orton et al. (2013).

KN6-3C (3013:1714). Taxa: Elephantulus edwardii; Felis silvestris; Lepus capensis; Otocyon megalotis; Parotomys brantsii; Raphicerus campestris. References: Dewar (2007).

Knysna Heads (3423BB). Taxa: Homo sapiens. References: Dusseldorp et al. (2013).

Komkans 2 (3115:1803). Taxa: Bathyergus suillus; Bos taurus; Canis; Caracal caracal; Equus; Felis silvestris; Hystrix africaeaustralis; Ictonyx striatus; Mellivora capensis; Orycteropus afer; Papio ursinus; Procavia capensis; Raphicerus campestris; Sylvicapra grimmia. References: Orton (2014).

Kommando Kop. See Commando Kop

Kommetjie (3409:1819). Taxa: Homo sapiens. References: Pfeiffer (2013).

KoNomtjarhelo (2529). Taxa: Capra hircus; Lepus; Ovis aries. References: Nelson (2008). 
Kouga (3323). Taxa: Homo sapiens. References: Steyn et al. (2007).

Kougha Dam (3325DA). Taxa: Homo sapiens. References: De Villiers (1965).

Kreeftebaai (3308:1759). Taxa: Bathyergus suillus; Bos taurus; Procavia capensis. References: Smith et al. (1991).

Kruger Cave (2545:2715). Taxa: Antidorcas bondi. References: Brown and Verhagen (1985).

Kuidas Spring (2114). Taxa: Antidorcas marsupialis; Equus; Lepus; Oryx gazella cf.; Petromus typicus; Procavia capensis; Tragelaphus strepsiceros cf. References: Badenhorst (2014); Badenhorst et al. (2016b).

Kumukams (2541:1716). Taxa: Bathyergus suillus; Canis mesomelas; Cynictis penicillata; Ovis aries; Procavia capensis; Raphicerus campestris. References: Cruz-Uribe and Klein (1981-1983).

KV502 (2930:1703). Taxa: Canis; Elephantulus edwardii; Gerbillurus paeba; Myotomys unisulcatus; Parotomys brantsii; Pedetes capensis; Rhabdomys pumilio. References: Dewar (2007); Dewar and Jerardino (2007).

KwaGandaganda (2941:3050). Taxa: Aepyceros melampus; Atilax paludinosus; Bos taurus; Canis lupus; Capra hircus; Chlorocebus aethiops; Equus burchellii; Felis silvestris; Genetta tigrina; Hippopotamus amphibius; Loxodonta africana; Otolemur crassicaudatus; Ovis aries; Panthera pardus; Phacochoerus africanus; Philantomba monticola; Potamochoerus larvatus; Procavia capensis; Raphicerus campestris; Smutsia temminckii; Sylvicapra grimmia; Syncerus caffer. References: Whitelaw (1994).

KwaMaza A \& B (2529BD). Taxa: Aepyceros melampus cf.; Bos taurus; Caracal caracal cf.; Equus; Genetta genetta; Ovis aries; Syncerus caffer; Taurotragus oryx. References: Nelson (2008, 2009).

KwaThwaleyakhe (2855:3027). Taxa: Alcelaphus buselaphus; Aonyx capensis; Canis mesomelas; Caracal caracal; Chlorocebus aethiops; Connochaetes gnou cf.; Connochaetes taurinus; Damaliscus pygargus; Hippotragus; Homo sapiens; Hystrix africaeaustralis; Oreotragus oreotragus; Orycteropus afer; Ourebia ourebi; Ovis aries; Panthera pardus; Papio ursinus; Parahyaena brunnea; Pelea capreolus; Phacochoerus africanus; Philantomba monticola; Potamochoerus larvatus; Procavia capensis; Raphicerus campestris; Redunca arundinum; Redunca fulvorufula; Smutsia temminckii; Sylvicapra grimmia; Taurotragus oryx; Thryonomys swinderianus. References: Mazel (1993); Plug (1993b).

Lame Sheep Shelter (3127:2439). Taxa: Alcelaphus buselaphus; Antidorcas australis; Atilax paludinosus; Canis mesomelas; Connochaetes gnou; Connochaetes taurinus cf.; Damaliscus pygargus; Diceros bicornis; Equus quagga; Ictonyx striatus; Oreotragus oreotragus; Ovis aries; Pelea capreolus; Phacochoerus africanus; Procavia capensis; Raphicerus campestris; Suricata suricatta; Sylvicapra grimmia; Taurotragus oryx; Vulpes chama. References: Plug (1993a, 1999b); Plug and Sampson (1996).

Langdraai (2530:3020). Taxa: Aepyceros melampus; Bos taurus; Canis mesomelas; Connochaetes taurinus; Panthera leo; Pelea capreolus; Phacochoerus africanus; Raphicerus campestris; Syncerus caffer. References: Plug (1999a).

Langebaan (3305:1802). Taxa: Homo sapiens. References: Pfeiffer (2013).

Lanlory (1658:2941). Taxa: Phacochoerus africanus; Potamochoerus larvatus; Thryonomys swinderianus?. References: Huffman (1979a).

Le 6, 7a and 7b (2350:3140). Taxa: Aepyceros melampus; Aonyx capensis; Bos taurus; Chlorocebus aethiops; Civettictis civetta; Connochaetes taurinus; Cryptomys hottentotus; Damaliscus lunatus; Equus burchellii; Gerbillurus paeba; Giraffa camelopardalis; 
Hippopotamus amphibius; Hippotragus equinus; Hippotragus niger; Kobus ellipsiprymnus; Orycteropus afer; Orycteropus afer; Panthera leo; Papio ursinus; Pedetes capensis; Phacochoerus africanus; Potamochoerus larvatus; Pronolagus; Raphicerus campestris; Redunca arundinum; Sylvicapra grimmia; Syncerus caffer; Taurotragus oryx; Thryonomys swinderianus. References: Plug (1989).

Leeuhoek (3130:2435). Taxa: Antidorcas australis; Bos taurus; Canis lupus; Canis mesomelas; Capra hircus; Connochaetes gnou; Damaliscus pygargus; Equus quagga; Oreotragus oreotragus; Ovis aries; Papio ursinus; Phacochoerus africanus; Procavia capensis; Raphicerus campestris; Vulpes chama. References: Plug (1999b); Plug and Sampson (1996).

Leeukop (2212:2858). Taxa: Aepyceros melampus; Connochaetes taurinus; Crocuta crocuta; Equus burchellii; Felis silvestris?; Hystrix africaeaustralis; Lepus saxatilis cf.; Mastomys; Panthera leo; Panthera pardus; Papio ursinus; Paraxerus cepapi; Pedetes capensis; Phacochoerus africanus; Procavia capensis cf.; Raphicerus campestris; Saccostomus; Sylvicapra grimmia; Syncerus caffer; Thryonomys swinderianus. References: Gautier and Van Waarden (1981).

Leholamogoa (2850:2316). Taxa: Procavia capensis; Redunca fulvorufula. References: Holt (2009).

Lekkerwater (1805:3142). Taxa: Aepyceros melampus; Bos taurus; Capra hircus; Connochaetes taurinus; Cryptomys hottentotus; Equus burchellii; Lepus; Papio ursinus; Potamochoerus larvatus; Procavia capensis; Sylvicapra grimmia. References: G. Turner (1984).

Leliehoek (2921:2726). Taxa: Alcelaphus; Antidorcas marsupialis; Connochaetes; Damaliscus pygargus; Felis silvestris; Genetta genetta; Hystrix africaeaustralis; Lepus; Otomys irroratus; Pedetes capensis; Phacochoerus africanus; Procavia capensis; Sylvicapra grimmia. References: Esterhuysen et al. (1994).

Lemoenfontein (2924). Taxa: Phacochoerus africanus; Raphicerus campestris. References: Klein (1979a).

Leopard Cave (2134:1533). Taxa: Ovis aries. References: Pleurdeau et al. (2012). Leqhetsoana (2927:2736). Taxa: Antidorcas marsupialis; Bos taurus; Capra hircus; Connochaetes gnou; Damaliscus pygargus; Hystrix africaeaustralis; Oreotragus oreotragus; Pedetes capensis; Phacochoerus africanus; Procavia capensis; Sylvicapra grimmia; Taurotragus oryx. References: Mitchell et al. (1994); Plug (1997b).

Letsibogo Dam (2127). Taxa: Bos taurus; Equus burchellii; Lepus cf.; Procavia capensis. References: Huffman and Kinahan (2002/2003).

Likoaeng (2944:2845). Taxa: Aonyx capensis; Atilax paludinosus; Bos taurus; Canis mesomelas; Caracal caracal; Chlorocebus aethiops; Cryptomys hottentotus cf.; Homo sapiens cf.; Hystrix africaeaustralis; Lepus saxatilis; Mastomys natalensis; Mellivora capensis cf.; Oreotragus oreotragus; Otomys irroratus; Ovis aries cf.; Panthera pardus; Papio ursinus; Pedetes capensis; Pelea capreolus; Phacochoerus africanus; Procavia capensis; Raphicerus campestris; Redunca fulvorufula; Sylvicapra grimmia; Taurotragus oryx. References: Plug et al. (2003).

Limerock 1 and 2 (2833:2408). Taxa: Aethomys chrysophilus; Alcelaphus buselaphus; Connochaetes gnou; Crocidura cyanea cf.; Crocidura fuscomurina; Crocidura hirta; Cryptomys hottentotus; Cynictis penicillata; Desmodillus auricularis; Eptesicus hottentotus; Equus quagga cf.; Felis silvestris; Gerbilliscus brantsii; Graphiurus murinus; Hippotragus; Malacothrix typica; Mastomys natalensis; Miniopterus natalensis; Mystromys albicaudatus; Neoromicia capensis; Papio ursinus?; Pedetes capensis; Phacochoerus africanus; Procavia capensis; Raphicerus campestris; Rhabdomys pumilio; Saccostomus campestris; Sylvicapra grimmia; Tragelaphus strepsiceros. References: Humphreys and Thackeray (1983); Klein (1979a). 
Liphofung (2944:2827). Taxa: Atilax paludinosus; Bos taurus; Canis mesomelas; Connochaetes gnou; Damaliscus pygargus; Felis silvestris; Hystrix africaeaustralis; Oreotragus oreotragus; Ourebia ourebi; Ovis aries; Panthera pardus; Papio ursinus; Pelea capreolus; Procavia capensis; Raphicerus campestris; Redunca fulvorufula; Sus scrofa; Sylvicapra grimmia; Taurotragus oryx; Xerus. References: Plug (1997b).

Lithakong (2923:2810). Taxa: Caracal caracal cf.; Chlorocebus; Crocidura; Damaliscus pygargus cf.; Hippotragus; Myosorex; Oreotragus oreotragus; Panthera pardus; Papio ursinus; Pelea capreolus; Procavia capensis; Raphicerus campestris; Redunca fulvorufula; Sylvicapra grimmia; Taurotragus oryx. References: Brink (2012).

Little Witkrans (2740:2437). Other names: Klein Witkrans. Taxa: Equus burchellii. References: Humphreys (1978); Klein (1979a).

LK2004-011 (3022:1718). Taxa: Antidorcas marsupialis; Caracal caracal; Elephantulus edwardii; Felis silvestris; Genetta genetta; Gerbillurus paeba; Micaelamys namaquensis; Parotomys brantsii; Raphicerus campestris; Sylvicapra grimmia; Vulpes chama. References: Dewar (2007).

LK5-1 (3023:1718). Taxa: Felis silvestris; Lepus capensis; Raphicerus campestris. References: Dewar (2007).

Lotshitshi (1923CD). Taxa: Bos taurus; Canis mesomelas; Connochaetes; Equus burchellii; Giraffa camelopardalis; Hystrix africaeaustralis; Lepus; Pedetes capensis; Phacochoerus africanus; Sylvicapra grimmia. References: G. Turner (1987b).

Lower Numas Cave (2114). Taxa: Equus; Procavia capensis. References: Cruz-Uribe and Klein (1981-1983).

Lydenburg Heads Site (2506:3030). Taxa: Bos taurus; Ovis aries cf.; Procavia; Sylvicapra grimmia. References: De Villiers (1982); Voigt (1982).

Ma 38 (2305:3130). Taxa: Canis mesomelas; Connochaetes taurinus; Equus burchellii; Giraffa camelopardalis; Hippotragus equinus; Hystrix africaeaustralis; Orycteropus afer; Phacochoerus africanus; Potamochoerus larvatus; Pronolagus; Raphicerus campestris. References: Plug (1989).

Ma 4 (2330:3150). Taxa: Aepyceros melampus; Bos taurus; Equus burchellii. References: Plug (1989).

Mabjanamatshwana (2627). Taxa: Aepyceros melampus; Bos taurus; Canis lupus; Capra hircus; Connochaetes taurinus; Diceros bicornis; Equus quagga; Giraffa camelopardalis; Hippotragus equinus; Loxodonta africana; Ovis aries; Phacochoerus africanus; Procavia capensis; Raphicerus campestris; Sylvicapra grimmia; Syncerus caffer; Tragelaphus strepsiceros. References: Plug and Badenhorst (2006).

Mabveni (1956:3046). Taxa: Bos taurus; Equus burchellii. References: Huffman (1975); Thorp (1979).

Magogo (2853:3017). Taxa: Aethomys chrysophilus; Bos taurus; Canis adustus; Canis lupus; Canis mesomelas; Capra hircus; Homo sapiens; Mastomys natalensis; Ovis aries; Panthera pardus; Pelea capreolus; Potamochoerus larvatus; Sylvicapra grimmia; Tragelaphus angasii; Tragelaphus scriptus. References: Arnold (2008); Voigt (1984).

Maguams Andalusia (2516DB). Taxa: Canis mesomelas; Equus; Hystrix africaeaustralis; Oreotragus oreotragus; Otocyon megalotis; Ovis aries; Pedetes capensis; Procavia capensis; Raphicerus campestris. References: Cruz-Uribe and Klein (1981-1983).

Maguams Elefant (2516DB). Taxa: Equus; Papio ursinus; Procavia capensis; Raphicerus campestris. References: Cruz-Uribe and Klein (1981-1983). 
Makgabeng (2328BB). Taxa: Aepyceros melampus; Alcelaphus buselaphus; Bos taurus; Canis mesomelas; Capra hircus; Connochaetes taurinus; Damaliscus pygargus; Equus burchellii; Felis silvestris; Hippotragus equinus; Lepus saxatilis; Oreotragus oreotragus; Orycteropus afer; Ovis aries; Panthera pardus; Pelea capreolus; Procavia capensis; Raphicerus campestris; Redunca arundinum; Sylvicapra grimmia. References: Van Schalkwyk (2000).

Malumba (2231). Taxa: Aepyceros melampus; Bos taurus; Caracal caracal; Connochaetes taurinus; Equus burchellii; Felis silvestris; Heterohyrax brucei; Hippotragus niger; Lepus saxatilis; Loxodonta africana; Oreotragus oreotragus; Ovis aries; Potamochoerus larvatus; Procavia capensis; Raphicerus campestris; Raphicerus melanotis; Rattus rattus cf.; Sylvicapra grimmia; Taurotragus oryx; Thryonomys swinderianus; Tragelaphus strepsiceros. References: Manyanga (2001); Manyanga et al. (2000); Shenjere-Nyabezi et al. (2013).

Mamaetla (2852:2314). Taxa: Pedetes capensis; Procavia capensis. References: Holt (2009).

Mamba (2857:3103). Taxa: Aepyceros melampus; Atilax paludinosus; Bos taurus; Canis lupus; Canis mesomelas; Capra hircus; Felis silvestris; Hippopotamus amphibius; Kobus ellipsiprymnus; Loxodonta africana; Ovis aries; Redunca fulvorufula; Sylvicapra grimmia; Tragelaphus scriptus. References: Arnold (2008); Voigt and Peters (1994b).

Mananzve Hill (2136:2857). Taxa: Aepyceros melampus; Bos taurus; Oreotragus oreotragus; Sylvicapra grimmia; Tragelaphus strepsiceros. References: Nyamushosho (2016).

Manjowe Rock Shelters (1932). Taxa: Bos taurus cf.; Equus burchellii; Heterohyrax brucei; Homo sapiens; Lepus saxatilis; Otolemur crassicaudatus; Ovis aries; Rattus rattus; Sylvicapra grimmia; Thryonomys swinderianus. References: Katsamudanga (2007a, 2007b); Mupira and Katsmudanga (2007).

Mantenge Shelter (2027). Taxa: Heterohyrax brucei; Pedetes capensis; Phacochoerus africanus; Procavia capensis; Pronolagus. References: De Villiers (1987); Walker (1994).

Manyikeni (2214:3448). Taxa: Bos taurus; Capra hircus; Diceros bicornis; Equus burchellii; Hippopotamus amphibius; Loxodonta africana; Ovis aries; Phacochoerus; Syncerus caffer. References: Sigvallius (1988).

Mapotini Hill (2355:3105). Taxa: Aepyceros melampus; Connochaetes taurinus; Equus burchellii; Homo sapiens; Phacochoerus africanus. References: Plug and Pistorius (1999).

Mapungubwe (2225:2915). Taxa: Aepyceros melampus; Aethomys chrysophilus; Bos taurus; Canis lupus; Canis mesomelas; Capra hircus; Chlorocebus aethiops; Connochaetes; Crocidura cyanea; Cryptomys; Equus burchellii; Felis silvestris; Hippopotamus amphibius; Homo sapiens; Hystrix africaeaustralis; Lepus; Loxodonta africana; Lycaon pictus; Mastomys natalensis; Oreotragus oreotragus; Ovis aries; Papio ursinus; Parahyaena brunnea; Pedetes capensis; Phacochoerus africanus; Potamochoerus larvatus; Pronolagus randensis; Raphicerus campestris; Suncus lixus; Sylvicapra grimmia; Thryonomys swinderianus; Tragelaphus strepsiceros. References: Badenhorst et al. (2011a); Plug (2000); Voigt (1983).

Maqonqo (2821:3025). Taxa: Aepyceros melampus; Amblysomus hottentotus?; Canis mesomelas; Caracal caracal; Chlorocebus aethiops; Connochaetes taurinus; Crocidura flavescens; Cryptomys hottentotus; Cynictis penicillata cf.; Damaliscus pygargus; Dasymys incomtus; Elephantulus myurus?; Equus burchellii; Felis silvestris; Genetta; Gerbilliscus brantsii?; Giraffa camelopardalis cf.; Hippotragus equinus; Homo sapiens; Hystrix africaeaustralis; Lepus saxatilis; Lycaon pictus; Mellivora capensis; Mystromys; Oreotragus oreotragus; Orycteropus afer; Otomys irroratus; Ourebia ourebi; Ovis aries; Panthera leo; Panthera pardus; Papio ursinus; Parahyaena brunnea; Paraxerus cepapi; Pedetes capensis; Pelea capreolus; Phacochoerus africanus; Philantomba monticola; Potamochoerus larvatus; 
Procavia capensis; Pronolagus rupestris; Proteles cristata; Raphicerus campestris; Redunca arundinum; Redunca fulvorufula; Rhabdomys pumilio; Smutsia temminckii; Sylvicapra grimmia; Syncerus caffer; Taurotragus oryx; Thryonomys swinderianus; Tragelaphus scriptus; Tragelaphus strepsiceros. References: Avery (1996); Plug (1996a).

Marupale Hill (2355:3105). Taxa: Aepyceros melampus; Bos taurus; Canis lupus; Capra hircus; Connochaetes taurinus; Equus burchellii; Equus caballus; Felis silvestris; Giraffa camelopardalis; Hippotragus; Homo sapiens; Hystrix africaeaustralis; Kobus ellipsiprymnus cf.; Lepus capensis; Phacochoerus africanus; Procavia capensis; Raphicerus campestris; Redunca arundinum cf.; Sylvicapra grimmia; Syncerus caffer; Taurotragus oryx; Thryonomys swinderianus; Tragelaphus strepsiceros cf. References: Plug and Pistorius (1999).

Maselspoort (2902:2625). Taxa: Connochaetes gnou; Homo sapiens. References: Brink (1993, 2005).

Matanga (2052:2737). Taxa: Bos taurus; Homo sapiens. References: De Villiers (1987); Van Waarden (1987).

Matjies River Rock Shelter (3401:2325). Taxa: Homo sapiens; Phacochoerus africanus; Rhinolophus clivosus. References: Dreyer (1933); L'Abbé et al. (2008); Louw (1960).

Matlapaneng (1923CD). Taxa: Aepyceros melampus; Bos taurus; Canis mesomelas cf.; Capra hircus; Connochaetes; Crocidura hirta cf.; Equus burchellii; Gerbilliscus brantsii cf.; Giraffa camelopardalis; Hystrix africaeaustralis; Lepus; Lycaon pictus cf.; Otocyon megalotis cf.; Ovis aries; Pedetes capensis; Redunca arundinum; Sylvicapra grimmia; Syncerus caffer; Taurotragus. References: G. Turner (1987b).

Mauermanshoek (2851:2714). Taxa: Aepyceros melampus; Alcelaphus; Antidorcas marsupialis; Bos taurus; Canis mesomelas; Connochaetes; Crocuta crocuta; Cynictis penicillata; Damaliscus pygargus; Lepus; Oreotragus oreotragus; Otomys irroratus; Procavia capensis; Raphicerus campestris; Raphicerus melanotis; Sylvicapra grimmia. References: Wadley (2001).

MB2005-005 (3028:1722). Other names: Seal Midden. Taxa: Felis silvestris; Parotomys brantsii; Raphicerus campestris. References: Dewar (2007).

MB2005-119 (3028:1722). Taxa: Raphicerus campestris. References: Dewar (2007).

Mbabane (2845:3022). Taxa: Crocidura cyanea cf.; Crocidura flavescens; Cryptomys hottentotus; Mastomys natalensis; Mus minutoides; Myosorex; Otomys irroratus cf.; Rhabdomys pumilio; Steatomys pratensis. References: Avery (1991b); Mazel (1986b).

Meerkat Shelter (3108:2513). Taxa: Cryptomys hottentotus; Desmodillus auricularis; Gerbilliscus brantsii; Myosorex varius; Mystromys albicaudatus; Otomys angoniensis; Otomys irroratus; Parotomys brantsii; Steatomys krebsii. References: Avery (unpublished).

Melkboom 1 (2902:2101). Taxa: Genetta genetta; Oreotragus oreotragus; Pedetes capensis; Procavia capensis; Raphicerus campestris; Sylvicapra grimmia. References: Badenhorst et al. (2015).

Melkbostrand (3343:1827). Taxa: Homo sapiens. References: Pfeiffer (2013).

Melkhoutboom (3319:2517). Taxa: Alcelaphus caama cf.; Canis mesomelas; Caracal caracal; Chlorocebus aethiops; Connochaetes gnou cf.; Damaliscus pygargus cf.; Equus quagga cf.; Felis silvestris; Hippotragus leucophaeus cf.; Homo sapiens; Hystrix africaeaustralis; Lepus capensis cf.; Oreotragus oreotragus; Orycteropus afer; Ourebia ourebi; Panthera pardus; Papio ursinus; Pelea capreolus; Phacochoerus africanus; Philantomba monticola; Potamochoerus larvatus; Procavia capensis; Proteles cristata; Raphicerus; Redunca arundinum cf.; Redunca fulvorufula cf.; Smutsia; Sylvicapra grimmia; Syncerus caffer; Taurotragus oryx; Tragelaphus scriptus; Tragelaphus strepsiceros. References: Deacon (1976); Pearce (2008). 
Messum (2114AD). Taxa: Antidorcas australis; Procavia capensis. References: Cruz-Uribe and Klein (1981-1983).

Mgede (2810:2941). Taxa: Amblysomus hottentotus; Aonyx capensis; Atilax paludinosus; Canis; Chrysospalax villosus; Crocidura cyanea; Crocidura flavescens; Cryptomys hottentotus; Dendromus mesomelas; Equus burchellii cf.; Felis silvestris; Galerella sanguinea; Hippotragus; Homo sapiens; Hystrix africaeaustralis; Lepus; Mellivora capensis; Micaelamys namaquensis; Mus minutoides; Myosorex varius; Mystromys albicaudatus; Oreotragus oreotragus; Orycteropus afer; Otomys irroratus cf.; Ourebia ourebi; Panthera pardus; Papio ursinus; Pelea capreolus; Potamochoerus larvatus; Procavia capensis; Raphicerus; Redunca fulvorufula; Rhabdomys pumilio. References: Avery (1991b); Mazel (1986a).

Mgoduyanuka (2843:2924). Taxa: Aepyceros melampus; Cryptomys hottentotus; Hippopotamus amphibius; Ovis aries; Pelea capreolus. References: Plug and Brown (1982).

Mhandambiri (2231). Taxa: Canis mesomelas cf.; Cephalophus monticola cf.; Connochaetes taurinus?; Georychus; Lepus saxatilis; Syncerus caffer cf.; Sylvicapra grimmia; Taurotragus oryx?; Thryonomys swinderianus; Tragelaphus scriptus; Tragelaphus strepsiceros. References: Shenjere-Nyabezi and Pwiti (2015).

Mhlopeni (2903:3024). Taxa: Capra hircus; Ovis aries. References: De Villiers (1984); Voigt (1984).

Mhlwazini (2903:2923). Taxa: Aepyceros melampus; Antidorcas australis; Antidorcas marsupialis; Atilax paludinosus; Canis mesomelas; Caracal caracal; Cephalophus natalensis; Crocidura flavescens; Cryptomys hottentotus; Felis silvestris; Hystrix africaeaustralis; Lepus; Myosorex; Oreotragus oreotragus; Otomys irroratus; cf.; Otomys laminatus; Ourebia ourebi; Ovis aries; Papio ursinus; Pedetes capensis; Pelea capreolus; Phacochoerus africanus; Philantomba monticola; Procavia capensis; Pronolagus rupestris; Raphicerus campestris; Redunca fulvorufula; Rhabdomys pumilio; Smutsia temminckii; Sylvicapra grimmia; Taurotragus oryx. References: Avery (1991b); Mazel (1990); Plug (1990, 1997b).

Milnerton (3353:1830). Taxa: Homo sapiens. References: Pfeiffer (2013).

Mirabib (2327:1519). Taxa: Crocidura; Desmodillus auricularis?; Elephantulus intufi?; Elephantulus rupestris?; Eremitalpa granti; Gerbillurus paeba?; Gerbillurus vallinus; Macroscelides proboscideus?; Malacothrix typica; Petromus typicus cf.; Petromyscus; Rhabdomys. References: Brain and Brain (1977).

Mitford Park (3220:2630). Taxa: Homo sapiens. References: Pearce (2008).

Mmatshetschele Mountain (2535:2720). Taxa: Bos taurus; Capra hircus; Cryptomys; Equus; Lepus; Ovis aries; Procavia capensis. References: Badenhorst and Plug (2001).

Mo 8 (2325:3155). Taxa: Aepyceros melampus; Connochaetes taurinus; Crocuta crocuta; Equus burchellii; Giraffa camelopardalis; Lepus saxatilis; Syncerus caffer. References: Plug (1989).

Modder River Mouth (3328:1818). Taxa: Homo sapiens. References: Pfeiffer (2013); Pfeiffer and Van der Merwe (2004).

Modipe Hill (2439:2610). Taxa: Homo sapiens. References: Owens (1995).

Mogapelwa (2022). Taxa: Aepyceros melampus; Equus burchellii; Felis silvestris; Herpestes ichneumon; Orycteropus afer; Pedetes capensis; Phacochoerus africanus; Raphicerus campestris; Sylvicapra grimmia. References: Robbins et al. (2009).

Molokwane (2527). Taxa: Aepyceros melampus; Alcelaphus buselaphus; Bos taurus; Canis lupus; Capra hircus; Chlorocebus pygerythrus; Connochaetes taurinus; Damaliscus pygargus; Equus quagga cf.; Genetta tigrina cf.; Giraffa camelopardalis; Hippotragus niger; Homo sapiens; Loxodonta africana; Ovis aries; Pedetes capensis; Phacochoerus africanus; Raphicerus 
campestris; Redunca fulvorufula; Sylvicapra grimmia; Syncerus caffer; Taurotragus oryx; Tragelaphus strepsiceros. References: Plug and Badenhorst (2006).

Moritsane (2439:2547). Taxa: Aepyceros melampus; Alcelaphus buselaphus; Bos taurus; Caracal caracal; Connochaetes taurinus cf.; Equus burchellii; Hippotragus equinus; Hippotragus niger; Kobus leche; Lepus; Procavia capensis; Raphicerus campestris; Sylvicapra grimmia; Syncerus caffer; Taurotragus oryx; Tragelaphus strepsiceros. References: Reed Cohen (2010).

Mpambanyoni (3017:3044). Taxa: Aethomys chrysophilus; Bos taurus?; Cryptomys hottentotus; Dasymys incomtus; Herpestes ichneumon?; Lepus; Loxodonta africana; Mastomys natalensis; Mungos mungo; Myosorex; Otomys irroratus; Otomys laminatus; Philantomba monticola; Potamochoerus larvatus; Procavia capensis; Thryonomys swinderianus; Tragelaphus scriptus. References: Robey (1980).

Mphekwane (2850:2316). Taxa: Oreotragus oreotragus; Raphicerus campestris; Smutsia temminckii; Sylvicapra grimmia; Tragelaphus strepsiceros. References: Holt (2009).

Msuluzi Confluence (2845:3008). Taxa: Aepyceros melampus; Bos taurus; Capra hircus; Ovis aries; Papio ursinus. References: Arnold (2008); De Villiers (1984); Voigt (1980a).

Mt Ziwa (1808:3238). Taxa: Bos taurus. References: Thorp (1979).

Mtanye (2129AD). Taxa: Bos taurus; Capra hircus. References: Huffman (2008).

Mud River (3318AD). Taxa: Homo sapiens. References: Pfeiffer (2013).

Muela (2944:2827). Taxa: Aepyceros melampus; Alcelaphus buselaphus; Antidorcas australis; Bos taurus; Canis mesomelas; Caracal caracal; Chlorocebus aethiops; Connochaetes gnou; Damaliscus pygargus; Hystrix africaeaustralis; Lepus; Oreotragus oreotragus; Ourebia ourebi; Ovis aries; Papio ursinus; Pelea capreolus; Phacochoerus africanus; Philantomba monticola; Procavia capensis; Pronolagus rupestris; Raphicerus campestris; Redunca fulvorufula; Sylvicapra grimmia; Taurotragus oryx. References: Plug (1997b).

Muhululu Hill (2331). Taxa: Aepyceros melampus; Loxodonta africana. References: Plug and Pistorius (1999).

Muozi (1750:3255). Taxa: Bos taurus; Canis lupus; Capra hircus; Cryptomys; Homo sapiens; Hystrix africaeaustralis; Lepus saxatilis; Ovis aries; Potamochoerus larvatus; Rattus rattus. References: Plug and Badenhorst (2002); Plug et al. (1997).

Murahwa's Hill (1858:3239). Taxa: Aepyceros melampus; Bos taurus cf.; Canis sp.; Capra hircus; Caracal caracal; Genetta; Lepus saxatilis; Ovis aries; Potamochoerus larvatus; Procavia capensis; Redunca arundinum; Sylvicapra grimmia cf.; Taurotragus oryx; Thryonomys swinderianus; Tragelaphus strepsiceros. References: Katsamudanga (2007a); Shenjere-Nyabezi et al. (2013).

Mutshilachokwe (2207:2931). Taxa: Bos taurus; Genetta genetta; Lepus saxatilis; Pedetes capensis; Sylvicapra grimmia. References: Manyanga (2006); Shenjere-Nyabezi et al. (2013).

Mwenezi Farm (2231). Taxa: Aepyceros melampus; Alcelaphus buselaphus; Bos taurus; Capra hircus; Cephalophus natalensis; Connochaetes taurinus; Equus burchellii; Giraffa camelopardalis; Hippotragus niger; Kobus ellipsiprymnus; Lepus saxatilis; Ovis aries; Phacochoerus africanus; Potamochoerus larvatus; Procavia capensis; Raphicerus campestris; Rattus rattus cf.; Redunca arundinum; Sylvicapra grimmia; Taurotragus oryx; Thryonomys gregorianus; Thryonomys swinderianus; Tragelaphus strepsiceros. References: Manyanga (2001); Manyanga et al. (2000); Shenjere-Nyabezi et al. (2013).

Mzinyashana 1 (2819:3028). Taxa: Aepyceros melampus; Aethomys chrysophilus; Alcelaphus buselaphus; Amblysomus hottentotus; Antidorcas marsupialis; Atilax paludinosus; Bos taurus; Canis mesomelas; Caracal caracal; Cephalophus natalensis; Chlorocebus aethiops; 
Chrysospalax villosus; Connochaetes taurinus; Crocidura cyanea; Crocidura flavescens; Crocuta crocuta; Cryptomys hottentotus; Cynictis penicillata; Dasymys incomtus; Dendromus melanotis; Equus burchellii; Felis silvestris; Gerbilliscus brantsii; Hippotragus; Homo sapiens; Hystrix africaeaustralis; Lepus saxatilis; Lycaon pictus; Mastomys natalensis; Micaelamys namaquensis; Mystromys albicaudatus; Neotragus moschatus; Oreotragus oreotragus; Orycteropus afer; Otomys irroratus; Ourebia ourebi; Ovis aries; Papio ursinus; Paraxerus cepapi cf.; Pedetes capensis; Pelea capreolus; Phacochoerus africanus; Philantomba monticola; Potamochoerus larvatus; Procavia capensis; Pronolagus rupestris; Proteles cristata; Raphicerus campestris; Redunca arundinum; Redunca fulvorufula; Rhabdomys pumilio; Saccostomus campestris; Smutsia temminckii; Steatomys pratensis; Suncus infinitesimus; Suncus varilla; Sylvicapra grimmia; Syncerus caffer; Taurotragus oryx; Thryonomys swinderianus; Tragelaphus scriptus; Tragelaphus strepsiceros cf.; Vulpes chama. References: Avery (1997a); Plug (2002).

Mzonjani (2944:3103). Taxa: Bos taurus; Philantomba monticola. References: Voigt (1980c).

Namib 2 (2347:1520). Taxa: Connochaetes taurinus?; Equus sp.; Oryx gazella?; Raphicerus campestris?. References: Shackley (1985).

Namtib (2602:1615). Taxa: Caracal caracal cf.; Equus zebra cf.; Lepus; Oreotragus oreotragus; Oryx gazella; Petromus typicus cf.; Procavia; Pronolagus randensis; Raphicerus campestris. References: Thackeray (1979).

Nanda (2940:3051). Taxa: Aethomys chrysophilus cf.; Atilax paludinosus; Bos taurus; Canis lupus cf.; Capra hircus; Chlorocebus aethiops; Cryptomys hottentotus; Felis silvestris; Genetta tigrina; Homo sapiens; Mastomys natalensis; Otomys irroratus; Ovis aries; Papio ursinus; Philantomba monticola; Potamochoerus larvatus; Procavia capensis; Raphicerus campestris; Rattus rattus; Redunca; Sylvicapra grimmia; Syncerus caffer; Tragelaphus scriptus. References: Plug (1993c).

Ndondondwane (2850:3102). Taxa: Bos taurus; Canis lupus; Canis mesomelas; Capra hircus; Hippopotamus amphibius; Ourebia ourebi; Ovis aries; Pelea capreolus; Rattus rattus; Redunca arundinum; Sylvicapra grimmia; Thryonomys swinderianus; Tragelaphus angasii. References: Arnold (2008); Voigt and Von den Driesch (1984).

Ndongo (2045:3209). Taxa: Aepyceros melampus; Capra hircus; Genetta genetta; Georychus capensis; Lepus saxatilis; Oreotragus oreotragus; Ovis aries; Raphicerus campestris; Sylvicapra grimmia; Syncerus caffer; Thryonomys swinderianus; Tragelaphus strepsiceros. References: Shenjere-Nyabezi (2017); Shenjere-Nyabezi et al. (2013).

Nelson Bay Cave (3406:2322). Taxa: Alcelaphus caama cf.; Aonyx capensis; Atilax paludinosus; Canis mesomelas; Caracal caracal cf.; Felis silvestris; Galerella pulverulenta; Herpestes ichneumon; Hippopotamus amphibius; Hippotragus; Homo sapiens; Hystrix africaeaustralis; Orycteropus afer; Ourebia ourebi; Panthera pardus; Papio ursinus; Phacochoerus africanus; Philantomba monticola; Potamochoerus larvatus; Procavia capensis; Raphicerus; Redunca arundinum; Redunca fulvorufula; Sylvicapra grimmia; Syncerus caffer; Taurotragus oryx; Tragelaphus scriptus. References: Brophy et al. (2014); Klein (1972, 1974b); Pearce (2008).

Nkupe (2809:2956). Taxa: Amblysomus hottentotus; Aonyx capensis; Atilax paludinosus; Canis mesomelas cf.; Caracal caracal cf.; Chlorocebus aethiops; Chrysospalax villosus; Connochaetes gnou cf.; Crocidura cyanea; Crocidura flavescens; Cryptomys hottentotus; Damaliscus pygargus; Dasymys incomtus; Dendromus melanotis; Dendromus mesomelas; Elephantulus myurus cf.; Eptesicus hottentotus?; Equus burchellii cf.; Felis silvestris; Galerella sanguinea; Genetta; Gerbilliscus brantsii cf.; Grammomys dolichurus; Graphiurus murinus; 
Hippotragus; Homo sapiens; Hystrix africaeaustralis; Kerivoula argentata; Lycaon pictus; Mastomys natalensis; Mellivora capensis; Micaelamys namaquensis; Miniopterus natalensis; Mus minutoides; Myosorex; Mystromys albicaudatus; Oreotragus oreotragus; Orycteropus afer; Otomys angoniensis; Otomys irroratus cf.; Ourebia ourebi; Ovis aries; Panthera leo; Panthera pardus; Papio ursinus; Pelea capreolus; Phacochoerus africanus; Potamochoerus larvatus; Raphicerus campestris cf.; Redunca fulvorufula; Rhabdomys pumilio; Rhinolophus clivosus cf.; Rhinolophus simulator?; Suncus infinitesimus; Syncerus caffer. References: Avery (1988, 1991b); Mazel (1988a).

Noordhoek (3406:1823). Taxa: Homo sapiens. References: Pfeiffer (2013).

North Brabant Shelter (2355:2806). Taxa: Aepyceros melampus; Cephalophus; Equus burchellii; Hystrix; Ictonyx; Lycaon pictus; Panthera pardus; Phacochoerus africanus; Procavia capensis; Syncerus caffer. References: Schoonraad and Beaumont (1968). Norvalspont (3038:2527). Taxa: Equus zebra. References: Lundholm (1952). Nos (2530:1530). Taxa: Canis; Procavia. References: Thackeray (1979).

Nqoma (1845:2145). Taxa: Aepyceros melampus; Bos taurus; Canis mesomelas cf.; Capra hircus; Civettictis civetta; Connochaetes; Cryptomys hottentotus; Equus burchellii; Gerbilliscus; Giraffa camelopardalis; Homo sapiens; Kobus ellipsiprymnus cf.; Kobus leche; Lepus; Loxodonta africana; Otocyon megalotis; Ovis aries; Pedetes capensis; Phacochoerus africanus; Redunca arundinum cf.; Sylvicapra grimmia; Syncerus caffer; Taurotragus. References: G. Turner (1987a).

Ntloana Tsoana (2910:2749). Taxa: Antidorcas australis; Canis mesomelas; Connochaetes gnou; Connochaetes taurinus cf.; Equus burchellii; Hippotragus leucophaeus cf.; Oreotragus oreotragus; Papio ursinus; Parahyaena brunnea cf.; Pelea capreolus; Phacochoerus africanus; Procavia capensis; Raphicerus campestris; Redunca fulvorufula; Taurotragus oryx. References: Plug (1997b).

Ntshekane (2858:3024). Taxa: Bos taurus; Canis lupus; Connochaetes taurinus; Felis silvestris cf.; Lepus; Lycaon pictus; Ourebia ourebi; Ovis aries; Phacochoerus africanus; Philantomba monticola cf.; Potamochoerus larvatus; Procavia capensis; Raphicerus campestris cf.; Sylvicapra grimmia; Tragelaphus angasii. References: Arnold (2008); Maggs and Michael (1976).

Numas 25 (2107:1425). Taxa: Antidorcas marsupialis; Canis mesomelas; Crocuta crocuta; Equus; Suricata suricatta. References: Van Neer and Breunig (1999).

Nuwekloof Shelter (3331:2339). Taxa: Lepus; Papio ursinus; Procavia capensis; Raphicerus melanotis; Sylvicapra grimmia. References: Binneman (2000).

Oakhurst (3401:2240). Taxa: Genetta?; Hippotragus leucophaeus?; Philantomba monticola; Potamochoerus larvatus; Procavia capensis; Raphicerus?; Syncerus caffer. References: Drennan (1937); Fagan (1960); Goodwin (1937a, 1937b); Pearce (2008).

Ochre Cave (2724). Taxa: Antidorcas marsupialis; Equus burchellii; Hystrix africaeaustralis; Phacochoerus; Procavia capensis; Redunca fulvorufula. References: Humphreys (1978).

OFD1 (2924DD). Taxa: Antidorcas marsupialis; Cynictis penicillata?; Herpestes ichneumon?; Procavia capensis. References: Klein (1979a).

OI 20 (2410:3105). Taxa: Aepyceros melampus; Bos taurus; Canis mesomelas; Civettictis civetta; Connochaetes taurinus; Crocuta crocuta; Equus burchellii; Giraffa camelopardalis; Hippopotamus amphibius; Hippotragus niger; Kobus ellipsiprymnus; Lepus saxatilis; Papio ursinus; Pedetes capensis; Phacochoerus africanus; Raphicerus campestris; Sylvicapra grimmia; Syncerus caffer. References: Plug (1989).

Olieboompoort (2359:2740). Taxa: Aepyceros melampus; Bos taurus; Canis mesomelas; Civettictis civetta; Connochaetes gnou cf.; Connochaetes taurinus cf.; Crocidura; Crocuta 
crocuta; Damaliscus lunatus cf.; Damaliscus pygargus cf.; Diceros bicornis; Equus quagga; Felis silvestris; Galerella sanguinea; Genetta tigrina; Gerbilliscus leucogaster cf.; Giraffa camelopardalis; Hippopotamus amphibius; Hippotragus equinus; Hystrix africaeaustralis; Kobus ellipsiprymnus cf.; Lepus saxatilis; Mungos mungo; Oreotragus oreotragus; Orycteropus afer cf.; Papio ursinus; Paraxerus cepapi; Pedetes capensis; Pelea capreolus; Phacochoerus africanus; Potamochoerus larvatus; Procavia capensis; Pronolagus randensis; Raphicerus campestris; Raphicerus sharpei; Rattus rattus cf.; Redunca arundinum; Redunca fulvorufula; Sylvicapra grimmia; Syncerus caffer; Taurotragus oryx; Thryonomys swinderianus cf.; Tragelaphus scriptus cf.; Tragelaphus strepsiceros cf. References: Van der Ryst (2006).

OND 2 and 3 (2901:2722). Taxa: Bos taurus; Connochaetes gnou cf.; Damaliscus pygargus cf. References: Maggs (1975).

Ondini (2819:3128). Taxa: Bos taurus; Capra hircus; Equus asinus; Felis silvestris; Hippopotamus amphibius; Hystrix africaeaustralis; Leptailurus serval; Ovis aries; Panthera leo; Pedetes capensis; Pelea capreolus; Procavia capensis; Proteles cristata; Raphicerus campestris; Redunca arundinum; Redunca fulvorufula; Saccostomus; Sylvicapra grimmia; Thryonomys swinderianus. References: Watson and Watson (1990).

001 (2757:2804). Taxa: Alcelaphus caama cf.; Antidorcas marsupialis; Bos taurus; Canis lupus; Damaliscus pygargus cf.; Pedetes capensis; Procavia capensis. References: Maggs (1975).

Orabes Upper (2114). Taxa: Felis silvestris cf.; Hystrix africaeaustralis; Oreotragus oreotragus cf.; Ovis aries; Panthera pardus; Procavia capensis. References: Cruz-Uribe and Klein (1981-1983).

Orange River Railway Station (2940:2412). Taxa: Aepyceros melampus; Antidorcas marsupialis; Bos taurus; Canis lupus; Capra hircus cf.; Damaliscus pygargus; Equus; Homo sapiens; Lepus saxatilis cf.; Oreotragus oreotragus; Ovis aries; Pedetes capensis; Pelea capreolus; Procavia capensis; Raphicerus campestris; Redunca arundinum; Sylvicapra grimmia. References: Badenhorst and Boshoff (2015).

Oshilongo (2110:1430). Taxa: Antidorcas marsupialis; Bos taurus; Canis; Equus; Felis silvestris; Oryx gazella; Pronolagus randensis; Xerus princeps. References: Van Neer and Breunig (1999).

Ostrich Shelter (2440:2535). Taxa: Connochaetes taurinus; Ovis aries; Procavia capensis. References: Sadr and Plug (2001).

OU1 (2720:2845). Taxa: Alcelaphus caama cf.; Bos taurus; Connochaetes gnou cf.; Damaliscus pygargus cf.; Pedetes capensis; Proteles cristata. References: Maggs (1975).

OU2 (2711:2908). Taxa: Alcelaphus caama cf.; Antidorcas marsupialis; Bos taurus; Connochaetes gnou cf.; Damaliscus pygargus cf.; Pedetes capensis. References: Maggs (1975).

Oudepost 1 (3308:1802). Taxa: Bathyergus suillus; Canis; Caracal caracal; Diceros bicornis; Felis; Galerella pulverulenta; Genetta; Herpestes ichneumon; Hippopotamus amphibius; Hystrix africaeaustralis; Ictonyx striatus; Mellivora capensis; Orycteropus afer; Ovis aries; Panthera leo; Panthera pardus; Parahyaena brunnea; Raphicerus campestris; Raphicerus melanotis; Sus scrofa; Sylvicapra grimmia; Taurotragus oryx; Vulpes chama. References: Cruz-Uribe and Schrire (1991).

Pa 8.1 (2245:3121). Taxa: Aepyceros melampus; Equus burchellii; Kobus ellipsiprymnus; Pronolagus; Sylvicapra grimmia; Syncerus caffer. References: Plug $(1989,2000)$.

Pancho's Kitchen Midden (3219:1819). Taxa: Atilax paludinosus; Bathyergus suillus; Canis; Felis silvestris; Galerella pulverulenta; Ictonyx striatus; Lepus capensis; Mellivora capensis; Panthera pardus; Papio ursinus; Procavia capensis; Pronolagus rupestris; Raphicerus campestris; Sylvicapra grimmia; Taurotragus oryx. References: Jerardino (1998, 2012); Jerardino et al. (2013). 
Paradise Main House (3340:1810). Taxa: Bos taurus; Canis lupus; Oreotragus oreotragus; Oryctolagus cuniculus; Ovis aries; Papio ursinus; Procavia capensis; Sus scrofa. References: Avery (1989).

Paternoster (3248:1753). Taxa: Bathyergus suillus; Canis mesomelas; Dendromus melanotis; Galerella pulverulenta; Hystrix africaeaustralis; Myotomys unisulcatus cf.; Raphicerus; Rhabdomys pumilio; Sylvicapra grimmia; Taurotragus oryx. References: Robertshaw (1977).

Penge (2422:1758). Taxa: Bos taurus; Oreotragus oreotragus cf.; Ovis aries; Panthera pardus; Phacochoerus africanus; Syncerus caffer cf.; Thryonomys swinderianus. References: Antonites et al. (2014).

Ph 9 (2325:3155). Taxa: Aepyceros melampus; Connochaetes taurinus; Equus burchellii; Pronolagus. References: Plug (1989).

Pjene Hill (2331). Taxa: Hippotragus equinus. References: Plug and Pistorius (1999).

Pockenbank (2713:1631). Taxa: Equus zebra cf.; Hystrix africaeaustralis; Lepus; Oreotragus oreotragus; Oryx gazella; Petromus typicus cf.; Procavia; Raphicerus campestris. References: Thackeray (1979); Wadley (2015).

Pomongwe (2032:2830). Taxa: Connochaetes; Damaliscus lunatus; Equus; Hippotragus niger; Oreotragus oreotragus; Orycteropus afer; Panthera pardus; Pedetes capensis; Phacochoerus africanus; Potamochoerus larvatus; Procavia capensis; Raphicerus campestris; Raphicerus melanotis; Sylvicapra grimmia; Tragelaphus scriptus. References: Brain (1981); Wadley (2015).

Pont Drift (2213:2909). Taxa: Aepyceros melampus; Bos taurus; Canis adustus; Canis lupus; Capra hircus; Chlorocebus aethiops; Diceros bicornis; Equus burchellii; Giraffa camelopardalis; Heterohyrax brucei; Hippopotamus amphibius; Homo sapiens; Loxodonta africana; Oreotragus oreotragus; Orycteropus afer; Ovis aries; Pedetes capensis; Pelea capreolus cf.; Phacochoerus africanus; Procavia capensis; Raphicerus campestris; Rattus rattus; Sylvicapra grimmia; Syncerus caffer; Xerus. References: De Villiers (1980); Hanisch (1980); Plug (2000); Plug et al. (1979); Steyn and Nienaber (2000); Voigt (1980b).

Potgietersrust (2429AA). Taxa: Oreotragus oreotragus. References: Wells (1951).

Powerhouse Cave (2737:2438). Taxa: Antidorcas marsupialis; Canis mesomelas; Caracal caracal; Connochaetes gnou; Cynictis penicillata cf.; Diceros bicornis; Equus burchellii; Equus quagga; Felis silvestris cf.; Hystrix africaeaustralis; Mellivora capensis; Oreotragus oreotragus cf.; Papio ursinus; Pedetes capensis; Phacochoerus africanus; Procavia capensis; Raphicerus campestris; Redunca fulvorufula; Sylvicapra grimmia; Taurotragus oryx?; Vulpes chama. References: Humphreys (1978); Klein (1979a).

Pramberg (2915:2445). Taxa: Alcelaphus buselaphus; Antidorcas marsupialis; Bos taurus; Canis mesomelas; Connochaetes gnou; Damaliscus pygargus; Hippopotamus amphibius; Orycteropus afer; Ovis aries; Parahyaena brunnea cf.; Pedetes capensis; Phacochoerus africanus; Raphicerus campestris. References: Brink et al. (1992).

Putslaagte 8 (3218). Taxa: Bathyergus suillus; Galerella pulverulenta; Hystrix africaeaustralis; Pelea capreolus; Procavia capensis; Raphicerus; Sylvicapra grimmia. References: Mackay et al. (2015).

Qugana (1822). Taxa: Aepyceros melampus; Connochaetes; Equus burchellii; Gerbilliscus brantsii cf.; Giraffa camelopardalis; Hippopotamus amphibius; Hippotragus niger cf.; Mastomys natalensis; Pedetes capensis; Redunca arundinum; Sylvicapra grimmia; Syncerus; Taurotragus. References: G. Turner (1987b).

QwaQwa Museum Site (2829:2844). Taxa: Bos taurus; Capra hircus; Lepus capensis cf.; Procavia capensis; Proteles cristata; Sylvicapra grimmia. References: Brink and Holt (1992). 
Radiepolong (2440:2535). Taxa: Alcelaphus buselaphus; Antidorcas australis; Bos taurus; Canis mesomelas; Capra hircus; Connochaetes taurinus; Equus burchellii; Hystrix africaeaustralis; Lepus; Oreotragus oreotragus; Oryx gazella; Ovis aries; Parahyaena brunnea; Paraxerus cepapi; Pedetes capensis; Phacochoerus africanus; Procavia capensis; Raphicerus campestris; Sylvicapra grimmia; Taurotragus oryx; Xerus inauris. References: Sadr and Plug (2001).

Randjies (2257:2843). Taxa: Aepyceros melampus; Alcelaphus buselaphus; Aonyx capensis; Atilax paludinosus; Bos taurus; Canis lupus; Canis mesomelas cf.; Capra hircus; Caracal caracal; Chlorocebus aethiops; Connochaetes taurinus; Crocuta crocuta; Equus burchellii; Felis silvestris; Giraffa camelopardalis; Hippotragus niger cf.; Homo sapiens; Hystrix africaeaustralis; Lepus saxatilis; Oreotragus oreotragus; Orycteropus afer; Ovis aries; Panthera leo; Pedetes capensis; Pelea capreolus; Phacochoerus africanus; Procavia capensis; Raphicerus campestris; Rattus rattus; Redunca arundinum cf.; Suricata suricatta; Sus scrofa; Sylvicapra grimmia; Syncerus caffer; Taurotragus oryx; Tragelaphus strepsiceros. References: Badenhorst and Plug (2003); Plug (1994).

Ratho Kroonkop (2229). Taxa: Aepyceros melampus; Bos taurus cf.; Ceratotherium simum cf.; Diceros bicornis; Equus quagga; Giraffa camelopardalis; Potamochoerus larvatus; Procavia capensis; Raphicerus campestris; Redunca arundinum; Sylvicapra grimmia; Syncerus caffer; Taurotragus oryx cf.; Tragelaphus strepsiceros. References: Brunton et al. (2013).

Rautenbach's Cave (3331:2342). Taxa: Equus zebra cf.; Oreotragus oreotragus; Papio ursinus; Procavia capensis; Raphicerus melanotis; Sylvicapra grimmia; Taurotragus oryx. References: Binneman (2000).

Ravenscraig (3000:2747). Taxa: Equus; Hippotragus; Oreotragus oreotragus; Panthera pardus; Papio ursinus; Pelea capreolus; Procavia capensis; Taurotragus oryx. References: Plug (1997b).

Reception Shelter (3132:1836). Other names: VR001. Taxa: Alcelaphus buselaphus cf.; Bathyergus janetta; Bathyergus suillus; Bos taurus; Canis; Chrysochloris asiatica; Crocidura flavescens; Cryptomys hottentotus; Dendromus melanotis; Desmodillus auricularis; Eremitalpa granti; Felis silvestris; Gerbilliscus afra; Gerbillurus paeba; Hystrix africaeaustralis; Macroscelides proboscideus; Malacothrix typica; Micaelamys namaquensis; Mus minutoides; Myosorex varius; Myotomys unisulcatus; Mystromys albicaudatus; Nycteris thebaica; Panthera leo; Parotomys brantsii; Procavia capensis; Raphicerus campestris; Rhabdomys pumilio; Rhinolophus clivosus; Steatomys krebsii; Suncus varilla; Sylvicapra grimmia. References: Avery (unpublished); Orton et al. (2011).

Renbaan (3214:1852). Taxa: Acomys subspinosus; Chlorotalpa sclateri; Chrysochloris asiatica; Crocidura cyanea; Crocidura flavescens; Cryptomys hottentotus; Dendromus melanotis; Elephantulus edwardii; Georychus capensis; Gerbilliscus afra; Gerbillurus paeba; Graphiurus ocularis; Micaelamys namaquensis; Mus minutoides; Myomyscus verreauxii; Myosorex varius; Myotomys unisulcatus; Mystromys albicaudatus; Nycteris thebaica; Otomys irroratus; Otomys saundersiae; Rhabdomys pumilio; Steatomys krebsii; Suncus varilla; Vulpes chama. References: Avery (unpublished); Kaplan (1987).

Rhenosterkloof (2439:2743). Taxa: Bos taurus; Equus burchellii; Procavia. References: Plug (1985).

Riversmead (3040:2520). Taxa: Canis; Cynictis penicillata; Equus; Felis silvestris; Herpestes ichneumon; Hystrix africaeaustralis; Orycteropus afer; Panthera pardus; Papio ursinus; Pedetes capensis; Pelea capreolus; Phacochoerus africanus; Procavia capensis; Raphicerus campestris; Redunca fulvorufula; Smutsia temminckii; Vulpes chama. References: Klein (1979a). 
Rooiberg (2327). Taxa: Homo sapiens. References: Steyn and Broekhuizen (1993).

Rooiels (3418:1849). Taxa: Alcelaphus buselaphus cf.; Aonyx capensis; Canis mesomelas cf.; Equus burchellii; Herpestes ichneumon; Hippopotamus amphibius; Oreotragus oreotragus; Ovis aries; Panthera pardus; Papio ursinus; Procavia capensis; Pronolagus rupestris cf.; Raphicerus campestris; Sylvicapra grimmia. References: Smith (1981).

Rooikrans Hilltop (2453:2739). Taxa: Bos taurus; Homo sapiens; Hystrix africaeaustralis; Oreotragus oreotragus; Ovis aries; Parahyaena brunnea; Pedetes capensis; Phacochoerus africanus; Raphicerus campestris; Redunca arundinum; Redunca fulvorufula; Sylvicapra grimmia. References: Plug (1985).

Rooikrans Shelter (2907:2725). Taxa: Antidorcas marsupialis; Bos taurus; Canis lupus; Canis mesomelas; Connochaetes gnou; Damaliscus pygargus; Felis silvestris; Hystrix africaeaustralis; Lepus; Ovis aries; Panthera pardus; Papio ursinus; Pedetes capensis; Pelea capreolus; Phacochoerus africanus; Procavia capensis; Raphicerus campestris; Redunca fulvorufula; Sylvicapra grimmia; Taurotragus oryx. References: Plug (1997b).

Rooiwal Hollow and Midden (3027:1721). Taxa: Bathyergus suillus; Canis; Lepus. References: Orton et al. (2005).

Roosfontein (2849:2744). Taxa: Aepyceros melampus; Connochaetes gnou; Taurotragus oryx. References: Klatzow (1994).

Rose Cottage Cave (2913:2728). Taxa: Alcelaphus buselaphus; Amblysomus hottentotus; Antidorcas australis; Antidorcas bondi; Antidorcas marsupialis; Aonyx capensis; Atilax paludinosus; Bos taurus; Canis mesomelas; Capra hircus; Caracal caracal; Chlorocebus aethiops; Connochaetes gnou; Connochaetes taurinus cf.; Crocidura cyanea; Crocidura flavescens; Cryptomys hottentotus; Cynictis penicillata; Damaliscus pygargus; Dendromus melanotis; Equus burchellii; Felis silvestris; Gerbilliscus brantsii; Graphiurus murinus; Hippotragus equinus cf.; Hippotragus leucophaeus cf.; Hystrix africaeaustralis; Lepus saxatilis; Mastomys natalensis sl.; Micaelamys namaquensis; Myosorex varius; Mystromys albicaudatus; Oreotragus oreotragus; Otomys irroratus; Otomys saundersiae; Ovis aries; Panthera pardus; Papio ursinus; Parahyaena brunnea; Pedetes capensis; Pelea capreolus; Phacochoerus africanus; Procavia capensis; Raphicerus campestris; Rattus rattus; Redunca fulvorufula; Rhabdomys pumilio; Steatomys krebsii; Suncus varilla; Suricata suricatta; Sylvicapra grimmia; Taurotragus oryx; Tragelaphus strepsiceros; Vulpes chama; Xerus inauris. References: Avery (1997b); Brophy et al. (2014); Plug (1997b); Plug and Engela (1992); Welbourne (1988); Wells (2006).

Ruanga Ruin (1702:3141). Taxa: Bos taurus. References: Pellatt (1972).

Saldanha (3317). Taxa: Homo sapiens. References: Pfeiffer (2013).

Salem Commonage (3328:2628). Taxa: Homo sapiens. References: Pearce (2008).

Sandy Bay (3400:1821). Taxa: Homo sapiens. References: Pfeiffer (2013).

Schoemansdal (2300:2955). Taxa: Aepyceros melampus; Alcelaphus buselaphus; Antidorcas marsupialis cf.; Bos taurus; Capra hircus; Connochaetes taurinus; Equus asinus; Equus burchellii; Equus caballus; Giraffa camelopardalis; Hippotragus; Loxodonta africana; Ovis aries; Panthera leo; Phacochoerus africanus; Raphicerus campestris; Sus scrofa; Sylvicapra grimmia; Syncerus caffer; Taurotragus oryx; Tragelaphus strepsiceros. References: Plug et al. (2000).

Schroda (2211:2925). Taxa: Aepyceros melampus; Bos taurus; Canis lupus; Capra hircus; Equus burchellii; Felis silvestris; Giraffa camelopardalis; Heterohyrax brucei; Hippopotamus amphibius; Hippotragus; Homo sapiens; Hystrix africaeaustralis; Ichneumia albicauda; Lepus; Loxodonta africana; Mungos mungo; Oreotragus oreotragus; Otocyon megalotis; Ovis aries; Panthera leo; Panthera pardus; Papio ursinus; Pedetes capensis; Phacochoerus 
africanus; Potamochoerus larvatus; Procavia capensis; Raphicerus campestris; Syncerus caffer; Taurotragus oryx; Thryonomys swinderianus. References: De Villiers (1980); Hanisch (1980); Plug (2000); Steyn and Nienaber (2000); Voigt (1980a).

Scott's Cave (2543:3344). Taxa: Caracal caracal cf.; Felis silvestris; Galerella pulverulenta; Genetta genetta cf.; Homo sapiens; Lepus; Loxodonta africana; Mellivora capensis; Oreotragus oreotragus; Ovis; Philantomba monticola; Potamochoerus larvatus; Procavia capensis; Raphicerus melanotis; Redunca fulvorufula; Syncerus caffer; Tragelaphus scriptus; Tragelaphus strepsiceros. References: Klein and Scott (1974).

Sea Point Cape Town (3355:1823). Taxa: Homo sapiens. References: Pfeiffer (2013). Sea Vista (3411:2450). Taxa: Homo sapiens. References: Pearce (2008).

Seal Midden. See MB2005-005.

Seal Point (3417:2446). Taxa: Homo sapiens. References: Pearce (2008).

Sebatini Hill (2355:3105). Taxa: Aepyceros melampus; Atilax paludinosus; Bos taurus; Capra hircus; Connochaetes taurinus; Homo sapiens; Lepus saxatilis; Phacochoerus africanus; Sylvicapra grimmia. References: Plug and Pistorius (1999).

Sehonghong (2946:2847). Taxa: Alcelaphus buselaphus; Antidorcas marsupialis; Bos taurus; Canis mesomelas; Caracal caracal; Chlorocebus pygerythrus; Connochaetes gnou; Cryptomys; Damaliscus pygargus; Equus asinus; Equus quagga; Felis silvestris; Hippotragus equinus; Hippotragus leucophaeus; Hystrix africaeaustralis; Ichneumia albicauda; Ictonyx striatus; Lepus saxatilis; Lycaon pictus; Oreotragus oreotragus; Ourebia ourebi; Ovis aries; Papio ursinus; Pedetes capensis; Pelea capreolus; Phacochoerus africanus; Procavia capensis; Pronolagus rupestris; Proteles cristata; Raphicerus campestris; Redunca arundinum; Redunca fulvorufula; Suricata suricatta; Sylvicapra grimmia; Taurotragus oryx; Vulpes chama; Xerus inauris. References: Horsburgh et al. (2016); Plug and Mitchell (2008); Wadley (2015).

Selongwe Hill (2355:3105). Taxa: Aepyceros melampus; Bos taurus; Damaliscus lunatus; Equus burchellii; Giraffa camelopardalis; Homo sapiens; Lepus saxatilis; Phacochoerus africanus; Proteles cristata; Raphicerus campestris; Raphicerus sharpei; Sylvicapra grimmia. References: Plug and Pistorius (1999).

Sentinel Ranch (2200:3015). Taxa: Aepyceros melampus; Raphicerus. References: Plug (2000). Seroromeng (2852:2315). Taxa: Aepyceros melampus; Giraffa camelopardalis; Oreotragus oreotragus; Pedetes capensis; Procavia capensis; Raphicerus campestris; Sylvicapra grimmia; Syncerus caffer. References: Holt (2009).

Serotwe Hill (2355:3105). Taxa: Aepyceros melampus; Capra hircus; Equus burchellii; Felis silvestris; Homo sapiens; Lepus saxatilis; Oreotragus oreotragus; Orycteropus afer; Procavia capensis; Raphicerus campestris; Redunca arundinum; Sylvicapra grimmia; Syncerus caffer; Tragelaphus strepsiceros. References: Plug and Pistorius (1999).

Sh 14a (2310:3125). Taxa: Aepyceros melampus; Connochaetes taurinus. References: Plug (1989).

Shankare Hill (2355:3105). Taxa: Aepyceros melampus; Bos taurus; Canis; Capra hircus; Damaliscus lunatus; Equus burchellii; Giraffa camelopardalis; Oreotragus oreotragus; Phacochoerus africanus; Raphicerus campestris; Redunca arundinum; Sylvicapra grimmia; Syncerus caffer; Tragelaphus strepsiceros. References: Plug and Pistorius (1999).

Sibudu (2931:3105). Taxa: Atilax paludinosus; Bos taurus; Cephalophus natalensis; Chlorocebus pygerythrus; Crocuta crocuta; Equus burchellii; Felis silvestris cf; Homo sapiens cf.; Hystrix africaeaustralis; Lycaon pictus; Mellivora capensis; Oreotragus oreotragus; Papio ursinus; Pelea capreolus; Phacochoerus africanus; Philantomba monticola; Potamochoerus larvatus; Procavia capensis; Pronolagus; Raphicerus; Redunca arundinum; Smutsia 
temminckii; Sylvicapra grimmia; Taurotragus oryx cf; Thryonomys swinderianus. References: Cain (2006); Clark and Plug (2008); Glenny (2006); Le Roux and Badenhorst (2016); Plug (1997d, 2004); Wadley et al. (2008); Wells (2006).

Sikhanyisweni (2828:3024). Taxa: Canis mesomelas; Equus burchellii cf.; Felis silvestris; Galerella sanguinea; Hippopotamus amphibius; Hippotragus; Hystrix africaeaustralis; Lycaon pictus; Oreotragus oreotragus; Orycteropus afer; Ourebia ourebi; Panthera pardus; Papio ursinus; Phacochoerus africanus; Philantomba monticola; Potamochoerus larvatus; Procavia capensis; Raphicerus campestris cf.; Redunca fulvorufula; Syncerus caffer; Taurotragus oryx; Tragelaphus scriptus. References: Mazel (1988b).

Simamwe (2129AD). Taxa: Aepyceros melampus; Bos taurus; Equus burchellii. References: Huffman (2008).

Simonstown (3411:1826). Taxa: Homo sapiens. References: Pfeiffer (2013).

Simunye (2609:3152). Taxa: Aepyceros melampus; Bos taurus; Capra hircus; Ceratotherium simum; Equus burchellii; Gerbilliscus leucogaster; Suncus; Sylvicapra grimmia. References: Badenhorst and Plug (2002).

Sk 17 (2431). Taxa: Aepyceros melampus; Bos taurus; Canis mesomelas; Connochaetes taurinus; Crocuta crocuta; Damaliscus lunatus; Equus burchellii; Genetta; Giraffa camelopardalis; Hystrix africaeaustralis; Kobus ellipsiprymnus; Mastomys; Panthera leo; Papio ursinus; Phacochoerus africanus; Pronolagus; Sylvicapra grimmia; Syncerus caffer. References: Plug (1989).

Skorpion Cave (2754:1639). Taxa: Capra hircus; Oreotragus oreotragus; Ovis aries; Parotomys brantsii; Procavia capensis; Raphicerus campestris cf. References: Kinahan and Kinahan (2003).

Skutwater (2211:2933). Taxa: Aepyceros melampus; Atelerix frontalis; Bos taurus; Capra hircus; Connochaetes taurinus; Crocidura; Equus burchellii; Giraffa camelopardalis; Homo sapiens; Hystrix africaeaustralis; Lepus capensis; Lepus saxatilis; Ovis aries; Raphicerus campestris; Sylvicapra grimmia; Syncerus caffer; Taurotragus oryx; Thryonomys swinderianus. References: Steyn and Nienaber (2000); Van Ewyk (1987).

Smitswinkelbaai (3416:1828). Taxa: Alcelaphus buselaphus; Bathyergus suillus; Bos taurus; Galerella pulverulenta; Genetta; Homo sapiens; Hystrix africaeaustralis; Lepus; Oreotragus oreotragus; Ovis aries; Papio ursinus; Pelea capreolus; Procavia capensis; Raphicerus; Sylvicapra grimmia; Taurotragus oryx. References: Marean (1985).

Snuifklip (3417:2155). Taxa: Homo sapiens. References: Pearce (2008).

Somerset Strand (3406:1850). Taxa: Homo sapiens. References: Pfeiffer (2013).

Sonkoanini Hill (2355:3105). Taxa: Aepyceros melampus; Bos taurus; Canis lupus; Capra hircus; Chlorocebus aethiops; Equus burchellii; Gerbilliscus leucogaster; Giraffa camelopardalis; Hystrix africaeaustralis; Kobus ellipsiprymnus; Lepus capensis; Procavia capensis; Raphicerus campestris; Redunca arundinum; Sylvicapra grimmia; Tragelaphus strepsiceros. References: Plug and Pistorius (1999).

Spitzkop Cave (3320:2613). Taxa: Homo sapiens. References: Pearce (2008).

Spoeg River (3018:1716). Taxa: Alcelaphus buselaphus; Antidorcas marsupialis; Bathyergus janetta; Bathyergus suillus; Canis mesomelas; Caracal caracal; Crocidura cyanea; Crocidura flavescens; Cryptomys hottentotus; Dendromus melanotis; Equus burchellii; Eremitalpa granti; Felis silvestris; Galerella pulverulenta; Gerbillurus paeba; Graphiurus platyops?; Hystrix africaeaustralis; Ictonyx striatus; Lepus saxatilis; Mellivora capensis; Micaelamys namaquensis; Mus minutoides; Myosorex varius; Myotomys unisulcatus; Mystromys albicaudatus; Oreotragus oreotragus; Oryx gazella; Otocyon megalotis; Otomys irroratus; 
Otomys saundersiae; Ovis aries; Panthera leo; Panthera pardus; Parahyaena brunnea; Parotomys brantsii; Procavia capensis; Raphicerus campestris; Raphicerus melanotis; Rhabdomys pumilio; Smutsia temminckii; Steatomys krebsii; Suncus varilla; Suricata suricatta; Sylvicapra grimmia; Taurotragus oryx; Vulpes chama. References: Avery (1992c); Dewar (2007); Webley (1992a, 1992b, 2001a, 2001b).

Spring Cave (3218:1820). Taxa: Alcelaphus buselaphus; Bathyergus suillus; Canis; Caracal caracal; Felis silvestris; Galerella pulverulenta; Genetta; Herpestes ichneumon; Hystrix africaeaustralis; Ictonyx striatus; Ovis aries; Procavia capensis; Raphicerus; Sylvicapra grimmia. References: Klein and Cruz-Uribe (1987).

St Francis Bay 2 (3424). Taxa: Bos taurus; Canis mesomelas cf.; Equus; Hippopotamus amphibius; Hystrix africaeaustralis; Homo sapiens; Raphicerus melanotis; Syncerus caffer. References: Binneman (2004/2005).

St Lucia (2817:3225). Taxa: Genetta maculata; Grammomys cometes; Homo sapiens. References: Galloway (1936).

Stayt (2210:3030). Taxa: Aepyceros melampus; Bos taurus; Capra hircus; Equus burchellii; Lepus saxatilis; Procavia capensis. References: Plug (2000); Steyn and Nienaber (2000).

Steenbokfontein (3210:1820). Taxa: Bathyergus suillus; Bos taurus; Canis mesomelas; Caracal caracal; Chrysochloris asiatica; Crocidura cyanea; Crocidura flavescens; Cryptomys hottentotus; Dendromus melanotis; Dendromus mesomelas; Desmodillus auricularis; Elephantulus edwardii; Eremitalpa granti; Felis silvestris; Galerella pulverulenta; Gerbilliscus afra; Gerbillurus paeba; Graphiurus ocularis; Herpestes ichneumon; Homo sapiens; Hystrix africaeaustralis; Malacothrix typica; Micaelamys namaquensis; Mus minutoides; Myosorex varius; Myotomys unisulcatus; Mystromys albicaudatus; Orycteropus afer; Otomys irroratus; Otomys saundersiae; Ovis aries; Parotomys brantsii; Procavia capensis; Raphicerus campestris; Rhabdomys pumilio; Steatomys krebsii; Suncus varilla; Sylvicapra grimmia. References: Avery (1999); Jerardino and Yates (1996); Jerardino et al. (2013); Pfeiffer (2013).

Steenbras Bay (2640:1509). Taxa: Antidorcas marsupialis; Raphicerus campestris. References: Thackeray (1979).

Steinaecker's Horse (2324:3137). Taxa: Aepyceros melampus; Bos taurus; Equus asinus; Equus burchellii; Leptailurus serval; Lepus; Ovis aries; Panthera pardus; Raphicerus campestris; Sylvicapra grimmia; Thryonomys swinderianus. References: Badenhorst et al. (2002).

Stofbergsfontein (3309:1804). Taxa: Bathyergus suillus; Canis mesomelas; Elephantulus edwardii cf.; Felis silvestris; Galerella pulverulenta; Myotomys unisulcatus cf.; Procavia capensis; Raphicerus campestris; Rhabdomys pumilio. References: Robertshaw (1978).

Striped Giraffe Shelter (2148:1542). Taxa: Capra hircus; Diceros bicornis; Hystrix africaeaustralis; Oreotragus oreotragus; Otocyon megalotis; Ovis aries; Procavia capensis cf. References: Plug (1979a).

Tandjesberg (2905:2737). Taxa: Alcelaphus buselaphus; Antidorcas marsupialis; Canis mesomelas; Cryptomys; Damaliscus pygargus; Equus; Otomys irroratus; Ovis aries; Papio ursinus; Parahyaena brunnea; Pelea capreolus; Phacochoerus africanus; Procavia capensis; Raphicerus campestris; Sylvicapra grimmia; Taurotragus oryx; Xerus inauris. References: Wadley and McLaren (1998).

Tautswemogala (2153:2713). Taxa: Aepyceros melampus; Atelerix frontalis; Bos taurus; Canis lupus; Capra hircus; Caracal caracal cf.; Ceratotherium simum; Connochaetes taurinus; Equus burchellii; Galerella sanguinea; Giraffa camelopardalis; Hystrix africaeaustralis; Lepus saxatilis cf.; Loxodonta africana; Oryx gazella cf.; Ovis aries; Panthera pardus; Pedetes 
capensis; Phacochoerus africanus; Raphicerus campestris; Rattus rattus; Sylvicapra grimmia; Taurotragus oryx. References: De Villiers (1976b); Welbourne (1975).

Tavhatshene (2309:2958). Taxa: Aepyceros melampus; Alcelaphus; Bos taurus; Canis mesomelas; Capra hircus; Cephalophus natalensis; Chlorocebus aethiops; Connochaetes taurinus; Cynictis penicillata; Equus burchellii; Felis silvestris; Galerella sanguinea; Hippotragus equinus; Homo sapiens; Lepus saxatilis; Loxodonta africana; Ovis aries; Pelea capreolus; Potamochoerus larvatus; Raphicerus campestris; Rattus rattus; Redunca fulvorufula; Sylvicapra grimmia; Syncerus caffer; Tragelaphus angasii; Tragelaphus scriptus. References: De Wet-Bronner (1994); Plug (2000).

Te Vrede (3128). Taxa: Crocidura flavescens; Cryptomys hottentotus; Damaliscus; Equus; Georychus capensis; Hippotragus; Oreotragus oreotragus; Otomys irroratus; Papio ursinus; Pelea capreolus; Procavia capensis; Taurotragus oryx. References: Opperman (1987).

Thamanga Rock Shelter 1 (2440:2535). Taxa: Equus burchellii?; Galerella sanguinea; Hystrix africaeaustralis; Orycteropus afer; Pedetes capensis; Phacochoerus africanus; Raphicerus campestris cf.; Redunca arundinum; Sylvicapra grimmia. References: Robbins (1986).

The Havens Cave (3341:2434). Taxa: Lepus; Oreotragus oreotragus; Papio ursinus; Potamochoerus larvatus; Procavia capensis; Raphicerus campestris; Redunca fulvorufula; Sylvicapra grimmia. References: Binneman (1997); Pearce (2008).

Thulamela (2210:3130). Taxa: Aepyceros melampus; Alcelaphus buselaphus; Bos taurus; Canis lupus; Capra hircus; Caracal caracal; Chlorocebus aethiops; Connochaetes taurinus; Crocuta crocuta; Equus burchellii; Genetta genetta; Gerbilliscus; Hippopotamus amphibius; Hippotragus equinus; Hippotragus niger; Homo sapiens; Hystrix africaeaustralis; Kobus ellipsiprymnus; Lepus saxatilis; Loxodonta africana; Oreotragus oreotragus; Ovis aries; Panthera leo; Panthera pardus; Papio ursinus; Parahyaena brunnea; Paraxerus cepapi; Pedetes capensis; Phacochoerus africanus; Potamochoerus larvatus; Procavia capensis; Raphicerus campestris; Raphicerus sharpei cf.; Rattus rattus; Redunca arundinum; Sylvicapra grimmia; Syncerus caffer; Taurotragus oryx; Thryonomys swinderianus; Tragelaphus angasii; Tragelaphus scriptus; Tragelaphus strepsiceros. References: Plug (1997c, 2000); Steyn and Nienaber (2000).

Tiara Shelter (2114). Taxa: Canis mesomelas; Procavia capensis. References: Cruz-Uribe and Klein (1981-1983).

Tierfontein 2, 4A and 7 (2826AA). Taxa: Antidorcas marsupialis; Aonyx capensis; Atilax paludinosus; Cynictis penicillata; Damaliscus pygargus; Homo sapiens; Hystrix africaeaustralis; Orycteropus afer; Panthera pardus; Phacochoerus africanus; Procavia capensis; Raphicerus campestris; Redunca arundinum; Redunca fulvorufula. References: Klein (1979a).

Tiras (2613:1634). Taxa: Canis; Lepus; Oryx gazella; Procavia. References: Thackeray (1979). Tloutle (2928:2746). Taxa: Alcelaphus buselaphus; Antidorcas australis; Antidorcas marsupialis; Atilax paludinosus; Bos taurus; Canis mesomelas; Capra hircus; Connochaetes gnou; Damaliscus pygargus; Equus burchellii; Hippotragus; Homo sapiens; Hystrix africaeaustralis; Lepus; Oreotragus oreotragus; Otomys irroratus cf.; Panthera pardus; Papio ursinus; Pedetes capensis; Pelea capreolus; Phacochoerus africanus; Procavia capensis; Raphicerus campestris; Redunca arundinum; Redunca fulvorufula; Sylvicapra grimmia; Taurotragus oryx; Xerus inauris. References: Mitchell (1993); Plug (1993d, 1997b).

Tortoise Cave (3218:1821). Taxa: Atelerix frontalis; Bathyergus suillus; Canis mesomelas; Caracal caracal; Felis silvestris; Galerella pulverulenta; Homo sapiens; Hystrix 
africaeaustralis; Ictonyx striatus; Mellivora capensis; Ovis aries; Panthera pardus; Papio ursinus; Procavia capensis; Raphicerus campestris; Raphicerus melanotis; Sus scrofa; Sylvicapra grimmia; Syncerus caffer; Taurotragus oryx. References: Jerardino et al. (2013); Klein and Cruz-Uribe (1987); Pfeiffer (2013).

Toteng (2023:2257). Taxa: Aepyceros melampus; Alcelaphus buselaphus cf.; Bos taurus; Canis lupus cf.; Chlorocebus pygerythrus; Hippopotamus amphibius; Hystrix africaeaustralis; Kobus leche cf.; Orycteropus afer; Otomys angoniensis cf.; Ovis aries; Panthera leo; Pedetes capensis; Raphicerus campestris; Saccostomus campestris; Sylvicapra grimmia; Syncerus caffer; Tragelaphus spekeii. References: Robbins et al. (2008).

TP2004-04 (2930:1700). Taxa: Oryx gazella. References: Dewar (2007).

Tsh 1 (2457:3138). Taxa: Aepyceros melampus; Atelerix frontalis; Canis mesomelas; Connochaetes taurinus; Damaliscus lunatus; Equus burchellii; Giraffa camelopardalis; Hippotragus; Homo sapiens; Hystrix africaeaustralis; Ictonyx striatus; Phacochoerus africanus; Pronolagus; Sylvicapra grimmia; Thryonomys swinderianus. References: Plug (1989).

Tshirululuni (2301:2955). Taxa: Acinonyx jubatus cf.; Aepyceros melampus; Atilax paludinosus; Bos taurus; Capra hircus; Civettictis civetta; Connochaetes taurinus cf.; Equus burchellii; Felis silvestris; Giraffa camelopardalis; Hippotragus niger cf.; Homo sapiens; Leptailurus serval; Lepus saxatilis; Loxodonta africana; Oreotragus oreotragus; Ovis aries; Panthera leo; Papio ursinus; Pelea capreolus; Phacochoerus africanus; Procavia capensis; Raphicerus campestris; Redunca arundinum; Redunca fulvorufula; Smutsia temminckii; Sylvicapra grimmia; Syncerus caffer; Taurotragus oryx cf.; Tragelaphus angasii cf.; Tragelaphus scriptus cf.; Tragelaphus strepsiceros. References: De Wet-Bronner (1995b); Plug (2000).

Tshisiku Shelter (2213:2909). Taxa: Equus burchellii; Lepus capensis cf.; Pedetes capensis cf.; Phacochoerus africanus. References: Van Doornum (2007).

Tshitheme (2309:2958). Taxa: Aepyceros melampus; Bos taurus; Equus burchellii; Lepus saxatilis; Panthera leo; Papio ursinus; Pronolagus randensis cf.; Sylvicapra grimmia; Syncerus caffer. References: De Wet-Bronner (1995a); Plug (2000).

Tshobwane (2206:2933). Taxa: Bos taurus; Lepus saxatilis; Pedetes capensis; Sylvicapra grimmia. References: Manyanga (2006).

Tshwane (2546:2822). Taxa: Homo sapiens. References: Pelser et al. (2004/2005).

Twyfelpoort Shelter (2837:2734). Taxa: Alcelaphus; Antidorcas marsupialis; Canis mesomelas; Connochaetes; Damaliscus pygargus; Equus; Hystrix; Lepus; Oreotragus oreotragus; Orycteropus afer; Ovis aries; Papio; Parahyaena brunnea; Pelea capreolus; Phacochoerus africanus; Procavia capensis; Raphicerus campestris; Redunca; Sylvicapra grimmia. References: Backwell et al. (1996).

uMgungundlovu (3131:2832). Taxa: Bos taurus; Cercopithecus; Lepus; Oreotragus oreotragus; Raphicerus campestris; Sylvicapra grimmia. References: Plug and Roodt (1990).

Umhlatuzana (2948:3045). Taxa: Aethomys chrysophilus; Amblysomus hottentotus; Atilax paludinosus; Canis; Cephalophus natalensis; Chlorocebus pygerythrus; Chrysospalax villosus; Crocidura flavescens; Cryptomys hottentotus; Dasymys incomtus; Dendromus; Genetta; Georychus capensis; Grammomys dolichurus; Herpestes ichneumon; Hippotragus; Homo sapiens; Hystrix africaeaustralis cf.; Mastomys natalensis; Mellivora capensis; Mus minutoides; Myosorex varius; Oreotragus oreotragus; Orycteropus afer; Otomys irroratus; Otomys laminatus; Ourebia ourebi; Panthera pardus; Philantomba monticola; Procavia capensis; Redunca fulvorufula; Rhabdomys pumilio; Smutsia temminckii; Steatomys 
pratensis; Suncus varilla; Syncerus caffer; Thryonomys; Tragelaphus scriptus. References: Avery (1991b); Kaplan (1990).

Umhloti (2931CA). Taxa: Homo sapiens. References: Galloway (1936).

UmKlaarmaak (2529BD). Taxa: Capra hircus; Lepus; Ovis aries. References: Nelson (2008, 2009).

Umuab (2110:1430). Taxa: Pronolagus randensis; Suricata suricatta. References: Van Neer and Breunig (1999).

Uri Hauchub 4 (2515). Taxa: Panthera pardus. References: Cruz-Uribe and Klein (1981-1983).

Vaalhoek (2835:1705). Other names: /hei-/khomas. Taxa: Bos taurus; Canis mesomelas; Capra hircus; Caracal caracal cf.; Felis silvestris; Galerella pulverulenta; Oreotragus oreotragus; Ovis aries; Procavia capensis; Pronolagus rupestris; Raphicerus campestris. References: Webley (2001b).

Van Zyl's Rus Shelter (3130:2435). Taxa: Antidorcas australis; Canis mesomelas; Capra hircus; Connochaetes gnou; Damaliscus pygargus; Equus quagga; Ovis aries; Papio ursinus; Pelea capreolus; Phacochoerus africanus; Procavia capensis; Raphicerus campestris; Redunca fulvorufula. References: Plug (1999b); Plug and Sampson (1996).

Velddrif (3247:1811). Taxa: Homo sapiens. References: Pfeiffer (2013).

Ventershoek (2927CA). Taxa: Antidorcas marsupialis; Aonyx capensis; Canis; Cynictis penicillata; Damaliscus pygargus; Equus; Felis silvestris; Oreotragus oreotragus; Pelea capreolus; Phacochoerus africanus; Procavia capensis; Raphicerus campestris; Redunca arundinum; Redunca fulvorufula; Taurotragus oryx; Vulpes chama. References: Klein (1979a).

Village 16 (2229). Taxa: Bos taurus; Lepus saxatilis; Pedetes capensis; Procavia capensis. References: Manyanga (2006).

Vlaeberg (3306:1800). Taxa: Bos taurus; Procavia capensis. References: Smith et al. (1991).

Vlermuisgat Cave (3101:2219). Taxa: Antidorcas marsupialis; Canis mesomelas; Connochaetes gnou; Crocidura; Cynictis penicillata; Equus zebra; Felis nigripes; Galerella sanguinea; Gerbilliscus leucogaster; Hystrix africaeaustralis; Lepus capensis; Oreotragus oreotragus; Ovis aries; Pedetes capensis; Procavia capensis; Raphicerus campestris; Redunca fulvorufula; Saccostomus campestris; Sylvicapra grimmia; Xerus inauris. References: Badenhorst et al. (2015).

Voëlvlei (3324:1904). Taxa: Alcelaphus buselaphus; Bathyergus suillus; Caracal caracal; Elephantulus; Felis silvestris; Hystrix africaeaustralis; Oreotragus oreotragus; Panthera pardus; Procavia capensis; Raphicerus. References: Morris et al. (2004/2005); Smith et al. (1991).

Volstruisfontein (3124). Taxa: Antidorcas australis; Atilax paludinosus; Bos taurus; Canis mesomelas; Connochaetes gnou; Damaliscus pygargus; Equus quagga; Galerella; Ictonyx striatus; Ovis aries; Parahyaena brunnea; Procavia capensis; Raphicerus campestris; Suricata suricatta; Taurotragus oryx; Vulpes chama. References: Plug (1999b); Plug and Sampson (1996).

VR001. See Reception Shelter.

VR005. See Buzz Shelter.

Vredenburg (3254:1759). Taxa: Homo sapiens. References: Pfeiffer (2013).

Vungu Vungu (1755:1940). Taxa: Hippopotamus amphibius. References: Sandelowsky (1979).

Vygeboom (3315:2537). Taxa: Homo sapiens. References: Pearce (2008).

Waterbakke (3124:1757). Taxa: Homo sapiens. References: Pfeiffer (2013).

Watervalrivier (3222:1857). Taxa: Homo sapiens. References: Pfeiffer (2013). 
Welgegund (2547:2754). Taxa: Homo sapiens. References: De Villiers (1972b).

Welgeluk Shelter (3259:2631). Taxa: Homo sapiens. References: Pearce (2008).

White Paintings Rock Shelter (1825:2130). Taxa: Aepyceros melampus; Caracal caracal cf.; Chlorocebus aethiops; Connochaetes taurinus; Crocidura hirta cf.; Equus burchellii; Felis silvestris; Fukomys damarensis; Genetta; Gerbilliscus leucogaster; Gerbillurus paeba; Giraffa camelopardalis; Hippotragus equinus cf.; Hystrix africaeaustralis; Kobus leche cf.; Lepus; Mellivora capensis; Orycteropus afer; Otocyon megalotis; Otomys angoniensis cf.; Ovis aries; Panthera pardus; Pedetes capensis; Phacochoerus africanus; Potamochoerus larvatus; Raphicerus campestris; Redunca arundinum cf.; Saccostomus campestris; Sylvicapra grimmia; Taurotragus oryx; Tragelaphus scriptus; Tragelaphus strepsiceros. References: Robbins (1990); Robbins et al. (2000).

Widcome (3320:2656). Taxa: Homo sapiens. References: Pearce (2008).

Wildebeestfontein (2627:2909). Taxa: Bos taurus; Canis lupus; Gerbilliscus cf.; Homo sapiens; Sylvicapra grimmia. References: Plug (1979b).

Wilton Cave (3322:2618). Taxa: Homo sapiens. References: Pearce (2008).

Wilton Large Rock Shelter (3319:2608). Taxa: Bos taurus; Dendromus mesomelas; Homo sapiens; Loxodonta africana; Pelea capreolus; Potamochoerus larvatus. References: Brain (1981); Pearce (2008).

Witklip (3255:1759). Taxa: Bathyergus suillus; Caracal caracal; Felis silvestris; Galerella pulverulenta; Hystrix africaeaustralis; Mellivora capensis; Raphicerus campestris; Sylvicapra grimmia; Syncerus caffer. References: Smith et al. (1991).

Witkrans Cave (2736:2436). Taxa: Alcelaphus caama?; Atilax paludinosus; Damaliscus pygargus?; Equus burchellii; Hippotragus equinus?; Papio ursinus; Phacochoerus; Procavia capensis; Raphicerus campestris; Redunca fulvorufula. References: Davis (1961); Dusseldorp et al. (2013); Humphreys (1978); McCrossin (1992); Meester (1961a).

Witputs North (2716). Taxa: Ovis aries; Procavia capensis. References: Cruz-Uribe and Klein (1981-1983).

Wonderwerk (2751:2333). Taxa: Antidorcas marsupialis; Crocidura cyanea; Crocidura flavescens; Crocidura fuscomurina; Crocidura hirta; Cryptomys hottentotus; Dendromus melanotis; Desmodillus auricularis; Equus capensis; Felis silvestris; Gerbilliscus; Gerbillurus paeba; Graphiurus murinus; Hippotragus equinus; Hystrix africaeaustralis; Kobus ellipsiprymnus; Malacothrix typica; Micaelamys namaquensis; Miniopterus natalensis; Mus indutus; Mus minutoides; Myosorex varius; Mystromys albicaudatus; Neoromicia capensis; Oreotragus oreotragus; Orycteropus afer; Otomys angoniensis cf.; Otomys irroratus; Papio ursinus; Phacochoerus africanus; Raphicerus campestris; Redunca; Rhabdomys pumilio; Rhinolophus capensis; Rhinolophus clivosus; Saccostomus campestris; Scotophilus nigrita; Steatomys krebsii; Suncus lixus; Suncus varilla; Sylvicapra grimmia; Syncerus caffer; Tadarida aegyptiaca; Taurotragus oryx; Thallomys paedulcus; Zelotomys woosnami. References: Avery (1981, 1995b, 2007); Brink et al. (2015b, 2016); Fernandez-Jalvo and Avery (2015); Klein (1988); Malan and Cooke (1941); Malan and Wells (1943); Thackeray (1984, 2015); Wells et al. (1942); Vrba (1973); Wadley (2015).

Wosi (2854:3102). Taxa: Aepyceros melampus; Aonyx capensis; Atilax paludinosus; Bos taurus; Canis lupus; Canis mesomelas; Capra hircus; Caracal caracal; Cephalophus natalensis; Chlorocebus aethiops; Connochaetes taurinus; Equus burchellii; Felis silvestris; Genetta genetta; Hippopotamus amphibius; Homo sapiens; Hystrix africaeaustralis; Loxodonta africana; Orycteropus afer; Ovis aries; Papio ursinus; Phacochoerus africanus; Philantomba monticola; Potamochoerus larvatus; Redunca arundinum; Redunca fulvorufula; Sylvicapra 
grimmia; Thryonomys swinderianus; Tragelaphus scriptus. References: Arnold (2008); Morris (1994); Voigt and Peters (1994a).

Xaro (1821). Taxa: Canis lupus cf.; Equus quagga; Hippopotamus amphibius cf.; Hydrictis maculicollis; Kobus leche; Ovis aries; Tragelaphus spekeii. References: Van Zyl et al. (2013).

Ysterfontein (3320:1809). Taxa: Homo sapiens. References: Pfeiffer (2013).

Zaayfontein (3040:2520). Taxa: Antidorcas marsupialis; Raphicerus campestris; Vulpes chama cf. References: Klein (1979a).

Zais (2401:1608). Taxa: Antidorcas australis; Oreotragus oreotragus; Papio ursinus; Procavia capensis; Raphicerus campestris. References: Cruz-Uribe and Klein (1981-1983).

Zebrarivier (2431:1716). Taxa: Atelerix frontalis; Crocidura cyanea; Desmodillus auricularis; Elephantulus rupestris cf.; Equus; Gerbilliscus brantsii cf.; Gerbillurus paeba; Homo sapiens; Macroscelides proboscideus; Micaelamys namaquensis; Mus indutus; Neoromicia capensis; Oreotragus oreotragus; Otomys irroratus cf.; Parotomys littledalei cf.; Petromus typicus; Petromyscus collinus; Procavia capensis; Raphicerus campestris; Rhabdomys pumilio; Rhinolophus capensis; Saccostomus campestris; Steatomys parvus; Suncus varilla. References: Avery (1984b); Broom (1928); Cruz-Uribe and Klein (1981-1983). Zoovoorbij (2839:2101). Taxa: Crocidura cyanea; Crocidura hirta; Cryptomys hottentotus; Desmodillus auricularis; Elephantulus; Gerbilliscus brantsii cf.; Gerbillurus paeba; Graphiurus murinus; Macroscelides proboscideus; Malacothrix typica; Micaelamys namaquensis; Miniopterus schreibersii; Mus; Parotomys brantsii; Petromyscus collinus; Rhabdomys pumilio; Rhinolophus fumigatus cf.; Saccostomus campestris; Thallomys paedulcus; Zelotomys woosnami. References: Avery (unpublished); Smith (1995). 


\section{CHAPTER 7}

\section{Present and Future}

Almost 200 years of dedicated work by increasing numbers of people has brought us to our present state of knowledge concerning the history of land mammals in southern Africa. This sheer weight of information has made it increasingly difficult to keep track of what is known and to find any piece of information, particularly for non-specialists. For this reason, it seemed useful to begin developing a record of what is known about the taxa involved and where they have been found until now. While such a stock-taking can only ever be backward-looking, it does have the merit not only of reporting the status quo but also of providing a basis for any similar assessments in the future.

\subsection{THE CURRENT SITUATION}

The number of various taxa recorded so far from southern Africa is considerable (Table 7.1). There is no overall increase with time, but the reasons for this may be somewhat artificial, having to do with not only the number of sites found, which rises from five in the Eocene to 415 in the Holocene, but also with the amount of work done on each epoch. There is, however, a discernible overall pattern in which Orders became essentially stable by the Miocene, families by the Pliocene, genera (though less clearly) by the Pleistocene and species not until the Holocene, when only five species became

Table 7.1 Numbers of Orders, families, genera and species in each epoch, with percentage representation of extinct taxa.

\begin{tabular}{|c|c|c|c|c|c|c|c|c|}
\hline & \multicolumn{4}{|c|}{ Number } & \multicolumn{4}{|c|}{ Percentage extinct } \\
\hline & Order & Family & Genus & Species & Order & Family & Genus & Species \\
\hline Eocene & 9 & 19 & 29 & 22 & 33,3 & 63,2 & 100 & 100 \\
\hline Miocene & 14 & 51 & 108 & 116 & 7.1 & 37.3 & 85.2 & 98.3 \\
\hline Pliocene & 13 & 31 & 114 & 130 & 0 & 3.2 & 33.3 & 63.8 \\
\hline Pleistocene & 15 & 38 & 166 & 290 & 0 & 0 & 21.7 & 37.2 \\
\hline Holocene & 15 & 37 & 130 & 193 & 0 & 0 & 0 & 3.1 \\
\hline TOTAL & 19 & 72 & 314 & 478 & 15.8 & 34.4 & 53.2 & 55.6 \\
\hline
\end{tabular}


Table 7.2 Families and genera previously occurring in southern Africa but no longer found in the region. Current distributions after Wilson and Reeder (2005).

\begin{tabular}{|l|l|l|}
\hline Family & & Madagascar; East, Central and West Africa \\
\hline Tenrecidae & Elephas & Southeast Asia \\
\hline Lorisidae & & Southeast Asia, Central Africa \\
\hline & Cercocebus & East, Central and West Africa \\
\hline Spalacidae & & East Africa; Eastern Europe; Asia Minor \\
\hline Ochotonidae & & North Asia; North America \\
\hline & Erinaceus & Europe; Asia Minor \\
\hline & Viverra & Southeast Asia \\
\hline Chiroptera & Asellia & Pakistan and Afghanistan to Asia Minor; West and North Africa \\
\hline Ursidae & & Europe; Asia; America \\
\hline Tragulidae & & East, Central and West Africa; Southeast Asia; Pacific islands \\
\hline Bovidae & Beatragus & East Africa \\
\hline & Gazella & East and North Africa; Asia Minor \\
\hline
\end{tabular}

extinct. Of these, two springboks and the Cape horse became extinct earlier in the Holocene, but the blue antelope and the quagga were only extirpated during the last century by European settlers. There are also a few families and genera that, although not extinct worldwide, no longer occur in southern Africa (Table 7.2). The family Ursidae, represented by the Pliocene Agriotherium africanum from Langebaanweg, tends to be thought of as the most surprising such occurrence but, in fact, the picas (Ochotonidae) are also today restricted to the northern hemisphere (Wilson and Reeder 2005), as is the hedgehog genus Erinaceus. However, the absence of Erinaceus and of the carnivore genus Viverra is effectively an artefact of taxonomy. At one time the extant species frontalis and civetta, which still occur in southern Africa, were assigned to Erinaceus and Viverra respectively, whereas they are now considered to belong to Atelerix and Civettictis. The present absence of Gazella is also an artefact of taxonomy. Additionally, there are several taxa that have been introduced by humans, either deliberately as domesticates or accidentally during the Holocene (Table 7.3). The Holocene southern African fauna appears less diverse than that of the Pleistocene, especially in terms of genera and species. When, however, the extinct taxa are removed from consideration, there is very little difference (Table 7.4). In-depth analysis of the temporal relationship between extinct and extant forms will undoubtedly shed light on the relative diversity of the two epochs, but the Pleistocene appears to have been a period of major faunal turnover at the species level. 
Table 7.3 Taxa introduced by humans intentionally or accidentally.

\begin{tabular}{|l|l|l|}
\hline Order & Genus and species & Black rat \\
\hline Rodentia & Rattus norvegicus & Roof rat \\
\hline Carnivora & Rattus rattus & Domestic dog \\
\hline Perissodactyla & Canis lupus & Domestic donkey \\
\hline & Equus asinus & Domestic horse \\
\hline Artiodactyla & Equus caballus & Domestic pig \\
\hline & Sus scrofa & Domestic cattle \\
\hline & Bos taurus & Domestic goat \\
\hline & Capra hircus & Domestic sheep \\
\hline
\end{tabular}

Table 7.4 Numbers of genera and species represented in each Order during the Pleistocene and Holocene. PE: Pleistocene. HO: Holocene. All Holocene genera are extant.

\begin{tabular}{|c|c|c|c|c|c|c|c|}
\hline & \multicolumn{3}{|c|}{ Genus } & \multicolumn{4}{|c|}{ Species } \\
\hline & PE all & PE extant & $\mathrm{HO}$ all & PE all & PE extant & $\mathrm{HO}$ all & HO extant \\
\hline Cimolesta & 0 & 0 & 0 & 0 & 0 & 0 & 0 \\
\hline Afrosoricida & 6 & 5 & 6 & 8 & 6 & 6 & 6 \\
\hline Macroscelidea & 3 & 2 & 2 & 10 & 7 & 5 & 5 \\
\hline Tubulidentata & 1 & 1 & 1 & 1 & 1 & 1 & 1 \\
\hline Embrithopoda & 0 & 0 & 0 & 0 & 0 & 0 & 0 \\
\hline Hyracoidea & 2 & 2 & 2 & 4 & 2 & 2 & 2 \\
\hline Proboscidea & 3 & 2 & 1 & 6 & 1 & 1 & 1 \\
\hline Primates & 12 & 4 & 6 & 23 & 5 & 6 & 7 \\
\hline Rodentia & 40 & 37 & 36 & 65 & 53 & 59 & 59 \\
\hline Lagomorpha & 3 & 3 & 4 & 6 & 6 & 7 & 6 \\
\hline Erinaceomorpha & 2 & 1 & 1 & 2 & 1 & 1 & 1 \\
\hline Soricopmorpha & 3 & 3 & 3 & 13 & 11 & 9 & 9 \\
\hline Chiroptera & 12 & 12 & 9 & 18 & 18 & 13 & 13 \\
\hline Pholidota & 1 & 1 & 1 & 1 & 1 & 1 & 1 \\
\hline Creodonta & 0 & 0 & 0 & 0 & 0 & 0 & 0 \\
\hline Carnivora & 36 & 28 & 27 & 56 & 32 & 33 & 33 \\
\hline Perissodactyla & 4 & 3 & 3 & 9 & 4 & 8 & 6 \\
\hline Artiodactyla & 38 & 26 & 28 & 68 & 34 & 41 & 38 \\
\hline TOTAL & 166 & 130 & 130 & 290 & 182 & 193 & 188 \\
\hline
\end{tabular}




\subsection{THE WAY FORWARD}

There are two potential shortcomings of any compilation that must be acknowledged: it is both effectively a snap-shot in time and almost certainly incomplete even at the time of its publication. This being the case, to increase or, at least, prolong its usefulness some attempt must be made to offset both failings. In the past this was difficult to do and could only be effected by the publication of periodic updates. There are various major examples such as the taxonomic work Mammal Species of the World, edited by Don E. Wilson and DeeAnn M. Reeder, of which the third edition has so far been published (Wilson and Reeder 2005) and a fourth edition is imminent (www.mammalsociety.org/uploads/ report_files/Checklist\%20Committee_0.pdf). This work is also an example of the explosion in knowledge on mammal taxonomy over the 35 years since the first edition was published. Now, online resources have made it much easier to update content and Mammal Species of the World has taken increasing advantage of this facility (www.departments.bucknell.edu/biology/resources/msw3).

Of course, it is in no way suggested the current work is comparable to this major exercise, but the principle is the same. For one thing, it is hoped that it will be unnecessary for anyone to begin at the beginning again. Instead, what appears here is very much the starting point to what can most usefully be considered a work in progress. The implication of such an approach is that it is not static but will require ongoing input and correction. To do this effectively it will be essential to establish an online database, such as Fossilworks (www.fossilworks.org), which will allow workers (both taxonomists and excavators) to add and/or correct data and keep information current. Other examples of similar databases are The East African Mammals Dentition Database (http://humanorigins.si.edu/education/website/east-african-mammals-dentitiondatabase) and The Copenhagen Database of African Vertebrates (http://macroecology .ku.dk/resources/african-vertebrates). None of these examples is directly comparable to what is proposed here, but each has aspects that could be considered and/or included. For instance, the East African database indicates the importance of adding illustrations of the taxa while the Copenhagen site shows that dedicated software can improve maps considerably. Indeed, use of the latest technology can only improve accuracy and presentation in general. It is also to be hoped that the site could include archived copies of as many relevant publications as possible. This, of course, depends on solving any copyright issues, but it should be possible to archive many of the early, hard-to-access papers. At the least, URLs should be provided so that all can benefit from the searches of others. It is to be hoped that a southern African institution will take up the challenge to provide this service to the community. 


\section{References}

Note: Although it proved impossible to obtain copies of a few publications they have been included for the sake of completeness.

Ackermann, R. R., Mackay, A. \& Arnold, M. L. (2016). The hybrid origin of 'modern' humans. Evolutionary Biology, 43, 1-11.

Adams, J. W. (2012a). Craniodental and postcranial remains of the extinct porcupine Hystrix makapanensis Greenwood, 1958 (Rodentia: Hystricidae) from Gondolin, South Africa. Annals of the Ditsong National Museum of Natural History, 2, 7-17.

Adams, J. W. (2012b). A revised listing of fossil mammals from the Haasgat cave system ex situ deposits (HGD), South Africa. Palaeontologia Electronica, 15, 1-88.

Adams, J. W. \& Conroy, G. C. (2005). Plio-Pleistocene faunal remains from the Gondolin GD 2 in situ assemblage, North West Province, South Africa. In D. Lieberman, R. J. Smith \& J. Kelley, eds. Interpreting the Past: Essays on Human, Primate and Mammal Evolution in Honor of David Pilbeam. Boston, MA: Brill Academic, pp. 243-261.

Adams, J. W., Hemingway, J., Kegley, A. D. T. \& Thackeray, J. F. (2007a). Luleche, a new paleontological site in the Cradle of Humankind, North-West Province, South Africa. Journal of Human Evolution, 53, 751-754.

Adams, J. W., Herries, A. I. R., Kuykendall, K. I. \& Conroy, G. C. (2007b). Taphonomy of a South African cave: geological and hydrological influences on the GD 1 fossil assemblage at Gondolin, a Plio-Pleistocene paleocave system in the Northwest Province,
South Africa. Quaternary Science Reviews, 26, 2526-2543.

Adams, J. W., Herries, A. I. R., Hemingway, J., et al. (2010). Initial fossil discoveries from Hoogland, a new Pliocene primate-bearing karstic system in Gauteng Province, South Africa. Journal of Human Evolution, 59, 685-691.

Adams, J. W., Kegley, A. D. T. \& Krigbaum, J. (2013). New faunal stable carbon isotope data from the Haasgat HGD assemblage, South Africa, including the first reported values for Papio angusticeps and Cercopithecoides haasgati. Journal of Human Evolution, 64, 693-698.

Afzelius, A. (1815). De antilopis in genere et speciatim guineensibus commentatio. Nova Acta Reginae Societatis Scientarium Upsaliensis, Series 2, 7, 195-270.

Aguilar, G. H. (1993). The karyotype and taxonomic status of Cryptomys hottentotus darlingi (Rodentia: Bathyergidae). South African Journal of Zoology, 28, 201-204.

Alba, D. M., Vinuesa, V. \& Madurell-Malapeira, J. (2015). On the original author and year of description of the extinct hyaenid Pachycrocuta brevirostris. Acta Palaeontologica Polonica, 60, 573-576.

Allen, G. M. (1939). A checklist of African mammals. Bulletin of the Museum of Comparative Zoology Harvard, 83, 1-763.

Andersen, K. (1904). Five new Rhinolophi from Africa. Annals and Magazine of Natural History, Series 7, 14, 378-388.

Andersen, K. (1905). Further descriptions of new Rhinolophi from Africa. Annals and Magazine of Natural History, Series 7, 15, 70-76. 
Andrews, C. W. (1911). On a new species of Deinotherium from British East Africa. Proceedings of the Zoological Society of London, 1911, 943-945.

Andrews, C. W. (1914). Appendix II: on the lower Miocene vertebrates from British East Africa, collected by Dr Felix Oswald. The Quarterly Journal of the Geological Society of London, 70, 163-186.

Andrews, C. W. (1916). Note on a new baboon (Simopithecus oswaldi gen. et sp. nov.) from the (?)Pliocene of British East Africa. Annals and Magazine of Natural History, Series 8, 18, 410-419.

Andrews, C. W. \& Beadnell, H. J. L. (1902). A Preliminary Note on Some New Mammals from the Upper Eocene of Egypt. Cairo: National Printing Department.

Angas, G. F. (1848). Description of Tragelaphus angasii, Gray, with some account of its habits. Proceedings of the Zoological Society of London, 16, 89-90.

Anonymous (1827). [Review of] Histoire Naturelle des Mammifères, avec des figures originales, dessinées d'après des animaux vivants; et. Par MM Geoffroy Saint-Hilaire et F. Cuvier. Livraisons 52de et 53ème. Zoological Journal, 3, 140-143.

Antonites, A., Antonites, A. R., Kruger, N. \& Roodt, F. (2014). Report on excavations at Penge, a first-millennium Doornkop settlement. Southern African Humanities, 26, 177-192.

Arambourg, C. (1942). L'Elephas recki Dietrich: Exposition systématique et ses affinités. Bulletin de la Société Géologique de France, Series 5, 12, 73-87.

Arambourg, C. (1947). Fascicle 3: Contribution à l'étude géologique et paléontologique du basin du lac Rodolphe et de la basse vallée de l'Omo. 2. Paléontologie. In C. Arambourg, ed. Mission Scientifiques de l'Omo, 1932-1933, Vol. 1. Géologie-Anthropologie. Paris: Muséum National d'Histoire Naturelle, pp. 232-562.

Arambourg, C. (1949). Numidocapra crassicornis, nov. gen, nov. sp., un Ovicapriné nouveau du Villafranchien constaninois. Compte Rendu Sommaire des Séances de la Société Géologique de France, Series 5, 13, 290-291.
Armstrong, A. (2016). Small mammal utilization by Middle Stone Age humans at Die Kelders Cave 1 and Pinnacle Point Site 5-6, Western Cape Province, South Africa. Journal of Human Evolution, 101, 17-44.

Arnold, E. A. (2008). A consideration of livestock exploitation during the Early Iron Age in the Thukela valley, KwaZulu-Natal. In S. Badenhorst, P. Mitchell, \& J. C. Driver, eds. Animals and People: Archaeozoological Papers in Honour of Ina Plug. Oxford: British Archaeological Reports, pp. 152-168.

Asher, R. J. \& Avery, D. M. (2010). New golden moles (Afrotheria, Chrysochloridae) from the Early Pliocene of South Africa. Palaeontologia Electronica, 13, 1-12.

Astre, G. (1929). Sur un félin a particularités ursoïdes des limons pliocènes du Roussillon. Compte Rendu Sommaire et Bulletin de la Société Géologique de France, Series 4, 29, 199-204.

Avery, D. M. (1977). Past and present distribution of some rodent and insectivore species in the southern Cape Province, South Africa: new information. Annals of the South African Museum, 74, 201-209.

Avery, D. M. (1979). Appendix 2: the micromammalian fauna from the Late Stone Age levels at Die Kelders. In F. R. Schweitzer. Excavations at Die Kelders, Cape Province, South Africa. The Holocene deposits. Annals of the South African Museum, 78, 229-232.

Avery, D. M. (1981). Holocene micromammalian faunas from the northern Cape Province, South Africa. South African Journal of Science, 77, 265-273.

Avery, D. M. (1982a). The micromammalian fauna from Border Cave, KwaZulu, South Africa. Journal of Archaeological Science, 9, 187-204.

Avery, D. M. (1982b). Micromammals as palaeoenvironmental indicators and an interpretation of the late Quaternary in the southern Cape Province, South Africa. Annals of the South African Museum, 85, 183-374.

Avery, D. M. (1984a). Appendix 2: the micromammalian fauna from Fairview Shelter. In P. T. Robertshaw. Fairview Rockshelter: a contribution to the prehistory of the eastern Cape Province of South Africa. 
Annals of the Cape Provincial Museums (Human Sciences), 1, 87-88.

Avery, D. M. (1984b). Micromammals and environmental change at Zebrarivier, central Namibia. Journal of the South West Africa Scientific Society, 38, 79-86.

Avery, D. M. (1985). The dispersal of brown rats Rattus norvegicus and new specimens from 19 th century Cape Town. Mammalia, 49, 573-576.

Avery, D. M. (1987a). Micromammalian evidence for natural vegetation and the introduction of farming during the Holocene in the Magaliesberg, Transvaal. South African Journal of Science, 83, 221-225.

Avery, D. M. (1987b). Late Pleistocene coastal environment of the southern Cape Province of South Africa: micromammals from Klasies River Mouth. Journal of Archaeological Science, 14, 405-421.

Avery, D. M. (1988). Comments on micromammalian fauna from Nkupe Shelter. Mazel, A.D. Nkupe Shelter: report on excavations in the eastern Biggarsberg, Thukela Basin, Natal, South Africa. Annals of the Natal Museum, 29, 376-377.

Avery, D. M. (1989). Remarks concerning vertebrate faunal remains from the main house at Paradise. South African Archaeological Bulletin, 44, 114-116.

Avery, D. M. (1991a). Micromammals, owls and vegetation change in the eastern Cape Midlands, South Africa, during the last millennium. Journal of Arid Environments, 20, 357-369.

Avery, D. M. (1991b). Late Quaternary incidence of some micromammals in Natal. Durban Museum Novitates, 16, 1-11.

Avery, D. M. (1992a). The environment of early modern humans at Border Cave, South Africa: micromammalian evidence. Palaeogeography, Palaeoclimatology, Palaeoecology, 91, 71-87.

Avery, D. M. (1992b). Micromammals from Collingham Shelter. Natal Museum Journal of Humanities, 4, 61-63.

Avery, D. M. (1992c). Micromammals and the environment of early pastoralists at Spoeg River, western Cape Province, South Africa. South African Archaeological Bulletin, 47, 116-121.
Avery, D. M. (1995a). A preliminary assessment of the micromammalian remains from Gladysvale Cave, South Africa. Palaeontologia Africana, 32, 1-10.

Avery, D. M. (1995b). Southern savannas and Pleistocene hominid adaptations: the micromammalian perspective. In E. S. Vrba, G. H. Denton, T. C. Partridge \& L. H. Burckle, eds. Paleoclimate and Evolution with Emphasis on Human Origins. New Haven, CT: Yale University Press, pp. 459-478.

Avery, D. M. (1996). Appendix 1: comments on micromammals from Maqonqo, KwaZuluNatal. In A. D. Mazel, Maqonqo Shelter: the excavation of Holocene deposits in the eastern Biggarsberg, Thukela Basin, South Africa. Natal Museum Journal of Humanities, 8, 39.

Avery, D. M. (1997a). Micromammals and the Holocene environment of Mzinyashana Shelter 1. Natal Museum Journal of Humanities, 9, 37-46.

Avery, D. M. (1997b). Micromammals and the Holocene environment of Rose Cottage Cave. South African Journal of Science, 93, 445-448.

Avery, D. M. (1998). An assessment of the lower Pleistocene micromammalian fauna from Swartkrans Members 1-3, Gauteng, South Africa. Geobios, 31, 393-414.

Avery, D. M. (1999). Holocene coastal environments in the Western Cape Province, South Africa: micromammalian evidence from Steenbokfontein. Archaeozoologia, 10, 163-180.

Avery, D. M. (2000). Notes on the systematics of micromammals from Sterkfontein, Gauteng, South Africa. Palaeontologia Africana, 36, 83-90.

Avery, D. M. (2001). The Plio-Pleistocene vegetation and climate of Sterkfontein and Swartkrans, South Africa, based on micromammals. Journal of Human Evolution, 41, 113-132.

Avery, D. M. (2004). Size variation in the common molerat Cryptomys hottentotus from southern Africa and its potential for palaeoenvironmental reconstruction. Journal of Archaeological Science, 31, 273-282.

Avery, D. M. (2007). Pleistocene micromammals from Wonderwerk Cave, South Africa: 
practical issues. Journal of Archaeological Science, 34, 613-625.

Avery, D. M. \& Avery, G. (2011). Micromammals in the Northern Cape Province of South Africa, past and present. African Natural History, 7, 9-39.

Avery, G. (1992). Appendix 1: faunal remains from Klein Kliphuis Shelter, Clanwilliam District, South Africa. In W. J. van Rijssen. The Late Holocene deposits at Klein Kliphuis Shelter, Cedarberg, Western Cape Province. South African Archaeological Bulletin, 47, 40-43.

Avery, G. (1997). 2nd report on work completed at Spreeuwal and proposed new work (NMC 80/97/12/004/51 Dec. 1997). Cape Town: Iziko South African Museum. Unpublished Report.

Avery, G., Cruz-Uribe, K., Goldberg, P., et al. (1997). The 1992-1993 excavations at the Die Kelders Middle and Later Stone Age cave site, South Africa. Journal of Field Archaeology, 24, 263-291.

Avery, G., Halkett, D., Orton, J., et al. (2008). The Ysterfontein 1 Middle Stone Age rock shelter and the evolution of coastal foraging. South African Archaeological Society Goodwin Series, 10, 66-89.

Aymard, A. (1846). Essaie monographique sur un nouveau genre de mammifère fossile trouvé dans la Haute-Loire, et nommé Entelodon. Annales de la Société d'Agriculture, Sciences, Arts et Commerce du Puy, 12, 227-268.

Azanza, B., Morales, J. \& Pickford, M. (2003). On the nature of the multibranched cranial appendages of the climacoceratid Orangemeryx hendeyi. Memoirs of the Geological Survey of Namibia, 19, 345-357.

Backwell, L., Bhagwandas-Jogibhai, K., Fenn, G., et al. (1996). Twyfelpoort Shelter: a Later Stone Age sequence. Southern African Field Archaeology, 5, 84-95.

Backwell, L., McCarthy, T. S., Wadley, L., et al. (2014). Multiproxy record of late Quaternary climate change and Middle Stone Age human occupation at Wonderkrater, South Africa. Quaternary Science Reviews, 99, 42-59.

Badenhorst, S. (2003). The archaeofauna from iNkolimahashi Shelter, a Later Stone Age shelter in the Thukela Basin, KwaZulu-Natal, South Africa. Southern African Humanities, 15, 45-57.

Badenhorst, S. (2014). Appendix B: hunters or herders? The fauna from Kuidas Spring, a Late Holocene stone circle site in Namibia. In A. Veldman. The archaeology of a rock shelter and a stone circle at Kuidas Spring, northwest Namibia. Unpublished MA thesis, University of Johannesburg, pp. 164-188.

Badenhorst, S. \& Boshoff, W. S. (2015). Animal remains from an early twentieth century rural farming community at Orange River Railway Station, South Africa. Navorsinge van die Nasionale Museum Bloemfontein, 31, 49-64.

Badenhorst, S. \& Plug, I. (2001). Appendix: the faunal remains from Mmatshetshele, a Late Iron Age site in the Rustenburg District. In J. C. C. Pistorius. Late Iron Age sites on Mmatshetshele Mountain in the Central Bankenveld of the North West Province, South Africa. South African Archaeological Bulletin, 56, 55-56.

Badenhorst, S. \& Plug, I. (2002). Appendix: animal remains from recent excavations at a Late Iron Age site, Simunye, Swaziland. In F. Ohinata. The beginning of 'Tsonga' archaeology: excavations at Simunye, northeastern Swaziland. Southern African Humanities, 14, 45-50.

Badenhorst, S. \& Plug, I. (2003). The archaeozoology of goats, Capra hircus (Linnaeus, 1758): their size variation during the last two millennia in southern Africa (Mammalia: Artiodactyla: Caprini). Annals of the Transvaal Museum, 40, 91-121.

Badenhorst, S. \& Plug, I. (2004/2005). Boleu: faunal analysis from a 19th century site in the Groblersdal area, Mpumalanga, South Africa. Southern African Field Archaeology, 13-14, 13-18.

Badenhorst, S. \& Plug, I. (2012). The faunal remains from the Middle Stone Age levels of Bushman Rock Shelter in South Africa. South African Archaeological Bulletin, 67, 16-31.

Badenhorst, S., Plug, I., Pelser, A. J. \& Van Vollenhoven, A. C. (2002). Faunal analysis from Steinaecker's Horse, the northernmost British military outpost in the Kruger 
National Park during the South African War. Annals of the Transvaal Museum, 39, 57-63.

Badenhorst, S., Plug, I. \& Boshoff, W. S. (2011a). Faunal remains from test excavations at Middle and Late Iron Age sites in the Limpopo Valley, South Africa. Annals of the Ditsong National Museum of Natural History, 1, 23-31.

Badenhorst, S., Sénégas, F., Gommery, D., et al. (2011b). Pleistocene faunal remains from Garage Ravine Cave on Bolt's Farm in the Cradle of Humankind, South Africa. Annals of the Ditsong National Museum of Natural History, 1, 33-40.

Badenhorst, S., Sinclair, P., Ekblom, A. \& Plug, I. (2011c). Faunal remains from Chibuene, an Iron Age coastal trading station in central Mozambique. Southern African Humanities, 23, 1-15.

Badenhorst, S., Parsons, I. \& Voigt, E. A. (2015). Fauna from five Later Stone Age sites in the Bushmanland region of South Africa. Annals of the Ditsong National Museum of Natural History, 5, 1-10.

Badenhorst, S., Van Niekerk, K. L. \& Henshilwood, C. S. (2016a). Large mammal remains from the $100 \mathrm{Ka}$ Middle Stone Age layers of Blombos Cave, South Africa. South African Archaeological Bulletin, 71, 46-52.

Badenhorst, S., Veldman, A. \& Lombard, M. (2016b). Late Holocene fauna from Kuidas Spring in Namibia. African Archaeological Review, 33, 29-44.

Bain, A. G. (1839). [Ox from the Modder River]. Proceedings of the Geological Society of London, 3, 152.

Baker, C. M. (1992). Atilax paludinosus. Mammalian Species, 408, 1-6.

Barry, R. E. \& Shoshani, J. (2000). Heterohyrax brucei. Mammalian Species, 645, 1-7.

Bate, D. M. A. (1949). A new African longhorned buffalo. Annals and Magazine of Natural History, Series 12, 11, 396-398.

Beaumont, P. B. \& Boshier, A. K. (1974). Report on test excavations in a prehistoric pigment mine near Postmasburg, northern Cape. South African Archaeological Bulletin, 29, 41-59.

Beaumont, P. B., De Villiers, H. \& Vogel, J. C. (1978). Modern man in sub-Saharan Africa prior to 49000 years BP: a review and evaluation with particular reference to Border Cave. South African Journal of Science, 74, 409-419.

Beck, R. (1906). Mastodon in the Pleistocene of South Africa. Geological Magazine, New Series 5, 3, 49-50.

Bender, P. A. (1990). A reconsideration of the fossil Suidae of the Makapansgat Limeworks, Potgietersrus, northern Transvaal.

Unpublished MSc thesis, University of the Witwatersrand.

Bender, P. A. (1992). A reconsideration of the fossil suid, Potamochoeroides shawi, from the Makapansgat Limeworks, Potgietersrus, Northern Transvaal. Navorsinge van die Nasionale Museum Bloemfontein, 8, 1-66.

Bender, P. A. \& Brink, J. S. (1992). Preliminary report on new large-mammal fossil finds from the Cornelia-Uitzoek site, in the northeastern Orange Free State. South African Journal of Science, 88, 512-515.

Benefit, B. R. \& Pickford, M. (1986). Miocene fossil cercopithecoids from Kenya. American Journal of Physical Anthropology, 69, 441-464.

Bennett, N. C., Maree, S. \& Faulkes, C. G. (2006). Georychus capensis. Mammalian Species, 799, 1-4.

Bennett, N. C., Faulkes, C. G., Hart, L. \& Jarvis, J. U. M. (2009). Bathyergus suillus (Rodentia: Bathyergidae). Mammalian Species, 828, 1-7. Bennett, N., Jarvis, J., Visser, J. \& Maree, S. (2016). A conservation assessment of Georychus capensis. In M. F. Child, L. Roxburgh, E. Do Linh San, D. Raimondo \& H. T. Davies-Mostert, eds. The Red List of Mammals of South Africa, Swaziland and Lesotho. South Africa: South African National Biodiversity Institute and Endangered Wildlife Trust.

Berger, L. R. (1992). Early hominid fossils discovered at Gladysvale Cave, South Africa. South African Journal of Science, 88, 362.

Berger, L. R. (1993). A preliminary estimate of the age of the Gladysvale australopithecine site. Palaeontologia Africana, 30, 51-55.

Berger, L. R. \& Brink, J. (1996). Late Middle Pleistocene fossils, including a human patella, from the Riet River gravels, Free State, South Africa. South African Journal of Science, 92, 1-3. 
Berger, L. R. \& Brink, J. (2001). An Atlas of Southern African Mammalian Fossil Bearing Sites: Late Miocene to Late Pleistocene. Johannesburg: University of the Witwatersrand.

Berger, L. R. \& Lacruz, R. (2003). Preliminary report on the first excavations at the new fossil site of Motsetse, Gauteng, South Africa. South African Journal of Science, 99, 279-282.

Berger, L. R. \& Parkington, J. E. (1995). Brief communication: a new Pleistocene hominidbearing locality at Hoedjiespunt, South Africa. American Journal of Physical Anthropology, 98, 601-609.

Berger, L. R. \& Tobias, P. V. (1994). New discoveries at the early hominid site of Gladysvale, South Africa. South African Journal of Science, 90, 223-226.

Berger, L. R., Keyser, A. W. \& Tobias, P. V. (1993). Gladysvale: first early hominid site discovered in South Africa since 1949. American Journal of Physical Anthropology, 92, 107-111.

Berger, L. R., Pickford, M. \& Thackeray, J. F. (1995). A Plio-Pleistocene hominid upper central incisor from the Cooper's sites, South Africa. South African Journal of Science, 91, 541-542.

Berger, L. R., Lacruz, R. \& De Ruiter, D. J. (2002). Brief communication: revised age estimates of Australopithecus-bearing deposits at Sterkfontein, South Africa. American Journal of Physical Anthropology, 119, 192-197.

Berger, L. R., De Ruiter, D. J., Steininger, C. M. \& Hancox, J. (2003). Preliminary results of excavations at the newly investigated Coopers D deposit, Gauteng, South Africa. South African Journal of Science, 99, 276-278.

Berger, L. R., De Ruiter, D., Churchill, S. E., et al. (2010). Australopithecus sediba: a new species of Homo-like australopith from South Africa. Science, 328, 195-204.

Berger, L. R., Hawks, J., De Ruiter, D. J., et al. (2015). Homo naledi, a new species of the genus Homo from the Dinaledi Chamber, South Africa. eLIFE, 2015, 1-35.

Berkenhout, J. (1769). Outlines of the Natural History of Great Britain and Ireland. London: Elmsly.
Bernor, R. L. \& Kaiser, T. L. (2006). Systematics and paleoecology of the earliest Pliocene Equid, Eurygnathohippus hooijeri n. sp. from Langebaanweg, South Africa. Mitteilungen aus dem Hamburgischen Zoologischen Museum und Institut, 103, 149-185.

Bernor, R. L., Armour-Chelu, A., Gilbert, H., Kaiser, T. M. \& Schulz, E. (2010). Equidae. In L. Werdelin \& W. J. Sanders, eds. Cenozoic Mammals of Africa. Berkeley, CA: University of California Press, pp. 685-721.

Berta, A. \& Galiano, H. (1983). Megantereon hesperus from the Late Hemphillian of Florida, with remarks on the phylogenetic relationships of machairodonts (Mammalia, Felidae, Machairodontinae). Journal of Paleontology, 57, 892-899.

Biedermann, W. G. A. (1863). Petrefakten aus der Umgegend von Winterthur. Winterthur: Bleuler-Hausheer.

Binneman, J. (1997). Results from a test excavation at The Havens Cave, Cambria Valley, south-eastern Cape. Southern African Field Archaeology, 6, 93-105.

Binneman, J. (1999). Results from a test excavation at Groot Kommandokloof Shelter, in the Baviaanskloof/Kouga region, Eastern Cape. Southern African Field Archaeology, 8, 100-107.

Binneman, J. (2000). Results from two test excavations in the Baviaanskloof Mountains, Eastern Cape Province. Southern African Field Archaeology, 9, 83-96.

Binneman, J. (2004/2005). Archaeological research along the south-eastern Cape Coast Part I: open-air shell middens. Southern African Field Archaeology, 13-14, 49-77.

Binneman, J. (2006/2007). Archaeological research along the south-eastern Cape coast part 2, caves and shelters: Kabeljous River Shelter 1 and associated stone tool industries. Southern African Field Archaeology, 15-16, 57-74.

Birungi, J. \& Arctander, P. (2001). Molecular systematics and phylogeny of the Reduncini (Artiodactyla: Bovidae) inferred from the analysis of mitochondrial cytochrome $b$ gene sequences. Journal of Mammalian Evolution, 8, 125-147. 
Bishop, L. C. (2010). Suoidea. In L. Werdelin \& W. J. Sanders, eds. Cenozoic Mammals of Africa. Berkeley, CA: University of California Press, pp. 823-842.

Blumenbach, J. F. (1797). D. Joh. Fr. Blumenbach's Handbuch der Naturgeschichte, 5th edition. Göttingen: Johann Christian Dieterich.

Boddaert, P. (1784 [1785]). Elenchus Animalium. Rotterdam: C.R. Hake.

Boisserie, J.-R. (2005). The phylogeny and taxonomy of Hippopotamidae (Mammalia: Artiodactyla): a review based on morphology and cladistic analysis. Zoological Journal of the Linnean Society, 143, 1-26.

Bonaparte, C. L. (1832-1841). Iconografia della Fauna Italica: per le quattro classi degli animali vertebrati. Rome: Salviucci.

Boné, E. L. \& Dart, R. A. (1955). A catalog of the australopithecine fossils found at the Limeworks, Makapansgat. American Journal of Physical Anthropology, 13, 621-624.

Boné, E. L. \& Singer, R. (1965). Hipparion from Langebaanweg, Cape Province and a revision of the genus in Africa. Annals of the South African Museum, 48, 273-397.

Boshoff, W. \& Steyn, M. (2000). A human grave from the farm Hamilton in the Limpopo River Valley (South Africa). Southern African Field Archaeology, 9, 68-74.

Boshoff, A., Landman, M. \& Kerley, G. (2016). Filling the gaps on the maps: historical distribution patterns of some larger mammals in part of southern Africa. Transactions of the Royal Society of South Africa, 71, 23-87.

Botha, J. \& Gaudin, T. (2007). An Early Pliocene pangolin (Mammalia; Pholidota) from Langebaanweg, South Africa. Journal of Vertebrate Paleontology, 27, 484-491.

Bousman, B. \& Brink, J. (2014). Excavation of Middle and Later Stone Age sites at Erfkroon, South Africa. Unpublished NSF Final Report 0918074.

Brain, C. K. (1969). Faunal remains from the Bushman Rock Shelter, Eastern Transvaal. South African Archaeological Bulletin, 24, 52-55.

Brain, C. K. (1970). New finds at the Swartkrans australopithecine site. Nature, 225, 112-1117.
Brain, C. K. (1974). Human food remains from the Iron Age at Zimbabwe. South African Journal of Science, 70, 303-309.

Brain, C. K. (1976). A re-interpretation of the Swartkrans site and its remains. South African Journal of Science, 72, 141-146.

Brain, C. K. (1981). The Hunters or the Hunted? Chicago, IL: University of Chicago Press.

Brain, C. K. \& Brain, V. (1977). Microfaunal remains from Mirabib: some evidence of palaeoecological changes in the Namib. Madoqua, 10, 285-293.

Brain, C. K. \& Watson, V. (1992). A guide to the Swartkrans early hominid site. Annals of the Transvaal Museum, 35, 343-365.

Brain, C. K., Vrba, E. S. \& Robinson, J. T. (1974). A new hominid innominate bone from Swartkrans. Annals of the Transvaal Museum, 29, 55-63.

Brain, C. K., Churcher, C. S., Clark, J. D., et al. (1988). New evidence of early hominids, their culture and environment from the Swartkrans cave, South Africa. South African Journal of Science, 84, 828-835.

Brants, A. (1827). Het Geslacht der Muizen door Linnaeus Opgesteld. Berlin: Akademische Boekdrukkery.

Bräuer, G. \& Singer, S. (1996). The Klasies zygomatic bone: archaic or modern? Journal of Human Evolution, 30, 161-165.

Bräuer, G., Deacon, H. J. \& Zipfel, B. (1992). Comment on the new maxilliary finds from Klasies River, South Africa. Journal of Human Evolution, 23, 419-422.

Braun, D. R., Levin, N. E., Stynder, D., et al. (2013). Mid-Pleistocene Hominin occupation at Elandsfontein, Western Cape, South Africa. Quaternary Science Reviews, 82, 145-166.

Brink, J. S. (1987). The archaeozoology of Florisbad, Orange Free State. Memoirs of the National Museum Bloemfontein, 24, 1-151.

Brink, J. S. (1988). The taphonomy and palaeoecology of the Florisbad spring fauna. Palaeoecology of Africa, 19, 169-179.

Brink, J. S. (1993). Postcranial evidence for the evolution of the black wildebeest, Connochaetes gnou: an exploratory study. Palaeontologia Africana, 30, 61-69. 
Brink, J.S. (1994). An ass, Equus (Asinus sp.), from the late Quaternary Mammalian assemblages of Florisbad and Vlakkraal, central southern Africa. South African Journal of Science, 90, 497-500.

Brink, J. S. (2004). The taphonomy of an Early/ Middle Pleistocene hyaena burrow at Cornelia-Uitzoek, South Africa. Revue de Paléobiologie, 23, 731-740.

Brink, J. S. (2005). The evolution of the Black Wildebeest, Connochaetes gnou, and modern large mammal faunas in central southern Africa. Unpublished DPhil thesis, University of Stellenbosch.

Brink, J. S. (2012). Appendix: the fauna from Lithakong. In J. Kaplan \& P. Mitchell. The archaeology of the Lesotho Highlands Water Project Phases IA and IB. Southern African Humanities, 24, 30-32.

Brink, J. S. \& Deacon, H. J. (1982). A study of a last interglacial shell midden and bone accumulation at Herold's Bay, Cape Province, South Africa. Palaeoecology of Africa, 15, 31-40.

Brink, J. S. \& Holt, S. (1992). A small goat, Capra hircus, from a Late Iron Age site in the eastern Orange Free State. Southern African Field Archaeology, 1, 88-91.

Brink, J. S. \& Rossouw, L. (2000). New trial excavations at the Cornelia-Uitzoek type locality. Navorsinge van die Nasionale Museum Bloemfontein, 16, 141-156.

Brink, J. S. \& Stynder, D. (2009). Morphological and trophic distinction in the dentitions of two early alcelaphine bovids from Langebaanweg (genus Damalacra). Palaeontologia Africana, 44, 139-193.

Brink, J. S. \& Webley, L. (1996). Faunal evidence for pastoral settlement at Jakkalsberg, Richtersveld, Northern Cape Province. Southern African Field Archaeology, 5, 70-78.

Brink, J. S., Dreyer, J. J. B. \& Loubser, J. H. N. (1992). Rescue excavations at Pramberg, Jacobsdal, south-western Orange Free State. Southern African Field Archaeology, 1, 54-60.

Brink, J. S., De Bruiyn, H., Rademeyer, L. B. \& Van der Westhuizen, W. A. (1995). A new find of Megalotragus priscus (Alcelaphini, Bovidae) from the central Karoo, South Africa. Palaeontologia Africana, 32, 17-22.
Brink, J. S., Berger, L. R. \& Churchill, S. E. (1999). Mammalian fossils from erosional gullies (dongas) in the Doring River drainage, central Free State Province, South Africa. In C. Becker, J. Manhart, J. Peters \& J. Schibler, eds. Historium animalium ex ossibus. Beiträge zur Paläoanatomie, Archäologie, Ägyptologie, Ethnologie und Geschichte der Tiermedizin: Festschrift für Angela von den Driesch. Rahden/Westfalen: Verlag Marie Leidorf, pp. 79-90.

Brink, J. S., Herries, A. I. R., Moggi-Cecchi, J., et al. (2012). First hominine remains from a 1.0 million year old bone bed at Cornelia-Uitzoek, Free State Province, South Africa. Journal of Human Evolution, 63, 527-535.

Brink, J. S., Bousman, C. B. \& Grün, R. (2015a). A reconstruction of the skull of Megalotragus priscus (Broom, 1909), based on a find from Erfkroon, Modder River, South Africa, with notes on the chronology and biogeography of the species. Palaeoecology of Africa, 33, 71-94.

Brink, J. S., Holt, S. \& Kolska Horwitz, L. (2015b). Preliminary findings on macro-faunal taxonomy, taphonomy, biochronology and palaeoecology from the basal layers of Wonderwerk Cave, South Africa. Preserving African Cultural Heritage, 93, 137-147.

Brink, J. S., Holt, S. \& Kolska Horwitz, L. (2016). The Oldowan and Early Acheulean Mammalian fauna of Wonderwerk Cave (Northern Cape Province, South Africa). African Archaeological Review, 33, 223-250.

Brisson, M.-J. (1762). Regnum Animale in Classes IX. Distributum, sive Synopsis Methodica, 2nd edition. Lugduni Batavorum: T. Haak.

Britton-Davidian, J., Catalan, J., Granjon, L. \& Duplantier, J. (1995). Chromosomal phylogeny and evolution in the genus Mastomys (Mammalia, Rodentia). Journal of Mammalogy, 76, 248-262.

Britton-Davidian, J., Robinson, T. J. \& Veyrunes, F. (2012). Systematics and evolution of the African pygmy mice, subgenus Nannomys: a review. Acta Oecologica, 42, 41-49.

Bronner, G. N. (1995a). Cytogenetic properties of nine species of golden moles. Journal of Mammalogy, 76, 957-971. 
Bronner, G. N. (1995b). Systematic revision of the golden mole genera Amblysomus, Chlorotalpa and Calcochloris (Insectivora: Chrysochloromorpha; Chrysochloridae). Unpublished PhD thesis, University of Natal (Durban).

Bronner, G. N. (1996). Non-geographic variation in morphological characteristics of the Hottentot golden mole Amblysomus hottentotus (Insectivora: Chrysochloridae). Mammalia, 60, 707-727.

Bronner, G. N. (2013). Neamblysomus gunningi. In J. Kingdon, D. Happold, T. Butynski, et al., eds. Mammals of Africa, Vol. 1. London: Bloomsbury Publishing, pp. 239-240.

Bronner, G. N. \& Jenkins, E. (2005). Order Afrosoricida. In D. E. Wilson \& D. M. Reeder, eds. Mammal Species of the World, Vol. 1. Baltimore, MD: Johns Hopkins University Press, pp. 71-81.

Bronner, G. N. \& Meester, J. A. J. (1988). Otomys angoniensis. Mammalian Species, 306, 1-6.

Bronner, G., Gordon, S. \& Meester, J. (1988). Otomys irroratus. Mammalian Species, 308, 1-6.

Bronner, G. N., Hoffmann, M., Taylor, P. J., et al. (2003). A revised systematic checklist of the extant mammals of the southern African subregion. Durban Museum Novitates, 28, 56-96.

Bronner, G. N., Van der Merwe, M. \& Njobe, K. (2007). Nongeographic cranial variation in two medically important rodents from South Africa, Mastomys natalensis and Mastomys coucha. Journal of Mammalogy, 88, 1179-1194.

Brook, G. A., Cherkinsky, A., Marias, E. \& Todd, N. B. (2014). Rare elephant molar (Loxodonta africana zulu) from the Windhoek Spring Deposit, Namibia. Transactions of the Royal Society of South Africa, 69, 145-150.

Brookes, J. (1827). III. Lycaon. In E. Griffiths, C. Hamilton Smith \& E. Pidgeon, eds. Animal Kingdom Arranged in Conformity with its Organization by the Baron Cuvier, with Additional Descriptions of All the Species Hitherto named and of Many Not Before Noticed, Vol. 5. London: Whittaker.

Brookes, J. (1828). A Catalogue of the Anatomical \& Zoological Museums of Joshua Brookes, Esq., F.R.S., F.L.S. etc., Part 1. London: Gold \& Walton.
Brooks, A. S. \& Yellen, J. E. (1977).

Archaeological excavations at $\neq$ gi: a preliminary report on the first two field seasons. Botswana Notes and Records, 9, 21-30.

Brooks, A. S., Crowell, A. L. \& Yellen, J. E. (1980). Gi: a Stone Age archaeological site in the northern Kalahari Desert, Botswana. In R. E. F. Leakey \& B. A. Bogot, eds. Eighth Panafrican Congress of Prehistory and Quaternary Studies (Nairobi, 1977). Nairobi: TILLMIAP, pp. 304-309.

Broom, R. (1907a). On some new species of Chrysochloris. Annals and Magazine of Natural History, Series 7, 19, 262-268.

Broom, R. (1907b). A contribution to the knowledge of the Cape golden moles. Transactions of the South African Philosophical Society, 18, 283-311.

Broom, R. (1908). Further observations on the Chrysochloridae. Annals of the Transvaal Museum, 1, 14-16.

Broom, R. (1909a). On a large extinct species of Bubalis. Annals of the South African Museum, 7, 279-280.

Broom, R. (1909b). On evidence of a large horse recently extinct in South Africa. Annals of the South African Museum, 7, 281-282.

Broom, R. (1909c). Some observations on the dentition of Chrysochloris and on the tritubercular theory. Annals of the Natal Government Museum, 2, 129-139.

Broom, R. (1910). On Chrysochloris namaquensis, Broom. Transactions of the Royal Society of South Africa, 2, 41-43.

Broom, R. (1913a). A new species of golden mole. Proceedings of the Zoological Society of London, 1913, 546-548.

Broom, R. (1913b). Note on Equus capensis Broom. Bulletin of the American Museum of Natural History, 32, 437-439.

Broom, R. (1913c). Man contemporaneous with extinct animals in South Africa. Annals of the South African Museum, 12, 13-16.

Broom, R. (1918). The evidence afforded by the Boskop skull of a new species of primitive man (Homo capensis). Anthropological Papers of the American Museum of Natural History, 23, 63-79. 
Broom, R. (1925). On evidence of a giant pig from the Late Tertiaries of South Africa. Records of the Albany Museum, 3(4), 307-308.

Broom, R. (1928). On some new mammals from the Diamond Gravels of the Kimberley District. Annals of the South African Museum, 22, 439-444.

Broom, R. (1929). The Transvaal fossil human skeleton. Nature, 123, 415-416.

Broom, R. (1931). An extinct giant pig from the gravels of Windsorton, South Africa. Records of the Albany Museum, 4, 167-168.

Broom, R. (1934). On the fossil remains associated with Australopithecus africanus. South African Journal of Science, 31, 471-480.

Broom, R. (1936a). A new fossil anthropoid skull from South Africa. Nature, 138, 486-488.

Broom, R. (1936b). A new fossil baboon from the Transvaal. Annals of the Transvaal Museum, 18, 393-396.

Broom, R. (1937a). On some new Pleistocene mammals from limestones caves of the Transvaal. South African Journal of Science, 33, 750-768.

Broom, R. (1937b). Notices of a few more new fossil mammals from the caves of the Transvaal. Annals and Magazine of Natural History, Series 10, 20, 509-514.

Broom, R. (1938a). The Pleistocene anthropoid apes of South Africa. Nature, 142, 377-379.

Broom, R. (1938b). Note on the premolars of the elephant shrews. Annals of the Transvaal Museum, 19, 251-252.

Broom, R. (1939a). The dentition of the Transvaal Pleistocene anthropoids, Plesianthropus and Paranthropus. Annals of the Transvaal Museum, 19, 303-314.

Broom, R. (1939b). The fossil rodents of the limestone cave at Taungs. Annals of the Transvaal Museum, 19, 315-317.

Broom, R. (1939c). A preliminary account of the Pleistocene carnivores of the Transvaal Caves. Annals of the Transvaal Museum, 19, 331-338.

Broom, R. (1939d). On the affinities of the South African Pleistocene anthropoids. South African Journal of Science, 36, 408-411.

Broom, R. (1939e). A restoration of the Kromdraai skull. Annals of the Transvaal Museum, 19, 327-329.
Broom, R. (1940). The South African Pleistocene cercopithecid apes. Annals of the Transvaal Museum, 20, 89-100.

Broom, R. (1941). On two Pleistocene golden moles. Annals of the Transvaal Museum, 20, 215-216.

Broom, R. (1946). Some new and some rare golden moles. Annals of the Transvaal Museum, 20, 329-335.

Broom, R. (1948a). Some South African Pliocene and Pleistocene mammals. Annals of the Transvaal Museum, 21, 1-38.

Broom, R. (1948b). The giant rodent mole, Gypsorhychus. Annals of the Transvaal Museum, 21, 47-49.

Broom, R. (1949a). Another new type of fossil ape-man (Paranthropus crassidens). Nature, 163, 903.

Broom, R. (1949b). The extinct blue buck of South Africa. Nature, 164, 1097-1098.

Broom, R. (1950). Some further advance in our knowledge of the Cape golden moles. Annals of the Transvaal Museum, 21, 234-241.

Broom, R. \& Jensen, J. S. (1946). A new fossil baboon from the caves at Potgietersrust. Annals of the Transvaal Museum, 20, 337-340.

Broom, R. \& Robinson, J. T. (1948). A new subfossil baboon from Kromdraai, Transvaal. Annals of the Transvaal Museum, 21, 242-245.

Broom, R. \& Robinson, J. T. (1949a). A new mandible of the ape-man Plesianthropus transvaalensis. American Journal of Physical Anthropology, 7, 123-127.

Broom, R. \& Robinson, J. T. (1949b). A new type of fossil baboon, Gorgopithecus major.

Proceedings of the Zoological Society of London, 119, 379-387.

Broom, R. \& Robinson, J. T. (1949c). A new type of fossil man. Nature, 164, 322-323.

Broom, R. \& Robinson, J. T. (1950a). Note on the skull of the Swartkrans ape-man Paranthropus crassidens. American Journal of Physical Anthropology, 8, 295-304.

Broom, R. \& Robinson, J. T. (1950b). One of the earliest types of man. South African Journal of Science, 47, 55-57.

Broom, R. \& Robinson, J. T. (1952). Swartkrans ape-man, Paranthropus crassidens. Transvaal Museum Memoir, 6, 1-123. 
Broom, R. \& Schepers, G. W. H. (1946). The South African fossil ape-men: the Australopithecinae. Transvaal Museum Memoir, 2, 1-272.

Brophy, J. K., De Ruiter, D. J., Lewis, P. J., Churchill, S. E. \& Berger, L. R. (2006). Preliminary investigation of the new Middle Stone Age site of Plovers Lake, South Africa. Current Research in the Pleistocene, 23, 41-43.

Brophy, J. K., De Ruiter, D. J., Athreya, S. \& DeWitt, T. J. (2014). Quantitative morphological analysis of bovid teeth and implications for paleoenvironmental reconstruction of Plovers Lake, Gauteng Province, South Africa. Journal of Archaeological Science, 41, 376-388.

Brown, A. J. V. (1981). Appendix 3: an analysis of faunal remains at Broederstroom 24/73. In R. J. Mason. Early Iron Age settlement at Broederstroom 24/73, Transvaal, South Africa. South African Journal of Science, 77, 416.

Brown, A. J. V. (1988). The faunal remains from Kalkbank, northern Transvaal. Palaeoecology of Africa, 19, 205-212.

Brown, A. J. V. \& Verhagen, B. T. (1985). Two Antidorcas bondi individuals from the Late Stone Age site of Kruger Cave 35/83, Olifantsnek, Rustenburg District, South Africa. South African Journal of Science, 81, 102.

Brunton, S., Badenhorst, S. \& Schoeman, M. H. (2013). Ritual fauna from Ratho Kroonkop: a second millennium $\mathrm{AD}$ rain control site in the Shashe-Limpopo Confluence area of South Africa. Azania, 48, 111-132.

Burchell, W. J. (1817). Note sur une nouvelle espèce de Rhinoceros. Bulletin des Sciences par la Société Philomathique de Paris, 1817-1819, 96-97.

Burchell, W. J. (1824). Travels in the Interior of Southern Africa. London: Longman, Hurst, Rees, Orme, Brown \& Green.

Burmeister, H. (1837). Handbuch der Naturgeschichte: zum Gebrauch bei Vorlesungen. Berlin: Enslin.

Butler, P. M. (1956). Erinaceidae from the Miocene of East Africa. Fossil Mammals of Africa, 11, 1-75.

Butler, P. M. (1965). Fossil Mammals of Africa No. 18: East African Miocene and Pleistocene chalicotheres. Bulletin of the British Museum (Natural History) Geology, 10, 163-237.

Butler, P. M. (1984). Macroscelidea, Insectivora and Chiroptera from the Miocene of East Africa. Palaeovertebrata, 14, 117-200.

Butler, P. M. (2010). Neogene Insectivora. In L. Werdelin \& W. J. Sanders, eds. Cenozoic Mammals of Africa. Berkeley, CA: University of California Press, pp. 573-580.

Butler, P. M. \& Greenwood, M. (1973). The early Pleistocene hedgehog from Olduvai, Tanzania. Fossil Vertebrates of Africa, 3, 7-42.

Butler, P. M. \& Greenwood, M. (1979). Soricidae (Mammalia) from the early Pleistocene of Olduvai Gorge, Tanzania. Zoological Journal of the Linnean Society, 67, 329-379.

Butler, P. M. \& Hopwood, A. T. (1957). Insectivora and Chiroptera from the Miocene rocks of Kenya Colony. Fossil Mammals of Africa, 13, 1-35.

Butler, P. M., Thorpe, R. S. \& Greenwood, M. (1989). Interspecific relations of African crocidurine shrews (Mammalia: Soricidae) based on multivariate analysis of mandibular data. Zoological Journal of the Linnean Society, 96, 373-412.

Butzer, K. W. (1973). On the geology of a late Pliocene Mammuthus site, Virginia, Orange Free State. Navorsinge van die Nasionale Museum Bloemfontein, 2, 386-393.

Cable, J. H. C., Scott, K. \& Carter, P. L. (1980). Excavations at Good Hope Shelter, Underberg District, Natal. Annals of the Natal Museum, 24, 1-34.

Cain, C. (2006). Human activity suggested by the taphonomy of $60 \mathrm{ka}$ and $50 \mathrm{ka}$ faunal remains from Sibudu Cave. Southern African Humanities, 18, 241-260.

Cain, J. W., Krausman, P. R. \& Germaine, H. L. (2004). Antidorcas marsupialis. Mammalian Species, 753, 1-7.

Capanna, E., Civitelli, M. V., Hickman, G. C. \& Nevo, E. (1989). The chromosomes of Amblysomus hottentotus (Smith 1829) and A. iris Thomas; Schwann 1905: first report for the golden moles of Africa (Insectivora, Chrysochloridae). Tropical Zoology, 2, 318-322.

Carlson, K. B., De Ruiter, D. J., DeWitt, T. J., et al. (2016). Developmental simulation of the 
adult cranial morphology of Australopithecus sediba. South African Journal of Science, 112, 1-9.

Castiglia, R., Solano, E., Makundi, R. H., et al. (2012). Rapid chromosomal evolution in the mesic four-striped grass rat Rhabdomys dilectus (Rodentia, Muridae) revealed by mtDNA phylogeographic analysis. Journal of Zoological Systematics and Evolutionary Research, 50, 165-172.

Cavallini, P. (1992). Herpestes pulverulentus. Mammalian Species, 409, 1-4.

Chacornac, M. (1999). Etude de quelques Gerbillinae (Mammalia, Rodentia): données moléculaires et morphologiques.

Unpublished MSc thesis, Université de Montpellier II.

Chatterton, J. F., Collett, D. P. \& Swan, J. T. (1979). A Late Iron Age village site in the Letaba District, Northeast Transvaal. South African Archaeological Society Goodwin Series, 3, 109-119.

Chevret, P., Denys, C., Jaeger, J.-J., Michaux, J. \& Catzeflis, F. (1993a). Molecular evidence that the spiny mouse (Acomys) is more closely related to the gerbils (Gerbillinae) than to true mice (Murinae). Proceedings of the National Academy of Science of the United States of America, 90, 3433-3436.

Chevret, P., Denys, C., Jaeger, J.-J., Michaux, J. \& Catzeflis, F. (1993b). Molecular and paleontological aspects of the tempo and mode of evolution in Otomys (Otomyinae: Muridae: Mammalia). Biochemical Systematics and Ecology, 21, 123-131.

Chimimba, C. T. (1997). A systematic revision of southern African Aethomys Thomas, 1915 (Rodentia: Muridae). Unpublished PhD thesis, University of Pretoria.

Chimimba, C. T. (1998). A taxonomic synthesis of southern African Aethomys (Rodentia: Muridae) with a key to species. Mammalia, 62, 427-437.

Chimimba, C. T. (2000). Geographic variation in Aethomys chrysophilus (Rodentia: Muridae) from southern Africa. Zeitschrift für Säugetierkunde, 65, 157-171.

Chimimba, C. T. (2001). Infraspecific morphometric variation in Aethomys namaquensis (Rodentia: Muridae) from southern Africa. Journal of Zoology, 253, 191-210.

Chimimba, C. T. (2005). Phylogenetic relationships in the genus Aethomys (Rodentia: Muridae). African Zoology, 40, 271-284.

Chimimba, C. T. \& Dippenaar, N. J. (1994). Non-geographic variation in Aethomys chrysophilus (De Winton, 1897) and $A$. namaquensis (A. Smith, 1834) (Rodentia: Muridae) from southern Africa. South African Journal of Zoology, 29, 107-117.

Chimimba, C. T., Dippenaar, N. J. \& Robinson, T. J. (1999). Morphometric and morphological delineation of southern African species of Aethomys (Rodentia: Muridae). Biological Journal of the Linnaean Society, 67, 501-527.

Churcher, C. S. (1956). The fossil Hyracoidea of the Transvaal and Taungs deposits. Annals of the Transvaal Museum, 22, 477-501.

Churcher, C. S. (1970). The fossil Equidae of the Krugersdorp caves. Annals of the Transvaal Museum, 26, 144-168.

Churcher, C. S. (1974). Sivatherium maurisium (Pomel) from the Swartkrans australopithecine site, Transvaal (Mammalia: Giraffidae). Annals of the Transvaal Museum, 29, 65-69.

Churcher, C. S. (2000). Extinct equids from Limeworks Cave and Cave of Hearths, Makapansgat, Northern Province and a consideration of variation in the cheek teeth of Equus capensis Broom. Palaeontologia Africana, 36, 97-117.

Churcher, C. S. (2006). Distribution and history of the Cape zebra (Equus capensis) in the Quaternary of Africa. Transactions of the Royal Society of South Africa, 61, 89-95.

Churcher, C. S. \& Watson, V. (1993). 5. Additional fossil Equidae from Swartkrans. In C. K. Brain, ed. Swartkrans: a cave's chronicle of early man. Transvaal Museum Monographs, 8, 137-150.

Churchill, S. E., Pearson, O. M., Grine, F. E., Trinkaus, E. \& Holliday, T. W. (1996). Morphological affinities of the proximal tibia from Klasies River main site: archaic or modern? Journal of Human Evolution, 31, 213-237. 
Churchill, S. E., Berger, L. R. \& Parkington, J. E. (2000a). A middle Pleistocene human tibia from Hoedjiespunt, Western Cape, South Africa. South African Journal of Science, 96, 367-368.

Churchill, S. E., Brink, J. S., Berger, L. R., et al. (2000b). Erfkroon: a new Florisian fossil locality from fluvial contexts in the western Free State, South Africa. South African Journal of Science, 96, 161-163.

Clark, H. O. (2005). Otocyon megalotis. Mammalian Species, 766, 1-5.

Clark, J. L. \& Plug, I. (2008). Animal exploitation strategies during the South African Middle Stone Age: Howiesons Poort and postHowiesons Poort fauna from Sibudu Cave. Journal of Human Evolution, 54, 886-898.

Clarke, R. J. (1988). A new Australopithecus cranium from Sterkfontein and its bearing on the ancestry of Paranthropus. In F. E. Grine, ed. Evolutionary History of the Robust Australopithecines. New York: Aldine de Ruyter, pp. 285-292.

Clarke, R. J. (1999). Discovery of complete arm and hand of the 3.3. million-year-old Australopithecus skeleton from Sterkfontein. South African Journal of Science, 95, 477-480.

Clarke, R. J. (2002). Newly revealed information on the Sterkfontein Member 2 Australopithecus skeleton. South African Journal of Science, 98, 523-526.

Clarke, R. J. (2008). Latest information on Sterkfontein's Australopithecus skeleton and a new look at Australopithecus. South African Journal of Science, 104, 443-449.

Clarke, R. J. \& Tobias, P. V. (1995). Sterkfontein Member 2 foot bones of the oldest South African hominid. Science, 269, 521-524.

Clarke, R. J., Howell, F. C. \& Brain, C. K. (1970). More evidence of an advanced hominid at Swartkrans. Nature, 225, 1219-1222.

Cohen, K. M., Finney, S. C., Gibbard, P. L. \& Fan, J.-X. (2013, updated). The ICS International Chronostratigraphic Chart. Episodes, 36, 199-204.

Collett, D. P. (1982). Excavations of stonewalled ruin types in the Badfontein Valley, Eastern Transvaal, South Africa. South African Archaeological Bulletin, 37, 34-43.
Collings, G. E. (1972). A new species of machaerodont from Makapansgat. Palaeontologia Africana, 14, 87-92.

Collings, G. E. (1973). Some new machaerodonts from Makapansgat Limeworks. Unpublished MSc thesis, University of the Witwatersrand.

Collings, G. E., Cruickshank, A. R. I., Maguire, J. M. \& Randall, R. M. (1976). Recent faunal studies at Makapansgat Limeworks, Transvaal, South Africa. Annals of the South African Museum, 71, 153-165.

Collins, B., Wilkins, J. \& Ames, C. (2017). Revisiting the Holocene occupations at Grassridge Rockshelter, Eastern Cape, South Africa. South African Archaeological Bulletin, 72, 162-170.

Commission on Zoological Nomenclature (2003). Opinion 2030 (Case 3178). Bulletin of Zoological Nomenclature, 60, 90-91.

Conard, N. J. \& Kandel, A. W. (2006). The economics and settlement dynamics of the later Holocene inhabitants of near coastal environments in the West Coast National Park (South Africa). In H.-P. Wotzka, ed. Grundlegungen. Beiträge zur europäischen und afrikanischen Archäologie für Manfred K. H. Eggert. Tübingen: Francke, pp. 329-355.

Conroy, G. C., Pickford, M., Senut, B., Van Couvering, J. \& Mein, P. (1992). Otavipithecus namibensis, first Miocene hominoid from southern Africa. Nature, 356, 144-148.

Conroy, G. C., Pickford, M., Senut, B. \& Mein, P. (1993). Additional Miocene primates from the Otavi Mountains, Namibia. Comptes Rendus de l'Académie des Sciences, Paris, Série II, 317, 987-990.

Conroy, G. C., Senut, B., Gommery, D., Pickford, M. \& Mein, P. (1996). Brief communication: new primate remains from the Miocene of Namibia southern Africa. American Journal of Physical Anthropology, 99, 487-492.

Cooke, H. B. S. (1939). On a collection of fossil Mammalian remains from the Vaal River gravels at Pniel. South African Journal of Science, 36, 412-416.

Cooke, H. B. S. (1947). Some fossil hippotragine antelopes from South Africa. South African Journal of Science, 43, 226-231. 
Cooke, H. B. S. (1949a). Fossil mammals of the Vaal River deposits. Memoirs of the Geological Society of South Africa, 35, 1-108.

Cooke, H. B. S. (1949b). The fossil Suina of South Africa. Transactions of the Royal Society of South Africa, 32, 1-44.

Cooke, H. B. S. (1950). A critical revision of the Quaternary Perissodactyla of southern Africa. Annals of the South African Museum, 31, 393-479.

Cooke, H. B. S. (1955). Some fossil mammals in the South African Museum collections. Annals of the South African Museum, 42, 161-168.

Cooke, H. B. S. (1961). Further revision of the fossil Elephantidae of southern Africa. Palaeontologia Africana, 46-58.

Cooke, H. B. S. (1962). Appendix 1: notes on the faunal material from the Cave of Hearths and Kalkbank. In R. Mason, ed. Prehistory of the Transvaal. Johannesburg: University of the Witwatersrand Press, pp. 447-453.

Cooke, H. B. S. (1974). The fossil mammals of the Cornelia Beds, O.F.S. Memoirs of the National Museum Bloemfontein, 9, 63-84.

Cooke, H. B. S. (1985). Ictonyx bolti, a new mustelid from cave breccias at Bolt's Farm, Sterkfontein area, South Africa. South African Journal of Science, 81, 618-619.

Cooke, H. B. S. (1988). The larger mammals from the Cave of Hearths. In R. Mason, ed. Cave of Hearths, Makapansgat, Transvaal.

Johannesburg: Archaeological Research Unit, pp. 507-523.

Cooke, H. B. S. (1990). Taung fossils in the University of California collections. In G. H. Sperber, ed. Apes to Angels: Essays in Anthropology in Honour of Phillip V. Tobias. New York: Wiley-Liss, pp. 119-134.

Cooke, H. B. S. (1991). Dinofelis barlowi (Mammalia, Carnivora, Felidae) cranial material from Bolt's Farm, collected by the University of California African Expedition. Palaeontologia Africana, 28, 9-21.

Cooke, H. B. S. (1993a). Undescribed suid remains from Bolt's Farm and other Transvaal cave deposits. Palaeontologia Africana, 30, 7-23.

Cooke, H. B. S. (1993b). Fossil proboscidean remains from Bolt's Farm and other Transvaal cave brecchias. Palaeontologia Africana, 30, 25-34.

Cooke, H. B. S. (1994). Phacochoerus modestus from Sterkfontein Member 5. South African Journal of Science, 90, 99-100.

Cooke, H. B. S. (1997). The status of the African fossil suids Kolpochoerus limnetes (Hopwood, 1926), K. phacochoeroides (Thomas, 1884) and 'K.' afarensis (Cooke, 1978). Geobios, 30, 121-126.

Cooke, H. B. S. (2005). Makapansgat suids and Metridiochoerus. Palaeontologia Africana, 41, 131-140.

Cooke, H. B. S. \& Hendey, Q. B. (1992). Nyanzachoerus (Mammalia: Suidae: Tetraconodontinae) from Langebaanweg, South Africa. Durban Museum Novitates, 17, $1-20$.

Cooke, H. B. S. \& Wells, L. H. (1946). The Power collection of mammalian remains from the Vaal River deposits at Pneil. South African Journal of Science, 42, 224-235.

Cooke, H. B. S. \& Wells, L. H. (1951). Fossil remains from Chelmer, near Bulawayo, Southern Rhodesia. South African Journal of Science, 47, 205-209.

Cooke, H. B. S., Malan, B. D. \& Wells, L. H. (1945). Fossil man in the Lebombo Mountains, South Africa: the 'Border Cave', Ingwavuma District, Zululand. Man, 43, 6-13.

Coombs, M. C. \& Cote, S. M. (2010). Chalicotheriidae. In L. Werdelin \& W. J. Sanders, eds. Cenozoic Mammals of Africa. Berkeley, CA: University of California Press, pp. 659-667.

Coppens, Y. (1971). Une nouvelle espèce de Suidé du Villafranchien du Tunisie, Nyanzachoerus jaegeri nov. sp. Comptes Rendus Hebdomadaires des Séances, Académie des Sciences, 272, 3264-3267.

Corbet, G. B. \& Hanks, J. (1968). A revision of the elephant shrews, family Macroscelididae. Bulletin of the British Museum (Natural History) Zoology, 16, 47-111.

Corvinus, G. (1978). Palaeontological and archaeological investigations in the lower Orange valley from Arrisdrift to Orib, in the concession area of the Consolidated 
Diamond Mines of South West Africa (Proprietary Limited). Palaeoecology of Africa, 10, 75-91.

Cote, S. M. (2010). Pecora Incertae Sedis. In L. Werdelin \& W. J. Sanders, eds. Cenozoic Mammals of Africa. Berkeley, CA: University of California Press, pp. 731-739.

Cotterill, F. P. D. (2003). Insights into the taxonomy of tsessebe antelopes Damaliscus lunatus (Bovidae: Alcelaphini) with a description of a new evolutionary species in south-central Africa. Durban Museum Novitates, 28, 11-30.

Crawford-Cabral, J. \& Fernandes, A. C. (2001). The rusty-spotted genets as a group with three species in Southern Africa (Carnivora: Viverridae). In C. Denys, L. Granjon \& A. Poulet, eds. Proceedings of the 8th International Symposium on African Small Mammals. Paris: I.R.D., pp. 65-80.

Cretzschmar, P. J. (1826). Atlas zu der Reise im nördlichen Afrika von Eduard Rüppell, 1. Zoologie, Säugethiere. Gedruckt und in Commission bei Heinr. Frankfurt am Main: Ludw. Brönner.

Croizet, J. B. \& Jobert, A. C. G. (1828). Recherches sur les Ossemens Fossiles du Département du Puy-de-Dome. Paris: Delahays.

Cruz-Uribe, K. (1983). The mammalian fauna from Redcliff Cave, Zimbabwe. South African Archaeological Bulletin, 38, 7-16.

Cruz-Uribe, K. (1991). Distinguishing hyena from hominid bone accumulations. Journal of Field Archaeology, 18, 467-486.

Cruz-Uribe, K. \& Klein, R. G. (1981-1983). Faunal remains from some Middle and Later Stone Age sites in South West Africa. Journal of the South West Africa Scientific Society, 3637, 91-114.

Cruz-Uribe, K. \& Schrire, C. (1991). Analysis of faunal remains from Oudepost I, an early outpost of the Dutch East India Company, Cape Province. South African Archaeological Bulletin, 46, 92-106.

Cruz-Uribe, K., Klein, R. G., Avery, G., et al. (2003). Excavation of buried Late Acheulean (Mid-Quaternary) land surfaces at Duinefontein 2, Western Cape Province, South Africa. Journal of Archaeological Science, 30, 559-575.
Curnoe, D. (2001). Early Homo from southern Africa: a cladistic perspective. South African Journal of Science, 97, 186-190.

Curnoe, D. (2010). A review of early Homo in southern Africa focusing on cranial, mandibular and dental remains, with the description of a new species (Homo gautengensis sp. nov.). Homo: Internationale Zeitschrift fur die vergleichende Forschung am Menschen, 61, 151-177.

Curnoe, D. \& Tobias, P. V. (2006). Description, reconstruction, comparative anatomy and classification of the Sterkfontein Stw 53 cranium, with discussions about the taxonomy of other southern African early Homo remains. Journal of Human Evolution, 50, 36-77.

Curnoe, D., Herries, A., Brink, J., et al. (2006). Discovery of Middle Pleistocene fossil and stone tool-bearing deposits at Groot Kloof, Ghaap escarpment, Northern Cape Province. South African Journal of Science, 102, 180-184.

Cuvier, F. (1821). Vervet. In E. GeoffroySaint-Hilaire \& F. Cuvier, eds. Histoire Naturelle des Mammifères, Vol. 5. Paris: Belin, 2 pp.

Cuvier, F. (1822). Du sanglier à masque et des Phacochoeres. Mémoires du Muséum d'Histoire Naturelle, 8, 447-455.

Cuvier, F. (1823). [Lievre des rochers, Lepus saxatilis]. Dictionnaire des Sciences Naturelles, 26, 309-310.

Cuvier, F. (1824). Vansire. In E. Geoffroy-SaintHilaire \& F. Cuvier, eds. Histoire Naturelle des Mammifères, Vol. 5. Paris: Belin, pl. XLVIII \& $2 \mathrm{pp}$.

Cuvier, F. (1825). Des Dents des Mammifères Considérées comme Caractères Zoologiques. Strasbourg: Levrault.

Cuvier, F. (1826). [Phacochoeres, Phacochoerus]. Dictionnaire des Sciences Naturelles, 39, 383-386.

Cuvier, F. (1829). Otomys cafre. In E. Geoffroy Saint-Hilaire \& F. Cuvier, eds. Histoire Naturelle des Mammifères Vol. 6. Paris: Belin, Pl. LX \& pp. 2.

Cuvier, F. (1841). Mémoire sur les gerboises et les gerbilles. Transactions of the Zoological Society of London, 2, 131-149. 
Cuvier, G. (1798). Tableau Elémentaire de l'Histoire Naturelle des Animaux. Paris: Baudouin.

Cuvier, G. (1817). Le Règne Animal distribué d'après son Organisation, Vol. 1. Paris: Detervile.

Cuvier, G. (1829). Le Règne Animal distribué d'après son Organisation, Vol. 1. Nouvelle édn. Paris: Detervile.

Daams, R. \& De Bruijn, H. (1995). A classification of the Gliridae (Rodentia) on the basis of dental morphology. Proc. II. Conf. on Dormice. Hystrix, 6, 3-50.

Dagg, A. I. (1971). Giraffa camelopardalis. Mammalian Species, 5, 1-8.

Dale, M. M. (1948). New fossil Suidae from Limeworks Quarry, Makapansgat, Potgietersrust. South African Science, 2, 114-117.

Dalton, D. L., Linden, B., Wimberger, K., et al. (2015). New insights into Samango monkey speciation in South Africa. PLoS ONE, 10. DOI: 10.1371/journal.pone.0117003.

Dart, R. A. (1925). Australopithecus africanus: the man-ape of South Africa. Nature, 115, 195-199.

Dart, R. A. (1927). Mammoths and man in the Transvaal. Nature, 120, 41-48.

Dart, R. A. (1929a). A note on the Taungs skull. South African Journal of Science, 26, 648-658.

Dart, R. A. (1929b). Mammoths and other fossil elephants of the Vaal and Limpopo watersheds. South African Journal of Science, 26, 698-731.

Dart, R. A. (1940). Recent discoveries bearing on human history in southern Africa. Journal of the Royal Anthropological Institute of Great Britain and Ireland, 70, 13-27.

Dart, R. A. (1948a). The Makapansgat protohuman Australopithecus prometheus. American Journal of Physical Anthropology, 6, 259-284.

Dart, R. A. (1948b). The first human mandible from the Cave of Hearths, Makapansgat. South African Archaeological Bulletin, 3, 96-98.

Dart, R. A. (1954). The adult female lower jaw from Makapansgat. American Anthropologist, NS, 56, 884-888.

Dart, R. A. (1959). A tolerably complete australopithecine cranial from the Makapansgat Pink Breccia. South African Journal of Science, 55, 325-327.
Davies, C. (1987). Fossil Pedetidae (Rodentia) from Laetoli. In M. D. Leakey \& J. M. Harris, eds. Laetoli: A Pliocene Site in Northern Tanzania. Oxford: Clarendon Press, pp. 171-189.

Davis, D. H. S. (1949). The affinities of the South African gerbils of the genus Tatera.

Proceedings of the Zoological Society of London, 118, 1002-1018.

Davis, D. H. S. (1961). Appendix B: report on the microfauna in the University of California collections from the South African cave breccias (excluding the Soricidae). Unpublished report, Transvaal Museum.

Davis, D. H. S. (1965). The affinities of the South African gerbils of the gerbils Tatera: corrections and notes. Proceedings of the Zoological Society of London, 144, 323-326.

De Blainville, H. (1816). Sur plusieurs espèces d'animaux mammifères, de l'ordre des ruminans. Bulletin des Sciences par la Société Philomathique de Paris, 1816, 72-82.

De Blainville, H. (1839). Sur l'Hyaenodon leptorhynchus (De Laizer) nouveau genre de Carnassiers fossiles d'Auvergne. Annales François et Etrange d'Anatomie et Physiologie, 3, 17-31.

De Christol, J. (1832). [Description of Hipparion]. Annales des Sciences et de l'Industrie du Midi de la France, 1, 180-181.

De Graaff, G. (1958). A new chrysochlorid from Makapansgat. Palaeontologia Africana, 5, 21-27.

De Graaff, G. (1961a). A preliminary investigation of the Mammalian microfauna in Pleistocene deposits in the Transvaal System. Palaeontologia Africana, 7, 59-118.

De Graaff, G. (1961b). A short survey of investigations of fossil rodents in African deposits. South African Journal of Science, 57, 191-196.

De Graaff, G. (1961c). On the fossil mammalian microfauna collected at Kromdraai by Draper in 1895. South African Journal of Science, 57, 259-260.

De Graaff, G. (1965). A Systematic Revision of the Bathyergidae (Rodentia) of Southern Africa. Unpublished PhD thesis, University of Pretoria. 
De Graaff, G. (1988). The smaller mammals of the Cave of Hearths from basal guano underlying the Acheulean deposits (circa 200 000 BP). In R. Mason, ed. Cave of Hearths, Makapansgat, Transvaal. Johannesburg: Archaeological Research Unit, pp. 535-548.

De Meneses Cabral, J. C. (1966). Note on the taxonomy of Genetta. Zoologica Africana, 2, 25-26.

De Ruiter, D. J. (2003). Revised faunal lists for Members 1-3 of Swartkrans, South Africa. Annals of the Transvaal Museum, 40, 29-41.

De Ruiter, D. J. (2004). Undescribed hominin fossils from the Transvaal Museum faunal collections. Annals of the Transvaal Museum, 41, 29-40.

De Ruiter, D. J., Steininger, C. M. \& Berger, L. R. (2006). A cranial base of Australopithecus robustus from the Hanging Remnant of Swartkrans, South Africa. American Journal of Physical Anthropology, 130, 435-444.

De Ruiter, D. J., Brophy, J. K., Lewis, P. J., Churchill, S. E. \& Berger, L. R. (2008a). Faunal assemblage composition and paleoenvironment of Plovers Lake, a Middle Stone Age locality in Gauteng Province, South Africa. Journal of Human Evolution, 55, 1102-1117.

De Ruiter, D. J., Sponheimer, M. \& Lee Thorp, J. A. (2008b). Indications of habitat association of Australopithecus robustus in the Bloubank Valley, South Africa. Journal of Human Evolution, 55, 1015-1030.

De Ruiter, D. J., Pickering, R., Steininger, C. M., et al. (2009). New Australopithecus robustus fossils and associated U-Pb dates from Cooper's Cave (Gauteng, South Africa). Journal of Human Evolution, 56, 497-513.

De Ruiter, D. J., Brophy, J. K., Lewis, P. J., et al. (2010). Preliminary investigation of Matjhabeng, a Pliocene fossil locality in the Free State of South Africa. Palaeontologia Africana, 45, 11-22.

De Ruiter, D. J., Churchill, S. E., Brophy, J. K. \& Berger, L. R. (2011). Regional survey of Middle Stone Age fossil vertebrate deposits in the Virginia-Theunissen area of the Free State, South Africa. Navorsinge van die Nasionale Museum Bloemfontein, 27, $1-20$.
De Ruiter, D. J., DeWitt, T. J., Carlson, K. B., et al. (2013). Mandibular remains support taxonomic validity of Australopithecus sediba. Science, 340. DOI: 10.1126/science.1232997.

De Villiers, H. (1965). Part II: skeletal remains from the Gamtoos Valley. South African Archaeological Bulletin, 20, 201-205.

De Villiers, H. (1972a). The first fossil human skeleton from South West Africa. Transactions of the Royal Society of South Africa, 40, 187-196.

De Villiers, H. (1972b). Appendix 1: the Welgegund human skeleton - physical description. In E. A. Voigt. Preliminary report on Welgegund: an Iron Age burial site. South African Archaeological Bulletin, 27, 163.

De Villiers, H. (1973). Human skeletal remains from Border Cave, Ingwavuma District, KwaZulu, South Africa. Annals of the Transvaal Museum, 28, 229-246.

De Villiers, H. (1974). Human skeletal remains from Cape St Francis, Cape Province. South African Archaeological Bulletin, 29, 89-91.

De Villiers, H. (1976a). A second adult human mandible from Border Cave, Ingwavuma District, KwaZulu, South Africa. South African Journal of Science, 72, 121-125.

De Villiers, H. (1976b). Human skeletal remains from Tautswemogala Hill, Botswana. Botswana Notes and Records, 8, 7-24.

De Villiers, H. (1980). Appendix: human skeletal remains from Iron Age burials in the Limpopo/Shashi Valley. In E. O. M. Hanisch. An archaeological interpretation of certain Iron Age sites in the Limpopo/Shashi Valley. Unpublished MA thesis, University of Pretoria, pp. 1-20.

De Villiers, H. (1982). Appendix II: report on human skeletal remains: 2539 AB4, JVIIm5. In T. M. Evers. Excavations at the Lydenburg Heads site, Eastern Transvaal, South Africa. South African Archaeological Bulletin, 37, 33.

De Villiers, H. (1984). Appendix: Early Iron Age human skeletal remains from Mhlopeni and Msuluzi Confluence. In T. Maggs \& V. Ward. Early Iron Age sites in the Muden area of Natal. Annals of the Natal Museum, 26, 138-140.

De Villiers, H. (1987). Appendix 1: report on human skeletal remains from Matanga 
(07-D3-9), Botswana. In C. Van Waarden. Matanga, a late Zimbabwe cattle post. South African Archaeological Bulletin, 42, 123-124.

De Villiers, H. \& Wilson, M. L. (1982). Human burials from Byneskranskop, Bredasdorp District, Cape Province, South Africa. Annals of the South African Museum, 88, 205-248.

De Wet-Bronner, E. (1994). The faunal remains from four Late Iron Age sites in the Soutpansberg region: Part 1 - Tavhatshena. Southern African Field Archaeology, 3, 33-43.

De Wet-Bronner, E. (1995a). The faunal remains from four Late Iron Age sites in the Soutpansberg region: Part II - Tshitheme and Dzata. Southern African Field Archaeology, 4, 18-29.

De Wet-Bronner, E. (1995b). The faunal remains from four Late Iron Age sites in the Soutpansberg region: Part III - Tshirululuni. Southern African Field Archaeology, 4, 109-119.

De Winton, W. E. (1896). On collections of rodents made by $\mathrm{Mr} \mathrm{J}$. ffolliot Darling in Mashunaland and Mr F.C. Selous in Matabeleland with short field notes by the collectors. Proceedings of the Zoological Society of London, 1896, 798-809.

De Winton, W. E. (1898). On the nomenclature and distribution of some of the rodents of South Africa, with descriptions of new species. Annals and Magazine of Natural History, Series 7, 2, 1-8.

Deacon, H. J. (1976). Where Hunters Gathered. Claremont Cape: South African Archaeological Society.

Dechow, P. C. \& Singer, R. (1984). Additional fossil Theropithecus from Hopefield, South Africa: a comparison with other African sites and a re-evaluation of its taxonomic status. American Journal of Physical Anthropology, 63, 405-435.

Dembo, M., Radovčić, D., Garvin, H. M., et al. (2016). The evolutionary relationships and age of Homo naledi: an assessment using dated Bayesian phylogenetic methods. Journal of Human Evolution, 97, 17-26.

Dempster, E. R., Perrin, M. R. \& Downs, C. T. (1999). Gerbillurus vallinus. Mammalian Species, 605, 1-4.
Denbow, J. (2011). Excavations at Divuyu, Tsodilo Hills. Botswana Notes and Records, 43, 76-94.

Denbow, J., Smith, J. D. B., Ndobochani, N. M., Atwood, K. \& Miller, D. (2008).

Archaeological excavations at Bosutswe, Botswana: cultural chronology, paleo-ecology and economy. Journal of Archaeological Science, 35, 459-480.

Denys, C. (1988a). Nouvelles observations de la structure dentaire de spécimens juvéniles de Cryptomys hottentotus (Rongeurs, Batherygidés). Mammalia, 52, 292-294.

Denys, C. (1988b). Apports de l'analyse morphologiques à la determination des espèces actuelles et fossiles du genre Saccostomus (Cricetomyinae, Rodentia). Mammalia, 52, 497-532.

Denys, C. (1990a). The oldest Acomys (Rodentia, Muridae) from the Lower Pliocene of South Africa and the problem of its murid affinities. Palaeontographica Abt A, 210, 79-91.

Denys, C. (1990b). Deux nouvelles espèces d'Aethomys (Rodentia, Muridae) à Langebaanweg (Pliocène, Afrique du Sud): implications phylogénétiques. Annales de Paléontologie, 76, 41-69.

Denys, C. (1991). Un nouveau rongeur Mystromys pocockei sp. nov. (Cricetinae) du Pliocène inférieur de Langebaanweg (Région du Cap, Afrique du Sud). Comptes Rendus de l'Académie des Sciences de Paris, Série IIa, 313, 1335-1341.

Denys, C. (1994a). Nouvelles espèces de Dendromus (Rongeurs, Muroidea) à Langebaanweg (Pliocène, Afrique du Sud). Conséquences stratigraphiques et paléoécologiques. Palaeovertebrata, 23, 153-176.

Denys, C. (1994b). Affinités systématiques de Stenodontomys (Mammalia, Rodentia) rongeur Muroidea du Pliocène de Langebaanweg (Afrique du Sud). Comptes Rendus de l'Académie des Sciences de Paris, Série IIa, 318, 411-416.

Denys, C. (1998). Phylogenetic implications of the existence of two modern genera of Bathyergidae (Mammalia, Rodentia) in the Pliocene site of Langebaanweg (South Africa). 
Annals of the South African Museum, 105, 265-286.

Denys, C. (1999). Of mice and men. In T. G. Bromage \& F. Schrenk, eds. African Biogeography, Climate Change and Human Evolution. New York: Oxford University Press, pp. 226-257.

Denys, C. \& Jaeger, J. J. (1992). Rodents of the Miocene site of Fort Ternan (Kenya): first part - phiomyids, bathyergids, sciurids and anomalurids. Neues Jahrbuch für Geologie und Paläontologie, Abhandlungen, 185, 63-84.

Denys, C. \& Matthews, T. (2017). A new Desmodillus (Gerbillinae, Rodentia) species from the early Pliocene site of Langebaanweg (South-western Cape, South Africa). Palaeovertebrata, 41. DOI: 10.18563/ pv.41.1.e1.

Denys, C., Michaux, J. \& Hendey, B. (1987). Les rongeurs (Mammalia) Euryotomys et Otomys: un exemple d'évolution parallèle en Afrique tropicale. Comptes Rendus de l'Academie des Sciences de Paris, Série IIa, 305, 1389-1395.

Depéret, C. (1897). Découverte du Mastodon angustidens dans l'étage cartennien de Kabylie. Bulletin de la Société Géologique de France, Series 3, 25, 518-521.

Deraniyagala, P. E. P. (1951). A hornless rhinoceros from the Mio-Pliocene deposits of East Africa. Spolia Zeylanica, 26, 133-135.

DeSilva, J. M., Steininger, C. \& Patel, B. A. (2013). Cercopithecoid primate postcranial fossils from Cooper's D, South Africa. Geobios, 46, 381-394.

Desmarest, A. G. (1822). Mammalogie ou Descriptions des Espèces de Mammifères. Paris: Mme Veuve Agasse.

Dewar, G. I. (2007). The archaeology of the coastal desert of Namaqualand, South Africa: a regional synthesis. Unpublished PhD thesis, University of Cape Town.

Dewar, G. \& Jerardino, A. (2007). Micromammals: when humans are the hunters. Journal of Taphonomy, 5, 1-14.

Dewar, G. \& Stewart, B. A. (2012). Preliminary results of excavations at Spitzkloof Rockshelter, Richtersveld, South Africa. Quaternary International, 270, 30-39.

Dewar, G. \& Stewart, B. A. (2016). Paleoenvironments, sea levels and land use in
Namaqualand, South Africa, during MIS6-2. In S. C. Jones \& B. A. Stewart, eds. Africa from MIS 6-2: Population Dynamics and Paleoenvironments. Dordrecht: Springer Science+Business Media, pp. 195-212. Dewar, G. \& Stewart, B. A. (2017). Early Maritime desert dwellers in Namaqualand, South Africa: a Holocene perspective on Pleistocene peopling. Journal of Island \& Coastal Archaeology, 12, 44-64.

Dewar, G., Halkett, D., Hart, T., Orton, J. \& Sealy, J. (2006). Implications of a mass kill site of springbok (Antidorcas marsupialis) in South Africa: hunting practices, gender relations and sharing in the Later Stone Age. Journal of Archaeological Science, 33, 1266-1275.

Dietrich, W. O. (1915). Elephas antiquus recki n.f. aus dem Diluvium Deutch-Ostafrikas. In H. Reck, ed. Wissenschaftliche Ergebnisse, Oldoway Expedition. Leipzig: Boerntraeger, pp. 1-80.

Dietrich, W. O. (1926). Fortschritte der Säugetierpaläontologie Afrikas. Forschungen und Fortschritte, 15, 121-122.

Dietrich, W. O. (1928). Pleistocäne DeutschOstafrikaische Hippopotamus-reste. In $\mathrm{H}$. Reck, ed. Wissenschaftliche Ergeebnisse des Oldoway Expedition herausgeben von Prof. Dr. Reck. Neue Folge, Heft 3. Leipzig: Boerntraeger, pp. 2-41.

Dietrich, W. O. (1942). Altestquartare Saugetiere aus der sudlichen Serengeti, DeutschOstafrika. Palaeontographica (A), 94, 43-133.

Dineur, H. (1982). Le genre Brachyodus, Anthracotheriidae (Artiodactyla, Mammalia) du Miocene inférieur d'Europe et d'Afrique. Mémoires des Sciences de la Terre, Université de Paris, 6, 1-186.

Dippenaar, N. J. (1977). Variation in Crocidura mariquensis (A. Smith, 1844) in southern Africa. Part 1 (Mammalia: Soricidae). Annals of the Transvaal Museum, 30, 163-206.

Dippenaar, N. J. (1979). Variation in Crocidura mariquensis (A. Smith, 1844) in southern Africa, Part 2 (Mammalia: Soricidae). Annals of the Transvaal Museum, 32, 1-34.

Dippenaar, N. J. \& Rautenbach, I. L. (1986). Morphometrics and karyology of the southern African species of the genus Acomys Geoffroy Saint-Hilaire, 1838 
(Rodentia: Muridae). Annals of the Transvaal Museum, 34, 129-183.

Dippenaar, N. J., Swanepoel, P. \& Gordon, D. H. (1993). Diagnostic morphometrics of two medically important southern African rodents, Mastomys natalensis and M. coucha (Rodentia: Muridae). South African Journal of Science, 89, 300-303.

Dirks, P. H. G., Kibii, J. M., Kuhn, B. F., et al. (2010). Geological age and setting of Australopithecus sediba from southern Africa. Science, 328, 205-208.

Discamps, E. \& Henshilwood, C. S. (2015). Intrasite variability in the Still Bay fauna at Blombos Cave: implications for explanatory models of the Middle Stone Age cultural and technological evolution. PLoS ONE, 10, 1-21.

Drennan, M. R. (1937). Archaeology of the Oakhurst Shelter, George: Part III. The cavedwellers. Transactions of the Royal Society of South Africa, 25, 259-280.

Drennan, M. R. (1953). The Saldanha skull and its associations. Nature, 172, 791-793.

Drennan, M. R. (1955). The special features and status of the Saldanha skull. American Journal of Physical Anthropology, 13, 625-634.

Dreyer, T. F. (1933). The archaeology of the Matjies River Rock Shelter. Transactions of the Royal Society of South Africa, 21, 187-209.

Dreyer, T. F. (1935). A human skull from Florisbad. Proceedings of the Academy of Sciences, Amsterdam, 38, 119-128.

Dreyer, T. F. \& Lyle, A. (1931). New Fossil Mammals and Man from South Africa. Bloemfontein: Nasionale Pers.

Dubois, E. (1894). Pithecanthropus erectus: ein meschenähnliche Übergangsform aus Java. Batavia: Landesdruckerei.

Dusseldorp, G., Lombard, M. \& Wurz, S. (2013). Pleistocene Homo and the updated Stone Age sequence of South Africa. South African Journal of Science, 109. DOI: 10.1590/ sajs.2013/20120042.

Duvernoy, G. L. (1838). Supplément au mémoire sur les musaraignes. Mémoire de la Société d' Histoire Naturelle de Strasbourg, 2, $1-7$.

Duvernoy, G. L. (1851). Note sur une espèce de Buffle fossile [Bubalus (Arni) antiquus], découverte en Algérie. Comptes Rendus
Hebdomadaires des Séances de l'Académie des Sciences, 33, 595-597.

Edwards, S. (2009). Phylogeographic variation of the Karoo bush rat, Otomys unisulcatus: a molecular and morphological perspective. Unpublished MSc thesis, University of Stellenbosch.

Edwards, S., Claude, J., Van Vuuren, B. J. \& Matthee, C. A. (2011). Evolutionary history of the Karoo bush rat, Myotomys unisulcatus (Rodentia: Muridae): disconcordance between morphology and genetics. Biological Journal of the Linnean Society, 102, 510-526.

Éhik, J. (1930). Prodinotherium hungaricum n. g., n. sp. Geologica Hungarica Series Palaeontologica, 6, 1-24.

Ehrenberg, C. G. (1832). Herpestes leucurus H. et E. Colloraria. In F. W. Hemprich \& C. G. Ehrenberg. Symbolae Physicae, seu, Icones et Descriptiones Corporum Naturalium Novorum aut Minus Cognitorum Zoologica 1,

Mammalium 2 . Berlin: Officina Academica.

Ehrenberg, C. G. (1833). Sciurus, Xerus, bracchyotus H. et E. In F. W. Hemprich \& C. G. Ehrenberg. Symbolae Physicae, seu, Icones et Descriptiones Corporum Naturalium Novorum aut Minus Cognitorum Zoologica 1, Mammalium 1. Berlin: Officina Academica.

Eisenmann, V. (2000). Equus capensis

(Mammalia, Perissodactyla) from

Elandsfontein. Palaeontologia Africana, 36, 91-96.

Eisenmann, V. \& Baylac, M. (2000). Extant and fossil Equus (Mammalia, Perissodactyla) skulls: a morphometric definition of the subgenus Equus. Zoologica Scripta, 29, 89-100.

Eisenmann, V. \& Brink, J. S. (2000). Koffiefontein quaggas and true Cape quaggas: the importance of basic skull morphology. South African Journal of Science, 96, 529-533.

Ellerman, J. R. (1941). The Families and Genera of Living Rodents. London: British Museum (Natural History).

Ellerman, J. R., Morrison-Scott, T. C. S. \& Hayman, R. W. (1953). Southern African Mammals 1758-1951: A Reclassification. London: British Museum (Natural History). Engelbrecht, A., Taylor, P. J., Daniels, S. R. \& Rambau, R. V. (2011). Chromosomal polymorphisms in African Vlei Rats, Otomys 
irroratus (Muridae: Otomyini), detected by banding techniques and chromosome painting: inversions, centromeric shifts and diploid number variation. Cytogenetic and Genome Research, 133, 8-15.

Ennouchi, E. (1953). Un nouveau genre d'ovicapriné dans le gisement pléistocène de Rabat. Compte Rendu Sommaire des Séances de la Société Géologique de France, 8, 126-128.

Erxleben, J. C. P. (1777). Systema Regni Animales per Classes, Ordines, Genera, Species, Varietates cum Synomia et Historia Animalium. Lipsiae [Leipzig]: Impensis Weygandianis.

Esterhuysen, A. B., Behrens, J. \& Harper, P. (1994). Leliehoek Shelter: a Holocene sequence from the eastern Orange Free State. South African Archaeological Bulletin, 49, 73-78.

Evans, F. G. (1942). The osteology and relationships of the elephant shrews (Macroscelididae). Bulletin of the American Museum of Natural History, 80, 85-125.

Evers, T. M. (1980). Klingbeil Early Iron Age sites, Lydenburg, Eastern Transvaal, South Africa. South African Archaeological Bulletin, $35,46-57$.

Ewer, R. F. (1954). The fossil carnivores of the Transvaal caves: the Hyaenidae of Kromdraai. Proceedings of the Zoological Society of London, 124, 565-585.

Ewer, R. F. (1955a). The fossil carnivores of the Transvaal caves: the Hyaenidae, other than Lycyaena, of Swartkrans and Sterkfontein. Proceedings of the Zoological Society of London, 124, 815-837.

Ewer, R. F. (1955b). The fossil carnivores of the Transvaal caves: the Lycyaenas of Sterkfontein and Swartkrans, together with some general considerations of the Transvaal fossil hyaenids. Proceedings of the Zoological Society of London, 124, 839-857.

Ewer, R. F. (1955c). The fossil carnivores of the Transvaal caves: Machairodontinae.

Proceedings of the Zoological Society of London, 125, 587-615.

Ewer, R. F. (1956a). The fossil carnivores of the Transvaal caves: two new viverrids, together with some general considerations. Proceedings of the Zoological Society of London, 126, 259-274.
Ewer, R. F. (1956b). The fossil suids of the Transvaal caves. Proceedings of the Zoological Society of London, 127, 527-544.

Ewer, R. F. (1957a). Some fossil carnivores from the Makapansgat Valley. Palaeontologia Africana, 4, 57-67.

Ewer, R. F. (1957b). The fossil pigs of Florisbad. Navorsinge van die Nasionale Museum Bloemfontein, 1, 239-257.

Ewer, R. F. (1958a). A collection of Phacochoerus aethiopicus teeth from the Kalkbank Middle Stone Age site, central Transvaal. Palaeontologia Africana, 5, 5-20.

Ewer, R. F. (1958b). Appendix A: faunal lists for the sites of Sterkfontein, Swartkrans, Kromdraai A and Makapansgat Limeworks. In C. K. Brain, ed. The Transvaal ape-manbearing cave deposits. Transvaal Museum Memoir, 11, 128-130.

Ewer, R. F. (1958c). A note on some South African fossil otters. Navorsinge van die Nasionale Museum Bloemfontein, 1, 275-280.

Ewer, R. F. (1958d). The fossil Suidae of Makapansgat. Proceedings of the Zoological Society, 130, 329-372.

Ewer, R. F. (1962). Appendix 2: Kalkbank Suidae. In R. J. Mason, ed. Prehistory of the Transvaal. Johannesburg: Witwatersrand University Press, p. 454.

Ewer, R. F. \& Singer, R. (1956). Fossil Carnivora from Hopefield. Annals of the South African Museum, 42, 335-342.

Fagan, B. M. (1960). The Glentyre shelter and Oakhurst re-examined. South African Archaeological Bulletin, 15, 80-94.

Fagan, B. M. (1966). Appendix: Vertebrate fauna from Harleigh Farm. In P. A. Robins \& A. Whitty. Excavations at Harleigh Farm, near Rusape, Rhodesia, 1958-1962. South African Archaeological Bulletin, 21, 78-80.

Faith, J. T. (2013). Taphonomic and paleoecological change in the large mammal sequence from Boomplaas Cave, western Cape, South Africa. Journal of Human Evolution, 65, 715-730.

Faith, J. T., Potts, R., Plummer, T. W., et al. (2012). New perspectives on middle Pleistocene change in the large mammal faunas of East Africa: Damaliscus hypsodon sp. nov. (Mammalia, Artiodactyla) from 
Lainyamok, Kenya. Palaeogeography, Palaeoclimatology, Palaeoecology, 361-362, 84-93.

Falconer, H. \& Cautley, P. T. (1836). Sivatherium giganteum, a new fossil ruminant gnu, from the Valley of the Markanda, in the Sivalik branch of the Sub-Himalayan Mountains. Journal of the Asiatic Society of Bengal, 5 , 38-50.

Faulkes, C. G., Bennett, N. C., Bruford, M. W., et al. (1997). Ecological constraints driving social evolution in the African mole-rat. Proceedings of the Royal Society of London B: Biological Sciences, 264, 1619-1627.

Faulkes, C. G., Verheyen, E., Verheyen, W., Jarvis, J. U. M. \& Bennett, N. C. (2004). Phylogeographical patterns of genetic divergence and speciation in African mole-rats (Family: Bathyergidae). Molecular Ecology, 13, 613-629.

Feakins, S. J. \& DeMenocal, P. (2010). Global and African climate during the Cenozoic. In L. Werdelin \& W. J. Sanders, eds. Cenozoic Mammals of Africa. Berkeley, CA: University of California Press, pp. 45-55.

Fernandez-Jalvo, Y. \& Avery, D. M. (2015). Pleistocene micromammals and their predators at Wonderwerk Cave, South Africa. African Archaeological Review, 32, 751-791.

Ficcarelli, G., Torre, D. \& Turner, A. (1984). First evidence of a species of raccoon dog, Nyctereutes Temminck, 1838, in South African Plio-Pleistocene deposits. Bolletino della Società Palaeontologica Italiana, 23, 125-130.

Fitzinger, L. J. (1867). Versuch einer natürlichen Anordnung der Nagthiere (Rodentia). Sitzungsberichte der Akademie der Wissenschafliche Wein, 56, 57-168.

Flynn, L. J. \& Sabatier, M. (1984). A muroid rodent of Asian affinity from the Miocene of Kenya. Journal of Paleontology, 3, 160-165.

Forssman, T. (2014). Dzombo Shelter: a contribution to the Later Stone Age sequence of the Greater Mapungubwe Landscape. South African Archaeological Bulletin, 69, 182-191.

Forster, J. R. (1778). Beskrifning på Djuret Yerbua Capensis, med Anmårkningar (*) om Genus Yerbuae. Kungl. Svenska Vetenskaps Akademiens Handlingar Series 1, 39, 108-119.
Forster, J. R. (1790). Le Vaillant's Erste Reise in das Innere von Afrika, während der Jahre 1780 bis 1782, Bd 1(v 3). Berlin: Fleischer.

Fourtau, R. (1918). Contribution à l'Etude des Vertébrés Miocènes de l'Egypte. Cairo: Geological Survey of Egypt.

Fourvel, J.-B. (2018). Civettictis braini nov. sp. (Mammalia: Carnivora), a new viverrid from the hominin-bearing site of Kromdraai (Gauteng, South Africa). Comptes Rendus Palevol, 17, 366-377.

Fraas, E. (1907). Pleistocäne Fauna aus den Diamantseifen von Südafrika. Zeitschrift der Deutschen Geologischen Gesellschaft, 59, 232-243.

Franz-Odendaal, T. A. \& Salounias, N. (2004). Comparative dietary evaluations of an extinct giraffid (Sivatherium hendeyi) (Mammalia, Giraffidae, Sivatheriinae) from Langebaanweg, South Africa (early Pliocene). Geodiversitas, 26, 675-685.

Franz-Odendaal, T., Kaiser, T. M. \& Bernor, R. L. (2003). Systematics and dietary evaluation of a fossil equid from South Africa. South African Journal of Science, 99, 453-459.

Freedman, L. (1954). The status of Papio rhodesiae (Haagner) 1918. Annals of the Transvaal Museum, 22, 267-270.

Freedman, L. (1957). The fossil Cercopithecoidea of South Africa. Annals of the Transvaal Museum, 23, 8-262.

Freedman, L. (1961). Some new fossil cercopithecoid specimens from Makapansgat, South Africa. Palaeontologia Africana, 7, 7-45.

Freedman, L. (1965). Fossil and subfossil primates from the limestone deposits at Taungs, Bolt's Farm and Witkrans, South Africa. Palaeontologia Africana, 9, 19-48.

Freedman, L. (1970). A new checklist of fossil Cercopithecoidea of South Africa. Palaeontologia Africana, 13, 109-110.

Freedman, L. (1976). South African fossil Cercopithecoidea: a re-assessment including a description of new material from Makapansgat, Sterkfontein and Taung. Journal of Human Evolution, 5, 297-315.

Freedman, L. \& Brain, C. K. (1972). Fossil cercopithecoid remains from the Kromdraai 
australopithecine site (Mammalia: Primates). Annals of the Transvaal Museum, 28, 1-16.

Freedman, L. \& Brain, C. K. (1977). A reexamination of the cercopithecoid fossils from Swartkrans (Mammalia: Cercopithecidae). Annals of the Transvaal Museum, 30, 211-218.

Freedman, L. \& Stenhouse, N. S. (1972). The Parapapio species of Sterkfontein, Transvaal, South Africa. Palaeontologia Africana, 14, 93-111.

Frisch, J. L. (1775). Das Natur-System der Vierfüssigen Thiere in Tabellen darinnen alle Ordnungen, Geschlechte und Arten, nicht nur mit bestimmenden Benennungen, sondern beygesetzten unterschiedenden Kennzeichen angezeigt werden, zum Nutzen der erwachsenen Schuljugend. Glogau: Günther.

Frost, S., Saanane, C., Starkovich, B., et al. (2017). New cranium of the large cercopithecid primate Theropithecus oswaldi leakeyi (Hopwood, 1934) from the paleoanthropological site of Makuyuni, Tanzania. Journal of Human Evolution, 109, 45-56.

Galloway, A. (1936). Some prehistoric skeletal material from the Natal coast. Transactions of the Royal Society of South Africa, 23, 277-295.

Galloway, A. (1937a). Man in Africa in the light of recent discoveries. South African Journal of Science, 34, 89-120.

Galloway, A. (1937b). The characteristics of the skull of the Boskop physical type. American Journal of Physical Anthropology, 32, 31-47.

Galloway, A. \& Wells, L. H. (1934). Report on the human skeletal remains from the Karridene site. Transactions of the Royal Society of South Africa, 22, 225-233.

Gaubert, P., Tranier, M., Veron, G., et al. (2003a). Case 3204: Viverra maculata Gray, 1830 (currently Genetta maculata; Mammalia, Carnivora) proposed conservation of the specific name. Bulletin of Zoological Nomenclature, 60, 45-47.

Gaubert, P., Tranier, M., Veron, G., et al. (2003b). Nomenclatural comments on the rusty-spotted genet (Carnivora, Viverridae) and designation of a neotype. Zootaxa, 160, 1-14.
Gaubert, P., Taylor, P. J., Fernandes, C. A., Bruford, M. W. \& Veron, G. (2005). Patterns of cryptic hybridization revealed using an integrative approach: a case study on genets (Carnivora, Viverridae, Genetta spp.) from the southern African subregion. Biological Journal of the Linnaean Society, 86, 11-33.

Gaudin, T. (2010). Pholidota. In L. Werdelin \& W. J. Sanders, eds. Cenozoic Mammals of Africa. Berkeley, CA: University of California Press, pp. 599-602.

Gaudin, T. J., Emry, R. J. \& Wible, J. R. (2009). The phylogeny of living and extinct pangolins (Mammalia, Pholidota) and associated taxa: a morphology based analysis. Journal of Mammalogy, 16, 235-305.

Gaudry, A. (1863). Animaux Fossiles et Géologie de l'Attique. Paris: Savy.

Gautier, A. \& Van Waarden, C. (1981). The subsistence patterns at the Leeukop site, eastern Tuli Block. Botswana Notes and Records, 13, 1-11.

Gear, J. H. S. (1926). A preliminary account of the baboon remains from Taungs. South African Journal of Science, 23, 731-747.

Gentry, A. W. (1965). New evidence on the systematic position of Hippotragus niro Hopwood, 1936 (Mammalia). Journal of Natural History, Series 13, 8, 335-338.

Gentry, A. W. (1970). Revised classification for Makapania broomi Wells and Cooke (Bovidae, Mammalia). Palaeontologia Africana, 13, 63-67.

Gentry, A. W. (1974). A new genus and species of the Pliocene boselaphine (Bovidae, Mammalia) from South Africa. Annals of the South African Museum, 65, 145-188.

Gentry, A. W. (1980). Fossil Bovidae (Mammalia) from Langebaanweg, South Africa. Annals of the South African Museum, 79, 213-337.

Gentry, A. W. (2006). A new bovine (Bovidae, Artiodactyla) from the Hadar Formation, Ethiopia. Transactions of the Royal Society of South Africa, 61, 41-50.

Gentry, A. W. (2010). Bovidae. In L. Werdelin \& W. J. Sanders, eds. Cenozoic Mammals of Africa. Berkeley, CA: University of California Press, pp. 741-796. 
Gentry, A. W. \& Gentry, A. (1978). Fossil Bovidae (Mammalia) of Olduvai Gorge, Tanzania: Part I. Bulletin of the British Museum (Natural History) Geology, 29, 289-446.

Gentry, A. W., Gentry, A. \& Mayr, H. (1995). Rediscovery of fossil antelope holotypes (Mammalia, Bovidae) collected from Olduvai Gorge, Tanzania, in 1913. Mitteilungen aus dem Bayerischen Staatssammlung für Paläontologie und historische Geologie, 35, 125-135.

Geoffroy Saint-Hilaire, E. (1796). Mammifères : Mémoire sur les rapports naturels des Makis Lemur. Magasin Encyclopedique, 1, 20-50.

Geoffroy Saint-Hilaire, E. (1803). Catalogue des Mammifères du Muséum National d'Histoire Naturelle. Paris: [publisher not given].

Geoffroy Saint-Hilaire, E. (1810). Description des roussettes et des cephalotes, deux nouveaux genres de la famille des chauvesouris. Annales du Muséum d'Histoire Naturelle, 15, 86-108.

Geoffroy Saint-Hilaire, E. (1812). Tableau des quadrumanes, ou des animaux composant le premier Order de la Classe des Mammifères. Annales du Muséum d'Histoire Naturelle, 19, 85-170.

Geoffroy Saint-Hilaire, E. (1813). Sur un genre de chauve-souris, sous le nom de rhinolopes (1). Annales du Muséum d'Histoire Naturelle Paris, 20, 254-266.

Geoffroy Saint-Hilaire, E. (1818). Des chauvesouris. In Description des Mammifères qui se trouvent en Egypte. Déscription de l'Egypte, Vol. 2 Histoire Naturelle. Paris: Imprimerie Imperiale, pp. 99-144.

Geoffroy Saint-Hilaire, E. \& Cuvier, F. (1795). Mémoire sur une nouvelle division des Mammifères, et sur les principes qui doivent servis de base dans cette sorte de travail. Magasin Encyclopedique, 1, 164-190.

Geoffroy Saint-Hilaire, I. (1827). Musaraigne. Dictionnaire Classique d'Histoire Naturelle, 11, 313-329.

Geoffroy Saint-Hilaire, I. (1832). II. Déscription de trois espèces du genre Lièvre. Magasin de Zoologie, 2, cl. 1, pl. 9-10.

Geoffroy Saint-Hilaire, I. (1837). Notice sur deux nouveaux genres de Mammifères carnassiers, les Ichneumies, du continent africain, et les
Galidies, de Madagascar. Annales des Sciences Naturelles (Zoologie) Paris, Series 2, 8, 249-252.

Geoffroy Saint-Hilaire, I. (1838). Notice sur les rongeurs épineux désignés par les auteurs sous les noms d'Echumys, Lonchères, Heteromys et Nelomys. Annales des Sciences Naturelles (Zoologie) Paris, Series 2, 10, 122-127.

George, M. (1950). A chalicothere from the Limeworks Quarry of the Makapan Valley, Potgietersrust District. South African Journal of Science, 46, 241-242.

Geraads, D. (1992). Phylogenetic analysis of the tribe Bovini (Mammalia: Artiodactyla). Zoological Journal of the Linnean Society, 104, 193-207.

Geraads, D. (1998). Rongeurs du Miocène supérieur de Chorora (Ethiopie): Cricetidae, Rhizomyidae, Phiomyidae, Thryonomyidae, Sciuridae. Palaeovertebrata, 27, 203-216.

Geraads, D. (2001). Rongeurs du Miocene supérieur de Chorora, Ethiopia: Murinae, Dendromurinae et conclusions. Palaeovertebrata, 30, 89-109.

Geraads, D. (2005). Pliocene Rhinocerotidae (Mammalia) from Hadar and Dikika (lower Awash, Ethiopia) and a revision of the origin of modern African rhinos. Journal of Vertebrate Paleontology, 25, 451-461.

Geraads, D. (2010a). Rhinocerotidae. In L. Werdelin \& W. J. Sanders, eds. Cenozoic Mammals of A frica. Berkeley, CA: University of California Press, pp. 669-683.

Geraads, D. (2010b). Tragulidae. In L. Werdelin $\&$ W. J. Sanders, eds. Cenozoic Mammals of Africa. Berkeley, CA: University of California Press, pp. 723-729.

Gervais, P. (1848-1852). Zoologie et Paléontologie Françaises. Nouvelles recherches sur les animaux vivants et fossiles de la France. Paris: Betrand.

Gheerbrandt, E., Schmitt, A. \& Kocsis, L. (2018). Early African fossils elucidate the origin of embrithopod mammals. Current Biology, 28, 2167-2173.

Gilbert, C. C. (2007a). Craniomandibular morphology supporting the diphyletic origin of mangabeys and a new genus of the Cercocebus/Mandrillus clade, Procercocebus. Journal of Human Evolution, 53, 69-102. 
Gilbert, C. C. (2007b). Identification and description of the first Theropithecus (Primates: Cercopithecidae) material from Bolt's Farm. Annals of the Transvaal Museum, 44, 1-10.

Gilbert, C. C. (2013). Cladistic analysis of extant and fossil African papionins using craniodental data. Journal of Human Evolution, 64, 399-433.

Gilbert, C. C., Steininger, C. M., Kibii, J. M. \& Berger, L. R. (2015). Papio cranium from the hominin-bearing site of Malapa: implications for the evolution of modern baboon cranial morphology and South African PlioPleistocene biochronology. PLOS ONE, 10, 1-15.

Gilbert, C. C., Frost, P. \& Delson, E. (2016a). Reassessment of Olduvai Bed I cercopithecoids: a new biochronological and biogeographical link to the South African fossil record. Journal of Human Evolution, 92, 50-59.

Gilbert, C. C., Takahashi, M. Q. \& Delson, E. (2016b). Cercopithecoid humeri from Taung support the distinction of major papionin clades in the South African fossil record. Journal of Human Evolution, 90, 88-104.

Gill, T. (1884). Order III: Insectivora. In J. S. Kingsley, ed. The Standard Natural History, Vol. 5. Mammals. Boston, MA: Cassino.

Gingerich, P. D. (1974). Proteles cristatus Sparrman from the Pleistocene of South Africa, with a note on tooth replacement in the aardwolf (Mammalia: Hyaenidae). Annals of the Transvaal Museum, 29, 49-54.

Ginsburg, L. (1965). L'Amphicyon ambiguus des phosphorites du Quercy. Bulletin de Museum National d'Histoire Naturelle, Series 2, 37, 724-730.

Glenny, W. (2006). Report on the micromammal assemblage analysis from Sibudu Cave, KwaZulu-Natal. South African Humanities, 18, 279-288.

Gmelin, J. F., ed. (1788). Linnaeus, C. Systema Naturae, 1, 13th edition. Lipsiae [Leipzig]: G. E. Beer.

Gommery, D. (2000). Superior cervical vertebrae of a Miocene hominoid and a PlioPleistocene hominid from southern Africa. Palaeontologia Africana, 36, 139-145.
Gommery, D. (2008). A new hominid hip bone from Swartkrans (SKW 8012) in relation to the anatomy of the anterior inferior iliac spine. Annals of the Transvaal Museum, 45, 55-66.

Gommery, D. \& Bento da Costa, L. (2016). Les primates non-humains pliocènes et pliopléistocènes d'Afrique du Sud. Revue de Primatologie, 7. DOI: 10.4000/ primatologie.2698.

Gommery, D., Sénégas, F., Thackeray, J. F., et al. (2008a). Plio-Pleistocene fossils from Femur Dump, Bolt's Farm, Cradle of Humankind World Heritage Site. Annals of the Transvaal Museum, 45, 67-76.

Gommery, D., Thackeray, J. F., Potze, S. \& Braga, J. (2008b). The first recorded occurrence of honey badger of the genus Mellivora (Carnivora: Mustelidae) at Kromdraai B, South Africa: scientific notes. Annals of the Transvaal Museum, 45, 145-148.

Gommery, D., Thackeray, J. F., Sénégas, F., Potze, S. \& Kgasi, L. (2008c). The earliest primate (Parapapio sp.) from the Cradle of Humankind World Heritage site (Waypoint 160, Bolt's Farm, South Africa). South African Journal of Science, 104, 405-408.

Gommery, D., Thackeray, J. F., Sénégas, F., Potze, S. \& Kgasi, L. (2009). Additional fossils of Parapapio sp. from Waypoint 160 (Bolt's Farm, South Africa), dated between 4 and 4.5 million years ago. Annals of the Transvaal Museum, 46, 63-72.

Gommery, D., Badenhorst, S., Potze, S., et al. (2012a). Preliminary results concerning the discovery of new fossiliferous sites at Bolt's Farm (Cradle of Humankind, South Africa). Annals of the Ditsong National Museum of Natural History, 2, 33-45.

Gommery, D., Badenhorst, S., Sénégas, F., Potze, S. \& Kgasi, L. (2012b). Minnaar's Cave: a PlioPleistocene site in the Cradle of Humankind, South Africa - its history, location and fauna. Annals of the Ditsong National Museum of Natural History, 2, 19-31.

Gommery, D., Sénégas, F., Potze, S., Kgasi, L. \& Thackeray, J. F. (2014). Cercopithecoidea material from the Middle Pliocene site, Waypoint 160, Bolt's Farm, South Africa. Annals of the Ditsong National Museum of Natural History, 4, 1-8. 
Gommery, D., Sénégas, F., Kgazi, L., et al. (2016). Bolt's Farm cave system dans le Cradle of Humankind (Afrique du Sud): un exemple d'approche multidisciplinaire dans l'étude des sites à primates fossiles. Revue de Primatologie, 7. DOI: 10.4000/ primatologie. 2725.

Goodwin, A. J. H. (1937a). Archaeology of the Oakhurst Shelter, George. Part I. Course of the excavation. Transactions of the Royal Society of South Africa, 25, 229-245.

Goodwin, A. J. H. (1937b). Archaeology of the Oakhurst Shelter, George. Part II.

Disposition of the skeletal remains. Transactions of the Royal Society of South Africa, 25, 247-257.

Goodwin, A. J. H. \& Van Riet Lowe, C. (1929). The Stone Age cultures of South Africa. Annals of the South African Museum, 27, 1-289.

Gordon, D. H. (1991). Chromosomal variation in the water rat Dasymys incomtus (Rodentia: Muridae). Journal of Mammalogy, 72, 411-414.

Granjon, L., Duplantier, J.-M., Catalan, J. M. \& Britton-Davidian, J. (1997). Systematics of the genus Mastomys (Thomas, 1915) (Rodentia: Muridae), a review. Belgian Journal of Zoology, 127 (Suppl.), 7-18.

Gray, J. E. (1824). A revision of the family Equidae. Zoological Journal, 1, 241-248.

Gray, J. E. (1830). Spicilegia Zoologica; or Original Figures and Short Systematic Descriptions of New and Unfigured Animals. London: Treüttel, Würtz.

Gray, J. E. (1831). Zoological Miscellany. London: Treüttel, Würtz.

Gray, J. E. (1837). [Arrangement of the Sorices]. Proceedings of the Zoological Society of London, 5, 123-126.

Gray, J. E. (1838). A revision of the genera of bats (Vespertilionidae) and the description of some new genera and species. Magazine of Zoology and Botany, 2, 483-505.

Gray, J. E. (1842). Descriptions of some new genera and fifty unrecorded species of Mammalia. Annals and Magazine of Natural History, 10, 255-267.

Gray, J. E. (1849). Description of a new species of Herpestes. Annals and Magazine of Natural History, 17, 11.
Gray, J. E. (1850a). Synopsis of the species of antelopes and strepsiceres, with descriptions of some new species. Proceedings of the Zoological Society of London, 18, 111-146.

Gray, J. E. (1850b). Gleanings from the Menagerie and Aviary at Knowsley Hall. Knowsley: [private publication].

Gray, J. E. (1862a). Notice of a new species of bosh-buck (Cephalophus bicolor) from Natal. Annals and Magazine of Natural History, Series 3, 10, 400 .

Gray, J. E. (1862b). Description of some new species of Mammalia. Proceedings of the Zoological Society of London, 1862, 261-265.

Gray, J. E. (1864a). Notice of a new species of zorilla. Proceedings of the Zoological Society of London, 1864, 69-70.

Gray, J. E. (1864b). Notes on the species of sandmoles (Georychus). Proceedings of the Zoological Society of London, 1864, 123-125.

Gray, J. E. (1864c). A revision of the genera and species of viverrine animals (Viverridae), founded on the collection in the British Museum. Proceedings of the Zoological Society of London, 1864, 502-578.

Gray, J. E. (1865a). A revision of the species of golden moles (Chrysochloris). Proceedings of the Zoological Society of London, 1865, 678-680.

Gray, J. E. (1865b). Revision of the genera and species of entomophagous Edentata, founded on the examination of the specimens in the British Museum. Proceedings of the Zoological Society of London, 1865, 359-386.

Gray, J. E. (1866). Notice of a new bat (Scotophilus welwitschii) from Angola. Proceedings of the Zoological Society of London, 1866, 211.

Gray, J. E. (1867). Observations on the preserved specimens and skeletons of the Rhinocerotidae in the collection of the British Museum and Royal College of Surgeons, including the descriptions of three new species. Proceedings of the Zoological Society of London, 1867, 1003-1032.

Gray, J. E. (1868). Revision of the species of Hyrax, founded on the specimens in the British Museums. Annals and Magazine of Natural History, Series 4, 1, 35-51. 
Gray, J. E. (1873). On the boomdas (Dendrohyrax arboreus). Annals and Magazine of Natural History, Series 4, 11, 154-155.

Gray, P. A., Fenton, M. B. \& Van Cakenberghe, V. (1999). Nycteris thebiaca. Mammalian Species, 612, 1-8.

Green, C. A., Keogh, H., Gordon, D. H., Pinto, M. \& Hartwig, E. K. (1980). The distribution, identification and naming of the Mastomys natalensis species complex in southern Africa (Rodentia: Muridae). Journal of Zoology, 192, 17-23.

Greenwood, M. (1955). Fossil Hystricoidea from the Makapan Valley. Palaeontologia Africana, 3, 77-85.

Greenwood, M. (1958). Fossil Hystricoidea from the Makapan Valley, Transvaal: Hystrix makapanensis nom. nov. for Hystrix major Greenwood. Annals and Magazine of Natural History, Series 13, 1, 365.

Griffin, M. (1990). A review of taxonomy and ecology of gerbilline rodents of the central Namib Desert, with keys to the species (Rodentia: Muridae). In M.K. Seeley. Namib ecology: 25 years of Namib Research. Transvaal Museum Monograph, 7, 83-98.

Grine, F. E. (1981). Description of some juvenile hominid specimens from Swartkrans, Transvaal. Annals of the South African Museum, 86, 43-71.

Grine, F. E. (1982). A new juvenile hominid (Mammalia: Primates) from Member 3, Kromdraai Formation, Transvaal, South Africa. Annals of the Transvaal Museum, 33, 165-239.

Grine, F. E. (1989). New hominid fossils from the Swartkrans Formation (1979-1986 excavations): cranio-dental specimens. American Journal of Physical Anthropology, 79, 409-450.

Grine, F. E. (1993). Description and preliminary analysis of new hominid craniodental fossils from the Swartkrans Formation. In C. K. Brain, ed. Swartkrans: a cave's chronicle of early man. Transvaal Museum Monograph, 8, 75-116.

Grine, F. E. (1998). Additional human fossils from the Middle Stone Age of Die Kelders Cave South Africa: 1995 excavation. South African Journal of Science, 94, 229-235.
Grine, F. E. (2000). Middle Stone Age human fossils from Die Kelders Cave 1, Western Cape Province, South Africa. Journal of Human Evolution, 38, 129-145.

Grine, F. E. (2005). Early Homo at Swartkrans, South Africa: a review of the evidence and an evaluation of recently proposed morphs. South African Journal of Science, 101, 43-52.

Grine, F. E. (2012). Observations on Middle Stone Age human teeth from Klasies River Main Site, South Africa. Journal of Human Evolution, 63, 750-758.

Grine, F. E. \& Daegling, W. L. (1993). New mandible of Paranthropus robustus from Member 1, Swartkrans Formation, South Africa. Journal of Human Evolution, 24, 319-333.

Grine, F. E. \& Hendey, Q. B. (1981). Earliest primate remains from South Africa. Journal of Mammalogy, 20, 62-64.

Grine, F. E. \& Klein, R. G. (1985). Pleistocene and Holocene human remains from Equus Cave, South Africa. Anthropology, 8, 55-98.

Grine, F. E. \& Klein, R. G. (1993). Late Pleistocene human remains from the Sea Harvest site, Saldanha Bay, South Africa. South African Journal of Science, 89, 145-152.

Grine, F. E. \& Strait, D. (1994). New hominid fossils from Member 1 Hanging Remnant, Swartkrans Formation, South Africa. Journal of Human Evolution, 26, 57-75.

Grine, F. E. \& Susman, R. L. (1991). Radius of Paranthropus robustus from Member 1, Swartkrans Formation, South Africa. American Journal of Physical Anthropology, 84, 229-248.

Grine, F. E., Klein, R. G. \& Volman, T. P. (1991). Dating, archaeology and human fossils from the Middle Stone Age levels of Die Kelders, South Africa. Journal of Human Evolution, 21, 363-395.

Grine, F. E., Jungers, W. L., Tobias, P. V. \& Pearson, O. M. (1995). Fossil Homo femur from Berg Aukas, northern Namibia. American Journal of Physical Anthropology, 97, 151-185.

Grine, F. E., Pearson, O. M., Klein, R. G. \& Rightmire, G. P. (1998). Additional human fossils from Klasies River Mouth, South 
Africa. Journal of Human Evolution, 35, 95-107.

Grine, F. E., Bailey, R. M., Harvati, K., et al. (2007). Late Pleistocene human skull from Hofmeyr, South Africa and modern human origins. Science, 315, 226-229.

Grine, F. E., Gunz, P., Betti-Nash, L., Neubauer, S. \& Morris, A. G. (2010). Reconstruction of the late Pleistocene human skull from Hofmeyr, South Africa. Journal of Human Evolution, 59, 1-15.

Grine, F. E., Jacobs, R. L., Reed, K. E. \& Plavcan, J. M. (2012). The enigmatic molar from Gondolin, South Africa: implications for Paranthropus paleobiology. Journal of Human Evolution, 63, 597-609.

Groves, C. P. (1972). Ceratotherium simum. Mammalian Species, 8, 1-6.

Groves, C. P. \& Grubb, P. (2011). Ungulate Taxonomy. Baltimore, MD: Johns Hopkins University Press.

Grubb, P. (1981). Equus burchelli. Mammalian Species, 157, 1-9.

Grubb, P. (2004). Controversial scientific names of African mammals. African Zoology, 39, 91-109.

Grubb, P. \& d'Huart, J.-P. (2010). Rediscovery of the Cape warthog Phacochoerus aethiopicus: a review. Journal of the East African Natural History Society, 99, 77-102.

Guérin, C. (1987). Fossil Rhinocerotidae (Mammalia, Perissodactyla) from Laetoli. In M. D. Leakey \& J. M. Harris, eds. Laetoli: A Pliocene site in northern Tanzania. Oxford: Clarendon Press, pp. 320-348.

Guérin, C. (2000). The Neogene rhinoceroses of Namibia. Palaeontologia Africana, 36, 119-138.

Guérin, C. (2003). Miocene Rhinocerotidae of the Orange River valley, Namibia. Memoirs of the Geological Survey of Namibia, 19, 257-281.

Guérin, C. (2008). The Miocene Rhinocerotidae (Mammalia) from the northern Sperrgebiet, Namibia. Memoirs of the Geological Survey of Namibia, 20, 331-341.

Haas, S. K., Hatssen, V. \& Krausman, P. R. (2005). Panthera leo. Mammalian Species, 762, 1-11.

Halkett, D., Hart, T., Yates, R., et al. (2003). First excavation of intact Middle Stone Age layers at Ysterfontein, Western Cape Province, South Africa: implications for Middle Stone Age ecology. Journal of Archaeological Science, 30, 955-971.

Hamilton, W. R. (1973). A Lower Miocene Mammalian fauna from Siwa, Egypt.

Palaeontology, 16, 275-281.

Hamilton, W. R. (1978). Fossil giraffes from the Miocene of Africa and a revision of the phylogeny of the Giraffoidea. Philosophical Transactions of the Royal Society Series B, 283, 165-229.

Hamilton, W. R. \& Van Couvering, J. A. (1977). Lower Miocene mammals from South West Africa. Bulletin of Desert Ecological Research Unit, 2, 9-11.

Hanisch, E. O. M. (1980). An archaeological interpretation of certain Iron Age sites in the Limpopo/Shashi valley. Unpublished MA thesis, University of Pretoria.

Harris, J. M. (1976). Pliocene Giraffoidea (Mammalia, Artiodactyla) from the Cape Province. Annals of the South African Museum, 69, 325-353.

Harris, J. M. (1977). Deinotheres from southern Africa. South African Journal of Science, 73, 281-282.

Harris, J. M. \& White, T. D. (1979). Evolution of the Plio-Pleistocene African Suidae.

Transactions of the American Philosophical Society, 69, 1-128.

Harris, J. M., Solounias, N. \& Geraads, D. (2010). Giraffoidea. In L. Werdelin \& W. J. Sanders, eds. Cenozoic Mammals of Africa. Berkeley, CA: University of California Press, pp. 797-811.

Harris, W. C. (1838a). [Aigererus niger]. Atheneum, $535,71$.

Harris, W. C. (1838b). [A new antelope from the Cape]. Proceedings of the Zoological Society of London, 1838, 1-3.

Harrison, T. (2010). Dendropithecoidea, Proconsuloidea and Hominoidea. In L. Werdelin \& W. J. Sanders, eds. Cenozoic Mammals of Africa. Berkeley, CA: University of California Press, pp. 429-469.

Hartstone-Rose, A., De Ruiter, D., Berger, L. R. \& Churchill, S. E. (2007). A sabre-tooth felid from Coopers Cave (Gauteng, South Africa) and its implications for Megantereon (Felidae: 
Machairodontinae) taxonomy. Palaeontologia Africana, 42, 99-108.

Hartstone-Rose, A., Werdelin, L., De Ruiter, D., Berger, L. R. \& Churchill, S. E. (2010). The Plio-Pleistocene ancestor of wild dogs, Lycaon sekoweni n. sp. Journal of Paleontology, 84, 299-308.

Hartstone-Rose, A., Kuhn, B. F., Nalla, S. \& Werdelin, L. (2013). A new species of fox from the Australopithecus sediba type locality, Malapa, South Africa. Transactions of the Royal Society of South Africa, 68, 1-9.

Harvati, K., Bauer, C. C., Grine, F. E., et al. (2015). A human deciduous molar from the Middle Stone Age (Howiesons Poort) of Klipdrift Shelter, South Africa. Journal of Human Evolution, 82, 190-196.

Haughton, S. H. (1921). A note on some fossils from the Vaal River Gravels. Transactions of the Geological Society of South Africa, 24, 11-16.

Haughton, S. H. (1924). A note on the occurrence of a species of baboon in limestone deposits near Taungs. Transactions of the Royal Society of South Africa, 12, lxviii.

Haughton, S. H. (1932a). The fossil Equidae of South Africa. Annals of the South African Museum, 28, 407-427.

Haughton, S. H. (1932b). On some South African fossil Proboscidea. Transactions of the Royal Society of South Africa, 21, 1-18.

Haughton, S. H., Thomson, R. B. \& Péringuey, L. (1917). Preliminary note on the ancient human skull-remains from the Transvaal. Transactions of the Royal Society of South Africa, 6, 1-13.

Häusler, M. \& Berger, L. R. (2001). StW 441/465: a new fragmentary ilium of a small-bodied Australopithecus africanus from Sterkfontein, South Africa. Journal of Human Evolution, 40, 411-417.

Hautier, L., Mackaye, H. T., Lihoreau, F. \& Tassy, P. (2009). New material of Anancus kenyensis (Proboscidea, Mammalia) from TorosMenalla (Late Miocene, Chad): contribution to the systematics of African anancines. Journal of African Earth Sciences, 53, 171-176.

Hawks, J., Elliott, M., Schmid, P., et al. (2017). New fossil remains of Homo naledi from the Lesedi Chamber, South Africa. eLife, 6, e24232.
Heaton, J. L. (2006). Taxonomy of the Sterkfontein fossil Cercopithecinae: the Papionini of Members 2 and 4 (Gauteng, South Africa). Unpublished PhD thesis, Indiana University.

Heissig, K. (1971). Brachypotherium aus dem Miozän von Südwestafrikas. Mitteilungen aus dem Bayerischen Staatssammlung für Paläontologie und historische Geologie, 11, 125-128.

Helbing, H. (1924). Das Genus Hyaenaelurus Biedermann. Eclogae Geologicae Helvetiae, 19, 214-245.

Heller, E. (1912a). New genera and races of African ungulates. Smithsonian Miscellaneous Collections, 60(8), 1-16.

Heller, E. (1912b). New races of insectivores, bats and lemurs from British East Africa.

Smithsonian Miscellaneous Collections, 60(12), 1-13.

Helm, C., Cawthra, H. C., Cowling, R., et al. (2018). Palaeoecology of giraffe tracks in Late Pleistocene aeolianites on the Cape south coast. South African Journal of Science, 114, 8.

Hemmer, H. (1965). Zur nomenklatur und verbreitung des genus Dinofelis Zdansky, 1924 (Therailurus Piveteau, 1948).

Palaeontologia Africana, 9, 75-89.

Hemprich, F. W. \& Ehrenberg, C. G. (1828-1845). Symbolae Physicae, seu, Icones et Descriptiones Corporum Naturalium Noorum aut Minus Cognitorum. Berlin: Officina Academica.

Henderson, Z. (1992). The context of some Middle Stone Age hearths at Klasies River Shelter 1B: implications for understanding human behaviour. Southern African Field Archaeology, 1, 14-26.

Hendey, Q. B. (1967). A specimen of 'Archidiskodon' cf transvaaalensis from the south-western Cape Province. South African Archaeological Bulletin, 22, 53-56.

Hendey, Q. B. (1968). The Melkbos site: an upper Pleistocene fossil occurrence in the south-western Cape Province. Annals of the South African Museum, 52, 89-119.

Hendey, Q. B. (1969). Quaternary vertebrate fossil sites in the south-western Cape Province. South African Archaeological Bulletin, 24, 96-105. 
Hendey, Q. B. (1970). A review of the geology and palaeontology of the Plio/Pleistocene deposits at Langebaanweg, Cape Province. Annals of the South African Museum, 56, 75-117.

Hendey, Q. B. (1972). A Pliocene ursid from South Africa. Annals of the South African Museum, 59, 115-132.

Hendey, Q. B. (1973a). Carnivore remains from the Kromdraai australopithecine site (Mammalia: Carnivora). Annals of the Transvaal Museum, 28, 99-112.

Hendey, Q. B. (1973b). Fossil occurrences at Langebaanweg, Cape Province. Nature, 244, 13-14.

Hendey, Q. B. (1974a). The Late Cenozoic Carnivora of the south-western Cape Province. Annals of the South African Museum, 63, 1-369.

Hendey, Q. B. (1974b). New fossil carnivores from the Swartkrans australopithecine site (Mammalia: Carnivora). Annals of the Transvaal Museum, 29, 27-48.

Hendey, Q. B. (1976a). Fossil pecary from the Pliocene of South Africa. Science, 192, 787-789.

Hendey, Q. B. (1976b). The Pliocene fossil occurrences in 'E' Quarry, Langebaanweg, South Africa. Annals of the South African Museum, 69, 215-247.

Hendey, Q. B. (1978a). Late Tertiary Hyaenidae from Langebaanweg, South Africa and their relevance to the phylogeny of the family. Annals of the South African Museum, 76, 265-297.

Hendey, Q. B. (1978b). Late Tertiary Mustelidae (Mammalia, Carnivora) from Langebaanweg, South Africa. Annals of the South African Museum, 76, 329-357.

Hendey, Q. B. (1978c). Preliminary report on the Miocene vertebrates from Arrisdrift, South West Africa. Annals of the South African Museum, 76, 1-41.

Hendey, Q. B. (1978d). The age of the fossils from Baard's Quarry, Langebaanweg, South Africa. Annals of the South African Museum, 75, 1-24.

Hendey, Q. B. (1980). Agriotherium (Mammalia, Ursidae) from Langebaanweg, South Africa and relationships of the genus. Annals of the South African Museum, 81, 1-109.
Hendey, Q. B. (1981). Palaeoecology of the Late Tertiary fossil occurrences in 'E' Quarry, Langebaanweg, South Africa and a reinterpretation of their geological context. Annals of the South African Museum, 84, 1-104.

Hendey, Q. B. (1984). Southern African late Tertiary vertebrates. In R. G. Klein, ed. Southern African Prehistory and Paleoenvironments. Rotterdam: Balkema, pp. 81-106.

Hendey, Q. B. \& Cooke, H. B. S. (1985). Kolpochoerus paiceae (Mammalia, Suidae) from Skuurwerug, near Saldanha, South Africa and its palaeoenvironmental implications. Annals of the South African Museum, 97, 9-56.

Hendey, Q. B. \& Hendey, H. (1968). New Quaternary fossil sites near Swartklip, Cape Province. Annals of the South African Museum, 52, 43-73.

Hendey, Q. B. \& Singer, R. (1965). Part III: The faunal assemblages from the Gamtoos Valley shelters. South African Archaeological Bulletin, 20, 206-213.

Henshilwood, C. S. (1995). Holocene archaeology of the coastal Garcia State Forest, southern Cape, South Africa. Unpublished $\mathrm{PhD}$ thesis, University of Cambridge.

Henshilwood, C. S. (1996). A revised chronology for pastoralism in southernmost Africa: new evidence of sheep at c. 2000 b.p. from Blombos Cave, South Africa. Antiquity, 70, 945-949.

Henshilwood, C. S., Sealy, J. C., Yates, R., et al. (2001). Blombos Cave, southern Cape, South Africa: preliminary report on the 1992-1999 excavations of the Middle Stone Age levels. Journal of Archaeological Science, 28, 421-448.

Henshilwood, C. S., Van Niekerk, K. L., Wurz, S., et al. (2014). Klipdrift Shelter, southern Cape, South Africa: preliminary report on the Howiesons Poort layers. Journal of Archaeological Science, 45, 284-303.

Herries, A. I. R., Adams, J. W., Kuykendall, K. I. \& Shaw, J. (2006). Speleology and magnetobiostratigraphic chronology of the GD 2 locality of the Gondolin homininbearing paleocave deposits, North West Province, South Africa. Journal of Human Evolution, 51, 617-631. 
Heuglin, T. von (1863). Beiträge zur Zoologie Afrika's. Über einige Säugethiere des BäschloGebietes. Novorum Actorum Academiae Caesareae Leopoldino-Carolinae Germanicae Naturae Curiosorum, 30, 1-14.

Heuglin, T. von (1865). Beschreibung eines centralafrikanischen Leporinen. Novorum Actorum. Academiae Caesareae LeopoldinoCarolinae Germanicae Naturae Curiosorum. Leopoldina, 32-24, 32-36.

Hill, J. \& Harrison, D. L. (1987). The baculum in the Vespertilioninae (Chiroptera:

Vespertilionidae) with a systematic review, a synopsis of Pipistrellus and Eptesicus and the descriptions of a new genus and subgenus. Bulletin of the British Museum (Natural History) Zoological Series, 52, 225-305.

Hillestad Nel, T. \& Henshilwood, C. S. (2016). The small mammal sequence from the c. 76-72 ka Still Bay levels at Blombos Cave, South Africa - taphonomic and palaeoecological implications for human behaviour. PLOS ONE, 11. DOI: 10.1371/ journal.pone.0159817.

Hillman-Smith, A. K. K. \& Groves, C. P. (1994). Diceros bicornis. Mammalian Species, 455, 1-8.

Hodgson, B. H. (1847). On the various genera of the ruminants. Journal of the Asiatic Society of Bengal, Series 3, 16, 685-711.

Hoffman, A. C. (1953). The fossil alcelaphines of South Africa: genera Peloceras, Lunatoseras and Alcelaphus. Navorsinge van die Nasionale Museum Bloemfontein, 1, 41-56.

Holden, M. E. (1996). Systematic revision of subSaharan African dormice (Rodentia: Myoxidae: Graphiurus) part 1: an introduction to the generic revision and a revision of Graphiurus surdus. American Museum Novitates, 3157, 1-44

Holloway, R. L., Hurst, S. D., Garvin, H. M., et al. (2018). Endocast morphology of Homo naledi from the Dinaledi Chamber, South Africa. Proceedings of the National Academy of Sciences of the United States of America. DOI: 10.1073/ pnas.1720842115.

Holroyd, P. A. (1999). New Pterodontinae (Creodonta: Hyaenodontidae) from the late Eocene-early Oligocene Jebel Qatrani Formation, Fayum province, Egypt. PaleoBios, 19, 1-18.
Holroyd, P. A. (2010a). Macroscelidea. In L. Werdelin \& W. J. Sanders, eds. Cenozoic Mammals of Africa. Berkeley, CA: University of California Press, pp. 89-98.

Holroyd, P. A. (2010b). Tubulidentata. In L. Werdelin \& W. J. Sanders, eds. Cenozoic mammals of Africa. Berkeley, CA: University of California Press. pp. 107-111.

Holroyd, P. A., Lihoreau, F., Gunnell, G. F. \& Miller, E. R. (2010). Anthracotheriidae. In L. Werdelin \& W. J. Sanders, eds. Cenozoic Mammals of Africa. Berkeley, CA: University of California Press, pp. 843-851.

Holt, S. (2009). The faunal remains from the Makgabeng Plateau, Limpopo Province. Unpublished MSc thesis, University of the Witwatersrand.

Hooijer, D. A. (1945). Note on the subfossil teeth of Equus zebra L. from the Orange Free State. Zoologische Mededelingen Museum Leiden, 25, 101-108.

Hooijer, D. A. (1958). Pleistocene remains of hippopotamus from the Orange Free State. Navorsinge van die Nasionale Museum Bloemfontein, 1, 259-266.

Hooijer, D. A. (1959). Fossil rhinoceroses from the Limeworks Cave, Makapansgat. Palaeontologia Africana, 6, 1-13.

Hooijer, D. A. (1963). Miocene Mammalia of Congo. Annales du Musée Royal de l'Afrique Centrale Sciences Géologiques, 46, 1-77.

Hooijer, D. A. (1971). A new rhinoceros from the Late Miocene of Loporot, Turkana District, Kenya. Bulletin of the Museum of Comparative Zoology, 142, 339-392.

Hooijer, D. A. (1972). A late Pliocene rhinoceros from Langebaanweg. Annals of the South African Museum, 59, 151-191.

Hooijer, D. A. (1973). Additional Miocene to Pleistocene rhinoceroses of Africa. Zoologische Mededelingen, 46, 149-177.

Hooijer, D. A. (1975). Miocene to Pleistocene hipparions of Kenya, Tanzania and Ethiopia. Zoologische Verhandelingen, 142, 3-80.

Hooijer, D. A. (1976). The late Pliocene Equidae of Langebaanweg, Cape Province, South Africa. Zoologische Verhandelingen, 148, 3-39.

Hooijer, D. A. \& Patterson, B. (1972). Rhinoceroses from the Pliocene of 
Northwestern Kenya. Bulletin on the Museum of Comparative Zoology, 144, 1-26.

Hooijer, D. A. \& Singer, R. (1960). Fossil rhinoceroses from Hopefield, South Africa. Zoologische Mededelingen, 37, 113-128.

Hooijer, D. A. \& Singer, R. (1961). The fossil hippopotamus from Hopefield, South Africa. Zoologische Mededelingen, 37, 157-165.

Hopley, P. J., Latham, A. G. \& Marshall, J. D. (2006). Palaeoenvironments and palaeodiets of mid-Pliocene micromammals from Makapansgat Limeworks, South Africa: a stable isotope and dental microwear approach. Palaeogeography, Palaeoclimatology, Palaeoecology, 233, 235-251.

Hopwood, A. T. (1926). Some Mammalia from the Pliocene of Homa Mountain, Victoria Nyanza. Annals and Magazine of Natural History, Series 9, 18, 266-272.

Hopwood, A. T. (1929). New and little known mammals from the Miocene of Africa. American Museum Novitates, 76, 1-9.

Hopwood, A. T. (1934). New fossil mammals from Olduvai, Tanganyika Territory. Annals and Magazine of Natural History, Series 10, 14, 546-550.

Hopwood, A. T. (1936). New and little-known fossil mammals from Kenya Colony and Tanganyika Territory I. Annals and Magazine of Natural History, Series 10, 17, 636-641.

Horsburgh, K. A. \& Moreno-Mayar, V. J. (2015). Molecular identification of sheep at Blydefontein Rock Shelter, South Africa. Southern African Humanities, 27, 65-80.

Horsburgh, K. A. \& Rhines, A. (2010). Genetic characterization of an archaeological sheep assemblage from South Africa's Western Cape. Journal of Archaeological Science, 37, 2906-2910.

Horsburgh, K. A., Orton, J. \& Klein, R. G. (2016). Beware the springbok in sheep's clothing: how secure are the faunal identifications upon which we build our models? African Archaeological Review, 33, 353-361.

Huchon, D., Catzeflis, F. \& Douzery, E. J. P. (2000). Variance of molecular datings, evolution of rodents, and the phylogenetic affinities between Ctenodactylidae and Hystricognathi. Proceedings of the Royal Society of London B: Biological Sciences, 267, 393-402.
Huchon, D., Madsen, O., Sibbald, M. J. J. B., et al. (2002). Rodent phylogeny and a timescale for the evolution of Glires: evidence from an extensive taxon sampling using three nuclear genes. Molecular Biology and Evolution, 19, 1053-1065.

Huffman, T. N. (1975). Cattle from Mabveni. South African Archaeological Bulletin, 30, 23-24.

Huffman, T. N. (1979a). Test excavations at Naba and Lanlory, Northern Mashonaland. South African Archaeological Society Goodwin Series, 3, 14-46.

Huffman, T. N. (1979b). Test excavations at Chamabvefva, southern Mashonaland. South African Archaeological Bulletin, 34, 57-70.

Huffman, T. N. (2008). Zhizo and Leopard's Kopje: test excavations at Simamwe and Mtanye, Zimbabwe. In S. Badenhorst, P. Mitchell \& J. C. Driver, eds. Animals and People: Archaeozoological Papers in Honour of Ina Plug. Oxford: Archaeopress, pp. 200-214.

Huffman, T. N. \& Kinahan, J. (2002/2003). Archaeological mitigation of the Letsibogo Dam: agropastoralism in southeastern Botswana. Southern African Field Archaeology, 11-12, 4-63.

Hughes, A. (1990). The Tuinplaas human skeleton from the Springbok Flats, Transvaal. In G. H. Sperber, ed. From Apes to Angels: Essays in Honour of Phillip V. Tobias. New York: Wiley-Liss, pp. 197-214.

Humphreys, A. J. B. (1974). A preliminary report on test excavations at Dikbosch Shelter 1, Herbert District, northern Cape. South African Archaeological Bulletin, 29, 115-119.

Humphreys, A. J. B. (1975). Burchell's Shelter: the history and archaeology of a Northern Cape rock shelter. South African Archaeological Bulletin, 30, 3-18.

Humphreys, A. J. B. (1978). The re-excavation of Powerhouse Cave and an assessment of Dr Frank Peabody's work on Holocene deposits in the Taung area. Annals of the Cape Provincial Museums, 11, 217-244.

Humphreys, A. J. B. \& Thackeray, A. I. (1983). Ghaap and Gariep. Cape Town: South African Archaeological Society.

Hutson, J. M. (2006). Taphonomy at Kalkbank: a Late Pleistocene site in the Limpopo 
Province, South Africa. Unpublished MSc thesis, University of the Witwatersrand.

Hutson, J. M. (2016). The faunal remains from Bundu Farm and Pniel 6: examining the problematic Middle Stone Age archaeological record within the southern African interior. Quaternary International. DOI: 10.1016/j. quaint.2016.04.030.

Hutson, J. M. \& Cain, C. R. (2008). Reanalysis and reinterpretation of the Kalkbank faunal accumulation, Limpopo Province, South Africa. Journal of Taphonomy, 6, 399-428.

Hutterer, R. (1983). Taxonomy and distribution of Crocidura fuscomurina (Heuglin, 1865). Mammalia, 47, 221-227.

Illiger, J. K. W. (1811). Prodromus Systematis Mammaliam et Avium. Berlin: C. Salfeld.

Illiger, J. K. W. (1815). Ueberblick der Säugethiere nach ihrer Vertheilung über die Welheile. Abhandlungen der physikalischen Klasse der Königlich-Preussischen Akademie der Wissenschaften, 1804-1811, 39-159.

Ingram, C. M., Burda, H. \& Honeycutt, R. L. (2004). Molecular phylogenetics and taxonomy of the African mole-rats, genus Cryptomys and the new genus Coetomys Gray, 1864. Molecular Phylogenetics and Evolution, 31, 997-1014.

Jablonsky, N. G. \& Frost, S. (2010). Cercopithecoidea. In L. Werdelin \& W. J. Sanders, eds. Cenozoic Mammals of Africa. Berkeley, CA: University of California Press, pp. 393-428.

Jacobs, B. F., Pan, A. D. \& Scotese, C. R. (2010). A review of the Cenozoic vegetation history of Africa. In L. Werdelin \& W. J. Sanders, eds. Cenozoic Mammals of Africa. Berkeley, CA: University of California Press, pp. 57-72.

Jacobson, L. (1978). Report on archaeological and palaeoecological studies in the Gobabis District, South West Africa. Palaeoecology of Africa, 10, 93-94.

Jaeger, J. J., Michaux, J. \& Sabatier, M. (1980). Premières données sur les rongeurs de la Formation de Cho'rora (Ethiopie) d'âge Miocène supérieur. I: Thryonomyidés. Palaeovertebrata, 9 Ext. Mémoire Jubilaire en Hommage à R. Lavocat, 365-374.

Jaeger, J. J., Denys, C. \& Coiffait, B. (1985). New Phiomorpha and Anomaluridae from the late
Eocene of north-west Africa: phylogenetic implications. In W. P. Luckett \& J.-L. Hartenberger, eds. Evolutionary Relationships among Rodents, a Multidisciplinary Analysis. New York: Plenum, pp. 567-588.

Jaeger, J. J., Marivaux, L., Salem, M., et al. (2010). New rodent assemblages from the Eocene Dur At-Talah escarpment (Sahara of central Libya): systematic, biochronological and palaeobiogeographical implications. Zoological Journal of the Linnean Society, 160, 195-213.

Jameson, H. L. (1907). On a new hare from the Transvaal. Annals and Magazine of Natural History, Series 7, 20, 404-406.

Jameson, H. L. (1909). On a sub-fossil hare from a cave deposit at Godwan River. Annals of the Transvaal Museum, 1, 195-196.

Jansen van Vuuren, B. (1999). Molecular phylogeny of duiker antelope (Mammalia: Cephalophini). Unpublished PhD thesis, University of Pretoria.

Jenkins, P., Ruedi, M. \& Catzeflis, F. (1998). A biochemical and morphological investigation of Suncus dayi (Dobson, 1888) and discussion of relationships in Suncus Hemprich \& Ehrenberg, 1833, Crocidura Wagler, 1832 and Sylvisorex Thomas, 1904 (Insectivora: Soricidae). Bonner Zoologische Beitragen, 47, 257-276.

Jerardino, A. (1998). Excavations at Pancho's Kitchen Midden, western Cape coast, South Africa: further observations into the Megamidden Period. South African Archaeological Bulletin, 53, 16-25.

Jerardino, A. (2007). Excavations at a huntergatherer site known as 'Grootrif G' Shell Midden, Lamberts Bay, Western Cape Province. South African Archaeological Bulletin, 62, 162-170.

Jerardino, A. (2012). Large shell middens and hunter-gatherer resource intensification along the west coast of South Africa: the Elands Bay case study. Journal of Island and Coastal Archaeology, 7, 76-101.

Jerardino, A. \& Yates, R. (1996). Preliminary results from excavations at Steenbokfontein Cave: implications for past and future research. South African Archaeological Bulletin, 51, 7-16. 
Jerardino, A., Yates, R., Morris, A. G. \& Sealy, J. C. (1992). A dated human burial from the Namaqualand coast: observations on culture, biology and diet. South African Archaeological Bulletin, 47, 75-81.

Jerardino, A., Dewar, G. \& Navarro, R. (2009a). Opportunistic subsistence strategies among Late Holocene coastal hunter-gatherers, Elands Bay, South Africa. Journal of Island and Coastal Archaeology, 4, 37-60.

Jerardino, A., Kolska Horwitz, L., Mazel, A. \& Navarro, R. (2009b). Just before Van Riebeeck: glimpses into terminal LSA lifestyle at Connies Limpet Bar, west coast of South Africa. South African Archaeological Bulletin, 64, 75-86.

Jerardino, A., Klein, R. G., Navarro, R., Orton, J. \& Kolska Horwitz, L. (2013). Settlement and subsistence patterns since the terminal Pleistocene in the Elands Bay and Lamberts Bay areas. In A. Jerardino, A. Malan \& D. Braun, eds. The Archaeology of the West Coast of South Africa. Oxford: Archaeopress, pp. 85-108.

Jerardino, A., Kaplan, J., Navarro, R. \& Nilssen, P. (2016). Filling-in the gaps and testing past scenarios on the central West Coast: huntergatherer subsistence and mobility at 'Deurspring 16' shell midden, Lamberts Bay, South Africa. South African Archaeological Bulletin, 71, 71-86.

Jones, T. R. (1937). A new fossil primate from Sterkfontein, Krugersdorp, Transvaal. South African Journal of Science, 33, 709-728.

Kandel, A. W. \& Conard, N. J. (2012). Settlement patterns during the Earlier and Middle Stone Age around Langebaan Lagoon, Western Cape (South Africa). Quaternary International, 270, 15-29.

Kaplan, J. (1987). Settlement and subsistence at Renbaan Cave. In J. Parkington \& M. Hall, eds. Papers in the Prehistory of the Western Cape, South Africa. Oxford: Archaeopress, pp. 237-261.

Kaplan, J. (1990). The Umhlatuzana Rock Shelter sequence: 100000 years of Stone Age history. Natal Museum Journal of Humanities, 2, 1-94.

Katsamudanga, S. (2007a). Environment and culture: a study of prehistoric settlement patterns in the Eastern Highlands of
Zimbabwe. Unpublished PhD thesis, University of Zimbabwe.

Katsamudanga, S. (2007b). Archaeological surveys in Zimunya, Burma Valley, Vumba and Tsetsera Mountains, central Eastern Highlands of Zimbabwe. Zimbabwea, 9, 9-19.

Kaup, J. J. (1828). Ueber Hyaena, Uromastix, Basiliscus, Corythaeolus, Acontoias. Isis von Oken, 21, 1144-1150.

Kaup, J. J. (1829). Skizzirte Entwickelungs-Geschichte und natürliches System der europäischen Thierwelt, Vol. 1. Darmstadt: Leske.

Kaup, J. J. (1833). Déscription d'Ossements Fossiles de Mammiferes Inconnus jusqu'à Présent, qui se trouvent au Museum Grand-Ducal de Darmstadt, Vol. 2. Darmstadt: Stahl and Bekker.

Kaup, J. J. (1835). Das Thierreich in seinen Hauptformen. Darmstadt: J. P. Biehl.

Kearney, T. \& Van Schalkwyk, E. (2009). Variation in the position within the tooth row of the minute premolars of Cistugo lesueuri and C. seabrae (Chiroptera: Vespertilionidae) and re-identification of some museum voucher specimens. Annals of the Transvaal Museum, 46, 117-120.

Kearney, T., Volleth, M., Contrafatto, G. \& Taylor, P. (2002). Systematic implications of chromosome GTG-band and bacula morphology for southern African Eptesicus and Pipistrellus and several other species of Vespertilioninae (Chiroptera: Vespertilionidae). Acta Chiropterologica, 4, 55-76.

Keen, E. N. \& Singer, R. (1956). Further fossil Suidae from Hopefield. Annals of the South African Museum, 42, 351-360.

Kegley, A. D. T., Hemingway, J. \& Adams, J. W. (2011). Odontometric analysis of the reanalyzed and expanded Cercopithecoides from the Haasgat fossil assemblage, Cradle of Humankind, South Africa. American Journal of Physical Anthropology, S144, 183.

Keith, A. (1933). A descriptive account of the human skulls from Matjes River Cave, Cape Province. Transactions of the Royal Society of South Africa, 21, 151-185.

Kerr, R. (1792). The Animal Kingdom, or Zoological System of the Celebrated Sir Charles Linnaeus. Edinburgh: Strahan \& Cadell and Creech. 
Keyser, A. (1991). The palaeontology of Haasgat: a preliminary account. Palaeontologia Africana, 28, 29-33.

Keyser, A. W. (2000). The Drimolen skull: the most complete australopithecine cranium and mandible to date. South African Journal of Science, 96, 189-193.

Keyser, A. W. \& Martini, J. E. J. (1991). Haasgat: a new Plio-Pleistocene fossil occurrence. Palaeoecology of Africa, 21, 119-129.

Keyser, A. W., Menter, C., Moggi-Cecchi, J., Pickering, T. R. \& Berger, L. R. (2000). Drimolen: a new hominid-bearing site in Gauteng, South Africa. South African Journal of Science, 96, 193-197.

Kiberd, P. (2006). Bundu Farm: a report on archaeological and palaeoenvironmental assemblages from a pan site in Bushmanland, Northern Cape, South Africa. South African Archaeological Bulletin, 61, 189-201.

Kibii, J. M. (2006). Comparative taxonomic, taphonomic and palaeoenvironmental analysis of 4-2.3 million year old australopithecine cave infills at Sterkfontein. Unpublished PhD thesis, University of the Witwatersrand.

Kinahan, J. \& Kinahan, J. (2003). Excavation of a late Holocene cave deposit in the southern Namib Desert, Namibia. Cimbebasia, 18, 1-10.

Kitching, J. W. (1963). A fossil Orycteropus from the Limeworks quarry, Makapansgat, Potgietersrus. Palaeontologia Africana, 8, 119-121.

Kitching, J. W. (1965). A new giant hyracoid from the Limeworks Quarry, Makapansgat, Potgietersrus. Palaeontologia Africana, 9, 91-96.

Klatzow, S. (1994). Roosfontein, a contact site in the Eastern Orange Free State. South African Archaeological Bulletin, 49, 9-15.

Klein, R. G. (1972). The Late Quaternary Mammalian fauna of Nelson Bay Cave (Cape Province, South Africa): its implications for megafaunal extinctions and for environmental and cultural change. Quaternary Research, 2, 135-142.

Klein, R. G. (1974a). On the taxonomic status, distribution and ecology of the blue antelope, Hippotragus leucophaeus (Pallas, 1766). Annals of the South African Museum, 65, 99-143.
Klein, R. G. (1974b). Environment and subsistence of prehistoric man in the southern Cape Province. World Archaeology, 5, 249-284.

Klein, R. G. (1975a). Paleoanthropological implications of the non-archeological bone assemblage from Swartklip 1, south-western Cape Province, South Africa. Quaternary Research, 5, 275-288.

Klein, R. G. (1975b). Middle Stone Age mananimal relationships in southern Africa: evidence from Die Kelders and Klasies River Mouth. Science, 190, 265-267.

Klein, R. G. (1976a). The mammalian fauna of the Klasies River Mouth sites, southern Cape Province, South Africa. South African Archaeological Bulletin, 31, 75-98.

Klein, R. G. (1976b). A preliminary report on the 'Middle Stone Age' open-air site of Duinefontein 2 (Melkbosstrand, southwestern Cape Province, South Africa). South African Archaeological Bulletin, 31, 12-20.

Klein, R. G. (1976c). The fossil history of Raphicerus H. Smith, 1827 (Bovidae, Mammalia) in the Cape Biotic Zone. Annals of the South African Museum, 71, 169-191.

Klein, R. G. (1977). The mammalian fauna from the Middle and Later Stone Age (Later Pleistocene) levels of Border Cave, Natal Province, South Africa. South African Archaeological Bulletin, 32, 14-27.

Klein, R. G. (1978a). A preliminary report on the larger mammals from the Boomplaas Stone Age cave site, Cango Valley, Oudtshoorn District, South Africa. South African Archaeological Bulletin, 33, 66-75.

Klein, R. G. (1978b). Preliminary analysis of the mammalian fauna from the Redcliff Stone Age site, Rhodesia. Occasional Papers of the National Museums and Monuments of Rhodesia Series A Human Sciences, 4, 74-80.

Klein, R. G. (1978c). The fauna and overall interpretation of the Cutting 10 Acheulean site at Elandsfontein (Hopefield), southwestern Cape Province, South Africa. Quaternary Research, 10, 69-83.

Klein, R. G. (1978d). Appendix: vertebrate fauna from the Buffelskloof Rock Shelter. In $\mathrm{H}$. Opperman, Excavations in the Buffelskloof Rock Shelter near Calitzdorp, Southern Cape. 
South African Archaeological Bulletin, 33, 35-38.

Klein, R. G. (1979a). Palaeoenvironmental and cultural implications of late Holocene archaeological faunas from the Orange Free State and north-central Cape Province, South Africa. South African Archaeological Bulletin, 34, 34-49.

Klein, R. G. (1979b). Appendix III: macromammals. In C. K. Cooke, Excavations at Diana's Vow, Rock Shelter: Makomi District, Zimbabwe-Rhodesia. Salisbury: National Museums and Monuments, 147-148.

Klein, R. G. (1980). Appendix 1: larger mammals. In T. Maggs \& V. Ward, Driel Shelter: rescue at a Late Stone Age site on the Tugela River. Annals of the Natal Museum, 24, 62-67.

Klein, R. G. (1981). Later Stone Age subsistence at Byeneskranskop Cave, South Africa. In R. S. O. Harding \& G. Teleki, eds. Omnivorous Primates: Gathering and Hunting in Human Evolution. New York: Columbia University Press, pp. 166-190.

Klein, R. G. (1982). Appendix 1: Byneskranskop 1 - mammals. Tables of the minimum numbers of individuals represented by various skeletal parts. In F. R. Schweitzer \& M. L. Wilson. Byneskranskop 1: a late Quaternary living site in the southern Cape Province, South Africa. Annals of the South African Museum, 82, 189-196.

Klein, R. G. (1984a). Later Stone Age faunal samples from Heuningneskrans Shelter (Transvaal) and Leopard's Hill Cave (Zambia). South African Archaeological Bulletin, 39, 109-116.

Klein, R. G. (1984b). Appendix 1: the remains of larger mammals from Fairview Shelter. In P. T. Robertshaw. Fairview Rockshelter: a contribution to the prehistory of the eastern Cape Province of South Africa. Annals of the Cape Provincial Museums (Human Sciences), 1, 82-86.

Klein, R. G. (1986). The prehistory of Stone Age herders in the Cape Province of South Africa. South African Archaeological Society Goodwin Series, 5, 5-12.

Klein, R. G. (1988). The archaeological significance of animal bones from Acheulean sites in southern Africa. The African Archaeological Review, 6, 3-25.

Klein, R. G. (1994a). The long-horned African buffalo (Pelorovis antiquus) is an extinct species. Journal of Archaeological Science, 21, 725-733.

Klein, R. G. (1994b). Southern Africa before the Iron Age. In R. S. Corruccini \& R. L. Ciochon, eds. Integrative Paths to the Past:

Paleoanthropological Advances in Honor of

F. Clark Howell. Englewood Cliffs, NJ: Prentice Hall, pp. 471-519.

Klein, R. G. \& Cruz-Uribe, K. (1987). Large mammal and tortoise bones from Elands Bay Cave and nearby sites, western Cape Province, South Africa. In J. Parkington \& M. Hall, eds. Papers in the Prehistory of the Western Cape, South Africa. Oxford: Archaeopress, pp. 132-163.

Klein, R. G. \& Cruz-Uribe, K. (1989). Faunal evidence for prehistoric herder-forager activities at Kasteelberg, Western Cape Province, South Africa. South African Archaeological Bulletin, 44, 82-97.

Klein, R. G. \& Cruz-Uribe, K. (1991). The bovids from Elandsfontein, South Africa and their implications for the age, palaeoenvironment and origins of the site. African Archaeological Review, 9, 21-79.

Klein, R. G. \& Cruz-Uribe, K. (1999). Craniometry of the genus Equus and the taxonomic affinities of the extinct South African quagga. South African Journal of Science, 95, 81-86.

Klein, R. G. \& Cruz-Uribe, K. (2000). Middle and Later Stone Age large mammal and tortoise remains from Die Kelders Cave 1, Western Cave Province, South Africa. Journal of Human Evolution, 38, 169-195.

Klein, R. G. \& Scott, K. (1974). The fauna of Scott's Cave, Gamtoos Valley, south-eastern Cape Province. South African Journal of Science, 70, 186-187.

Klein, R. G. \& Steele, T. E. (2008). The faunal remains from Diepkloof Rock Shelter, South Africa. In P.-J. Texier. Diepkloof (Western Cape, République d'Afrique du Sud). Rapport sur les travaux effectués du 16 octobre 2007 au 15 octobre 2008. Unpublished CNRS report. 
Klein, R. G., Cruz-Uribe, K. \& Beaumont, P. B. (1991). Environmental, ecological and paleoanthropological implications of the Late Pleistocene mammalian fauna from Equus Cave, northern Cape Province, South Africa. Quaternary Research, 36, 94-119.

Klein, R. G., Avery, G., Cruz-Uribe, K., et al. (1999a). Duinefontein 2: an Acheulean site in the Western Cape Province of South Africa. Journal of Human Evolution, 37, 153-190.

Klein, R. G., Cruz-Uribe, K., Halkett, D., Hart, T. \& Parkington, J. E. (1999b).

Paleoenvironmental and human behavioral implications of the Boegoeberg 1 Late Pleistocene hyena den, Northern Cape Province, South Africa. Quaternary Research, 52, 393-403.

Klein, R. G., Avery, G., Cruz-Uribe, K., et al. (2004). The Ysterfontein 1 Middle Stone Age site, South Africa and early human exploitation of coastal resources. Proceedings of the National Academy of Sciences of the United States of America, 101, 5708-5715.

Klein, R. G., Avery, G., Cruz-Uribe, K. \& Steele, T. E. (2007). The mammalian fauna associated with an archaic hominin skullcap and later Acheulian artifacts at Elandsfontein, Western Cape Province, South Africa. Journal of Human Evolution, 52, 164-186.

Kock, D., Ingram, C. M., Frabotta, L. J., Honeycutt, R. L. \& Burda, H. (2006). On the nomenclature of Bathyergidae and Fukomys n. gen. (Mammalia: Rodentia). Zootaxa, 1142, 51-55.

Koehler, C. E. \& Richardson, P. R. K. (1990). Proteles cristatus. Mammalian Species, 363, 1-6.

Kolbe, F. F. (1948). On a hitherto unrecorded subspecies of South African bush hare (Lepus saxatilis orangensis $\mathrm{n}$. subsp.). Annals of the Transvaal Museum, 21, 71-72.

Korsman, S. \& Plug, I. (1994). Two Later Stone Age sites on the Farm Honingklip in the Eastern Transvaal. South African Archaeological Bulletin, 49, 24-32.

Krausman, P. R. \& Morales, S. M. (2005). Acinonyx jubatus. Mammalian Species, 771, 1-6.

Kretzoi, N. (1929). Materialien zur phylogenetischen Klassifikation der
Aeluroideen. In 10th International Congress of Zoology (1927), Vol. 2. Budapest:

Stephaneum, pp. 1293-1355.

Kryštufek, B., Haberl, W., Baxter, R. M. \& Zima, J. (2004). Morphology and karyology of two populations of the woodland dormouse Graphiurus murinus in the Eastern Cape, South Africa. Folia Zoologica, 53, 339-350.

Kryštufek, B., Baxter, R. M., Haberl, W., Zima, J. \& Bužan, E. V. (2008). Systematics and biogeography of the Mozambique thicket rat, Grammomys cometes, in Eastern Cape Province, South Africa. Journal of Mammalogy, 89, 325-335.

Kuhl, H. (1817). Die Deutschen Fledermäuse. Hanau: Universitätsbibliothek Johann Christian Senckenberg.

Kuhn, B. F., Werdelin, L., Hartstone-Rose, A., Lacruz, R. \& Berger, L. R. (2011). Carnivoran remains from the Malapa Hominin Site, South Africa. PLoS ONE, 6. DOI: 10.1371/ journal.pone.0026940.

Kuhn, B. F., Herries, A. I. R., Price, G. J., et al. (2016). Renewed investigations at Taung: 90 years after the discovery of Australopithecus africanus. Palaeontologia Africana, 51, 10-26.

Kuhn, B. F., Werdelin, L. \& Steininger, C. (2017). Fossil Hyaenidae from Cooper's Cave, South Africa and the palaeoenvironmental implications. Palaeobiodiversity and Palaeoenvironments, 97, 355-365.

Kuman, K. \& Clarke, R. J. (1986). Florisbad: new investigations at a Middle Stone Age site in South Africa. Geoarchaeology, 1, 103-125.

Kurtén, B. (1976). Fossil Carnivora from the Late Tertiary of Bled Douarah and Cherichira, Tunisia. Notes du Service Géologique de Tunisie, 42, 177-214.

Kuykendall, K. L. \& Conroy, G. C. (1999). Description of the Gondolin teeth: hyperrobust hominids in South Africa? American Journal of Physical Anthropology, 28, 176-177.

Kuykendall, K. L. \& Rae, T. C. (2008). Presence of the maxillary sinus in fossil Colobinae (Cercopithecoides williamsi) from South Africa. The Anatomical Record, 291, 1499-1506.

Kuykendall, K. L., Toich, C. A. \& McKee, J. K. (1995). Preliminary analysis of the fauna from Buffalo Cave, northern Transvaal, South Africa. Palaeontologia Africana, 32, 27-31. 
L'Abbé, E. N., Loots, M. \& Keough, N. (2008). The Matjes River Rock Shelter: a description of the skeletal assemblage. South African Archaeological Bulletin, 63, 61-68.

Lacépède, E. B. G. (1799). Tableau des Divisions, Sous-divisions, Ordres, et Genres des Mammifères. Paris: Plasson.

Lacruz, R. (2009). Panthera leo (Mammalia: Felidae) remains from the Gladysvale Cave, South Africa: scientific note. Annals of the Transvaal Museum, 46, 121-124.

Lacruz, R. S., Brink, J. S., Hancox, P. J., et al. (2002). Palaeontology and geological context of a Middle Pleistocene faunal assemblage from the Gladysvale Cave, South Africa. Palaeontologia Africana, 38, 99-114.

Lacruz, R., Ungar, P., Hancox, P. J., Brink, J. S. \& Berger, L. R. (2003). Gladysvale: fossils, strata and GIS analysis. South African Journal of Science, 99, 283-285.

Lacruz, R., Turner, A. \& Berger, L. R. (2006). New Dinofelis (Carnivora: Machairodontinae) remains from Sterkfontein Valley sites and a taxonomic revision of the genus in southern Africa. Annals of the Transvaal Museum, 43, 89-106.

Larivière, S. (2001a). Aonyx capensis. Mammalian Species, 671, 1-6.

Larivière, S. (2001b). Poecilogale albinucha. Mammalian Species, 681, 1-4.

Larivière, S. (2002). Ictonyx striatus. Mammalian Species, 698, 1-5.

Larivière, S. \& Calzada, J. (2001). Genetta genetta. Mammalian Species, 680, 1-6.

Laubscher, N. F., Steffens, F. E. \& Vrba, E. (1972). Statistical evaluation of the taxonomic status of a fossil member of the Bovidae (Mammalia: Artiodactyla). Annals of the Transvaal Museum, 28, 17-26.

Laurillard, C. L. (1842). Antilope. In C. D. d'Orbigny, ed. Dictionnaire Universel d'Histoire Naturelle, Vol. 1. Paris: Bureau Principal des Editeurs, pp. 612-626.

Lavocat, R. (1952). Sur une faune de mammifères miocènes découverte à BeniMellal (Atlas Marocain). Comptes Rendus de l'Academie des Sciences, Paris, 235, 189-191.

Lavocat, R. (1956). La faune des rongeurs des grottes à Australopithèques. Palaeontologia Africana, 4, 69-75.
Lavocat, R. (1957). Sur l'âge des faunes de rongeurs des grottes à Australopithèques. In J. D. Clark \& S. Cole, eds. Third Pan-African Congress of Prehistory, Livingstone, 1955. Livingstone: Chatto \& Windus, pp. 133-134. Lavocat, R. (1961). Le gisement de vertébrés Miocènes de Beni Mallal (Maroc), 2. Etude systématique de la faune de mammifères. Notes et Mémoires du Service Géologique du Maroc, 155, 29-94.

Lavocat, R. (1973). Les Rongeurs du Miocene d'Afrique Orientale, 1. Miocene Inférieur. Mémoires et travaux de l'Institut de Montpellier de l'Ecole Pratique des Hautes Etudes, 1, 1-284.

Lawes, M. J. (1990). The distribution of the samango monkey (Cercopithecus mitis erythrarchus Peters, 1852 and Cercopithecus mitis labiatus I. Geoffroy, 1843) and the forest history in southern Africa. Journal of Biogeography, 17, 669-680.

Le Grange, A., Bastos, A. D. S., Brettschneider, H. \& Chimimba, C. T. (2015). Evidence of a contact zone between two Rhabdomys dilectus (Rodentia: Muridae) mitotypes in Gauteng province, South Africa. African Zoology, 50, 63-68.

Le Roux, A. \& Badenhorst, S. (2016). Iron Age fauna from Sibudu Cave in KwaZulu-Natal, South Africa. Azania, 51, 307-326.

Le Roux, A., Badenhorst, S., Esterhuysen, A. \& Cain, C. (2013). Faunal remains from the 1854 siege of Mugombane, Makapans Valley, South Africa. Journal of African Archaeology, 11, 97-110.

Leakey, L. S. B. (1942). Fossil Suidae of Oldoway. Journal of the East Africa Natural History Society, 16, 178-196.

Leakey, L. S. B. (1943a). Notes on Simopithecus oswaldi Andrews from the type site. Journal of the East Africa Natural History Society, 17, $39-44$.

Leakey, L. S. B. (1943b). New fossil Suidae from Shungura, Omo. Journal of the East Africa Natural History Society, 17, 45-61.

Leakey, L. S. B. (1958). Some East African fossil Suidae. Fossil Mammals of Africa, 14, 1-133.

Leakey, L. S. B. (1961). A new Lower Pliocene fossil primate from Kenya. Annals and Magazine of Natural History, Series 13, 4, 689-696. 
Leakey, L. S. B. (1965). Olduvai Gorge, Vol. 1. Cambridge: Cambridge University Press.

Leece, A. B., Kegley, A. D. T., Lacruz, R. S., et al. (2016). The first hominin from the early Pleistocene paleocave of Haasgat, South Africa. PeerJ, 4. DOI: 10.7717/peerj.2024. eCollection 2016.

Lehmann, T. (2004). Fossil aardvark (Orycteropus) from Swartkrans Cave, South Africa. South African Journal of Science, 100, 311-314.

Lehmann, T. (2007). Amended taxonomy of the order Tubulidentata (Mammalia, Eutheria). Annals of the Transvaal Museum, 44, 179-196.

Lehmann, T. (2009). Phylogeny and systematics of the Orycteropodidae (Mammalia, Tubulidentata). Zoological Journal of the Linnean Society, 155, 649-702.

Lesson, R. P. (1826). Voyage autour du Monde sur la Coquille pendant 1822. Paris: Bertrand.

Lesson, R. P. (1827). Manuel de Mammalogie ou l'Histoire Naturelle des Mammifères. Paris: Roret.

Lesson, R. P. (1842). Nouveau Tableau du Règne Animal. Paris: Bertrand.

Lewis, M. E. \& Morlo, M. (2010). Creodonta. In L. Werdelin \& W. J. Sanders, eds. Cenozoic Mammals of Africa. Berkeley, CA: University of California Press, pp. 543-560.

Lewis, P. J., Brink, J. S., Kennedy, A. M. \& Campbell, T. L. (2011). Examination of the Florisbad microvertebrates. South African Journal of Science, 107, 1-4.

Lichtenstein, W. H. C. (1812a). Reisen im südlichen Africa in en Jahren 1803, 1804, 1805 und 1806. Berlin: Salfeld.

Lichtenstein, W. H. C. (1812b). Die Gattung Antilope. Sitzungsberichte der Gesellschaft Naturforschender Freunde zu Berlin, 6, 147-160.

Lichtenstein, W. H. C. (1823). Verzeichniss der Doubletten des zoologischen Museums der Königl. Universität zu Berlin. Berlin: Trautwein.

Lichtenstein, W. H. C. (1835). Ueber Lutra maculicollis Lichtenst: aus dem Kafferlande. Archiv für Naturgeschichte, 1, 89-92.

Lihoreau, F. \& Ducrocq, S. (2007). Family Anthracotheriidae. In D. R. Prothero \& S. E. Foss, eds. The Evolution of Artiodactyls. Baltimore, MD: Johns Hopkins University Press, pp. 89-105.
Lindsay, E. H. (1988). Cricetid rodents from Siwalik deposits near Chinji village: part I. Megacricetodontinae, Myocricetodontinae and Dendromurinae. Palaeovertebrata, 18, 95-154.

Linnaeus, C. (1758). Systema Naturae Regnum Animale, 10th edition. Leipzig: Engelmann.

Lockwood, C. A. \& Tobias, P. V. (1999). A large male cranium from Sterkfontein, South Africa and the status of Australopithecus africanus. Journal of Human Evolution, 36, 637-685.

Lockwood, C. A. \& Tobias, P. V. (2002). Morphology and affinities of new hominin cranial remains from Member 4 of the Sterkfontein Formation, Gauteng Province, South Africa. Journal of Human Evolution, 42, 389-450.

Loubser, J. H. N. (1985). Buffelshoek: an ethnoarchaeological consideration of a Late Iron Age settlement in the southern Transvaal. South African Archaeological Bulletin, 40, 81-87.

Loubser, J. H. N. (1994). Ndebele archaeology of the Petersburg area. Navorsinge van die Nasionale Museum Bloemfontein, 10, 61-147.

Louw, J. T. (1960). Prehistory of the Matjes River Rock Shelter. Bloemfontein: National Museum Bloemfontein.

Low, M. E. Y. \& Evenhuis, N. L. (2014). Additional dates of Sir Andrew Smith's illustrations of the zoology of South Africa. Zootaxa, 3795, 483-488.

Lundholm, B. (1951). A skull of the true quagga (Equus quagga) in the collection of the Transvaal Museum. South African Journal of Science, 47, 307-312.

Lundholm, B. G. (1952). Equus zebra greatheadi, n. subsp., a new South African fossil zebra. Annals of the Transvaal Museum, 22, 25-27.

Lundholm, B. G. (1954). A taxonomic study of Cynictis penicillata (G. Cuvier). Annals of the Transvaal Museum, 22, 305-319.

Lynch, C. D. (1981). The status of the Cape grey mongoose Herpestes pulverulentus Wagner 1839 (Mammalia: Viverridae). Navorsinge van die Nasionale Museum Bloemfontein, 4, 121-168. 
Lyon, M. W. (1904). Classification of the hares and their allies. Smithsonian Miscellaneous Collections, 45, 321-447.

MacFadden, B. J. (1984). Systematics and phylogeny of Hipparion, Neohipparion, Nannippus and Cormohipparion (Mammalia, Equidae) from the Miocene and Pliocene of the New World. Bulletin of the American Museum of Natural History, 179, 1-195.

MacInnes, D. G. (1936). A new genus of fossil deer from the Miocene of Africa. Zoological Journal of the Linnean Society, 39, 521-530.

MacInnes, D. G. (1942). Miocene and postMiocene Proboscidia [sic] from East Africa. Transactions of the Zoological Society of London, 25, 33-106.

MacInnes, D. G. (1951). Miocene Anthracotheriidae from East Africa. Fossil Mammals of Africa, 4, 1-24.

Mackay, A., Jacobs, Z. \& Steele, T. E. (2015). Pleistocene archaeology and chronology of Putslaagte 8 (PL8) Rockshelter, Western Cape, South Africa. Journal of African Archaeology, 13, 71-98.

MacLatchy, L., DeSilva, J. M., Sanders, W. J. \& Wood, B. (2010). Hominini. In L. Werdelin \& W. J. Sanders, eds. Cenozoic Mammals of Africa. Berkeley, CA: University of California Press, pp. 473-540.

Madden, C. T., Schmidt, D. L. \& Whitmore, F. C. (1983). Mastritherium (Artiodactyla, Anthracotheriidae) from Wadi Sabya, southwestern Saudi Arabia: an earliest Miocene age for continental Rift-Valley volcanic deposits of the Red Sea margin. US Department of the Interior Geological Survey Report 83-83-488. Washington, DC: USGS.

Maggs, T. M. O. C. (1975). Faunal remains and hunting patterns from the Iron Age of the southern highveld. Annals of the Natal Museum, 22, 449-459.

Maggs, T. M. O. C. \& Michael, M. A. (1976). Ntshekane: an Early Iron Age site in the Tugela Basin, Natal. Annals of the Natal Museum, 22, 705-740.

Maglio, V. J. \& Hendey, Q. B. (1970). New evidence relating to the supposed stegolophodont ancestry of the Elephantidae. South African Archaeological Bulletin, 25, 85-87.
Maguire, J. M. (1976). A taxonomic and ecological study of the living and fossil Hystricidae with particular reference to southern Africa. Unpublished PhD thesis, University of the Witwatersrand.

Maguire, J. M. (1985). Recent geological, stratigraphic and palaeontological studies at Makapansgat Limeworks. In P. V. Tobias, ed. Hominid Evolution: Past, Present and Future. New York: Liss, pp. 151-164.

Maier, W. (1970). New fossil Cercopithecoidea from the lower Pleistocene cave deposits of the Makapansgat Limeworks, South Africa. Palaeontologia Africana, 13, 69-107.

Maier, W. (1971). Two new skulls of Parapapio antiquus from Taung and a suggested phylogenetic arrangement of the genus Parapapio. Annals of the South African Museum, 59, 1-16.

Maier, W. (1972). The first complete skull of Simopithecus darti from Makapansgat, South Africa and its systematic position. Journal of Human Evolution, 1, 395-405.

Malan, B. D. (1970). Remarks and reminiscences on the history of archaeology in South Africa. South African Archaeological Bulletin, 25, 88-92.

Malan, B. D. \& Cooke, H. B. S. (1941). A preliminary account of the Wonderwerk Cave, Kuruman District, South Africa. South African Journal of Science, 37, 300-312.

Malan, B. D. \& Wells, L. H. (1943). A further report on the Wonderwerk Cave, Kuruman. South African Journal of Science, 40, 258-270.

Manhire, A. (1993). A report on the excavations at Faraoskop Rock Shelter in the Graafwater District of the south-western Cape. Southern African Field Archaeology, 2, 3-23.

Manthi, F. K. (2002). Saldanha Bay Yacht Club micromammals. Unpublished MA thesis, University of Cape Town.

Manyanga, M. (2001). Choices and constraints: animal resource exploitation in south-eastern Zimbabwe c. AD 900-1500. Studies in African Archaeology, 18, 1-139.

Manyanga, M. (2006). Resilient landscapes. Unpublished PhD thesis, Uppsala University.

Manyanga, M., Pikirayi, I. \& Ndoro, W. (2000). Coping with dryland environments: preliminary results from Mapungubwe and 
Zimbabwe phase sites in the Mateke Hills, south-eastern Zimbabwe. South African Archaeological Society Goodwin Series, 8, 69-77.

Marean, C. W. (1985). The faunal remains from Smitswinkelbaai Cave, Cape Peninsula. South African Archaeological Bulletin, 40, 100-102.

Marean, C. W., Abe, Y., Frey, C. J. \& Randall, R. C. (2000). Zooarchaeological and taphonomic analysis of the Die Kelders Cave 1 layers 10 and 11 Middle Stone Age larger mammal fauna. Journal of Human Evolution, 38, 197-233.

Marean, C. W., Nilssen, P. J., Brown, K. S., Jerardino, A. \& Stynder, D. D. (2004). Palaeoanthropological investigations of Middle Stone Age sites at Pinnacle Point, Mossel Bay (South Africa): archaeology and hominid remains from the 2000 field season. Paleoanthropology, 1, 14-83.

Maree, S. (2002). Phylogenetic relationships and mitochondrial DNA sequence evolution in the African rodent subfamily Otomyinae (Muridae). Unpublished PhD thesis, University of Pretoria.

Maree, S. \& Faulkes, C. (2016). Cryptomys hottentotus. The IUCN Red List of Threatened Species, 2016, e.T5755A115079767. DOI: 10.2305/IUCN.UK.2016-3. RLTS.T5755A22185187.en.

Maree, S., Visser, J., Bennett, N. C. \& Jarvis, J. (2017). Georychus capensis. The IUCN Red List of Threatened Species, 2017, e. T9077A110019425. DOI: 10.2305/ IUCN.UK.2017-2.RLTS.T9077A110019425. en.

Marivaux, L., Adaci, M., Bensalah, M., et al. (2011). Zegdoumyidae (Rodentia, Mammalia), stem anomaluroid rodents from the Early to Middle Eocene of Algeria (Gour Lazib, Western Sahara): new dental evidence. Journal of Systematic Palaeontology, 9, 563-588.

Marivaux, L., Essid, E. M., Marzougui, W., et al. (2014). A new and primitive species of Protophiomys (Rodentia, Hystricognathi) from the late middle Eocene of Djebel el Kébar, Central Tunisia. Palaeovertebrata, 38, 1-17.

Marivaux, L., Essid, E. M., Marzougui, W., et al. (2015). The early evolutionary history of anomaluroid rodents in Africa: new dental remains of a zegdoumyid (Zegdoumyidae, Anomaluroidea) from the Eocene of Tunisia. Zoologica Scripta, 44, 117-134.

Martínez-Navarro, B., Pérez-Claros, J. A., Palombo, M. R., Rook, L. \& Palmqvist, P. (2007). The Olduvai buffalo Pelorovis and the origin of Bos. Quaternary Research, 68, 220-226.

Maswanganye, K. A., Cunningham, M. J., Bennett, N. C., Chimimba, C. T. \& Bloomer, P. (2017). Life on the rocks: multilocus phylogeography of rock hyrax (Procavia capensis) from southern Africa. Molecular Phylogenetics and Evolution, 114, 49-62.

Matthews, T. \& Stynder, D. D. (2011a). An analysis of the Aethomys (Murinae) community from Langebaanweg (Early Pliocene, South Africa) using geometric morphometrics. Palaeogeography, Palaeoclimatology, Palaeoecology, 302, 230-242.

Matthews, T. \& Stynder, D. D. (2011b). An analysis of two Myosorex species (Soricidae) from the Early Pliocene site of Langebaanweg (West coast, South Africa) using geometric morphometrics, linear measurements and non-metric characters. Geobios, 44, 87-99.

Matthews, T., Denys, C. \& Parkington, J. E. (2005). The palaeoecology of the micromammals from the late middle Pleistocene site of Hoedjiespunt 1 (Cape Province, South Africa). Journal of Human Evolution, 49, 432-451.

Matthews, T., Parkington, J. E. \& Denys, C. (2006). The taphonomy of the micromammals from the Late Middle Pleistocene site of Hoedjiespunt 1 (Cape Province, South Africa). Journal of Taphonomy, 4, 11-26.

Matthews, T., Denys, C. \& Parkington, J. E. (2007). Community evolution of Neogene micromammals from Langebaanweg 'E' Quarry and other west coast fossil sites, south-western Cape, South Africa. Palaeogeography, Palaeoclimatology, Palaeoecology, 245, 332-352.

Matthews, T., Marean, C. \& Nilssen, P. J. (2009). Micromammals from the Middle Stone Age (92-167 ka) at Cave PP13B, Pinnacle Point, 
south coast, South Africa. Palaeontologia Africana, 44, 112-120.

Matthews, T., Rector, A., Jacobs, Z., Herries, A. I. R. \& Marean, C. W. (2011). Environmental implications of micromammals accumulated close to the MIS 6 to MIS 5 transition at Pinnacle Point Cave 9 (Mossel Bay, Western Cape Province, South Africa). Palaeogeography, Palaeoclimatology, Palaeoecology, 302, 213-229.

Mazel, A. D. (1984a). Gehle Shelter: report on excavations in the uplands ecological zone, Tugela Basin, Natal, South Africa. Annals of the Natal Museum, 26, 1-24.

Mazel, A. D. (1984b). Diamond I and Clarke's Shelter: report on excavations in the northern Drakensberg, Natal, South Africa. Annals of the Natal Museum, 26, 25-70.

Mazel, A. D. (1986a). Mgede Shelter: a mid- and late Holocene observation in the western Biggarsberg, Thukela Basin, South Africa. Annals of the Natal Museum, 27, 357-387.

Mazel, A. D. (1986b). Mbabane Shelter and eSinhlonhweni Shelter: the last two thousand years of hunter-gatherer settlement in the central Thukela Basin, Natal, South Africa. Annals of the Natal Museum, 27, 389-453.

Mazel, A. D. (1988a). Nkupe Shelter: report on excavations in the eastern Biggarsberg, Thukela Basin, Natal, South Africa. Annals of the Natal Museum, 29, 321-377.

Mazel, A. D. (1988b). Sikhanyisweni Shelter: report on excavations in the Thukela Basin, Natal, South Africa. Annals of the Natal Museum, 29, 379-406.

Mazel, A. D. (1990). Mhlawazini Cave: the excavation of Late Holocene deposits in the northern Natal Drakensberg, Natal, South Africa. Natal Museum Journal of Humanities, 2, 95-133.

Mazel, A. D. (1992). Collingham Shelter: the excavation of late Holocene deposits, Natal, South Africa. Natal Museum Journal of Humanities, 4, 1-51.

Mazel, A. D. (1993). KwaThwaleyakhe Shelter: the excavation of mid and late Holocene deposits in the central Thukela Basin, Natal, South Africa. Natal Museum Journal of Humanities, 5, 1-36.
McCrae, C. \& Potze, S. (2006). Analysis of microfauna-bearing breccia from Kromdraai A in the Cradle of Humankind World Heritage Site. Annals of the Transvaal Museum, 43, 107-110.

McCrossin, M. L. (1992). Human molars from Later Pleistocene deposits of Witkrans Cave, Gaap Escarpment, Kalahari Margin. Human Evolution, 7, 1-10.

McCrossin, M. L. \& Benefit, B. R. (1997). On the relationships and adaptations of

Kenyapithecus, a large-bodied hominoid from the Middle Miocene of eastern Africa. In D. R. Begun, C. V. Ward \& M. D. Rose, eds. Function, Phylogeny and Fossils: Miocene Hominoid Evolution and Adaptations. New York: Plenum, pp. 241-267.

McGrath, J. R., Cleghorn, N., Gennari, B., et al. (2015). The Pinnacle Point shell midden complex: a Mid- to Late Holocene record of Later Stone Age coastal foraging along the southern Cape coast of South Africa. South African Archaeological Bulletin, 70, 209-219.

McKee, J. K. (1991). Palaeo-ecology of the Sterkfontein hominids: a review and synthesis. Palaeoecology of Africa, 28, 41-51.

McKee, J. K. (1993a). Faunal dating of the Taung fossil hominid deposit. Journal of Human Evolution, 25, 363-376.

McKee, J. K. (1993b). Taxonomic and evolutionary affinities of Papio izodi fossils from Taung and Sterkfontein. Palaeontologia Africana, 30, 43-49.

McKee, J. K. (1994). Catalogue of fossil sites at the Buxton Limeworks, Taung. Palaeontologia Africana, 31, 73-81.

McKee, J. K. \& Keyser, A. W. (1994). Craniodental remains of Papio angusticeps from the Haasgat cave site, South Africa. International Journal of Primatology, 15, 823-841.

McKee, J. K., Thackeray, J. F. \& Berger, L. R. (1995). Faunal assemblage seriation of southern African Pliocene and Pleistocene fossil deposits. American Journal of Physical Anthropology, 96, 235-250.

McKee, J. K., Von Mayer, A. \& Kuykendall, K. I. (2011). New species of Cercopithecoides from Haasgat, North West Province, South Africa. Journal of Human Evolution, 60, 83-93. 
McKenna, M. C. \& Bell, S. K. (1997). Classification of Mammals above the Species Level. New York: Columbia University Press.

McMahon, C. R. \& Thackeray, J. F. (1994). PlioPleistocene Hyracoidea from Swartkrans Cave, South Africa. South African Journal of Zoology, 29, 40-45.

Meester, J. (1953a). The genera of African shrews. Annals of the Transvaal Museum, 22, 205-214.

Meester, J. (1953b). A new golden mole from Spitzkop, Sabie. South African Journal of Science, 49, 207-208.

Meester, J. (1954). Fossil shrews of South Africa. Annals of the Transvaal Museum, 22, 271-278.

Meester, J. (1958). Variation in the shrew genus Myosorex in southern Africa. Journal of Mammalogy, 39, 325-339.

Meester, J. (1961a). Appendix A: report on the fossil shrews in the University of California collections from the South African cave breccias. Transvaal Museum unpublished report, $2 \mathrm{pp}$.

Meester, J. (1961b). A taxonomic revision of southern African Crocidura (Mammalia: Insectivora). Annals and Magazine of Natural History, Series 13, 4, 561-571.

Meester, J. (1963). A systematic revision of the shrew genus Crocidura in southern Africa. Transvaal Museum Memoir, 13, 1-127.

Meester, J. (1964a). Revision of the Chrysochloridae: 1 . The desert golden mole, Eremitalpa Roberts. Scientific Papers of the Namib Desert Research Station, 26, 1-9.

Meester, J. (1964b). The status of Crocidura mariquensis (Smith) (Mammalia: Insectivora). Puku, 2, 78-80.

Meester, J. (1980). Appendix 2: small mammals. In T. M. Maggs \& V. Ward. Driel Shelter: rescue at a Late Stone Age site on the Tugela River. Annals of the Natal Museum, 24, 67.

Meester, J. \& Lambrechts, A. von W. (1971). The southern African species of Suncus Ehrenberg (Mammalia: Soricidae). Annals of the Transvaal Museum, 27, 1-14.

Meester, J. \& Meyer, I. J. (1972). Fossil Suncus (Mammalia: Soricidae) from southern Africa. Annals of the Transvaal Museum, 27, 269-277.

Meester, J., Richardson, E. J. \& Work, D. (1985). Multivariate analysis of southern African
Crocidura (Soricidae). Acta Zoologica Fennica, 173, 219-222.

Meester, J., Taylor, P. J., Confratto, G.-C., et al. (1992). Chromosomal speciation in southern African Otomyinae (Rodentia: Muridae): a review. Durban Museum Novitates, 17, 58-63.

Mein, P. \& Pickford, M. (2003a). Fossil bat (Microchiroptera, Mammalia) from Arrisdrift, Namibia. Memoirs of the Geological Survey of Namibia, 19, 115-117.

Mein, P. \& Pickford, M. (2003b). Insectivora from Arrisdrift, a basal Middle Miocene locality in southern Namibia. Memoirs of the Geological Survey of Namibia, 19, 143-146.

Mein, P. \& Pickford, M. (2003c). Rodentia (other than Pedetidae) from the Orange River deposits, Namibia. Memoirs of the Geological Survey of Namibia, 19, 147-160.

Mein, P. \& Pickford, M. (2003d). Fossil picas (Ochotonidae, Lagomorpha, Mammalia) from the basal Middle Miocene of Arrisdrift, Namibia. Memoirs of the Geological Survey of Namibia, 19, 171-176.

Mein, P. \& Pickford, M. (2008a). Early Miocene insectivores from the northern Sperrgebiet, Namibia. Memoirs of the Geological Survey of Namibia, 20, 169-183.

Mein, P. \& Pickford, M. (2008b). Early Miocene Lagomorpha from the northern Sperrgebiet, Namibia. Memoirs of the Geological Survey of Namibia, 20, 227-233.

Mein, P. \& Pickford, M. (2008c). Early Miocene Rodentia from the northern Sperrgebiet, Namibia. Memoirs of the Geological Survey of Namibia, 20, 235-290.

Mein, P. \& Pickford, M. (2018).

Reithroparamyine rodent from the Eocene of Namibia. Communications of the Geological Survey of Namibia, 18, 38-47.

Mein, P. \& Senut, B. (2003). The Pedetidae from the Miocene site of Arrisdrift (Namibia). Memoirs of the Geological Survey of Namibia, 19, 161-170.

Mein, P., Pickford, M. \& Senut, B. (2000a). Late Miocene micromammals from the Harasib karst deposits, Namibia. Part 1 - large muroids and non-muroid rodents. Communications of the Geological Survey of Namibia, 12, 375-390. 
Mein, P., Pickford, M. \& Senut, B. (2000b). Late Miocene micromammals from the Harasib karst deposits, Namibia. Part 2a Myocricetodontinae, Petromyscinae and Namibimyinae (Muridae, Gerbillidae). Communications of the Geological Survey of Namibia, 12, 391-401.

Mein, P., Pickford, M. \& Senut, B. (2004). Late Miocene micromammals from the Harasib karst deposits, Namibia. Part $2 \mathrm{~b}-$ Cricetomyidae, Dendromuridae and Muridae, with an addendum on the Myocricetodontinae. Communications of the Geological Survey of Namibia, 13, 43-61.

Meiring, A. J. D. (1955). Fossil proboscidean teeth and ulna from Virginia, O.F.S. Navorsinge van die Nasionale Museum Bloemfontein, 1, 187-201.

Mendrez, C. (1966). On Equus (Hippotigris) cf. burchelli (Gray) from 'Sterkfontein extension', Transvaal, South Africa. Annals of the Transvaal Museum, 25, 91-97.

Menter, C., Kuykendall, K. I., Keyser, A. W. \& Conroy, G. C. (1999). First record of hominid teeth from the Plio-Pleistocene site of Gondolin, South Africa. Journal of Human Evolution, 37, 299-307.

Miller, E. R., Gunnell, G. F., Gawad, M. A., et al. (2014). Anthracotheres from Wadi Moghra, early Miocene, Egypt. Journal of Paleontology, 88, 967-981.

Miller-Butterworth, C. M., Eick, G., Jacobs, D. S., Schoeman, M. C. \& Harley, E. C. (2005). Genetic and phenotypic differences between South African long-fingered bats, with a global miniopterine phylogeny. Journal of Mammalogy, 86, 1121-1135.

Miller-Butterworth, C. M., Murphy, W. J., O'Brien, S. J., et al. (2007). A family matter: conclusive resolution of the taxonomic position of the long-fingered bats, Miniopterus. Molecular Biology and Evolution, 24, 1553-1561.

Mills, M. G. L. (1982). Hyaena brunnea. Mammalian Species, 194, 1-5.

Mitchell, P. J. (1993). The archaeology of Tloutle rockshelter, Maseru District, Lesotho. Navorsinge van die Nasionale Museum Bloemfontein, 9, 77-132.
Mitchell, P. J., Parkington, J. E. \& Yates, R. (1994). Recent Holocene archaeology in western and southern Lesotho. South African Archaeological Bulletin, 49, 33-52.

Moggi-Cecchi, J., Tobias, P. V. \& Benyon, A. D. (1998). The mixed dentition and associated skull fragments of a juvenile fossil hominid from Sterkfontein, South Africa. American Journal of Physical Anthropology, 106, 425-466.

Moggi-Cecchi, J., Grine, F. E. \& Tobias, P. V. (2006). Early hominid dental remains from Members 4 and 5 of the Sterkfontein Formation (1966-1996 excavations): catalogue, individual associations, morphological descriptions and initial metrical analysis. Journal of Human Evolution, 50, 239-328.

Moggi-Cecchi, J., Menter, C., Boccone, S. \& Keyser, A. (2010). Early hominin dental remains from the Plio-Pleistocene site of Drimolen, South Africa. Journal of Human Evolution, 58, 374-405.

Mollett, O. D. van der Spuy. (1947). Fossil mammals from the Makapan Valley, Potgietersrust: I. Primates. South African Journal of Science, 43, 295-303.

Montgelard, C., Matthee, C. A. \& Robinson, T. J. (2003). Molecular systematics of dormice (Rodentia, Gliridae) and the radiation of Graphiurus in Africa. Proceedings of the Royal Society of London Series B, 270, 1947-1955.

Montoya, P., Morales, J. \& Abella, J. (2011). Musteloidea (Carnivora, Mammalia) from the Late Miocene of Venta del Moro (Valencia, Spain). Estudios Geológicos, 67, 193-206.

Morales, J. \& Pickford, M. (2018). A new barbourofelid mandible (Carnivora, Mammalia) from the Early Miocene of Grillental-6, Sperrgebiet, Namibia. Communications of the Geological Survey of Namibia, 18, 113-123.

Morales, J., Soria, D. \& Pickford, M. (1995). Sur les origines de la famille des Bovidae (Artiodactyla, Mammalia). Comptes Rendus de l'Académie des Sciences de Paris, Sciences de la Terre et des Planètes, 321, 1211-1217.

Morales, J., Pickford, M. \& Soria, D. (1998a). A new creodont Metapterodon stromeri nov. sp. (Hyaenodontidae, Mammalia) from the Early 
Miocene of Langental (Sperrgebiet, Namibia). Comptes Rendus de l'Académie des Sciences de Paris, Sciences de la Terre et des Planètes, 327, 633-638.

Morales, J., Pickford, M., Soria, D. \& Fraile, S. (1998b). New carnivores from the basal Middle Miocene of Arrisdrift, Namibia. Eclogae Geologicae Helvetiae, 91, 27-40.

Morales, J., Soria, D. \& Pickford, M. (1999). New stem giraffoid ruminants from the early and middle Miocene of Namibia. Geodiversitas, 21, 229-253.

Morales, J., Pickford, M., Soria, D. \& Fraile, S. (2001a). New Viverrinae (Carnivora: Mammalia) from the basal Middle Miocene of Arrisdrift, Namibia. Palaeontologia Africana, 37, 99-102.

Morales, J., Salesa, M. J., Pickford, M. \& Soria, D. (2001b). A new tribe, new genus and two new species of Barbourofelinae (Felidae, Carnivora, Mammalia) from the Early Miocene of East Africa and Spain. Transactions of the Royal Society of Edinburgh: Earth Sciences, 92, 97-102.

Morales, J., Pickford, M., Fraile, S., Salesa, M. \& Soria, D. (2003a). Creodonta and Carnivora from Arrisdrift, early Middle Miocene of southern Namibia. Memoirs of the Geological Survey of Namibia, 19, 177-194.

Morales, J., Soria, D., Pelaez-Campomanes, P. \& Pickford, M. (2003b). New data regarding Orangemeryx hendeyi Morales et al., 2000, from the type locality, Arrisdrift, Namibia. Memoirs of the Geological Survey of Namibia, 19, 305-344.

Morales, J., Soria, D., Pickford, M. \& Nieto, M. (2003c). A new genus and species of Bovidae (Artiodactyla, Mammalia) from the early Middle Miocene of Arrisdrift, Namibia and the origins of the family Bovidae. Memoirs of the Geological Survey of Namibia, 19, 371-384.

Morales, J., Soria, D., Sánchez, I. M., Quiralte, V. $\&$ Pickford, M. (2003d). Tragulidae from Arrisdrift, basal Middle Miocene, southern Namibia. Memoirs of the Geological Survey of Namibia, 19, 359-369.

Morales, J., Pickford, M. \& Soria, D. (2005). Carnivores from the late Miocene and basal Pliocene of the Tugen Hills, Kenya. Revista de la Sociedad Geológica de España, 18, 39-61.
Morales, J., Pickford, M. \& Salesa, M. J. (2008a). Creodonta and Carnivora from the early Miocene of the northern Sperrgebiet, Namibia. Memoirs of the Geological Survey of Namibia, 20, 291-310.

Morales, J., Soria, D. \& Pickford, M. (2008b). Pecoran ruminants from the Early Miocene of the Sperrgebiet, Namibia. Memoirs of the Geological Survey of Namibia, 20, 397-464.

Morales, J., Senut, B. \& Pickford, M. (2011). Crocuta dietrichi from Meob, Namibia: implications for the age of the Tsondab Sandstone in the coastal part of the Namib Desert. Estudios Geológicos, 67, 207-215.

Morales, J., Pickford, M. \& Valenciano, A. (2016). Systematics of African Amphicyonidae, with descriptions of new material from Napak (Uganda) and Grillental (Namibia). Journal of Iberian Geology, 42, 131-150.

Morlo, M., Miller, E. R. \& El-Barkooky, A. N. (2007). Creodonta and Carnivora from Wadi Moghra, Egypt. Journal of Vertebrate Paleontology, 27, 145-159.

Morris, A. G. (1994). Appendix 1: human skeletal remains from Wosi - an Early Iron Age site in the Thukela Basin, Natal. In L. Van Schalkwyk. Wosi: an Early Iron Age village in the lower Thukela Basin, Natal. Natal Museum Journal of Humanities, 6, 97-104.

Morris, A. G., Louw, G. H., Van Wyk, E. \& Cooper, C. (1995). A brief report on the rescue excavation on a human skeleton from Nooitgedacht, Northern Cape Province, South Africa. Southern African Field Archaeology, 4, 120-123.

Morris, A. G., Dlamini, N., Joseph, J., et al. (2004/2005). Later Stone Age burials from the Western Cape Province, South Africa Part 1: Voëlvlei. Southern African Field Archaeology, 13-14, 19-26.

Mullin, S. K., Pillay, N. \& Taylor, P. J. (2004). Skull size and shape of Dasymys (Rodentia, Muridae) from sub-Saharan Africa.

Mammalia, 68, 185-220.

Mupira, P. \& Katsamudanga, S. (2007). Excavations at Manjowe and Gwenzi rock shelters in Zimunya Communal Lands, central Eastern Highlands of Zimbabwe. Zimbabwea, 9, 21-42. 
Musser, G. G. \& Carleton, M. D. (2005). Superfamily Muroidea. In D. E. Wilson \& D. M. Reeder, eds. Mammal Species of the World, Vol. 2. Baltimore, MD: Johns Hopkins University Press, pp. 894-1599.

Mutter, R. J., Berger, L. R. \& Schmid, P. (2001). New evidence of the giant hyaena, Pachycrocuta brevirostris (Carnivora, Hyaenidae), from the Gladysvale Cave deposit (Plio-Pleistocene, John Nash Nature Reserve, Gauteng, South Africa).

Palaeontologia Africana, 37, 103-113.

Mynhardt, S., Maree, S., Pelser, I., et al. (2015). Phylogeography of a morphologically cryptic golden mole assemblage from south-eastern Africa. PLOS ONE, 10. DOI: 10.1371/journal. pone.0144995.

Nelson, C. (2008). An archaeozoological and ethnographic investigation into animal utilisation practices of the Ndzundza Ndebele of the Steelpoort River Valley, South Africa, 1700 AD-1900 AD. Unpublished MA thesis, University of Pretoria.

Nelson, C. (2009). An archaeozoology of the Ndzundza Ndebele in the Steelpoort River valley. Mpumulanga, South Africa. c. 1700 AD - 1883 AD. South African Archaeological Bulletin, 64, 184-192.

Noack, T. (1887). Beiträge zur Kenntniss der Säugethier-Fauna von Ost- und CentralAfrika. Zoologische Jahrbücher Systematik, 2, 193-302.

Noli, D. (1988). Results of the 1986 excavation at Hailstone Midden (HSM), Eland's Bay, Western Cape Province. South African Archaeological Bulletin, 43, 43-48.

Nyamushosho, R. T. (2016). Living on the margin? The Iron Age communities of Mananzve Hill, Shashi region, South-western Zimbabwe. Unpublished MPhil thesis, University of Cape Town.

Ogilby, W. (1833). [Antilope ellipsyprymnus and Cynictis steedmani in the collection of $\mathrm{Mr}$ Steedman]. Proceedings of the Zoological Society of London, 1, 47-49.

Ogilby, W. (1836). [On the generic characters of ruminants]. Proceedings of the Zoological Society of London, 4, 131-139.

Ogilby, W. (1838). On a collection of Mammalia, procured by Captain Alexander during his journey into the country of the Damaras, on the south-west African coast. Proceedings of the Zoological Society of London, 6, 5-6.

Ogola, C. A. (2009). The Sterkfontein western breccias: stratigraphy, fauna and artefacts. Unpublished PhD thesis, University of the Witwatersrand.

Opperman, H. (1982). Some research results of excavations in the Colwinton Rock Shelter, north-eastern Cape. South African Archaeological Bulletin, 37, 51-56.

Opperman, H. (1987). The Later Stone Age of the Drakensberg Range and its Foothills. Oxford: British Archaeological Reports.

Opperman, H. (1992). A report on the results of a test pit in Strathalan Cave B: Maclear District, north-eastern Cape. Southern African Field Archaeology, 1, 98-102.

Opperman, H. (1996). Strathalan Cave B, northwestern Cape Province, South Africa: evidence for human behaviour 29,000-26,000 years ago. Quaternary International, 33, 45-53.

O'Regan, H. J. (2007). A revision of the Carnivora from Member 5, Sterkfontein, South Africa, based on a reassessment of published material and site stratigraphy. Annals of the Transvaal Museum, 44, 209-214.

O’Regan, H. J. \& Menter, C. G. (2009). Carnivora from the Plio-Pleistocene hominin site of Drimolen, Gauteng, South Africa. Geobios, 42, 329-350.

O’Regan, H. J. \& Steininger, C. (2017). Felidae from Cooper's Cave, South Africa (Mammalia: Carnivora). Geodiversitas, 39, 315-332.

O’Regan, H. J., Cohen, B. F. \& Steininger, C. M. (2013). Mustelid and viverrid remains from the Pleistocene site of Cooper's D, Gauteng, South Africa. Palaeontologia Africana, 48, 19-23.

Orlando, L., Metcalf, J. L., Alberdi, M. T., et al. (2009). Revising the recent evolutionary history of equids using ancient DNA. Proceedings of the National Academy of Sciences of the United States of America, 106, 21754-21759.

Orton, J. (2014). The late pre-colonial site of Komkans 2 (KK002) and an evaluation of the 
evidence for indigenous copper smelting in Namaqualand, southern Africa. Azania, 47, 386-410.

Orton, J., Hart, T. \& Halkett, D. (2005). Shell middens in Namaqualand: two Later Stone Age sites at Rooiwalbaai, Northern Cape Province, South Africa. South African Archaeological Bulletin, 60, 24-32.

Orton, J., Klein, R. G., Mackay, A., Schwortz, S. \& Steele, T. E. (2011). Two Holocene rock shelter deposits from the Knersvlakte, southern Namaqualand, South Africa. Southern African Humanities, 23, 109-150.

Orton, J., Mitchell, P., Klein, R., Steele, T. \& Horsburgh, K. A. (2013). An early date for cattle from Namaqualand, South Africa: implications for the origins of herding in southern Africa. Antiquity, 87, 108-120.

Orton, J., Halkett, D., Hart, T., Patrick, M. \& Pfeiffer, S. (2015). An unusual pre-colonial burial from Bloubergstrand, Table Bay, South Africa. South African Archaeological Bulletin, 70, 106-112.

Osborn, H. F. (1928). Mammoths and man in the Transvaal. Nature, 121, 672-673.

Osborn, H. F. (1934). Primitive Archidiskodon and Palaeoloxodon of South Africa. American Museum Novitates, 741, 1-15.

Osborn, H. F. (1942). Proboscidea. New York: American Museum of Natural History.

Osgood, W. H. (1910). Diagnoses of new East African mammals, including a new genus of Muridae. Publications of the Field Museum of Natural History, Zoological Series, 10, 5-13.

Owens, M. D. (1995). Archaeological research at Modipe Hill, Kgatleng District: the burials from 1994 excavations. Botswana Notes and Records, 27, 41-48.

Pallas, P. S. (1766). P.S. Pallas Medecinae Doctoris Miscellanea Zoologica. La Haye: Van Cleef.

Pallas, P. S. (1767). De Antilopibus generatim. Spicilegia Zoologica, 1, 1-44.

Pallas, P. S. (1778). Novae Species Quadrupedum e Glirium Ordine. Erlangen, Walther.

Pappas, L. A. (2002). Taurotragus oryx. Mammalian Species, 689, 1-5.

Partridge, T. C. (1966). Ficus Cave: an Iron Age living site in the central Transvaal. South African Archaeological Bulletin, 21, 125-132.
Partridge, T. C. (2010). Tectonics and geomorphology of Africa during the Phanerozoic. In L. Werdelin \& W. J. Sanders, eds. Cenozoic Mammals of Africa. Berkeley, CA: University of California Press, pp. 3-17.

Patterson, B. (1965). The fossil elephant shrews (family Macroscelididae). Bulletin of the Museum of Comparative Zoology Harvard, 133, 295-335.

Pavlinov, I. J. A. (2001). Current concepts in gerbillid phylogeny and classification. In C. Denys, L. Granjon \& A. Poulet, eds.

Proceedings of the 8th International Symposium on African Small Mammals. Paris: I.R.D., pp. 141-149.

Pearce, D. G. (2008). Later Stone Age burial practice in the Eastern Cape Province, South Africa. Unpublished DPhil thesis, University of the Witwatersrand.

Pearson, O. M. \& Grine, F. E. (1996). Morphology of the Border Cave hominid ulna and humerus. South African Journal of Science, 92, 231-236.

Pellatt, A. (1972). Appendix: report on skeletal material from Ruanga. In P. S. Garlake. Excavations at the Nhunguza and Ruanga Ruins in northern Mashonaland. South African Archaeological Bulletin, 27, 139-140.

Pelser, A. J., Teichert, F. \& Steyn, M. (2004/ 2005). A Late Iron Age/Contact Period burial at Stand 1610, Hillside Street, Silver Lakes, Tshwane. Southern African Field Archaeology, 13-14, 27-35.

Penzhorn, B. L. (1988). Equus zebra. Mammalian Species, 314, 1-7.

Péringuey, L. (1911). The Stone Ages of South Africa as represented in the collection of the South African Museum. Annals of the South African Museum, 8, 1-201.

Perrin, M. R. \& Fielden, L. J. (1999). Eremitalpa granti. Mammalian Species, 629, 1-4.

Perrin, M. R., Dempster, E. R. \& Downs, C. T. (1999). Gerbillurus paeba. Mammalian Species, 606, 1-6.

Perry, G. (1811). Arcana, or, the Museum of Natural History: Containing the Most Recent Discovered Objects. London: Smicton.

Peters, J. \& Brink, J. S. (1992). Comparative postcranial osteomorphology and osteometry of springbok, Antidorcas 
marsupialis (Zimmerman, 1780) and Grey rhebok, Pelea capreolus (Forster, 1790) (Mammalia, Bovidae). Navorsinge van die Nasionale Museum Bloemfontein, 8, 162-207.

Peters, W. C. H. (1846). Über neue Säugethiergattungen aus den Ordnungen der Insectenfresser und Nagelthiere. Bericht über die zur Bekanntmachung geeigneten Verhandlungen der Koniglichen Preussische Akademie der Wissenschaften zu Berlin, 1846, 257-259.

Peters, W. C. H. (1851). Derselbe machte eine Mittheilung über zwei neue Insectivoren aus Mossambique. Bericht über die zur Bekanntmachung geeigneten Verhandlungen der Koniglichen Preussische Akademie der Wissenschaften zu Berlin, 1851, 467-468.

Peters, W. C. H. (1852a). Einige neue Säugethiere und Flussfische aus Mossambique vor. Bericht über die zur Bekanntmachung geeigneten Verhandlungen der Koniglichen Preussische Akademie der Wissenschaften zu Berlin, 1852, 273-276.

Peters, W. C. H. (1852b). Naturwissenschaftliche Reise nach Mossambique. Berlin: Reimer.

Peters, W. C. H. (1866). Über einige neue oder weniger bekannte Flederthiere.

Monatsberichte der Königlichen Preussische Akademie des Wissenschaften zu Berlin, 1866, 16-25.

Peters, W. C. H. (1875). Über Dasymys, eine neue Gattung von murinen Nagethieren aus Südafrika. Monatsberichte der Königlichen Preussische Akademie des Wissenschaften zu Berlin, 1875, 12-14.

Peters, W. C. H. (1878). Über die von Hrn. J.M. Hildebrandt während seiner letzen ostafrikaischen Reise gesammelten Säugethiere und Amphibien. Monatsberichte der Königlichen Preussische Akademie des Wissenschaften zu Berlin, 1878, 194-209.

Pether, J. (1994). The sedimentology, palaeontology and stratigraphy of coastal plain deposits at Hondeklip Bay, Namaqualand, South Africa. Unpublished MSc thesis, University of Cape Town.

Petter, F. (1967). Particularités dentaires des Petromyscinae Roberts 1951 (Rongeurs, Cricetides). Mammalia, 31, 217-224.
Petter, F. (1981). Remarques sur la systématique des Chrysochloridés. Mammalia, 45, 49-53.

Petter, G. (1963). Étude de quelques Viverridés (Mammifères, Carnivores) du Pléistocène inférìeur du Tanganyika (Afrique orientale). Bulletin de la Société Géologique de France, S7-V, 265-274.

Petter, G. (1987). Small carnivores (Viverridae, Mustelidae, Canidae) from Laetoli. In M. D. Leakey \& J. M. Harris, eds. Laetoli: A Pliocene Site in Northern Tanzania. Oxford: Clarendon Press, pp. 194-234.

Petter, G. \& Howell, F. C. (1989). Une nouvelle espèce du genre Crocuta Kaup (Mammalia: Carnivora: Hyaenidae) dans la faune pliocène de Laetoli (Tanzanie): Crocuta dietrichi nov. sp.; origine du genre. Comptes Rendus de l'Académie des Sciences, Paris, Série II, 308, 1031-1038.

Pfeiffer, S. (2013). Population dynamics in the Southern African Holocene: Human burials from the West Coast. In A. Jerardino, A. Malan \& D. Braun, eds. The Archaeology of the West Coast of South Africa. Oxford:

Archaeopress, pp. 143-154.

Pfeiffer, S. \& Van der Merwe, N. J. (2004). Cranial injuries to Later Stone Age children from the Modder River Mouth, Western Cape Province, South Africa. South African Archaeological Bulletin, 59, 59-65.

Pia, J. (1930). Eine neue quartäre Warzenschwein art aus Südwestafrika. Centralblatt für Mineralogie, Geologie und Paläontologie, 1930 B, 76-83.

Pickering, R., Dirks, P. H. G. M., Jinnah, Z., et al. (2011). Australopithecus sediba at $1.977 \mathrm{Ma}$ and implications for the origins of the genus Homo. Science, 333, 1421-1423.

Pickering, T. R., Heaton, J. L., Clarke, R. J., et al. (2012). New hominid fossils from Member 1 of the Swartkrans formation, South Africa. Journal of Human Evolution, 62, 618-628.

Pickford, M. (1975). New fossil Orycteropodidae (Mammalia, Tubulidentata) from East Africa: Orycteropus minutus sp. nov. and Orycteropus chemeldoi sp. nov. Netherlands Journal of Zoology, 25, 57-88.

Pickford, M. (1984). A revision of the Sanitheriidae (Suiformes, Mammalia). Geobios, 17, 133-154. 
Pickford, M. (1986). A revision of the Miocene Suidae and Tayassuidae (Artiodactyla, Mammalia) of Africa. Tertiary Research Special Paper, 7, 1-83.

Pickford, M. (1987). Miocene Suidae from Arrisdrift, South West Africa - Namibia. Annals of the South African Museum, 97, 283-314.

Pickford, M. (1988). Un étrange suide nain du Neogene supérieur de Langebaanweg. Annales de Paléontologie, 74, 229-250.

Pickford, M. (1990). Some fossiliferous PlioPleistocene cave systems of Ngamiland, Botswana. Botswana Notes and Records, 22, 1-15.

Pickford, M. (1991a). Late Miocene anthracothere (Mammalia, Artiodactyla) from tropical Africa. Comptes Rendus de l'Académie des Sciences de Paris, Série IIa 313, 709-715.

Pickford, M. (1991b). Revision of the Neogene Anthracotheriidae of Africa. In M. J. Salem, ed. The Geology of Libya, Vol. 4, Amsterdam: Elsevier, pp. 1491-1525.

Pickford, M. (1994). A new species of Prohyrax (Mammalia, Hyracoidea) from the middle Miocene of Arrisdrift, Namibia. Communications of the Geological Survey of Namibia, 9, 43-62.

Pickford, M. (1995). Suidae (Mammalia, Artiodactyla) from the Early Middle Miocene of Arrisdrift, Namibia: Namachoerus (gen. nov.) moruoroti and Nguruwe kijivium. Comptes Rendus de l'Académie des Sciences de Paris, Sciences de la Terre et des Planètes, 320, 319-326.

Pickford, M. (1996a). Pliohyracids (Mammalia, Hyracoidea) from the upper Middle Miocene at Berg Aukas, Namibia. Comptes Rendus de l'Académie des Sciences de Paris, Série IIa 322, 501-505.

Pickford, M. (1996b). Tubulidentata

(Mammalia) from the middle and upper Miocene of southern Namibia. Comptes Rendus de l' Académie des Sciences de Paris, Sciences de la Terre et des Planètes, 322, 805-810.

Pickford, M. (1997). Lower Miocene Suiformes from the northern Sperrgebiet, Namibia, including new evidence for the systematic position of the Sanitheriidae. Comptes Rendus de l' Académie des Sciences de Paris, Sciences de la Terre et des Planètes, 325, 285-292.

Pickford, M. (2001a). Afrochoerodon nov. gen. kisumuensis (MacInnes) (Proboscidea, Mammalia) from Cheparawa. Middle Miocene, Kenya. Annales de Paléontologie, 87, 99-117.

Pickford, M. (2001b). Africa's smallest ruminant: a new tragulid from the Miocene of Kenya and the biogeography of East African Tragulidae. Geobios, 34, 437-447.

Pickford, M. (2003a). Minute species of Orycteropus from the early Middle Miocene at Arrisdrift, Namibia. Memoirs of the Geological Survey of Namibia, 19, 195-198.

Pickford, M. (2003b). Middle Miocene Hyracoidea from the lower Orange River valley, Namibia. Memoirs of the Geological Survey of Namibia, 19, 199-205.

Pickford, M. (2003c). New Proboscidea from the Miocene strata in the lower Orange River Valley, Namibia. Memoirs of the Geological Survey of Namibia, 19, 207-256.

Pickford, M. (2003d). Early and Middle Miocene Anthracotheriidae (Mammalia, Artiodactyla) from the Sperrgebiet, Namibia. Memoirs of the Geological Survey of Namibia, $19,283-289$.

Pickford, M. (2003e). Suidae from the Middle Miocene of Arrisdrift, Namibia. Memoirs of the Geological Survey of Namibia, 19, 291-303.

Pickford, M. (2003f). Giant dassie (Hyracoidea, Mammalia) from the middle Miocene of South Africa. South African Journal of Science, 99, 366-367.

Pickford, M. (2004). Miocene Sanitheriidae (Suiformes, Mammalia) from Namibia and Kenya: systematic and phylogenetic implications. Annales de Paléontologie, 90, 223-278.

Pickford, M. (2005a). Choerolophodon pygmaeus (Proboscidea: Mammalia) from the Middle Miocene of Southern Africa. South African Journal of Science, 101, 175-177.

Pickford, M. (2005b). Orycteropus (Tubulidentata, Mammalia) from Langebaanweg and Baard's Quarry, Early Pliocene of South Africa. Comptes Rendus Paléovol, 4, 715-726. 
Pickford, M. (2005c). Fossil hyraxes (Hyracoidea: Mammalia) from the Late Miocene and PlioPleistocene of Africa and the phylogeny of the Procaviidae. Palaeontologia Africana, 41, 141-161.

Pickford, M. (2007). New mammutid proboscidean teeth from the Middle Miocene of tropical and southern Africa. Palaeontologia Africana, 42, 29-35.

Pickford, M. (2008a). Tubulidentata from the northern Sperrgebiet, Namibia. Memoirs of the Geological Survey of Namibia, 20, 311-313.

Pickford, M. (2008b). Hyracoidea from the Early Miocene of the northern Sperrgebiet, Namibia. Memoirs of the Geological Survey of Namibia, 20, 315-325.

Pickford, M. (2008c). Proboscidea from the Early Miocene of the northern Sperrgebiet, Namibia. Memoirs of the Geological Survey of Namibia, 20, 327-329.

Pickford, M. (2008d). Anthracotheriidae from the Early Miocene deposits of the northern Sperrgebiet, Namibia. Memoirs of the Geological Survey of Namibia, 20, 343-347.

Pickford, M. (2008e). Suidae from the Early Miocene of the northern Sperrgebiet, Namibia. Memoirs of the Geological Survey of Namibia, 20, 349-363.

Pickford, M. (2008f). Early Miocene Santheriidae from the northern Sperrgebiet, Namibia. Memoirs of the Geological Survey of Namibia, 20, 365-385.

Pickford, M. (2012). Ancestors of Broom's pigs. Transactions of the Royal Society of South Africa, 67, 17-35.

Pickford, M. (2013a). The diversity, age, biogeographic and phylogenetic relationships of Plio-Pleistocene suids from Kromdraai, South Africa. Annals of the Ditsong National Museum of Natural History, 3, 11-32.

Pickford, M. (2013b). Locomotion, diet, body weight, origin and geochronology of Metridiochoerus andrewsi from the Gondolin Karst Deposits, Gauteng, South Africa. Annals of the Ditsong National Museum of Natural History, 3, 33-47.

Pickford, M. (2015a). Chrysochloridae (Mammalia) from the Lutetian (Middle Eocene) of Black Crow, Namibia.
Communications of the Geological Survey of Namibia, 16, 105-113.

Pickford, M. (2015b). Late Eocene Potamogalidae and Tenrecidae (Mammalia) from the Sperrgebiet, Namibia. Communications of the Geological Survey of Namibia, 16, 114-152.

Pickford, M. (2015c). Late Eocene Chrysochloridae (Mammalia) from the Sperrgebiet, Namibia. Communications of the Geological Survey of Namibia, 16, 153-193.

Pickford, M. (2015d). Late Eocene lorisiform primate from Eocliff, Sperrgebiet, Namibia. Communications of the Geological Survey of Namibia, 16, 194-199.

Pickford, M. (2015e). New Titanohyracidae (Hyracoidea: Afrotheria) from the Late Eocene of Namibia. Communications of the Geological Survey of Namibia, 16, 200-214.

Pickford, M. (2015f). Bothriogenys (Anthracotheriidae) from the Bartonian of Eoridge, Namibia. Communications of the Geological Survey of Namibia, 16, 215-222.

Pickford, M. (2018a). New Zegdoumyidae (Rodentia, Mammalia) from the Middle Eocene of Black Crow, Namibia: taxonomy, dental formula. Communications of the Geological Survey of Namibia, 18, 48-63.

Pickford, M. (2018b). Fossil fruit bat from the Ypresian/Lutetian of Black Crow, Namibia. Communications of the Geological Survey of Namibia, 18, 64-71.

Pickford, M. (2018c). Additional material of Namahyrax corvus from the Ypresian/Lutetian of Black Crow, Namibia. Communications of the Geological Survey of Namibia, 18, 81-86.

Pickford, M. (2018d). Tenrecoid mandible from Elisabethfeld (Early Miocene) Namibia. Communications of the Geological Survey of Namibia, 18, 87-92.

Pickford, M. (2018e). Characterising the zegdoumyid rodent Tsaukhaebmys from the Ypresian/Lutetian of Black Crow, Namibia. Communications of the Geological Survey of Namibia, 19, 66-70.

Pickford, M. (2018f). Tufamyidae, a new family of hystricognath rodents from the Palaeogene and Neogene of the Sperrgebiet, Namibia. Communications of the Geological Survey of Namibia, 19, 71-109. 
Pickford, M. \& Fischer, M. S. (1987).

Parapliohyrax ngororaensis, a new hyracoid from the Miocene of Kenya, with an outline of the classification of Neogene Hyracoidea. Neue Jahrbuch für Geologie und PaläontologieAbhandlungen, 175, 207-234.

Pickford, M. \& Gommery, D. (2016). Fossil Suidae (Artiodactyla, Mammalia) from Aves Cave I and nearby sites in Bolt's Farm Palaeokarst System, South Africa. Estudios Geológicos, 72, e059.

Pickford, M. \& Mein, P. (1988). The discovery of fossiliferous Plio-Pleistocene cave fillings in Ngamiland, Botswana. Comptes Rendus de l'Académie des Sciences de Paris, Série IIa, 307, 1681-1686.

Pickford, M. \& Mein, P. (2011). New Pedetidae (Rodentia: Mammalia) from the Mio-Pliocene of Africa. Estudios Geológicos, 67, 455-469.

Pickford, M. \& Senut, B. (1997). Cainozoic mammals from coastal Namaqualand. Palaeontologia Africana, 34, 199-217.

Pickford, M. \& Senut, B. (1998). Orange River Man, an archaic Homo sapiens from Namibia. South African Journal of Science, 94, 312.

Pickford, M. \& Senut, B. (1999). Geology and palaeobiology of the Namib Desert, southwestern Africa. Memoirs of the Geological Survey of Namibia, 18, 1-155.

Pickford, M. \& Senut, B. (2002). The Fossil Record of Namibia. Windhoek: Geological Survey of Namibia.

Pickford, M. \& Senut, B. (2003). Miocene paleobiology of the Orange River Valley, Namibia. Memoirs of the Geological Survey of Namibia, 19, 1-22.

Pickford, M. \& Senut, B. (2008). Geology and palaeobiology of the northern Sperrgebiet: general conclusions and summary. Memoirs of the Geological Survey of Namibia, 20, 555-573.

Pickford, M. \& Senut, B. (2010). Karst geology and palaeobiology of northern Namibia. Memoirs of the Geological Survey of Namibia, 21, 1-74.

Pickford, M. \& Senut, B. (2018). Afrohyrax namibensis (Hyracoidea, Mammalia) from the Early Miocene of Elisabethfeld and Fiskus, Sperrgebiet, Namibia. Communications of the Geological Survey of Namibia, 18, 93-112.
Pickford, M. \& Tassy, P. (1980). A new species of Zygolophodon (Mammalia, Proboscidea) from the Miocene hominoid localities of Meswa Bridge and Moroto (East Africa). Neues Jahrbuch für Geologie und PaläontologieAbhandlungen, 4, 235-251.

Pickford, M. \& Uhen, M. D. (2014). Namaia Pickford et al., 2008, preoccupied by Namaia Green, 1963: proposal of a replacement name. Communications of the Geological Survey of Namibia, 15, 91.

Pickford, M., Senut, B. \& Mein, P. (1992). Otavipithecus namibiensis, first Miocene hominoid from southern Africa. Nature, 356, 144-148.

Pickford, M., Senut, B., Mein, P. \& Conroy, G. C. (1993). Premiers gisements fossilifères postmiocènes dans le Kaokoland, nord-ouest de la Namibie. Comptes Rendus de l'Académie des Sciences de Paris, Série IIa, 317, 719-720.

Pickford, M., Mein, P. \& Senut, B. (1994). Fossiliferous Neogene karst fillings in Angola, Botswana and Namibia. South African Journal of Science, 90, 227-230.

Pickford, M., Senut, B., Mein, P., et al. (1995). The discovery of lower and middle Miocene vertebrates at Auchas, southern Namibia. Comptes Rendus de l'Academie des Sciences de Paris, Série IIa, 322, 901-906.

Pickford, M., Senut, B., Mein, P., et al. (1996). Preliminary results of new excavations at Arrisdrift, middle Miocene of southern Namibia. Comptes Rendus Hebdomadaires des Séances de l'Académie des Sciences de Paris, 322, 991-996.

Pickford, M., Mein, P., Moyà-Solà, S. \& Köhler, M. (1997). Phylogenetic implications of the first African middle Miocene hominoid frontal bone from Otavi, Namibia. Comptes Rendus de l'Académie des Sciences de Paris, Sciences de la Terre et des Planètes, 325, 459-466.

Pickford, M., Eisenmann, V. \& Senut, B. (1999). Timing of landscape development and calcrete genesis in northern Namaqualand, South Africa. South African Journal of Science, 95, 357-359.

Pickford, M., Senut, B., Morales, J., Mein, P. \& Sánchez, I. M. (2008). Mammalia, from the 
Lutetian of Namibia. Memoirs of the Geological Survey of Namibia, 20, 465-514.

Pickford, M., Senut, B., Hipondoka, M., et al. (2014). Mio-Plio-Pleistocene geology and palaeobiology of the Etosha Pan, Namibia. Communications of the Geological Survey of Namibia, 15, 16-68.

Piveteau, J. (1948). Un félide du Pliocène de Roussillon. Annales de Paléontologie, 43, 97-124.

Pleurdeau, D., Imalwa, E., Détroit, F., et al. (2012). 'Of sheep and men': earliest direct evidence of caprine domestication in southern Africa at Leopard Cave (Erongo, Namibia). PLoS ONE, 7. DOI: 10.1371/ journal.pone.0040340.

Plug, I. (1979a). Appendix: Striped Giraffe Shelter faunal report. In L. Wadley. Big Elephant Shelter and its role in the prehistory of central South West Africa. Cimbebasia Series B, 3, 71-72.

Plug, I. (1979b). The faunal remains from Wildeebeestfontein. South African Archaeological Society Goodwin Series, 3, 130-132.

Plug, I. (1981). Some research results on the late Pleistocene and early Holocene deposits of Bushman Rock Shelter, eastern Transvaal. South African Archaeological Bulletin, 36, 14-21.

Plug, I. (1985). Appendix 1: the faunal remains from two Iron Age sites, Rooikrans and Rhenosterkloof, central Transvaal. In S. L. Hall. Later Iron Age sites in the Rooiberg area of the Transvaal. Annals of the Cape Provincial Museums: Human Sciences, 1, 201-210.

Plug, I. (1989). Aspects of life in the Kruger National Park during the Early Iron Age. South African Archaeological Society Goodwin Series, 6, 62-68.

Plug, I. (1990). The macrofaunal remains from Mhlwazini Cave, a Holocene site in the Natal Drakensberg. Natal Museum of Humanities, 2, 135-142.

Plug, I. (1992). The macrofaunal remains of Collingham Shelter, a Late Stone Age site in Natal. Natal Museum Journal of Humanities, 4, 53-59.

Plug, I. (1993a). The macrofaunal remains of wild animals from Abbot's Cave and Lame
Sheep Shelter, Seacow Valley, Cape. Koedoe, 36, 15-26.

Plug, I. (1993b). The faunal remains from Nanda, an Early Iron Age site in Natal. Natal Journal of Humanities, 5 , 99-107.

Plug, I. (1993c). KwaThwaleyakhe Shelter: the faunal remains from a Holocene site in the Thukela Basin, Natal. Natal Journal of Humanities, 5, 37-45.

Plug, I. (1993d). The macrofaunal and molluscan remains from Tloutle, a Later Stone Age site in Lesotho. Southern African Field Archaeology, 2, 44-48.

Plug, I. (1994). Randjies: the faunal remains from a Late Iron Age site, northern Transvaal. Research by the National Cultural History Museum, 3, 119-129.

Plug, I. (1996a). The hunter's choice: faunal remains from Maqonqo Shelter, South Africa. Natal Journal of Humanities, 8, 41-52.

Plug, I. (1996b). Seven centuries of Iron Age traditions at Bosutswe, Botswana: a faunal perspective. South African Journal of Science, 92, 91-97.

Plug, I. (1997a). Early Iron Age buffalo hunters on the Kadzi River, Zimbabwe. African Archaeological Review, 14, 85-105.

Plug, I. (1997b). Late Pleistocene and Holocene hunter-gatherers in the Eastern Highlands of South Africa and Lesotho: a faunal interpretation. Journal of Archaeological Science, 24, 715-727.

Plug, I. (1997c). The faunal samples from Thulamela 2231AC2, Kruger National Park, South Africa. Research by the National Cultural History Museum, 6, 78-93.

Plug, I. (1997d). Resource exploitation: animal use during the Middle Stone Age at Sibudu Cave, KwaZulu-Natal. South African Journal of Science, 100, 151-158.

Plug, I. (1999a). Some early Iron Age communities of the Eastern escarpment and lowveld, South Africa: a faunal perspective. Archaeozoologia, 10, 189-199.

Plug, I. (1999b). The fauna from Later Stone Age and contact sites in the Karoo, South Africa. In C. Becker, H. Manhart, J. Peters \& J. Schibler, eds. Historia animalium ex ossibus: Beiträge zur Paläoanatomie, Archäologie, 
Ethnologie und Geschichte der Tiermedizin. Festschrift für Angela von den Driesch zum 65 Geburstag. Rahden/Westfalen: Marie Leidorf, pp. 343-353.

Plug, I. (2000). Overview of Iron Age fauna from the Limpopo Valley. South African Archaeological Society Goodwin Series, 8, 117-126.

Plug, I. (2002). Faunal remains from Mzinyashana, a Later Stone Age site in KwaZulu-Natal, South Africa. Southern African Humanities, 14, 51-63.

Plug, I. (2004). Resource exploitation: animal use during the Middle Stone Age at Sibudu Cave, KwaZulu-Natal. South African Journal of Science, 100, 151-158.

Plug, I. \& Badenhorst, S. (2001). The distribution of macromammals in southern Africa over the past 30,000 years. Transvaal Museum Monograph, 21, 1-234.

Plug, I. \& Badenhorst, S. (2002). Appendix B: bones from Muozi Midden Trench II. In R. Soper. Nyanga: Ancient Fields, Settlements and Agricultural History in Zimbabwe. Oxford: British Institute in Eastern Africa, pp. 242-248.

Plug, I. \& Badenhorst, S. (2006). Notes on the fauna from three Late Iron Age mega-sites, Boitsemagano, Molokwane and Mabjanamatshwana, North West Province, South Africa. South African Archaeological Bulletin, 61, 57-67.

Plug, I. \& Brown, A. (1982). Mgoduyanuka: faunal remains. Annals of the Transvaal Museum, 25, 115-121.

Plug, I. \& Engela, R. (1992). The macrofaunal remains from recent excavations at Rose Cottage Cave, Orange Free State. South African Archaeological Bulletin, 47, 16-25.

Plug, I. \& Keyser, A. W. (1993). Haasgat Cave, a Pleistocene site in the central Transvaal: geomorphological, faunal and taphonomic considerations. Annals of the Transvaal Museum, 36, 139-145.

Plug, I. \& Mitchell, P. (2008). Sehonghong: hunter-gatherer utilization of animal resources in the highlands of Lesotho. Annals of the Transvaal Museum, 45, 31-53.

Plug, I. \& Peters, J. (1991). Osteomorphological differences in the appendicular skeleton of
Antidorcas marsupialis (Zimmerman, 1780) and Antidorcas bondi (Cooke \& Wells, 1951) (Mammalia: Bovidae) with notes on the osteometry of Antidorcas bondi. Annals of the Transvaal Museum, 35, 253-264.

Plug, I. \& Pistorius, J. C. C. (1999). Animal remains from industrial Iron Age communities in Phalaborwa, South Africa. African Archaeological Review, 16, 155-184.

Plug, I. \& Roodt, F. (1990). The faunal remains from recent excavations at uMgungundlovu. South African Archaeological Bulletin, 45, 47-52.

Plug, I. \& Sampson, C. G. (1996). European and Bushman impacts on Karoo fauna in the nineteenth century: an archaeological perspective. South African Archaeological Bulletin, 51, 26-31.

Plug, I. \& Voigt, E. A. (1985). Archaeozoological studies of Iron Age communities in southern Africa. Advances in World Archaeology, 4, 189-238.

Plug, I., Dippenaar, N. J. \& Hanisch, E. O. M. (1979). Evidence of Rattus rattus (house rat) from Pont Drift, an Iron Age site in the northern Transvaal. South African Journal of Science, 75, 82.

Plug, I., Bollong, C. A., Hart, T. J. G. \& Sampson, C. G. (1994). Context and direct dating of pre-European livestock in the upper Seecow River valley. Annals of the South African Museum, 104, 31-48.

Plug, I., Soper, R. \& Chirawu, S. (1997). Pits, tunnels and cattle in Nyanga, Zimbabwe: new light on an old problem. South African Archaeological Bulletin, 52, 89-94.

Plug, I., Scott, K. \& Fish, W. (2000). Schoemansdal: faunal remains from selected sites in an historic village. Annals of the Transvaal Museum, 37, 125-130.

Plug, I., Mitchell, P. \& Bailey, G. (2003). Animal remains from Likoaeng, an open-air river site and its place in the post-classic Wilton of Lesotho and eastern Free State, South Africa. South African Journal of Science, 99, 143-152.

Pocock, T. N. (1969). Appendix 1: micro-fauna provisionally identified from sieved material recovered thus far from Dump 8 at Sterkfontein. 1967-1969. South African Archaeological Bulletin, 24, 168-169. 
Pocock, T. N. (1976). Pliocene mammalian microfauna from Langebaanweg: a new fossil genus linking the Otomyinae with the Murinae. South African Journal of Science, 72, 58-60.

Pocock, T. N. (1985). Plio-Pleistocene mammalian microfauna in southern Africa. Annals of the Geological Survey of South Africa, 19, 65-67.

Pocock, T. N. (1987). Plio-Pleistocene mammalian microfauna in southern Africa: a preliminary report including description of two new fossil muroid genera (Mammalia: Rodentia). Palaeontologia Africana, 26, 69-91.

Pohle, H. (1928). Die Raubtiere von Oldoway. In H. Reck, ed. Wissenschaftliche Ergebnisse der Oldoway-Expedition 1913, New Series, 3, 45-54.

Pomel, A. (1848). Etudes sur les carnassiers insectivores (Extrait): Premiere partie Insectivores fossiles. Archives des Sciences Physiques et Naturelles, Genève, 9, 159-165.

Pomel, A. (1879). Ossements d'Eléphants et d'Hippopotames découvertes dans une station préhistorique de la plaine d'Eglis (Provence d'Oran). Bulletin de la Société Géologique de France, Series 3, 7, 44-51.

Pomel, A. (1892). Sur Libytherium maurisium, grand ruminant du terrain pliocène plaisancien d'Algérie. Comptes Rendus Hebdomadaires des Séances de l'Académie des Sciences, 115, 100-102.

Pomel, A. (1893). Bubalus antiquus: Carte Géologique de l'Algérie. Alger: Fontana.

Pomel, A. (1895). Les éléphants Quaternaires: Carte Géologique de l'Algérie. Alger: Fontana.

Pomel, A. (1897). Les Equides: Carte Géologique de l'Algérie. Alger: Fontana.

Power, J. H. (1955). Power's Site, Vaal River. South African Archaeological Bulletin, 10, 96-101.

Prat, S. \& Gommery, D. (2012). First partial skeleton of Paranthropus robustus from Swartkrans (South Africa). In 2nd Annual Meeting of the European Society for the Study of Human Evolution, Bordeaux, p. 142.

Prat, S., Jashajsvili, T., Gommery, D. \& Thackeray, J. F. (2014). A specimen of Paranthropus robustus from Bolt's Farm,
Cradle of Humankind, South Africa? In 4th Annual Meeting European Society for the Study of Human Evolution, Florence,

p. 134.

Pwiti, G. (1996). Continuity and change. Studies in African Archaeology, 13, 1-180.

Pycraft, W. P. (1925). On the calvaria found at Boskop, Transvaal, in 1913 and its relationship to Cromagnard and Negroid skulls. Journal of the Royal Anthropological Institute of Great Britain and Ireland, 55, 179-198.

Quérouil, S., Hutterer, R., Barrière, P., et al. (2001). Phylogeny and evolution of African shrews (Mammalia: Soricidae) inferred from 16s rRNA sequence. Molecular Phylogenetics and Evolution, 20, 185-195.

Quiralte, V., Sánchez, I. M., Morales, J. \& Pickford, M. (2008). Tragulidae (Artiodactyla, Ruminantia) from the Early Miocene of the Sperrgebiet, Southern Namibia. Memoirs of the Geological Survey of Namibia, 20, 387-396.

Qumsiyeh, M. B. (1986). Phylogenetic studies of the rodent family Gerbillidae: 1 .

Chromosomal evolution in the southern African complex. Journal of Mammalogy, 67, 680-692.

Qumsiyeh, M. B., Hamilton, M. J., Dempster, E. R. \& Baker, R. J. (1991). Cytogenetics and systematics of the rodent genus Gerbillurus. Journal of Mammalogy, 72, 89-96.

Rafinesque, C. S. (1814). Précis des découvertes et travaux somiologiques. Palerme: Royale Typographie Militaire.

Rafinesque, C. S. (1820). I Class. Mastosia - the Sucklers. Annals of Nature, 1, 2-4.

Ramsay, P. J., Smith, A. M., Lee-Thorp, J. A., et al. (1993). 130 000-year-old fossil elephant found near Durban, South Africa: preliminary report. South African Journal of Science, 89, 165.

Rasmussen, D. T. \& Gutiérrez, M. (2010). Hyracoidea. In L. Werdelin \& W. J. Sanders, eds. Cenozoic Mammals of Africa. Berkeley, CA: University of California Press, pp. 123-145.

Rasmussen, D. T., Pickford, M., Mein, P., Senut, B. \& Conroy, G. C. (1996). Earliest known procaviid hyracoid from the late Miocene of Namibia. Journal of Mammalogy, 77, 745-754. 
Ratcliffe, J. M. (2002). Myotis welwitschii. Mammalian Species, 701, 1-3.

Ray, J. C. (1995). Civettictis civetta. Mammalian Species, 488, 1-7.

Reck, H. (1928). Pelorovis oldowayensis nov. gen. nov. sp. Wissenschaft Ergebnisse der Oldoway-Expedition 1913, New Series, 3, 56-67.

Rector, A. L. \& Reed, K. E. (2010). Middle and Late Pleistocene faunas of Pinnacle Point and their paleoecological implications. Journal of Human Evolution, 59, 340-357.

Reed, K. E., Kitching, J. W., Grine, F. E., Jungers, W. L. \& Sokoloff, L. (1993). Proximal femur of Australopithecus africanus from member 4, Makapansgat, South Africa. American Journal of Physical Anthropology, 92, 1-15.

Reed Cohen, D. (2010). Hunting and herding at Moritsane, a village in southeastern Botswana, c. AD 1165-1275. South African Archaeological Bulletin, 65, 154-163.

Renard, A., Lavoie, M., Pitt, J. A. \& Larivière, S. (2015). Felis nigripes. Mammalian Species, 47, 78-83.

Repenning, C. A. (1965). An extinct shrew from the early Pleistocene of South Africa. Journal of Mammalogy, 46, 189-196.

Reynard, J. P., Discamps, E., Badenhorst, S., Van Niekerk, K. \& Henshilwood, C. S. (2016a). Subsistence strategies in the southern Cape during the Howiesons Poort: taphonomic and zooarchaeological analyses of Klipdrift Shelter, South Africa. Quaternary International, 404, 2-19.

Reynard, J. P., Discamps, E., Wurz, S., et al. (2016b). Occupational intensity and environmental changes during the Howiesons Poort at Klipdrift Shelter, southern Cape, South Africa. Palaeogeography, Palaeoclimatology, Palaeoecology, 449, 349-364.

Reynolds, S. C. (2012). Nyctereutes terblanchei: the raccoon dog that never was. South African Journal of Science, 108. DOI: 10.4102/sajs. v108i1/2.589.

Reynolds, S. C. (2010a). Morphological evaluation of genetic evidence for a Pleistocene extirpation of eastern African impala. South African Journal of Science, 106. DOI: 10.4102/sajs.v106i11/12.325.
Reynolds, S. C. (2010b). Where the wild things were: spatial and temporal distribution of carnivores in the Cradle of Humankind (Gauteng, South Africa) in relation to the accumulation of mammalian and hominin assemblages Journal of Taphonomy, 8, 233-257.

Reynolds, S. C. \& Bishop, L. C. (2006). Craniodental variability in modern and fossil plains zebra (Equus burchellii Gray 1824) from East and southern Africa. In M. Mashkour, ed. Equids in Time and Space. Oxford: Oxbow Books, pp. 49-60.

Reynolds, S. C. \& Kibii, J. M. (2011). Sterkfontein at 75: review of palaeoenvironments, fauna and archaeology from the hominin site of Sterkfontein (Gauteng Province, South Africa). Palaeontologia Africana, 46, 59-88.

Reynolds, S. C., Vogel, J. C., Clarke, R. J. \& Kuman, K. (2003). Preliminary results of excavations at Lincoln Cave, Sterkfontein, South Africa. South African Journal of Science, 99, 286-288.

Reynolds, S. C., Clarke, R. J. \& Kuman, K. (2007). The view from the Lincoln Cave: mid- to late Pleistocene fossil deposits from Sterkfontein hominid site, South Africa. Journal of Human Evolution, 53, 160-271.

Rhoads, S. N. (1896). Mammals collected by Dr A. Donaldson Smith during his expedition to Lake Rudolf, Africa. Proceedings of the Academy of Natural Sciences of Philadelphia, 48, 517-546.

Riccucci, M. \& Lanza, B. (2008). Neoromicia Roberts, 1926 (Mammalia, Vespertilionidae): correction of gender and etymology. Italian Journal of Zoology, 19, 175-177.

Rightmire, G. P. (1978). Florisbad and human population succession in southern Africa. American Journal of Physical Anthropology, 48, 475-486.

Rightmire, G. P. (1979a). Appendix 3: human skeletal remains from Die Kelders, Cape. In F. R. Schweitzer. Excavations at Die Kelders, Cape Province, South Africa: the Holocene deposits. Annals of the South African Museum, 78, 233.

Rightmire, G. P. (1979b). Implications of Border Cave skeletal remains for Later Pleistocene 
human evolution. Current Anthropology, 20, 23-35.

Rightmire, G. P. \& Deacon, H. J. (1991). Comparative studies of Late Pleistocene human remains from Klasies River Mouth, South Africa. Journal of Human Evolution, 20, 131-156.

Rightmire, G. P., Deacon, H. J., Schwartz, J. H. \& Tattersall, I. (2006). Human foot bones from Klasies River main site, South Africa. Journal of Human Evolution, 50, 96-103.

Robbins, C. B. (1978). Taxonomic identification of Scotophilus nigrita (Schreber) (Chiroptera: Vespertilionidae). Journal of Mammalogy, 59, 212-213.

Robbins, C. B., De Vree, F. \& Cakenberghe, V. van (1985). A review of the systematics of the African bat genus Scotophilus

(Vespertilionidae). Annales du Musée Royal de l'Afrique Centrale Série in-8vo Sciences Zoologiques, 237, 53-84.

Robbins, L. H. (1986). Recent archaeological research in southeastern Botswana: the Thamaga site. Botswana Notes and Records, 18, 1-13.

Robbins, L. H. (1990). Excavation at the White Paintings Rock-Shelter Tsodilo Hills. Nyame Akuma, 34, 2-4.

Robbins, L. H., Murphy, M. L., Stevens, N. J., et al. (1996). Paleoenvironment and archaeology of Drotsky's Cave: western Kalahari Desert, Botswana. Journal of Archaeological Science, 23, 7-22.

Robbins, L. H., Murphy, M. L., Brook, G. A., et al. (2000). Archaeology, palaeoenvironment and chronology of the Tsodilo Hills White Paintings Rock Shelter, northwest Kalahari Desert, Botswana. Journal of Archaeological Science, 27, 1085-1113.

Robbins, L. H., Campbell, A. C., Murphy, M. L., et al. (2008). Recent archaeological research at Toteng, Botswana: early domesticated livestock in the Kalahari. Journal of African Archaeology, 6, 131-149.

Robbins, L. H., Campbell, A. C., Murphy, M. L., et al. (2009). Mogapelwa: archaeology, palaeoenvironment and oral traditions at Lake Ngami, Botswana. South African Archaeological Bulletin, 64, 13-32.
Roberts, A. (1913). Supplement to list of mammals in the Transvaal Museum. Annals of the Transvaal Museum, 4, 108-109.

Roberts, A. (1914). Supplementary list of African mammals in the collection of the Transvaal Museum, with descriptions of some new species. Annals of the Transvaal Museum, 5, 180-186.

Roberts, A. (1919). Descriptions of some new mammals. Annals of the Transvaal Museum, 6, 112-115.

Roberts, A. (1924). Some additions to the list of South African mammals. Annals of the Transvaal Museum, 10, 59-76.

Roberts, A. (1926). Some new S. African mammals and some changes in nomenclature. Annals of the Transvaal Museum, 11, 245-263.

Roberts, A. (1929). New forms of African mammals. Annals of the Transvaal Museum, 13, 82-121.

Roberts, A. (1931). New forms of South African mammals. Annals of the Transvaal Museum, 14, 221-236.

Roberts, A. (1932). Preliminary description of fiftyseven new forms of South African mammals. Annals of the Transvaal Museum, 15, 1-19.

Roberts, A. (1933). Eleven new forms of South African mammals. Annals of the Transvaal Museum, 15, 265-270.

Roberts, A. (1938). Description of new forms of mammals. Annals of the Transvaal Museum, 19, 231-245.

Roberts, A. (1946). Descriptions of numerous new subspecies of mammals. Annals of the Transvaal Museum, 20, 303-328.

Roberts, A. (1951). The Mammals of South Africa. Johannesburg: The Trustees of the Mammals of South Africa Book Fund.

Robertshaw, P. T. (1977). Excavations at Paternoster, South-Western Cape. South African Archaeological Bulletin, 32, 63-73.

Robertshaw, P. T. (1978). Archaeological investigations at Langebaan Lagoon, Cape Province. Palaeoecology of Africa, 10-11, 139-148.

Robertshaw, P. T. (1979). Excavations at Duiker Eiland, Vredenburg District, Cape Province. Annals of the Cape Provincial Museums (Human Sciences), 1, 1-26. 
Robey, T. (1980). Mpambanyoni: a Late Iron Age site on the Natal south coast. Annals of the Natal Museum, 24, 147-164.

Robinson, J. T. (1970). Two new early hominid vertebrae from Swartkrans. Nature, 225, 1217-1219.

Robinson, T. J. \& Dippenaar, N. J. (1983). The status of Lepus saxatilis, L. whytei and $L$. crawshayi in southern Africa. Acta Zoologica Fennica, 174, 35-39.

Robinson, T. J. \& Dippenaar, N. J. (1987). Morphometrics of the South African Leporidae. II: Lepus Linnaeus, 1758 and Bunolagus Thomas, 1929. Annals of the Transvaal Museum, 34, 379-404.

Robinson, T. J. \& Matthee, C. A. (2005). Phylogeny and evolutionary origins of the Leporidae: a review of cytogenetics, molecular analyses and a supermatrix analysis. Mammal Review, 35, 231-247.

Robinson, T. J. \& Skinner, J. D. (1983). Karyology of the riverine rabbit, Bunolagus monticularis and its taxonomic implications. Journal of Mammalogy, 64, 678-681.

Roger, O. (1902). Wirbeltierreste aus dem Obermiocän der bayerischschwäbischen Hochebene. Bericht des Naturwissenschaftlichen Vereines für Schwaben und Neuburg, 1902, 1-22.

Rookmaker, L. C. (1989). The Zoological Exploration of Southern Africa 1650-1790. Rotterdam: Balkema.

Rookmaker, L. C. \& Meester, J. (1988). Case 2605: Euryotis brantsii A. Smith, 1834 (currently Parotomys brantsii; Mammalia, Rodentia) - proposed conservation of the specific name. Bulletin of Zoological Nomenclature, 45, 43-44.

Rossouw, L. (2006). Florisian mammal fossils from erosional gullies along the Modder River at Mitasrust Farm, central Free State, South Africa. Navorsinge van die

Nasionale Museum Bloemfontein, 22, 145-162.

Rovinsky, D. S., Herries, A. I. R., Menter, C. G. \& Adams, J. W. (2015). First description of in situ primate and faunal remains from the Plio-Pleistocene Drimolen Makondo palaeocave infill, Gauteng, South Africa. Palaeontologia Electronica, 18.2.34A, 1-21.
Rüppell, E. (1835). Neue Wirbelthiere zu der Fauna von Abyssinien gehörig. Frankfurt am Main: Schmerber.

Rüppell, E. (1842a). Über Säugthiere aus der Ordnung der Nager. Abhandlungen aus dem Gebiete der beschreibenden Naturgeschichte Museum Senckenbergianum, 1833-1845, 91-101.

Rüppell, E. (1842b). Beschreibung mehrerer neuer Säugethiere, in der zoologischen Sammlung der Senckenbergische naturforschenden Gesellschaft befindlich. Museum Senckenbergianum: Abhandlungen aus dem Gebiete der beschreibenden Naturgeschichte, 3, 129-144.

Russo, I. M. (2009). Patterns and processes underlying genetic diversity in the Namaqua rock mouse Micaelamys namquensis Smith, 1834 (Rodentia: Muridae) from southern Africa. Unpublished PhD thesis, University of Pretoria.

Sach, V. J. \& Heizmann, E. P. J. (2001). Stratigraphy and mammal faunas of the Brackwassermolasse in the surroundings of Ulm (Southwest Germany). Stuttgarter Beiträge zur Naturkunde Serie B (Geologie und Paläontologie), 310, 1-95.

Sadr, K. (2007). Early first millennium pastoralists on Kasteelberg? The UB/UCT excavation at KBA. South African Archaeological Bulletin, 62, 154-161.

Sadr, K. \& Plug, I. (2001). Faunal remains in the transition from hunting to herding in Southeastern Botswana. South African Archaeological Bulletin, 56, 76-82.

Sallam, H. M. \& Seiffert, E. R. (2016). New phiomorph rodents from the latest Eocene of Egypt and the impact of Bayesian 'clock'based phylogenetic methods on estimates of basal hystricognath relationships and biochronology. PeerJ, 4, 1-53.

Sandelowsky, B. H. (1979). Kapako and Vungu Vungu: Iron Age sites on the Kavango River. South African Archaeological Society Goodwin Series, 3, 52-61.

Sanders, W. J. (2007). Taxonomic review of fossil Proboscidea (Mammalia) from Langebaanweg, South Africa. Transactions of the Royal Society of South Africa, 62, 1-16. 
Sanders, W. J., Gheerbrandt, E., Harris, J. M., Saegusa, H. \& Delmer, C. (2010a). Proboscidea. In L. Werdelin \& W. J. Sanders, eds. Cenozoic Mammals of Africa. Berkeley, CA: University of California Press, pp. 161-251.

Sanders, W. J., Rasmussen, D. T. \& Kappelman, J. (2010b). Embripothoda. In L. Werdelin \& W. J. Sanders, eds. Cenozoic Mammals of Africa. Berkeley, CA: University of California Press, pp. 115-122.

Sardella, R. \& Werdelin, L. (2007). Amphimachairodus (Felidae, Mammalia) from Sahabi (Latest Miocene-Earliest Pliocene, Libya), with a review of African Miocene Machairodontinae. Rivista Italiana di Paleontologia e Stratigrafia, 113, 67-77.

Savage, R. J. G. (1965). The Miocene Carnivora of East Africa. Fossil Mammals of Africa, 19, 239-316.

Schepers, G. W. H. (1941). The mandible of the Transvaal human skeleton from Springbok Flats. Annals of the Transvaal Museum, 20, 253-271.

Schinz, H. R. (1821-1825). Das Thierreich eingetheilt nach dem Bau der Thiere als Grundlage ihrer Naturgeschichte und der vergleichenden Anatomie von dem Herrn Ritter von Cuvier. Stuttgart: Cotta'schen.

Schoonraad, M. \& Beaumont, P. B. (1968). The North Brabant Shelter, north western Transvaal. South African Journal of Science, 64, 319-331.

Schreber, J. C. D. von (1775-1792). Die Säugethiere in Abbildungen nach der Natur, mit Beschreibungen. Erlangen: Walther.

Schwann, H. (1906). A list of the mammals obtained by Messrs R.B. Woosnam and R.E. Dent in Bechuanaland. Proceedings of the Zoological Society of London, 1906, 101-111.

Schwartz, G. T. (1997). Re-evaluation of the PlioPleistocene hyraxes (Mammalia, Procaviidae) from South Africa. Neues Jahrbuch für Geologie und Paläontologie, Abhandlungen, 206, 365-383.

Schwarz, E. (1932). Neue diluviale Antilopen aus Ostafrika. Zentralblatt für Mineralogie, Geologie und Paläontologie B, 1932, 1-4.

Schweitzer, F. R. (1974). Archaeological evidence for sheep at the Cape. South African Archaeological Bulletin, 29, 75-82.
Schweitzer, F. R. (1979). Excavations at Die Kelders, Cape Province, South Africa: the Holocene deposits. Annals of the South African Museum, 78, 101-233.

Schweitzer, F. R. \& Scott, K. (1973). Early occurrence of domestic sheep in sub-Saharan Africa. Nature, 241, 547.

Sclater, P. L. \& Thomas, O. (1894). The Book of Antelopes. London: Porter.

Scott, K. \& Klein, R. G. (1981). A hyenaaccumulated bone assemblage from Late Holocene deposits at Deelpan, Orange Free State. Annals of the South African Museum, 86, 217-227.

Scott, L. \& Brink, J. S. (1992). Quaternary palaeoenvironments of pans in central South Africa: palynological and palaeontological evidence. South African Geographer, 19, 22-34.

Scott, W. B. (1907). A collection of fossil mammal bones from the coast of Zululand. Geological Survey of Natal and Zululand Third and Final Report.

Sealy, J., Maggs, T., Jerardino, A. \& Kaplan, J. (2004). Excavations at Melkbosstrand: variability among herder sites on Table Bay, South Africa. South African Archaeological Bulletin, 59, 17-28.

Seeley, H. G. (1891). On Bubalis bainii (Seeley). Geological Magazine, New series 3, 8, 199-202.

Sénégas, F. (1996). Introduction à l'étude des faunes de rongeurs du Plio-Pleistocène du sud de l'Afrique: analyse d'un échantillon de brêches fossilifères de gisements du Transvaal (S. Afr.) et du site de Friesenberg (Namibie). Montpellier: Diplôme d'Etudes Aprofondies (DEA) Paléontologie, Montpellier II.

Sénégas, F. (2001). Interpretation of the dental pattern of the South African fossil Euryotomys (Rodentia, Murinae) and the origin of otomyine dental morphology. In C. Denys, L. Granjon \& A. Poulet, eds. African Small Mammals. Paris: I.R.D., pp. 151-160.

Sénégas, F. (2004). A new species of Petromus (Rodentia, Hystricognatha, Petromuridae) from the Early Pliocene of South Africa and its paleoenvironmental implications. Journal of Vertebrate Paleontology, 24, 757-763.

Sénégas, F. \& Avery, D. M. (1998). New evidence for the murine origin of the Otomyinae (Mammalia, Rodentia) and the age of Bolt's 
Farm. South African Journal of Science, 94, 503-507.

Sénégas, F. \& Michaux, J. (2000). Boltimys broomi gen nov.; sp. nov. (Rodentia, Mammalia), nouveau Muridae d'affinité incertaine du Pliocène inférieur d'Afrique du Sud. Comptes Rendus de l' Académie des Sciences de Paris, Sciences de la Terre et des Planètes, 330, 521-525.

Sénégas, F., Paradis, E. \& Michaux, J. (2005). Homogeneity of fossil assemblages extracted from mine dumps: an analysis of PlioPleistocene fauna from South African caves. Lethaia, 38, 315-322.

Senut, B. (1996). Plio-Pleistocene Cercopithecoidea from the Koanaka Hills (Ngamiland, Botswana). Comptes Rendus de l'Academie des Sciences de Paris, Série IIa, 322, 423-428.

Senut, B. (2003). The Macroscelididae from the Miocene of the Orange River, Namibia. Memoirs of the Geological Survey of Namibia, 19, 119-141.

Senut, B. (2008). Macroscelididae from the lower Miocene of the northern Sperrgebiet, Namibia. Memoirs of the Geological Survey of Namibia, 20, 185-225.

Senut, B. \& Georgalis, G. (2014).

Brevirhynchocyon gen. nov., a new name for the genus Brachyrhynchocyon Senut, 2008 (Mammalia, Macroscelidea) preoccupied by Brachyrhynchocyon Loomis, 1936 (Mammalia, Carnivora). Communications of the Geological Survey of Namibia, 15, 69.

Senut, B., Pickford, M., Mein, P., Conroy, G. \& Van Couvering, J. (1992). Discovery of 12 new Late Cainozoic fossiliferous sites in paleokarst of the Otavi Mountains, Namibia. Comptes Rendus de l'Académie des Sciences de Paris, Série IIa, 314, 727-733.

Senut, B., Pickford, M., De Wit, M., et al. (1996). Biochronology of sediments at Bosluis Pan, Northern Cape Province, South Africa. South African Journal of Science, 92, 249-251.

Senut, B., Pickford, M. \& Wessels, D. (1997). Panafrican distribution of Lower Miocene Hominoidea. Comptes Rendus de l' Académie des Sciences de Paris, Sciences de la Terre et des Planètes, 325, 741-746.
Senut, B., Pickford, M., Braga, M., Marais, D. \& Coppens, Y. (2000). Découverte d'un Homo sapiens archaïque à Oranjemund, Namibie. Comptes Rendus de l' Académie des Sciences de Paris, Sciences de la Terre et des Planètes, 330, 813-819.

Shackley, M. (1980). An Acheulean industry with Elephas recki fauna from Namib IV, South West Africa (Namibia). Nature, 284, 340-341.

Shackley, M. (1985). Palaeolithic archaeology of the central Namib Desert. Cimbebasia, 6, $1-84$.

Shaw, G. (1800). General Zoology, or Systematic Natural History. London: Kearsley.

Shaw, J. C. M. (1937). Evidence concerning a large fossil hyrax. Journal of Dental Research, $16,37-40$.

Shaw, J. C. M. (1938). The teeth of the South African fossil pig (Notochoerus capensis syn. meadowsi) and their geological significance. Transactions of the Royal Society of South Africa, 26, 25-37.

Shaw, J. C. M. \& Cooke, H. B. S. (1940). New fossil pig remains from the Vaal River gravels. Transactions of the Royal Society of South Africa, 28, 293-299.

Shee, J. C. (1963). Appendix A: skeletal remains from Gokomere. In K. R. Robinson. Further excavations in the Iron Age deposits at the Tunnel Site, Gokomere Hill, Southern Rhodesia. South African Archaeological Bulletin, 18, 170.

Shenjere-Nyabezi, P. (2017). Ndongo: a Zimbabwe culture site in the Middle Save. In M. Manyanga \& S. Chirikure, eds. Archives, Objects, Places and Landscapes. Cameroon: Bamenda, pp. 137-167.

Shenjere-Nyabezi, P. \& Pwiti, G. (2015). Late Stone Age economies of the Lower Save Valley, south eastern Zimbabwe: an archaeozoological perspective. Zimbabwea, 11, 26-35.

Shenjere-Nyabezi, P., Pwiti, G. \& Manyanga, M. (2013). Making the most out of rubbish: trends in archaeozoological studies in postindependence Zimbabwe. In M. Manyanga \& S. Katsamudanga, eds. Archaeology in the PostIndependence Era. Harare: Sapes Books, pp. 117-142. 
Shortridge, G. C. (1942). Field notes on the first and second expeditions of the Cape museums' mammal survey of the Cape Province: descriptions of some new subgenera and subspecies. Annals of the South African Museum, 36, 27-100.

Shortridge, G. C. \& Carter, D. (1938). A new genus and new species and subspecies of mammals from Little Namaqualand and the north-west Cape Province; and a new subspecies of Gerbillus paeba from the eastern Cape Province. Annals of the South African Museum, 32, 281-291.

Shoshani, J., Goldman, C. A. \& Thewissen, J. G. M. (1988). Orycteropus afer. Mammalian Species, 300, 1-8.

Shrubsall, F. C. (1911). A note on craniology. In L. Péringuey. The Stone Ages of South Africa as represented in the collections of the South African Museum. Annals of the South African Museum, 8, 202-206.

Sigvallius, B. (1988). The faunal remains from Manyikeni. Studies in African Archaeology, 2, 23-34.

Sinclair, P. J. J. (1991). Excavations at Chivowa Hill, south central Zimbabwe. Zimbabwea, 3, 23-50.

Singer, R. (1954). The Saldanha skull from Hopefield, South Africa. American Journal of Physical Anthropology, 12, 345-362.

Singer, R. (1956). Man and animals in South Africa. Journal of the Palaeontological Society of India, 1, 122-130.

Singer, R. \& Boné, E. L. (1960). Modern giraffes and the fossil giraffids of Africa. Annals of the South African Museum, 45, 375-548.

Singer, R. \& Fuller, A. O. (1962). The geology and description of a fossiliferous deposit near Zwartklip in False Bay. Transactions of the Royal Society of South Africa, 36, 205-211.

Singer, R. \& Keen, E. N. (1955). Fossil Suiformes from Hopefield. Annals of the South African Museum, 42, 169-179.

Singleton, M. (2000). The phylogenetic affinities of Otavipithecus namibiensis. Journal of Human Evolution, 38, 537-573.

Singleton, M., Gilbert, C. C., Frost, S. R. \& Seitelman, B. C. (2016). Comparative morphometric analysis of a juvenile papionin (Primates: Cercopithecidae) from
Kromdraai A. Annals of the Ditsong National Museum of Natural History, 6, 1-17.

Skead, C. J. (2011). Historical Incidence of the Larger Land Mammals in the Broader Western and Northern Cape, 2nd edition. Port Elizabeth: Centre for African Conservation Ecology.

Skinner, J. D. \& Chimimba, C. T. (2005). The Mammals of the Southern African Subregion, 3rd edition. Cambridge: Cambridge University Press.

Skurski, D. A. \& Waterman, J. M. (2005). Xerus inauris. Mammalian Species, 781, 1-4.

Smit, A. A. \& Van der Bank, H. F. H. (2001). Isozyme and allozyme markers distinguishing two morphologically similar, medically important Mastomys species (Rodentia: Muridae). BMC Genetics, 2. DOI: 10.1186/1471-2156-2-15.

Smith, A. (1827). Description of two quadrupeds inhabiting the South of Africa, about the Cape of Good Hope. Transactions of the Linnean Society of London, 15, 460-470.

Smith, A. (1829). Contributions to the natural history of South Africa. Zoological Journal, 4, 433-444.

Smith, A. (1830). [New species of Macroscelides and Erinaceus]. Proceedings of the Committee of Science and Correspondence of the Zoological Society of London, 1, 11.

Smith, A. (1831). Contributions to the natural history of South Africa, etc. No. 1. South African Quarterly Journal, 1(5), 10-24.

Smith, A. (1833a). African Zoology, continued. South African Quarterly Journal, Series 2, 1, 49-64.

Smith, A. (1833b). African Zoology, continued. South African Quarterly Journal, Series 2, 1, 81-96.

Smith, A. (1834a). African Zoology, continued. South African Quarterly Journal, Series 2, 2, 145-160.

Smith, A. (1834b). African Zoology, continued. South African Quarterly Journal, Series 2, 2, 169-192.

Smith, A. (1834c). African Zoology, continued. South African Quarterly Journal, Series 2, 3, 209-224.

Smith, A. (1836 [1834d]). Report of the Expedition for Exploring Central Africa from the Cape of 
Good Hope, June 23rd, 1834. Cape Town: Government Gazette.

Smith, A. (1838-1849). Illustrations of the Zoology of South Africa. London: Smith, Elder.

Smith, A. B. (1981). An archaeological investigation of Holocene deposits at Rooiels Cave, southwestern Cape. South African Archaeological Bulletin, 36, 75-83.

Smith, A. B. (1995). Archaeological observations along the Orange River and its hinterland. In A. B. Smith, ed. Einiqualand: Studies of the Orange River Frontier. Cape Town: University of Cape Town Press, pp. 265-300.

Smith, A. B. \& Jacobson, L. (1995). Excavations at Geduld and the appearance of early domestic stock in Namibia. South African Archaeological Bulletin, 50, 3-20.

Smith, A. \& Mütti, B. (2013). The past 3000 years of human habitation and coastal resource exploitation on the Vredenburg Peninsula. In A. Jerardino, A. Malan \& D. Braun, eds. The Archaeology of the West Coast of South Africa. Oxford: Archaeopress, pp. 68-84.

Smith, A. B. \& Ripp, M. R. (1978). An archaeological reconnaissance of the Doorn/ Tanqua Karoo. South African Archaeological Bulletin, 33, 118-133.

Smith, A. B., Sadr, K., Gribble, J. \& Yates, R. (1991). Excavations in the south-western Cape, South Africa and the archaeological identity of prehistoric hunter-gatherers within the last 2000 years. South African Archaeological Bulletin, 46, 71-91.

Smith, A. B., Yates, R., Miller, D., Jacobson, L. \& Evans, G. (1995). Excavations at Geduld and the appearance of early domestic stock in Namibia. South African Archaeological Bulletin, 50, 3-20.

Smith, C. H. (1827). Synopsis of the species of the Class Mammalia. In E. Griffiths, ed. The Animal Kingdom Arranged in Conformity with its Organization by the Baron Cuvier with Additional Descriptions, Vol. 5. London: Whittaker.

Smith, H. F. \& Grine, F. E. (2008). Cladistic analysis of early Homo crania from Swartkrans and Sterkfontein, South Africa. Journal of Human Evolution, 54, 684-704.

Smith, P., Nshimirimana, R., De Beer, F., et al. (2012). Canteen Kopje: a new look at an old skull. South African Journal of Science, 108. DOI: $10.4102 /$ sajs.v108i1/2.738.

Smuts, J. (1832). Dissertation Zoologica, Ennumerationem Mammalium Capensium. London: Cyfveer.

Sparrman, A. (1779). Bos caffer, et nytt Species af Buffel, från Caput Bonæ Spei. Kungl. Svenska Vetenskapsakademiens Handlingar, Series 1, 40, 79-84.

Sparrman, A. (1783). Resa Goda-Hopps-Udden, Soedra Pol-kretsen Och Omkring Jordklotet, Samt till Hottentott-och Caffer-Landen, Aren 1772-76. Stockholm: Nordstroem.

Sparrman, A. (1784). Mus pumilio, en ny Râtta från det fódra af Africa, uptåkt och belrifven. Kungl. Svenska Vetenskapsakademiens Handlingar, Series 2, 5, 236-237.

Speke, J. H. (1863). Journal of the Discovery of the Source of the Nile. Edinburgh: Blackwood \& Sons.

Steele, T. E. \& Klein, R. G. (2013). The Middle and Later Stone Age faunal remains from Diepkloof Rock Shelter, Western Cape, South Africa. Journal of Archaeological Science, 40, 3453-3462.

Steele, T. E., Mackay, A., Orton, J. \& Schwortz, S. (2012). Varsche Rivier 003, a new Middle Stone Age site in southern Namaqualand, South Africa. South African Archaeological Bulletin, 67, 108-119.

Steininger, C., Berger, L. R. \& Kuhn, B. F. (2008). A partial skull of Paranthropus robustus from Cooper's Cave, South Africa. South African Journal of Science, 104, 143-146.

Steyn, M. \& Broekhuizen, T. (1993). Report on human skeletal remains from Rooiberg (Transvaal). Southern African Field Archaeology, 2, 53-55.

Steyn, M. \& Nienaber, W. C. (2000). Iron Age human skeletal remains from the Limpopo Valley and Soutpansberg area. South African Archaeological Society Goodwin Series, 8, 112-116.

Steyn, M., Meyer, A. \& Loots, M. (1998). Report on isolated human remains from $\mathrm{K} 2$, South Africa. Southern African Field Archaeology, 7, 53-58.

Steyn, M., Nienaber, W. C., Loots, M. \& Meiring, J. H. (1999). An infant grave from K2 
(Greefswald). South African Archaeological Bulletin, 54, 104-106.

Steyn, M., Binneman, J. \& Loots, M. (2007). The Kouga mummified human remains. South African Archaeological Bulletin, 62, 3-8.

Storr, G. C. C. (1780). Prodromus Methodi Mammalium. Tübingen: Reissianis.

Stromer, E. (1921). Erste Mitteilung úber Tertiare Wirbeltier-Reste aus Deutsch-Sudwestafrika. Sitzungsberichte der Bayern Akademie der Wissenschaften zu München, 1921 (II), 331-340.

Stromer, E. (1923). Ergebnisse der Bearbeitung mitteltertiarer Wirbeltier-Reste aus DeutschSudwestafrika. Sitzungsberichte der Bayern Akademie der Wissenschaften München, 1923 (II), 253-270.

Stromer, E. (1926). Reste land- und süsswasserbewohnender Wirbeltiere aus den Diamenten-feldern Deutsch-Südwestafrikas. In E. Kaiser, ed. Die Diamentenwuste südwestafrikas. Vol. 2. Berlin: Dietrich Reimer (Ernst Volsen), pp. 107-153.

Stromer, E. (1931a). Reste Susswasser- und Landbewohnender Wirbeltiere aus den Diamantfeldern Klein-Namaqualandes (Sudwestafrika). Sitzungsberichte der Bayern Akademie der Wissenschaften München. Mathematisch-Naturwissenschaftliche Abteilung, 1931, 17-47.

Stromer, E. (1931b). Palaeothentoides africanus nov. gen., nov. spec., ein erstes Beuteltier aus Afrika. Sitzungsberichte der Bayern Akademie der Wissenschaften München. MathematischNaturwissenschaftliche Abteilung, 1931, 177-190.

Stynder, D. D. (1997). The use of faunal evidence to reconstruct site history at Hoedjiespunt 1 (HDP1), Western Cape. Unpublished MA thesis, University of Cape Town.

Stynder, D., Moggi-Cecchi, J., Berger, L. R. \& Parkington, J. E. (2001). Human mandibular incisors from the late Middle Pleistocene locality of Hoedjiespunt 1, South Africa. Journal of Human Evolution, 41, 369-383.

Sundevall, C. J. (1842). Öfversigt af slagtet Manis. Kungl. Svenska Vetenskapsakademiens Handlingar Stockholm, Series 3, 30, 245-282.

Sundevall, C. J. (1845). Methodisk öfversigt af Idislande djuren, Linnés Pecora. Fam. 5
Antilopina cont. Kungl. Svenska

Vetenskapsakademiens Handlingar, Series 3, 33, 267-330.

Sundevall, C. J. (1846). Nya Mammalia, frän Sydafrika: Öfversigt af Kongl. VetenskapsAkademiens Förhandlingar, 3, 118-123.

Susman, R. L. (1993). Hominid postcranial remains from Swartkrans. In C. K. Brain, ed. Swartkrans. A cave's chronicle of early man. Transvaal Museum Monograph, 8, 117-136.

Susman, R. L. \& De Ruiter, D. (2004). New hominin first metatarsal (SK 1813) from Swartkrans. Journal of Human Evolution, 47, 171-181.

Susman, R. L., De Ruiter, D. \& Brain, C. K. (2001). Recently identified postcranial remains of Paranthropus and an early Homo from Swartkrans Cave, South Africa. Journal of Human Evolution, 41, 607-629.

Swan, L. (2002). Excavations at Copper Queen Mine, Northwestern Zimbabwe. South African Archaeological Bulletin, 57, 64-79.

Swanepoel, E. (2003). An analysis of the faunal remains of Kemp's Caves and an investigation into possible computerized classification of bones. Unpublished MSc thesis, University of Pretoria.

Sydow, W. (1969). The discovery of a Boskop skull at Otjiseva, near Windhoek, South West Africa. South African Journal of Science, 65, 77-82.

Sykes, W. H. (1831). [Cercopithecus albogularis]. Proceedings of the Committee of Science and Correspondence of the Zoological Society of London, 1, 106-107.

Tassy, P. (1986). Nouveau Elephantoidea (Mammalia) dans le Miocene de Kenya. Cahiers de Paléontologie, 10, 1-230.

Taylor, M. E. (1972). Ichneumia albicauda. Mammalian Species, 12, 1-4.

Taylor, M. E. (1975). Herpestes sanguineus. Mammalian Species, 65, 1-5.

Taylor, P. J. (2000). Patterns of chromosomal variation in southern African rodents. Journal of Mammalogy, 81, 317-331.

Taylor, P. J. \& Meester, J. (1993). Cynictis penicillata. Mammalian Species, 432, 1-7.

Taylor, P. J., Campbell, G. K., Meester, J., Willan, K. \& Van Dyk, D. (1989). Genetic variation in the African rodent subfamily Otomyinae (Muridae): 1. Allozyme divergence among 
four species. South African Journal of Science, 85, 257-262.

Taylor, P. J., Meester, J. \& Kearney, T. (1993). The taxonomic status of Saunders' vlei rat, Otomys saundersiae Roberts (Rodentia: Muridae: Otomyinae). Journal of African Zoology, 107, 571-596.

Taylor, P. J., Rautenbach, I. L., Gordon, D., Sink, K. \& Lotter, P. (1995). Diagnostic morphometrics and southern African distribution of two sibling species of tree rat, Thallomys paedulcus and Thallomys nigricauda (Rodentia: Muridae). Durban Museum Novitates, 20, 49-62.

Taylor, P. J., Denys, C. \& Mukerjee, M. (2004). Phylogeny of the African murid tribe Otomyini (Rodentia), based on morphological and allozyme evidence. Zoologica Scripta, 33, 389-402.

Taylor, P. J., Maree, S., Van Sandwyk, J., Baxter, R. M. \& Rambau, R. V. (2009). When is a species not a species? Uncoupled phenotypic, karyotypic and genotypic divergence in two species of South African laminate-toothed rats (Murinae: Otomyini). Journal of Zoology, 277, 317-332.

Taylor, P. J., Stoffberg, S., Monadjem, A., et al. (2012). Four new bat species (Rhinolophus hildebrandtii complex) reflect Plio-Pleistocene divergence of dwarfs and giants across an Afromontane Archipelago. PLoS ONE, 7. DOI: 10.1371/journal.pone.0041744.

Temminck, C. J. (1820). Sur le genre hyène, et description d'une espèce nouvelle, découverte en Afrique. Annales Générales des Sciences Physiques, 3, 46.

Temminck, C. J. (1827). Monographies de Mammalogie. Paris: Dufour \& d'Ocagne.

Temminck, C. J. (1832). In J. Smuts. Dissertatio Zoologica, Ennumerationem Mammalium Capensium. Leiden: Cyfveer.

Thackeray, A. I., Thackeray, J. F. \& Beaumont, P. B. (1983). Excavations at the Blinkklipkop specularite mine near Postmasburg, northern Cape. South African Archaeological Bulletin, 38, 17-25.

Thackeray, J. F. (1979). An analysis of faunal remains from archaeological sites in southern South West Africa (Namibia). South African Archaeological Bulletin, 34, 18-33.
Thackeray, J. F. (1984). Man, animals and extinctions: the analysis of Holocene faunal remains from Wonderwerk Cave, South Africa. Unpublished PhD thesis, Yale University.

Thackeray, J. F. (2010). Ancient DNA from fossil equids: a milestone in palaeogenetics. South African Journal of Science, 106. DOI: 10.4102/ sajs.v106i1/2.111.

Thackeray, J. F. (2015). Faunal remains from Holocene deposits: Excavation 1, Wonderwerk Cave, South Africa. African Archaeological Review, 32, 729-750.

Thackeray, J. F. \& Brink, J. S. (2004). Damaliscus niro horns from Wonderwerk Cave and other Pleistocene sites: morphological and chronological considerations. Palaeontologia Africana, 40, 89-93.

Thackeray, J. F. \& Myer, S. (2004). Parapapio broomi and Parapapio jonesi from Sterkfontein: males and females of one species? Annals of the Transvaal Museum, 41, 79-82.

Thackeray, J. F. \& Watson, V. (1994). A preliminary account of faunal remains from Plover's Lake. South African Journal of Science, 90, 231-233.

Thackeray, J. F., De Ruiter, D. J., Berger, L. R. \& Van der Merwe, N. J. (2001). Fossil hominins from Kromdraai: a revised list of specimens discovered since 1938. Annals of the Transvaal Museum, 38, 43-56.

Thackeray, J. F., Braga, J., Sénégas, F., et al. (2005). Discovery of a humerus shaft from Kromdraai B: part of the skeleton of the type specimen of Paranthropus robustus Broom, 1938? Annals of the Transvaal Museum, 42, 92-93.

Thackeray, J. F., Gommery, D., Sénégas, F., et al. (2008). A survey of past and present work on Plio-Pleistocene deposits on Bolt's Farm, Cradle of Humankind, South Africa. Annals of the Transvaal Museum, 45, 83-89.

Thomas, H. (1981). Les bovides miocènes de la formation de Ngorara du Bassin de Baringo (Rift Valley, Kenya): 2. Proceedings of the Koninklijke Nederlandse Akademie van Wetenschappen Series B Physical Sciences, 84, 357-375. 
Thomas, O. (1883). On Mustela albinucha, Gray. Annals and Magazine of Natural History, Series 5, 11, 370-371.

Thomas, O. (1892). On the mammals of Nyasaland: (first notice). Proceedings of the Zoological Society of London, 1892, 546-554.

Thomas, O. (1894). Description of a new species of reed-rat (Aulacodus) from East Africa, with remarks on the milk-dentition of the genus. Annals and Magazine of Natural History, Series 6, 13, 202-204.

Thomas, O. (1895a). Description of five new African shrews. Annals and Magazine of Natural History, Series 6, 16, 51-55.

Thomas, O. (1895b). On African mole-rats of the genera Georychus and Myoscalops. Annals and Magazine of Natural History, Series 6, 16, 238-241.

Thomas, O. (1896). On the mammals of Nyasaland: fourth notice. Proceedings of the Zoological Society of London, 1896, 788-798.

Thomas, O. (1897a). Small mammals collected by Mr Alexander Whyte during his expedition to the Nyika Plateau and the Masuku Mountains, N. Nyasa. Proceedings of the Zoological Society of London, 1897, 430-436.

Thomas, O. (1897b). On a new dormouse from Mashonaland. Annals and Magazine of Natural History, Series 6, 19, 388-389.

Thomas, O. (1897c). On the mammals obtained by Mr A. Whyte in Nyasaland and presented to the British Museum by Sir H.H. Johnston, K.C.B.; being a fifth contribution to the mammal fauna of Nyasaland. Proceedings of the Zoological Society of London, 1897, 925-939.

Thomas, O. (1902). On some new forms of Otomys. Annals and Magazine of Natural History, Series 7, 10, 311-314.

Thomas, O. (1903). On a remarkable new hare from Cape Colony. Annals and Magazine of Natural History, Series 7, 11, 78-79.

Thomas, O. (1904a). Three new bats, African and Asiatic. Annals and Magazine of Natural History, Series 7, 13, 384-388.

Thomas, O. (1904b). On mammals from northern Angola collected by Dr W.J. Ansorge. Annals and Magazine of Natural History, Series 7, 13, 405-421.
Thomas, O. (1909). New African mammals. Annals and Magazine of Natural History, Series 8, 4, 542-553.

Thomas, O. (1910). New African mammals. Annals and Magazine of Natural History, Series 8, 5, 83-92.

Thomas, O. (1915a). New African rodents and insectivores, mostly collected by $\mathrm{Dr}$ C. Christy for the Congo Museum. Annals and Magazine of Natural History, Series 8, 16, 146-152.

Thomas, O. (1915b). List of mammals (excluding Ungulata) collected on the Upper Congo by Dr Christy for the Congo Museum, Tervuren. Annals and Magazine of Natural History, Series 8, 16, 465-481.

Thomas, O. (1916). On the rats usually included in the genus Arvicanthis. Annals and Magazine of Natural History, Series 8, 18, 67-70.

Thomas, O. (1917). Notes on Georychus and its allies. Annals and Magazine of Natural History, Series 8, 20, 441-444.

Thomas, O. (1918a). New species of Gerbillus and Taterillus. Annals and Magazine of Natural History, Series 9, 2, 146-151.

Thomas, O. (1918b). A revised classification of the Otomyinae, with descriptions of new genera and species. Annals and Magazine of Natural History, Series 9, 2, 203-211.

Thomas, O. (1920). The generic position of Mus migricauda, Thos. and woosnami, Schwann. Annals and Magazine of Natural History, Series 9, 5, 140-142.

Thomas, O. (1926). The generic position of certain African Muridae hitherto referred to Aethomys and Praomys. Annals and Magazine of Natural History, Series 9, 17, 174-179.

Thomas, O. (1929). On mammals from the Kaokoveld, South West Africa, obtained during Capt Shortridge's fifth Percy Sladen and Kaffrarian Museum Expedition.

Proceedings of the Zoological Society of London, 1929, 99-111.

Thomas, O. \& Hinton, M. A. C. (1925). On mammals collected in 1923 by Capt G.C. Shortridge during the Percy Sladen and Kaffrarian Museum Expedition to South West Africa. Proceedings of the Zoological Society of London, 1925, 221-246. 
Thomas, O. \& Schwann, H. (1904a).

[A collection of mammals from

Namaqualand presented to the British

Museums by Mr C.D. Rudd]. Abstract of the

Proceedings of the Zoological Society of London, 2, 5-6.

Thomas, O. \& Schwann, H. (1904b). On a collection of mammals from British Namaqualand presented to the National Museum by Mr C.D. Rudd. Proceedings of the Zoological Society of London, 1904, 171-183.

Thomas, O. \& Schwann, H. (1905). [Mammals from Zululand]. Abstract of the Proceedings of the Zoological Society of London, 18, 23.

Thomas, O. \& Schwann, H. (1906a). The Rudd exploration of South Africa: V. List of mammals obtained by Mr Grant in NE Transvaal. Proceedings of the Zoological Society of London, 1906, 575-591.

Thomas, O. \& Schwann, H. (1906b). [Mammals from the Zoutpansberg]. Abstract of the Proceedings of the Zoological Society of London, 33, 10.

Thomas, O. \& Wroughton, R. C. (1908). The Rudd exploration of South Africa: X. List of mammals obtained by Mr Grant near Tette, Zambesia. Proceedings of the Zoological Society of London, 1908, 535-552.

Thorp, C. (1979). Cattle from the Early Iron Age of Zimbabwe-Rhodesia. South African Journal of Science, 95, 461.

Thorp, C. (1984). A cultural interpretation of the faunal remains from Khami Hill ruin. In M. Hall, G. Avery, D. M. Avery, M. L. Wilson \& A. J. B. Humphreys, eds. Frontiers: Southern African Archaeology Today. Oxford: Archaeopress, pp. 266-276.

Thorp, C. (2009). Excavations at Hlamba Mlonga Hill, Malilangwe Trust, south-eastern Zimbabwe. Journal of African Archaeology, 7, 191-218.

Thorp, C. (2010). Induna Cave, southeastern Zimbabwe: a 'contact period' assemblage in a changing social landscape. Southern African Humanities, 22, 113-147.

Thunberg, C. P. (1788-1789). Resa uti Europa Africa, Asia, forrattad aren 1770-1779. Uppsala: Edman.
Thunberg, C. P. (1811). Mammalia Capensia, recensita et illustrata. Mémoires de l'Académie impériale des sciences de St. Pétersbourg, 3, 299-323.

Thunberg, C. P. (1820). Beskrifning och techning på ett nytt species, Hyaena Brunnea. Kungl. Svenska Vetenskapsakademiens Handlingar, Series 3, 8, 59-65.

Tobias, P. V. (1971). Human skeletal remains from the Cave of Hearths, Makapansgat, northern Transvaal. American Journal of Physical Anthropology, 34, 335-368.

Todd, N. E. (2005). Reanalysis of African Elephas recki: implications for time, space and taxonomy. Quaternary International, 126-128, 65-72.

Todd, N. E. (2010). New phylogenetic analysis of the Family Elephantidae based on cranialdental morphology. The Anatomical Record, 293, 74-90.

Toerien, M. J. (1952). The fossil hyaenas of the Makapansgat Valley. South African Journal of Science, 48, 293-300.

Toerien, M. J. (1955). A sabre-tooth cat from the Makapansgat valley. Palaeontologia Africana, $3,43-46$.

Tomes, R. F. (1861). Notes on a collection of bats made by Mr Andersson in the Damara country, south-western Africa, with notices of some other African species. Proceedings of the Zoological Society of London, 1861, 31-40.

Toussaint, M., Macho, G. A., Tobias, P. V., Partridge, T. C. \& Hughes, A. R. (2003). The third partial skeleton of a late Pliocene hominin (Stw 431) from Sterkfontein, South Africa. South African Journal of Science, 99, 215-223.

Trinkaus, E., Ruff, C. B. \& Conroy, G. C. (1999). The anomalous archaic Homo femur from Berg Aukas, Namibia: a biomechanical assessment. American Journal of Physical Anthropology, 110, 379-391.

Troussaert, E.-L. (1881). Catalogue des mammifères vivants et fossiles. Bulletin de la Société d'Etudes Scientifiques d'Angers, 10, 58-212.

Turner, A. (1984). The interpretation of variation in fossil specimens of spotted hyaena (Crocuta crocuta Erxleben, 1777) from Sterkfontein Valley sites (Mammalia: 
Carnivora). Annals of the Transvaal Museum, 33, 399-418.

Turner, A. (1986). Miscellaneous carnivore remains from Plio-Pleistocene deposits in the Sterkfontein Valley (Mammalia: Carnivora). Annals of the Transvaal Museum, 34, 203-226.

Turner, A. (1987a). New fossil carnivore remains from the Sterkfontein hominid site (Mammalia: Carnivora). Annals of the Transvaal Museum, 34, 319-347.

Turner, A. (1987b). Megantereon cultridens (Cuvier) (Mammalia, Felidae, Machairodontinae) from Plio-Pleistocene deposits in Africa and Eurasia, with comments on dispersal and the possibility of a New World origin. Journal of Paleontology, 61, 1256-1268.

Turner, A. (1988). On the claimed occurrence of the hyaenid genus Hyaenictis at Swartkrans (Mammalia: Carnivora). Annals of the Transvaal Museum, 34, 523-533.

Turner, A. (1993). New fossil carnivore remains from Swartkrans. In C. K. Brain, ed. Swartkrans. A cave's chronicle of early man. Transvaal Museum Monograph, 8, 151-165.

Turner, A. (1997). Further remains of Carnivora (Mammalia) from the Sterkfontein Hominid Site. Palaeontologia Africana, 34, 115-126.

Turner, A. \& Antón, M. (1996). The giant hyaena Pachycrocuta brevirostris (Mammalia, Carnivora, Hyaenidae). Geobios, 29, 455-468.

Turner, A., Bishop, L. C., Denys, C. \& McKee, J. K. (1999). Appendix: a locality-based listing of African Plio-Pleistocene mammals. In T. G. Bromage \& F. Schrenk, eds. African Biogeography, Climate Change, \& Human Evolution. New York: Oxford University Press, pp. 369-399.

Turner, G. (1984). Vertebrate remains from Lekkerwater. South African Archaeological Bulletin, 39, 106-108.

Turner, G. (1986). Faunal remains from Jubilee Shelter. South African Archaeological Bulletin, 41, 63-68.

Turner, G. (1987a). Hunters and herders of the Okavango delta, Botswana. Botswana Notes and Records, 19, 25-40.

Turner, G. (1987b). Early Iron Age herders in northwestern Botswana: the faunal evidence. Botswana Notes and Records, 19, 7-23.
Underhill, D. (2011). A history of Stone Age archaeological study in South Africa. South African Archaeological Bulletin, 66, 3-14.

Val, A. \& Stratford, D. J. (2015). The macrovertebrate fossil assemblage from the Name Chamber, Sterkfontein: taxonomy, taphonomy and implications for site formation processes. Palaeontologia Africana, 50, 1-17.

Val, A., Carlson, K. J., Steininger, C. M., et al. (2011). 3D techniques and fossil identification: an elephant shrew hemimandible from the Malapa site. South African Journal of Science, 107. DOI: 10.4102/sajs. v107i11/12.583 .

Val, A., Dirks, P. H. G., Backwell, L., D’Errico, F. \& Berger, L. R. (2014). Taphonomic analysis of the faunal assemblage associated with the hominins (Australopithecus sediba) from the Early Pleistocene cave deposits of Malapa, South Africa. PLoS ONE, 10. DOI: 10.1371/ journal.pone.0126904.

Van Aardt, A. C., Bousman, C. B., Brink, J. S., et al. (2016). First chronological, palaeoenvironmental and archaeological data from the Baden-Baden fossil spring complex in the western Free State, South Africa. Palaeoecology of Africa, 33, 117-152.

Van der Horst, C. J. (1944). Remarks on the systematics of Elephantulus. Journal of Mammalogy, 25, 77-82.

Van der Merwe, M. (2007). Discriminating between Thryonomys swinderianus and Thryonomys gregorianus. African Zoology, 42, 165-171.

Van der Ryst, M. M. (2006). Seeking shelter: Later Stone Age hunters, gatherers and fishers of Olieboomspoort in the western Waterberg, south of the Limpopo. Unpublished PhD thesis, University of the Witwatersrand.

Van Doornum, B. (2007). Tshisiku Shelter and the Shashe-Limpopo confluence area huntergatherer sequence. Southern African Humanities, 19, 17-67.

Van Ewyk, J. F. (1987). The prehistory of an Iron Age site on Skutwater. Unpublished MA thesis, University of Pretoria.

Van Hoepen, E. C. N. (1930a). Vrystaatse fossiele perde. Paleontologiese Navorsinge van die Nasionale Museum Bloemfontein, 2, 1-11. 
Van Hoepen, E. C. N. (1930b). Fossiele perde van Cornelia, O.V.S. Paleontologiese Navorsinge van die Nasionale Museum Bloemfontein, 2, 13-34.

Van Hoepen, E. C. N. (1932a). Die stamlyn van die Sebras. Paleontologiese Navorsinge van die Nasionale Museum Bloemfontein, 2, 25-37.

Van Hoepen, E. C. N. (1932b). Voorlopige beskrywing van Vrystaatse soogdiere.

Paleontologiese Navorsinge van die Nasionale Museum Bloemfontein, 2, 63-65.

Van Hoepen, E. C. N. (1947). A preliminary description of new Pleistocene mammals of South Africa. Paleontologiese Navorsinge van die Nasionale Museum Bloemfontein, 2, 103-106.

Van Hoepen, E. C. N. \& Van Hoepen, H. E. (1932). Vrystaatse wilde varke. Paleontologiese Navorsinge van die Nasionale Museum Bloemfontein, 2, 39-62.

Van Neer, W. \& Breunig, P. (1999). Contribution to the archaeozoology of the Brandberg. Cimbebasia, 15, 127-140.

Van Noten, F. L. (1974). Excavations at the Gordon's Bay Shell Midden, South-Western Cape. South African Archaeological Bulletin, 29, 122-142.

Van Pletzen, L. (2000). The large mammal fauna from Klasies River. Unpublished MA thesis, University of Stellenbosch.

Van Schalkwyk, J., Pelser, A. \& Teichert, F. (1999). Archaeological investigation of a Late Iron Age Tswana settlement on the farm Hoekfontein 432JQ, Odi District, North West Province. National Cultural History Museum Unpublished Report No. 98KH21.

Van Schalkwyk, J. A. (2000). Excavation of a Late Iron Age site in the Makgabeng, Northern Province. Southern African Field Archaeology, 9, 75-82.

Van Staaden, M. J. (1994). Suricata suricatta. Mammalian Species, 483, 1-8.

Van Waarden, C. (1987). Matanga, a Late Zimbabwe cattle post. South African Archaeological Bulletin, 42, 107-124.

Van Zyl, W. J., Badenhorst, S., Taljaard, E., Denbow, J. R. \& Wilmsen, E. N. (2013). The archaeofauna from Xaro on the Okavango Delta in northern Botswana. Annals of the Ditsong National Museum of Natural History, 3, 49-58.
Van Zyl, W. J., Badenhorst, S. \& Brink, J. (2016). Pleistocene Bovidae from X Cave on Bolt's Farm in the Cradle of Humankind in South Africa. Annals of the Ditsong National Museum of Natural History, 6, 39-73.

Verheyen, W. N., Huiselmans, J. L. J., Dierckx, T., et al. (2003). A craniometric and genetic approach to the systematics of the genus Dasymys Peters, 1875, selection of a neotype and description of three new taxa (Rodentia, Muridae, Africa). Bulletin de L'Institut Royal des Sciences Naturelles de Belgique, Biologie, 73, 27-71.

Veyrunes, F., Britton-Davidian, J., Robinson, T. J., et al. (2005). Molecular phylogeny of the African pygmy mice, subgenus Nannomys (Rodentia, Murinae, Mus): implications for chromosomal evolution. Molecular Phylogenetics and Evolution, 36, 358-369.

Viret, J. (1939). Monographie paléontologique de la faune de vertébrés des Sables de Montpellier. III. Carnivora, Fissipedia. Travaux du Laboratoire de Géologie de la Faculté des Sciences de Lyon, 37, 5-26.

Viriot, L., Pelaez-Campomanes, P., Vignaud, P., et al. (2011). A new Xerinae (Rodentia, Sciuridae) from the Late Miocene of TorosMenalia (Chad). Journal of Vertebrate Paleontology, 31, 844-848.

Visser, D. S. \& Robinson, T. J. (1986). Cytosystematics of the South African Aethomys (Rodentia: Muridae). South African Journal of Zoology, 21, 264-268.

Vogelsang, R., Richter, J., Jacobs, Z., et al. (2010). New excavations of Middle Stone Age deposits at Apollo 11 Rockshelter, Namibia: stratigraphy, archaeology, chronology and past environments. Journal of African Archaeology, 8, 185-218.

Voigt, E. A. (1979). The faunal remains from Icon. South African Archaeological Society Goodwin Series, 3, 80-85.

Voigt, E. A. (1980a). Appendix. the faunal sample from Msuluzi Confluence. In T. Maggs. Msuluzi Confluence: a seventh century Early Iron Age site on the Tugela River. Annals of the Natal Museum, 24, 140-145. 
Voigt, E. A. (1980b). Reconstructing Iron Age economies of the Northern Transvaal: a preliminary report. South African Archaeological Bulletin, 35, 39-45.

Voigt, E. A. (1980c). Appendix 1: mammalian remains from Mzonjani. In T. Maggs. Mzonjani and the beginning of the Iron Age in Natal. Annals of the Natal Museum, 24, 94-95.

Voigt, E. A. (1982). Appendix I: faunal report on the Lydenberg Heads Site, 2539 AB4. In T. M. Evers. Excavations at the Lydenburg Heads Site, Eastern Transvaal, South Africa. South African Archaeological Bulletin, 37, 31-32.

Voigt, E. A. (1983). Mapungubwe: an Archaeozoological Interpretation of an Iron Age Community. Pretoria: Transvaal Museum.

Voigt, E. A. (1984). The faunal remains from Magogo and Mhlopeni: small stock herding in the Early Iron Age of Natal. Annals of the Natal Museum, 26, 141-163.

Voigt, E. A. \& Peters, J. H. (1994a). Appendix 2: the faunal assemblage from Wosi in the Thukela Valley. In L. van Schalkwyk. Wosi: an Early Iron Age village in the lower Thukela Basin, Natal. Natal Museum Journal of Humanities, 6, 105-117.

Voigt, E. A. \& Peters, J. H. (1994b). Appendix: the faunal assemblage from the Early Iron Age site of Mamba 1 in the Thukela Valley. In L. van Schalkwyk. Mamba confluence: a preliminary report on an Early Iron Age industrial centre in the lower Thukela Basin, Natal. Natal Journal of Humanities, 6, 145-152.

Voigt, E. A. \& Plug, I. (1981). Early Iron Age Herders of the Limpopo Valley. Pretoria: Transvaal Museum.

Voigt, E. A. \& Plug, I. (1984). Happy Rest: the earliest Iron Age from the Soutpansberg. South African Journal of Science, 80, 221-227.

Voigt, E. A. \& Von den Driesch, A. (1984). Preliminary report on the faunal assemblage from Ndondondwane, Natal. Annals of the Natal Museum, 26, 95-104.

Voigt, E. A., Plug, I. \& Sampson, C. G. (1995). European livestock from rock shelters in the upper Seacow valley. Southern African Field Archaeology, 4, 37-49.

Von den Driesch, A. \& Deacon, H. J. (1985). Sheep remains from Boomplaas, South Africa.
South African Archaeological Bulletin, 40, 39-44.

Von Dueben, W. (1846). Om Nesotragus moschatus n. sp. In C. J. Sundevall. Nya Mammalia, från Sydafrika. Öfversigt af Kongl. Vetenskaps-akademiens forhandlingar, Series 3, 1846, 221-222.

Von Mayer, A. (1998). A reassessment of Cercopithecoides in southern Africa. Unpublished MSc thesis, University of the Witwatersrand.

Von Richter, W. (1974). Connochaetes gnou. Mammalian Species, 50, 1-6.

Vrba, E. S. (1970). Evaluation of springbok-like fossils: measurement and statistical treatment of the teeth of the springbok Antidorcas marsupialis Zimmerman (Artiodactyla: Bovidae). Annals of the Transvaal Museum, 26, 285-299.

Vrba, E. S. (1971). A new fossil alcelaphine (Artiodactyla: Bovidae) from Swartkrans. Annals of the Transvaal Museum, 27, 59-82.

Vrba, E. S. (1973). Two new species of Antidorcas Sundevall at Swartkrans (Mammalia: Bovidae). Annals of the Transvaal Museum, 28, 287-352.

Vrba, E. S. (1974a). Chronological and ecological implications of the fossil Bovidae at the Sterkfontein australopithecine site. Nature, 250, 19-23.

Vrba, E. S. (1974b). The fossil Bovidae of Sterkfontein, Swartkrans and Kromdraai. Unpublished PhD thesis, University of Cape Town.

Vrba, E. S. (1976). The Fossil Bovidae of Sterkfontein, Swartkrans and Kromdraai. Memoir of the Transvaal Museum, 21.

Vrba, E. S. (1977). New species of Parmularius Hopwood and Damaliscus Sclater (Alcelaphini, Bovidae, Mammalia) from Makapansgat. Palaeontologia Africana, 20, 137-151.

Vrba, E. S. (1978). Problematical alcelaphine fossils from the Kromdraai faunal site (Mammalia, Bovidae). Annals of the Transvaal Museum, 31, 21-28.

Vrba, E. S. (1981). The Kromdraai australopithecine site revisited in 1980: recent investigations and results. Annals of the Transvaal Museum, 33, 17-60. 
Vrba, E. S. (1987a). A revision of the Bovini (Bovidae) and a preliminary revised checklist of Bovidae from Makapansgat (Transvaal: South Africa). Palaeontologia Africana, 26, 33-46.

Vrba, E. S. (1987b). New species and a new genus of Hippotragini (Bovidae) from Makapansgat Limeworks. Palaeontologia Africana, 26, 47-58.

Vrba, E. S. (1997). New fossils of Alcelaphini and Caprinae (Bovidae: Mammalia) from Awash, Ethiopia and phylogenetic analysis of Alcelaphini. Palaeontologia Africana, 34, 127-198.

Vrba, E. S. \& Panagos, D. C. (1978). A new limestone cave breccia from Vlakplaats near Pretoria. Annals of the Transvaal Museum, 31, 177-183.

Wadley, L. (1979). Big Elephant Shelter and its role in the Holocene prehistory of central South West Africa. Cimbebasia B, 3, 1-76.

Wadley, L. (2001). Who lived in Mauermanshoek Shelter, Korannaberg, South Africa? African Archaeological Review, 18, 153-179.

Wadley, L. (2015). Those marvellous millennia: the Middle Stone Age of Southern Africa. Azania, 50, 155-226.

Wadley, L. \& Laue, G. (2000). Adullam Cave, eastern Free State, South Africa: test excavations at a multiple-occupation Oakhurst Industry site. Natal Journal of Humanities, 12, 1-13.

Wadley, L. \& McLaren, G. (1998). Tandjesberg Shelter, eastern Free State, South Africa. Natal Museum Journal of Humanities, 10, 19-32.

Wadley, L. \& Turner, G. (1987). Hope Hill Shelter: a Later Stone Age site in the southern Transvaal. South African Journal of Science, 83, 98-105.

Wadley, L., Plug, I. \& Clark, J. L. (2008). The contribution of Sibudu fauna to an understanding of KwaZulu-Natal environments at $\sim 60 \mathrm{ka}, \sim 50 \mathrm{ka}$ and $\sim 37 \mathrm{ka}$. In S. Badenhorst, P. Mitchell \& J. C. Driver, eds. Animals and People: Archaeozoological Papers in Honour of Ina Plug. Oxford: Archaeopress, pp. 34-45.

Wagler, J. G. (1832). Mittheilungen über werkwürbige Thiere. Isis von Oken, 25, 275.
Wagner, J. A. (1839). Ueber die Berwandtschafts = Berhältnisse der Pharaonstratte. Gelehrte Anzeigen Königlich Bayerische Akademie der Wissenschaften zu München, 9, cols 425-432.

Wagner, J. A. (1841). Gruppirung der Gattungen der Nager. Gelehrte Anzeigen Königlich Bayerische Akademie der Wissenschaften zu München, 12, cols 433-440.

Wagner, J. A. (1843). Malacothrix. Die Didmaus. In Die Säugthiere in Abbildungen nach der Natur von Dr Johann Christian Daniel von Schreber, Supplement 3. Erlangen: Expedition des Schreber'schen säugthier- und des Esper'schen Schmetterlingswerkes, pp. 496-499.

Wagner, J. A. (1845). Diagnosen einiger neuen Arten von Nagern und Handflüglern. Archiv für Naturgeschichte, 11, 145-149.

Wagner, J. A. (1855). Antilope strepsicerinae. Schrauben-Antipolen. In Die Säugthiere in Abbildungen nach der Natur, mit Beschreibungen von Dr Johann Christian Daniel von Schreber, Vol. 5. Leipzig: Weigel, pp. $438-461$

Walker, N. (1994). The Late Stone Age of Botswana: some recent excavations. Botswana Notes and Records, 26, 1-35.

Walker, N. J. (1983). The significance of an early date for pottery and sheep in Zimbabwe. South African Archaeological Bulletin, 38, 88-92.

Waterhouse, G. R. (1837). [A species of mouse from the Cape of Good Hope]. Proceedings of the Zoological Society of London, 2, 104-105.

Watson, E. J. \& Watson, V. (1990). Of commoners and kings: faunal remains from Ondini. South African Archaeological Bulletin, $45,33-46$.

Watson, V. (1993a). Composition of the Swartkrans bone accumulations, in terms of skeletal parts and animals represented. In C. K. Brain, ed. Swartkrans: A cave's chronicle of early man. Transvaal Museum Monograph 8, 35-73.

Watson, V. (1993b). Glimpses from Gondolin: a faunal analysis of a fossil site near Broederstroom, Transvaal, South Africa. Palaeontologia Africana, 30, 35-42.

Watson, V. \& Plug, I. (1995). Oreotragus major Wells and Oreotragus oreotragus (Zimmerman) 
(Mammalia: Bovidae): two species? Annals of the Transvaal Museum, 36, 183-191.

Webb, G. L. (1965). Notes on some chalicothere remains from Makapansgat. Palaeontologia Africana, 9, 49-68.

Webb, P. I. \& Skinner, J. D. (1995). The dormice (Myoxidae) of southern Africa. Hystrix, New Series, 6, 287-293.

Webley, L. (1992a). Early evidence for sheep from Spoeg River Cave, Namaqualand. Southern African Field Archaeology, 1, 3-13.

Webley, L. E. (1992b). The history and archaeology of pastoralist and huntergatherer settlement in the north-western Cape, South Africa. Unpublished PhD thesis, University of Cape Town.

Webley, L. (2001a). Excavations at /hei-/khomas (Vaalhoek) in the Richtersveld, Northern Cape. Southern African Field Archaeology, 10, 46-74.

Webley, L. (2001b). The re-excavation of Spoegrivier Cave on the West Coast of South Africa. Annals of the Eastern Cape Museums, 2, 19-49.

Webley, L., Archer, F. \& Brink, J. (1993). Die Toon: a Late Holocene site in the Richtersveld National Park, northern Cape. Koedoe, 36, 1-9.

Weithofer, K. A. (1889). Ueber die tertiären Landsäugethiere Italiens. Jahrbuch der Kaiserlich Königlichen Geologischen Reichsanstalt, 39, 55-82.

Welbourne, R. (1973). Identification of animal remains from the Broederstroom 24/73 Early Iron Age site. South African Journal of Science, 69, 325.

Welbourne, R. G. (1974). Appendix C: animal remains from the Harmony 24 Iron Age sites. In T. M. Evers. Three Iron Age industrial sites in the Eastern Transvaal Lowveld. Unpublished MA thesis, University of the Witwatersrand, pp. 100-104.

Welbourne, R. G. (1975). Tautswe Iron Age Site: its yield of bones. Botswana Notes and Records, 7, 1-16.

Welbourne, R. G. (1979). Animals remains from Harmony Salt Factory, Northern Transvaal. South African Archaeological Society Goodwin Series, 3, 108.
Welbourne, R. G. (1985). Faunal analysis. In P. J. J. Sinclair. Excavations at Chivowa Hill, south central Zimbabwe. Zimbabwea, 9, 38-44.

Welbourne, R. G. (1988). Appendix 1: an analysis of the animal remains recovered from Rose Cottage Cave. In J. Kohary, ed. Rose Cottage Cave. Johannesburg: Archaeological Research Unit, University of the Witwatersrand, pp. 133-140.

Wells, C. R. (2006). A sample integrity analysis of faunal remains from the RSp layer at Sibudu Cave. Southern African Humanities, 18, 261-277.

Wells, L. H. (1940). A fossil horse from Koffiefontein, O.F.S. Transactions of the Royal Society of South Africa, 28, 301-306.

Wells, L. H. (1951). Large fossil klipspringer from Potgietersrust. South African Journal of Science, 47, 167-168.

Wells, L. H. (1959a). The nomenclature of South African fossil equids. South African Journal of Science, 55, 64-66.

Wells, L. H. (1959b). The Quaternary giant hartebeests of South Africa. South African Journal of Science, 55, 123-128.

Wells, L. H. (1959c). Mammalian fossils from Barkly West in the Natal Museums, Pietermaritzburg. South African Journal of Science, 55, 146.

Wells, L. H. (1960). Mammalian remains from Late Stone Age sites in the George-Knysna area. South African Journal of Science, 56, 306.

Wells, L. H. (1964). A large extinct antelope skull from the Younger Gravels at Sydney-on-Vaal, C.P. South African Journal of Science, 60, 88-91.

Wells, L. H. (1965). Antelopes in the Pleistocene of southern Africa. Zoologica Africana, 1, $115-120$.

Wells, L. H. (1970a). The fauna of the Aloes Bone Deposit: a preliminary note. South African Archaeological Bulletin, 25, 22-23.

Wells, L. H. (1970b). A Late Pleistocene faunal assemblage from Driefontein, Cradock District, C.P. South African Journal of Science, 66, 59-61.

Wells, L. H. (1988). Pre-occupation microfauna of the Cave of Hearths. In R. Mason, ed. Cave of Hearths, Makapansgat, Transvaal. Johannesburg: Archaeological Research Unit, 
University of the Witwatersrand, pp. 549-550.

Wells, L. H. \& Cooke, H. B. S. (1955). Fossil remains from Chelmer, near Bulawayo, S. Rhodesia: a further note. South African Journal of Science, 52, 49.

Wells, L. H. \& Cooke, H. B. S. (1957). Fossil Bovidae from the Limeworks Quarry, Makapansgat, Potgietersrus. Palaeontologia Africana, 4, 1-55.

Wells, L. H. \& Gear, J. H. (1931). Skeletal material from early graves in the Riet river valley. South African Journal of Science, 28, 435-443.

Wells, L. H., Cooke, H. B. S. \& Malan, B. D. (1942). The associated fauna and culture of the Vlakkraal Thermal Springs, O.F.S. Transactions of the Royal Society of South Africa, 29, 203-233.

Werdelin, L. \& Cote, S. M. (2010). Prionogalidae (Mammalia, Incertae Sedis). In L. Werdelin \& W. J. Sanders, eds. Cenozoic Mammals of Africa. Berkeley, CA: University of California Press, pp. 561-562.

Werdelin, L. \& Lewis, M. E. (2001). A revision of the genus Dinofelis (Mammalia, Felidae). Zoological Journal of the Linnean Society, 32, 147-258.

Werdelin, L. \& Peigné, S. (2010). Carnivora. In L. Werdelin \& W. J. Sanders, eds. Cenozoic Mammals of Africa. Berkeley, CA: University of California Press, pp. 603-657.

Werdelin, L. \& Sanders, H. L. (2010). Cenozoic Mammals of Africa. Berkeley, CA: University of California Press.

Werdelin, L. \& Sardella, R. (2006). The Homotherium from Langebaanweg, South Africa and the origin of Homotherium. Palaeontographica Abt A, 277, 123-130.

Werdelin, L. \& Solounias, N. (1991). The Hyaenidae: taxonomy, systematics and evolution. Fossils and Strata, 30, 1-104.

Werdelin, L., Turner, A. \& Solounias, N. (1994). Studies of fossil hyaenids: the genera Hyaenictis Gaudry and Chasmaporthetes Hay, with a reconsideration of the Hyaenidae of Langebaanweg, South Africa. Zoological Journal of the Linnean Society, 111, 197-217.

Weston, E. \& Boisserie, J.-R. (2010).

Hippopotamidae. In L. Werdelin \& W. J.
Sanders, eds. Cenozoic Mammals of Africa. Berkeley, CA: University of California Press, pp. 853-871.

Whitelaw, G. (1994). KwaGandaganda: settlement patterns in the Natal Early Iron Age. Natal Journal of Humanities, 6, 1-64.

Whitworth, T. (1958). Miocene ruminants of East Africa. Fossil Mammals of Africa, 15, 1-50.

Wilkinson, A. F. (1976). The lower Miocene Suidae of Africa. Fossil Vertebrates of Africa, 4, 173-282.

Williams, B. A., Ross, C. A., Frost, S. R., et al. (2012). Fossil Papio cranium from !Ncumtsa (Koanaka) Hills, western Ngamiland, Botswana. American Journal of Physical Anthropology, 149, 1-17.

Willows-Munro, S. \& Matthee, C. A. (2009). The evolution of the southern African members of the shrew genus Myosorex: understanding the origin and diversification of a morphologically cryptic group. Molecular Phylogenetics and Evolution, 51, 394-398.

Willows-Munro, S., Robinson, T. J. \& Matthee, C. A. (2005). Utility of nuclear DNA intron markers at lower taxonomic levels: phylogenetic resolution among nine Tragelaphus spp. Molecular Phylogenetics and Evolution, 35, 624-636.

Wilmsen, E. N. (1989). The antecedents of contemporary pastoralism in western Ngamiland. Botswana Notes and Records, 20, 29-39.

Wilson, D. E. \& Reeder, D. M. (2005). Mammal Species of the World, 3rd edition. Baltimore, MD: Johns Hopkins University Press.

Wilson, M. L. (1988). Forest Hall Shelter: an early excavation on the southern Cape coast. South African Archaeological Bulletin, 43, 53-55.

Wood, A. E. (1968). Early Cenozoic Mammalian faunas, Fayum Province, Egypt. Part II: the African Oligocene Rodentia. Bulletin of the Peabody Museum of Natural History, Yale University, 28, 23-105.

Woodward, A. S. (1921). A new cave man from Rhodesia, South Africa. Nature, 108, 371-372.

Wroughton, R. C. (1905). Notes on the various forms of Arvicanthis pumilio Sparrm. Annals and Magazine of Natural History, Series 7, 16, 629-639. 
Wroughton, R. C. (1906). Notes on the genus Otomys. Annals and Magazine of Natural History, Series 7, 18, 264-278.

Wroughton, R. C. (1909). New species of Dendromus \& Tatera. Annals and Magazine of Natural History, Series 8, 3, 246-249.

Yellen, J. E., Brooks, A. S., Stuckenrath, R. \& Welbourne, R. (1987). A Terminal Pleistocene assemblage from Drotsky's Cave, western
Ngamiland, Botswana. Botswana Notes and Records, 19, 1-6.

Zdansky, O. (1924). Jungtertiäre Carnivoren Chinas. Palaeontologia Sinica Series C, 2, 1-149.

Zimmermann, J. C. (1779-1783). Geographische Geschichte des Menschen und der Allgemein Verbreiteten Vierfüssigen Thiere. Leipzig: Weygandschen. 


\section{Index}

!Ncumtsa Hills, 123

/hei-/khomas, 183

$\neq \mathrm{Gi}, 123$

2229AD5, 183

2329CD, 183

Aar I and II, 183

Abbot's Cave, 183

Abenab, 123

Abrahamskraal, 123

Aceratatherium, 32

Acinonyx, 54, 68

Acinonyx jubatus, 69, 100, 127, 129, 139, 141, 167, 187, 196-197, 215

Acomys, 46, 69, 84, 123, 125-126, 132, 134, 143

Acomys mabele, 46, 69

Acomys spinosissimus, 46, 70, 84, 124, 128, 130, 140-141

Acomys subspinosus, 84, 124-125, 127, 132-133, 153, 184, 186, 191, 197, 209

Acronotus, 115, 177

Adelphailurus, 56

Adullam, 184

Aepyceros helmoedi, 113, 127

Aepyceros melampus, 113, 124-127, 129-130,

132-134, 136-139, 141, 143, 176, 183-196, 198-215, 217

Aepycerotinae, 113, 176

Aethomys, 48, 85, 87, 154

Aethomys adamanticola, 47, 69

Aethomys chrysophilus, 85, 123, 125-126, 128,

130, 137, 140-142, 154, 185-186, 189-191,

195-196, 199-201, 204-205, 215

Aethomys modernis, 47, 69

Afaromys guillemoti, 20, 37

Africanictis hyaenoides, 29, 35

Africanictis meini, 29, 35

Africanictis schmidtkittleri, 29, 35

Afrochoerodon kisumuensis, 16, 36
Afrocricetodontinae, 19

Afrohyrax namibiensis, 15, 36-37

Afromastodon coppensi, 16, 35

Afrosmilus africanus, 28, 35-37

Afrosoricida, 6, 12, 39, 71, 145, 221

Agnotherium, 30, 37

Agriotherium africanum, 59, 69, 220

Aigamas I and II, 123

Aigoceros, 67, 121, 181

Alcelaphinae, 34, 63, 114, 176

Alcelaphus, 114, 125-126, 129, 143, 176, 188, 191-192, 199, 214-215

Alcelaphus buselaphus, 114, 124-134, 136, 138-139, 142, 176, 183-191, 193, 195-200, 203-204, 208-216

Alcelaphus caama, 63, 69, 114, 123, 126, 130, 133-135, 137, 142, 176, 193, 202, 205, 207, 217

Alcelaphus robustus, 64, 69, 114, 126, 134

Aloes Bone Deposit, 123

Amblysomus, 72, 140

Amblysomus hamiltoni, 39, 69

Amblysomus hottentotus, 72, 125, 133, 146, 186, 188, 192, 201, 203-205, 210, 215

Amblysomyinae, 39, 72, 146

Amebelodontinae, 16

Amis, 123, 184

Amphechinus rusingensis, 26, 35, 37

Amphicyon giganteus, 30, 35

Amphicyonidae, 30

Amphicyoninae, 30

Amphimachairodus, 54, 69

Amphiorycteropus, 14, 35, 38

Anancinae, 16, 42

Anancus capensis, 42, 69

Anancus kenyensis, 16, 37-38

Ancylotherium, 60, 70

Andriesgrond, 123, 184

Andrieskraal, 184 
Anomaluromorpha, 9, 21, 50, 91, 159

Anthracotheriidae, 11, 33

Antidorcas, 116, 123-124, 126-128, 134-135, 138, 143

Antidorcas australis, 116, 127-129, 132-133, 135, 139, 141, 178, 183, 185, 187, 190, 194, 197-199, 203-204, 206, 209-210, 214, 216, 218

Antidorcas bondi, 65, 68, 116, 124-133, 135-138, 140-141, 143, 178, 183, 194, 198, 210

Antidorcas marsupialis, 116, 123-127, 129-143, 178, 184-200, 202-204, 206-208, 210-218

Antidorcas recki, 65, 68, 116, 127-131, 137, 140, 143

Antilope, 63, 114, 118, 121, 176, 181

Antilopinae, 35, 65, 116, 178

Anyskop Blowout, 123

Aonyx, 108

Aonyx capensis, 108, 124, 129-130, 132-133, 135, 137-139, 141, 173, 188-190, 192, 197-199, 203, 205, 209-210, 214, 216-217

Apodecter, 24, 36-37

Apodecter stromeri, 10-11, 24, 35-37

Apollo 11, 123, 184

Archidiskodon, 41, 75, 148

Areb, 61, 68

Arenagale calcareus, 7, 11

Arrisdrift, 12, 14-16, 19, 22-23, 25-26, 28-32, 34-35

Arsinoitheriidae, 7

Artiodactyla, 11, 32, 61, 111, 175, 221

Arvicanthis, 85, 134, 140

Asellia, 26, 36, 220

Asis Ost, 123

Aspoort, 184

Atelerix frontalis, 94, 124, 127-129, 137, 162, 186, 191-192, 194, 212-215, 218

Atelerix major, 94

Atilax mesotes, 56, 69, 103, 135, 141

Atilax paludinosus, 103, 127, 129-130, 132-133, $135,137,139,141,168,183-184,188-190$, 192-194, 196, 198-201, 203-205, 207, 209-211, 214-217

Atlantic Beach, 184

Auchas, 16, 36

Austerlitz, 184

Austin's Rush, 123

Australopithecus, 44, 68, 80, 139

Australopithecus africanus, 1, 44, 69-70, 79, 127, 130, 140
Australopithecus sediba, 79, 135

Austrolagomys hendeyi, 25, 35

Austrolagomys inexpectatus, 25, 36-37

Aves Cave 168

Awasib, 68

Baard's Quarry, 124

Baden-Baden, 184

Badfontein Valley, 184

Baken, 36

Bambata, 184

Barbourofelidae, 28

Barbourofelinae, 28

Barkly West, 124

Bathyergidae, 23, 25, 50, 91, 159

Bathyerginae, 23, 50, 91, 159

Bathyergoides, 9, 11, 23, 35

Bathyergoides neotertiarius, 23, 36-37

Bathyergoididae, 9, 23

Bathyergus, 91, 141

Bathyergus hendeyi, 50, 69

Bathyergus janetta, 91, 124, 159, 209, 212

Bathyergus suillus, 91, 123-124, 127-128, 130, 132-133, 135-138, 140-141, 143, 159, 184, 186-187, 189-192, 194, 196-198, 207-210, 212-214, 216-217

Beatragus, 114, 141, 220

Berg Aukas, 36, 124

Berg Aukas I, 15, 17, 21

Bestpan, 124

Biesje Poort 2, 184

Big Elephant Shelter, 185

Blaauheuvel, 185

Black Crow, 6-9, 11

Black Earth Cave, 124

Blinkklipkop, 185

Bloembos, 124

Bloemhof, 124

Bloemhof Site, 124

Blombos Cave, 124, 185

Bloubergstrand, 185

Bloubos, 185

Blue Pool Cave, 185

Blydefontein, 185

Boegoeberg 1, 124

Bogenfels, 36

Bohrloch des Betriebes 4, 36

Boitsemangano, 185

Bokbaai, 185

Bokvasmaak 3, 185

Bolahla, 186 
Boleu, 186

Boltimys broomi, 49, 70

Bolt's Farm, 40, 47, 72, 86-87, 94, 103, 109, 124

Bonawe, 186

Boomplaas Cave, 125, 186

Border Cave, 125, 186

Borrow Pit Midden, 186

Bos, 66, 119, 179

Bos makapani, 118, 126

Bos taurus, 179, 183-217, 221

Boselaphus, 118

Boskop, 126

Bosluis Pan, 36

Bosutswe, 186

Bothriogenys gorringei, 11

Boundary Shelter, 187

Bovidae, 34, 63, 113, 176, 220

Bovinae, 35, 65, 118, 179

Brachygnathopithecus peppercorni, 43, 78

Brachyodus, 33

Brachyodus aequatorialis, 33, 37

Brachyodus depereti, 33, 36-37

Brachypotherium, 31, 36

Brachypotherium heinzelini, 31, 37

Brachyrhynchocyon, 14

Brachyuromys, 18

Brakfontein, 126

Bremen, 187

Brevirhynchocyon gariepensis, 14

Brevirhynchocyon jacobi, 14, 35-36

Broederstroom 24/73, 187

Bubalis, 66, 115, 118, 179

Bubalus, 63, 114-115, 176-177

Buffalo Cave, 118, 126

Buffelshoek, 187

Bulawayo, 126

Bundu Farm, 126

Bunolagus monticularis, 93, 125, 161, 187

Burchell's Shelter, 187

Bushman Hill, 68

Bushman Rockshelter, 126, 187

Buzz Shelter, 187

Byneskranskop, 188

CaeCae, 188

Cainochoerus africanus, 61, 69

Calabria 630, 126

Cango Caves, 126

Canidae, 59, 106, 172

Caniformia, 30, 59, 106, 172
Caninae, 59, 106, 172

Canis, 106, 123-124, 128, 136, 172, 187-189, 191, 193-194, 196-197, 203-204, 206-207, 209, 211, 213-214, 216

Canis adustus, 106, 124, 172, 197, 200, 208

Canis antiquus, 106, 134, 140

Canis atrox, 106, 134

Canis lupus, 172, 183, 185-186, 188-189, 191-210, 212-215, 217-218, 221

Canis mesomelas, 59, 68, 70, 107, 124-137, 139-143, 172, 183-206, 208-217

Canteen Koppie, 126

Cape of Good Hope, 58

Cape Point, 188

Cape St Francis, 188

Cape Town, 188

Capra hircus, 180, 183-191, 194-217, 221

Caprinae, 66, 119, 180

Caracal caracal, 55, 68-69, 100, 123-129, 132-133, 135-137, 139, 141-143, 167, 183-184, 186-189, 191-192, 194-205, 207-214, 216-217

Carnivora, 28, 54, 100, 167, 221

Catoblepas, 64, 115, 177

Cave of Hearths, 126

Cephalophinae, 67, 120, 181

Cephalophus, 66, 69, 120, 130, 181, 206

Cephalophus natalensis, 120, 138-139, 181, 188, 203-204, 211, 214-215, 217

Cephalophus parvus, 67, 70

Ceratotherium, 31, 61, 124

Ceratotherium simum, 61, 69, 110, 123, 127-133, $135,137,139,141,143,175,186,209$, 212-213

Cercocebus, 42, 69, 220

Cercopithecidae, 17, 42, 76, 148

Cercopithecinae, 42, 76, 148

Cercopithecoides, 128

Cercopithecoides haasgati, 78, 131

Cercopithecoides williamsi, 44, 69-70, 78, 124, 127, 130-131, 140-141

Cercopithecus, 76, 148, 215

Cercopithecus albogularis, 76, 139

Cervicapra, 122, 183

Chalicotheriidae, 60

Chamabvefva, 188

Chapattimyidae, 8

Chasmaporthetes, 57, 104

Chasmaporthetes australis, 57, 69

Chasmaporthetes nitidula, 57, 68, 104, 127-128, 131, 140-141 
Chelmer, 65, 116, 126, 178

Chibuene, 188

Chilotheridium pattersoni, 31, 35, 37

Chiroptera, 26, 53, 96, 164, 220-221

Chivowa Hill, 188

Chlorocebus, 149, 200

Chlorocebus aethiops, 76, 125, 137, 139, 149, 188-189, 192, 196, 198-199, 201-202, 204-205, 208-210, 212, 214, 217

Chlorocebus pygerythrus, 76, 139, 142, 149, 203, 211, 215

Chlorotalpa duthiae, 71, 124, 133

Chlorotalpa sclateri, 71, 125, 140, 143, 145, 184, 186, 191, 197, 209

Chlorotalpa spelea, 71, 140

Choerolophodon, 16

Choerolophodon pygmaeus, 35-36

Choerolophodontinae, 16

Christiana, 126

Chrysochloridae, 7, 12, 39, 71, 145

Chrysochloridea, 7, 12, 39, 71, 145

Chrysochlorinae, 39, 71, 145

Chrysochloris arenosa, 39, 69

Chrysochloris asiatica, 71, 127, 138, 143, 145, 189, 191, 209, 213

Chrysochloris bronneri, 39, 69

Chrysochloris villosa, 72

Chrysospalax, 72, 134, 143

Chrysospalax villosus, 72, 140-141, 145, 188, 192, 203, 205, 215

Chrysosticha, 39

Cimolesta, 6, 221

Cistugo lesueuri, 99, 186

Civettictis braini, 105

Civettictis civetta, 171, 186, 196-198, 206, 215

Clanwilliam, 188

Clarke's Shelter, 188

Claviglis, 80

Climacoceras, 32

Climacoceratidae, 32

Collingham, 188

Colobinae, 17, 44, 78

Colwinton, 188

Commando Kop, 189

Connies Limpet Bar, 189

Connochaetes, 114-115, 123-124, 126, 128, 131, 133-135, 137, 142-143, 177, 190, 196, 199-202, 206, 208

Connochaetes antiquus, 114, 130, 135, 138
Connochaetes gnou, 114, 124-127, 129-143, 177, 183-184, 187-190, 194-196, 198-200, 202, 204-208, 210-211, 214, 216

Connochaetes laticornutus, 114, 177

Connochaetes taurinus, 64, 68-69, 115, 123-131, 133-142, 177, 183, 185-187, 189-192, 194-195, 197-215, 217

Cooper's, 101, 107, 127

Copper Queen Mine, 189

Cornelia, 31, 60, 110-111, 113, 127

Creodonta, 10, 27, 221

Cricetodontinae, 20

Cricetomyinae, 19, 81, 151

Cricetomys ansorgei, 81, 138-139

Crocidura, 94, 123-124, 132, 136, 138, 162, 184, 190, 200, 203, 206, 212

Crocidura cyanea, 94, 124-125, 128, 132-133, $136,138,143,163,183-186,188,190-191$, 197, 199, 201-203, 205, 209-210, 212-213, 217-218

Crocidura flavescens, 95, 123-125, 127-129, 133, 136, 138, 142-143, 163, 183-184, 186-192, 197, 201-203, 205, 209-210, 212-215, 217

Crocidura fuscomurina, 52, 70, 95, 124-125, 130, 143, 163, 184-186, 196, 199, 217

Crocidura hirta, 95, 125, 128, 143, 163, 185-186, 196, 199, 202, 217-218

Crocidura mariquensis, 95, 125, 163, 186, 196

Crocidura silacea, 95, 137, 140

Crocidura taungensis, 53, 70, 95, 126

Crocidurinae, 52, 94, 162

Crocuta, 104, 123

Crocuta crocuta, 57, 68, 104, 123-124, 126-135, 137, 139-143, 170, 184, 186, 192, 195-196, 199, 202-203, 205-206, 209, 211-212, 214

Crocuta dietrichi, 57, 70

Crocuta ultra, 105, 127, 130, 134

Crossarchus transvaalensis, 103, 124, 134

Cryptochloris zyli, 146, 191

Cryptomys, 92, 124, 130, 132, 136, 142, 159, 186-187, 196, 201, 203-204, 213

Cryptomys broomi, 50, 69

Cryptomys hottentotus, 50, 69, 92, 123, 125 , 128-130, 132-133, 136-143, 159, 183-193, 195-199, 201-206, 209-210, 212-215, 217-218

Cryptomys robertsi, 51, 69-70, 92, 124, 126, 130, $134,140-141$ 
Cynictis, 135, 169, 196

Cynictis penicillata, 56, 68-69, 103, 127, 129, 137, 141, 169, 183-185, 187, 190, 192-194, 196, 198-199, 201-202, 205-206, 208-210, 214, 216

Daberas Dune, 68

Dakkamyoides, 21, 36

Damalacra, 64, 70

Damalacra acalla, 34, 36, 64, 69

Damalacra neanica, 64, 69

Damalis, 66, 119, 180

Damaliscus, 115, 120, 126-128, 130-132, 135-136, 141-143, 177, 186, 193, 214

Damaliscus dorcas, 193

Damaliscus gentryi, 64, 69

Damaliscus lunatus, 115, 126, 129-131, 133, $137,177,187,194-195,198,207-208$, 211-212, 215

Damaliscus niro, 115, 127-131, 133-137, 140-141

Damaliscus pygargus, 115, 124-143, 177, 183-196, 198-205, 207-208, 210-211, 213-217

Darling, 189

Dasymys, 85, 134, 136, 141

Dasymys bolti, 86, 125, 128, 130

Dasymys broomi, 86, 140

Dasymys incomtus, 47, 69, 86, 124-125, 127, 130, 133, 140, 143, 154, 186, 192, 196, 201, 204-205, 215

Dasymys lavocati, 47, 70, 86, 125

David's Drift, 127

De Hoop 120, 127

Deelpan, 189

Deinotheriidae, 15, 41

Deinotheriinae, 15, 41

Deinotherium, 15, 41

Deinotherium bozasi, 68, 70

Delanymyinae, 44, 81

Delport's Hope, 127

Dendrohyrax arboreus, 74, 137

Dendromurinae, 19, 44, 82, 151

Dendromus, 82, 124, 130, 134, 136, 151, 190-191, 215

Dendromus antiquus, 44, 70

Dendromus averyi, 45, 69

Dendromus darti, 45, 69

Dendromus denysae, 19, 37

Dendromus melanotis, 82, 124-125, 127-128, 130, 133, 137-138, 140, 143, 151, 184-186, 188-189, 191, 197, 205, 208-210, 212-213, 217
Dendromus mesomelas, 45, 69, 82, 124-127, 133, $138,140,151,184,186,188-189,192,203$, 205, 213, 217

Dendromus mystacalis, 82, 125, 136, 152, 186

Dendromus nyikae, 82, 141

Deomyinae, 20, 46, 84, 153

Desmodillus, 46, 84, 123, 134, 138

Desmodillus auricularis, 46, 70, 84, 125, 128, 130, $143,153,183,185,187,190,199,202-203$, 209, 213, 217-218

Desmodillus magnus, 46, 69

Deurspring 16, 189

Diamant, 189

Diamantochloris inconcessus, 7, 11

Diamantofelis, 36

Diamantofelis ferox, 28, 35

Diamantohyus africanus, 34, 36-37

Diamantomyidae, 9, 22

Diamantomyinae, 22

Diamantomys luederitzi, 22, 35-37

Diamond 1, 189

Diana's Vow, 189

Dicerorhinus, 31

Diceros australis, 31, 35

Diceros bicornis, 110, 123-124, 126-131, 133, $135-136,140,142-143,175,184,186-193$, 196, 198, 200-201, 207-209, 213

Diceros praecox, 31, 37-38, 61, 68-69

Die Kelders, 127, 189

Die Toon, 189

Diepkloof, 127, 190

Dikbosch 1, 128, 190

Dinaledi Chamber, 79, 128, 134

Dinofelis, 68, 131, 141

Dinofelis aronoki, 101, 127

Dinofelis barlowi, 55, 69, 101, 125, 130, 135, 140

Dinofelis diastemata, 55, 69

Dinofelis piveteaui, 101, 128, 130, 135

Dinopithecus, 77

Dinopithecus ingens, 42, 70, 76, 141

Dinotherium, 15, 41

Diplomesodon fossorius, 53, 69

Divuyu, 190

Doonside, 190

Doornfontein, 190

Dorcatherium moruorotensis, 34, 37

Dorcatherium parvum, 34, 37

Dorcatherium pigotti, 34-35

Dorcatherium songhorensis, 34, 37

Doring River, 128 
Doringbaai, 190

DP2004-014, 190

Draaihoek, 128

Drie Susters, 190

Driebos, 190

Driefontein, 128

Driekoppen, 190

Driel Shelter, 190

Drimolen, 128

Drotsky's Cave, 128, 191

Duiker Eiland, 191

Duinefontein, 128, 191

Durban Country Club, 191

Dzata, 191

Dzombo Shelter, 191

E-Bay, 36

Efeldomys, 10-11

Efeldomys loliae, 25, 36

Eiland Salt Works, 191

Ekuma, 68

Elands Bay Cave, 128, 191

Elands Bay Open, 191

Elandsfontein, 104, 111, 122, 129

Elephantidae, 15, 41, 75, 148

Elephantinae, 15, 41, 75, 148

Elephantomys langi, 40, 73

Elephantulus, 13, 36, 72, 124-125, 128, 130, 132, 135-138, 143, 146, 184, 186, 190, 196, 213, 216, 218

Elephantulus antiquus, 40, 70, 72, 125, 134, 140

Elephantulus brachyrhynchus, 40, 69-70, 72, 125, 134, 140

Elephantulus broomi, 40, 69-70, 73, 125-126, 134, 140-141

Elephantulus edwardii, 73, 123, 129, 136, 138, 146, 184, 191, 197-198, 200, 209, 213

Elephantulus fuscus, 40, 73, 140-141

Elephantulus intufi, 73, 140, 146, 203

Elephantulus myurus, 73, 125, 130, 139, 143, 146, 186, 201, 205

Elephantulus rupestris, 73, 129, 132, 147, 185, 191, 203, 218

Elephas, 41, 69, 75, 131, 136, 141, 220

Elephas iolensis, 75, 124, 126-127, 136-139, 142

Elephas recki, 75, 125, 133, 135, 137, 140, 142

Elisabethfeld, 10, 12, 14, 21, 23, 25, 27-28, 32, 36,50

Emballonuridae, 27, 98

Embrithopoda, 7, 221

Enhydriodon, 60
Eocliff, 6-8, 11

Eoridge, 8, 11

Eozygodon morotoensis, 16, 35-36

Eptesicus, 98, 165

Eptesicus hottentotus, 54, 69, 98, 125, 131, 134, 141, 143, 165, 185, 199, 205

Equidae, 60, 109

Equus, 109, 126, 131-133, 135, 137, 141-142, 174, 184-187, 190, 192-194, 196-198, 200, 203, 205-209, 214, 216, 218

Equus asinus, 130, 142, 174, 184, 194, 207, 210-211, 213, 221

Equus burchellii, 109, 124-143, 174, 183-215, 217

Equus caballus, 174, 202, 210, 221

Equus capensis, 109, 123-144, 174, 191, 196, 217

Equus Cave, 129, 192

Equus quagga, 109, 123-124, 126-141, 143, 174, 183-191, 194-200, 202-203, 207-209, 211, 216, 218

Equus sandwithi, 109, 123, 134, 137, 139, 142

Equus zebra, 110, 123, 136-137, 142, 174, 183-184, 186-188, 190, 193, 205-206, 208-209, 216

Eremitalpa granti, 146, 191, 203, 209, 212-213

Erfkroon, 129

Erinaceidae, 26, 94, 162

Erinaceinae, 26, 94, 162

Erinaceomorpha, 26, 94, 162, 221

Erinaceus, 220

Erinaceus broomi, 94, 125

Erinaceus capensis, 94, 162

Esikhunjini, 192

eSinhlonhweni, 192

Etemba, 192

Etosha Pan, 36, 68, 129

Euryboas, 57

Eurygnathohippus, 60, 70, 110, 126, 131, 143

Eurygnathohippus cornelianus, 31, 37, 60, 69, 110, 125-128, 131, 134, 140-141

Eurygnathohippus hooijeri, 61, 69, 110, 124

Eurygnathohippus namaquense, 61, 68

Euryotis, 89-90, 158

Euryotomys bolti, 49, 70

Euryotomys pelomyoides, 49, 69

Fackelträger, 192

Fairview, 192

Faraoskop Rock Shelter, 130, 192

Felidae, 28, 54, 100, 167 
Feliformia, 28, 54, 100, 167

Felinae, 29, 54, 100, 167

Felis, 55, 68, 100, 102, 167, 193, 207

Felis catus, 102, 168

Felis crassidens, 101, 134

Felis issiodorensis, 55, 69

Felis nigripes, 101, 129, 132, 135, 167, 185, 192, 216

Felis shawi, 101, 125

Felis silvestris, 68, 101, 124-125, 127-129, 132-133, 135-139, 141-143, 168, 183-203, 205-217

Femur Dump, 130

Ficus Cave, 192

Fiskus, 36

Florisbad, 91, 114, 122, 130

Forest Hall, 192

Forlorn Hope, 130

Friesenberg Hilltop, 130

Fukomys damarensis, 51, 92, 159, 217

Galagidae, 148

Galago, 148, 195

Galago senegalensis, 148, 186

Galerella, 169, 183, 194, 216

Galerella pulverulenta, 103, 124-125, 127-128,

133, 136, 139, 169, 184, 186-189, 191-194, 197, 205, 207-208, 211-214, 216-217

Galerella sanguinea, 103, 129-130, 137-139, 141, 169, 189, 197, 203, 205, 207, 212-214, 216

Galericinae, 26

Galerix, 26, 36

Garage Ravine Cave, 130

Gaudeamus, 10-11

Gazella, 65, 70, 113, 116, 129, 140-141, 143, 220

Gazella praethomsoni, 117, 124

Gazella vanhoepeni, 65, 69, 117, 128

Gcwihaba C Hill, 130

Geduld, 192

Geelbek Dunes, 130

Geelwal Karoo, 130

Gehle, 192

Genetta, 58, 69, 105, 124-125, 127-129, 133, 135-136, 171, 184-186, 188-194, 197, 201, 204-207, 212-213, 215, 217

Genetta genetta, 58, 69, 106, 134, 171, 184, 198-200, 202, 204-205, 211, 214, 217

Genetta maculata, 106, 171, 213
Genetta tigrina, 106, 132, 137, 139, 141, 171, 195, 198, 203, 205, 207

Geniohyidae, 8

Geofossor corvinusae, 23, 35

Geofossor moralesi, 23, 36-37

Georychus, 92, 130, 203

Georychus capensis, 51, 92, 124, 127, 133, 136, 139-140, 160, 188, 191-193, 197, 205, 209, 214-215

Geosciurus capensis, 80, 150

Gerbillinae, 21, 46, 84, 153

Gerbilliscus, 47, 69, 84, 123-125, 128, 130-131, 136-138, 141, 143, 153, 185, 190, 192-193, 195-196, 206, 214, 217

Gerbilliscus afra, 84, 123-125, 127, 129, 132, 136, 138, 153, 184, 186, 189, 191, 197, 209, 213

Gerbilliscus brantsii, 47, 70, 84, 126, 128, 130, 140, 153, 183, 186-188, 190-191, 199, 201-202, 205, 208, 210, 218

Gerbilliscus leucogaster, 85, 125, 134, 140, 154, 186, 190-191, 207, 212, 216-217

Gerbillurus, 85, 123, 130, 132, 136, 141

Gerbillurus paeba, 85, 123, 125, 128-129, 138, 140, 143-144, 154, 184-187, 190-191, 194-195, 197-198, 200, 203, 209, 212-213, 217-218

Gerbillurus vallinus, 154, 203

Gerbillus, 46-47, 84, 153

Ghoenkop, 193

Gigantohyrax maguirei, 41, 69

Ginsburgsmilus napakensis, 28, 37

Giraffa, 63, 69, 113, 141

Giraffa camelopardalis, 113, 123-124, 129-131, 133, 135, 138-139, 176, 186, 189-190, 195-196, 198, 200-204, 206, 208-213, 215, 217

Giraffa stillei, 63, 69, 113

Giraffidae, 63, 113, 176

Giraffinae, 63, 113, 176

Girls' School, 193

Gladysvale, 130, 193

Glastal, 37

Glauconycteris variegata, 98, 125, 186

Glen Elliott, 193

Glennel, 193

Glentyre, 193

Glibia namibiensis, 9, 11

Gliridae, 18, 80, 150

Gliriscus, 80

Gobabis townlands, 131

Godwan River, 193 
Goedgekloof Middens, 193

Gokomere Tunnel, 193

Gomphotheriidae, 16, 42

Gomphotherium pygmaeus, 16

Gondolin, 131

Gong-Gong, 131

Good Hope Shelter, 193

Gordons Bay, 193

Gorgopithecus, 42-43, 77

Gorgopithecus major, 77, 134

GP Pan North, 37

Grammomys, 86, 124, 131

Grammomys cometes, 155, 213

Grammomys dolichurus, 47, 69, 86, 125, 128, 133-134, 136, 142, 155, 186, 196, 205, 215

Graphiurinae, 18, 80, 150

Graphiurus, 80, 123-124, 130, 134, 136, 138, 140-141

Graphiurus microtis, 81, 143

Graphiurus murinus, 81, 125, 150, 186, 189, 196, 199, 205, 210, 217-218

Graphiurus ocularis, 81, 125, 128, 133, 151, 184, 190-191, 197, 209, 213

Graphiurus platyops, 151, 212

Grassridge, 193

Great Zimbabwe, 193

Green Point Cape Town, 193

Grillental, 14-15, 23, 30, 37

Griquatherium, 113

Groenrivier, 37

Groenriviermond, 193

Groot Kloof, 131

Groot Kommandokloof Shelter, 193

Grootrif G, 194

Gwenzi II Hill, 194

Gymnurechinus leakeyi, 26, 37

Gypsorhychus darti, 51, 70

Gypsorhychus makapani, 51, 69

Gypsorhychus minor, 51, 70

Ha Makotoko, 194

Haalenberg, 194

Haasgat, 78, 131

Haaskraal, 194

Hadeco, 131

Hailstone Midden, 194

Halliwell's Workings, 131

Hamilton, 194

Hapi Pan, 194

Haplorrhini, 17, 42, 76, 148

Happy Rest, 194
Harasib 3a, 17-21, 25, 37

Harasibomys, 18, 36-37

Harasibomys petteri, 18, 37

Harimyscus hoali, 20, 37

Harleigh Farm, 194

Harmony Salt Factory, 194

Hawston, 131

Hecubides euryodon, 30, 36-37

Hennops River, 131

Herolds Bay, 132

Herpestes, 56, 69, 103, 138, 168-169

Herpestes ichneumon, 103, 125-129, 132-133, 136, 139-141, 169, 186, 188-189, 191-192, 197, 203-207, 209, 213, 215

Herpestes pulverulentus, 193

Herpestidae, 56, 103, 168

Heterocephalus, 51, 69

Heterohyrax, 74, 147

Heterohyrax auricampensis, 15, 36

Heterohyrax brucei, 147, 189, 194, 196, 201, 208, 210

Heteroxerus karsticus, 17, 37

Hettie 582, 132

Heuningneskrans, 132, 194

Highlands Rock Shelter, 194

Hill X, 194

Hipparion, 31, 60, 68, 110

Hipparion baardi, 61

Hipparion sitifense, 31, 110

Hippopotamidae, 63, 112, 176

Hippopotamus, 63, 68, 70, 112, 124, 127, 130-132, 136-137, 141

Hippopotamus amphibius, 63, 69, 112, 123-143, 176, 184-199, 201, 203, 205-208, 210, 212-218

Hippopotamus gorgops, 113, 127

Hipposideridae, 26, 97

Hipposideros, 97, 124

Hipposideros caffer, 97, 131

Hipposideros gigas, 26, 36, 97

Hipposideros vittatus, 97, 144

Hippotigris, 110

Hippotraginae, 67, 120, 181

Hippotragoides, 66, 119

Hippotragus, 66, 115, 119-120, 125-127, 129-130, 132-138, 140-143, 181, 186, 189, 191-192, 194, 198-200, 202, 205, 209-210, 212, 214-215

Hippotragus cookei, 67, 69, 120, 140

Hippotragus equinus, 121, 125-126, 131, 137, 139-140, 181, 183, 187, 190, 192-193, 
195, 199-201, 204, 207-208, 210-211, 214, 217

Hippotragus gigas, 121, 129, 141

Hippotragus leucophaeus, 121, 124-125, 127-130, 132-133, 136-141, 143, 181, 188-189, 191-194, 202, 206, 210-211

Hippotragus niger, 67, 69, 121, 124, 126, 131, 133-135, 137, 140-141, 182, 187, 190-191, 195-196, 199, 201, 203-204, 206, 208-209, 214-215

Historic Cave, 195

Hlamba Mlonga Hill, 195

Hoedjiesbaai, 132

Hoedjiespunt 1, 132

Hoekfontein, 195

Hofmeyr Cave, 132

Homestead Area, 132

Hominidae, 17, 44, 79, 149

Homininae, 17, 44, 79, 149

Homo, 79, 127-128, 131, 138, 142

Homo erectus, 79, 134, 140

Homo gautengensis, 79, 140-141

Homo naledi, 79, 128, 134

Homo rhodesiensis, 79, 129, 132

Homo sapiens, 79, 124-127, 129-130, 132-133, 136-139, 142-143, 149, 183-218

Homoioceras, 118

Homoiodorcas, 35

Homotherium, 54, 141

Homotherium crenatidens, 55, 69, 102, 139-140

Homotherium problematicum, 55, 69

Hondeklip Bay, 37

Honingklip I and V, 195

Hoogland, 68

Hoogstede, 132

Hope Hill Shelter, 195

Hout Bay, 195

Hyaena, 58, 105, 170, 185, 196

Hyaena bellax, 58, 105

Hyaena hyaena, 57, 69, 105, 134, 141

Hyaena makapani, 57, 69

Hyaenictis, 58

Hyaenictis hendeyi, 57, 69

Hyaenictis preforfex, 57, 69

Hyaenictitherium namaquensis, 58, 69

Hyaenidae, 57, 104, 170

Hyaeninae, 57, 104, 170

Hyaenodontidae, 10, 27

Hyaenodontinae, 27

Hyainailourinae, 10, 28

Hyainailouros sulzeri, 28, 35
Hydrictis maculicollis, 173, 218

Hyotherium, 33

Hypsorhynchocyon burrelli, 14, 37

Hyracoidea, 8, 14, 41, 74, 147, 221

Hystricidae, 51, 92, 160

Hystricinae, 51, 92, 160

Hystricomorpha, 9, 22, 50, 91, 159

Hystrix, 124, 134, 142, 160, 189, 206

Hystrix africaeaustralis, 51, 69-70, 92, 123-129, 131-144, 160, 183-217

Hystrix cristata, 92, 140

Hystrix makapanensis, 51, 69, 92, 131

Ichneumia albicauda, 103, 133, 138, 169, 183, 194, 210-211

Ichneumon, 169

Icon, 195

Ictitherium, 57

Ictonyx, 109, 173, 206

Ictonyx striatus, 108, 124, 127-129, 132, 136-137, 139, 141, 173, 183, 188-191, 194-198, 207, 211-213, 215-216

Ikelohyaena abronia, 58, 69

Induna Cave, 195

iNkolomahashi, 195

Isohyaenodon, 27

Jack's Camp, 132

Jacovec Cavern, 68

Jägersquelle, 132

Jagt Pan 7, 196

Jakkalsberg, 196

Jakkalsfontein, 132

Jubilee Shelter, 196

K2, 196

Kabeljous River Cave 1, 196

Kadzi River, 196

Kalk Bay, 132

Kalk Plateau, 132

Kalkbank, 133

Kalkoenkrans 225, 133

Kamukombe, 196

Kapako, 196

Kareepan, 196

Karingarab, 37

Karnimata, 20

Karridene, 196

Kasteelberg, 196

Katarakt, 197

Kathu Pan, 133 
Keeble's Paddock, 121, 133

Kenyapithecinae, 17

Kenyapithecus, 17, 38

Kenyasus, 33

Kenyasus namaquensis, 33, 38

Kenyasus rusingensis, 33, 38

Kerivoula argentata, 166, 206

Khami Hill, 197

Khartoum 1, 197

Klasies River, 133, 197

Klein Kliphuis, 197

Klein Spitzkoppe, 197

Klein Witkrans, 197

Kleinzee, 13, 69

Kleinzzee, 40

Klingbeil, 197

Klipdrift Complex, 133

Klipfonteinrand, 197

Klipspruit, 197

KN2005-0041, 197

KN6-3C, 197

Knysna Heads, 197

Koanaka Hills, 133

Kobus, 67-68, 121, 133, 142

Kobus altidens, 121, 133

Kobus ellipsiprymnus, 122, 125, 129-133, 135, 137-139, 182-183, 187-188, 190-191, 194-196, 199, 201-202, 204, 206-207, 212, 214, 217

Kobus leche, 122, 124, 126, 129-131, 135, 137-138, 141, 182, 187, 190, 204, 206, 215, 217-218

Kobus subdolus, 67, 69

Koffiefontein, 133

Kolpochoerus heseloni, 111, 127

Kolpochoerus paiceae, 62, 70, 111, 127, 129, 131, 137, 143

Kolpohippus, 109, 174

Kombat E900, 134

Komkans 2, 197

Kommando Kop, 197

Kommetjie, 197

KoNomtjarhelo, 197

Kouga, 198

Kougha Dam, 198

Kraanvogelvallei Breakwater, 134

Kramleeg, 134

Kranskraal, 134

Kreeftebaai, 198

Kromdraai, 77, 80, 101, 105-108, 116, 134

Kruger Cave, 198
Kubanochoerinae, 33

Kuidas Spring, 198

Kumukams, 198

KV502, 198

KwaGandaganda, 198

KwaMaza A and B, 198

KwaThwaleyakhe, 198

Lagomorpha, 25, 52, 93, 161, 221

Lame Sheep Shelter, 198

Langdraai, 198

Langebaan, 198

Langebaanweg, 15, 34-35, 39, 41, 44, 46, 49-50, 56-61, 63-65, 67-69, 76, 110, 113, 118, 220

Langental, 13, 15, 27, 29, 32, 37

Lanlory, 198

Larsen's, 64, 114, 134

Le 6, 7a and $7 \mathrm{~b}, 198$

Leecyaena forfex, 57

Leeuhoek, 199

Leeukop, 199

Leggada, 48, 87, 156

Leholamogoa, 199

Lekkerwater, 199

Leliehoek, 199

Lemniscomys, 47, 69, 134

Lemniscomys rosalia, 86, 125, 131, 143, 155, 186, 196

Lemoenfontein, 199

Leopard Cave, 199

Leporidae, 52, 93, 161

Leptailurus serval, 55, 69, 102, 125, 128, 134, 139, 141, 168, 189, 196-197, 207. 213, 215

Leptoplesictis namibiensis, 29, 37

Leptoplesictis senutae, 30, 37

Lepus, 52, 70, 93, 123, 125-129, 135, 137-138, 142-144, 161, 184-186, 188-190, 192-200, 202-204, 206, 208-210, 212-215, 217

Lepus capensis, 52, 69, 93, 124-125, 127-130, 132-133, 136, 140, 143, 161, 185, 187-190, 193-197, 200, 202, 207-208, 212, 215-216

Lepus saxatilis, 93, 124, 126, 132-134, 139-140, 161, 183-184, 187, 191-192, 194-197, 199, 201, 203-207, 209-216

Leqhetsoana, 199

LeRoux 717, 134

Letsibogo Dam, 199

Libytherium, 113 
Libytherium olduvaiensis, 63

Likoaeng, 199

Limerock 1 and 2, 199

Limeworks Makapansgat, 39, 41, 44-46, 51, 53, $55,57,60,64-69,78,81,83,92,117$, 119-120, 122

Lincoln Cave, 134

Linkerhandsgat, 134

Liphofung, 200

Lithakong, 200

Little England, 134

Little Witkrans, 200

LK2004-011, 200

LK5-1, 200

Longlands, 62, 112, 134

Lopholistriodon, 33

Lorisidae, 8, 220

Lotshitshi, 200

Lower Kemp's Cave, 134

Lower Numas Cave, 200

Loxodonta, 15, 37, 75, 131, 133, 137-138, 142, 189, 196-197, 211, 217

Loxodonta africana, 75, 123, 125-133, 135, 137-139, 142-143, 148, 184, 186-189, 191, 194-198, 200-201, 203-204, 206, 208, 210, 213-215, 217

Loxodonta atlantica, 75, 129

Loxodonta cookei, 15, 36, 41, 68-69, 76, 129

Lüderitz Bay, 10, 37

Lüderitz Bay (south of), 10, 24

Lukeino Stage, 15, 41, 76

Luleche, 135

Lunatoceras, 115

Lutra, 173

Lutrinae, 60, 108, 173

Lycaon, 107, 124, 130, 135

Lycaon pictus, 107, 125, 127, 129-130, 132-133, 137-139, 141-142, 172, 183, 187, 189, 192, 194, 196-197, 201-202, 205-206, 211-212

Lycaon sekowei, 107, 127

Lycyaena, 57, 104

Lycyaenops silberbergi, 57, 69, 104, 139-140

Lydenburg Heads Site, 200

Ma 4, 38, 200

Mabjanamatshwana, 200

Mabveni, 200

Machaerodus, 55, 101

Machairodontinae, 56, 102

Machairodus, 54-55, 102
Macroscelidea, 7, 13, 40, 72, 146, 221

Macroscelides, 40, 72-73, 123, 146

Macroscelides proboscideus, 40, 70, 73, 133-134, 140-141, 143, 147, 183, 185, 187, 203, 209, 218

Macroscelididae, 7, 13, 40, 72, 146

Macroscelidinae, 13, 40, 72, 146

Madoqua, 178, 192

Magogo, 200

Maguams Andalusia, 200

Maguams Elefant, 200

Mahemspan, 135

Makapania, 119, 141

Makapania broomi, 66, 69, 119, 131, 140

Makgabeng, 201

Malacothrix, 82, 123-124, 128, 130, 132, 134, 136, 138

Malacothrix makapani, 45, 69

Malacothrix typica , 45, 69-70, 82, 125, 140-141, 143, 152, 183, 185-187, 199, 203, 209, 213, $217-218$

Malapa, 79, 108, 135

Malumba, 201

Mamaetla, 201

Mamba, 201

Mammuthidae, 16

Mammuthus, 75

Mammuthus subplanifrons, 42, 69-70, 76, 124, 131, 142

Mananzve Hill, 201

Manidae, 54, 100, 166

Manis, 54, 100, 167

Manjowe Rock Shelters, 201

Mantenge Shelter, 201

Manyikeni, 201

Mapotini Hill, 201

Mapungubwe, 201

Maqonqo, 201

Marupale Hill, 202

Maselspoort, 202

Masritherium, 33

Mastodon, 16, 42, 76

Mastomys, 86, 124, 130-131, 136, 138, 141, 155, 199, 212

Mastomys coucha, 87, 133, 141, 143, 155, 190

Mastomys natalensis, 48, 69-70, 87, 125, 128, $134,137-140,155,185-187,192,195-196$, 199-202, 204-206, 208, 210, 215

Matanga, 202

Matjies River Rock Shelter, 202

Matjihabeng, 70 
Matlapaneng, 202

Mauermanshoek, 202

MB2005-005, 202

MB2005-119, 202

Mbabane, 202

Meerholtzkop, 135

Meerkat Shelter, 202

Megaderma, 27, 36

Megadermatidae, 27

Megalotragus, 64, 69-70, 115, 127-128, 131, 135-136, 138, 140-141

Megalotragus priscus, 115, 123-140, 142-143

Megantereon, 55

Megantereon gracile, 102, 129, 140

Megantereon obscura, 56

Megantereon whitei, 56, 70, 102, 127, 135, 139-141

Megapedetes, 22, 36

Megapedetes gariepensis, 22, 35, 37

Megatereon, 55, 101

Melkboom 1, 202

Melkbos, 135

Melkbostrand, 202

Melkhoutboom, 202

Mellivora, 134

Mellivora benfieldi, 60, 69

Mellivora capensis, 108, 124-125, 127-130, 132-133, 136-139, 141, 144, 173, 184-186, 188-189, 191, 196-197, 199, 201, 203, 206-208, 211-212, 215, 217

Mellivorinae, 108

Meob, 70

Meriones, 84, 153

Mesembriportax, 65, 118

Mesochoerus lategani, 36, 111

Messum, 203

Metailurus, 28-29, 35

Metailurus obscurus, 56, 69

Metaphiomyinae, 9

Metaphiomys schaubi, 9, 11

Metapterodon, 37

Metapterodon kaiseri, 27, 36

Metapterodon stromeri, 27, 37

Metaschizotherium transvaalensis, 60, 69

Metridiochoerus, 62, 111-112, 128, 130-131, 136, 138

Metridiochoerus andrewsi, 62, 69-70, 111, 125, 127, 129, 131, 134, 137, 141
Metridiochoerus compactus, 111, 127, 131, 134, 137,142

Metridiochoerus modestus, 111, 125, 127, 134, 140, 143

Metridochoerus andrewsi, 134

Mgede, 203

Mgoduyanuka, 203

Mhandambiri, 203

Mhlopeni, 203

Mhlwazini, 203

Micaelamys, 85, 87, 132, 154

Micaelamys namaquensis, 48, 69, 87, 123, 125, 129-130, 132-134, 137, 140-141, 143, 155, 183-187, 190, 192, 197, 200, 203, 205-206, 209-210, 212-213, 217-218

Microchiroptera, 26, 53, 96, 164

Microcolobus, 17, 37, 78

Microfossor biradiculatus, 23, 36

Milnerton, 203

Milo's Pit A, 70

Mimosa 559, 135

Minaar's Cave, 135

Miniopterinae, 54, 99, 166

Miniopterus, 54, 69, 99, 123, 132, 166

Miniopterus natalensis, 100, 124-126, 131, 140, 143, 166, 185-186, 199, 206, 217

Miniopterus schreibersii, 100, 166, 218

Mioharimys milleri, 21, 37

Mioharimys schneideri, 21, 37

Miorhynchocyon, 14

Miorhynchocyon gariepensis, 13, 35

Miotragoceros acrae, 65, 69, 118, 124

Mirabib, 203

Mitasrust Farm, 135

Mitford Park, 203

Mmatshetschele Mountain, 203

Mo 8, 203

Mockesdam, 135

Modder River, 115, 135

Modder River Mouth, 203

Modipe Hill, 203

Mogapelwa, 203

Molokwane, 203

Molossidae, 27, 98, 165

Molossinae, 27, 98, 165

Moritsane, 204

Morris Draai, 135

Motsetse, 135

Mpambanyoni, 204

Mphekwane, 204

Msuluzi Confluence, 204 
Mt Ziwa, 204

Mtanye, 204

Mud River, 204

Muela, 204

Muhululu Hill, 204

Mungos, 135

Mungos mungo, 104, 137, 140, 170, 204, 207, 210

Muozi, 204

Murahwa's Hill, 204

Muridae, 20, 46, 84-85, 153

Murinae, 47, 85, 154

Mus, 87, 90, 123-124, 128, 130, 132, 136, 138, 141,156

Mus indutus, 156, 217-218

Mus major, 87, 125

Mus minutoides, 48, 69, 87, 124-128, 131, 133, 136-137, 140, 143, 156, 184-186, 190-191, 196, 202-203, 206, 209, 212-213, 215,217

Mus musculus, 145, 156, 195

Mus triton, 88, 125

Mustelidae, 30, 59, 108, 173

Mustelinae, 108, 173

Mutshilachokwe, 204

Mwenezi Farm, 204

Mylomygale spiersi, 40, 70, 74, 140

Mylomygalinae, 40, 74

Myocricetodon, 21, 36-37, 89, 124

Myocricetodontinae, 21, 49, 89

Myohyracinae, 7, 13

Myohyrax, 7, 11, 35

Myohyrax oswaldi, 13, 35-37

Myohyrax pickfordi, 13, 37

Myomorpha, 18, 44, 80, 150

Myomys, 48, 88, 156

Myomyscus, 48, 69

Myomyscus verreauxii, 88, 124-125, 127, 132-133, 136, 156, 184, 186, 189, 191, 197, 209

Myophiomyidae, 9, 24

Myophiomyinae, 24

Myorycteropus minutus, 14

Myosorex, 96, 128, 130, 164, 193, 200, 202-203, 206

Myosorex cafer, 96, 125, 140-141

Myosorex robinsoni, 53, 69, 96, 125, 131, 134, 140-141

Myosorex varius, 53, 69, 96, 123-125, 127, 129, 132-133, 136, 138-139, 142-143, 164, 184, 186, 188-192, 196-197, 202-203, 209-210, 212-213, 215, 217
Myosoricinae, 53, 96, 164

Myotinae, 27, 99, 166

Myotis, 27, 36, 99, 125, 136, 138

Myotis tricolor, 99, 131, 134, 140-141, 166, 189

Myotis welwitschii, 99, 134

Myotomys campbelli, 49, 70, 89, 130

Myotomys sloggetti, 49, 69, 89, 130-132, 140, 158,188

Myotomys unisulcatus, 89, 123, 125, 129-130, 132, 138, 158, 183-184, 186-187, 189, 191, 194, 197-198, 208-209, 212-213

Myoxus, 80, 151

Mystromyinae, 45, 83, 152

Mystromys, 83, 124, 132, 136, 141, 201

Mystromys albicaudatus, 45, 69-70, 83, 123, 125, 127-129, 131-133, 136-138, 140-141, 143, 152, 183-186, 188-193, 197, 199, 202-203, 205-206, 209-210, 212-213, 217

Mystromys hausleitneri, 45, 69-70, 83, 125-126, 128, 130-131, 134, 140

Mystromys pocockei, 46, 69

Mzinyashana 1, 204

Mzonjani, 205

Nakalimys lavocati, 18, 37

Namacerus gariepensis, 34-35

Namachloris arenatans, 7, 11

Namachoerinae, 33, 61

Namachoerus moruoroti, 33, 35

Namafelis minor, 28, 35

Namagale grandis, 6, 11

Namahyrax corvus, 8, 11

Namaia, 8

Namalestes gheerbranti, 6, 11

Namaloris rupestris, 8, 11

Namaparamys inexpectatus, 8, 11

Namaphiomys, 10-11

Namasector soriae, 28, 36

Namatherium blackcrowense, 7, 11

Namib 2, 205

Namib IV, 135

Namibictis senuti, 30, 35

Namibimyinae, 21

Namibimys angustidens, 21, 36-37

Namibiomeryx senuti, 32, 36

Namibiomeryx spaggiarii, 32, 37

Namtib, 205

Nanda, 205

Ndondondwane, 205

Ndongo, 205

Neamblysomus gunningi, 72, 141 
Nelson Bay Cave, 135, 205

Neoromicia, 98

Neoromicia capensis, 99, 125, 131, 143, 165, 197, 199, 217-218

Neosciuromys, 25

Neosciuromys africanus, 24, 36-37

Neotragus moschatus, 178, 188, 205

Nesomyidae, 44, 81, 151

Nesotragus, 178

Nguruwe namibensis, 33, 35-38

Niekerk's Rush, 136

Nkupe, 205

Nooitgedacht, 136

Noordhoek, 206

North Brabant Shelter, 206

Norvalspont, 206

Nos, 136, 206

Nosib, 136

Notnamaia bogenfelsi, 8, 11

Notochoerus, 62, 111

Notochoerus capensis, 62, 68-70, 112, 134

Notochoerus jaegeri, 62, 69

Notocricetodon, 19, 36

Nova, 37

Nqoma, 206

Ntloana Tsoana, 206

Ntshekane, 206

Numas 25, 206

Numidocapra arambourgi, 119, 129

Numidocapra porrocornutus, 120, 141

Nuwekloof Shelter, 206

Nxazini Pans, 136

Nyanzachoerus, 62

Nyanzachoerus kanamensis, 33, 37, 62, 69

Nyctereutes terblanchei, 107, 131, 134

Nycteridae, 98, 165

Nycteris, 98, 138

Nycteris thebaica, 98, 125, 165, 184, 186, 209

Oakhurst, 206

Ochotonidae, 25, 220

Ochre Cave, 206

OFD1, 206

Ol 20, 206

Old Pont Site, 136

Oldrichpedetes brigittae, 22, 38

Oldrichpedetes pickfordi, 22, 35

Olieboompoort, 206

OND 2 and 3, 207

Ondera, 136

Ondini, 207
Ongers River, 136

Onotragus, 67, 121

OO1, 207

Opsiceros, 110, 175

Orabes Upper, 207

Orange River Railway Station, 207

Orangemeryx hendeyi, 32, 35

Orangictis gariepensis, 29, 36

Oranjemund, 136

Oreotragus, 128, 135, 142

Oreotragus oreotragus, $65,68,70,117,123-129$, 131-133, 135-143, 178, 183-218

Orycteropus, 14, 37, 74, 124, 126, 129, 132-133, 138-139, 141, 187, 189-190, 193-195, 199, 201-203, 208, 214, 217

Orycteropus afer, 74, 126, 128-129, 132-133, 136, 138-139, 141, 147, 186-187, 189-190, 192-203, 205-209, 211-215, 217

Oryctolagus cuniculus, 162, 208

Oryctopodidae, 14, 74, 147

Oryx, 121, 138

Oryx gazella, 121, 123-124, 128-129, 131, 137, 140, 143, 182-185, 187, 190, 195, 198, 205, 207-209, 212-215

Oshilongo, 207

Ostrich Shelter, 207

Otaviglis daamsi, 18, 37

Otavimyinae, 20

Otavimys senegasi, 20, 37

Otavipithecus namibiensis, 17, 35-36

Otjiseva, 136

Otocyon megalotis, 107, 124, 129, 134, 136-137, 140, 142-143, 172, 183, 185, 187-188, 190, 192, 197, 200, 202, 206, 210, 212-213, 217

Otocyon recki, 107, 141

Otolemur crassicaudatus, 148, 198, 201

Otomyinae, 49, 89, 158

Otomys, 45, 49, 89

Otomys angoniensis, 90, 125, 128, 139, 143, 158, 185-186, 190-191, 196, 202, 206, 215, 217

Otomys gracilis, 50, 69-70, 90, 125-126, 128, 130-131, 134, 140-141, 143

Otomys irroratus, 90, 124-125, 127, 129-130, 132-133, 136-140, 142, 158, 183-186, 188-193, 195, 197, 199, 201-206, 209-210, 212-215, 217-218

Otomys laminatus, 90, 125, 133, 139, 142, 158, 186, 188-189, 192, 203-204, 215

Otomys saundersiae, 90, 123-125, 127-129, 131-133, 136, 139-141, 158, 184, 186, 188-191, 197, 209-210, 213 
OU1, 207

OU2, 207

Oudepost 1, 207

Ourebia, 117, 126

Ourebia ourebi, 117, 125, 131, 133, 136, 138, 178, 186, 188-190, 192-196, 198, 200-206, 211-212, 215

Ovis aries, 180, 183-218, 221

Pa 8.1, 207

Pachycrocuta brevirostris, 58, 69, 105, 124, 130-131, 140

Pachyuromys, 84

Palaeoloxodon, 41, 75, 148

Palaeothentoides, 13, 36, 40

Palaeothentoides africanus, 40, 69

Palaeotomys, 50

Palaeotragiscus longiceps, 65, 117, 178

Palaepetromys, 52

Pancho's Kitchen Midden, 207

Panthera, 124

Panthera leo, 29, 36, 56, 68-70, 102, 124, 126-142, 168, 187, 189-190, 193-195, 197-199, 201, 206-207, 209-210, 212-215

Panthera pardus, 56, 68-69, 102, 125-129, 132-133, 135-142, 168, 183-203, 205-210, 212-217

Pantherinae, 29, 56, 102, 168

Papio, 42-43, 76-77, 125-127, 135, 139, 149, 187-190, 193, 199, 206-207, 217

Papio (hamadryas) angusticeps, 42, 77

Papio hamadryas, 42, 77

Papio izodi, 42, 70, 77, 125, 127, 131, 134-135, 140

Papio robinsoni, 77, 127-128, 130-131, 137, 140-141

Papio spelaeus, 77, 137

Papio ursinus, 77, 123-129, 132-136, 138-140, 142-143, 149, 184-218

Paracryptomys mackennae, 23

Paradise Main House, 208

Parahyaena, 68

Parahyaena brunnea, 58, 69, 105, 124-125, 127-130, 132-142, 170, 184, 187-188, 191-192, 194-196, 198, 201, 206-210, 213-216

Paramyidae, 8

Paranthropus robustus, 80, 127-128, 134, 140-141 Parapapio, 42-43, 69-70, 77-78, 132

Parapapio broomi, 43, 69-70, 78, 125, 131, 140-141

Parapapio jonesi, 43, 69, 78, 123, 140-141
Parapapio whitei, 43, 69-70, 78, 125, 140-141

Parapedetes, 21, 36

Parapedetes namaquensis, 22, 36

Parapedetidae, 21

Paraphiomys australis, 24, 37

Paraphiomys orangeus, 25, 36

Paraphiomys roessneri, 25, 37

Parapliohyrax, 14, 36

Parapliohyrax ngororaensis, 14, 37

Paraulacodus johanesi, 25, 37

Paraxerus cepapi, 150, 199, 201, 205, 207, 209, 214

Paraxerus palliatus, 80, 125

Parmularius, 116, 126, 129, 143

Parmularius braini, 64, 69, 114

Parmularius parvus, 116, 134

Parotomys, 90, 130, 141

Parotomys brantsii, 90, 132, 158, 190, 197-198, 200, 202, 209, 212-213, 218

Parotomys littledalei, 90, 144, 158, 218

Paternoster, 208

Pecarichoerus, 61

Pedetes, 22, 91, 127, 138, 141

Pedetes capensis, 50, 69-70, 91, 123, 126, 128-130, 133-138, 140, 143, 159, 187, 189-211, 214-217

Pedetes gracilis, 50, 68, 70

Pedetes hagenstadti, 91, 130

Pedetidae, 22, 50, 91, 159

Peers Cave, 136

Pelea, 127-128

Pelea capreolus, 122, 124-129, 131-143, 182, 184-190, 192-217

Peleoroceras, 115

Pelomys fallax, 48, 69, 88, 125, 140

Pelorovis, 66, 118, 179

Penge, 208

Percrocuta, 57

Perissodactyla, 31, 60, 109, 221

Petromuridae, 52, 93, 160

Petromus, 93, 138, 141, 160, 192

Petromus antiquus, 52, 70

Petromus minor, 52, 70

Petromus typicus, 93, 123, 144, 160, 183-185, 194, 198, 203, 205, 208, 218

Petromyscinae, 20, 83, 153

Petromyscus, 20, 37, 83, 138, 153, 203

Petromyscus collinus, 153, 194, 218

Pfeiffer, 197

Ph 9, 208

Phacochoerus, 111, 123, 126-127, 129, 133, 136, $138,143,175,184,196,201,206,217$ 
Phacochoerus antiquus, 131

Phacochoerus africanus, 112, 123-126, 128-140, 142-143, 175, 183-217

Phacochoerus altidens, 112, 134, 137

Phacochoerus antiquus, 112, 140-141

Philantomba monticola, 120, 133, 136, 138-139, 142, 181, 184, 188, 192, 194-198, 201-206, 211, 215, 217

Phiocricetomyinae, 9

Phiomyoides humilis, 24, 36-37

Phiomys, 9

Phiomys lavocati, 11

Phiomys phiomyoides, 10-11

Pholidota, 54, 100, 166, 221

Phthinylla fracta, 25, 36-37

Pinnacle Point, 136

Pjene Hill, 208

Plesianthropus, 44, 79

Plesiogulo monspessulanus, 59, 69

Pliohyracidae, 14

Plovers Lake, 137

Pneil, 112, 137

Pockenbank, 137, 208

Poecilogale albinucha, 109, 127

Poemys, 82

Pomongwe, 137, 208

Pomonomys dubius, 23, 37

Pont Drift, 208

Potamochoeroides hypsodon, 62, 68-69, 112, 125, 131, 134

Potamochoerus, 194

Potamochoerus larvatus, 62, 69, 112, 123, $125,127,129,131-133,135-139,175$, 184-186, 188-189, 191-206, 208-209, 211, 214, 217

Potamogalidae, 6

Potgietersrust, 208

Powerhouse Cave, 208

Power's Site, 137

Praia de Morrugusu, 70

Pramberg, 208

Praomys, 48, 86, 128, 153, 155

Preacomys griffini, 20, 37

Preacomys karsticus, 21, 37

Preacomys kikiae, 21, 37

Prepoecilogale bolti, 109

Prepomonomys bogenfelsi, 9, 11

Pretoria, 77, 137

Prieska, 137

Primates, 8, 17, 42, 76, 148, 221

Proamblysomys antiquus, 72, 125, 134, 140
Proboscidea, 15, 41, 75, 148, 221

Procavia, 74, 134, 136, 138, 142, 147, 183, 192, 200, 205-206, 208

Procavia antiqua, 41, 68-70, 74, 127, 131, 135, 140, 143

Procavia capensis, 74, 123-129, 131-144, 147, 183-218

Procavia pliocenica, 41, 69

Procavia transvaalensis, 41, 68, 70, 75, 127, 131, 134, 137, 140-141, 143

Procaviidae, 8, 15, 41, 74, 147

Procavis capensis, 214

Procercocebus antiquus, 43, 70

Prochrysochlorinae, 12

Prochrysochloris miocaenicus, 12, 36-37

Prodeinotherium hobleyi, 15, 36, 38, 41

Progomphotherium maraisi, 16, 36

Proheliophobius, 23, 36

Prohyrax, 41, 74

Prohyrax hendeyi, 15, 36

Prohyrax tertiarius, 15, 36-37

Promicrogale namibiensis, 12, 36

Pronolagus, 52, 69, 94, 123, 140-143, 162, 201, 207, 215

Pronolagus crassicaudatus, 94, 139

Pronolagus intermedius, 162, 193

Pronolagus randensis, 94, 123, 126, 162, 183-184, 196, 201, 205, 207, 215-216

Pronolagus rupestris, 94, 131, 139, 162, 188-189, 194-195, 202-205, 207, 210-211, 216

Pronotochoerus, 62, 112

Proodontomys cookei, 46, 69-70, 83, 125, 128, 130-131, 134, 140-141

Propalaeorycinae, 32

Propalaeoryx austroafricanus, 32, 36

Propalaeoryx stromeri, 32, 36-37

Propedetes, 22, 37, 68

Propedetes efeldensis, 22, 36-38, 50, 70

Propedetes laetoliensis, 22, 37-38, 50, 68, 70

Propoecilogale, 109

Propottininae, 6

Protarsomys, 19, 36

Protarsomys lavocati, 19, 36

Protarsomys macinnesi, 19, 36-37

Proteles cristata, 105, 125, 127, 170, 187, 190, 194-196, 202, 205, 207-208, 211

Proteles transvaalensis, 105, 141

Protelinae, 105, 170

Protenrec butleri, 12, 36-37 
Protenrecinae, 12

Protophiomyinae, 8

Protophiomys algeriensis, 5, 8, 11

Protypotheroides beetzi, 13, 36-37

Pseudaelurus, 28

Pseudocyon, 30

Pterodon, 10-11, 27

Putslaagte 8, 137, 208

Qugana, 208

QwaQwa Museum Site, 208

Rabaticeras, 119

Radiepolong, 209

Randjies, 209

Raphicerus, 65, 68, 117, 123-124, 127-128, 131-137, 143, 179, 186, 188-194, 205-206, 208, 212-213

Raphicerus campestris, 117, 124-126, 128-132, 135-137, 139-143, 179, 184-185, 187-218

Raphicerus melanotis, 117, 124-125, 127-129, 132-133, 136, 139, 141, 179, 184, 187-191, 193, 196-197, 201-202, 206-209, 211, 213, 215

Raphicerus paralus, 65, 69

Raphicerus sharpei, 118, 137, 179, 187, 196, 207, 211, 214

Ratho Kroonkop, 209

Rattus, 194

Rattus norvegicus, 145, 156, 188, 221

Rattus rattus, 145, 156, 187, 195-197, 201, 204-205, 207-210, 214, 221

Rautenbach's Cave, 209

Ravenscraig, 137, 209

Reception Shelter, 209

Redcliff, 137

Redunca, 67-68, 122, 126, 128, 133, 135, 137, 143, 182, 205, 215, 217

Redunca arundinum, 122, 124-133, 135-144, 182, 184, 186-188, 190-196, 198-199, 201-202, 204-212, 214-217

Redunca darti, 68-69, 122, 131, 140-141

Redunca fulvorufula, 122, 124-127, 129, 131-133, 136-139, 142, 183-188, 190, 192-212, 214-217

Reithroparamyinae, 8

Renbaan, 209

Reunion Rocks, 138

Rhabdomys, 48, 69-70, 88, 123, 136, 138, 141, 157, 192, 203

Rhabdomys dilectus, 88, 157
Rhabdomys pumilio, 48, 69, 88, 123-125, 127-134, 136, 139-143, 157, 184-193, 196-199, 202-203, 205-206, 208-210, 213, $215,217-218$

Rhenosterkloof, 209

Rhinchocyoninae, 14

Rhinoceros, 61, 110, 175

Rhinocerotidae, 31, 61, 110, 175

Rhinocerotinae, 31, 61, 110, 175

Rhinolophidae, 26, 53, 96, 164

Rhinolophus, 26, 36-37, 96-97, 124, 130, 132, 136, 138

Rhinolophus blasii, 96, 141

Rhinolophus capensis, 53, 69-70, 97, 124-126, 134, 140-141, 143-144, 164, 184-185, 189, 192, 217-218

Rhinolophus clivosus, 53, 69, 97, 124-126, 131-132, 136, 139-140, 164, 184-187, 189, 192, 202, 206, 209, 217

Rhinolophus contrarius, 26, 36

Rhinolophus darlingi, 54, 69-70, 97, 125, 140, 164, 186

Rhinolophus fumigatus, 165, 218

Rhinolophus hildebrandtii, 97, 125

Rhinolophus simulator, 165, 206

Rhizomyinae, 18

Rhynchogale, 104, 135

Rhynchogale melleri, 170, 196

Riet River, 138

Rietfontein, 138

River-bed above the Island, 138

River-bed below the Island, 138

Riversmead, 209

Riverton, 138

Rocky II and III, 138

Rodentia, 8, 17, 44, 80, 150, 221

Rooiberg, 210

Rooiels, 210

Rooikrans Hilltop, 210

Rooikrans Shelter, 210

Rooilepel, 38, 70

Rooiwal Hollow and Midden, 210

Roosfontein, 210

Rose Cottage Cave, 138, 210

Ruanga Ruin, 210

Rupestrohyrax palustris, 8, 11

Ryskop, 33, 38

SAAN-0005, 138

SAAN-0034, 0035, 138

SAAN-0036, 138 
SAAN-004, 138

SAAN-0042, 0043, 138, 142

Saccolaimus, 27

Saccostomus, 19, 36, 81, 123-125, 131, 138, 151, 184, 190, 192, 199, 205, 218

Saccostomus campestris, 81, 123, 125-126, 131, 136, 140, 143, 151, 184-186, 196, 199, 205, 215-218

Saccostomus geraadsi, 19, 37

Saldanha, 210

Saldanha Bay, 138

Saldanha Bay Yacht Club, 138

Salem Commonage, 210

Sand River, 139

Sandy Bay, 210

Sanitheriidae, 34

Schizotheriinae, 60

Schmidt's Drift, 139

Schoemansdal, 210

Schoolplaats No 1, 139

Schroda, 210

Schurveberg, 40, 42, 50-51, 56, 70, 73, 76, 83, 90, 92, 102

Sciuravida, 8

Sciuridae, 17, 80, 150

Sciuromorpha, 17, 80, 150

Sciurus, 150

Scotophilus, 99

Scotophilus nigrita, 99, 125, 131, 166, 186, 217

Scott's Cave, 211

Sea Harvest, 139

Sea Point Cape Town, 211

Sea Vista, 211

Seal Midden, 211

Seal Point, 211

Sebatini Hill, 211

Seekoei River, 109, 174

Sehonghong, 139, 211

Selongwe Hill, 211

Sentinel Ranch, 211

Seroromeng, 211

Serotwe Hill, 211

Sh 14a, 211

Shankare Hill, 211

Sheppard Island, 139

Sibudu, 139, 211

Sikhanyisweni, 212

Silberberg Grotto, 139

Silica, 11

Silica North, 9
Silicamys cingulatus, 9, 11

Simamwe, 212

Simatherium, 35, 66

Simatherium kohllarseni, 118, 127

Simonstown, 212

Simopithecus, 43, 78

Simunye, 212

Sivaonyx africanus, 60, 69

Sivaonyx hendeyi, 60, 69

Sivatheriinae, 63, 113

Sivatherium, 63, 70

Sivatherium hendeyi, 63, 69

Sivatherium maurisium, 113, 124, 127, 129-130, 142

Sk 17, 212

SK400, 140

Skorpion Cave, 212

Skutwater, 212

Skuurwerug, 70

Smitswinkelbaai, 212

Smutsia, 100, 129, 141, 166, 202

Smutsia gigantea, 54

Smutsia temminckii, 100, 136, 138-139, 167, 187, 193-196, 198, 202-205, 209, 212-213, 215

Smutsiinae, 54, 100, 166

Snuifklip, 212

Somerset Strand, 212

Sonkoanini Hill, 212

Sorex, 53, 95

Soricidae, 52, 94, 162

Soricomorpha, 52, 94, 162, 221

Spalacidae, 18-19, 220

Sperrgale minutus, 7, 11

Sperrgebiet, 9, 22, 24-25, 34

Sperrgebietomerycinae, 32

Sperrgebietomeryx wardi, 32, 36

Spitskop B, 140

Spitzkloof A Rockshelter, 140

Spitzkop Cave, 212

Spoeg River, 212

Spreeuwal, 140

Spring Cave, 213

Springbok Flats, 140

Springbok Midden, 140

St Francis Bay 2, 213

St Lucia, 213

Stayt, 213

Steatomys, 20, 36, 69, 82, 123-125, 128, 131-132, 134, 136, 138, 141, 152, 184, 190-192 
Steatomys harasibensis, 20, 37

Steatomys jaegeri, 20, 37

Steatomys krebsii, 83, 123-124, 129, 136, 139, 143, 152, 184-186, 189, 191, 197, 202, 209-210, 213, 217

Steatomys opimus, 45, 83, 152

Steatomys parvus, 152

Steatomys pratensis, 45, 69, 83, 125-126, 128, 130, 137, 140-141, 152, 186, 196, 202, 205, 216

Steenbokfontein, 213

Steenbras Bay, 213

Stegodon, 42, 76

Steinaecker's Horse, 213

Stenodontomys, 81, 123-124, 131, 136

Stenodontomys darti, 44, 69

Stenodontomys saldanhae, 44, 69

Stenoplesictidae, 29

Stenoplesictinae, 29

Sterkfontein, 43, 55, 57, 71-72, 78-79, 86, 101, $104,106,112,140$

Stofbergsfontein, 213

Strathalan B, 141

Strepsiceros, 66, 119, 180

Strepsirrhini, 8, 148

Striped Giraffe Shelter, 213

Stylochoerus, 111

Stylohipparion, 31, 60, 110

Suidae, 33, 61, 111, 175

Suinae, 62, 111, 175

Suncus, 53, 95, 128, 130, 163

Suncus infinitesimus, 53, 69, 95, 125, 131, 133, $136,163,186,205-206$

Suncus lixus, 96, 128, 164, 186, 196, 201, 217

Suncus varilla, 53, 69-70, 96, 124-125, 127, 129, 131, 134, 136, 139-143, 164, 185-186, 189-191, 197, 205, 209-210, 213, 216-218

Sunnyside Pan, 141

Suricata, 127, 132

Suricata major, 104, 129

Suricata suricatta, 104, 123-124, 129, 132, 134, 137, 140-141, 170, 184, 190, 194-195, 198, 206, 209-211, 213, 216

Sus, 175, 187

Sus scrofa, 175, 200, 207-210, 215, 221

Swart Duinen, 141

Swartklip, 116, 141, 178

Swartkrans, 53, 77, 96, 105, 120, 141

Swartlintjies 1, 38, 142

Sydney-on-Vaal, 42, 76, 142

Sylvicapra, 67-68, 120, 136
Sylvicapra grimmia, 120, 126-133, 136-140, 142-143, 181, 183-185, 187-217

Syncerus, 66, 69, 118, 126-127, 131, 135-136, 140-141, 143, 179, 193, 208

Syncerus aceolotus, 66, 70

Syncerus antiquus, 118, 123-124, 126-141, 143-144, 186

Syncerus caffer, 118, 124-127, 129, 132-139, 142-143, 179, 183, 185-217

Tadarida, 27, 36, 98, 125

Tadarida aegyptiaca, 98, 143, 165, 217

Talahphiomys, 11

Talahphiomys lavocati, 9

Tandjesberg, 213

Taphozous, 98, 134

Taphozous incognita, 27, 36

Tapinochoerus, 62, 111

Tatera, 47, 84, 154

Taung, 1, 40, 42, 44, 49-53, 59, 68, 70, 74, 77, $79,88-89,95$

Taurotragus, 127, 179, 190, 202, 208

Taurotragus oryx, 118, 123-143, 180, 184, 186-188, 190-217

Tautswemogala, 213

Tavhatshene, 214

Te Vrede, 214

Tenrecidae, 7, 12, 220

Tenrecomorpha, 6, 12

Tetraconodontinae, 33, 62, 112

Tetralophodon, 15

Thallomys, 49, 69, 88, 123, 138, 141, 157, 192

Thallomys debruyni, 49, 70, 88, 125, 131

Thallomys paedulcus, 89, 125-126, 133-134, 140, 157, 186, 192, 217-218

Thamanga Rock Shelter 1, 214

Thamnomys, 47, 86, 155

The Bend, 142

The Havens Cave, 214

Therailurus, 55, 101

Theropithecus darti, 43, 69

Theropithecus oswaldi, 43, 68, 78, 125, 127, 129, 131, 134, 140, 142

Thomeng, 142

Thos, 106

Thryonomyidae, 10, 24, 93, 161

Thryonomys, 93, 138, 142, 161, 216

Thryonomys gregorianus, 161, 204

Thryonomys swinderianus, 93, 139, 161, 187, 189, 194-196, 198-199, 201-205, 207-208, 211-215, 218 
Thulamela, 214

Tiara Shelter, 214

Tierfontein, 142

Tierfontein 2, 4A \& 7, 214

Tim's Cave, 142

Tiras, 214

Titanohyracidae, 15

Tloutle, 142, 214

Tobias Cave, 142

Todralestidae, 6

Tortoise Cave, 214

Toteng, 215

TP2004-04, 215

Tragelaphus, 66, 69-70, 119, 126, 128, 132-133, 139, 142

Tragelaphus angasii, 66, 70, 119, 125, 130-131, $135,140,142,180,191,200,205-206$, 214-215

Tragelaphus pricei, 66, 69

Tragelaphus scriptus, 119, 126-127, 133-140, 142-143, 180, 184, 187-190, 192-193, 195-196, 200-205, 207-208, 211-212, 214-218

Tragelaphus spekeii, 119, 129, 180, 187, 215, 218

Tragelaphus strepsiceros, 66, 68, 119, 125-129, 131-133, 135-142, 144, 180, 184, 186-196, 198-202, 204-205, 207, 209-212, 214-215, 217

Tragoportax, 65, 118

Tragulidae, 34, 220

Tree Pan, 70

Tsauchab, 70

Tsaukhaebmys calcareus, 9, 11

Tsh 1, 215

Tshirululuni, 215

Tshisiku Shelter, 215

Tshitheme, 215

Tshobwane, 215

Tshwane, 215

Tubulidentata, 14, 74, 147, 221

Tufamyidae, 10

Tufamys woodi, 10-11

Tuinplaas, 142

Turkanatherium acutirostrum, 32, 36

Twyfelpoort Shelter, 215

Ugandax demissum, 35, 37-38, 66, 69

Uitkomst, 142

Uitzoek, 127

uMgungundlovu, 215

Umhlatuzana, 142, 215
Umhloti, 216

UmKlaarmaak, 216

Umuab, 216

Uri Hauchub 4, 216

Ursidae, 59, 220

Usakos, 109, 142

Vaalhoek, 216

Van Zyl's Rus Shelter, 216

Vanwyksfontein, 142

Varsche Rivier 003, 142

Velddrif, 216

Ventershoek, 216

Vespertilio nigrita, 99, 166

Vespertilionidae, 27, 54, 98, 165

Vespertilioninae, 54, 98, 165

Village 16, 216

Virginia 751, 142

Viverra, 171, 220

Viverra capensis, 108, 173

Viverra leakeyi, 58, 69

Viverra tigrina, 106, 171

Viverridae, 29, 58, 105, 171

Viverrinae, 58, 105, 171

Vlaeberg, 216

Vlakkraal Thermal Springs, 142

Vlakplaats, 143

Vlermuisgat Cave, 216

Voëlvlei, 216

Volstruisfontein, 216

VR001, 216

VR005, 216

Vredenburg, 216

Vulcanisciurus, 17, 36-37

Vulcanisciurus africanus, 18, 36-37

Vulpes, 59, 70, 124

Vulpes chama, 59, 69, 107, 124, 128-129, 132-138, 140-143, 173, 184-185, 188-196, 198-200, 205, 207-211, 213, 216, 218

Vulpes pattisoni, 59, 70

Vulpes pulcher, 108, 134, 142

Vulpes skinneri, 108, 135

Vungu Vungu, 216

Vygeboom, 216

Waldeck's Plant, 143

Warrenton, 143

Waterbakke, 216

Watervalrivier, 216

Waypoint 160, 49, 52, 70 
Welgegund, 217

Welgeluk Shelter, 217

Wellsiana torticornuta, 67, 69

White Paintings Rock Shelter, 143, 217

Widcome, 217

Wildebeestfontein, 217

Willowbank, 143

Wilton Cave, 217

Wilton Large Rock Shelter, 217

Windsorton, 62, 111, 143

Winter's Rush, 143

Witklip, 217

Witkrans Cave, 143, 217

Witputs North, 217

Wonderkrater, 143

Wonderwerk, 143, 217

Wosi, 217

X Cave, 143

Xaro, 218

Xenohystrix crassidens, 52, 69

Xerinae, 17, 80, 150

Xerus, 150, 187, 194, 208
Xerus inauris, 80, 130, 150, 184-185, 187, 209-211, 213-214, 216

Xerus princeps, 150, 207

Yatala 73, 143

Ysengrinia, 30, 36-37

Ysengrinia ginsburgi, 30, 36

Ysterfontein, 143, 218

Ysterplaat, 109, 144, 174

Zaayfontein, 218

Zais, 144, 218

Zebra Hill, 22, 38

Zebrarivier, 144, 218

Zegdoumyidae, 9

Zegdoumys, 9

Zegdoumys namibiensis, 11

Zelotomys, 49, 69, 89, 123-124, 132, 134, 136, 138

Zelotomys woosnami, 89, 130, 132, 137, 140, 143, $157,185,217-218$

Zoovoorbij, 218

Zwartklip, 144

Zygolophodon aegyptensis, 16 
